Rolle der biologischen $\mathrm{N}_{2}$-Fixierung von Baumleguminosen im östlichen Amazonasgebiet, Brasilien

- Anwendung der ${ }^{15} \mathrm{~N}$ natural abundance Methode -

\author{
Dissertation \\ zur Erlangung des Doktorgrades \\ des Fachbereichs Agrarwissenschaften \\ (Landwirtschaftliche Fakultät) \\ der Georg-August-Universität Göttingen
}

vorgelegt von

\title{
Antje Thielen-Klinge
}

geboren in Gütersloh

Göttingen, im März 1997 
D7

1. Referent: Prof. Dr. P.L.G. Vlek

2. Korreferent: Prof. Dr. G. Wolf

Tag der mündlichen Prüfung: 29. 05. 1997 
Meinen Eltern 


\section{Inhaltsverzeichnis}

\section{Literaturüberblick} .4

Der Stickstoffkreislauf 4

Stickstoff in den Tropen. 8

Die Leguminosen. .11

Die ${ }^{15} \mathrm{~N}$ natural abundance - Methode $\left({ }^{15} \mathrm{NNAM}\right)$ .16

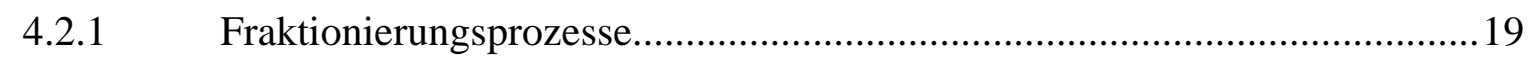

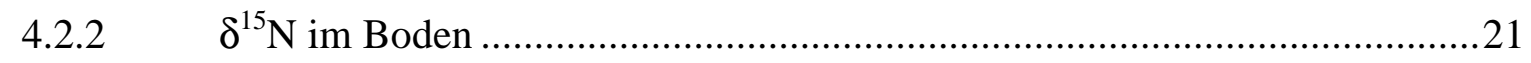

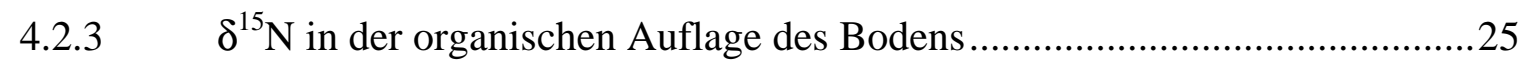

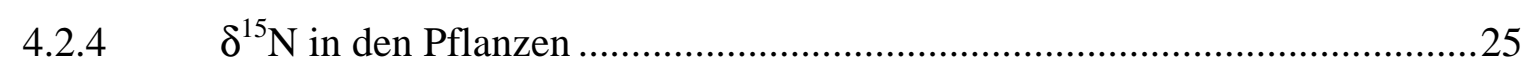

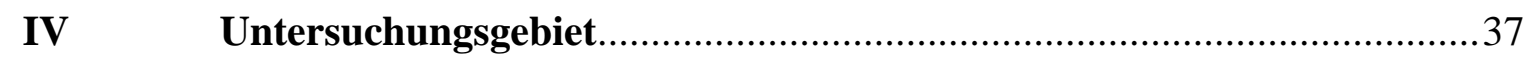

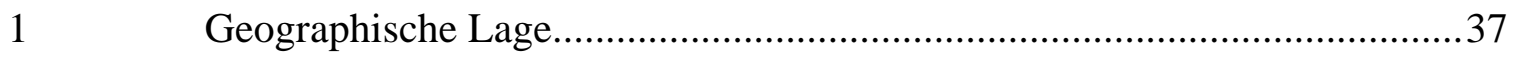

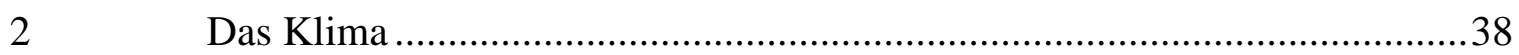

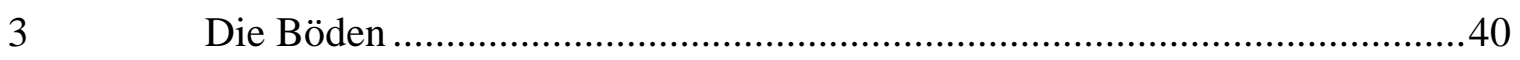

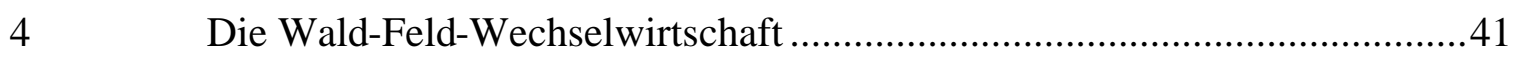

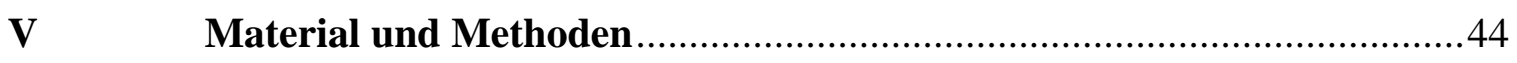

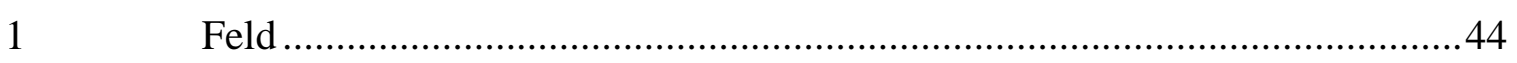

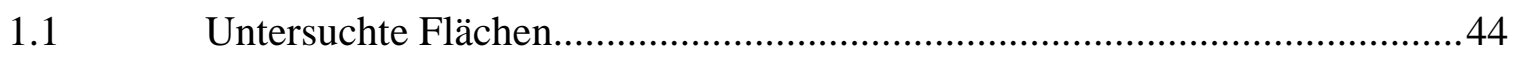

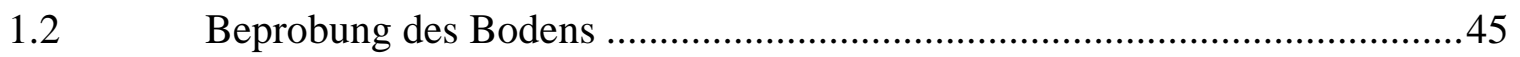

1.3 Physikalische und chemische Bodenanalysen der Probeflächen ...................46

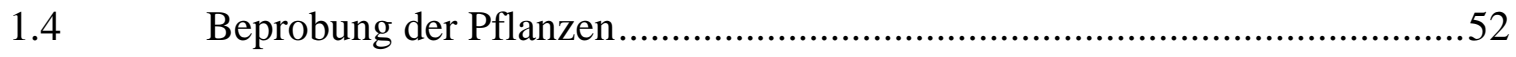

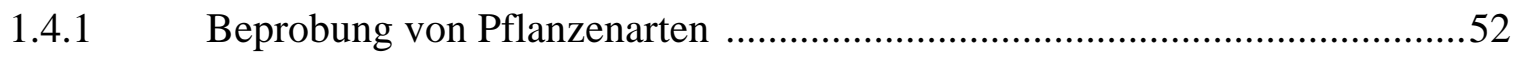

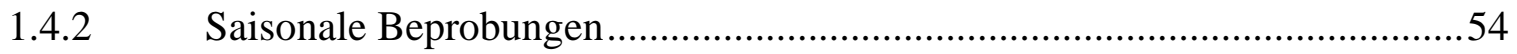

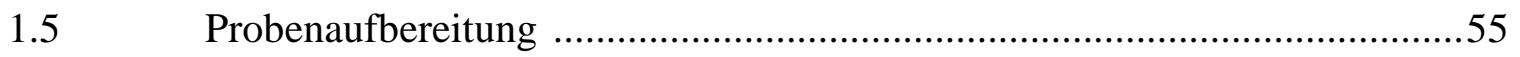




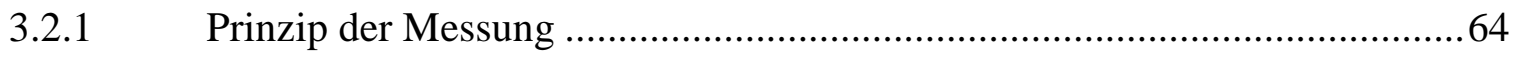

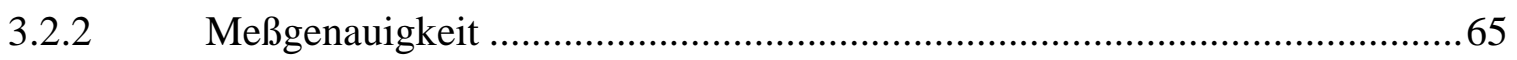

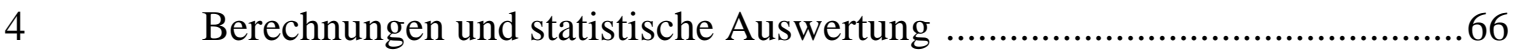

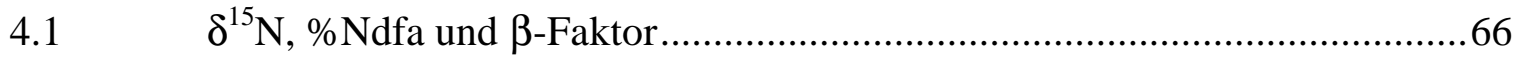

4.2 Berechnung der Stickstoffakkumulation über die BNF der Leguminosen ...67

$4.3 \quad$ Statisitik.

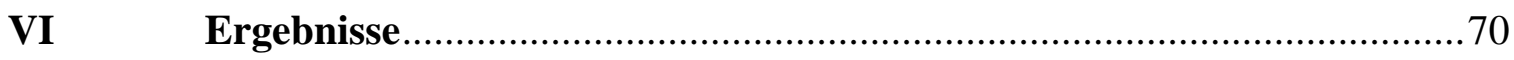

$1 \quad \delta^{15} \mathrm{~N}$ in der Sekundärvegetation der Bragantina-Region .............................. 70

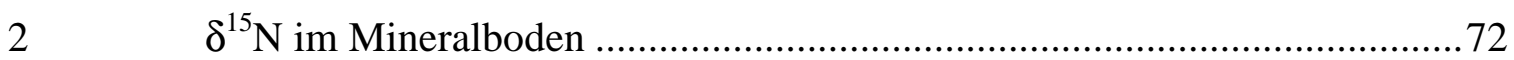

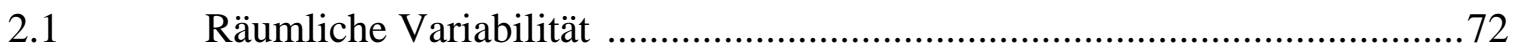

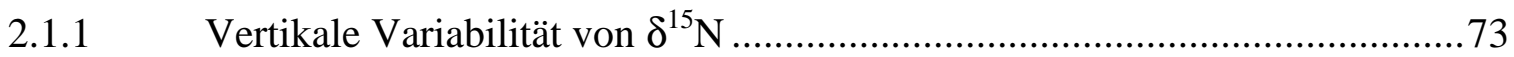

2.1.2 Zusammenhang des $\delta^{15} \mathrm{~N}$ mit bodenchemischen und -physikalischen

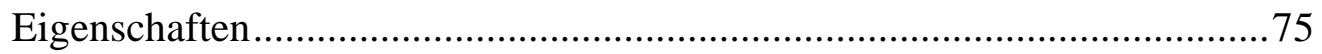

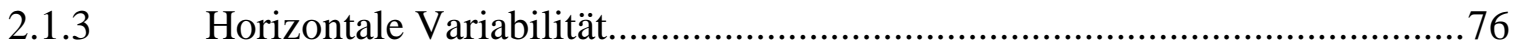

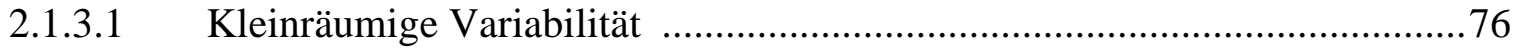

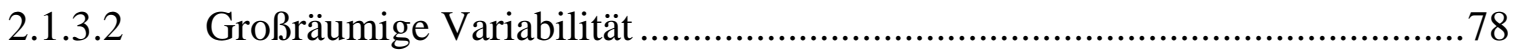

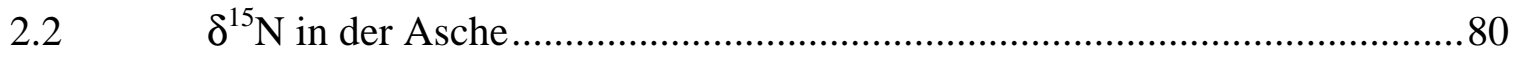

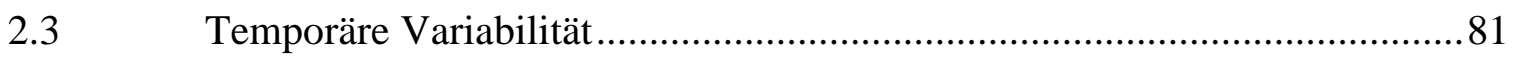

$3 \quad \delta^{15} \mathrm{~N}$ in der organischen Auflage der Sekundär - und Primärvegetation.........81

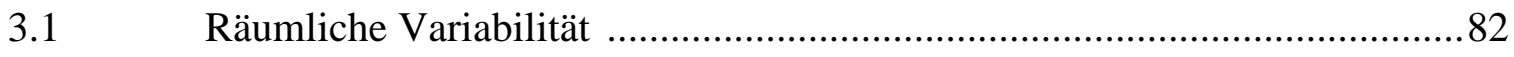

3.2 Temporäre Variabilität im $\delta^{15} \mathrm{~N}$ der organischen Auflage............................84

3.3 Beziehung von $\delta^{15} \mathrm{~N}$ der organischen Auflage zum $\delta^{15} \mathrm{~N}$ des Mineralbodens

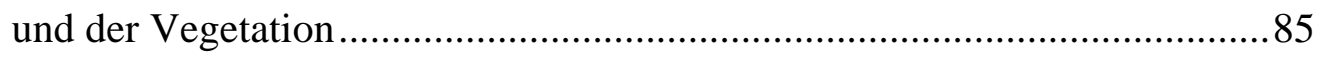

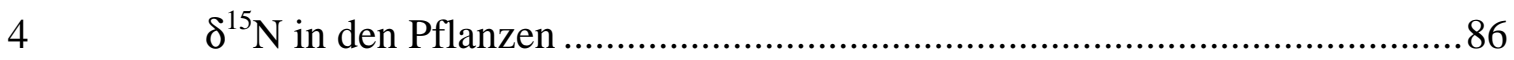

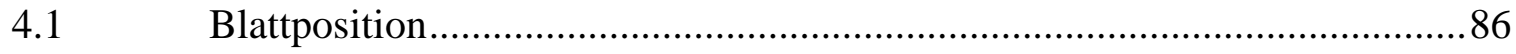

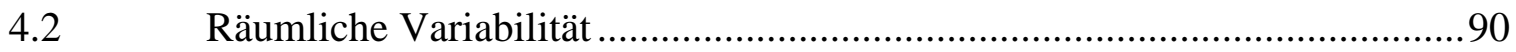




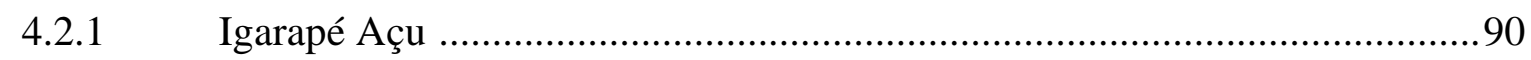

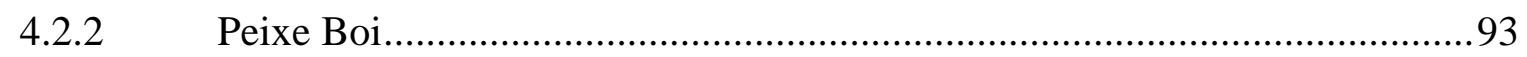

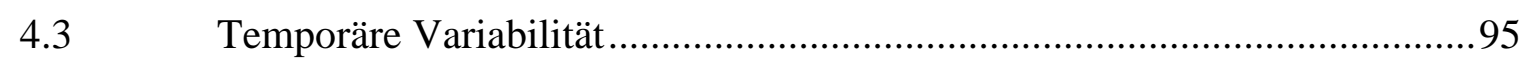

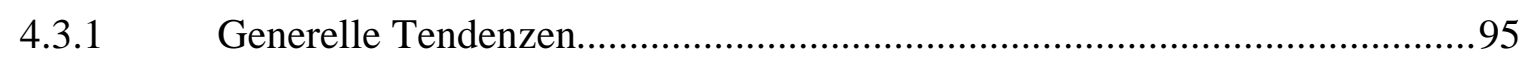

4.3.2 Unterschiede im zeitlichen $\delta^{15} \mathrm{~N}$-Muster der Arten ......................................

4.3.3 Pflanzengruppierung anhand zeitlicher Strukturen ................................... 100

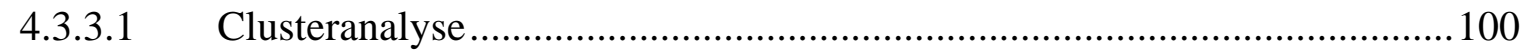

4.3.3.2 Differenzen zwischen Referenzpflanzen und Leguminosen ........................ 101

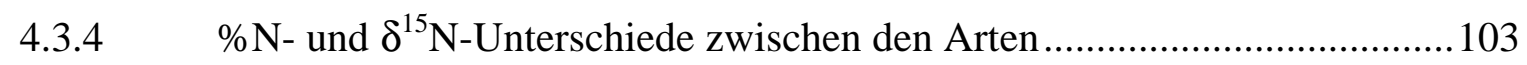

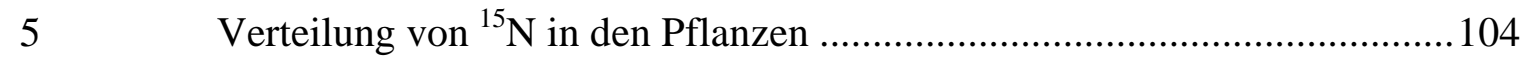

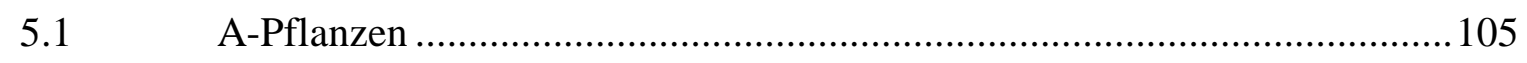

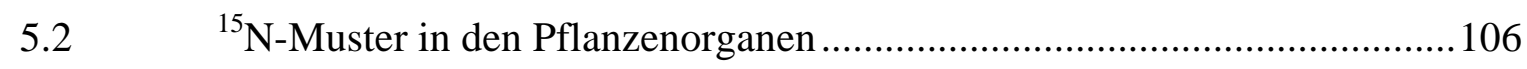

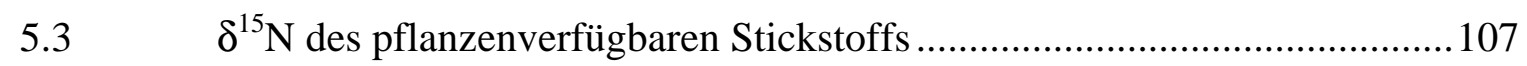

$6 \quad \%$ Ndfa der Leguminosen und die Präzision der Berechnung ....................... 108

$7 \quad$ Rolle der biologischen $\mathrm{N}_{2}$-Fixierung in der Bragantina-Region...................113

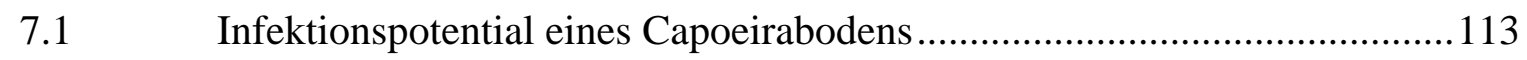

7.2 BNF in den verschiedenen Sukzessionsstadien der Entwicklung eines

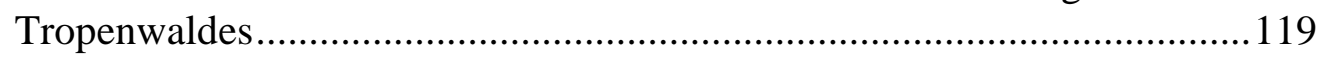

7.2.1 Beziehung von Baumgröße und Fixierungsleistung ..................................119

7.2.2 N-Akkumulation über BNF in verschieden alten Sekundärvegetationen und Primärwäldern ............................................................................... 122

7.2.3 Vergleich der $\delta^{15} \mathrm{~N}$-Werte von Primärwald und Sekundärwald ..................... 124

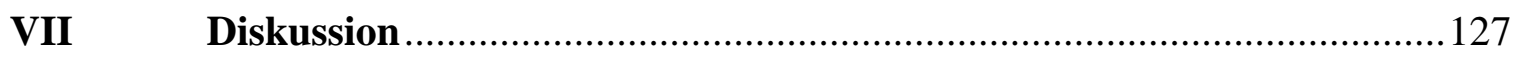

1 Die biologische $\mathrm{N}_{2}$-Fixierung in der Bragantina-Region ........................... 127

$2 \quad \delta^{15} \mathrm{~N}$ in den Böden der Bragantina-Region................................................ 133

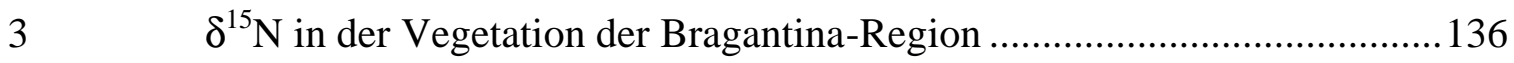

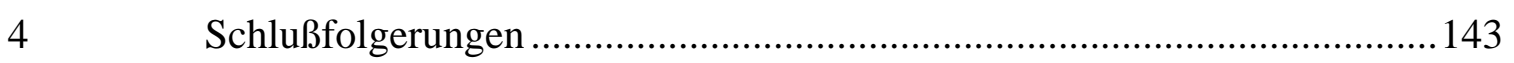

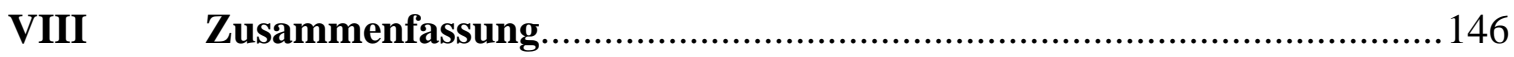

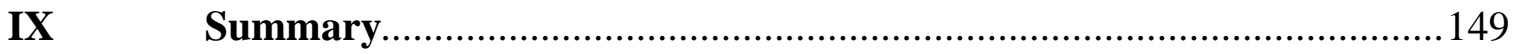

$\mathrm{X} \quad$ Resumo

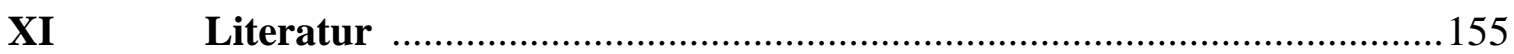

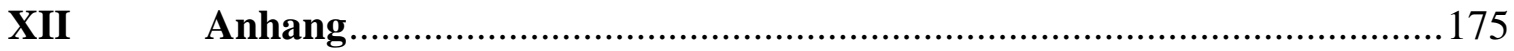



Abkürzungen
$\%$ Ndfa
$\%$ Nitrogen derived from atmosphere
${ }^{15}$ NNAM
${ }^{15} \mathrm{~N}$ natural abundance Methode
BMBF
Bundesministerium für Bildung, Wissenschaft, Forschung und Technologie
BNF Biologische $\mathrm{N}_{2}$-Fixierung
$\mathrm{CNPq}$ Conselho Nacional de Desenvolvimento Científico e Tecnológico
CPATU Centro de Pesquisa Agroflorestal da Amazonia Oriental
FG Freiheitsgrad
EMBRAPA Empresa Brasileira de Pesquisa Agropecuária
FCAP Faculdade das Ciências Agrárias do Pará
IBAMA Instituto Brasileiro do Meio Ambiente e dos Recursos Renováveis
LSD least significant difference
MW Mittelwert
MQS mittlere Quadratsumme
$\mathrm{n} \quad$ Anzahl Wiederholungen
NL Nährlösung
$\mathrm{N}_{\text {min. }} \quad$ mineralisierter Stickstoff
$\mathrm{N}_{\text {tot. }} \quad$ Gesamtstickstoff
$s \quad$ Standardabweichung
$\sigma \quad$ Standardfehler
SHIFT Studies on Human Impact on Forests and Floodplains in the Tropics 


\section{Einleitung}

Die Weltbevölkerung wächst jährlich um 95 Mio. Menschen und wird im Jahr 2025 voraussichtlich 8.5 Milliarden erreichen (Enquete-Kommission „Schutz der Erdatmosphäre“ des Deutschen Bundestages, 1994). Obwohl das Weltnahrungsmittelangebot rascher zugenommen hat als die Bevölkerung, leiden etwa 20\% der Menschen - hauptsächlich in den Entwicklungsländern - an Unterernährung. Werden zur Zeit etwa $2700 \mathrm{~m}^{2}$ Fläche zur Ernährung einer Person bebaut, kann im Jahr 2010 nur noch mit $1600 \mathrm{~m}^{2}$ pro Kopf gerechnet werden (Alexandratos, 1995).

Die Bereitstellung landwirtschaftlicher Flächen zur Sicherung der Ernährung ist zu 90\% Ursache für die Brandrodung tropischer Regenwälder (Amelung und Diehl, 1992, zitiert in: Enquete-Kommission „Schutz der Erdatmosphäre“ des Deutschen Bundestages, 1994). Allein in Latein-Amerika nimmt die Ackerfläche (ohne Weide) jährlich um 2.3\% zu (Myers, 1991).

Das traditionelle landwirtschaftliche Nutzungssystem im Gebiet dieser Untersuchung - der Bragantina-Region im Nordosten des brasilianischen Bundesstaates Pará - ist eine Feldumlagewirtschaft mit Brandrodung. Durch die circa alle 3-9 Jahre wiederkehrende Brandrodung der Flächen, kommt es zu erheblichen Nährstoffverlusten, verursacht durch den Stofftransfer in die Luft. Hierbei wird Stickstoff bis zu 96\%, Kohlenstoff sogar zu 98\%, Phosphor zu 47\% und andere Elemente zu 30-50\% in die Luft transferiert (Hölscher, 1995). Nach Hölschers Bilanz der Stoffvorräte kommt es zusätzlich noch zu Auswaschungsverlusten im Boden nach der Brandrodung, da freigesetzte Nährstoffe nicht durch die Wurzeln einer Vegetation aufgenommen werden können.

Der zwischen den Kulturphasen aufwachsenden Buschvegetation kommt die Funktion der Nährstoffakkumulation und Unkrauteindämmung zu. Ursprünglich ein Wanderfeldbau, bei dem zwischen 2 Bewirtschaftungsphasen Jahrzehnte liegen konnten, führten hoher Bevölkerungsdruck und die Nahrungsmittelversorgung der regionalen Bevölkerung im Nordosten Parás jedoch zu einem Bewirtschaftungszyklus von 2 Jahren Anbau und einer mehr und mehr verkürzten Bracheperiode von derzeit 3-7 Jahren mit Sekundärvegetation. Immer häufiger werden Marktfrüchte wie Maracuja, Pfeffer und Ölpalme angebaut, für die eine intensive Bodenbearbeitung nötig ist. Die Produktivität der Böden degradiert und neue Waldstücke müssen zur Landbearbeitung gerodet werden. Auf diese Art ist eine nachhaltige 
Nutzung der Böden nicht mehr gewährleistet und weitere Regenwaldflächen müssen angegriffen werden.

Stickstoff ist in diesen Regionen der neben Phosphor hauptsächlich wachstumslimitierende Faktor (Gehring, 1997). Die Atmosphäre besteht zu 78\% aus $\mathrm{N}_{2}$ und birgt damit $18 \%$ des gesamten Stickstoffvorkommens der Erde. Einen Beitrag zur Intensivierung des Ackerbaus können daher Leguminosen leisten. Leguminosen spielen in fast jedem landwirtschaftlichen und agroforstlichen System über ihre Wurzelsymbiose mit im Boden lebenden $\mathrm{N}_{2}$-fixierenden Bakterien der Gattung Rhizobium spp. eine große Rolle. Über diese biologische $\mathrm{N}_{2}$-Fixierung (BNF) können durchschnittlich in Abhängigkeit von Stickstoff- und Phosphor-Angebot oder anderen Faktoren etwa100-300 kg N zusätzlich aus der Atmoshäre in ein System eingetragen werden (Sanginga, 1995; Giller und Wilson, 1991).

Die Leguminosen in der Brachevegetation des Projektgebietes sind bisher wenig untersucht. In Abhängigkeit von der Nutzungsgeschichte nehmen sie mal nur 0.6\% der Biomasse einer Sekundärvegetation ein (nach mechanisierten Pfefferplantagen), in Sekundärvegetation nach traditionellem Anbau aber bis zu 25\% der Biomasse (Denich, 1989; Nunez, 1995). Bei einer Abundanz von maximal 12.9\% (Baar, 1997) ist diese Biomasseproduktion beachtenswert und läßt einen Zusammenhang mit der symbiontischen $\mathrm{N}_{2}$-Fixierung vermuten. Welchen Stellenwert die BNF im Stickstoffkreislauf der Sekundärvegetation einnimmt, ist jedoch unklar.

Informationen über den Stickstoffkreislauf in natürlichen Systemen können mit Hilfe der ${ }^{15} \mathrm{~N}$ natural abundance Methode $\left({ }^{15} \mathrm{NNAM}\right)$ gewonnen werden. Grundlage der Methode sind die unterschiedlichen Konzentrationen des schwereren Stickstoffisotopen ${ }^{15} \mathrm{~N}$ in der Luft, dem Boden und anderen N-Quellen, die in den Pflanzen ihrer jeweiligen N-Quelle entsprechend wiederzufinden sind. Über den Vergleich der ${ }^{15} \mathrm{~N}-$ Konzentrationen in nicht $\mathrm{N}_{2}$ fixierenden Referenzpflanzen mit denen in zu untersuchenden Leguminosen kann auf den Prozentsatz fixierten Stickstoffs im Gesamt-N der Leguminose geschlossen werden. Die ${ }^{15}$ NNAM wurde für Gewächshausbedingungen oder aber Feldexperimente auf kultivierten, homogenen Böden entwickelt (Shearer und Kohl, 1986). Sie ist nicht destruktiv, bedarf keiner besonderen Behandlungen der Versuchspflanzen, ermöglicht spontane Feldbeprobungen und kann sowohl qualitative als auch quantitative Informationen über die bestehende $\mathrm{N}_{2}$-Fixierung in einer Pflanze liefern. Die ${ }^{15} \mathrm{~N}$-Konzentrationsunterschiede bewegen sich in sehr kleinen Dimensionen. Daher wurde der $\delta^{15} \mathrm{~N}$-Wert geschaffen, der die ${ }^{15} \mathrm{~N}-$ Konzentration einer Probe 
mit dem $\delta^{15} \mathrm{~N}_{\text {Luft }}$ standardisiert. Positive $\delta^{15} \mathrm{~N}$-Werte signalisieren höhere und negative $\delta^{15} \mathrm{~N}-$ Werte niedrigere ${ }^{15} \mathrm{~N}-$ Konzentrationen als in der Luft.

Grundlegende Prozesse wie Nitrifikation, Denitrifikation und Immobilisierung von mineralischem Stickstoff haben Auswirkungen auf die Verteilung von ${ }^{15} \mathrm{~N}$ im System und lassen Schlüsse auf die Rolle und das Zusammenspiel dieser Prozesse zu.

Sogenannte Fraktionierungsprozesse während der Bodenevolution und des Stickstoffkreislaufes in Boden und Vegetation, eine ungleiche Beteiligung der beiden $\mathrm{N}$-Isotopen ${ }^{15} \mathrm{~N}$ und ${ }^{14} \mathrm{~N}$ an den Reaktionen im Stoffwechsel und anderen Umsetzungen, führten zu teilweise noch ungeklärten Isotopenverhältnissen (Handley und Raven 1992). Diese Prozesse nehmen in den unterschiedlichsten Regionen und Ökosystemen eine unterschiedliche Bedeutung ein. Für eine Feldumlagewirtschaft mit Brandrodung existieren bislang jedoch keine Erfahrungen dazu, was zum Beispiel die Auswahl von adäquaten Referenzpflanzen erschwert.

Aufgabe dieser Arbeit sollte die Abschätzung der Rolle der biologischen $\mathrm{N}_{2}$-Fixierung von Baumleguminosen der Brachevegetation sein. Hauptaugenmerk mußte jedoch zunächst auf die Überprüfung der Eignung der ${ }^{15} \mathrm{~N}$ natural abundance Methode für diese Aufgabe gelegt werden. Dazu sollte die Verteilung des ${ }^{15} \mathrm{~N}$ im slash and burn System der Bragantina-Region (Boden und Vegetation), die Aufnahme dieses Isotopes von einigen Pflanzenarten und mögliche Fraktionierungen während der Stickstoffumsetzung in Leguminosen und potentiellen Referenzpflanzen untersucht werden.

Die Arbeit wurde im Rahmen des Projektes „Sekundärwald und Brachevegetation in der Kulturlandschaft des östlichen Amazonasgebietes - Funktion und Manipulierbarkeit -,, durchgeführt. Das Projekt ist Bestandteil des Forschungsprogrammes „Studies on Human Impact on Forests and Floodplains in the Tropics (SHIFT)“, das im Rahmen einer vom BMBF und dem brasilianischen Partner CNPq/IBAMA vereinbarten wissenschaftlich-technologischen Zusammenarbeit steht. Beteiligte Institute waren das CPATU/EMBRAPA (Centro de Pesquisa Agroflorestal da Amazônia Oriental), die FCAP (Faculdade das Ciências Agrárias do Pará) und das Institut für Pflanzenbau und Tierproduktion in den Tropen und Subtropen der Universität Göttingen. 


\section{Literaturüberblick}

Durch die in der Untersuchungsregion üblichen Brandrodungen verliert das System Boden Pflanze bis zu 96\% des in der Biomasse konservierten Stickstoffs, der wieder in das System eingebracht werden muß. Die einzige Möglichkeit, auf natürlichem Wege ein großes Stickstoffreservoir - den Luftstickstoff - zu erschließen, ist die biologische $\mathrm{N}_{2}$-Fixierung. Es stellte sich somit die Frage, ob und wieviel Stickstoff in der auf die Kulturphasen folgenden Sekundärvegetation über die BNF der Leguminosen - einer Pflanzenfamilie mit Arten, die zur Symbiose mit $\mathrm{N}_{2}$-fixierenden Bakterien der Gattung Rhizobium befähigt sind - wieder dem System zugeführt wird.

Die ${ }^{15}$ NNAM ermöglicht eine nicht destruktive Untersuchung der BNF und des N-Kreislaufes eines Systems. Voraussetzungen für die Anwendung dieser Methode sind Kenntnisse über die Verteilung der Stickstoffisotope in den einzelnen Kompartimenten - Boden, mineralisches N und Pflanzen - dieses Systems.

Im folgenden wird ein Einblick in den N-Kreislauf, die BNF der Leguminosen und die Verteilung von ${ }^{15} \mathrm{~N}$ und ihre Mechanismen gegeben.

\section{$1 \quad$ Der Stickstoffkreislauf}

Stickstoff ist einer der wichtigsten Makronährstoffe, der zum Aufbau aller lebenden Organismen benötigt wird. Viele Autoren haben sich mit der Abschätzung der Stickstoffmengen in den einzelnen Sphären und Kompartimenten der Erde beschäftigt (Sanhueza, 1982; Clark, 1981; Tamm, 1990). Das Element N ist in allen Sphären vertreten. Der größte Teil liegt in der Lithosphäre fixiert vor $\left(16.4 \times 10^{19}\right)$. Nur $24 \times 10^{13} \mathrm{~kg} \mathrm{~N}$ sind als organische Substanz und in mineralischer Form verfügbar (Stephenson, 1986). Die Atmosphäre besteht zu $78 \%$ aus $\mathrm{N}_{2}$ und enthält mit $3.9 \times 10^{18} \mathrm{~kg} \mathrm{~N} 18 \%$ des gesamten $\mathrm{N}$ Vorkommens der Erde (Sanhueza, 1982)(Abb. 1).

\footnotetext{
Abb. 1: N-Kreislauf und N-Reserven der Erde. Mit roten Zahlen sind die $\delta^{15} \mathrm{~N}-$ Werte einiger N-Verbindungen eingezeichnet (Daten aus Brandes et al., 1996; France, 1995; Hauck, 1973; Heaton, 1987; Hoering, 1955; Kim und Graig, 1993; Minami et al., 1995; Nadelhoffer und Fry 1994; Peterson und Fry, 1987; Sanhueza et al., 1982; Stephenson, 1986; Wada et al., 1975;)
} 


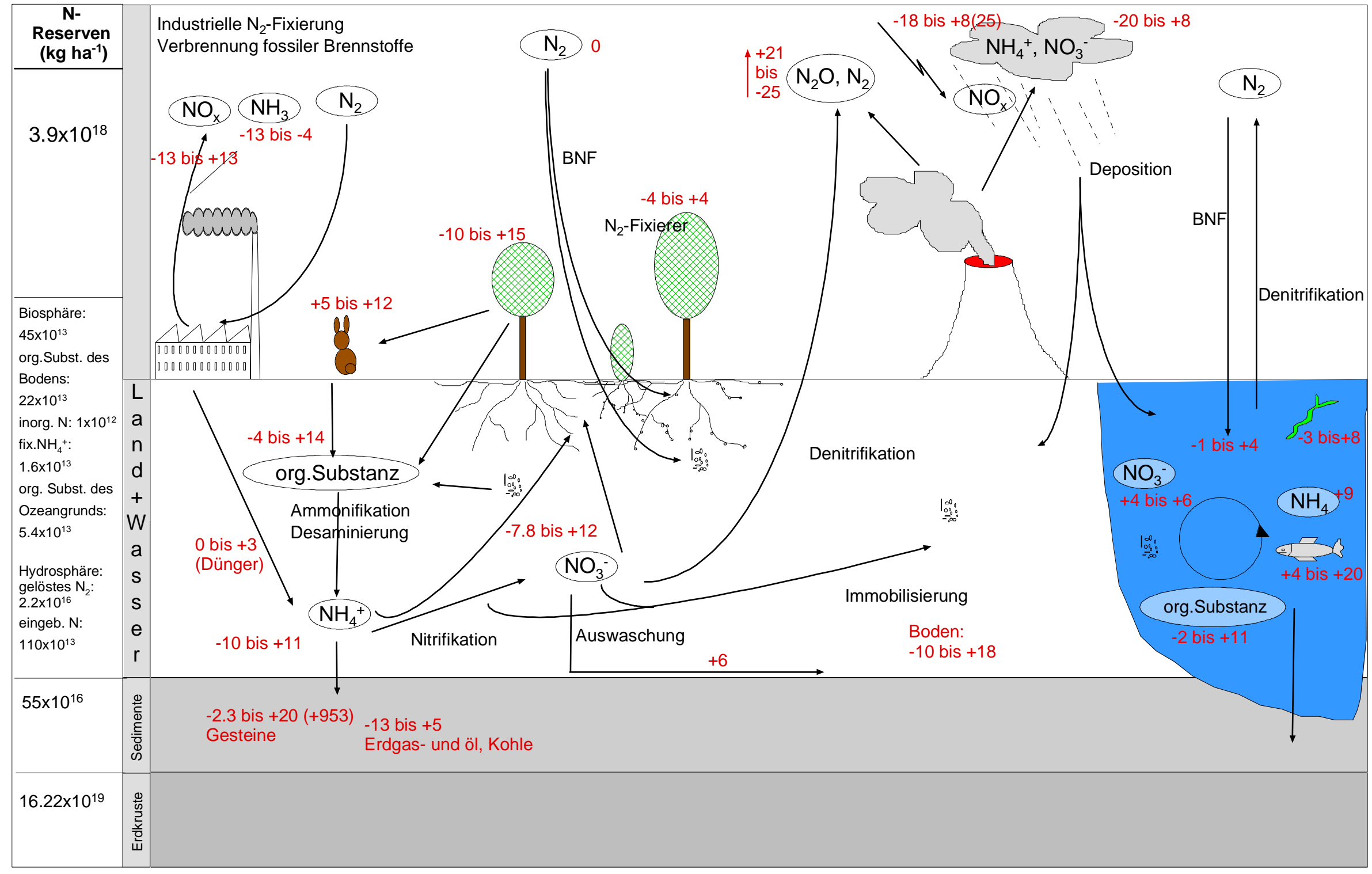


Um die verfügbaren Stickstoffresourcen des Bodens und der Luft aufzuschließen, sind in der Natur verschiedenste chemische und biologische Prozesse entstanden (Abb. 1).

Die stickstoffhaltige organische Substanz des Bodens wird durch extrazelluläre Enzyme der mikrobiellen Destruenten in Oligopeptide und Aminosäuren zersetzt. Diese Moleküle werden in die körpereigenen Substanzen der Mikroorganismen eingebaut (Immobilisierung) oder aber desaminiert $\mathrm{zu} \mathrm{NH}_{4}{ }^{+}$, welches als erstes Produkt der Mineralisation gilt.

Als Nitrifikation betrachtet man die nun folgende Oxidation von $\mathrm{NH}_{4}{ }^{+} \mathrm{Zu} \mathrm{NO}_{3}{ }^{-}$. Sie gliedert sich in zwei Schritte, die im Boden von einer Parabiose chemoautotropher, aerober Bakterien durchgeführt werden: Der erste Schritt der Oxidation des $\mathrm{NH}_{4}{ }^{+} \mathrm{zu} \mathrm{NO}_{2}^{-}$wird von Ammonium-oxidierenden Bakterien der Gattung Nitrosomonas, der zweite Schritt vom $\mathrm{NO}_{2}$ zum $\mathrm{NO}_{3}-$, von nitritoxidierenden Bakterien der Gattung Nitrobacter vollzogen. Nitrifikation wird reguliert über die $\mathrm{NH}_{4}{ }^{+}$- und $\mathrm{O}_{2}$ - Konzentration im Boden, das $\mathrm{pH}$-Optimum der Bakterien liegt bei etwa 7-9, die aktive Temperatur umfaßt 2-40 ${ }^{\circ} \mathrm{C}$ (Schlegel, 1992; Mosier und Schimel, 1993).

Neben dieser autotrophen Nitrifikation gibt es auch die heterotrophe Nitrifikation, die in sehr sauren Böden angetroffen wird. Ihre Bedeutung ist jedoch als gering einzuschätzen (Schlegel, 1992).

Denitrifikation benennt sowohl einen chemischen als auch biologischen Prozeß, bei dem es zum N-Austrag aus einem System kommt (global 140 x 109 $)$. Die chemische Denitrifikation findet bei $\mathrm{pH}$-Werten unter 5 statt und nutzt $\mathrm{HNO}_{2}$ als Substrat, welches bei der Nitrifikation als Zwischenprodukt entsteht. Dabei entstehen NO, $\mathrm{N}_{2} \mathrm{O}$ und Wasser (Smith und Chalk, 1980, zitiert in Tortoso und Hutchinson, 1990). Wesentlich bedeutender ist jedoch der biologische Prozeß, der zu einer Reduktion von $\mathrm{N}$-Oxiden wie $\mathrm{NO}_{3}{ }^{-}$und $\mathrm{NO}_{2}{ }^{-}$zu den Gasen $\mathrm{NO}, \mathrm{N}_{2} \mathrm{O}$ und $\mathrm{N}_{2}$ führt. Hauptsächlich fakultativ anaerobe, heterotrophe Mikroorganismen z.B. der Gattungen Paracoccus, Pseudomonas, Alcaligenes oder Azospirillum benutzen diese N-Oxide als Elektronenakzeptor, wenn $\mathrm{O}_{2}$ im Mangel vorliegt (Knowles, 1980, Gamble et al., 1977). Das pH-Optimum liegt zwischen 7 und 8. Diese Verhältnisse finden sich in Böden mit feiner Textur, schlechter Entwässerung oder hohem Regeneinfall. Denitrifikation ist auch abhängig von der Verfügbarkeit von $\mathrm{C}_{\text {und }} \mathrm{NO}_{3}{ }^{-}$, die wiederum in Abhängigkeit von der am Standort bestehenden Pflanzengesellschaft und ihrer Stickstoffnachfrage variiert (Groffman, 1995). 
Ein weiterer Verlust findet über den gasförmigen Austritt von $\mathrm{NH}_{3}$ aus dem Boden statt (Volatilisation). Er ist abhängig von der Differenz des Dampfdrucks zwischen der Bodenlösung und der Atmosphäre, der wiederum von Bodeneigenschaften wie KAK, pH,

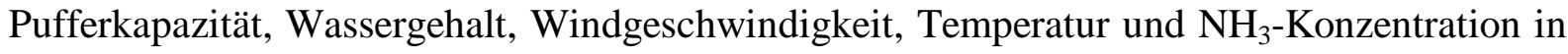
Boden und Luft beeinflußt wird. Als hauptsächliche N-Quelle fungieren hierbei tierische Exkretionen, N-Dünger, Dekomposition von organischem Material und die Verbrennung von Kohle. $\mathrm{NH}_{3}$-Volatilisation hat vor allem auf landwirtschaftlich genutzten Böden eine Bedeutung, wenn Dünger unsachgemäß ausgebracht wird, der Boden-pH zu hoch ist oder aber Vegetationen entfernt werden, die das gasförmige $\mathrm{NH}_{3}$ absorbieren könnten (Freney und Simpson, 1983).

Sowohl $\mathrm{NO}_{3}^{-}$als auch $\mathrm{NH}_{4}^{+}$können von den Pflanzen durch die Wurzeln aufgenommen werden. In der Regel wird das $\mathrm{NO}_{3}{ }^{-}$über die assimilatorische Nitratreduktase in $\mathrm{NH}_{4}{ }^{+}$ umgewandelt. $\mathrm{NH}_{4}{ }^{+}$wird über das GS/GOGAT - System (Glutaminsynthetase/Glutamin-2Oxoglutarat-Amino-Transferase) an Glutamat oder Aspartat gebunden und so mit dem Xylem-Flux in die einzelnen Kompartimente der Pflanzen transportiert, in denen es in andere organische Verbindungen eingebaut wird.

Die Atmosphäre birgt das größte Stickstoffreservoir, das Organismen mit der Befähigung zur biologischen $\mathbf{N}_{\mathbf{2}}$-Fixierung (BNF) nutzen können. $\mathrm{Zu}$ diesen Organismen zählen symbiontische Bakterien der Gattung Rhizobium und Bradyrhizobium die eine Symbiose in den Wurzeln oder manchmal auch im Pflanzenstamm von Arten der Familie Leguminosae eingehen, wobei die Pflanze Energie und Kohlenhydrate liefert und das Bakterium $\mathrm{NH}_{4}{ }^{+}$. Diese Art der Symbiose gehen auch einige Ulmaceae mit der Bakteriengattung Bradyrhizobium ein. Cyanobakterien gehen assoziative Symbiosen mit Pflanzen der Gattung Peltigera (Flechten), Moosen, dem Farn Azolla u.a. ein. Auch Actinomyceten (Gattung Franckia) assoziieren sich mit höheren sog. Aktinorrhiza-Pflanzen der Betulaceae, Casuarinaceae, Elaegnaceae, Myricaceae, Rhamnaceae, Rosaceae und anderen (z.B. Alnus spp., Hippophae spp. oder Casuarina spp.). Freilebende Bakterien wie z.B. Azotobacter paspalum, Klebsiella pneumonia, anoxygen phototrophe Purpurbakterien, Beijerinckia spp., Methylotrophe oder Desulfurikanten sind ebenfalls zu BNF befähigt. Ihnen allen gemeinsam ist die Entwicklung a) des sauerstoffempfindlichen Enzyms Nitrogenase, das für die Reduktion des $\mathrm{N}_{2} \mathrm{Zu} \mathrm{NH}_{4}{ }^{+}$verantwortlich ist und b) eines Mechanismus, der den auf die $\mathrm{N}_{2}{ }^{-}$ Fixierung hemmend wirkenden Sauerstoffpartialdruck niedrig hält. 
Die Nitrogenase besteht aus 3 Untereinheiten, dem zweifach vorkommenden Azoferredoxin und dem Molybdoferredoxin (Schlegel, 1992). Unter Aufwendung von 16 ATP (Adenosin-triphosphat) katalysiert der Enzymkomplex die Reduktion von $1 \mathrm{~N}_{2}$ zu $2 \mathrm{NH}_{3}$ und $\mathrm{H}_{2}$. Die Summenformel dieser Reaktion ist:

$$
\mathrm{N}_{2}+8 \mathrm{H}^{+}+8 \mathrm{e}^{-}+16 \mathrm{ATP} \rightarrow 2 \mathrm{NH}_{3}+\mathrm{H}_{2}+16 \mathrm{ADP}+16 \mathrm{P}_{\mathrm{i}}
$$

Rein rechnerisch werden für diesen Vorgang 16 ATP als Energiequelle benötigt, erfahrungsgemäß sind es aber wohl eher 20-30 ATP (Stacey et al., 1992). Die Enzyme enthalten, wie schon ihr Name sagt, neben schwefelhaltigen Proteinen auch Eisen und Molybdän, so daß Mikroorganismen nur dann $\mathrm{N}_{2}$ binden können, wenn Phosphor, Eisen und Molybdän vorhanden sind. Andere wichtige Mikronährstoffe sind Kobalt, Bor, Zink, Nickel, Selen und Kupfer (Giller und Wilson, 1991). Von Fixierungsaktivitäten bei arktischen Temperaturen (Nosko et al., 1994) oder aber in heißen Quellen (Wahlund und Madigan, 1993) wurde zwar berichtet, das Temperaturoptimum liegt jedoch bei $30^{\circ} \mathrm{C}$. Die BNF bringt den ausführenden Organismen immer dann Vorteile, wenn der Boden $\mathrm{N}$-arm, jedoch mit Phosphor und den o.g. Mikronährstoffen ausreichend versorgt ist.

\section{Stickstoff in den Tropen}

Die Mineralisation und Denitrifikation in tropischen Böden hängt sehr stark von deren Vegetation und Lage ab (Robertson, 1989). So unterliegt die Ammonifikation in Böden von terra firme Wäldern und Savannen im brasilianischen Bundesstaat Roraima einer Periodizität in Abhängigkeit von der Trocken- und Regenzeit (Marrs et al. 1991; Neill et al., 1995). Die Mineralisationsrate ist während des Überganges von der Trocken - zur Regenzeit am höchsten, in der Trockenzeit am niedrigsten. Nach Vegetationsbränden, landwirtschaftlichen Maßnahmen wie Hacken und Ernten kommt es zu einem Anstieg der Mineralisationsrate und damit der $\mathrm{NO}_{3}{ }^{-}$-Konzentrationen im Boden (Hölscher, 1995). Mit den $\mathrm{NO}_{3}{ }^{-}$-Konzentrationen im Boden eng verbunden ist die Denitrifikation. Groffman (1995) geht davon aus, daß in sehr jungen (z.B. dem Wiederaufwuchs der natürlichen Vegetation nach einer Kulturphase) oder alten Vegetationen (wie dem Primärwald) das Stickstoff- und Wasserangebot nicht von der Vegetation ausgeschöpft wird und so verfügbarer Stickstoff und Wasserüberschuß $\left(\mathrm{O}_{2^{-}}\right.$ Mangel fördernd) zu bedeutenden Denitrifikationsraten führen könnten. Mittelalte, schnellwüchsige Vegetationen regulieren über ihr Wachstum, das einen hohen $\mathrm{N}$ - und Wasserbedarf fordert, sowohl die Verfügbarkeit von Stickstoff als auch den Wasserhaushalt 
und unterdrücken damit die Denitrifikation. Robertson und Tiedje (1988, zitiert in Groffman, 1995) schätzten die Denitrifkationsraten für Primärwald auf $13.2 \mathrm{~kg} \mathrm{~N} \mathrm{ha}^{-1} \mathrm{Jahr}^{-1}$ und für Sekundärvegetationen mittleren Alters auf nur $3.6 \mathrm{~kg} \mathrm{~N} \mathrm{ha}^{-1} \mathrm{Jahr}^{-1}$.

Freney und Simpson (1983) messen der $\mathrm{NH}_{3}$-Volatilisation im slash and burn System über die Anhebung des Boden-pH-Wertes und die Entfernung der Vegetation eine gewisse Bedeutung zu. Die entscheidenden N-Verluste finden jedoch beim Stofftransfer in die Atmosphäre durch den Brand statt. Hölscher (1995) bestimmte den Stickstoffverlust durch den Brand einer 7jährigen Sekundärvegetation in Pará auf $200 \mathrm{~kg} \mathrm{~N} \mathrm{ha}^{-1}$, Kauffman et al. (1994, 1995) ermittelten Verluste durch den Brand einer Primärvegetation von 820 - $1600 \mathrm{~kg}$ $\mathrm{N}_{\text {ha }}{ }^{-1}$. Dies entspricht einem Verlust von $96 \%$ bzw. $64 \%$ des in der Biomasse enthaltenen Stickstoffs. Die Verluste von Phosphor mit $4.3 \mathrm{~kg} \quad \mathrm{P} \quad \mathrm{ha}^{-1}$ (47\%) der jungen Sekundärvegetation und $20 \mathrm{~kg} \mathrm{P} \mathrm{ha}{ }^{-1}(32 \%)$ der Primärvegetation fielen geringer als die N-Verluste aus. Gehring (1997) ermittelte als hauptsächlich das Wachstum der Sekundärvegetation limitierende Faktoren Phosphor und Stickstoff. Bis zu einem Vegetationsalter von circa 1.5 Jahren waren diese Elemente jedoch noch ausreichend vorhanden und stünden somit als „Startgabe“ für die BNF zur Verfügung.

Biologische $\mathrm{N}_{2}$-Fixierung in tropischen Regionen hat aufgrund der oft $\mathrm{N}$-armen Böden eine große Bedeutung. Mangel an Wasser, Phosphor, Molybdän und anderen Mikronährstoffen limitieren ihre Effektivität jedoch (Giller und Wilson, 1991; Sanginga et al., 1995). Um ihren hohen Energiebedarf (ATP) zu decken, benötigen die Pflanzen viel Phosphor, der in den tropischen Böden häufig nicht ausreichend vorhanden ist. Die meisten Leguminosen sind daher von Ecto- und (V)A-Mycorrhiza abhängig, um über die Vergrößerung des Wurzelsystems an schwerer verfügbare Nährstoffe zu gelangen (Alexander, 1989a und b). Molybdän, Teil des Nitrogenase-Komplexes, wird in sauren Böden ebenso wie Phosphor in nicht pflanzenverfügbarer Form fixiert. Auch Calcium und Magnesium spielen eine Rolle bei der $\mathrm{N}_{2}$-Fixierung, sind in sauren Böden aber oftmals mangelhaft vorhanden.

Die Rolle der BNF in den verschiedenen Sukzessionsstadien einer Vegetation wird sehr gegensätzlich eingeschätzt (Tab. 1). Nach den Zahlen von Kauffman et al. (1995) und Hölscher (1995) herrscht im Anfangsstadium der Sekundärvegetation N-Mangel, aber noch ein relativ gutes P-Angebot vor, also gute Voraussetzungen für die BNF. Mit zunehmendem Alter und Erreichen des stabilen Klimaxstadiums wird Stickstoff akkumuliert und Phosphor 
tritt als limitierender Faktor und somit die BNF reprimierend auf (Gorham et al., 1979; Stevens und Walker, 1970). Hierzu existieren jedoch nur sehr wenige Berechnungen.

Die nicht-symbiontische BNF über Flechten und freilebende Bakterien auf Rinden, in Humus und Boden eines Caatinga Primärwaldes (Rio Negro, Venezuela) wird auf etwa 35 bis 200 $\mathrm{kg} \mathrm{N} \mathrm{ha}^{-1} \mathrm{Jahr}^{-1}$ geschätzt ( Herrera und Jordan, 1981) (Tab.1) Die Berechnungen reichen von 0.1 bis $600 \mathrm{~kg} \mathrm{~N} \mathrm{ha}^{-1} \mathrm{Jahr}^{-1}$. In welcher Form dieser N-Input reguliert wird, ist unbekannt. Todd et al. (1978) gehen für ihren Standort (North Carolina, USA) davon aus, daß dieser nicht symbiontisch fixierte N-Eintrag zusammen mit der N-Deposition die Verluste durch Auswaschung und Denitrifikation ausgleicht.

Welche Bedeutung den Baumleguminosen in der N-Bilanz eines Primärwaldes zukommt, liegt vollkommen im Unklaren.

Tab. 1: Fixierungsraten einiger Primär - und Sekundärvegetationen der Amazonasregion

\begin{tabular}{|c|c|c|c|}
\hline \multirow[t]{2}{*}{ Ökosystem } & \multicolumn{3}{|c|}{ Fixierungsrate $\left(\mathrm{kg} \mathrm{N} \mathrm{ha}^{-1} \mathrm{Jahr}^{-1}\right)$} \\
\hline & $\begin{array}{l}\text { symbiontische } \\
\text { BNF }\end{array}$ & $\begin{array}{l}\text { BNF via Flechten und freilebenden Na-Fixierern } \\
\text { in Boden und Phyllosphäre }\end{array}$ & Referenz \\
\hline \multicolumn{4}{|l|}{ Primärvegetationen: } \\
\hline lehmiger Oxisol, Brasilien & 1.5 & $0-5$ & Salati et al., 1982 \\
\hline $\begin{array}{l}\text { Várzea, alluvialer Boden, } \\
\text { Brasilien }\end{array}$ & & 243 & $\begin{array}{l}\text { Sylvester-Bradley et al., } \\
1980\end{array}$ \\
\hline $\begin{array}{l}\text { Campinas, sandiger } \\
\text { Ultisol, Brasilien }\end{array}$ & & $5-20$ & Salati et al., 1982 \\
\hline $\begin{array}{l}\text { Caatinga, sandiger } \\
\text { Tropaquod, Venezuela }\end{array}$ & & $35-200$ & $\begin{array}{l}\text { Herrera und Jordan, } \\
1981\end{array}$ \\
\hline $\begin{array}{l}\text { Terra firme, sandiger } \\
\text { Oxisol, Rio Negro, } \\
\text { Venezuela, }\end{array}$ & & 16.2 & Jordan et al., 1982 \\
\hline Puerto Rico & & 88 & Todd et al., 1978 \\
\hline Costa Rica & & $1-8$ & \\
\hline La Selva, Costa Rica & $?$ & 0.1 & Carpenter, 1992 \\
\hline „Primärwald“ & & bis 600 & Ruinen, 1974 \\
\hline \multicolumn{4}{|l|}{ Sekundärvegetationen: } \\
\hline Oxisol, Brasilien & 2.45 & & Salati et al., 1982 \\
\hline sandiger Latosol, Brasilien & 20 & & $\begin{array}{l}\text { Sylvester-Bradley et al., } \\
1980\end{array}$ \\
\hline
\end{tabular}

Einen Hinweis auf die symbiontische $\mathrm{N}_{2}$-Fixierung gibt die Nodulation (Knöllchenbildung an den Wurzeln) der Leguminosen. Vitousek und Denslow (1986) fanden eine hohe Nodulation auf Lichtungen eines Primärwaldes. Magalhães et al. (1982) und Sylvester-Bradley et al. 
(1980) fanden eine unterschiedliche Nodulation in Abhängigkeit von der Nährstoffversorgung des Bodens (auf N-reicheren Böden keine oder ineffektive und auf N-armen Böden viele Knöllchen). Auch Moreira et al. (1992) bestätigten dieses Ergebnis und fügten noch die Beobachtung hinzu, daß generell ein Nodulationspotential vorhanden, aber durch Bodensubstanzen der Primärvegetation unterdrückt wird. Dies können sowohl hohe N-Gehalte, niedrige P-Gehalte als auch Tannine oder andere allelopathische Substanzen sein (Jordan et al., 1979; Rice, 1984). Norris (1969) berichtete aber auch von hoher Nodulation in Primärwäldern der brasilianischen Bundesstaaten Pará und Amazonas, die sich jedoch fast ausschließlich auf Keimlinge und Jungpflanzen bezogen. Diese Beobachtungen liefern eher einen Hinweis darauf, daß die Nodulation nicht von der Nährstoffsituation oder allelopathischen Substanzen limitiert wird, sondern von dem Alter der Bäume abhängt.

Durch Rodung und Brennen einer alten Sekundärvegetation oder eines Primärwaldes geht das Potential der nicht-symbiontischen $\mathrm{N}_{2}$-Fixierer zunächst einmal verloren (Jordan, 1989). Die im Boden freilebenden $\mathrm{N}_{2}$ fixierenden Bakterien regenerieren sich im Laufe eines Jahres (Vasquez et al., 1993), die in der Phyllosphäre, der Rinde und auf verrottender Substanz lebenden Bakterienpopulationen und Flechten etc. jedoch nur mit der sich über Jahre wieder aufbauenden Vegetation.

Welchen Beitrag die symbiontische BNF zur Regeneration der Vegetation beiträgt, wurde bisher nicht untersucht. Für die Anwendung tropischer Leguminosen als Acker - bzw. Mischkulturen hingegen gibt es viele Berichte, die die Bedeutung für die Landwirtschaft unterstreichen (siehe Tab. 2).

\section{Die Leguminosen}

„Die Leguminosae stellen das spektakulärste Beispiel für evolutionäre und ökonomische Entwicklung aller Angiosperm-Familien dar“ (McKey, 1994).

Zu dieser Aussage kam McKey (1994) aufgrund der hohen Diversität bezüglich Wuchsform, Lebenszyklus, Habitate, geographischen Ausbreitung, Tierassoziationen und Samenverbreitung der Leguminosen. Beispielsweise umspannt die Wuchsform krautige, kurzlebige Pflanzen bis hin zu großen Bäumen mit Blättern über $2 \mathrm{~m}$ Länge, es gibt Samen mit $0.1 \mathrm{~mm}$ (Pycnospora lutescens) bis hin zu 18cm Durchmesser (Mora oleifera). 


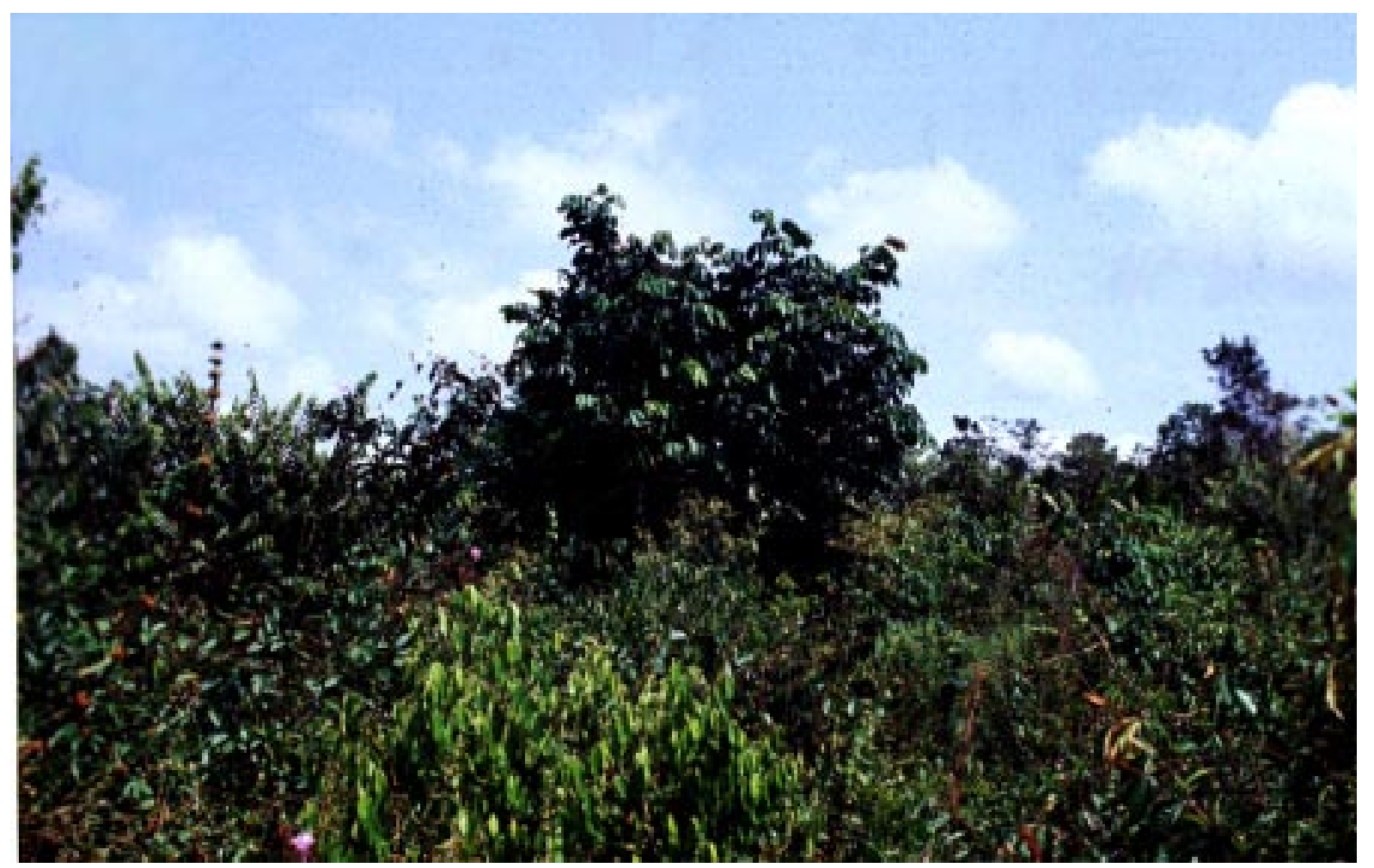

Abb. 2: Leguminosen können die Sekundärvegetation weit überragen. Inga macrophylla in einer etwa einjährigen Sekundärvegetation.

Damit sind sie sehr anpassungsfähig an ökologische Nischen, was besonders für die Verbreitung in den Tropen von Vorteil ist. Zur Zeit sind sie in 750 Genera mit etwa 1600019000 Spezies zusammengefaßt, unterteilt in drei Subfamilien - Papilionoideae, Caesalpinioideae und Mimosoideae (Giller und Wilson, 1991). Aus den Familien der Papilionoideae und Mimosoideae sind 95\%, in der Familie der Caesalpinioideae nur 23\% als nodulierend, d.h. in Symbiose mit Rhizobien lebend, bekannt (Faria et al. 1984, 1987, 1989; Moreira et al., 1992).

Man geht davon aus, daß die ersten Vorläufer der Leguminosen im frühen Tertiär (vor 60 70 Millionen Jahren) auftraten. Ihr Vorkommen vor 50 Mio. Jahren wurde in Form von Fossilien nachgewiesen (Sprent, 1994). Wahrscheinlich begann ihre Entwicklung mit Arten, den heutigen Caesalpinoideae ähnlich, in den Tropen, wo sie vermutlich von Bakterien der Gattung Rhizobium parasitär befallen und die Knöllchenbildung durch Tumorgene initiiert wurden. Die Rhizobien entwickelten sich später zu den 2 Gattungen Bradyrhizobium und Rhizobium. BNF in freilebenden Mikroorganismen ist älter. Sie entwickelte sich schon zwischen der Entstehung der Chemo-Autotrophie und der oxygenen Photosynthese (vor 700 Millionen Jahren), die auf Grund der $\mathrm{O}_{2}$-Empfindlichkeit der Nitrogenase zur Entwicklung 
von $\mathrm{O}_{2}$-Schutzmechanismen wie z.B. der Symbiose mit höheren Pflanzen führte (Raven und Sprent, 1989).

Aufgrund ihrer Befähigung, bis zu 90\% (durchschnittlich jedoch 60\%) ihres N-Bedarfes aus dem Luft-Stickstoff zu decken und ihrer Anpassungsfähigkeit sind Leguminosen heute in der tropischen Landwirtschaft sehr populär. Leguminosen sind auf Weiden und in agroforstlichen Systemen eine häufig verwendete Anreicherungskultur (Humphreys, 1995; Sanford et al., 1995; Sanginga et al.1995). Sie dienen der Holzgewinnung (Rosenholz - Genus Dalbergia-, Acacia-Arten und andere) oder als multipurpose trees für den Anbau von Fruchtbäumen wie z.B. Tamarinde (Tamarindus indica, Caesalpinioideae) oder Karop (Ceratonia siliqua, Caesalpinioideae)(Nair, 1986), als Schattenbaum und Hecken (Ladha et al., 1993; Stephenson und Raisson, 1988), als Erosionsschutz und Pioniergehölz (Döbereiner et al., 1979), zur Biogasproduktion (Döbereiner et al., 1995) oder in der Landwirtschaft als Futterpflanze (Blair et al., 1990). In Agroforstsystemen werden sie in Alley-Cropping-Systemen, Heckenpflanzungen und Mischkulturen eingesetzt. Hier werden hauptsächlich Baum-Leguminosen wie Gliricidia sepium, Leucaena leucocephala, Prosopis spp., Faidherbia albida, Sesbania rostrata, Calliandra calothyrsus, Acacia spp., Albizia falcataria und Aeschynomene indica (Peoples et al. 1995) gepflanzt. Als Bodendecker oder Unterwuchs werden aber auch Pueraria phaseoloides oder Medicago - und Trifolium - Arten verwendet (Lunaorea und Wagger, 1996; Vesterager et al., 1995). Einen Überblick über die Nutzung und die Biologie von Leguminosen in den Tropen und ihre Funktion geben Giller und Wilson (1991) und Ledgard und Giller (1995). In Tab. 2 sind einige Leguminosen, ihre Anwendung und $\mathrm{N}_{2}$-Fixierungsleistung zusammengefaßt.

Die BNF der Leguminosen fordert jedoch einen gesteigerten Energiebedarf, der sich in einem hohen Verbrauch an Zuckern und ATP widerspiegelt. Für die Nitrogenase, das gasförmiges $\mathrm{N}_{2}$ reduzierende Enzym, wird Mangan und Molybdän benötigt (siehe Kap. II.1). Somit sind sie abhängig von Standorten, die u.a. einen gesteigerten Lichtbedarf, Phosphor-, Manganund Molybdän-Anspruch decken. Sie sind teilweise gegenüber Beschattung empfindlich und ihr Vorkommen sinkt mit abnehmender Lichtintensität (Kessel und Roskosky, 1983; Izaguirre-Mayoral et al. 1995). Es kommt auch zu Konkurrenz - und allelopathischen Effekten, die sich negativ auf den Ertrag der eigentlich mit $\mathrm{N}$ anzureichernden Produktion auswirkt (Halsall et al., 1995; Gibson und Waring, 1994; Leigh et al., 1995, Rosecrance et al., 1992, Rice, 1984; Muhr et al., 1995). 
Die Rolle der BNF der Leguminosen in natürlichen Systemen wie der Sekundärvegetation und den Primärwäldern ist, wie schon in Kap. II.1 erläutert, bisher nicht geklärt. Die am häufigsten vorkommenden Arten dieser Systeme befinden sich jedoch in dieser Familie (Sprent, 1995). Sie können 45\% der Basalfläche eines Caatinga-Waldes am Rio Negro (Venezuela) einnehmen (Coomes und Grubb, 1996), aber auch nur 3.4\% der Basalfläche eines Primärwaldes in Roraima (Thompson et al., 1992). In einem Primärwald des Bundesstaates Pará gehörten 17\% der häufigsten Arten zu den Leguminosen (Vieira, 1996). Auch in kolumbianischen Wäldern war diese Familie mit den meisten Arten vertreten (Duivenvoorden, 1995).

In der Sekundärvegetation der Untersuchungsregion wurden Leguminosen in unterschiedlicher Häufigkeit gefunden. Für eine 5jährige Sekundärvegetation der Bragantina-Region bestimmte Denich (1989) 11\% der Individuen als Leguminosen, die mit $27 \%$ an der oberirdischen Biomasse beteiligt waren. Das entsprach 19\% der holzigen Arten. Vieira (1996) fand für eine gleich alte Vegetation sogar einen Anteil der Leguminosen an den häufigsten Arten von 45\% und 21\% der Individuen. Der Leguminosen-Bestand änderte sich jedoch drastisch mit der der Sekundärvegetation vorangehenden Nutzung (Nunez, 1996). In auf Pfefferanbau folgenden Sekundärvegetationen war der Leguminosenanteil an der oberirdischen Biomasse in einer einjährigen Vegetation 6-10\% und in einer zehnjährigen Vegetation nur $0.2-0.8 \%$.

Die Beobachtung, daß auf das traditionelle Bearbeitungssystem ein hoher Anteil der Leguminosen an der Biomasse in der Sekundärvegetation folgt, läßt einen Vorteil dieser Arten über ihre BNF gegenüber anderen Arten vermuten. Untersuchungen dazu liegen jedoch nicht vor. Diese Arbeit soll eine erste grobe Abschätzung der Rolle der symbiontischen $\mathrm{N}_{2}$-Fixierung von Baumleguminosen in der Sekundärvegetation geben. 
Tab. 2: Tropische Leguminosen, ihre $\mathrm{N}_{2}$-Fixierungsleistung und Anwendung (zusammengestellt aus Blair et al., $1990<1>$; Brewbaker et al., $1981<2>$; Depinto et al., $1995<3>$; Garrity und Mercado, $1994<4>$; Giller und Wilson, $1991<5>$; Gutteridge und Shelton, $1993<6>$; Herridge und Danso, 1995 <7>; Högberg und Kvarnström, $1982<8>$; Kadiata und Mulongoy, $1995<9\rangle$; Kessel et al., $1994<10>$; Lehmann et al., $1995<11\rangle$; National Academy of Science, $1979<12\rangle$; Ovalle et al., $1996<13\rangle$; Peoples et al., $1995<14>$; Rosecrance et al., $1992<15\rangle$; Sanford et al., $1993<16>$; Sanginga et al., $1990<17>$ )

\begin{tabular}{|c|c|c|c|}
\hline Genera & $\begin{array}{l}\text { Fixierungsrate } \\
\left(\mathrm{kg} \mathrm{N} \mathrm{ha}^{-1} \mathrm{Jahr}^{-1}\right)\end{array}$ & Funktion & Referenz \\
\hline \multicolumn{4}{|l|}{ Bäume: } \\
\hline Acacia & $\begin{array}{l}34-95 \% \text { vom } \\
\text { Gesamt-N }\end{array}$ & $\begin{array}{l}\text { Brennholz, lebender Zaun, Holzkohle, } \\
\text { Erosionsschutz, Futter }\end{array}$ & $2,1,8,14$ \\
\hline Albizia & $\begin{array}{l}34-80 \% \text { vom } \\
\text { Gesamt-N }\end{array}$ & Brennholz, Holzkohle & $2,9,14$ \\
\hline Calliandra & 11 & $\begin{array}{l}\text { Brennholz, Gründüngung, Alley-cropping, } \\
\text { Holzkohle }\end{array}$ & $2,6,16,14$ \\
\hline Chamaecrista & $8-82$ & Futter & 13 \\
\hline Gliricida & 99 & $\begin{array}{l}\text { Brennholz, Futter, Holzkohle, Hecke, } \\
\text { Gründüngung, Erosionsschutz, lebender Zaun }\end{array}$ & $\begin{array}{l}2,6,4,11,15,1 \text {, } \\
14\end{array}$ \\
\hline Inga & $?$ & Brennholz, Gründüngung & 2,8 \\
\hline Leucaena & $70-240$ & $\begin{array}{l}\text { Brennholz, Alley-cropping, lebender Zaun, } \\
\text { Holzkohle, Erosionsschutz }\end{array}$ & $\begin{array}{l}2,6,15,8,10,17, \\
14\end{array}$ \\
\hline Pithecellobium & $?$ & Brennholz, lebender Zaun, Futter, Harz & $2,6,1,3$ \\
\hline Prosopis & $\begin{array}{l}1.9(50 \% \text { vom } \\
\text { Gesamt-N) }\end{array}$ & Brennholz, Futter, Holzkohle, Erosionsschutz & $2,1,12,13$ \\
\hline Sesbania & $14-281$ & $\begin{array}{l}\text { Brennholz, Alley-cropping, lebender Zaun, } \\
\text { Reisanbau }\end{array}$ & $2,6,15$ \\
\hline \multicolumn{4}{|l|}{ Kräuter: } \\
\hline Cajanus & $68-88$ & Körnerleguminosen & 5 \\
\hline Calopogonium & $136-182$ & Weideleguminose, Gründüngung, Bodendecker & 5,14 \\
\hline Desmodium & $60-380$ & Gründüngung & 5 \\
\hline Glycine max & $26-450$ & Körnerleguminosen, Soja & 5,7 \\
\hline Macroptilium & $46-167$ & Gründüngung, Weideleguminose & 5 \\
\hline Phaseolus & $3-91$ & Körnerleguminose & 5 \\
\hline Pueraria & $9-115$ & Weideleguminose, Bodendecker & 5,14 \\
\hline Stylosanthes & $1-263$ & Gründüngung, Weideleguminose & 5 \\
\hline Trifolium & $2-206$ & Futter, Weide & 14,16 \\
\hline Vicia & $47-201$ & Körnerleguminose & 5 \\
\hline Vigna & $9-201$ & Futter, Körnerleguminose & 14 \\
\hline
\end{tabular}




\section{$4 \quad$ Die ${ }^{15} \mathrm{~N}$ natural abundance -Methode}

\subsection{Idee der ${ }^{15}$ NNAM-Methodik}

Mit Hilfe der ${ }^{15} \mathrm{~N}$ natural abundance -Methode kann die $\mathrm{N}_{2}$-Fixierungsleistung von Pflanzen ermittelt und der Weg des Stickstoffs in einem System verfolgt werden. Die Untersuchungen können ohne Beeinflussung des Wachstums der Pflanzen unternommen werden. Im Gegensatz zur Acetylen-Reduktions-Methode oder Total-Nitrogen-Difference-Methode müssen die Pflanzen nicht zerstört werden. Für einige Fragestellungen ist es sinnvoll, die zu untersuchenden Pflanzen, Böden oder andere Substrate mit ${ }^{15} \mathrm{~N}$ anzureichern $\left({ }^{15} \mathrm{~N}\right.$ dilution Methode). Mit der Stickstoffapplikation wird aber in das System eingegriffen und Faktoren wie Auswaschung, Bindung des Stickstoffs an Bodenkolloide, Veränderung des Mineralisationsverhaltens und der $\mathrm{N}_{2}$-Fixierung erschweren die Quantifizierung der BNF (Chalk, 1985; Peoples et al., 1989; Yoneyama et al. a, 1990).

Für die Fragestellung dieser Arbeit, ob Baumleguminosen in der natürlichen Sekundärvegetation $\mathrm{N}_{2}$ fixieren, war daher die ${ }^{15} \mathrm{NNAM}$ am besten geeignet.

Bisher wurde die ${ }^{15}$ NNAM hauptsächlich unter definierten Versuchsbedingungen wie dem Gewächshaus oder Feldversuchen durchgeführt. Neuerdings wird die Methode auch verstärkt zur Erforschung des N-Kreislaufes in natürlichen Ökosystemen eingesetzt (Altabet et al., 1995; Dauchez et al., 1995; France, 1995; Liu et al., 1996; Sanford et al., 1993).

In natürlichen Vegetationen, speziell in feuchttropischen Klimaregionen mit Brandrodungspraxis jedoch liegen wenige Erfahrungen vor (Högberg und Alexander, 1995; Sanginga et al., 1992 und 1995; Sprent et al., 1995 und 1996; Yoneyama et al., 1993b).

Die ${ }^{15}$ NNAM wurde verstärkt in den 80er Jahren u.a. von Amarger et al. (1979), sowie Delwiche und Steyn (1970), Kohl et al. (1980), Mariotti (1983) und Rennie und Rennie (1983), entwickelt. Basis der Methode sind die unterschiedlichen Konzentrationen von ${ }^{15} \mathrm{~N}$ in Boden und Luft, wobei im Boden die höheren Konzentrationen angetroffen werden. Bei vielen Untersuchungen wurde der Atomprozentanteil ${ }^{15} \mathrm{~N}$ an dem Luft- $\mathrm{N}$ mit 0,3663 als weltweit konstant ermittelt (Burris und Miller, 1941; Delwiche et al., 1979; Handley und Raven, 1992; Hauck et al., 1958; Hoering, 1955; Junk und Svek 1958; Mariotti 1983; Shearer et al., 1974b; Shearer und Kohl, 1988a und b; Wellmann et al., 1968). Schon 1941 wiesen Burris und Miller anhand der ${ }^{15} \mathrm{~N}$-Konzentrationen $\mathrm{N}_{2}$-Fixierung nach. Systematisch wurde ${ }^{15} \mathrm{~N}$ zur Bestimmung der $\mathrm{N}_{2}$-Fixierung erstmals von McAuliffe (1958) eingesetzt. 
Um die sehr geringen Unterschiede in den ${ }^{15} \mathrm{~N}$-Konzentrationen (Beispiel: ${ }^{15} \mathrm{~N}_{\text {Luft }}=0,3663$ Atom $\%$ und ${ }^{15} \mathrm{~N}_{\text {Boden }} \approx 0,3675$ Atom $\%$ ) in übersichtlicher Form zu erfassen, greift man auf eine sogenannte Deltanotation $\left(\delta^{15} \mathrm{~N}\right)$ zurück. Hierbei signalisieren positive $\delta^{15} \mathrm{~N}$-Werte höhere und negative $\delta^{15} \mathrm{~N}$-Werte niedrigere ${ }^{15} \mathrm{~N}$-Konzentrationen als in der Luft.

Die unterschiedlichen $\delta^{15} \mathrm{~N}-$ Werte von Pflanzen je nach ihrer N-Quelle (Luft, Boden, sonstige Substrate) macht man sich zur Bestimmung der $\mathrm{N}_{2}$-Fixierungsleistungen von Leguminosen zunutze.

Shearer und Kohl (1986) erklären die Ermittlung der $\mathrm{N}_{2}$-Fixierungsleistung mit der ${ }^{15} \mathrm{NNAM}$ mit folgendem Schaubild zweier Pole (Abb. 3):

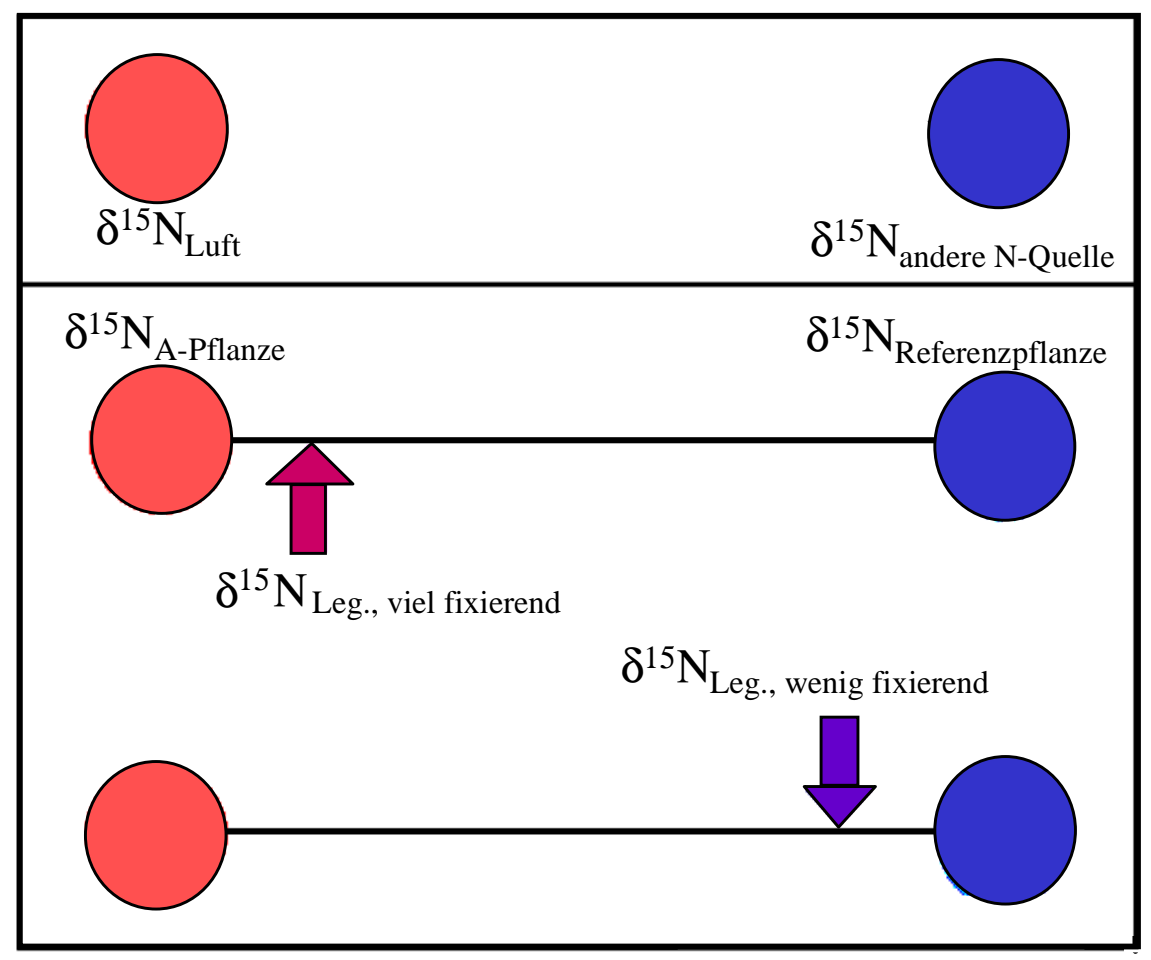

Abb. 3: Modell der Verteilung von ${ }^{15} \mathrm{~N}$ in Leguminosen, Referenzpflanzen und den N-Quellen (nach Shearer und Kohl, 1986).

Dabei nehmen sie zwei Stickstoff-Quellen für die Pflanzen an. Zum einen wird N über die Wurzeln aufgenommen, zum anderen über die Wurzelknöllchen mittels biologischer $\mathrm{N}_{2^{-}}$ Fixierung. Eine Pflanze, die ihren N-Bedarf nur aus dem Boden über die Wurzeln deckt, muß demnach den $\delta^{15} \mathrm{~N}-$ Wert der Boden-N-Quelle widerspiegeln. Eine Pflanze, die $100 \%$ ihres N aus der Luft bezieht, muß den $\delta^{15} \mathrm{~N}$-Wert des Luft-N widerspiegeln (als A-Wert bzw. A-Pflanze bezeichnet). Der $\delta^{15} \mathrm{~N}$-Wert einer Leguminose, die teils fixiert, aber auch einen Teil ihres $\mathrm{N}$ aus dem Boden aufnimmt, muß sich entsprechend dem Anteil fixierten $\mathrm{N}$ zwischen 
den beiden Polen ansiedeln. Aus dem Vergleich einer nicht-fixierenden sog. Referenzpflanze für den Boden-N-Pool, einer 100\% fixierenden Leguminose für den Luft-N-Pool (A-Pflanze) mit der untersuchten Leguminose kann nun auf ihre Fixierungsleistung, ausgedrückt in \% Anteil Luft-N am Gesamt-N (\%Ndfa) der betrachteten Leguminose, errechnet werden.

Von entscheidender Bedeutung für die Anwendung der Methode ist die Wahl der nicht $\mathrm{N}_{2}$ fixierenden Referenzpflanzen, die gerade in natürlichen Systemen sehr erschwert ist. Von ihrem $\delta^{15} \mathrm{~N}$-Wert hängt es letztlich ab, wie genau eine $\%$ Ndfa-Berechnung ist. Sie gibt die $\delta^{15} \mathrm{~N}$-Spanne vor, in die sich das oben beschriebene Schema Abb. 3 einpaßt (Unkovich et al., 1994). Je konstanter und größer die Differenz zwischen Referenzen und Leguminosen ist, desto genauer wird die Berechnung.

Die im Feld untersuchten Leguminosen müssen im Gewächshaus in N-freier Kultur angezogen werden, um den $\delta^{15} \mathrm{~N}-$ Wert der oben beschriebenen A-Pflanze zu erlangen.

In den letzten Jahren wurde immer deutlicher, daß reaktionskinetisch bedingte Fraktionierungsprozesse zwischen dem leichten $\left({ }^{14} \mathrm{~N}\right)$ und schweren Stickstoff-Isotop $\left({ }^{15} \mathrm{~N}\right)$ zur Verwischung der Differenzen zwischen den $\delta^{15} \mathrm{~N}-$ Werten einzelner Arten, oder aber zu Unterschieden in den einzelnen Pflanzenorganen und den einzelnen N-Fraktionen im Boden führen. Auf Grund dieser Schwankungen der $\delta^{15} \mathrm{~N}$-Werte im biotischen und abiotischen System können einerseits die Wege des Stickstoffs verfolgt werden, müssen aber andererseits besondere Maßnahmen zur Bestimmung der $\mathrm{N}_{2}$-Fixierungsleistung unternommen werden. Dazu schlagen einige Wissenschaftler vor, mehrere Methoden parallel zu verwenden (Chalk et al., 1996; Herridge et al., 1990; Hoghjensen und Kristensen, 1995; Hoghjensen und Schjoerring, 1994; Hossain et al., 1995; Peoples et al., 1996; Sanginga et al., 1989) oder Alternativen zur Wahl einer Referenzpflanze auszuprobieren (Chalk et al., 1996). In den meisten Fällen wurde die ${ }^{15}$ NNAM und die,${ }^{15} \mathrm{~N}$-dilution"-Methode parallel eingesetzt. Chalk et al. (1996) verbesserten die Methode zur Bestimmung des pflanzenverfügbaren $\mathrm{N}_{\min }$ im Boden. Stock et al. (1995) begaste die Wurzeln von Feldleguminosen mit $\mathrm{O}_{2}$, um so die $\mathrm{N}_{2}$-Fixierung zu unterdrücken und eine Referenzpflanze zu erlangen, die der fixierenden Pflanze physiologisch sehr ähnelte.

Einen detaillierten Überblick über die Methode und ihre Probleme geben u.a. Andreux et al. (1988), Handley und Raven (1992), Hauck (1973), Shearer und Kohl (1986, 1988, 1993) und Yoneyama (1996). 


\subsection{Vorkommen von ${ }^{15} \mathrm{~N}$}

In Abb. 1 des Kap. II.1 sind die einzelnen Prozesse des N-Kreislaufes dargestellt. Mit roten Zahlen sind die bisher gefundenen $\delta^{15} \mathrm{~N}$-Werte der betreffenden Substanzen und Kompartimente dargestellt. Abb. 4 faßt die bisher vorgefundenen $\delta^{15} \mathrm{~N}$-Werte in Boden und Vegetation zusammen.

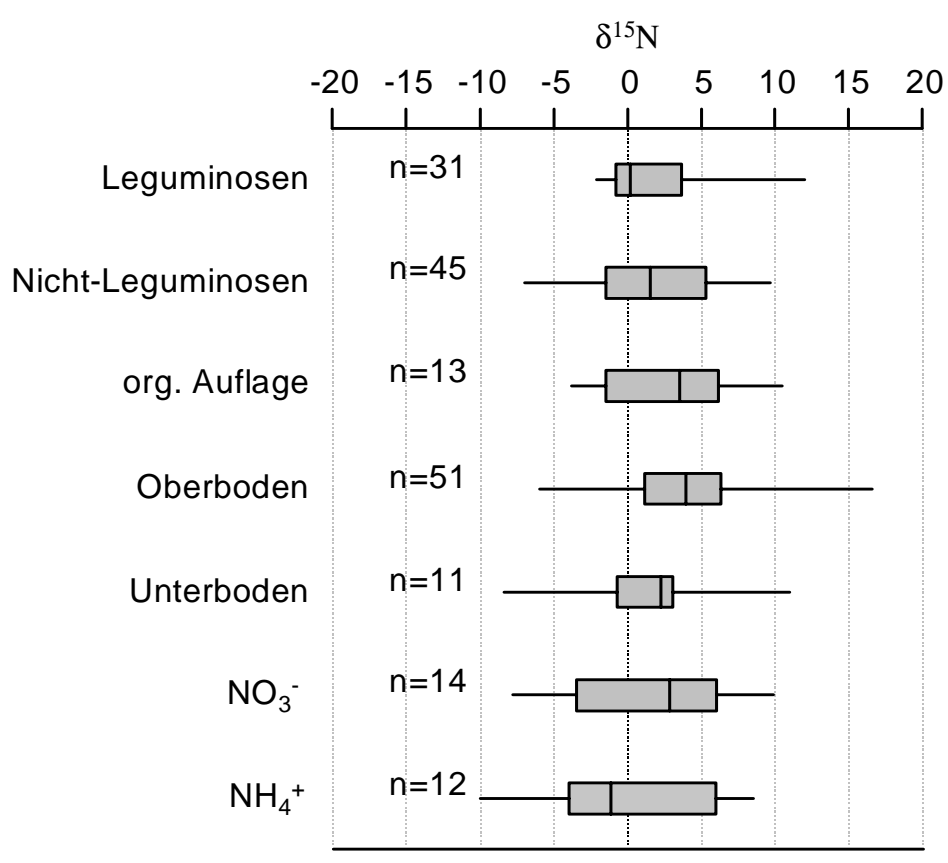

Abb. 4: Weltweites ${ }^{15} \mathrm{~N}$-Vorkommen in Pflanzen und Boden $(\mathrm{n}=$ Anzahl Referenzen - siehe Anhang Tab.51); $\mathrm{NH}_{4}{ }^{+}$bzw. $\mathrm{NO}_{3}{ }^{-}=$pflanzenverfügbares $\mathrm{NH}_{4}{ }^{+}$bzw. $\mathrm{NO}_{3}{ }^{-}$im Boden; Box-Whisker-Plots geben den Median, 25\% und 75\% Quartil und Spannweite an).

Generell reicht die Spanne an vorgefundenen $\delta^{15} \mathrm{~N}-$ Werten in etwa von -10 bis +17 . Der $\mathrm{N}_{2}$ der Luft wird - wie schon zuvor erwähnt - auf der ganzen Welt mit 0.3663 Atom\% ${ }^{15} \mathrm{~N}$ als Standard verwendet und hat demzufolge einen $\delta^{15} \mathrm{~N}$-Wert von 0.

\subsubsection{Fraktionierungsprozesse}

Die unterschiedlichen $\delta^{15} \mathrm{~N}$-Werte in biotischen und abiotischen Substanzen sind Folge einer Fraktionierung der Stickstoffisotope ${ }^{15} \mathrm{~N}$ und ${ }^{14} \mathrm{~N}$ während der einzelnen Phasen der biochemischen Umsetzung und Reaktionen im N-Kreislauf. Wellmann et al. (1968) weisen als eine der Ersten darauf hin, daß Moleküle mit dem leichteren Isotop ${ }^{14} \mathrm{~N}$ aufgrund reaktionskinetischer Gesetzmäßigkeiten meistens gegenüber denen mit dem schwereren Isotop ${ }^{15} \mathrm{~N}$ etwas bevorzugt werden. So sind als Ergebnis dieser Fraktionierung bei 
unvollständigen Prozessen unterschiedliche Anteile ${ }^{15} \mathrm{~N}$ (Atom\%) in Substrat und Produkt zu finden. Das Maß dieser Fraktionierung wird mit dem $\beta$-Faktor, dem Verhältniswert des ${ }^{15} \mathrm{~N}$ Gehaltes des Substrates zu dem des Produktes, quantifiziert (Tab. 3, Formel des $\beta$-Faktors siehe Kap. V.4.3). Ein $\beta$-Faktor > 1 bedeutet eine Anreicherung im Substrat, $<1$ eine Anreicherung im Produkt der jeweiligen Reaktion.

Tab. 3: Zusammenfassung der $\beta$-Faktoren einiger biochemischer Reaktionen (Referenzen: 1 - Wada et al. 1975, 2 - Handley und Raven, 1992, 3 - Shearer und Kohl, 1986 und 1993; 4 - Hoering und Ford, 1960)

\begin{tabular}{|c|c|c|c|}
\hline Biochemische Reaktion & $\beta$-Faktor & $\delta^{15} \mathrm{~N}$ des Produktes & Referenz \\
\hline Abbauprozesse von $\mathrm{N}_{\text {org. }}$. & 1.003 & 0 bis -2.3 & 1 \\
\hline Desaminierung & 1.003 bis 1.023 & ${ }^{15} \mathrm{~N}$-verdünnt & 2 \\
\hline Assimilation von $\mathrm{NH}_{4}^{+}$ & & -9.6 bis +7.3 & 1 \\
\hline Assimilation von $\mathrm{NH}_{4}^{+}$ & 1.0091 bis 1.0179 & ${ }^{15} \mathrm{~N}$-verdünnt & 2 \\
\hline Assimilation von $\mathrm{NO}_{3}^{-}$ & 1.005 & -0.9 bis -18.5 & 1 \\
\hline Assimilation von $\mathrm{NO}_{3}$ bis zum & 1.003 bis 1.03 & ${ }^{15} \mathrm{~N}$-verdünnt & 2 \\
\hline Einbau in org.Substanzen & & & \\
\hline Nitrifikation & 1.015 bis 1.035 & stark ${ }^{15} \mathrm{~N}$-verdünnt & 2 \\
\hline GS/GOGAT & 0.9808 bis 1.0125 & eher ${ }^{15} \mathrm{~N}$-angereichert & 2 \\
\hline Transaminierung & 1.0083 bis 1.0017 & schwach ${ }^{15} \mathrm{~N}$-verdünnt & 2 \\
\hline marin, $\mathrm{NH}_{4}^{+} \mathrm{zu} \mathrm{NO}{ }_{2}^{-}$ & & -5.4 bis -21.1 & 1 \\
\hline marine Denitrifikation & 1.021 & -1.9 bis -30 & 1 \\
\hline Diffusion & 1.000 & unverändert & 3 \\
\hline $\mathrm{NH}_{3}$-Diffusion in die Gasphase & 1.018 & schwach ${ }^{15} \mathrm{~N}$-verdünnt & 2 \\
\hline Denitrifikation & 1.028 bis 1.033 & stark ${ }^{15} \mathrm{~N}$-verdünnt & 2 \\
\hline Symbiontische $\mathrm{N}_{2}$-Fixierung & 0.9992 bis 1.0034 & schwach ${ }^{15} \mathrm{~N}$-verdünnt & 2 \\
\hline $\begin{array}{l}\mathrm{N}_{2} \text {-Fixierung durch freilebende } \\
\text { Bakterien }\end{array}$ & 0.9963 bis 1.0035 & $\begin{array}{l}\text { meistens schwach } \\
\text { verdünnend }\end{array}$ & 4 \\
\hline
\end{tabular}

Diese Fraktionierungen finden hauptsächlich im Boden bei der im weiteren erläuterten Transformation des Stickstoffs der organischen Substanz in mineralisches N, bzw. dem erneuten Einbau in mikrobielle oder pflanzliche Substanzen statt. In den Pflanzen kommt es an verschiedensten Stellen zu einer möglichen Fraktionierung, wie z.B. der Diffusion durch Zellmembrane, katalytische oder enzymatische Reaktionen. Wie der Tab. 3 zu entnehmen ist, wird dabei unterschiedlich stark fraktioniert, so daß z.B. eine klare Bestimmung des $\delta^{15} \mathrm{~N}-$ Wertes des pflanzenverfügbaren $\mathrm{N}$ des Bodens erschwert ist. Durch viele Faktoren, die im Folgenden erläutert werden, können diese Prozesse beeinflußt und damit undurchschaubar gemacht werden. Das Wissen über die $\delta^{15} \mathrm{~N}$-Werte der N-Quelle der Pflanzen im Feld ist jedoch sehr wichtig. Daher ist es unerläßlich, Hinweise auf die Prozesse im zu untersuchenden Boden und ihre Bedeutung für die Fraktionierung der Isotopen zu erlangen. 


\subsection{2 $\delta^{15} \mathrm{~N}$ im Boden}

Boden ist das Kompartiment, in dem eine intensive Umsetzung des Stickstoffs durch mannigfaltige Prozesse stattfindet, wodurch er zum Drehpunkt des N-Kreislaufes wird. Hier finden auch die Prozesse statt, die zu einer Fraktionierung von ${ }^{15} \mathrm{~N}$ und ${ }^{14} \mathrm{~N}$ führen.

Weltweit ist der Boden mit ${ }^{15} \mathrm{~N}$ angereichert. Fraktionierungsprozesse während der Mineralisation, Denitrifikation, Volatilisation, Aufnahme durch die Pflanzen und Mikroorganismen, Translokation und Assimilation verändern klein- und großräumig die Isotopenverhältnisse (Handley und Raven, 1992; Nadelhoffer und Fry, 1994; Shearer et al., 1974a, 1978, 1986, 1988a; Yoneyama, 1996). Diese Prozesse wiederum werden durch Bodenbearbeitung, Bodenstruktur, Wasserhaushalt, Mikrofauna und -flora, Temperatur, Niederschlag, Vegetation und ihren Streufall beeinflußt (Evans und Ehleringer, 1994; Focht, 1973; Handley und Raven,1992; Högberg et al., 1995; Nadelhoffer und Fry, 1988; Yoneyama, 1996).

\section{Stickstoffeintrag in den Boden}

Als N-Input in den Boden kommen zunächst die Deposition von $\mathrm{NH}_{4}{ }^{+}$und $\mathrm{NO}_{3}{ }^{-}$aus der Luft sowie die BNF in Frage. In den Südappalachen wurden $\delta^{15} \mathrm{~N}$-Werte für $\mathrm{NH}_{4}{ }^{+}$von -3.4 und für $\mathrm{NO}_{3}{ }^{-}+2.3$ gemessen (Garten, 1993).

Im Boden zeigt $\mathrm{NH}_{4}^{+}$überwiegend negative $\delta^{15} \mathrm{~N}$-Werte (Abb. 4), während für $\mathrm{NO}_{3}^{-}$auch häufiger positive Werte gefunden wurden.

Die $\delta^{15} \mathrm{~N}$-Werte des biologisch fixierten Stickstoffs bewegen sich zwischen 0 und -2 .

Industriell produzierter Dünger liegt in Bereichen von 0 bis +3 .

Aus der Vegetation wird Stickstoff in organischer Form durch Ernterückstände, Streufall und absterbende Organismen eingeführt. Diese Materialien sind im Vergleich zum Boden meistens ${ }^{15} \mathrm{~N}$-verdünnt (Sutherland et al., 1991 und 1993; Virginia et al., 1989). N-Einträge durch tierische Produkte hingegen steuern ${ }^{15} \mathrm{~N}$-angereicherte Substanzen bei (Hoering, 1955; Minami et al, 1995; Shearer et al. 1974a), denn die Nahrungskette führt zu einer sukzessiven Anreicherung der N-Verbindungen mit ${ }^{15} \mathrm{~N}$ (Minagawa und Wada, 1984). 


\section{Stickstoffaustrag aus dem Boden}

Durch partielle Verluste über Denitrifkation, Auswaschung oder Volatilisation kann der im Boden verbliebene Stickstoff mit ${ }^{15} \mathrm{~N}$ angereichert werden. Högberg und Johannison (1993) fanden eine mit zunehmenden N-Verlusten durch Volatilisation und Auswaschung aus einem Wald-Ökosystem positiv korrelierte ${ }^{15} \mathrm{~N}$-Anreicherung. Turner et al. (1983) ermittelte $\delta^{15} \mathrm{~N}$-Werte von -12 für $\mathrm{N}_{2}$, welches durch Denitrifikation entstand, und -45.7 für $\mathrm{NH}_{3}$ der Ammoniakverflüchtigung. Im verbliebenen Bodenstickstoff ermittelten Karamanos und Rennie (1980) und Karamanos et al. (1981) Werte zwischen +7 und +15 .

\section{Unterschied zwischen Gesamt-N und pflanzenverfügbarem $N$ des Bodens}

Sowohl die Ammonifikation als auch die Nitrifikation sind einer ${ }^{15} \mathrm{~N}$-Fraktionierung mit einem $\beta>1$ unterworfen (Mariotti et al., 1980a und 1982). Das resultierende $\mathrm{NH}_{4}{ }^{+}$und $\mathrm{NO}_{3}{ }^{-}$ (als Produkt) ist ${ }^{15} \mathrm{~N}$-verarmt und das verbliebene N (als Substrat) angereichert. So kommt es zu ${ }^{15} \mathrm{~N}$-verdünntem mineralisiertem $\mathrm{N}\left(\mathrm{N}_{\text {min. }}\right)$, während der Gesamt-N ( $\left.\mathrm{N}_{\text {tot. }}\right)$ höhere Werte annimmt. So erklären sich die schon von Cheng et al. (1964), aber auch von Binkley et al. (1985), Ledgard et al. (1984), Nadelhoffer und Fry (1988) und Vitousek et al. (1989) gemessenen 2 bis 4 Einheiten niedrigere $\delta^{15} \mathrm{~N}$-Werte des mineralischen $\mathrm{N}$ gegenüber dem $\mathrm{N}_{\text {tot. }}$ des Bodens.

${ }^{15} \mathrm{~N}$-Analysen des $\mathrm{N}_{\text {min. }}$ im Boden der Untersuchungsregion gaben ebenfalls Hinweise auf vom $\mathrm{N}_{\text {tot. }}$ verschiedene negative $\delta^{15} \mathrm{~N}$-Werte des pflanzenverfügbaren Stickstoffs. Paparcikova (1996) fand mit der Bodentiefe abnehmende $\delta^{15} \mathrm{~N}$-Werte, die zu $\delta^{15} \mathrm{~N}$-negativem $\mathrm{NH}_{4}{ }^{+}$ab einer Bodentiefe von $40 \mathrm{~cm}$ führten. In den obersten $40 \mathrm{~cm}$ jedoch war das mineralische $\mathrm{N}$ sogar mehr als das $\mathrm{N}_{\text {tot. }}$ mit ${ }^{15} \mathrm{~N}$ angereichert.

Das Ausmaß der Differenz zwischen $\mathrm{N}_{\text {tot. }}$ und $\mathrm{N}_{\text {min. }}$ ist von dem verfügbaren $\mathrm{N}$ des Bodens abhängig (Garten und van Miegrot, 1994). Ist wenig N im Boden verfügbar, wird der vorhandene Stickstoff häufiger umgesetzt und in der mikrobiellen Biomasse und den Pflanzen immobilisiert als in N-reicheren Böden. Der so zirkulierende $\mathrm{N}$ wird stärker ${ }^{15} \mathrm{~N}$-verdünnt als in einem Boden mit mehr verfügbarem $\mathrm{N}$ und einer höheren Nitrifikationsrate. 


\section{$\delta^{15} \mathrm{~N}$ im Bodenprofil}

Weltweit nimmt $\delta^{15} \mathrm{~N}$ mit der Bodentiefe zu. Dieser $\delta^{15} \mathrm{~N}$-Verlauf wurde von vielen Autoren beschrieben (Gebauer und Schulze, 1991; Ledgard et al., 1984; Mariotti et al., 1980b; Picollo et al., 1994a und 1996; Rennie et al., 1976; Shearer et al., 1978; Steele et al., 1981). Tiessen et al. (1984) und Ledgard et al. (1984) erklärten die Zunahme mit der Bodentiefe über eine Verlagerung der nach Abbauprozessen verbliebenen Humuspartikel. Feinere und somit stärker angereicherte Humuspartikel werden parallel zur Tonfraktion in tiefere Bodenschichten verlagert. Die gröberen, weniger intensiv abgebauten Partikel bleiben in den oberen Bodenschichten.

Chromatografische Prozesse können diesem Effekt entgegen wirken, indem ${ }^{15} \mathrm{NH}_{4}^{+}$eher an Ton und andere Kationenaustauscheroberflächen gebunden wird als ${ }^{14} \mathrm{NH}_{4}{ }^{+}$und dadurch eher ${ }^{14} \mathrm{~N}$ in tiefere Bodenschichten gelangt (Delwiche und Steyn, 1970; Karamanos und Rennie, 1978). Daher kann es unterhalb von 50cm Bodentiefe wieder zu einer Verminderung der $\delta^{15} \mathrm{~N}$-Werte kommen. Delwiche und Steyn (1970) und Karamanos und Rennie (1978) führen den Einfluß alluvialen Stickstoffs in tieferen Schichten mit geringen N-Konzentrationen als eine weitere Erklärung an, vermuten aber auch eine wenn auch schwache Immobilisierung ausgewaschenen ${ }^{15} \mathrm{~N}$ verdünnten Nitrates.

Negative $\delta^{15} \mathrm{~N}$-Werte werden in den obersten Bodenschichten selten gemessen (Bremner und Tabatabai, 1973; Michelsen et al., 1996; Nadelhoffer et al., 1996; Riga et al., 1971). Sie treten da auf, wo $\delta^{15} \mathrm{~N}$-negative Streu dominiert (Mariotti et al., 1980b), auf geologisch jungen Böden (Vitousek et al., 1989), einigen Waldböden (Broadbent et al., 1980; Gebauer et al., 1991 und 1994; Garten, 1993; Riga et al., 1971), wo Stickstoff hauptsächlich aus dem $\delta^{15} \mathrm{~N}$-negativen Niederschlag stammt (Wada et al.,1975) oder auf Böden mit Brandvorgeschichte (Herman und Rundel, 1989). Boden unter langjährigem Leguminosenbewuchs wies dem $\delta^{15} \mathrm{~N}_{\text {Luft }}$ nähere Werte auf als Boden, der nicht mit $\mathrm{N}_{2^{-}}$ Fixierern bewachsen war (Kessel et al., 1994; Peoples et al., 1991; Piccolo et al., 1994a).

\section{Auswirkung der Bodenbearbeitung auf den $\delta^{15} \mathrm{~N}$-Wert}

Die Art und Dauer der Bodenbearbeitung kann sich auf die $\delta^{15} \mathrm{~N}$-Werte im System auswirken. Boden unter natürlichen Systemen wie Wald oder auch Strauchvegetationen 
haben häufig niedrigere $\delta^{15} \mathrm{~N}$-Werte als Böden, die bearbeitet wurden (Bremner und Tabatabai, 1973; Peoples et al., 1991; Högberg und Alexander, 1995; Turner et al., 1987).

Wenn durch Kultivierungsmaßnahmen wie z.B. Ernten, Hacken oder Pflügen eine Vegetation zerstört wird, erhöht sich die mikrobielle Aktivität im Boden. Fraktionierungen während der Mineralisation führen zur Anreicherung der im Boden verbleibenden, stabileren organischen Substanz, während der mineralische Stickstoff von der Vegetation aufgenommen und mit der Ernte aus dem System entfernt wird (Turner et al., 1983; Mariotti et al., 1980b; Karamanos et al., 1981; Riga et al., 1971). Mit der Dauer dieser Art der Kultivierung reichert sich ${ }^{15} \mathrm{~N}$ immer mehr im Boden an (Nadelhoffer und Fry, 1988).

Im Amazonasgebiet fanden Piccolo et al. (1994 und 1996) niedrigere $\delta^{15} \mathrm{~N}-$ Werte unter Weiden als in benachbarten Böden mit Sekundärvegetation oder Primärvegetation, wo durch den Eintrag von $\mathrm{N}$ aus der Luft über die BNF eine Absenkung der $\delta^{15} \mathrm{~N}-$ Werte in der Vegetation und später über den Streufall im Boden folgte.

$\delta^{15} \mathrm{~N}$-Werte für Böden eines slash and burn-Systemes, wie die des Untersuchungsgebietes, werden sehr kontrovers dargestellt. Durch Brand und Kahlschlag oder auch andere Eingriffe kommt es nach einer kurzen Reduktion zu einem Anstieg der Mineralisationsprozesse im Boden (Herman und Rundel, 1989; Prieto-Fernandez, 1993). Vasquez et al. (1993) beobachteten für $1 \mathrm{Jahr}$ nach dem Brand einen Populationszuwachs aller aeroben heterotrophen Bakterien, während die Anzahl der Cyanobakterien, Pilze und Algen stark reduziert wurde. Solche Verschiebungen in der Mikroflora des Bodens bewirken eine Veränderung des $\delta^{15} \mathrm{~N}-$ Wertes, denn sie ändern auch die Bedeutung einzelner mikrobieller Prozesse.

Schulze et al. (1991) stellten einen Zusammenhang zwischen der Brandvorgeschichte einiger Standorte in Namibia und den erhöhten $\delta^{15} \mathrm{~N}$-Werten der untersuchten Böden her. In Australien fanden Pate et al. (1993) nach einem Vegetationsbrand in den $\mathrm{NO}_{3}{ }^{-}$aufnehmenden Pionierpflanzen ebenfalls höhere $\delta^{15} \mathrm{~N}$-Werte als in den anderen Arten und sehen dies als Hinweis für eine Anreicherung des $\mathrm{N}_{\text {min. }}$-Pools mit ${ }^{15} \mathrm{~N}$ nach einem Brand.

Im Widerspruch dazu stehen Ergebnisse von Herman und Rundel (1989). Sie verfolgten die Entwicklung der $\delta^{15} \mathrm{~N}$-Werte im $\mathrm{N}_{\text {min. }}$-Pool nach einem Brand in einem Inkubationsversuch. Nach der 1. Regenerationsphase entwickelten sich als erstes die Ammonifikanten und der Abbau der freigesetzten labilen organischen Substanz begann. Mit dem Einsatz der 
Nitrifikation erreichte $\mathrm{NO}_{3}^{-}$immer negativere $\delta^{15} \mathrm{~N}$-Werte (bis -11), und $\mathrm{NH}_{4}^{+}$immer positivere Werte (bis +15$)$.

\subsection{3 $\delta^{15} \mathrm{~N}$ in der organischen Auflage des Bodens}

Der $\delta^{15} \mathrm{~N}-$ Wert der organischen Auflage gilt als Bindeglied zwischen dem N in der Vegetation und dem $\mathrm{N}$ des Bodens.

In Eichenwäldern Wisconsins fanden Nadelhoffer und Fry (1988) eine Verdünnung des ${ }^{15} \mathrm{~N}$-Pools des $\mathrm{N}_{\text {tot }}$ durch den Laubfall. Sutherland et al. (1991 und 1993), Virginia et al. (1989) und Kessel et al. (1994) bestätigten dies für Weizenfelder bzw. Leucaena-Plantagen.

Vor dem Streufall finden Umlagerungsprozesse in den alten Blättern an der Pflanze statt (Buchmann et al., 1995; Domenach et al, 1992), trotzdem spiegelt sich in der frischen organischen Auflage der in der Vegetation vorherrschende $\delta^{15} \mathrm{~N}$-Wert wider.

Die auf den Blattfall folgenden biologischen und chemischen Prozesse beginnen mit unterschiedlicher Rate (Clinton et al., 1995; Kessel et al., 1994; Palm und Sanchez, 1990). In der älteren organischen Auflage finden sich die $\delta^{15} \mathrm{~N}$-Werte, die durch diese Vorgänge verändert wurden (Andreux et al., 1988). So fanden Turner et al. (1983) durch die Abbauprozesse Verschiebungen im organischen Material um durchschnittlich 12 Einheiten.

Die Art des organischen Materials beeinflußt die Entwicklung des $\delta^{15} \mathrm{~N}$-Wertes. So kommt es im Holz zu einer langsamen, in Fichtennadeln zu einer schnellen ${ }^{15} \mathrm{~N}$-Anreicherung, während sich z.B. das Laub von Birke und Buche nur sehr langsam und nur wenig mit ${ }^{15} \mathrm{~N}$ anreichert (Downs et al., 1996).

\subsection{4 $\delta^{15} \mathrm{~N}$ in den Pflanzen}

\section{Die Referenzpflanzen}

Für die Bestimmung des \%Ndfa werden nicht fixierende Referenzpflanzen benötigt, die den $\mathrm{zu}$ untersuchenden Leguminosen in Physiologie und Stoffwechsel ähneln und die gleiche Boden-N-Quelle nutzen. Von den $\delta^{15} \mathrm{~N}$-Werten dieser Referenzpflanzen hängt die Präzision der \%Ndfa-Bestimmung entscheidend ab (Domenach und Corman, 1984; Unkovich et al., 1994). 
In der Vegetation spiegeln sich die $\delta^{15} \mathrm{~N}$-Werte des pflanzenverfügbaren $\mathrm{N}$ des Bodens wider. Als N-Quelle dient der mineralische $\mathrm{N}\left(\mathrm{NO}_{3}{ }^{-}\right.$und $\left.\mathrm{NH}_{4}{ }^{+}\right)$sowie der biologisch fixierte $\mathrm{N}$ und der Stickstoff aus biologischer Fixierung, der über Transferwege im Boden in nicht fixierende Pflanzen gelangt. Unterschiedliche Aufnahmemechanismen, Durchwurzelungsstrategien und die Verteilung in der Pflanze tragen zu variierenden $\delta^{15} \mathrm{~N}$-Werten bei.

In natürlichen Systemen kann die Variabilität der $\delta^{15} \mathrm{~N}$-Werte in der Vegetation sehr groß sein und zu Fehlern in der \%Ndfa-Berechnung führen. So kann es durch die Anwendung verschiedener Referenzpflanzen zu unterschiedlichsten Abschätzungen des \%Ndfa kommen (Bremer et al., 1993; Danso et al., 1991; Ledgard et al., 1985; Pate et al., 1994; Sanginga et al., 1990a und 1996; Schöneberger et al., 1989;). Bei einer Wertedifferenz von beispielsweise nur $2 \delta^{15} \mathrm{~N}$-Einheiten zwischen der Leguminose und der Referenzpflanze für die Bestimmung des $\%$ Ndfa müssen die Schwankungen unter $0.2 \delta^{15} \mathrm{~N}$-Einheiten liegen, um eine $10 \%$ sichere Aussage machen zu können (Unkovich et al., 1994). Sanginga et al. (1996) fanden Unterschiede in der \%Ndfa-Berechnung von mehr als $20 \%$ in Abhängigkeit von der Referenzpflanze. Domenach et al. (1989) konnten keine exakten Fixierungsraten aufgrund der großen Streuung innerhalb der Vegetationen bestimmen. Högberg (1986) fand in Tanzania zwar stabile Differenzen zwischen Leguminosen und potentiellen Referenzpflanzen, konnte aber auf Grund der unterschiedlichen Wurzelsysteme keine quantitative Abschätzung geben.

Hier liegen noch große Probleme vor. Oft sind keine geeigneten Referenzen zur Hand und Methoden zu ihrer Erfassung fehlen (Awoneike et al., 1993; Hamilton et al., 1992). Bremer et al. (1993) schlugen hierzu Gewächshausversuche mit parallel angereicherten und natürlichen ${ }^{15} \mathrm{~N}$-Mengen vor, um darüber eine Selektion potentieller Referenzpflanzen vornehmen zu können. Oftmals wird eine Referenzpflanze ausgewählt, von der man Informationen über das Wurzelsystem, den N-Aufnahme - und Verteilmodus besitzt (Ladha, 1993; Liyanage et al., 1994; Shearer und Kohl, 1986 und 1988a). Für krautige Leguminosen diente dieselbe, aber nicht nodulierte Leguminose als Referenz. Fried et al. (1983) verwendete nicht nodulierte Sojabohnen neben Gerste und Sudangrass.

Für holzige Leguminosen gibt es die Möglichkeit, dieselbe Leguminose nicht-noduliert als Referenz einzusetzen, jedoch nur sehr eingeschränkt. Auf Feldern, die für das Fehlen von Franckia- bzw. bestimmten Rhizobium-Stämmen bekannt waren, konnten nicht-nodulierte Bäume der zu untersuchenden Leguminosen etabliert werden. Casuarina, Acacia und 
Leucaena-Arten wurden mit nicht-fixierenden Isolinien von Franckia und Rhizobium als Referenzen eingesetzt. Bei beiden Möglichkeiten ist jedoch eine nachträgliche Kontamination mit fixierenden Isolinien nicht auszuschließen, die zu Verfälschungen der Ergebnisse führen (Überblick in Sanginga et al., 1992). In Plantagen und agroforstlichen Systemen werden daher hauptsächlich Cassia (Senna)- oder Eucalyptus-Arten eingesetzt (Jonsson et al., 1996; Ladha et al., 1993; Parrotta et al., 1994; Peoples et al., 1996; Sanginga et al., 1990 und 1996;). Aber auch diese Arten können z.B. aufgrund von Wurzelexudaten inhibitorisch auf die BNF wirken, oder aber den Boden $-\delta^{15} \mathrm{~N}$-Wert verändern. In diesen Plantagen und Feldversuchen wurde meistens mit ${ }^{15} \mathrm{~N}$-Anreicherungen gearbeitet.

Die Vegetation der Bragantina-Region ist in ihrer Artenzusammensetzung sehr divers (Baar, 1997; Clausing, 1994; Denich, 1989). Das Wurzelsystem fast aller Arten ist bisher weitestgehend unbekannt, ebenso ihre Mykorrhizierung und physiologischen Besonderheiten. Die $\delta^{15} \mathrm{~N}$-Werte der Pflanzen sind bisher nicht bekannt. Daher beschäftigte sich die vorliegende Arbeit intensiv mit der Auswahl von Referenzpflanzen, dem zu beprobenden Pflanzenorgan, Standorten und Jahreszeiten.

\section{Abiotische Faktoren, die den $\delta^{15} N$-Wert in den Pflanzen beeinflussen}

Weltweit bewegen sich die $\delta^{15} \mathrm{~N}$-Werte der Blätter der Leguminosen zwischen -5 und +10 , für Nicht-Leguminosen zwischen -10 und +15 .

Ein räumliches Muster, daß sich auf Kontinente oder gar Regionen bezieht, gibt es nicht. Unterschiede der räumlichen Struktur finden sich in Abhängigkeit vom Ökosystem, Nährstoff- und Wasserverfügbarkeit, Bodenbearbeitung und Topographie.

Einen deutlichen Trend zu positiven $\delta^{15} \mathrm{~N}$-Werten fanden Wada et al. (1975) und Heaton (1987) entlang von Küsten, wo das über die marine Denitrifikation angereicherte Sprühwasser einen Einfluß auf die pflanzlichen $\delta^{15} \mathrm{~N}$-Werte nimmt. Die gleiche Wirkung üben Trockenheit und Salinität aus.

In Abhängigkeit vom geologischen Alter der Böden verändert sich die Isotopenzusammensetzung der Vegetation. Auf Hawaii können aufgrund der Vulkanaktivitäten verschiedene, im geologischen Alter unterschiedliche Böden untersucht werden (Vitousek et al., 1989). Vitousek fand auf geologisch jungen Böden negative $\delta{ }^{15} \mathrm{~N}$-Werte in der Vegetation, die sich mit zunehmendem Alter mit ${ }^{15} \mathrm{~N}$ anreicherten. Die 
$\delta^{15} \mathrm{~N}$-Werte der Vegetation waren auf Hawaii im Durchschnitt immer $4 \delta^{15} \mathrm{~N}$-Einheiten niedriger als der Bodenwert. In geologisch jungen Böden gibt es einen nur sehr kleinen $\mathrm{N}$-Pool, der über den $\delta^{15} \mathrm{~N}$-negativen Regen und die BNF langsam aufgefüllt wird. Erst mit der Entwicklung der Böden wird Stickstoff akkumuliert und Prozesse wie die Nitrifikation oder Denitrifikation setzen ein und führen über die Fraktionierung der N-Isotopen zu einer Anreicherung des Bodens mit ${ }^{15} \mathrm{~N}$.

Vegetationen, die häufigen Bränden unterliegen, zeigen ebenfalls auffällige $\delta^{15} \mathrm{~N}$-Werte. Virginia et al. (1989) fanden in dem mediterranen Chaparral System Kaliforniens ebenfalls negative $\delta^{15} \mathrm{~N}$-Werte, sowohl in der Vegetation als auch im Boden-N. Die Vegetation paßt sich an die für das Chaparral üblichen Brände an, indem sie tiefreichende Wurzelsysteme entwickelte. In tieferen Bodenschichten vermuten sie durch Auswaschung und Fraktionierungen negativere $\delta^{15} \mathrm{~N}$-Werte als im oberen Boden. Sprent et al. (1996) fanden im brasilianischen Cerrado ebenfalls negative $\delta^{15} \mathrm{~N}$-Werte wie auch Mordelet et al. (1996) in der Kapalga-Savanne Australiens.

Eine hohe räumliche Variabilität der $\delta^{15} \mathrm{~N}$-Werte auf einzelnen Standorten ist immer wieder ein Problem in natürlichen Vegetationen sowie landwirtschaftlich genutzten Flächen. Innerhalb von $2 \mathrm{~m}$ können Unterschiede von $2 \delta^{15} \mathrm{~N}$-Einheiten in Weizenkörnern auftreten, die sich aber auf größere Distanzen wieder ausgleichen (Sutherland et al., 1991). Bei ihrer Suche nach den Ursachen stießen Sutherland et al. (1991) auf Zusammenhänge mit der Topographie und dem Landschaftsmuster, die sie auf die biologische Aktivität im Boden, die $\mathrm{NO}_{3}^{-}$Verfügbarkeit, die Denitrifikation und die damit einhergehende ${ }^{15} \mathrm{~N}$-Fraktionierung zurückführten. In gleicher Weise erklärten sich Bremer und Kessel (1990) die Differenzen von $6 \delta^{15} \mathrm{~N}$-Einheiten über 40 bis $60 \mathrm{~m}$ in verschiedenen Kulturpflanzen. Garten (1993) führte die im Vergleich zur Bergkuppe erhöhten pflanzlichen $\delta^{15} \mathrm{~N}$-Werte in einer Talsohle der Süd-Appalachen auf eine erhöhte $\mathrm{NH}_{4}{ }^{+}$-Aufnahme der Pflanzen vom $\mathrm{N}_{\text {min. }}$-reicheren Talboden zurück. Im Talboden fand eine intensivere Nitrifikation statt, wodurch sich im Boden vergleichsweise mehr ${ }^{15} \mathrm{~N}$-angereichertes $\mathrm{NH}_{4}{ }^{+}$ansammelte.

Ein weiterer Faktor für die räumliche Variabilität ist die Bodenfeuchte, die die mikrobiellen Prozesse das Bodens und die Verfügbarkeit von Nährstoffen beeinflußt. Handley et al. (1994) konnten auf 4 kenianischen Standorten nur dann Unterschiede in der Vegetation finden, 
wenn auch unterschiedliche Bodenfeuchten vorlagen. Dann aber fanden sie herabgesetzte $\delta^{15} \mathrm{~N}-$ Werte für Arten, die auf Boden mit einem Acacia-Bestand wuchsen.

Ein für Primärwälder interessanter Aspekt ist die vertikale Verteilung von ${ }^{15} \mathrm{~N}$. Stewart et al. (1995) fanden auf 6 verschiedenen Primärwald-Standorten in Epiphyten immer niedrigere $\delta^{15} \mathrm{~N}$-Werte als in ihren Wirtsbäumen. Die N-Quelle könnte aus den Werten zu erkennen sein: Die Epiphyten scheinen einen Großteil ihres Stickstoffbedarfes aus luftfixiertem N und atmosphärischer Deposition, die Bäume hingegen aus bodenbürtigem N zu beziehen.

\section{Jahreszeiten}

Mikrobielle Prozesse des Bodens und die Biomasseakkumulation der Pflanzen verändern sich mit den Jahreszeiten und den jeweiligen Temperaturen, Bodenfeuchten und NVerfügbarkeiten (Nadelhoffer und Fry, 1994; Handley et al. 1994; Pate et al., 1994; Virginia, 1972). Saisonale Änderung der Wachstumsrate und des damit einhergehenden N-Bedarfs sind Ausdruck eines normalen biologischen Rhythmus. Damit kann das ${ }^{15} \mathrm{~N}-$ Vorkommen in einzelnen Arten während einer Wachstumsperiode je nach Klimaregion sehr unterschiedlich ausfallen.

Einige Autoren beobachteten eine Veränderung der $\delta^{15} \mathrm{~N}$-Werte in den Blättern mit der Jahreszeit. Virginia et al. (1989) wies für Prosopis glandulosa nach, daß sie ihren N-Bedarf im Frühjahr bei hoher Bodenfeuchte aus dem Oberboden deckte (die höheren $\delta^{15} \mathrm{~N}$-Werte entsprachen dem mineralischen $\mathrm{N}$ dieser Bodenschicht) und dann mit zunehmender Trockenheit auf $\mathrm{N}_{2}$-Fixierung und tiefere Bodenschichten mit niedrigeren $\delta^{15} \mathrm{~N}$-Werten zurückgriff. Bremer und Kessel (1990) konnten ein saisonales Muster in verschiedenen Getreidesorten und anderen Ackerpflanzen erkennen, bei dem der $\delta^{15} \mathrm{~N}-$ Wert in den Blättern mit der Aufnahme an mineralischem Stickstoff zunahm. Mögliche Änderungen treten mit Umlagerungsprozessen zur Blütezeit auf (Kessel et al., 1994). Garten und Miegrot (1994) wiesen eine zunehmende Diskrepanz zwischen den $\delta^{15} \mathrm{~N}$-Werten in den Blättern und dem $\mathrm{N}_{\text {tot }}$ des Bodens mit abnehmender Nitrifikationsrate - also N-Verfügbarkeit - nach.

In sommergrünen Baumarten fallen die N-Gehalte gegen Ende der Wachstumsperiode signifikant ab. Diese Tendenz war auch für die $\delta^{15} \mathrm{~N}$-Werte festzustellen, jedoch nur in sehr abgeschwächter, nicht signifikanter Form (Garten, 1993). 
Domenach et al. $(1989,1992)$ beobachtete keine deutliche saisonale Tendenz, dafür aber eine stärkere Streuung in Referenzpflanzen im Vergleich zu den $\mathrm{N}_{2}$-Fixierern. Trotz der geringen Saisonalität wurden die \%Ndfa von Alnus incana im Frühjahr überschätzt, da die $\delta^{15} \mathrm{~N}$-Werte am Anfang eines Jahres den Reservestoffen des Vorjahres glichen.

Um den Fehler durch die große Streuung zu umgehen, empfehlen Pate et al. (1994) Vergleichsbeprobungen zum Zeitpunkt der höchsten Biomasse- bzw. N-Aufnahme durchzuführen. $\mathrm{Zu}$ diesem Zeitpunkt ist die Differenz zwischen Leguminosen und Referenzpflanzen am größten.

\section{Unterschiede zwischen und innerhalb von Pflanzen}

In der Regel umfassen Leguminosen eine geringere Spannweite als die Nicht-Leguminosen und weisen ihrer N-Quelle entsprechend $\delta^{15} \mathrm{~N}$-Werte nahe 0 auf. Dieses Muster ist weltweit dasselbe, sofern die Leguminosen eine Symbiose mit den $\mathrm{N}_{2}$-fixierenden Knöllchenbakterien eingegangen sind. Fixieren sie jedoch keinen Stickstoff, so variieren ihre $\delta^{15} \mathrm{~N}$-Werte genauso, wie die der Referenzpflanzen. In Südafrika fand Stock (1995) in 2 AcacienMischwäldern einmal für alle Arten ähnliche $\delta^{15} \mathrm{~N}$-Werte vor und einmal in Leguminosen niedrigere als Nichtleguminosen. Er führt dies auf Fixierungsaktivitäten zurück, die im nährstoffarmen Sandboden, aber nicht im besser mit Nährstoffen versorgten Boden vorhanden sind. Vergleichbare Ergebnisse fanden Högberg und Alexander (1995) in einem Regenwald in Kamerun im Vergleich zu einem Miombowald in Zambia. Salati et al.(1982) erhielten in verschiedenen Waldökosystemen des Amazonasgebietes ebenfalls vergleichbare Ergebnisse in Abhängigkeit von der Nährstoffversorgung durch den Boden.

Durch die unterschiedliche Zusammensetzung der einzelnen Organe mit ihren jeweiligen $\mathrm{N}-$ Verbindungen entstehen unterschiedliche $\delta^{15} \mathrm{~N}$-Werte innerhalb der Pflanze (Yoneyama und Kaneko, 1989). Die Diskriminierung von ${ }^{15} \mathrm{~N}$ setzt sich bis in die feinsten Zellkompartimente fort. Bidigare et al. (1991) fanden z.B. höhere $\delta^{15} \mathrm{~N}$-Werte in den Lipidim Gegensatz zu den Chlorophyllmolekülen einer Pflanzenzelle.

Die Blätter werden als das repräsentative Organ eines Baumes für das Anzeigen des $\delta^{15} \mathrm{~N}$-Wertes der jeweiligen N-Quelle angesehen. Die ${ }^{15} \mathrm{~N}$-Gehalte in den einzelnen Organen einer Pflanze können aber sehr unterschiedlich sein. Innerhalb der Pflanze kann es Umlagerungsprozesse geben, die zu unterschiedlichen $\delta^{15} \mathrm{~N}$-Werten in z.B. neuen und alten 
Blättern oder holzigen Teilen führen (Gebauer et al., 1994; Mariotti et al., 1982; Nommik et al, 1994; Peoples et al., 1991; Domenach und Kurdali, 1989; Li et al., 1992). Dieser Effekt scheint aber in krautigen Pflanzen stärker ausgeprägt zu sein als in Bäumen (Peoples et al., 1991). Shearer et al. (1983) fanden im Stammholz von Prosopis glandulosa dennoch 3.4\% geringere $\delta^{15} \mathrm{~N}$-Werte als in den anderen Kompartimenten. In Leucaena leucocephala wurde eine sukzessive Anreicherung mit ${ }^{15} \mathrm{~N}$ vom Blatt bis zu den Wurzeln festgestellt (Sanginga et al., 1990b).

Arten lassen sich nach ihren N-Aufnahme-Modi unterscheiden (Pearson und Stewart, 1993). Einige Pflanzenarten bevorzugen $\mathrm{NH}_{4}{ }^{+}$. Dazu zählen langsam wachsend, perenne Arten, wie z.B. Koniferen, Erica-Gewächse, Eichen, Buchen oder Hainbuchen. $\mathrm{NH}_{4}{ }^{+}$aus dem Boden wird nicht im Xylem transportiert, sondern in der Wurzel via GS/GOGAT assimiliert und als Aminosäuren in die anderen Pflanzenorgane transportiert. Andere Arten assimilieren hauptsächlich $\mathrm{NO}_{3}{ }^{-}$in ihren Blättern. $\mathrm{Zu}$ ihnen gehören schnell wachsende Pionierpflanzen (z.B. Weizen, Gerste, Birke, Pappel, Holunder). Die Substrate $\mathrm{NH}_{4}{ }^{+}$und $\mathrm{NO}_{3}^{-}$haben unterschiedliche Anteile ${ }^{15} \mathrm{~N}$ und ${ }^{14} \mathrm{~N}$, so wirkt sich dies auch auf die Blätter der aufnehmenden Pflanzen aus.

Die Transportform innerhalb der Pflanzen kann unterschiedlich sein. Leguminosen z.B. transportieren den fixierten Stickstoff als Allantoinsäuren oder aber als Asparagin/Glutamin aus den Wurzeln in die einzelnen Kompartimente (Yoneyama et al., 1986). Die Arten, die Allantoinsäuren bilden, halten mehr ${ }^{15} \mathrm{~N}$ in den Knöllchen zurück und weisen damit negativere $\delta^{15} \mathrm{~N}$-Werte in ihren Blättern auf als die Arten, die $\mathrm{N}$ als Aminosäuren transportieren. Ledgard und Peoples (1988) ordnen die Baumleguminosen hauptsächlich dem Aminosäure-Transportmechanismus zu.

Schon bei der Aufnahme des Stickstoffs über die Wurzeln kann es zu einer ersten Veränderung der Isotopenzusammensetzung des Stickstoffs kommen. Reispflanzen sind z.B. dafür bekannt, bei der Aufnahme von $\mathrm{NH}_{4}^{+}$gegen ${ }^{15} \mathrm{~N}$ zu diskriminieren, was zu Blatt- $\delta^{15} \mathrm{~N}$-Werten unter denen des $\mathrm{NH}_{4}{ }^{+}$der N-Quelle führt (Yoneyama et al., 1991a). Vitousek et al. (1989) ermittelten unabhängig vom Standort in den Blättern von Metrosideros polymorpha, Myrtaceae, circa $4 \delta^{15} \mathrm{~N}$-Einheiten niedrigere Werte in den Blättern als im Boden. Solche Verschiebungen in der Isotopenzusammensetzung können durch die $\mathrm{NH}_{3}$-Volatilisation aus den Blättern wieder abgeschwächt werden (Freney und Simpson, 1983). 
Einen Zusammenhang der Wuchsform mit den $\delta^{15} \mathrm{~N}$-Werten in den Blättern konnten Virginia und Delwiche (1982) herstellen. Sie fanden eine Abnahme der $\delta^{15} \mathrm{~N}-$ Werte mit zunehmender Lebensdauer und Holzbildung, so daß annuelle Kräuter die höchsten $\delta^{15} \mathrm{~N}$-Werte zeigten. Für Leguminosen fanden Yoneyama et al. (1990) jedoch eher höhere Werte in den Bäumen als den Sträuchern. Sie begründeten dies mit dem Grad der BNF, denn Sträucher decken häufig einen größeren Teil ihres N-Bedarfs aus der Luft als Bäume.

Eine (vesikulär-) arbuskuläre Mykorrhiza ((V)AM), eine Ektomykorrhiza (ECM) oder Wurzelassoziationen haben eine Oberflächen vergrößernde Wirkung und fördern die Aufnahme von Nährstoffen, führen aber auch zu unterschiedlichen $\delta^{15} \mathrm{~N}$-Werten in den Pflanzen (Frey und Schüepp, 1992; Hamel et al, 1991; Haysted et al., 1988; Kessel und Roskosky, 1983; Mallarino et al., 1990a und b; Rao und Giller, 1993; Redmon et al., 1994; Yoneyama, 1987). Nicht immer gehen die gleichen Arten Symbiosen oder Assoziationen mit den gleichen Pilzen ein. So fand Högberg (1990) in Miombowäldern Tanzanias gegenüber (V)AM-Pflanzen um 1 bis $2 \delta^{15} \mathrm{~N}$-Einheiten erhöhte Werte für ektomykorrhizierte Pflanzen, in Zambia jedoch nicht (Högberg und Alexander, 1995). Handley et al.(1993) stellte eine unterschiedliche Verteilung des über ECM oder AM aufgenommenen Stickstoffs in der Pflanze fest, so daß eine mykorrhizierte Referenzpflanze für eine nicht-mykorrhizierte Leguminose $\mathrm{zu}$ falschen Berechnungen des \%Ndfa führen wird. Michelsen et al. (1996) erklärten unterschiedliche $\delta^{15} \mathrm{~N}$-Werte in Tundra-Pflanzen über ihren unterschiedlichen Mykorrhiza-Status (AM, erikoid, ECM), der den einzelnen Arten unterschiedliche N-Quellen des Bodens aufschließt. So konnte er auch einen Zusammenhang zwischen AM-infizierten Pflanzen, einer durch die verbesserte P-Verfügbarkeit erhöhten $\mathrm{N}_{2}$-Fixierung und den damit verbundenen niedrigen $\delta^{15} \mathrm{~N}$-Werten finden (Michelsen und Sprent, 1994). Högberg et al. (1994) und Handley et al. (1993) stellten die Zuverlässigkeit der Bestimmung der \%Ndfa mit Hilfe der ${ }^{15}$ NNAM daraufhin in Frage, denn eine Vergleichbarkeit der Pflanzen ist nicht gewährleistet.

Schließlich sei an dieser Stelle noch das Wurzelsystem der Pflanzen an sich erwähnt, das je nach der Tiefe ihrer Wurzeln unterschiedliche N-Quellen ausschöpfen und somit den $\delta^{15} \mathrm{~N}-$ Wert der oberirdischen Pflanzenteile mit beeinflussen kann (Mordelet et al., 1996; Virginia et al., 1989; Nadelhoffer et al., 1996) 


\section{A-Pflanze}

Für die Berechnung des $\%$ NdfA einer Leguminose muß man den $\delta^{15} \mathrm{~N}$-Wert derselben Leguminose kennen, wenn sie ihren N-Bedarf ausschließlich aus der Luft decken muß. Die Leguminosen werden zu diesem Zwecke im Gewächshaus ohne N-Quelle angezogen. Diese Pflanzen werden als A-Pflanzen bezeichnet.

Im Laufe der Nutzung der ${ }^{15}$ NNAM zur Bestimmung des \%Ndfa stellte sich heraus, daß die Knöllchen, in denen $\mathrm{N}_{2}$ fixiert wird, stark mit ${ }^{15} \mathrm{~N}$ angereichert waren und sich demzufolge in den Blättern der Leguminosen leicht negative $\delta^{15} \mathrm{~N}-$ Werte entwickelten (Domenach et al., 1988; Doughton et al., 1992; Högberg et al., 1994; Ledgard, 1989; Turner und Bergersen, 1983; Yoneyama, 1991b und 1986). Mit Hilfe der A-Pflanzen will man den Fehler in der $\%$ Ndfa-Berechnung vermeiden, der durch diese ungleiche ${ }^{15} \mathrm{~N}$-Verteilung auftritt. Das Spektrum an bisher gemessenen $\delta^{15} \mathrm{~N}$-Werten in den Blättern von A-Pflanzen reicht von $+2,6$ in krautigen $100 \%$ fixierenden Sojabohnen (Turner und Bergersen, 1983) bis zu -1.8 in Blättern einer 100\% fixierenden Erle (Domenach et al., 1988).

Generell sind die $\delta^{15} \mathrm{~N}$-Werte dieser A-Pflanzen unabhängig von der Wuchsform (Tab. 4a und b). Sie werden aber beeinflußt durch den die Knöllchen bildenden Bakterienstamm und das Stickstofftransportsystem der Pflanze.

Yoneyama et al. (1986) stellte einen Einfluß des Rhizobiumstammes auf die Fraktionierung in den Knöllchen von Phaseolus-Pflanzen fest, die zu Unterschieden von $1 \delta^{15} \mathrm{~N}$-Einheit führen kann. In Leucaena-Pflanzen waren Unterschiede aufgrund des Bakterienstammes jedoch nicht signifikant nachzuweisen (Sanginga et al., 1990).

Tab. 4a: $\delta^{15} \mathrm{~N}$ in den Blättern einiger A-Pflanzen von Baum und Straucharten

\begin{tabular}{lcccl}
\hline Art & Wuchsform & $\delta^{15} \mathrm{~N}$ & SE & Autor \\
\hline Alnus cordata & Baum & -1.9 & 0.2 & Kurdali et al., 1993 \\
Alnus glutinosa & & -2.0 & 0.2 & Kurdali et al., 1993 \\
Alnus glutinosa & & -1.9 & 0.4 & Domenach et al., 1988 \\
Alnus incana & -1.8 & 0.2 & Domenach et al., 1988 \\
Alnus rubra & -1.5 & 0.3 & Kurdali et al., 1993; \\
& & & & Binkley et al., 1985 \\
Prosopis glandulosa* & & -2.4 & & Virginia et al., 1989 \\
Prosopis spp. & -1.3 & 0.5 & Shearer et al., 1983 \\
\hline Aeschynomene indica & Strauch & -0.7 & & Yoneyama et al., 1991 \\
Senna (Cassia nomane) & & -0.95 & & Yoneyama et al., 1986 \\
Gliricidia sepium & & -1.45 & 0.02 & Ladha et al., 1993 \\
Sesbania rostrata & & -1.0 & & Yoneyama et al., 1991 \\
\hline
\end{tabular}


Je nach der Art des N-Transfers aus der Wurzel in die oberen Organe der Pflanze, kommt es zu negativen Werten in den Pflanzenorganen und positiven Werten in den Knöllchen (Shearer et al., 1980; Yoneyama, 1987; Yoneyama et al., 1991 und 1993a; Peoples et al., 1987 und 1989). Knöllchen der Arten, die den Stickstoff als Allantoinsäuren transportieren, sind hoch, solche mit dem Aminosäure-Transportsystem weniger hoch angereichert. Die ${ }^{15} \mathrm{~N}$-Akkumulation kann bis zu $12 \%$ o betragen (Yoneyama, 1987). Dabei kommt es innerhalb der Knöllchen wiederum zu einer Aufteilung. Turner und Bergersen (1983) fand im Bakteroid $\delta^{15} \mathrm{~N}+16.5$, im löslichen Protein +7.2 .

Tab. 4b: $\delta^{15} \mathrm{~N}$ in den Blättern einiger A-Pflanzen von krautigen Arten

\begin{tabular}{|c|c|c|c|c|}
\hline$A r t$ & $\begin{array}{l}\text { Wuchsfor } \\
\mathrm{m}\end{array}$ & $\delta^{15} \mathrm{~N}$ & SE & Autor \\
\hline Arachis hypogea & Kraut & -0.7 & 0.3 & Yoneyama, 1987 \\
\hline Astragalus sinicus & & -0.89 & 0.22 & Yoneyama et al., 1986 \\
\hline Centrosema pubescens & & -2.48 & & Yoneyama et al., 1986 \\
\hline Desmodium intortum & & -1.84 & & Yoneyama et al., 1986 \\
\hline Glycine $\max$ & & -1.45 & & Yoneyama et al., 1986 \\
\hline Glycine $\max$ & & +1.55 & 0.44 & Turner und Bergersen, 1983 \\
\hline Glycine max & & -1.5 & 0.4 & $\begin{array}{l}\text { Amarger et al., } 1979 \text {, } \\
\text { Shearer et al., } 1980\end{array}$ \\
\hline Glycine $\max$ & & -1.6 & & Yoneyama, 1987 \\
\hline Lotus pedunculatus & & -0.1 & 0.3 & Steele et al., 1983 \\
\hline Lupinus luteus & & -0.9 & 0.1 & Amarger et al., 1979 \\
\hline Lupinus luteus & & +4.20 & 0.62 & Turner und Bergersen, 1983 \\
\hline Lupinus angustifolium & & -0.2 & & Unkovich et al., 1994 \\
\hline Macroptilium & & -2.56 & & Yoneyama et al., 1986 \\
\hline \multicolumn{5}{|l|}{ atropurpureum } \\
\hline Medicago sativa & & +3.69 & 0.65 & Turner und Bergersen, 1983 \\
\hline Medicago sativa & & -0.92 & & Mariotti et al., 1980 \\
\hline Medicago sativa & & +0.97 & 0.13 & Ledgard, 1989 \\
\hline Medicago sativa & & +3.56 & 0.65 & Turner und Bergersen, 1983 \\
\hline Medicago sativa & & -0.82 & 0.26 & Yoneyama et al., 1986 \\
\hline Pisum sativum & & -0.3 & & Unkovich et al., 1994 \\
\hline Prophocarpus tetragonolobus & & -1.54 & 0.36 & Yoneyama et al., 1986 \\
\hline Stylosanthes humilis & & -0.28 & & Yoneyama et al., 1986 \\
\hline Trifolium hybridum & & -0.7 & 0.03 & Yoneyama et al., 1986 \\
\hline Trifolium medicago & & +2.83 & 1.06 & Turner und Bergersen, 1983 \\
\hline Trifolium pratense & & -0.36 & 0.32 & Yoneyama et al., 1986 \\
\hline Trifolium pratense, & & -0.88 & & Mariotti et al., 1980 \\
\hline Trifolium pratense, & & -1.07 & & Yoneyama et al., 1986 \\
\hline Trifolium repens & & +0.58 & 0.12 & Ledgard, 1984 \\
\hline Trifolium repens & & -1.9 & 0.2 & Steele et al., 1983 \\
\hline Trifolium repens & & -0.98 & & Yoneyama et al., 1986 \\
\hline Trifolium repens & & -1.18 & 0.08 & Ledgard, 1989 \\
\hline Trifolium repens & & -0.38 & 0.53 & Yoneyama et al., 1986 \\
\hline Trifolium subterraneum & & +2.58 & & Turner und Bergersen, 1983 \\
\hline Trifolium subterraneum & & -1.14 bis & +0.06 & Turner und Bergersen, 1983 \\
\hline Trifolium subterraneum & & -0.57 & & Unkovich et al., 1994 \\
\hline Trifolium subterraneum & & +0.59 & 0.11 & Ledgard, 1989 \\
\hline Trifolium spp. (mehrere Arten) & & -0.3 bis & -0.9 & Ledgard, 1989 \\
\hline Vicia sativa & & -0.79 & & Yoneyama et al., 1986 \\
\hline Vicia villosa & & -0.20 & 0.21 & Yoneyama et al., 1986 \\
\hline
\end{tabular}


Wie wichtig es ist, für jede Art unter den entsprechenden Versuchsbedingungen A-Pflanzen als Referenz heranzuziehen, veranschaulicht der Vergleich der $\delta^{15} \mathrm{~N}$-Werte der gleichen Art, von verschiedenen Arbeitsgruppen kultiviert (Tab. 4 b). Hier haben Nährlösung, Temperatur und Wasserhaushalt ihren Einfluß und können eine Differenz von bis zu $2 \delta^{15} \mathrm{~N}$-Einheiten verursachen. Mangelversorgung mit z.B. Molybdän, Phosphor oder Wasser führen zu negativeren $\delta^{15} \mathrm{~N}$-Werten in den Pflanzen als unter optimalen Bedingungen (Ledgard, 1989). 


\section{Zielsetzung}

Die Abschätzung von $\mathrm{N}_{2}$-Fixierungsraten mit Hilfe der ${ }^{15} \mathrm{~N}$ natural abundance Methode baut auf minimale Differenzen in den Atom $\%{ }^{15} \mathrm{~N}$ auf. Räumliche und jahreszeitlich bedingte Schwankungen, die Umsetzungs- und Auswaschungsprozesse im Boden, pflanzeninterne Stoffwechsel- und Transportprozesse, unterschiedliche Wurzelsysteme und Wuchsformen nehmen Einfluß auf die Isotopenzusammensetzung der Pflanzen.

Die Vielzahl der in Kap. II erläuterten Möglichkeiten der Beeinflussung des $\delta^{15} \mathrm{~N}$-Wertes macht deutlich, daß es entscheidend ist, welche Referenzpflanze, welches Kompartiment, welche Standorte und Jahreszeiten für die Bestimmung der $\mathrm{N}_{2}$-Fixierungsrate (\%Ndfa) gewählt werden. Für das natürliche System der Sekundärvegetation der Bragantina-Region lagen bisher keine Erfahrungen bezüglich dieser Faktoren vor. Daher war Ziel der vorliegenden Arbeit, im Vorfeld der Anwendung dieser Methode Informationen über die Isotopenverteilung in Boden und Vegetation und ihre möglichen Ursachen (Bodenbearbeitung, Fraktionierungsprozesse, pflanzenverfügbarer N, Pflanzenhabitus), die Variationsbreite der $\delta^{15} \mathrm{~N}$-Werte zwischen und innerhalb der zu untersuchenden Pflanzen und die für die Berechnung der \%Ndfa notwendigen A-Pflanzen zu sammeln.

Unter Berücksichtigung der gewonnenen Informationen sollte abschließend eine Abschätzung der $\mathrm{N}_{2}$-Fixierungsraten einiger Leguminosen in der Sekundär- und Primärvegetation der Bragantina-Region gegeben werden. 


\section{Untersuchungsgebiet}

\section{Geographische Lage}

Die Feldbeprobungen der vorliegenden Arbeit wurden in der Mikroregion Bragantina im Bundesstaat Pará, Brasilien durchgeführt (Abb. 5). Sie ist Teil der früheren „Zona Bragantina“, die die Großstadt Belém mit einschloß. Diese Region ist zwischen $0^{\circ} 45^{\prime}$ und $1^{\circ} 39^{\prime}$ südlicher Breite und $46^{\circ} 16^{\prime}$ und $48^{\circ} 15^{\prime}$ 'westlicher Länge lokalisiert).

Der Großteil der beprobten Standorte befand sich in der Nähe der Kleinstadt Igarapé Açu, circa $100 \mathrm{~km}$ ostnordöstlich von Belém, ein weiterer Teil in der Nähe der Kleinstädte Peixe Boi, 150 km östlich von Belém, und Iracema, 70 km ONO von Belém. Igarapé Açu wurde 1897 das erste Mal als Siedlungsschwerpunkt erwähnt.

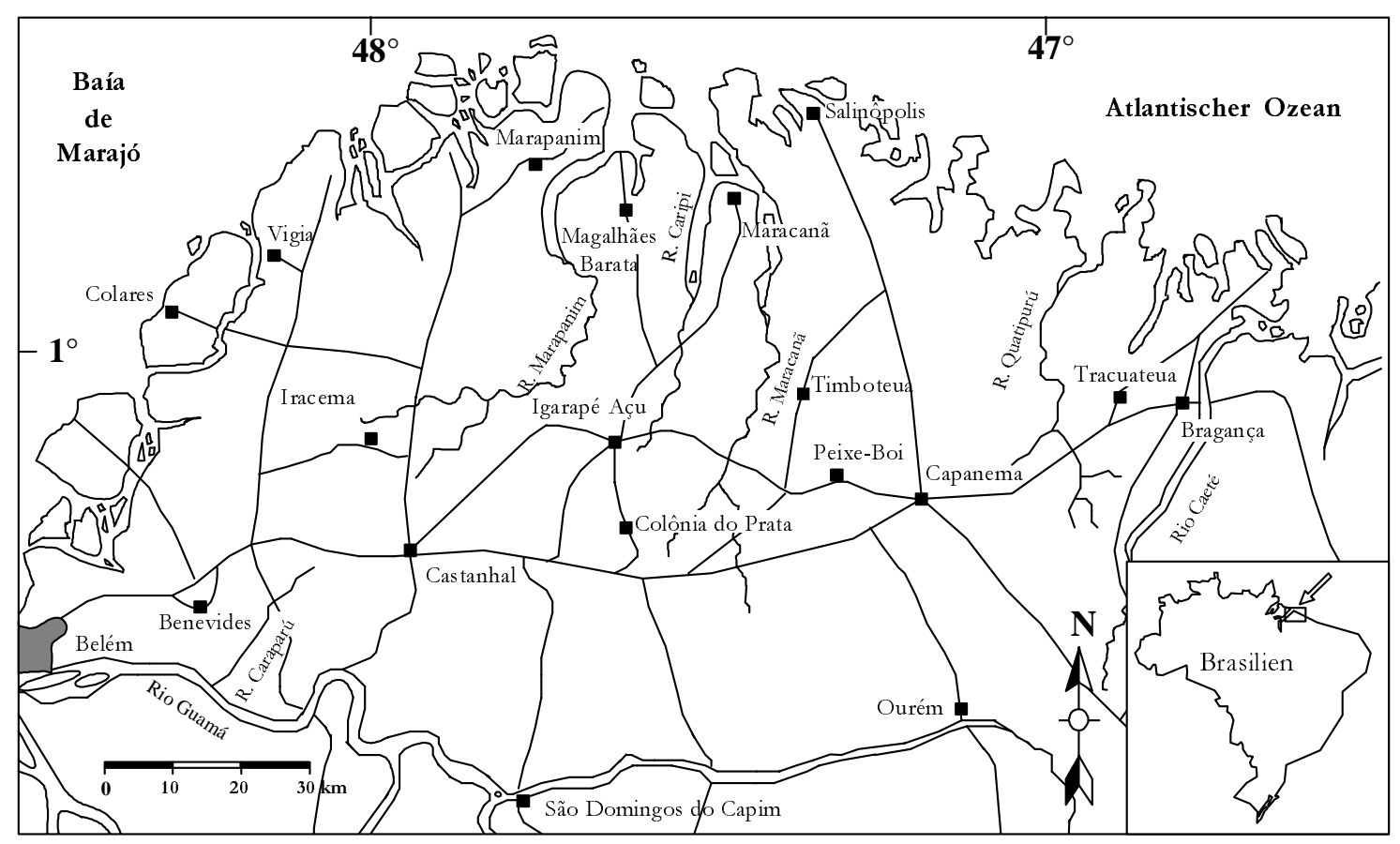

Abb. 5: Lage des Untersuchungsgebietes Bragantina im Nordosten von Pará, Brasilien (nach Denich, 1989)

Eine hohe Geburtenrate und die Dürrekatastrophen in anderen Teilen Brasiliens führten im Laufe der letzten 20 Jahre zu einer Verdoppelung der Bevölkerungsdichte auf 40.8 Einwohner pro $\mathrm{km}^{2}$ (IDESP, 1990). 


\section{Das Klima}

Nach Walter (1977) liegt die Bragantina-Region im Zono-Ökoton I/II (halbimmergrüner tropischer Regenwald) zwischen Zonobiom I (äquatoriales Tageszeitenklima mit immergrünem tropischen Regenwald) und Zonobiom II (tropisches Sommerregengebiet mit laubabwerfenden Wäldern). Dieses Gebiet ist durch weitgehend konstante Temperaturen, aber stark schwankende Niederschläge geprägt. Je nach Ausprägung der niederschlagsarmen Trockenzeit tendiert das Klima mehr zu Zonobiom I bzw. II. Von Belém nach Osten hin nimmt die Dauer der Trockenzeit zu. An dem Hauptstandort des Projektes nahe Igarapé Açu kommt es zu einer regenarmen Periode von mehr als 2 Monaten, die zu Trockenstreß der Kulturpflanzen führen kann. Die Klima-Diagramme nach Walter (Abb. 6) geben einen Überblick über die vorherrschenden Bedingungen in der Bragantina-Region.
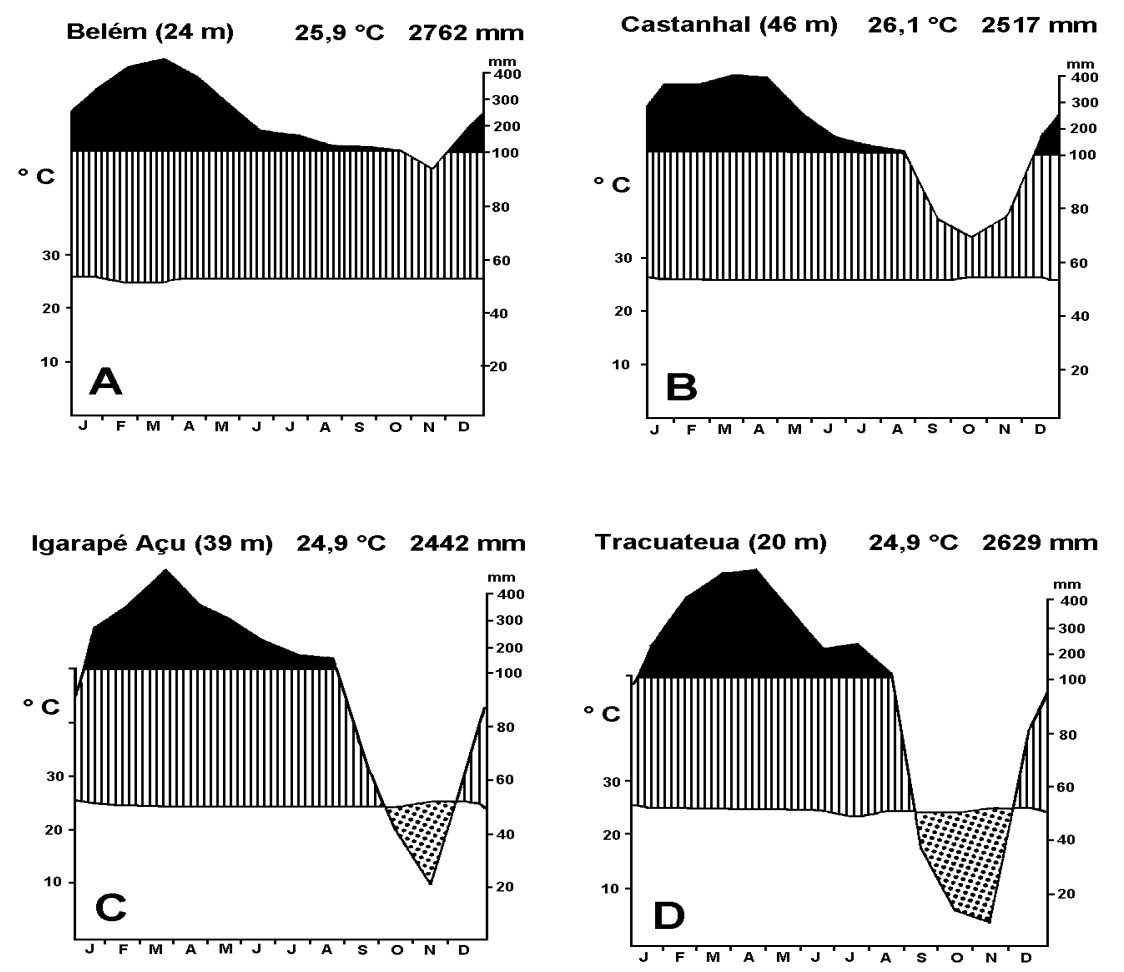

Abb. 6: Klimadiagramme nach Walter von Belém (A), Castanhal (B), Igarapé Açu (C) und Tracuateua (D) (Denich, 1989)

Nach Diniz (1986) beträgt die mittlere Jahrestemperatur $25-26^{\circ} \mathrm{C}$, die mittlere jährliche Niederschlagsmenge 2000-3000 $\mathrm{mm}$ und die mittlere jährliche Sonnenscheindauer 2200-2400 Stunden. Im langjährigen Mittel (1977-1987) wurde an einer Station in der Nähe von Castanhal, zwischen Belém und Igarapé Açu, an 16.1\% aller Tage Südost und an 76.1\% aller Tage Nordost als Hauptwindrichtung ermittelt (Boletim Agrometeorológico, 1977- 
1987). Im Untersuchungszeitraum wurde an einer Station in unmittelbarer Nähe der Beprobungsstandorte in der Umgebung von Igarapé Açu von Hölscher (1995) folgende Daten erhoben (Abb. 7):

Mittlere Jahrestemperatur:

Mittleres tägliches Minimum:

Mittleres tägliches Maximum:

Mittlerer Jahresniederschlag:

Regenärmste Monate:

Regenreichster Monat:

Mittlere jährliche Sonnenscheindauer: $26.6^{\circ} \mathrm{C}$

$20.9^{\circ} \mathrm{C}$

$31.8^{\circ} \mathrm{C}$

$2298 \mathrm{~mm}$

September bis Dezember 1992, unter 50mm

April 1993, 455mm

2213.6

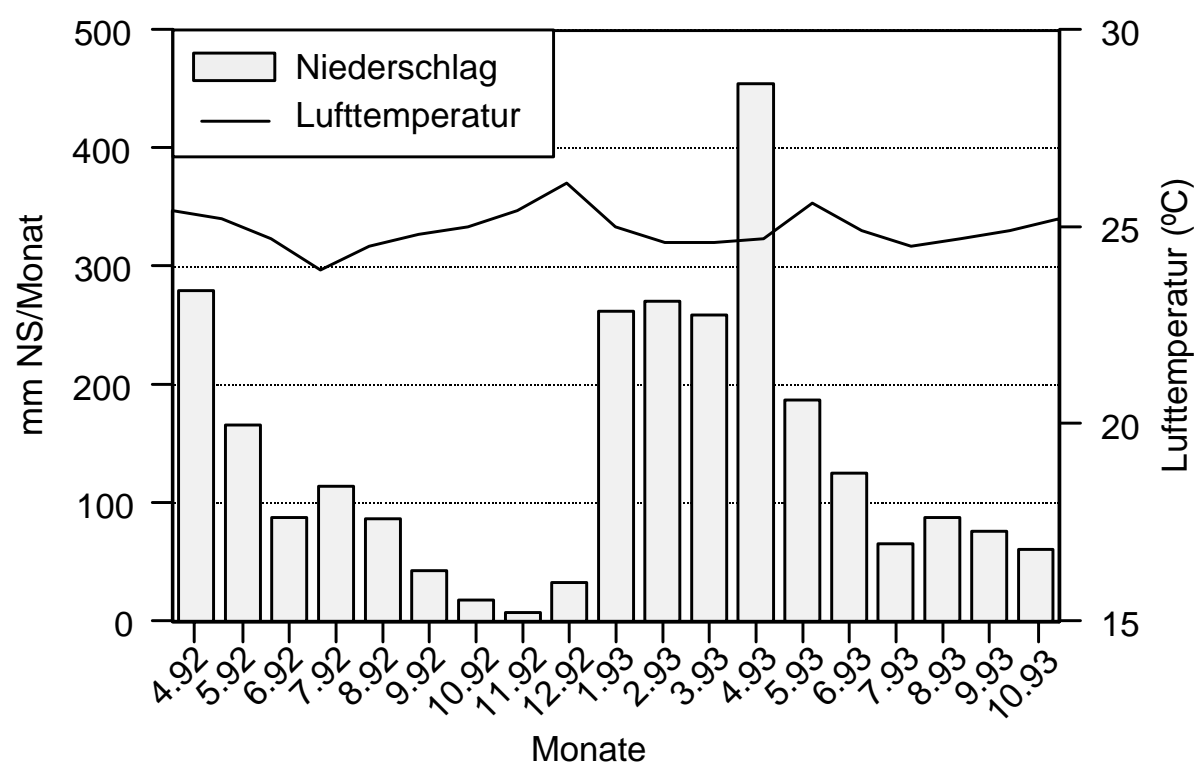

Abb. 7: Monatlicher Niederschlags - und Temperaturverlauf für den Untersuchungszeitraum auf einer Meßstation in unmittelbarere Nähe der Beprobungsflächen in der Umgebung von Igarapé Açu (Daten von Hölscher, 1995)

Die monatlichen Niederschläge des Untersuchungszeitraumes lagen generell unter dem langjährigen Mittel, bis auf April 1993, der mit seinen extremen Regenfällen sogar das langjährige Mittel übertraf (Abb. 7). 


\section{$3 \quad$ Die Böden}

Die Bragantina-Region liegt durchschnittlich 30m über NN, übersteigt 60m über NN aber nicht. Damit ist ihr Relief flach bis leicht gewellt. Die Böden entwickelten sich aus Süßwassersedimenten im Plio- bis Pleistozän. Diese Sedimente bestanden hauptsächlich aus Granit-, Gneis- und Sandsteinverwitterungsprodukten des guyanischen und brasilianischen Schildes (Barreiras-Sedimente) (Francisco et al., 1971). Zu dieser Zeit war die gesamte Amazonassenke von einem Binnensee bedeckt. Am Ende des Miozäns kam es zu einer starken Auffaltung des Andengebirges und gleichzeitigen Verschiebung der Kontinentscholle, die die Entwässerung in den Atlantik nach sich zog. Der trockengefallene Seegrund wurde zum Gebiet der rezenten terra firme Böden. Aufgrund des Ausgangsmaterials handelt es sich hier um ausgesprochen nährstoffarme Böden (Sioli, 1968).

Ein Drittel der Böden der Bragantina-Region ist von einer sog. Pirabas-Formation unterlegt, eine Kalkstein-Sand-Ton-Ablagerung marinen Ursprungs. In der Regel von o.g. Sedimenten überlagert, tritt sie an einzelnen Stellen an die Oberfläche. Auf solchen Flächen ist die Versorgung der Böden mit Spurenelementen deutlich verbessert (nach Denich, 1989).

Die Böden der Projektregion werden nach der USDA Soil Taxonomy als Oxisole oder Ultisole, nach dem brasilianischen „sistema brasileiro de classificacao de solos“ als Latossolos amarelos oder Podzólicos amarelos eingeordnet (Lenthe und Cordeiro, 1985). Sie zeichnen sich durch ein sehr weites Verhältnis im Tongehalt von Horizont B zum Horizont A (B/A; abrúptico) aus. Nach Schachtschabel et al. (1989) weisen diese für die feuchten Tropen typischen, aus Silikatgestein entwickelten Böden einen tiefgründig verwitterten B-Horizont auf, dessen Tonfraktion hauptsächlich aus Kaolinit sowie Al- und Fe-Oxiden besteht. Sie fixieren aufgrund des hohen Oxidgehaltes Phosphat, wodurch dieses Element häufig wachstumslimitierend ist (Schmidt-Lorenz, 1986). Die Al-Sättigung beträgt über $50 \%$. Bei niedrigen $\mathrm{pH}-$ Werten kann es zu toxischen Konzentrationen dieses Elementes kommen. Der Boden zeichnet sich weiterhin durch eine geringe Austauschkapazität, Nährstoff- und Basenarmut, hohe Infiltrationsrate und geringe Wasserhaltekapazität aus. Mit starken Auswaschungsprozessen muß gerechnet werden. Seine Dichte liegt durchschnittlich bei $1.40 \mathrm{~g} / \mathrm{cm}^{3}$.

Ausführlichere Bodenbeschreibungen der Projektregion sind in den Arbeiten von Bigarella und Ferreira (1985), Rego et al. (1993), Rosetti et al.(1990) und Sioli (1968) nachzulesen. 


\section{Die Wald-Feld-Wechselwirtschaft}

Das heutige Landschaftsbild der Bragantina-Region wird durch die kleinbäuerliche Wald-Feld-Wechselwirtschaft mit Brandrodung bestimmt. Drei Viertel dieser Umgebung ist von verschiedenen Sukzessionsstadien des Sekundärwaldes, der Brachevegetation bzw. Sekundärvegetation (regional Capoeira genannt), bedeckt (Watrin, 1994) (Abb. 8).

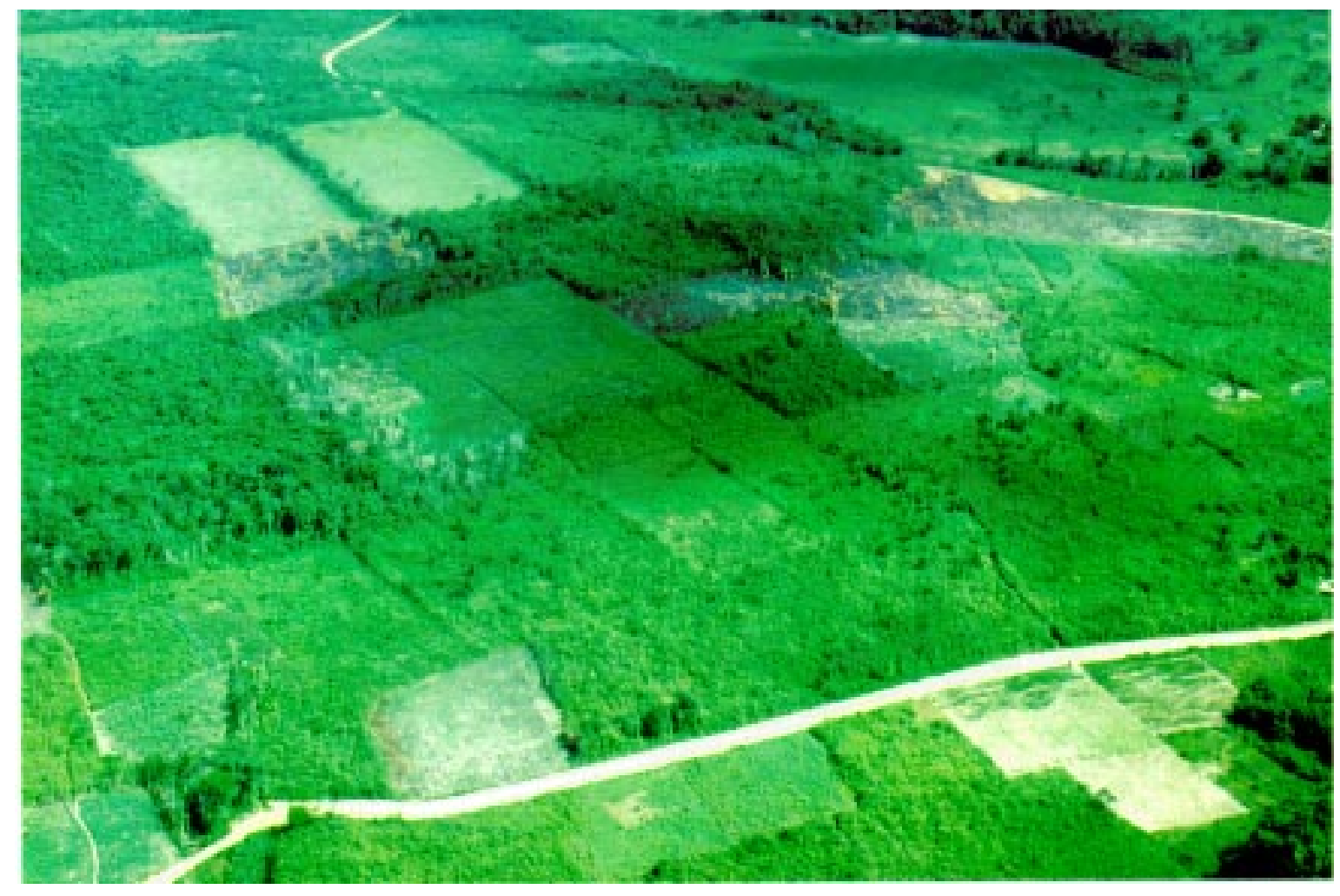

Abb. 8: Luftaufnahme der Umgebung von Igarapé Açu. Zu erkennen sind verschiedene Stadien des Sekundärwaldes, Maniokfelder, Maracujá-Plantage und Igapó (Flußwald)

Nur noch 5.3\% der Fläche entlang der Flußläufe sind mit ursprünglichem Primärwald bedeckt, der jedoch auch stark exploitiert wurde (Watrin, 1994) (Abb. 9). Es handelt sich dabei meistens um sog. Igapó Wälder, die auf überflutetem Boden stehen und somit für die landwirtschaftliche Nutzung ausscheiden. 21.5\% der Gesamtfläche wird ackerbaulich genutzt. Dabei wird diese Fläche zur Zeit von der Weidewirtschaft dominiert (74.5\%). Ein kleinerer Teil (6.4\%) wird mit annuellen (Maniok - Manihot esculentes, Trockenreis - Oryza sativa, Mais - Zea mays) und Bohnen - Vigna unguiculata) und semi-perennen Kulturen wie Pfeffer (Piper nigrum), Obstbau (Cupuacu - Theobroma grandiflorum, Papaya - Carica papaya, Citrusfrüchten - Citrus spp., Maracujá - Passiflora edulis, Murucí - Byrsonima 
crassiflora, Cashew - Anacardium occidentale) und 3.8\% mit der permanenten Kultur Ölpalme (Elaeis guianensis) bebaut.

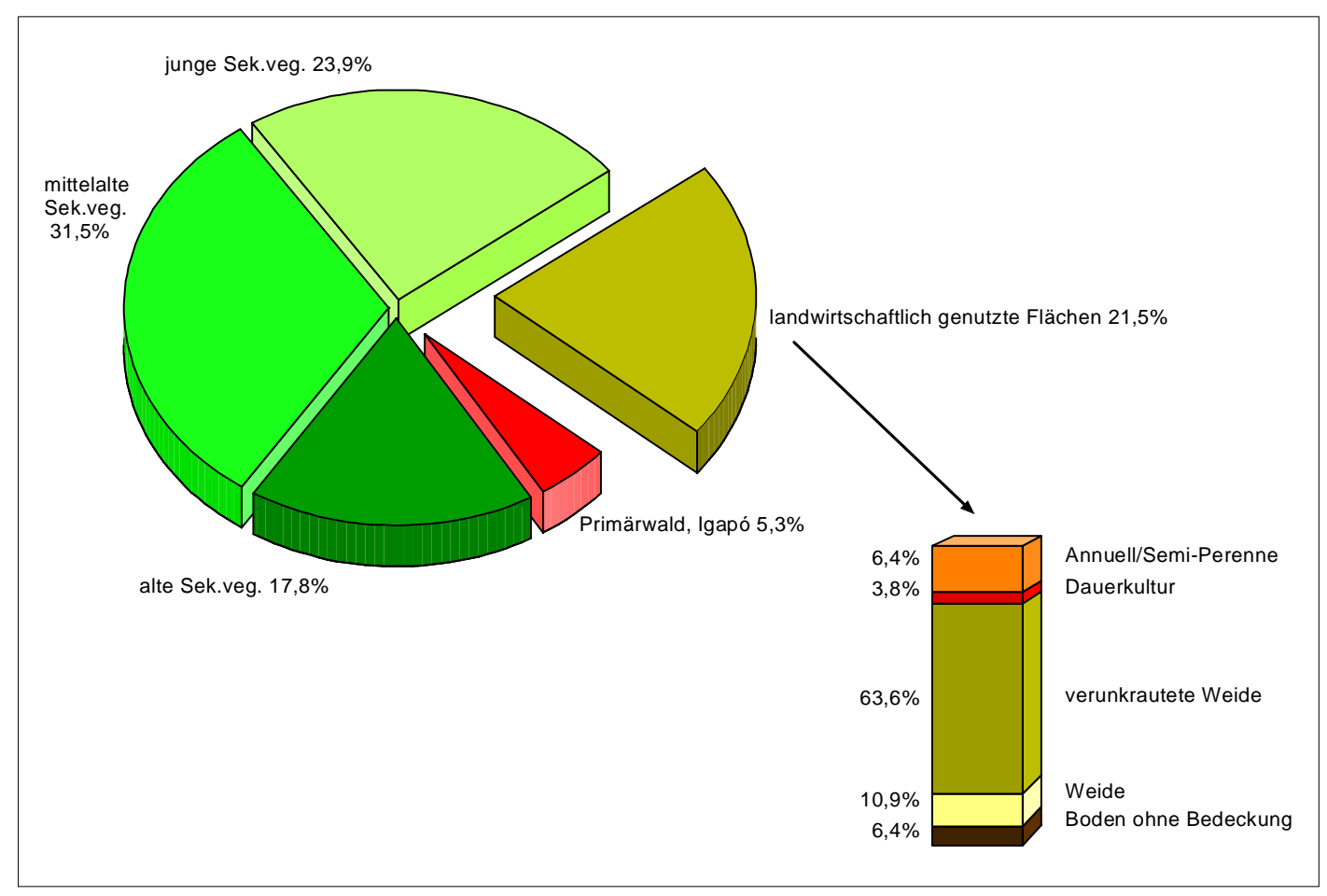

Abb. 9: Flächenanteil der verschiedenen Vegetationsdecken an der Gesamtfläche des Munizips Igarapé Açu im Jahre 1991 (nach Watrin, 1994)

Der einzelne Besitz eines Kleinbauern erstreckt sich meistens auf weniger als 100ha (im Durchschnitt 20-40ha). Mit 97\% aller landwirtschaftlichen Betriebe halten diese Kleinbauern aber 74\% der bewirtschafteten Fläche der Mikroregion (Denich und Kanashiro, 1995). Im Oktober, zu Beginn der Trockenzeit, wird ein kleiner Teil der Vegetation gerodet und nach einer Phase der Trocknung gebrannt (Abb. 10). Mit Beginn der Regenzeit wird dann als erstes Mais, bei guter Einschätzung der Bodenqualität auch Reis gepflanzt, gefolgt von Bohnen und/oder Maniok. Bohnen werden in der Regel mit einer geringen NPK-Menge im Pflanzloch gedüngt. Der Maniok wird sukzessive geerntet, die Unkrauthackungen werden etwa 3 Monate vor der letzten Ernte eingestellt. Die Wiederbesiedlung durch die Sekundärvegetation, die heute nach 3-7 Jahren wieder geschlagen und gebrannt wird, beginnt. Die Arbeiten werden im Familienbetrieb durchgeführt. Die Produktion reicht zum Erhalt des eigenen Lebensunterhaltes und einem geringen Einkommen über den Maniok- und Maracujá-Verkauf. 


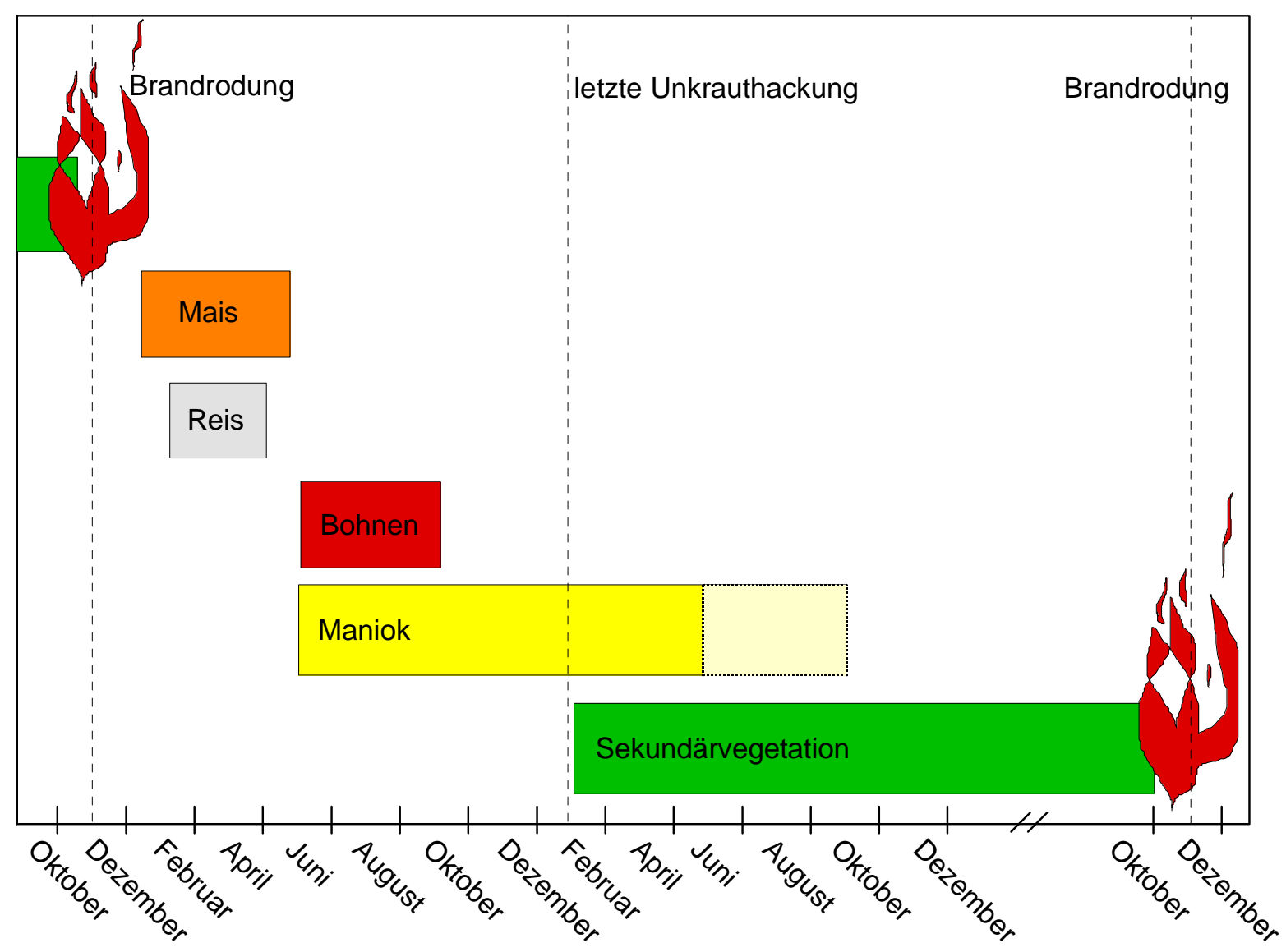

Abb. 10: Beispiel eines Nutzungszyklus der kleinbäuerlichen Wald-Feld-Umlagewirtschaft. Die Kulturphase beträgt 2 Jahre, die Brachephase mit Sekundärvegetation beträgt 3-7 Jahre. 


\section{Material und Methoden}

\section{$1 \quad$ Feld}

\subsection{Untersuchte Flächen}

Als Probeflächen wurden Brachevegetationen mit weitgehend identischer Nutzungsgeschichte ausgewählt. Die Flächen befanden sich hauptsächlich in der näheren Umgebung Igarapé Açus, zur Primärwaldbeprobung mußte auf Peixe Boi, circa 40 km östlich von Igarapé Açu ausgewichen werden. Eine Fläche befand sich in Iracema, circa $50 \mathrm{~km}$ westlich von Igarapé Açu. Die Lage der Flächen in und um Igarapé Açu ist auf der Karte in Abb. 11 eingezeichnet. Tab. 5 subsumiert die Nutzung und Vorgeschichte der Flächen, wie es von den Besitzern vermittelt wurde.

Tab. 5: Nutzungsvorgeschichte und Bezeichnung der Probeflächen $(M=$ Flächen, auf denen monatlich beprobt wurde, $S$ = Flächen, auf den zur Erfassung der räumlichen Variabilität beprobt wurde)

\begin{tabular}{|c|c|c|c|c|c|c|}
\hline Fläche & $\begin{array}{l}\text { letzte } \\
\text { Brandro- } \\
\text { dung }\end{array}$ & $\begin{array}{l}\text { Alter } \\
\text { der } \\
\text { Cap. }\end{array}$ & Düngung & Vorkulturen & $\begin{array}{c}\text { Alter der } \\
\text { vorherigen } \\
\text { Capoeira }\end{array}$ & $\begin{array}{l}\text { Abkürzung } \\
\text { der Flächen }\end{array}$ \\
\hline Ramal do Prata, km 10 A & 1986 & 5 & nein & Mais, Maniok & >30 Jahre & M1 S1 \\
\hline Ramal do Prata, km 10 B & 1984 & 7 & nein & $?$ & $>28$ Jahre & M2 S1 \\
\hline Igarapé Açu, Travessa 10 & 1986 & 5 & nein & $\begin{array}{l}\text { Baumwolle, } \\
\text { Maniok }\end{array}$ & $>30$ Jahre & M3 S3 \\
\hline Igarapé Açu, Travessa 12 & 1986 & 5 & nein & $\begin{array}{l}\text { Baumwolle, } \\
\text { Maniok }\end{array}$ & & M4 \\
\hline Curi, Travessa 14 & 1985 & 6 & nein & Maniok & $>25$ Jahre & M5 S5 \\
\hline $\begin{array}{l}\text { Jambu Açu, nahe des Sítios Santo } \\
\text { Antonio }\end{array}$ & 1984 & circa 7 & ja & Maniok & >30 Jahre & S2 \\
\hline Igarapé Açu, Travessa Cumaru & 1986 & 5 & ja & $\begin{array}{l}\text { Mais, Maniok, } \\
\text { Bohnen }\end{array}$ & 8 Jahre & S4 \\
\hline $\begin{array}{l}\text { Iracema, Raimundo und Ayrton } \\
\text { Marcello }\end{array}$ & 1986 & 5 & nein & Mais, Maniok & & S6 \\
\hline $\begin{array}{l}\text { Peixe Boi, Fazenda Monte Verde, } \\
\text { Primärwald }\end{array}$ & noch nie & & nein & ? & - & $\mathrm{PB}_{\mathrm{PW}}$ \\
\hline $\begin{array}{l}\text { Peixe Boi, Fazenda Monte Verde, } \\
\text { Sekundärwald }\end{array}$ & $\begin{array}{c}\text { zwischen } \\
1968 \text { und } \\
1978\end{array}$ & $15-25$ & nein & ? & ? & $\mathrm{PB}_{\mathrm{SW}}$ \\
\hline
\end{tabular}




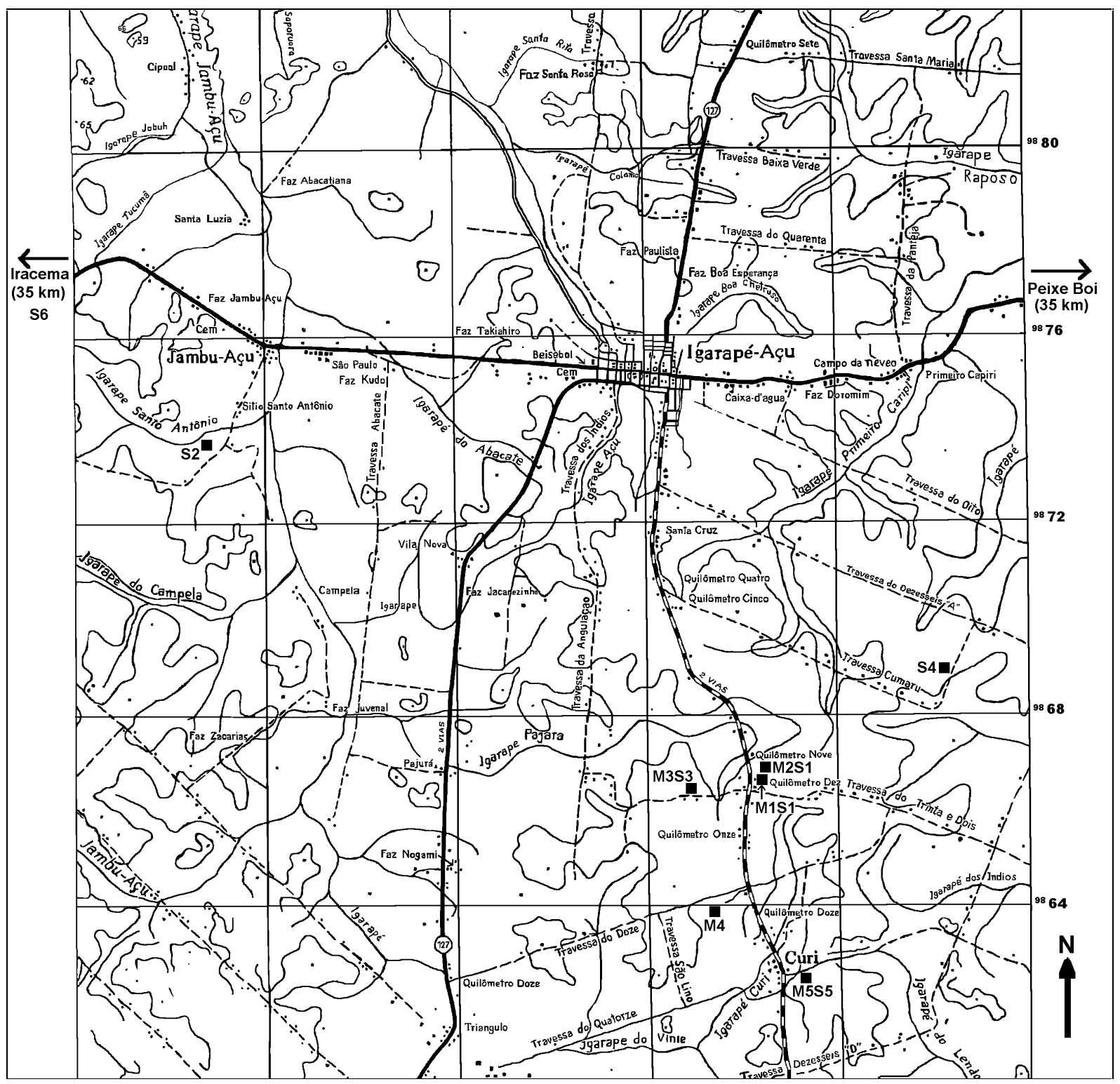

Abb. 11: Lage der Beprobungsflächen in der näheren Umgebung von Igarapé Açu (Abstand zwischen 2 Linien $=3.6 \mathrm{~km}$ )

\subsection{Beprobung des Bodens}

Die Bodenproben wurden bis zu 1m Tiefe mit einem Pürkhauer -Bohrstock, Proben bis 6m Tiefe mit einem verlängerbaren Bohrstock (Eigenbau) entnommen. Nach Trocknung an der Luft wurden sie für die massenspektrometrischen ${ }^{15} \mathrm{~N}$-Analysen in einer Kugelschlagmühle homogenisiert bzw. für die physikalischen und chemischen Analysen durch ein 2mm-Sieb passiert.

Für alle standortlichen und zeitlichen Beprobungen wurde aus folgenden Bodentiefen Proben gezogen: 0 - $5 \mathrm{~cm}, 5-10 \mathrm{~cm}, 10-20 \mathrm{~cm}, 20-40 \mathrm{~cm}, 40-60 \mathrm{~cm}, 60-100 \mathrm{~cm}$. 
Auf der Fläche M2S1 wurde eine einmalige Beprobung des Bodens bis in $6 \mathrm{~m}$ Tiefe vorgenommen. Dabei wurden folgende Tiefen zusammengefaßt:

$$
\begin{aligned}
& 0-50 \mathrm{~cm}, \quad 50-100 \mathrm{~cm} \\
& 100-150 \mathrm{~cm}, 150-200 \mathrm{~cm} \\
& 200-250 \mathrm{~cm}, 250-300 \mathrm{~cm} \\
& 300-350 \mathrm{~cm}, 350-400 \mathrm{~cm} \\
& 400-450 \mathrm{~cm}, 450-500 \mathrm{~cm} \\
& 500-550 \mathrm{~cm}, 550-600 \mathrm{~cm}
\end{aligned}
$$

\section{Beprobung verschiedener Standorte}

1. Die Flächen, die innerhalb von 5 bis $50 \mathrm{~km}$ um Igarapé Açu herum lagen - M1 bis M5 und die Standorte Iracema (S6) und Peixe Boi (PB) - wurden beprobt. Dabei wurden pro Fläche 3 bis 6 Wiederholungen, bestehend aus jeweils einer Mischprobe aus 6 bis 10 Einstichen, erstellt.

2. Auf den Flächen M1 bis M5 wurden Proben $50 \mathrm{~cm}$ von dem Stamm einer Leguminose und in $5 \mathrm{~m}$ Abstand von ihr genommen. Dabei wurden 5 Wiederholungen je Leguminose (Abarema jupunba, Inga heterophylla, Inga thibaudiana) durchgeführt.

\section{Beprobung zu verschiedenen Zeitpunkten}

Die Beprobung der Flächen M1 bis M5 im Februar 1993 - regenreiche Zeit - wurde in der trockeneren Jahreszeit im Juli 1993 wiederholt.

\section{Beprobung der organischen Auflage}

Die Beprobung der organischen Auflage erfolgte parallel zu der Bodenbeprobung, so daß Daten zu kleinräumger, großräumiger sowie temporärer Variabilität vorliegen.

\subsection{Physikalische und chemische Bodenanalysen der Probeflächen}

Die physikalischen und chemischen Analysen der Böden der Untersuchungsflächen wurden im bodenkundlichen Labor des CNPS (Centro National de Pesquisa dos Solos) der EMBRAPA in Rio de Janeiro durchgeführt. Analysen der tieferen Bodenschichten wurden im Zentrallabor des Instituts für Bodenkunde und Waldernährung, Göttingen durchgeführt. Die Bodenanalysen wurden gemäß der Methodenbeschreibung von Guimarães et al. (1970) durchgeführt. Eine Auflistung der verwendeten Analysemethoden befindet sich in Tab. 6 . 
Tab. 6: Physikalische und chemische Bodenanalysemethoden

\begin{tabular}{ll}
\hline Parameter & Analysemethode \\
\hline Korngrößenbestimmung & $\begin{array}{l}\text { Dispergierung mit 1N NaOH, Pipettanalyse, Trockensiebung } \\
\text { in Wert }\end{array}$ \\
organisch gebundenes C & $\begin{array}{l}\text { nasse Veraschung mit Kaliumdichromat, titrimetrisch, nach } \\
\text { Walkley/Black und Autoanalyzer }\end{array}$ \\
$\mathrm{N}_{\text {tot. }}$ & Kjeldahl-Aufschluß, titrimetrisch \\
$\mathrm{P}$ & saure Extraktionslösung Mehlich I (North Carolina) \\
$\mathrm{K}, \mathrm{Na}$ & Mehlich-Extraktionslösung, flammenphotometrisch \\
$\mathrm{Ca}, \mathrm{Mg}$ & 1N KCL-Extraktionslösung, atomabsorptionsspektrometrisch \\
$\mathrm{Al}, \mathrm{H}$ & 1N KCL- bzw. 1N Ca-Acetat-Extraktionlösung, pH 7, titrimetrisch \\
$\mathrm{Mn}, \mathrm{Zn}, \mathrm{Cu}$ & nach Singh und Möller (1984), Mehlich-Extraktion, \\
$\mathrm{AK}(\mathrm{pH} 7)$ & atomabsorptionsspektrometrisch \\
$\mathrm{AKe}$ & Summe der austauschbaren Kationen Na, K, Mg, Ca, Al und H \\
\hline
\end{tabular}

Die Böden der Untersuchungsflächen (Tab. 7 bis Tab. 10) können als typische Oxisole sandig-lehmiger Struktur bezeichnet werden. Im Vergleich mit Daten, die von Denich (1989) sowohl selbst erhoben als auch zitiert wurden, sind die Böden in fast allen chemischen Kenngrößen im unteren Bereich der in der Bragantina-Region vorkommenden Parameter anzusiedeln. Das heißt, daß sowohl an Makro- als auch an Mikronährstoffen Mangel herrscht. Die schon im Oberboden niedrige effektive Austauschkapazität (Ake) erreicht in $1.50 \mathrm{~m}$ Tiefe ihren Minimalwert von $0.1 \mathrm{mval} / 100 \mathrm{~g}$ Boden, der sich in den tieferen Schichten des B-Horizontes nicht mehr verändert. Aluminium nimmt Konzentrationen bis zur Pflanzentoxizität an. Lateritkonkretionen wurden vereinzelt auf der Primärwald-Fläche in Peixe Boi angetroffen. Die Bodenverhältnisse können als typisch für die Bragantina-Region angesehen werden. 
Tab. 7: Überblick über die physikalischen und chemischen Eckdaten der Böden von 0 bis $100 \mathrm{~cm}$ Tiefe der Flächen M1 bis M5 und S1 bis S5 (angegeben sind jeweils der Minimal- und Maximalwert der Mittelwerte aus 6 Mischproben je Bodentiefe und Fläche). Die Einzelwerte befinden sich in Tab. 53 bis 59 im Anhang.

\begin{tabular}{|c|c|c|c|c|c|c|}
\hline Bodentiefe & $0-5 \mathrm{~cm}$ & $5-10 \mathrm{~cm}$ & $10-20 \mathrm{~cm}$ & $20-40 \mathrm{~cm}$ & $40-60 \mathrm{~cm}$ & $60-100 \mathrm{~cm}$ \\
\hline Grobsand [\%] & $45.1-84.0$ & $38.3-79.0$ & $42.0-81.5$ & $33.5-74.5$ & $32-69.5$ & $33-67$ \\
\hline Feinsand [\%] & $12.0-38.0$ & $16.0-41.6$ & $12.5-39.0$ & $17.5-37.5$ & $19.5-36$ & $19-35.5$ \\
\hline Schluff [\%] & $1.0-8.5$ & $2.0-7.6$ & $1-10$ & $2.5-9$ & $4.0-7.5$ & $3-9$ \\
\hline Ton [\%] & $0.0-10.3$ & $3.0-14.6$ & $5.0-15.0$ & $5.5-23.5$ & $7.0-27.3$ & $11-31$ \\
\hline $\mathrm{pH}\left(\mathrm{H}_{2} \mathrm{O}\right)$ & $5.4-5.8$ & $5.2-5.6$ & $5.0-5.6$ & $4.9-5.4$ & $4.8-5.2$ & $4.8-5.3$ \\
\hline $\mathrm{pH}(\mathrm{KCl})$ & 4.3-4.6 & $4.0-4.5$ & $4.0-4.5$ & $4.0-4.4$ & $4.0-4.2$ & $4.05-4.3$ \\
\hline $\mathrm{C}[\%]$ & $0.60-1.60$ & $0.56-1.00$ & $0.57-0.78$ & $0.37-0.52$ & $0.25-0.41$ & $0.20-0.56$ \\
\hline $\mathrm{N}[\%]$ & $0.07-0.12$ & $0.06-0.10$ & $0.05-0.10$ & $0.04-0.08$ & $0.04-0.08$ & $0.03-0.08$ \\
\hline $\mathrm{C} / \mathrm{N}$ & $6.0-16.5$ & $3.0-16$ & $2.0-16.5$ & $1.0-13.0$ & $1.0-10.5$ & $1.0-9.0$ \\
\hline $\mathrm{P}[\mathrm{ppm}]$ & $2.5-5.0$ & $2.0-3.0$ & $1.5-3.0$ & $1.0-4.0$ & $0.2-2.5$ & $1.0-2.0$ \\
\hline $\mathrm{Ca}^{++}[\mathrm{mval} / 100 \mathrm{~g}]$ & $0.8-2.8$ & $0.4-1.2$ & $0.3-0.7$ & $0.1-0.7$ & $0.1-0.4$ & $0.1-0.2$ \\
\hline $\mathrm{Mg}^{++}[\mathrm{mval} / 100 \mathrm{~g}]$ & $0.40-1.60$ & $0.30-1.00$ & $0.15-0.85$ & $0.10-0.80$ & $0.1-0.35$ & $0.10-0.25$ \\
\hline $\mathrm{K}^{+}[\mathrm{mval} / 100 \mathrm{~g}]$ & $0.03-0.08$ & $0.02-0.04$ & $0.02-0.03$ & $0.01-0.04$ & $0.01-0.02$ & $0.01-0.02$ \\
\hline $\mathrm{Na}^{+}[\mathrm{mval} / 100 \mathrm{~g}]$ & $0.01-0.05$ & $0.02-0.04$ & $0.01-0.04$ & $0.02-0.03$ & $0.01-0.03$ & $0.01-0.03$ \\
\hline $\mathrm{Al}^{+++}[\mathrm{mval} / 100 \mathrm{~g}]$ & 0.03 & 0.70 & $0.03-0.70$ & $0.10-1.00$ & $0.40-1.20$ & $0.40-1.00$ \\
\hline $\mathrm{H}^{+}[\mathrm{mval} / 100 \mathrm{~g}]$ & $1.60-3.50$ & $1.20-3.00$ & $2.20-3.00$ & $1.30-2.50$ & $1.25-2.20$ & $1.20-1.95$ \\
\hline $\mathrm{Ak}[\mathrm{mval} / 100 \mathrm{~g}]$ & $3.2-7.4$ & $2.6-5.2$ & $2.2-4.4$ & $2.2-4.0$ & $2.0-4.1$ & $2.1-6.6$ \\
\hline Ake[mval/100g] & $1.6-4.1$ & $0.4-2.1$ & $0.4-1.35$ & $0.6-4.0$ & $0.2-0.9$ & $0.2-0.5$ \\
\hline Basensättigung [\%] & $34-55$ & $11.4-54$ & $11-46$ & $6-36$ & $2.5-27$ & $6-24$ \\
\hline $\mathrm{Zn}[\mathrm{ppm}]$ & $0.10-0.45$ & $0.06-0.59$ & $0.04-0.41$ & $0.15-0.74$ & $0.14-0.55$ & $0.08-0.52$ \\
\hline $\mathrm{Cu}[\mathrm{ppm}]$ & $0.001-0.01$ & $0.00-0.02$ & $0.00-0.10$ & $0.00-0.40$ & $0.01-0.04$ & $0.00-0.05$ \\
\hline Mn [ppm] & $1.60-5.70$ & $1.10-2.40$ & $0.19-1.24$ & $0.18-1.07$ & $0.12-0.64$ & $0.17-0.48$ \\
\hline
\end{tabular}


Tab. 8: Physikalische und chemische Eckdaten des Bodens von 0 bis $100 \mathrm{~cm}$ Tiefe der Fläche S6 in Iracema (Daten zum Teil von Ulrich Diekmann)

\begin{tabular}{|c|c|c|c|c|c|c|}
\hline Bodentiefe [\%] & $0-5$ & $0-10$ & $10-20$ & $20-40$ & $40-60$ & $60-100$ \\
\hline Grobsand [\%] & 45.1 & 38.3 & & & & \\
\hline Feinsand [\%] & 39.0 & 41.6 & & & & \\
\hline Schluff [\%] & 5.6 & 7.6 & & & & \\
\hline Ton [\%] & 10.3 & 12.6 & & & & \\
\hline $\mathrm{pH}\left(\mathrm{H}_{2} \mathrm{O}\right)$ & 5.5 & 5.2 & 5.0 & 5.0 & 5.1 & 5.0 \\
\hline $\mathrm{pH}(\mathrm{KCl})$ & 4.3 & 4.1 & 4.1 & 4.2 & 4.3 & 4.2 \\
\hline $\mathrm{C}[\%]$ & 1.12 & 0.7 & 0.72 & 0.46 & 0.33 & 0.22 \\
\hline $\mathrm{N}[\%]$ & 0.11 & 0.07 & 0.06 & 0.06 & 0.05 & 0.05 \\
\hline $\mathrm{C} / \mathrm{N}$ & 11 & 10 & 12 & 8 & 7 & 4 \\
\hline $\mathrm{P}[\mathrm{ppm}]$ & 3 & 3 & 2 & 1 & 1 & 1 \\
\hline $\mathrm{Ca}^{++}[\mathrm{mval} / 100 \mathrm{~g}]$ & 1.21 & 0.40 & 0.30 & 0.20 & 0.20 & 0.20 \\
\hline $\mathrm{Mg}^{++}[\mathrm{mval} / 100 \mathrm{~g}]^{*}$ & 0.37 & & & & & \\
\hline $\mathrm{K}^{+}[\mathrm{mval} / 100 \mathrm{~g}]$ & 0.05 & 0.03 & 0.02 & 0.02 & 0.01 & 0.01 \\
\hline $\mathrm{Na}^{+}[\mathrm{mval} / 100 \mathrm{~g}]$ & 0.03 & 0.02 & 0.04 & 0.03 & 0.02 & 0.02 \\
\hline $\mathrm{Al}^{+++}[\mathrm{mval} / 100 \mathrm{~g}]$ & 0.40 & 0.40 & 0.70 & 0.80 & 0.80 & 0.80 \\
\hline $\mathrm{H}^{+}[\mathrm{mval} / 100 \mathrm{~g}]$ & 3.20 & 2.9 & 2.60 & 2.20 & 1.50 & 1.20 \\
\hline $\mathrm{Ak}[\mathrm{mval} / 100 \mathrm{~g}]$ & 4.90 & 3.75 & 3.700 & 3.20 & 2.50 & 2.20 \\
\hline Ake $[\mathrm{mval} / \mathrm{g}]$ & 1.70 & 0.43 & 0.40 & 0.20 & 0.20 & 0.20 \\
\hline Basensättigung [\%] & 33.7 & 11.4 & 11 & 6 & 8 & 9 \\
\hline Zn [ppm] & 0.43 & 0.59 & 0.27 & 0.54 & 0.55 & 0.22 \\
\hline $\mathrm{Cu}[\mathrm{ppm}]$ & 0.001 & 0.02 & 0.02 & 0.01 & 0.03 & 0.05 \\
\hline Mn [ppm] & 1.65 & 0.55 & 0.19 & 0.19 & 0.20 & 0.28 \\
\hline
\end{tabular}

* $\overline{\text { leere Zellen }=\mathrm{Ca}++ \text { und } \mathrm{Mg}++ \text { wurden zusammen bestimmt }}$ 
Tab. 9: Physikalische und chemische Eckdaten des Bodens von 0 bis $100 \mathrm{~cm}$ Tiefe der Flächen in Peixe Boi (PW = Primärwaldfläche, SW = Sekundärwaldfläche)

\begin{tabular}{|c|c|c|c|c|c|c|c|c|c|c|}
\hline \multirow{2}{*}{$\begin{array}{l}\text { Bodentiefe [cm] } \\
\text { Standort }\end{array}$} & \multicolumn{2}{|c|}{$0-10$} & \multicolumn{2}{|c|}{$10-20$} & \multicolumn{2}{|c|}{$20-40$} & \multicolumn{2}{|c|}{$40-60$} & \multicolumn{2}{|c|}{$60-100$} \\
\hline & PW & SW & PW & SW & PW & SW & PW** & SW & $\begin{array}{ll}\text { PW } \\
\end{array}$ & SW \\
\hline Grobsand [\%] & 33 & 51 & 31 & 51 & 26 & 39 & 24 & 30 & 25 & 33 \\
\hline Feinsand [\%] & 38 & 32 & 34 & 32 & 37 & 35 & 37 & 33 & 37 & 30 \\
\hline Schluff [\%] & 9 & 7 & 13 & 7 & 9 & 6 & 9 & 9 & 8 & 5 \\
\hline Ton [\%] & 20 & 10 & 22 & 10 & 28 & 20 & 30 & 28 & 30 & 32 \\
\hline $\mathrm{pH}\left(\mathrm{H}_{2} \mathrm{O}\right)$ & 4.4 & 5.0 & 4.4 & 5 & 4.5 & 4.8 & 4.5 & 4.8 & 4.5 & 4.8 \\
\hline $\mathrm{pH}(\mathrm{KCl})$ & 3.5 & 3.9 & 3.6 & 3.9 & 3.8 & 3.9 & 3.9 & 4.0 & 4.0 & 4.0 \\
\hline $\mathrm{C}[\%]$ & 1.66 & 1.14 & 1.35 & 0.88 & 1.13 & 0.85 & 0.74 & 0.62 & 0.58 & 0.41 \\
\hline $\mathrm{N}[\%]$ & 0.16 & 0.11 & 0.15 & 0.10 & 0.11 & 0.09 & 0.09 & 0.08 & 0.08 & 0.06 \\
\hline $\mathrm{C} / \mathrm{N}$ & 10 & 10 & 9 & 9 & 10 & 9 & 8 & 8 & 7 & 7 \\
\hline $\mathrm{P}$ [ppm] & 7 & 2 & 4 & 2 & 2 & 2 & 2 & 1 & 1 & 1 \\
\hline $\mathrm{Ca}^{++}[\mathrm{mval} / 100 \mathrm{~g}]$ & 0.70 & 0.80 & 0.40 & 0.60 & 0.20 & 0.30 & 0.20 & 0.30 & 0.20 & 0.30 \\
\hline $\mathrm{Mg}^{++}[\mathrm{mval} / 100 \mathrm{~g}]^{*}$ & I & 0.30 & & 0.30 & & & 1 & & & \\
\hline $\mathrm{K}^{+}[\mathrm{mval} / 100 \mathrm{~g}]$ & 0.07 & 0.04 & 0.05 & 0.03 & 0.04 & 0.02 & 0.03 & 0.02 & 0.01 & 0.01 \\
\hline $\mathrm{Na}^{+}[\mathrm{mval} / 100 \mathrm{~g}]$ & 0.06 & 0.05 & 0.07 & 0.05 & 0.03 & 0.05 & 0.04 & 0.03 & 0.02 & 0.03 \\
\hline $\mathrm{Al}^{+++}[\mathrm{mval} / 100 \mathrm{~g}]$ & 2.20 & 0.80 & 2.70 & 0.80 & 2.70 & 1.40 & 2.40 & 1.20 & 2.20 & 1.20 \\
\hline $\mathrm{H}^{+}[\mathrm{mval} / 100 \mathrm{~g}]$ & 6.20 & 3.20 & 4.50 & 3.20 & 2.90 & 3.20 & 2.80 & 2.80 & 1.40 & 1.70 \\
\hline $\mathrm{Ak}[\mathrm{mval} / 100 \mathrm{~g}]$ & 9.20 & 5.20 & 7.70 & 5.00 & 5.90 & 5.00 & 5.50 & 4.30 & 3.80 & 3.20 \\
\hline Ake [mval/g] & 0.80 & 1.20 & 0.50 & 1.00 & 0.30 & 0.40 & 0.30 & 0.30 & 0.20 & 0.30 \\
\hline Basensättigung [\%] & 9 & 23 & 6 & 20 & 5 & 8 & 5 & 7 & 5 & 9 \\
\hline $\mathrm{Zn}[\mathrm{ppm}]$ & 0.30 & 0.14 & 0.28 & 0.27 & 0.70 & 0.39 & 0.63 & 0.42 & 0.87 & 0.23 \\
\hline $\mathrm{Cu}[\mathrm{ppm}]$ & 0.02 & 0.02 & 0.02 & 0.02 & 0.09 & 0.04 & 0.08 & 0.05 & 0.14 & 0.02 \\
\hline Mn [ppm] & 0.22 & 0.55 & 0.21 & 0.43 & 0.17 & 0.16 & 0.10 & 0.09 & 0.07 & 0.08 \\
\hline
\end{tabular}

$*$ leere Zellen $=\mathrm{Ca}++$ und $\mathrm{Mg}++$ wurden zusammen bestimmt

** ab $40 \mathrm{~cm}$ Bodentiefe wurden auf dieser Fläche Lateritkonkretionen angetroffen 
Tab. 10: Physikalische und chemische Eckdaten des Bodens der Untersuchungsfläche M2S1 für die Bodentiefen von 0 bis $600 \mathrm{~cm}$ (es sind die Mittelwerte aus 6 Einzelproben je Bodentiefe mit dem Standardfehler in Klammern angegeben, nur jede 2. Bodentiefe wurde analysiert)

\begin{tabular}{|c|c|c|c|c|c|c|c|}
\hline Bodentiefe $[\mathrm{cm}]$ & $0-50$ & $50-100$ & $150-200$ & $250-300$ & $350-400$ & $450-500$ & $550-600$ \\
\hline Grobsand [\%] & $44.5(2.5)$ & $36.0(1.1)$ & $41.0(1.0)$ & $42.5(0.5)$ & $36.0(0.0)$ & $41.0(2.1)$ & $45.0(7.0)$ \\
\hline Feinsand [\%] & $30.0(1.0)$ & $26.5(1.0)$ & $22.5(0.5)$ & $22.5(1.5)$ & $28.0(0.0)$ & $28.5(0.6)$ & $24.7(4.1)$ \\
\hline Schluff $[\%]$ & $4.5(1.5)$ & $6.5(0.6)$ & $3.5(2.5)$ & $2.5(0.5)$ & $4.0(0.0)$ & $3.5(0.6)$ & $3.7(0.7)$ \\
\hline Ton [\%] & $21.0(3.0)$ & $31.0(1.2)$ & $33.5(1.5)$ & $32.5(1.5)$ & $32.0(0.0)$ & $27.0(1.9)$ & $26.7(4.1)$ \\
\hline $\mathrm{pH}\left(\mathrm{H}_{2} \mathrm{O}\right)$ & $4.9(0.09)$ & $5.0(0.04)$ & $4.8(0.1)$ & $4.8(0.05)$ & $4.8(0.1)$ & $4.8(0.12)$ & $5.21(0.27)$ \\
\hline $\mathrm{pH}(\mathrm{KCl})$ & $4.0(0.05)$ & $4.1(0.02)$ & $4.1(0.0)$ & $4.20(0.03)$ & $4.2(0.04)$ & $4.2(0.04)$ & $4.53(0.33)$ \\
\hline $\mathrm{C}[\%]$ & $0.64(0.04)$ & $0.36(0.05)$ & $0.58(0.15)$ & $0.14 *$ & $0.1^{*}$ & $0.1^{*}$ & $0.1 *$ \\
\hline $\mathrm{N}[\%]$ & $0.09(0.02)$ & $0.06(0.01)$ & $0.06(0.02)$ & $0.01 *$ & $0.01 *$ & $\mathrm{nd}^{*}$ & $\mathrm{nd}^{*}$ \\
\hline $\mathrm{C} / \mathrm{N}$ & $7.5(1.2)$ & $6.7(0.7)$ & $10.0(1.8)$ & $8.5(1.2)$ & $7.6(0.8)$ & $7.7(0.8)$ & $7.7(1.1)$ \\
\hline $\mathrm{P}[\mathrm{mg} / 100 \mathrm{~g}]$ & $1.0(0.0)$ & $1.4(0.1)$ & $1.0(0.0)$ & $1.0(0.0)$ & $1.0(0.0)$ & $1.0(0.0)$ & $36.0(0.0)$ \\
\hline $\mathrm{Ca}^{++}[\mathrm{mval} / 100 \mathrm{~g}]$ & $0.35(0.11)$ & $0.29(0.04)$ & $0.10(0.00)$ & $0.10(0.00)$ & $0.10(0.00)$ & $0.12(0.01)$ & $0.10(0.00)$ \\
\hline $\mathrm{Mg}^{++}[\mathrm{mval} / 100 \mathrm{~g}]$ & $0.40(0.00)$ & 0.04 & 0.02 & 0.02 & 0.03 & 0.03 & 0.03 \\
\hline \multirow[t]{2}{*}{$\mathrm{K}^{+}[\mathrm{mval} / 100 \mathrm{~g}]$} & $0.02(0.001)$ & 0.01 & 0.009 & $0.01(0.00)$ & 0.01 & $0.01(0.003)$ & 0.003 \\
\hline & & $(0.001)$ & $(0.00)$ & & $(0.002)$ & & $(0.00)$ \\
\hline \multirow[t]{2}{*}{$\mathrm{Na}^{+}[\mathrm{mval} / 100 \mathrm{~g}]$} & $0.04(0.01)$ & 0.02 & 0.02 & 0.02 & 0.01 & $0.02(0.002)$ & $0.02(0.01)$ \\
\hline & & $(0.001)$ & $(0.004)$ & $(0.004)$ & $(0.002)$ & & \\
\hline $\mathrm{Al}^{+++}[\mathrm{mval} / 100 \mathrm{~g}]$ & $0.83(0.09)$ & $0.84(0.06)$ & $0.77(0.03)$ & $0.62(0.02)$ & $0.44(0.07)$ & $0.37(0.05)$ & $0.32(0.08)$ \\
\hline $\mathrm{H}^{+}[\mathrm{mval} / 100 \mathrm{~g}]$ & $2.51(0.8)$ & $1.67(0.09)$ & $1.00(0.06)$ & $0.80(0.07)$ & $0.70(0.04)$ & $0.68(0.08)$ & $0.48(0.06)$ \\
\hline Ak $[\mathrm{mval} / 100 \mathrm{~g}]$ & $3.80(0.09)$ & $3.15(0.38)$ & $1.86(0.07)$ & $1.52(0.05)$ & $1.25(0.06)$ & $1.12(0.11)$ & $0.92(0.13)$ \\
\hline Ake $[\mathrm{mval} / \mathrm{g}]$ & $0.45(0.16)$ & $0.29(0.04)$ & $0.10(0.00)$ & $0.10(0.00)$ & $0.10(0.00)$ & $0.12(0.02)$ & $0.12(0.02)$ \\
\hline Basensättigung [\%] & $11.7(4.1)$ & $9.8(1.4)$ & $5.0(0.0)$ & $6.5(0.3)$ & $8.0(0.4)$ & $10.4(0.9)$ & $15.2(4.0)$ \\
\hline $\mathrm{Zn}[\mathrm{ppm}]$ & $0.57(0.10)$ & $0.32(0.09)$ & $0.52(0.19)$ & $0.48(0.12)$ & $0.29(0.05)$ & $0.30(0.07)$ & $0.38(0.08)$ \\
\hline $\mathrm{Cu}[\mathrm{ppm}]$ & $0.03(0.003)$ & $0.04(0.01)$ & $0.04(0.02)$ & $0.05(0.02)$ & $0.04(0.01)$ & $0.02(0.01)$ & $0.03(0.01)$ \\
\hline Mn [ppm] & $0.24(0.04)$ & $0.32(0.03)$ & $0.01(0.03)$ & $0.05(0.04)$ & $0.03(0.01)$ & $0.08(0.04)$ & $4.55(4.47)$ \\
\hline
\end{tabular}

*Analyse-Ergebnisse der EMBRAPA durch Analysen des Elementaranalyzer ersetzt, da offensichtlich kontaminiert. 


\subsection{Beprobung der Pflanzen}

Im Anhang Tab. 60 befindet sich eine vollständige Liste der untersuchten Arten, ihre Familienzugehörigkeit, Wuchsform und Zuordnung zu Abkürzungen.

Es wurden 2 Blattpositionen, im Folgenden als alte und neue Blätter bezeichnet, beprobt. Jeweils die letzten drei Blätter eines Zweiges wurden als neue Blätter, die ersten drei Blätter vom Stamm aus als alte Blätter angesehen. Eine Probe bestand aus der Mischung von Blättern der gesamten Baumkrone. Eine Pflanze wurde als ein Individuum betrachtet, wenn sie einzeln stand oder die Triebe aus einem gemeinsamen Stubben hervorkamen oder sich eindeutig aus dem gleichen Wurzelstock entwickelten.

Holz und Rinde wurden getrennt nach folgenden Durchmesserklassen der Zweige und Baumstämme gesammelt: $0.0-0.5 \mathrm{~cm}, 0.5-1.0 \mathrm{~cm}, 1.0-2.0 \mathrm{~cm}, 2.0-3.0 \mathrm{~cm}, 3.0-4.0 \mathrm{~cm}, 4.0-$ $5.0 \mathrm{~cm}, 5.0-6.0 \mathrm{~cm}, 6.0-7.0 \mathrm{~cm}$.

Von Abarema jupunba wurden 10 verschieden große Individuen auf dem Feld mit Ausnahme der Wurzeln komplett geerntet, in neue und alte Blätter sowie Holz und Rinde verschiedener Durchmesserklassen zerlegt und die jeweilige Biomasse (Trockenmasse) bestimmt. Standort dieser Erhebung war Fläche M1S1.

\subsubsection{Beprobung von Pflanzenarten}

Die Sekundärvegetationen der Flächen S1 bis S6 in Igarapé Açu, sowie in Peixe Boi die Flächen $\mathrm{PB}_{\mathrm{PW}}$ und $\mathrm{PB}_{\mathrm{SW}}$ wurden beprobt. Die Entfernung der Flächen betrug 5-35 km.

Im Juli 1993 wurde Blattmaterial folgender Arten auf den Flächen S1 bis S6 gesammelt:

$\begin{array}{ll}\text { Abarema jupunba } & \text { (Mimosoideae) } \\ \text { Inga heterophylla } & \text { (Mimosoideae) } \\ \text { Inga thibaudiana } & \text { (Mimosoideae) } \\ \text { Vismia guianensis } & \text { (Guttiferae) } \\ \text { Lacistema pubescens } & \text { (Lacistemataceae) } \\ \text { Tapirira guianensis } & \text { (Anacardiaceae) } \\ \text { Banara guanensis } & \text { (Flacourtiaceae) }\end{array}$

Dabei wurde darauf geachtet, daß die Bäume annähernd gleich groß waren. Angestrebt waren 5 Wiederholungen je Art und Fläche, was aber nicht immer eingehalten werden konnte. Abb. 12 gibt einen Eindruck von der Streuung der vorgefundenen Baumdurchmesser und der 
Wiederholungen. Die Spanne an Durchmessern lag zwischen $\varnothing=2,8$ bis $4,4 \mathrm{~cm}$. Extreme Ausreißer wurden aus dem Datenmaterial eliminiert. Eine Korrelation zwischen den Stubbendurchmessern der Pflanzen und ihren $\delta^{15} \mathrm{~N}$-Werten $\left(\mathrm{r}_{\text {neue Blätter }}=-0.15, \mathrm{r}_{\text {alte Blätter }}=\right.$ $-0.08)$ bzw. den N-Gehalten $\left(r_{\text {neue Blätter }}=0.09, \mathrm{r}_{\text {alte Blätter }}=-0.03\right)$ bestand nicht.
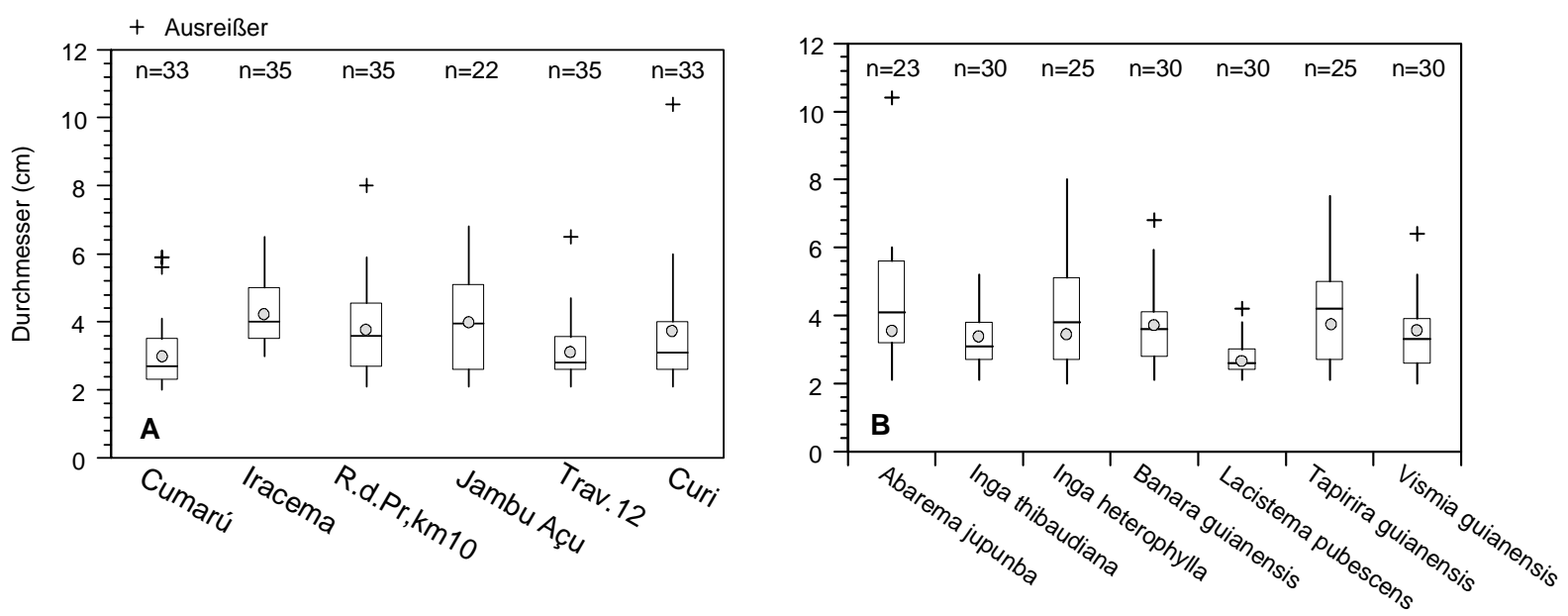

Abb. 12: Box - Whisker- Plots der Verteilung der Baumdurchmesser auf den Standorten (A) und der beprobten Arten (B) (Punkte markieren den Mittelwert)

Die Beprobung der Primär- und Sekundärvegetation in Peixe Boi wurde durch die freundliche Erlaubnis des Besitzers der Fazenda Monte Verde, Dr. Danilo Mendonça, ermöglicht. Hier wurde jedoch nicht auf Größenunterschiede der Probepflanzen geachtet, da es sich um eine einmalige Aktion ohne Wiederholungen handelte. In Tab. 11 sind die beprobten Arten aufgelistet.

Tab. 11: Liste der in Peixe Boi beprobten Arten

\begin{tabular}{|c|c|c|c|}
\hline Art & Familie & Primärwald & Sekundärwald \\
\hline$?$ & Melastomataceae & $\mathrm{X}$ & $\mathrm{X}$ \\
\hline Bagassa guianensis & Moraceae & $\mathrm{X}$ & \\
\hline Banara guianensis & Flacourtiaceae & & $X$ \\
\hline Cordia exaltata & Boraginaceae & $X$ & $\mathrm{X}$ \\
\hline Dipteryx odorata & Papilionoideae & $\mathrm{X}$ & \\
\hline Inga rubiginosa & Mimosoideae & $\mathrm{X}$ & \\
\hline Inga thibaudiana & Mimosoideae & $X$ & $X$ \\
\hline Lacistema pubescens & Lacistemataceae & & $\mathrm{X}$ \\
\hline Lecythis lurida & Lecythidaceae & & $X$ \\
\hline Memora allamandiflora & Bignoniaceae & $X$ & $X$ \\
\hline Myrcia sylvatica & Myrtaceae & $X$ & \\
\hline Neea oppositifolia & Nyctaginaceae & $\mathrm{X}$ & \\
\hline Rollinia exsucca & Annonaceae & & $\mathrm{X}$ \\
\hline Selaginella stellaria & Selaginellaceae & $X$ & \\
\hline Sterculia pruriens & Sterculiaceae & $\mathrm{X}$ & \\
\hline Tapirira guianensis & Anacardiaceae & $\mathrm{X}$ & $X$ \\
\hline Vismia guianensis & Guttiferae & & $\mathrm{X}$ \\
\hline
\end{tabular}




\subsubsection{Saisonale Beprobungen}

Von September 1992 bis Oktober 1993 wurden monatlich die jungen und alten Blätter von 3 Bäumen der Spezies Abarema jupunba, 5 Inga heterophylla und 5 Inga thibaudiana gesammelt. In unmittelbarer Umgebung jeder Leguminose (etwa ein 5m-Radius) wurden Nichtleguminosen in gleicher Weise beprobt. Tab. 12 gibt einen Überblick über Standort der Pflanzen, Höhe sowie Basalfläche als Maß für die Größe/Alter der Bäume. Zur Berechnung der Basalfläche $\left(\pi \times r^{2}\right)$ wurde der Radius aus der Summe der einzelnen Stockausschläge oder des Stammes der jeweiligen Pflanze in $30 \mathrm{~cm}$ Bodenhöhe genommen.

Tab. 12: Übersicht über die monatlich erfaßten Arten und ihre Größenverhältnisse (Basalfläche = $\left.\pi \times r^{2}\right)$

\begin{tabular}{|c|c|c|c|c|c|}
\hline Leguminose & Basalfläche $\left(\mathrm{cm}^{2}\right)$ & Höhe(cm) & nicht $\mathrm{N}_{2}$-fixierende Arten & Standort & Familie \\
\hline \multirow[t]{4}{*}{ Abarema jupunba } & 18.8 & 520 & & M1S1 & Mimosoideae \\
\hline & 8.4 & 300 & Lacistema pubescens & & Lacistemataceae \\
\hline & 2.3 & 300 & Mabea angustifolia & & Euphorbiaceae \\
\hline & 12.6 & 450 & Vismia guianensis & & Guttiferae \\
\hline \multirow[t]{6}{*}{ Abarema jupunba } & 15.9 & 400 & & M1S1 & Mimosoideae \\
\hline & 2.2 & 250 & Lacistema pubescens & & Lacistemataceae \\
\hline & 3.1 & 255 & Mabea angustifolia & & Euphorbiaceae \\
\hline & 1.1 & 280 & Myrcia sylvatica & & Myrtaceae \\
\hline & 0.5 & 280 & Myrcia sylvatica & & Myrtaceae \\
\hline & 2.5 & 350 & Vismia guianensis & & Guttiferae \\
\hline \multirow[t]{4}{*}{ Abarema jupunba } & 11.9 & 350 & & M1S1 & Mimosoideae \\
\hline & 7.1 & 270 & Nectandra cuspidata & & Lauraceae \\
\hline & 19.4 & 290 & Tapirira guianensis & & Anacardiaceae \\
\hline & 2.8 & 300 & Vismia guianensis & & Guttiferae \\
\hline \multirow[t]{7}{*}{ Inga heterophylla } & 2.3 & 400 & & M5S5 & Mimosoideae \\
\hline & 3.8 & 220 & Casearia arborea & & Flacourtiaceae \\
\hline & 3.8 & 240 & Casearia arborea & & Flacourtiaceae \\
\hline & 1.8 & 410 & Myrcia sylvatica & & Myrtaceae \\
\hline & 1.8 & 190 & Myrcia sylvatica & & Myrtaceae \\
\hline & 2.5 & 420 & Myrcia sylvatica & & Myrtaceae \\
\hline & 4.5 & 400 & Vismia guianensis & & Guttiferae \\
\hline \multirow[t]{4}{*}{ Inga heterophylla } & 13.2 & 300 & & M5S5 & Mimosoideae \\
\hline & 2.0 & 320 & Guatteria poeppigiana & & Anonaceae \\
\hline & 13.2 & 450 & Mabea angustifolia & & Euphorbiaceae \\
\hline & 4.3 & 300 & Sloanea guianensis & & Elaeocarpaceae \\
\hline \multirow[t]{4}{*}{ Inga heterophylla } & 10.0 & 310 & & M5S5 & Mimosoideae \\
\hline & 13.1 & 260 & Lecythis lurida & & Lecythidaceae \\
\hline & 8.0 & 350 & Myrcia sylvatica & & Myrtaceae \\
\hline & 4.0 & 240 & Vismia guianensis & & Guttiferae \\
\hline \multirow[t]{4}{*}{ Inga heterophylla } & 6.2 & 400 & & M2S1 & Mimosoideae \\
\hline & 4.9 & 210 & Lacistema pubescens & & Lacistemataceae \\
\hline & 5.5 & 290 & Nectandra cuspidata & & Lauraceae \\
\hline & 6.6 & 450 & Vismia guianensis & & Guttiferae \\
\hline \multirow[t]{2}{*}{ Inga heterophylla } & 3.3 & 250 & & $\mathrm{M} 2 \mathrm{~S} 1$ & Mimosoideae \\
\hline & 3.1 & 220 & Myrcia sylvatica & & Myrtaceae \\
\hline
\end{tabular}


Fortsetzung Tab. 12: Übersicht über die monatlich erfassten Arten und ihre Größenverhältnisse (Basalfläche $\left.=\pi \mathrm{xr}^{2}\right)$

\begin{tabular}{|c|c|c|c|c|c|}
\hline Leguminose & Basalfläche $\left(\mathrm{cm}^{2}\right)$ & Höhe(cm) & nicht $\mathrm{N}_{2}$-fixierende Arten & Standort & Familie \\
\hline \multirow[t]{10}{*}{ Inga thibaudiana } & 14.5 & 430 & & M3S3 & Mimosoideae \\
\hline & 2.8 & 300 & Cordia exaltata & & Boraginaceae \\
\hline & 5.9 & 395 & Lacistema pubescens & & Lacistemataceae \\
\hline & 24.6 & 600 & Trema micrantha & & Ulmaceae \\
\hline & 24.1 & 410 & Thyrsodium paraense & & Anacardiaceae \\
\hline & 2.8 & 340 & Thyrsodium paraense & & Anacardiaceae \\
\hline & 5.7 & 355 & Vismia guianensis & & Guttiferae \\
\hline & 4.9 & 385 & Vismia guianensis & & Guttiferae \\
\hline & 4.2 & 385 & Vismia guianensis & & Guttiferae \\
\hline & 18.4 & 270 & Vismia guianensis & & Guttiferae \\
\hline \multirow[t]{7}{*}{ Inga thibaudiana } & 32.2 & 560 & & M3S3 & Mimosoideae \\
\hline & 7.7 & 395 & Banara guianensis & & Flacourtiaceae \\
\hline & 3.2 & 225 & Lacistema pubescens & & Lacistemataceae \\
\hline & 3.5 & 360 & Myrcia sylvatica & & Myrtaceae \\
\hline & 3.5 & 310 & Myrcia sylvatica & & Myrtaceae \\
\hline & 2.0 & 400 & Myrcia sylvatica & & Myrtaceae \\
\hline & 2.0 & 330 & Vismia guianensis & & Guttiferae \\
\hline \multirow[t]{3}{*}{ Inga thibaudiana } & 47.6 & 390 & & M3S3 & Mimosoideae \\
\hline & 23.7 & 295 & Ocotea guianensis & & Lauraceae \\
\hline & 25.3 & 460 & Tapirira guianensis & & Anacardiaceae \\
\hline \multirow[t]{7}{*}{ Inga thibaudiana } & 15.6 & 415 & & M3S3 & Mimosoideae \\
\hline & 10.4 & 240 & Cordia exaltata & & Boraginaceae \\
\hline & 2.3 & 320 & Lacistema pubescens & & Lacistemataceae \\
\hline & 14.5 & 312 & Ocotea guianensis & & Lauraceae \\
\hline & 5.1 & 340 & Tapirira guianensis & & Anacardiaceae \\
\hline & 5.0 & 375 & Tapirira guianensis & & Anacardiaceae \\
\hline & 1.8 & 305 & Vismia guianensis & & Guttiferae \\
\hline
\end{tabular}

\subsection{Probenaufbereitung}

Alle Pflanzenproben wurden 3 Tage bei $60^{\circ} \mathrm{C}$ im Trockenschrank, die Bodenproben an der Luft getrocknet.

Die Pflanzenproben wurden in einer Mikro-Feinmühle Culatti MFC (IKA), Bodenproben in der am Institut für tropischen Pflanzenbau nach einer Vorlage von Rondon und Thomas (1994) hergestellten Kugelschlagmühle homogenisiert. $\mathrm{Zu}$ grob homogenisierte Pflanzenproben wurden in der Zentrifugalmühle des Types Rotor Speed Mill Pulverisette 14 (FRITSCH) nachbehandelt. Die typische Korngrößenzusammensetzung kann Tab. 13 entnommen werden. 
Tab. 13: Korngrößenverteilung (in \%) in den homogenisierten Pflanzen- und Bodenproben $($ Feinmühle $=$ Mikro-Feinmühle Culatti MFC (IKA). Zentrifugalmühle Rotor Speed Mill Pulverisette 14 (FRITSCH). Kugelschlagmühle (Eigenbau)

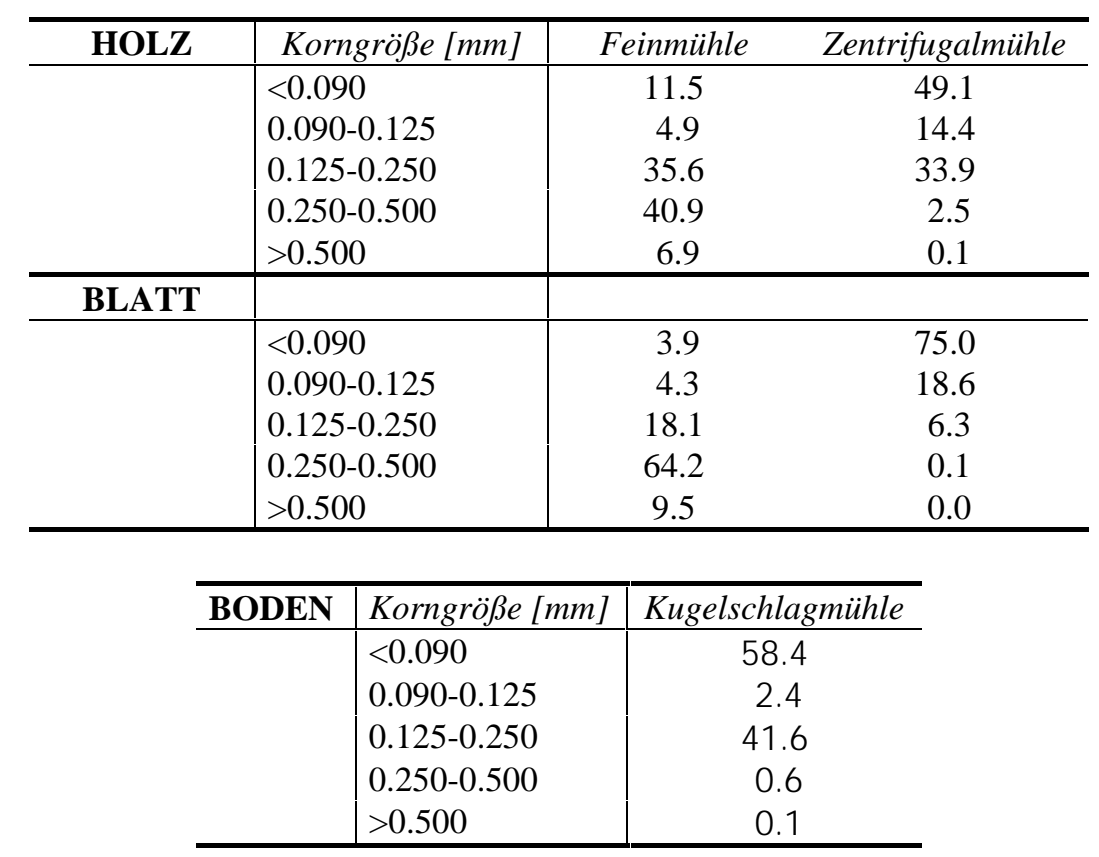

\section{Gewächshausversuche}

\subsection{Nodulation nativer Arten}

Es wurden Ausgrabungen von Wurzelsystemen an 9 Leguminosen in 6-8jähriger und 4 in 1jähriger Sekundärvegetation (Tab. 14) unternommen. Dazu wurde die Hälfte des Wurzelsystems halbkreisförmig in 3 Abschnitten bis 1.5m Abstand vom Stamm und bis auf $40 \mathrm{~cm}$ Bodentiefe freigelegt (Abb. 13).

Die Knöllchen wurden durch Sieben des Bodens extrahiert und gezählt. Durchmesser und Höhe der Bäume und die Lage der Wurzeln und Knöllchen wurde notiert. Aus den Knöllchen wurden Bakterienisolate gewonnen, die später charakterisiert, auf ihre Effektivität hin getestet und als Inokulum für Gewächshausversuche verwendet wurden. 


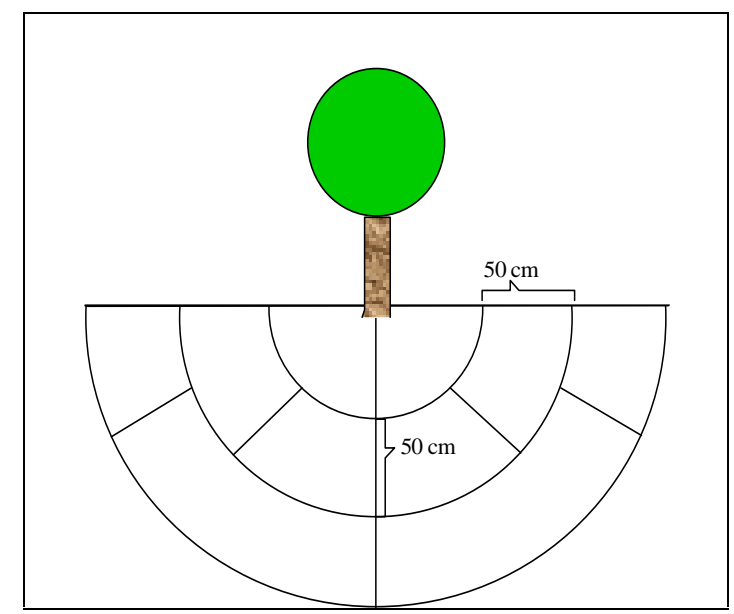

Abb. 13: Schema der Ausgrabungen

Tab. 14: Auf ihre Nodulation im Feld hin untersuchte Arten, von deren Knöllchen auch Rhizobien isoliert wurden

\begin{tabular}{lclccc}
\hline Art & $\begin{array}{c}\text { Alter der } \\
\text { Capoeira }\end{array}$ & \multicolumn{1}{c}{ Fläche } & Höhe (cm) & $\begin{array}{c}\text { Basalfläche } \\
\left.\text { (cm }^{2}\right)\end{array}$ & Stubben $\varnothing(\mathbf{c m})^{*}$ \\
\hline Inga alba & 10 & Curi, nahe M5S5 & 550 & 471.2 & 7.8 \\
Inga edulis & 4 & Curi, Feldrand & 200 & 49.0 & 2.5 \\
Inga heterophylla & 1 & neben M2S1 & 150 & 3,8 & 0.8 \\
Inga heterophylla & 6 & M5S5 & 350 & 4,5 & 3.3 \\
Inga heterophylla & 7 & M2 & 350 & 13,8 & 4.2 \\
Inga macrophylla & 7 & M2 & 400 & 463,5 & 2.9 \\
Inga nitida & 6 & M5S5 & 250 & 1748,9 & 1.4 \\
Inga thibaudiana & 1 & neben M2 & 150 & 0,9 & 1.1 \\
Inga thibaudiana & 6 & M5S5 & 400 & 3,5 & 2.1 \\
Abarema jupunba & 1 & neben M5 & 180 & 3,5 & 2.1 \\
Abarema jupunba & 7 & M2S2 & 420 & 2272,1 & 4.8 \\
Desmodium & 1 & neben M2S2 & Kraut & Kraut & \\
bracteata & & & & & \\
\hline
\end{tabular}

* Durchschnittswert der jeweiligen Austriebe, wenn mehrere vorhanden waren.

\subsection{Infektionspotential des Bodens}

An 6 Stellen der Fläche M2S1 wurden Bodenproben in $50 \mathrm{~cm}$ Abschnitten bis zu einer Bodentiefe von $6 \mathrm{~m}$ genommen. Von Stelle Nr.6 wurden ca. $6 \mathrm{~kg}$ Boden pro Tiefenstufe (0 $50 \mathrm{~cm}, 50-100 \mathrm{~cm}, 100-150 \mathrm{~cm}, 150-200 \mathrm{~cm}, 200-250 \mathrm{~cm}, 250-300 \mathrm{~cm}, 300-350 \mathrm{~cm}, 350$ $-400 \mathrm{~cm}, 400-450 \mathrm{~cm}, 450-500 \mathrm{~cm}, 500-550 \mathrm{~cm}, 550-600 \mathrm{~cm})$ extra entnommen. 
Beschreibung der Stellen:

Nr.1: $\quad$ Bewuchs mit Abarema jupunba

Nr.2: $\quad$ Bewuchs mit Inga heterophylla

Nr.3: $\quad$ Bewuchs mit Tapirira guianensis, keine Leguminosen in 50m Umkreis

Nr.4: Bewuchs mit Tapirira guianensis, keine Leguminosen in 50m Umkreis

Nr.5: $\quad$ Bewuchs mit Inga thibaudiana

Nr.6: $\quad$ Bewuchs mit Inga thibaudiana

Die Proben wurden gekühlt ins Labor gebracht und wie folgt verarbeitet:

- $100 \mathrm{~g}$ luftgetrocknet für ${ }^{15} \mathrm{~N}$-Analysen und Nährstoffanalysen.

- $50 \mathrm{~g}$ zur Wassergehaltsbestimmung verwendet.

- Der Boden der 6. Stelle wurde luftgetrocknet.

Ca. $340 \mathrm{~g}$ des frischen Bodens wurde mit $8 \mathrm{~g}$ autoklaviertem Vermiculit ( $/ \mathrm{v}=1 / 1)$ gemischt und in 11-Töpfe gefüllt. Samen der Augenbohne (Vigna unguiculata) der Sorte BR3 wurden mit $\mathrm{NaOCl}(3 \%)$ oberflächensterilisiert und 5 Samen pro Topf gepflanzt. Gewässert wurde die ersten 2 Wochen mit autoklaviertem destilliertem Wasser, danach wurde destilliertes Wasser unsteril benutzt. Da der Boden stark mit Pilzen befallen war, wurden sowohl Bohnen als auch der Boden in den Töpfen mit dem systemischen Fungizid „Benlate“ behandelt. Der Versuch wurde mit luftgetrocknetem Boden von Stelle 6 in 11-Pflanzsäcken wiederholt. Der 1. Versuch bestand aus 2 Wiederholungen pro Stelle und Bodentiefe, so daß insgesamt 12 Wiederholungen pro Bodentiefe vorhanden waren. Der 2. Versuch bestand aus 10 Wiederholungen je Bodentiefe, allerdings wurde er nur mit 250g Boden pro Pflanzsack durchgeführt. Nach dem Auskeimen wurde auf 2 Pflanzen pro Topf reduziert. Nährlösung ohne N (siehe Tab. 17) (anfangs ebenfalls autoklaviert) wurde wöchentlich verabreicht.

Nach 6 Wochen erfolgte die Auswertung durch Gewinnung von oberirdischer Blattmasse, Wurzeln und Knöllchen, und durch die Bestimmung von Frisch- und Trockengewicht.

\section{3 $\quad{ }^{15} \mathrm{~N}$-Verteilung innerhalb der Pflanzen}

Zur Erfassung der ${ }^{15} \mathrm{~N}-$ Verteilung in Pflanzen von Abarema jupunba, Abarema cochleatum und Tapirira guianensis während der Aufnahme, $\mathrm{N}_{2}$-Fixierung und des Wachstums wurden mehrere Gewächshausversuche angelegt. Die Abarema jupunba- und Tapirira guianensisPflänzchen wurden aus Keimlingen gewonnen, die entlang eines Flußlaufes in Curi, Travessa 14, wuchsen. Die Leguminose wies schon 1-6 Knöllchen auf. Abarema cochleatum-Samen 
wurden von einem Baum auf der Fläche M1S1 geerntet. Nach 8wöchiger Vorkeim-und Angleichphase (in 11-Töpfen mit Sand plus Nährlösung mit 15mg N/Woche bzw. ohne N, oder in Boden ohne Düngung) wurden die Pflänzchen in 10kg-Töpfe entsprechend ihrer Bestimmung überführt (Tab. 15).

Tab. 15: Übersicht über die Varianten zur Untersuchung der ${ }^{15} \mathrm{~N}$-Verteilung in Pflanzen

\begin{tabular}{lccc}
\hline \multicolumn{1}{c}{ Varianten } & Abarema cochleatum & Abarema jupunba & Tapirira guianensis \\
\hline Sand, Nährlösung, ohne N & $\mathrm{X}$ & $\mathrm{X}$ & $\mathrm{X}$ \\
Sand, Nährlösung, komplett & & $\mathrm{X}$ & $\mathrm{X}$ \\
Boden, ohne Nährlösung & & $\mathrm{X}$ & $\mathrm{X}$ \\
\hline
\end{tabular}

Die Töpfe waren je nach Variante wie folgt befüllt:

Sandvariante: Der Sand wurde circa 14 Stunden mit fließendem Wasser gewässert. In 10Liter-Eimern wurde per Hand 20-30 Mal mit Leitungswasser gewaschen, bis der Überstand vollkommen klar war. Als letztes wurde der Sand zweimal mit destilliertem Wasser gewaschen und getrocknet.

Mit Verunreinigung des destillierten Wassers von 0.04 mval N/l mußte zu Beginn der Versuche gerechnet werden. In der zweiten Hälfte des Versuches ab '2.93 wurde eine neue Destillieranlage montiert, so daß von $\mathrm{N}$-freiem Wasser ausgegangen werden konnte. Bei der N-Analyse des gesäuberten Sandes blieb der N-Gehalt immer unter der Nachweisgrenze.

Der Boden stammte von der Fläche M5S5 und entsprach somit einem typischen Boden der Bragantina-Region. Sowohl Sand als auch Boden wurden auf $30 \%$ ihrer $\mathrm{Wk}_{\max }$. (vor Versuchsbeginn einmal ermittelt) angefeuchtet und durch zweimaliges Gießen pro Woche ungefähr auf diesem Stand gehalten.

In jeden Topf wurde ein Pflänzchen gegeben. In das Pflanzloch für Abarema cochleatum wurden 10ml eines Inokulums mit den Rhizobium-Stämmen Nr. 50 und 3, in das Pflanzloch für Abarema jupunba Inokulum der Stämme Nr. 62 und 56 gegeben (Tab. 24). Die Inokulation wurde nach 3 Wochen wiederholt.

Die Düngung wurde dem Pflanzenwachstum angepasst. Je nach Variante wurde mit 100ml der Hoagland-Nährlösung (siehe Tab. 16 und Tab. 17) anfangs einmal die Woche, ab der 24. Versuchswoche alle 3 Tage gegossen. Die N-Konzentration der Nährlösung betrug von der 1. bis zur 14. Versuchswoche $11 \mathrm{mg} /$ Pflanze, danach $17 \mathrm{mg}$ N/Pflanze. 
Die äußeren Bedingungen des Gewächshauses entsprachen denen des jeweiligen Tagesklimas, da es ein offenes Gewächshaus war. Um den Schädlingsbefall einzuschränken, wurden Kästen über die Tische gestellt, die mit feinmaschigem Fliegennetz bezogen waren. Dadurch wurde die Lichtintensität jedoch um circa $25 \%$ vermindert.

Gegen Pilz- und Insektenbefall wurde regelmäßig gespritzt. Hierbei wurden folgende Chemikalien verwendet:

KUMULUS S.

Mancozeb, DITHANE PM, ROHM

HAAS Brazil

Diazinon 400PM CIBA Geigy.

K-Othrin

Benlate (Benomyl)
Schwefel. (500g/1001 $\left.\mathrm{H}_{2} \mathrm{O}\right)$

Dithiocarbamat $\left(1 \mathrm{~g} / 1 \mathrm{H}_{2} \mathrm{O}\right)$

Organophosphat (125g/1001 $\mathrm{H}_{2} \mathrm{O}$ )

Methyl-1-(buthylcarbamoyl)2benzimidazol-carbamat ( $1 \mathrm{~g} / \mathrm{l} \mathrm{H}_{2} \mathrm{O}$ )

\author{
Akarizid \\ Akarizid und Fungizid \\ Akarizid und Insektizid \\ Fungizid \\ systemisches Fungizid
}

Folgender Befall mit Insekten, Pilzen und Milben mußte protokolliert werden:

Befall der Blätter von Abarema jupunba mit:......

Befall der Sandtöpfe mit:.
Capimodium spp.

Curvularia spp

Migraspora spp.

weiße Fliege

Pseudococcus comstocki, Hemiptera, Pseudococcidae ("Cochinilhas")

Schlupfwespe, die ihre Eier zusammen mit einer betäubten Larve in Gänge legte. Sie fraßen Knöllchen

Die Akarizide mußten als Prevention eingesetzt werden, da im gleichen Gewächshaus an anderen Pflanzen ein Befall mit Milben zu verzeichnen war.

Die Auswertung fand nach 6, 10, 12 und 14 Monaten statt. Es wurden jeweils 3 Töpfe geerntet, die Biomasse (Trockengewicht) jedes einzelnen Pflanzenorgans sowie Höhe und Durchmesser wurden bestimmt. Eine Bodenprobe je Topf wurde genommen. Sowohl Pflanzen- als auch Bodenproben wurden wie in Kap. V.1.5 behandelt und auf ihre NKonzentration und ${ }^{15} \mathrm{~N}$-Gehalt untersucht. 


\subsection{Nährlösungen}

Tab. 16: Zusammensetzung der Komplett-Nährlösung, modifiziert nach Hoagland

\begin{tabular}{lccc}
\hline \multicolumn{1}{c}{ Chemikalie } & $\begin{array}{c}\text { Menge der } \\
\text { Stammlösung }\end{array}$ & Stammlösung & Konzentration in der NL \\
\hline $\mathrm{H}_{2} \mathrm{O}_{\text {dest. }}$ & auf 1000ml auffüllen & $1 \mathrm{M}$ & $0.15 \mathrm{~g} / 1$ \\
$\mathrm{KH}_{2} \mathrm{PO}_{4}$ & $1,1 \mathrm{ml}$ & $1 \mathrm{M}$ & $0.41 \mathrm{~g} / 1$ \\
$\mathrm{KNO}_{3}$ & $4,1 \mathrm{ml}$ & $1 \mathrm{M}$ & $0.69 \mathrm{~g} / 1$ \\
$\mathrm{Ca}\left(\mathrm{NO}_{3}\right)_{2}$ & $4,2 \mathrm{ml}$ & $1 \mathrm{M}$ & $0.24 \mathrm{~g} / 1$ \\
$\mathrm{MgSO}_{4}$ & $2 \mathrm{ml}$ & siehe Tab. 18 & \\
$\mathrm{Mikroelemente}_{\text {FeEDTA }}$ & $1 \mathrm{ml}$ & siehe unten & \\
\hline
\end{tabular}

Tab. 17: Zusammensetzung der Nährlösung ohne N, modifiziert nach Hoagland

\begin{tabular}{lccc}
\hline \multicolumn{1}{c}{ Chemikalie } & $\begin{array}{c}\text { Menge der } \\
\text { Stammlösung }\end{array}$ & Stammlösung & $\begin{array}{c}\text { Konzentration in der } \\
\text { Nährlösung }\end{array}$ \\
\hline $\mathrm{H}_{2}$ Odest. & auf $1000 \mathrm{ml}$ auffüllen & \\
$\mathrm{KH}_{2} \mathrm{PO}_{4}$ & $1,1 \mathrm{ml}$ & $1 \mathrm{M}$ & $0.15 \mathrm{~g} / 1$ \\
$\mathrm{KCl}_{\mathrm{CaCl}}$ & $5,0 \mathrm{ml}$ & $1 \mathrm{M}$ & $0.37 \mathrm{~g} / 1$ \\
$\mathrm{MgSO}_{4}$ & $5,0 \mathrm{ml}$ & $1 \mathrm{M}$ & $0.55 \mathrm{~g} / 1$ \\
$\mathrm{Mikroelemente}_{\text {FeEDTA }}$ & $2,0 \mathrm{ml}$ & $1 \mathrm{M}$ & $0.24 \mathrm{~g} / 1$ \\
\hline
\end{tabular}

Tab. 18: Mikroelemente-Nährlösung

\begin{tabular}{ll}
\hline Chemikalie & Konzentration [g/l] \\
\hline $\mathrm{H}_{2}$ Odest. & auf $1000 \mathrm{ml}$ auffüllen \\
$\mathrm{H}_{3} \mathrm{BO}_{3}$ & $2,86 \mathrm{~g}$ \\
$\mathrm{MnCl}_{2} \times 4 \mathrm{H}_{2} \mathrm{O}$ & $1,81 \mathrm{~g}$ \\
$\mathrm{ZnSO}_{4} \times 7 \mathrm{H}_{2} \mathrm{O}$ & $0,22 \mathrm{~g}$ \\
$\mathrm{CuSO}_{4} \times 5 \mathrm{H}_{2} \mathrm{O}$ & $0,08 \mathrm{~g}$ \\
$\mathrm{Na}_{2} \mathrm{MoO}_{4} \times 4 \mathrm{H}_{2} \mathrm{O}$ & $0,02 \mathrm{~g}$ \\
\hline
\end{tabular}

\section{FeEDTA}

Die FeEDTA-Lösung wurde für die gesamte Bodenkunde-Abteilung der FCAP nach folgendem Rezept hergestellt:

24.9 $\mathrm{g} \mathrm{FeSO}_{4} \times 7 \mathrm{H}_{2} \mathrm{O}$ mit $286 \mathrm{ml} \mathrm{KOH}$ mischen, 5 Minuten unter leichter Hitzezufuhr rühren lassen. 26.1g EDTA zur Lösung geben, ebenfalls etwas rühren lassen. Diese Suspension mindestens 8 Stunden belüften, dann das Volumen auf 1L auffüllen. 


\section{$3 \quad$ Laborarbeiten}

\subsection{Bakterienkultivierung}

Die im Feld gewonnenen Knöllchen wurden unter fließendem Wasser gereinigt. Durch die Transportzeit und Lagerung dehydrierte Knöllchen wurden durch Übernacht-Inkubation in sterilem Wasser aufgequollen. Unter sterilen Bedingungen wurden die Knöllchen 1 Minute in 95\%igem Ethanol, anschließend in 3\%iger NaOCl-Lösung oberflächensterilisiert. Nach 3maligem Waschen in sterilisiertem Wasser wurden die Knöllchen mazeriert und auf „roten“ Hefe-Mannitol-Agar (YMA) (siehe Tab. 19) mit Verdünnungsausstrich ausgebracht. Nach 2 Tagen Inkubation bei Raumtemperatur $\left(25^{\circ} \mathrm{C}\right)$ wurden einzeln gewachsene Kolonien, die den Farbstoff nicht absorbiert hatten, auf „grünen“ YMA übertragen und erneut Vereinzelungen vorgenommen. Nach 3 bzw. 7 Tagen wurde zur Identifizierung der Kolonien eine Charakterisierung nach Somasegaran und Hoben (1985) durchgeführt.

Tab. 19: Agarmedien zur Isolation und Kultur von Rhizobium spp.

\begin{tabular}{|c|c|c|}
\hline $\mathrm{K}_{2} \mathrm{HPO}_{4}($ Merck) & $0,5 \mathrm{~g}$ & \\
\hline 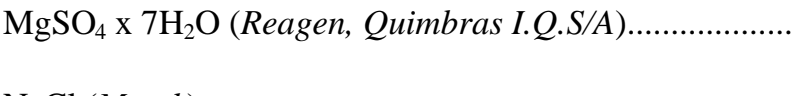 & $0,2 \mathrm{~g}$ & \\
\hline $\begin{array}{l}\mathrm{NaCl}(\text { Merck) } \\
\text { Mannitol (Reagen, Ouimbras Industrias Ouímicas S/A). }\end{array}$ & $0,1 \mathrm{~g}$ & \\
\hline $\begin{array}{l}\text { Mannitol (Reagen, Quimbras Industrias Químicas S/A).. } \\
\text { Hefeextract (Biobras, Bioquímica do Brasil) }\end{array}$ & $10 \mathrm{~g}$ & \\
\hline $\mathrm{H}_{2} \mathrm{Odest}$ & $\begin{array}{l}0,4 \mathrm{~g} \\
1000 \mathrm{ml}\end{array}$ & \\
\hline 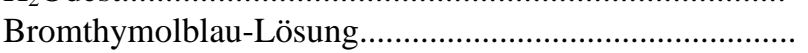 & circa $5 \mathrm{ml}$ & für ,grünen“ YMA \\
\hline 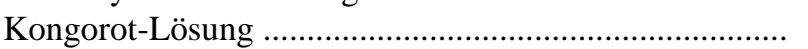 & circa $5 \mathrm{ml}$ & für „roten“ YMA \\
\hline 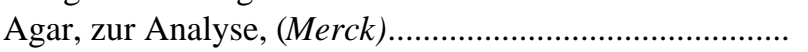 & $15 \mathrm{~g}$ & für festes Medium, Agarplatten etc. \\
\hline Agar...1... & $1,5 \mathrm{~g}$ & für Flüssigmedium, Inokulumproduktion \\
\hline
\end{tabular}

Der $\mathrm{pH}$ des Agarmediums wird mit 10\%iger $\mathrm{KOH}$ auf 6,8-7,0 eingestellt. Wurde Bromthymolblau (Tab. 20 oder Tab. 21) zugegeben, zeigte der Agar eine dunkelgrüne Färbung, bei Zugabe von Kongorot (Tab. 22) wurde auch der Agar dem Indikator gemäß rot.

Für kurzfristige Kulturen wurde der Agar in Petrischalen gegossen, für Dauerkulturen wurden mit demselben Agar Agarschrägröhrchen (sterilisierte Reagenzgläser mit 8ml Agar, zum Abkühlen schräg gelagert) hergestellt. 
Tab. 20: Bromthymol-Lösung in 20\%igem Ethanol $(0,5 \%)$

\begin{tabular}{|c|c|}
\hline Bromthymolblau...... & $0,1 \mathrm{~g}$ \\
\hline Ethanol, reinst, heiß. & $20 \mathrm{ml}$ \\
\hline $\begin{array}{l}\mathrm{H}_{2} \mathrm{O}_{\text {dest }} \ldots \ldots \ldots \ldots \ldots \ldots . . . . . . . . . \\
\text { nach Lösung des Farbstoffes } \\
\text { in Ethanol zugeben }\end{array}$ & $80 \mathrm{ml}$ \\
\hline
\end{tabular}

Tab. 22: Kongorot-Lösung $(0,25 \%)$

\begin{tabular}{ll}
\hline Kongorot & $125 \mathrm{mg}$ \\
$\mathrm{H}_{2}$ Odest. & $50 \mathrm{ml}$ \\
\hline
\end{tabular}

Tab. 21: Bromthymol-Lösung in $0,2 \mathrm{~N} \mathrm{KOH}$ $(0,5 \%)$

\begin{tabular}{lll}
\hline $\mathrm{KOH}($ Merck)................. & $5,61 \mathrm{~g}$ \\
Bromthymolblau (Merck) & $2,5 \mathrm{~g}$ \\
$\mathrm{H}_{2} \mathrm{O}_{\text {dest }} \ldots \ldots \ldots \ldots \ldots \ldots \ldots \ldots \ldots \ldots .$. & $500 \mathrm{ml}$
\end{tabular}

Zur Produktion von Inokulum wurden 100ml-Erlenmeyer-Kolben mit 50ml YMAFlüssigmedium befüllt und autoklaviert. Unter sterilen Bedingungen wurden die Kolben nach Abkühlen mit dem jeweiligen Bakterienstamm beimpft. Auf einem langsamen Schüttler wurden diese Kolben dann 5 Tage bei Raumtemperatur inkubiert. Dabei wurde eine Zelldichte von 1 bis 5,6 x 107 Zellen/ml Medium je nach Bakterienstamm erreicht.

Tab. 23: Auf grünem YMA zeigen typische Rhizobien nach Somasegaran und Hoben (1985) folgende Charakteristika:

\begin{tabular}{lll}
\hline Eigenschaft & Typ A & Typ B \\
\hline Säurereaktion & alkalisch (Blaufärbung des Agars) & sauer (Gelbfärbung des Agars) \\
Wachstum & langsam (maximaler $\varnothing$ nach 7-12 & schnell (maximaler $\varnothing$ nach 3-5 Tagen 4- \\
& Tagen 1-3mm) & $5 \mathrm{~mm}$ ) \\
Farbe & weiß-opak oder milchig-transparent & weiß-opak oder milchig-transparent \\
Kolonienform & diskret, rund, erhaben, glatter Rand & diskret, rund, erhaben, glatter Rand \\
Schleimproduktion & ja & viel \\
Indikatorabsorptio & nein & nein \\
n & & \\
\hline
\end{tabular}

Von 112 Isolaten zeigten 25 die typischen Charakteristika von Rhizobium (Typ B) und Bradyrhizobium (Typ A) (Tab. 23). In einem Gewächshausversuch mit Vigna unguiculata wurde deren Infektionspotential überprüft und ihre Zugehörigkeit zur Gattung Rhizobium verifiziert. Sowohl die Form der im Feld gesammelten Knöllchen als auch die Farbe ihrer 
Bakterienkolonien stimmten mit denen von Souza et al. (1994) gefundenen Charakteristika für die Arten Abarema jupunba und Inga thibaudiana überein.

Tab. 24: Isolate, die in den Versuchen verwendet wurden.

\begin{tabular}{lll}
\hline Bakterienstamm & Art & Typ \\
\hline $50(3)$ & Abarema cochleatum & $\mathrm{B}$ \\
62 & Abarema jupunba & $\mathrm{B}$ \\
64 & Abarema jupunba & $\mathrm{B}$ \\
56 & Abarema jupunba & $\mathrm{A}$ \\
\hline
\end{tabular}

\section{$3.2 \quad{ }^{15} \mathrm{~N}$-Analyse}

Die bei $60^{\circ} \mathrm{C}$ getrockneten, gemahlenen Proben wurden in einem Exsikkator abgekühlt. 97$104 \mathrm{mg}$ Boden, $6 \pm 1 \mathrm{mg}$ von Blatt, Wurzel oder Rinde, $10 \pm 1 \mathrm{mg}$ Holz, $15 \pm 1 \mathrm{mg}$ Streu oder 34 mg Knöllchen wurden in Zinnkapseln (Firma Lüdi AG) eingewogen (Sartorius Feinwaage M5P) und dem Isotopenlaboratorium für biologische und medizinische Forschung in Göttingen zur massenspektrometrischen Analyse übergeben.

\subsubsection{Prinzip der Messung}

Das Isotopenlaboratorium verwendete ein Massenspektrometer des Typs Finnigan MAT 251. Die Überführung der N-Verbindungen in die Gasphase geschieht durch Verbrennung. Das Gas wird zuerst chemisch mit $\mathrm{Mg}\left(\mathrm{ClO}_{4}\right)_{2}$ und $\mathrm{NaOH}$, dann physikalisch gereinigt, so daß pures $\mathrm{N}_{2}$ mit $2 \times 10^{-6} \mathrm{mbar}$ über die Ionisierungsfilamente geleitet wird. Diese Filamente produzieren Elektronen, die eine kleine definierte Menge des Gases bombardieren und so die Moleküle in $\left(\mathrm{N}_{2}\right)^{30+},\left(\mathrm{N}_{2}\right)^{29+}$ und $\left(\mathrm{N}_{2}\right)^{28+}$-Kationen konvertieren. Diese Kationen werden in einem Magnetfeld (4000V mit $10^{-5}$ torr) beschleunigt und ihrer Masse entsprechend dispergiert, mit einem Prisma polychromatischen Lichtes vergleichbar. Dieser Ionenstrom trifft nun je nach Masse des Ions zeitlich verschoben auf den Analysator (der Anode), der die unterschiedlichen Mengen mißt. Die Meßergebnisse werden in $\mathrm{m} / \mathrm{e}^{29}$ und $\mathrm{m} / \mathrm{e}^{28}$ ausgedrückt. $\left(\mathrm{N}_{2}\right)^{30}$ wird bei der Berechnung vernachlässigt. Vor und nach jeder Messung wird ein Standardgas (hier $\mathrm{N}_{2}$ ) durch das System geleitet. Kalibriert wird das System mit Acetanilid, das nach je 10 Proben analysiert wird. Das ${ }^{15} \mathrm{~N}$ im Luftstickstoff wird als 0-Wert mit in die Berechnung einbezogen. Dem Massenspektrometer am hiesigen Isotopenlabor war ein automatischer N-Analysator (Carlo Erba, NA 1500) vorgeschaltet. 


\subsubsection{Meßgenauigkeit}

Minimalmenge des eingewogenen Stickstoffes ist $10 \mu \mathrm{g}$, wobei die Standardabweichung bei den eingewogenen $\mathrm{N}_{\text {tot }}$-Mengen unter $200 \mu \mathrm{g} 0,2 \%$, bei $\mathrm{N}_{\text {tot. }}$-Mengen über $200 \mu \mathrm{g} 0,1 \%$ o beträgt (Reineking et al., 1993).

Daten aus den Meßserien des Jahres 1994 veranschaulichen die Abhängigkeit der Meßgenauigkeit von der N-Einwaage (Abb. 14). Ein Problem der Isotopen-Messungen geht daraus deutlich hervor: Die Pflanzenproben können nur beschränkt in höherer Menge als 10 mg Trockenmasse in die kleinen $\mathrm{Zn}$ - Kapseln eingewogen werden. Daraus resultiert eine zu analysierende N-Menge von 20 bis $70 \mu \mathrm{g}$. Diese N-Einwaage liegt jedoch in dem Meßbereich, in dem die Messungen noch stark streuen.

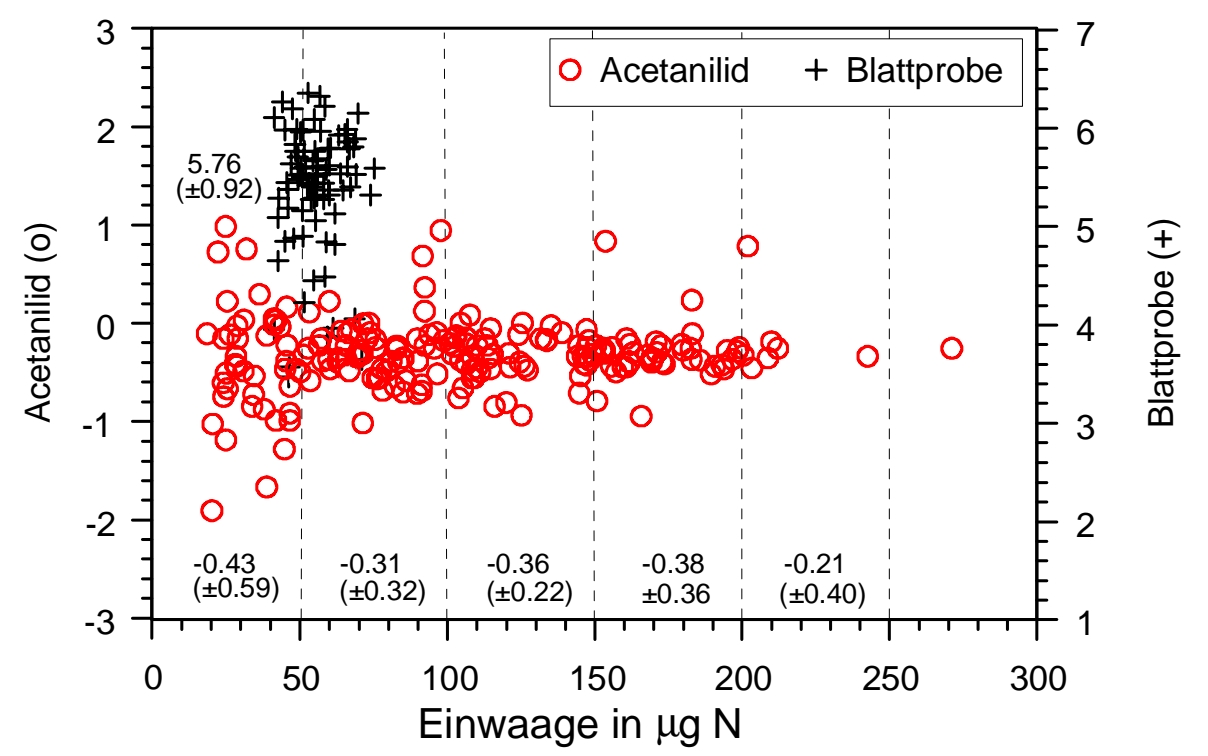

Abb. 14: Abhängigkeit der massenspektrometrischen ${ }^{15} \mathrm{~N}-$ Meßgenauigkeit von der N-Einwaage (Symbol 0 gibt Einzelmeßwerte des Standards Acetanilid an, Symbol + steht für den Meßwert einer Pflanzenprobe; Zahlen geben den Mittelwert der jeweiligen Messungen für die Einwaage von 0 $50 \mu \mathrm{g}, 50-100 \mu \mathrm{g}, 100-150 \mu \mathrm{g}, 150-200 \mu \mathrm{g}$ und $200-250 \mu \mathrm{g} \mathrm{N}$ an, Standardabweichung in Klammern)

In diesen niedrigen Meßbereichen können kleinere Stromschwankungen schon zu Meßfehlern führen. Solche Schwankungen sind zu bestimmten Tageszeiten, zu der auf dem Universitätsgelände häufiger Geräte ein - und ausgeschaltet werden, möglich. Anhand der Messung einer Pflanzenprobe verteilt über einen Tag wurden diese Unregelmäßigkeiten überprüft. Abb. 15 veranschaulicht diese Tagesschwankungen. Diesen Ungenauigkeiten wurde Rechnung getragen, indem Proben mit geringen ${ }^{15} \mathrm{~N}$-Konzentrationen vorwiegend nachts gemessen wurden. 


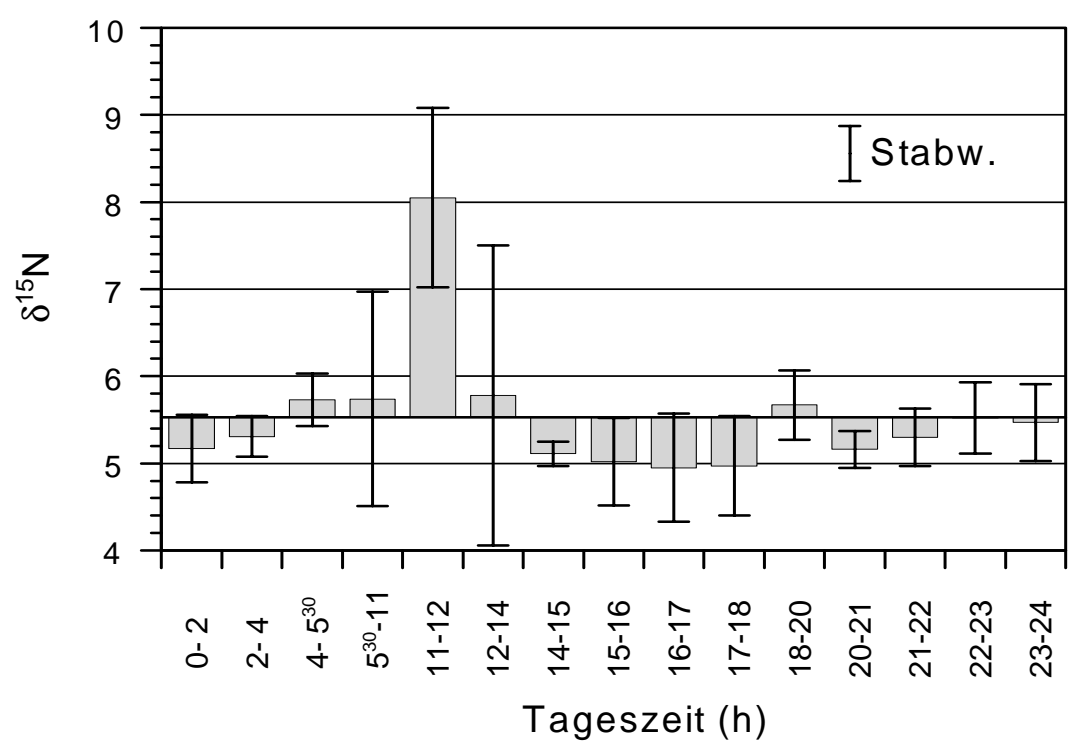

Abb. 15: Abweichungen vom Tagesmittel der ${ }^{15}$ N-Messungen einer Blattprobe im Tageslauf $(n \geq 4)$

\section{$4 \quad$ Berechnungen und statistische Auswertung}

\section{1 $\quad \delta^{15} \mathrm{~N}, \%$ Ndfa und $\beta$-Faktor}

Die Ratio des Ionenstromes $\left({ }^{14} \mathrm{~N}_{2} /{ }^{14} \mathrm{~N}^{15} \mathrm{~N}\right)=\mathrm{R}$ wird wie folgt ausgedrückt:

$$
\text { Atom\% }{ }^{15} N=100 \div(2 R+1)
$$

Die Anreicherung der Probe wird gegenüber dem Standard Luft in Promilleeinheiten angegeben und $\delta^{15} \mathrm{~N}$ genannt:

$\delta^{15} \mathrm{~N}=\left(\left(\%{ }^{15} \mathrm{~N}_{\text {Probe }}-\%{ }^{15} \mathrm{~N}_{\text {Standard }}\right) \div\left(\%{ }^{15} N_{\text {Standard }}\right)\right) \cdot 1000$

Zur Berechnung des Anteils fixierten $\mathrm{N}$ in einer Pflanze (\% Ndfa) oder einem Substrat wurde folgende Formel verwendet:

$$
\% N d f A=\left(\left(\delta^{15} \mathrm{~N}_{\text {Ref }}-\delta^{15} \mathrm{~N}_{\text {Leg }}\right) \div\left(\delta^{15} \mathrm{~N}_{\text {Ref }}-\delta^{15} \mathrm{~N}_{\mathrm{A}}\right)\right) \cdot 100
$$

Zur Beschreibung des Grades der Fraktionierung einer Reaktion bedient man sich des sog. $\beta$-Faktors, der vereinfacht als Quotient aus Substrat und Produkt beschrieben werden kann (Lajtha und Marshall, 1994; Shearer und Kohl, 1993) und nach folgender Formel errechnet wird: 


$$
\beta=\frac{\left(\mathrm{mgN}_{\text {Substrat }} \times \mathrm{At} \%^{14} \mathrm{~N}-\mathrm{mgN}_{\text {Produkt }} \times \mathrm{At} \%^{14} \mathrm{~N}\right) /\left(\mathrm{mgN}_{\text {Substrat }} \times \operatorname{At} \%^{14} \mathrm{~N}+\mathrm{mgN}_{\text {Produkt }} \times \operatorname{At} \%^{14} \mathrm{~N}\right)}{\left(\mathrm{mgN}_{\text {Substrat }} \times \mathrm{At} \%^{15} \mathrm{~N}-\mathrm{mgN}_{\text {Produkt }} \times \mathrm{At} \%^{15} \mathrm{~N}\right) /\left(\mathrm{mgN}_{\text {Substrat }} \times \mathrm{At} \%^{15} \mathrm{~N}+\mathrm{mgN}_{\text {Produkt }} \times \mathrm{At} \%{ }^{15} \mathrm{~N}\right)}
$$

\subsection{Berechnung der Stickstoffakkumulation über die BNF der Leguminosen}

Von Nunez (1995) wurden Stubben-Durchmesser und Biomasse-Erhebungen gezielt für einige Arten, unter anderem Leguminosen, in der Brachevegetation des Untersuchungsgebietes durchgeführt. Hieraus ist die Größenzusammensetzung der Arten für eine definiertes Feldareal bekannt.

Die Analyse verschieden großer Individuen von Abarema jupunba und nicht $\mathrm{N}_{2}$ fixierender Referenzarten ermöglicht Berechnungen, wieviel \% des Gesamt-N eines Individuums einer bestimmten Größe durch biologische $\mathrm{N}_{2}$-Fixierung erlangt wurde (die Maße der beprobten Individuen und ihre Referenzpflanzen sind in Tab. 25 zusammengefaßt). Mit den für Abarema jupunba gewonnenen Daten wurden Regressionen der Parameter $\mathrm{kg}$ Trockenmasse/Baum zu Basalfläche $\left(\pi \mathrm{r}^{2}\right)$, gN/Baum $\mathrm{zu} \mathrm{kg}$ TM/Baum und \%Ndfa zu Basalfläche mit dem Programm Xact der Firma SciLab gerechnet. Darüber kann die Stickstoffmenge, die pro Flächeneinheit von dieser Art biologisch gebunden wurde, kalkuliert werden und so eine Aussage über die Fixierungseffektivität dieser Art in der Sekundärvegetation gemacht werden.

Tab. 25: Größenzusammensetzung der einzelnen Individuen von Abarema jupunba und die in unmittelbarer Nähe dazu beprobten Referenzpflanzen

\begin{tabular}{|c|c|c|}
\hline Basalfläche $\left(\mathrm{cm}^{2}\right)$ & Höhe $(\mathrm{cm})$ & Referenzpflanzen \\
\hline 0.2 & 48 & $\begin{array}{l}\text { Banara guianensis. Myrcia cuprea. Myrcia deflexa. Solanum } \\
\text { carvuranum }\end{array}$ \\
\hline 0.8 & 61 & $\begin{array}{l}\text { Banara guianensis. Myrcia cuprea. Myrcia deflexa. Solanum } \\
\text { caavurana }\end{array}$ \\
\hline 5.4 & 160 & Casearia arborea. Mabea angustifolia. Ocotea spec. \\
\hline 10.2 & 280 & Aegiphila racemosa. Myrcia sylvatica. $2 x$ Vismia guianensis \\
\hline 13.8 & 340 & Aegiphila racemosa. Myrcia sylvatica. $2 \times$ Vismia guianensis \\
\hline 19.2 & 590 & $\begin{array}{l}\text { Myrcia sylvatica. Myrcia bracteata. Tapirira guianensis. Vismia } \\
\text { guianensis }\end{array}$ \\
\hline 27.2 & 460 & $\begin{array}{l}\text { Mabea angustifolia. Myrcia cuprea. Vismia guianensis. Tapirira } \\
\text { guianensis. }\end{array}$ \\
\hline 33.3 & 520 & $\begin{array}{l}\text { Myrcia sylvatica. Myrcia bracteata. Tapirira guianensis. Vismia } \\
\text { guianensis }\end{array}$ \\
\hline 36.4 & 640 & $\begin{array}{l}\text { Myrcia sylvatica. Myrcia bracteata. Tapirira guianensis. Vismia } \\
\text { guianensis }\end{array}$ \\
\hline 44.3 & 750 & $\begin{array}{l}\text { Myrcia sylvatica. Myrcia bracteata. Tapirira guianensis. Vismia } \\
\text { guianensis }\end{array}$ \\
\hline
\end{tabular}




\subsection{Statisitik}

Die Berechnungen wurden mit den Statistikprogrammen SAS (SAS Institute Inc., Cary, USA) und Statistica (Statsoft 1994) durchgeführt. Wie zuvor beschrieben, wurden Regressionen mit dem Programm Xact der Firma SciLab angepasst.

Der Verteilungsmodus der Daten wurde mit dem Chiquadrat-Vergleichstest und dem Kolmogorow-Smirnov-Test auf Anpassungsgüte ermittelt.

Wegen der geringen Anzahl an Wiederholungen der chemischen Bodenanalysen wurde hier nur der Mittelwert und der Standardfehler der jeweiligen Variante angegeben.

Bei Normalverteilung wurden einfache und mehrfaktorielle Analysen (ANOVA) durchgeführt, sofern der Levene's Test auf Homogenität gleiche Varianzen schließen ließ. Hierarchische und faktorielle ANOVA wiesen hierbei keine wesentlichen Unterschiede auf. Daher wurden im weiteren einzelne Tests auf der Basis faktorieller Varianzanalysen durchgeführt. Die ANOVA-Ergebnisse sind als allgemeiner Überblick der einzelnen Datensätze im Anhang zu finden.

Bei nicht normalverteilten Daten wurde transformiert, indem im Falle der N-Analysen logarithmiert, im Falle der Deltawerte 10 Deltaeinheiten dazu addiert und dann logarithmiert wurde. Erreichte man so keine Normalverteilung, wurde auf nicht-parametrische Tests zurückgegriffen.

Die Signifikanzgrenze wurde bei einer Irrtumswahrscheinlichkeit von $\mathrm{p} \leq 0.05$ festgelegt. Das Signifikanzniveau $\mathrm{p} \leq 0.05, \mathrm{p} \leq 0.01$ und $\mathrm{p} \leq 0.001$ wird im Folgenden mit *, **, *** gekennzeichnet.

In Tab. 26 ist ein Überblick über die angewendeten statistischen Verfahren gegeben.

Die ${ }^{15} \mathrm{~N}$-Daten im natürlichem Konzentrationsbereich in slash and burn Vegetationen waren durch eine enorme Streuung geprägt. Daher war es oft schwierig, Ergebnisse statistisch abzusichern. Auch nach Transformationen blieben die Daten trotz erzielter Normalverteilung in ihren Varianzen stark inhomogen. Unterschiedlichster Bewuchs, Anzahl an Bränden, Hackungen, Düngungen, Termiten oder Blattschneiderameisen etc. haben den Boden in Nuancen unterschiedlich verändert. Auch Schulze et al. (1991) oder Högberg und Alexander (1995) bestätigen diese Inhomogenität der $\delta^{15} \mathrm{~N}$-Werte in einer Vegetation mit Brand- 
Vorgeschichte. Bis auf wenige Ausnahmen wurde daher bei fast allen Statistikprozeduren auf eine Ausreißereliminierung verzichtet und zum Teil statistisch schlecht abgesicherte Ergebnisse toleriert.

Tab. 26: Übersicht über die statistischen Verrechnungen, die zu den jeweiligen Themen durchgeführt wurden.

\begin{tabular}{|c|c|c|c|}
\hline Datensatz & Verteilung & Statisitk & Fragestellung/Wirkung \\
\hline \multirow{2}{*}{$\begin{array}{l}\text { Bodenchemie - } \\
\text { und physik }\end{array}$} & \multirow{2}{*}{$\begin{array}{l}\text { nicht } \\
\text { normal }\end{array}$} & Standardfehler & Charakterisierung \\
\hline & & multiple Regression & $\begin{array}{l}\text { Zusammenhänge zwischen Bodenchemie } \\
\text { und } \delta^{15} \mathrm{~N}\end{array}$ \\
\hline \multirow{3}{*}{$\begin{array}{l}\text { Boden }-\delta^{15} \mathrm{~N} \\
\% \mathrm{~N}\end{array}$} & \multirow[t]{3}{*}{ normal } & ANOVA & generell \\
\hline & & Regressionen & Verlauf im Boden \\
\hline & & $t$-Test, Scheffé-Test & Asche, Distanz, Jahreszeit \\
\hline $\begin{array}{l}\text { Boden mit Peixe } \\
\text { Boi }\end{array}$ & $\begin{array}{l}\text { extreme } \\
\text { Ausreißer }\end{array}$ & $\begin{array}{l}\text { multipler } t \text {-Test } \\
\text { Kruskal-Wallis-,,ANOVA“ }\end{array}$ & $\begin{array}{l}\text { Absicherung der } \delta^{15} \mathrm{~N}-\text { Wert- } \\
\text { Unterschiede, nicht möglich }\end{array}$ \\
\hline \multirow[t]{3}{*}{$\begin{array}{l}\text { organische } \\
\text { Auflage }\end{array}$} & \multirow[t]{3}{*}{ normal } & $\begin{array}{l}\text { ANOVA, } t \text {-Test, Scheffé- } \\
\text { Test }\end{array}$ & Distanzen \\
\hline & & $t$-Test & Jahreszeit \\
\hline & & Pearson-Korrelation & Bez. Blatt-Boden \\
\hline Knöllchen & $\begin{array}{l}\text { nicht } \\
\text { normal }\end{array}$ & Wilcoxon-Rang-Test & Infektionspotential \\
\hline \multirow[t]{5}{*}{$\begin{array}{l}\delta^{15} \mathrm{~N}- \\
\text { Vegetation }\end{array}$} & \multirow[t]{5}{*}{ normal } & $\begin{array}{l}\text { ANOVA, LSD-Test, } \\
\text { Scheffé }\end{array}$ & Zeitliche und räumliche Variabilität \\
\hline & & Varianz & Blattauswahl \\
\hline & & $\begin{array}{l}\text { Korrelationsanalyse nach } \\
\text { Pearson, Regressionen }\end{array}$ & Relation $\delta^{15} \mathrm{~N}$ und $\mathrm{N}-$ Gehalt der Blätter \\
\hline & & LSD-Test & Standortunterschiede \\
\hline & & $\begin{array}{l}\text { Cluster- Analyse, } \\
\text { Ward'scher Algorithmus, } \\
\text { Euclid'sche Distanz }\end{array}$ & $\begin{array}{l}\text { räumliche und zeitliche } \\
\text { Artenunterschiede }\end{array}$ \\
\hline \multirow[t]{2}{*}{$\%$ NdfA } & & $\begin{array}{l}\text { Standardfehler für Summen } \\
\text { und Quotienten nach } \\
\text { Shearer und Kohl, } 1978\end{array}$ & $\mathrm{~N}_{2}$-Fixierungsrate \\
\hline & & Regressionsanalysen & $\begin{array}{l}\text { Quantifizierung biologisch fixierten } \mathrm{N} \text { in } \\
\text { einem Bestand }\end{array}$ \\
\hline
\end{tabular}




\section{Ergebnisse}

\section{$1 \quad \delta^{15} \mathrm{~N}$ in der Sekundärvegetation der Bragantina-Region}

Für einen ersten Eindruck der ${ }^{15} \mathrm{~N}$-Verteilung in der Vegetation wurden die $\delta^{15} \mathrm{~N}$-Werte in den Blättern von 31 Nicht-Fixierern und 9 Fixierern von den verschiedenen Beprobungen in Abb. 16 zusammengefaßt. Die $\delta^{15} \mathrm{~N}-$ Werte der Sekundärvegetation in der Bragantina-Region zeigten auf den ersten Blick ein typisches Bild (im Anhang Tab. 59 sind die Werte mit $s$ aufgelistet). Die $\mathrm{N}_{2}$-Fixierer stammen aus der Familie der Leguminosae, Unterfamilie Mimosoideae, und wiesen eine geringere Streuung als die Nicht-Fixierer auf und ihre $\delta^{15} \mathrm{~N}$-Werte bewegten sich mit einer Streuung von $\pm 2 \delta^{15} \mathrm{~N}$-Einheiten um den Nullpunkt herum. Für die hier vorgefundenen Arten der beiden anderen Unterfamilien, den Caesalpinoideae und Papilionoideae, ist keine Nodulation bekannt (Faria et al., 1989), sodaß sie in die Gruppe der Nicht-Fixierer eingeordnet wurden. Die $\delta^{15} \mathrm{~N}$-Werte der Nicht-Fixierer umfaßten ein Spanne von 10 $\delta^{15} \mathrm{~N}$-Einheiten, die als sehr hoch angesehen werden kann. Innerhalb einer Pflanzenfamilie kommen Arten mit hohen oder niedrigen $\delta^{15} \mathrm{~N}$-Werten vor. Die Streuung der $\delta^{15} \mathrm{~N}$-Werte der einzelnen Arten wird daher für die Anwendung der ${ }^{15}$ NNAM als problematisch angesehen und bedarf einer ausführlichen Untersuchung. Interessant ist auch der Schwerpunkt dieser $\delta^{15} \mathrm{~N}$-Werte. Sind weltweit hauptsächlich positive $\delta^{15} \mathrm{~N}$-Werte für Nicht-Fixierer anzutreffen, so zeigten sich hier mehr negative bis wenig von Null abweichende $\delta^{15} \mathrm{~N}$-Werte. Nur 7 Arten wurden mit deutlich positiven Werten angetroffen, während 10 Arten deutlich negativ waren. Der $\delta^{15} \mathrm{~N}$-Wert der restlichen 13 Arten bewegte sich im Bereich um 0 bis negativ. Ein Zusammenhang der $\delta^{15} \mathrm{~N}-$ Werte mit den $\mathrm{N}-$ Gehalten der Blätter war nicht zu erkennen. 


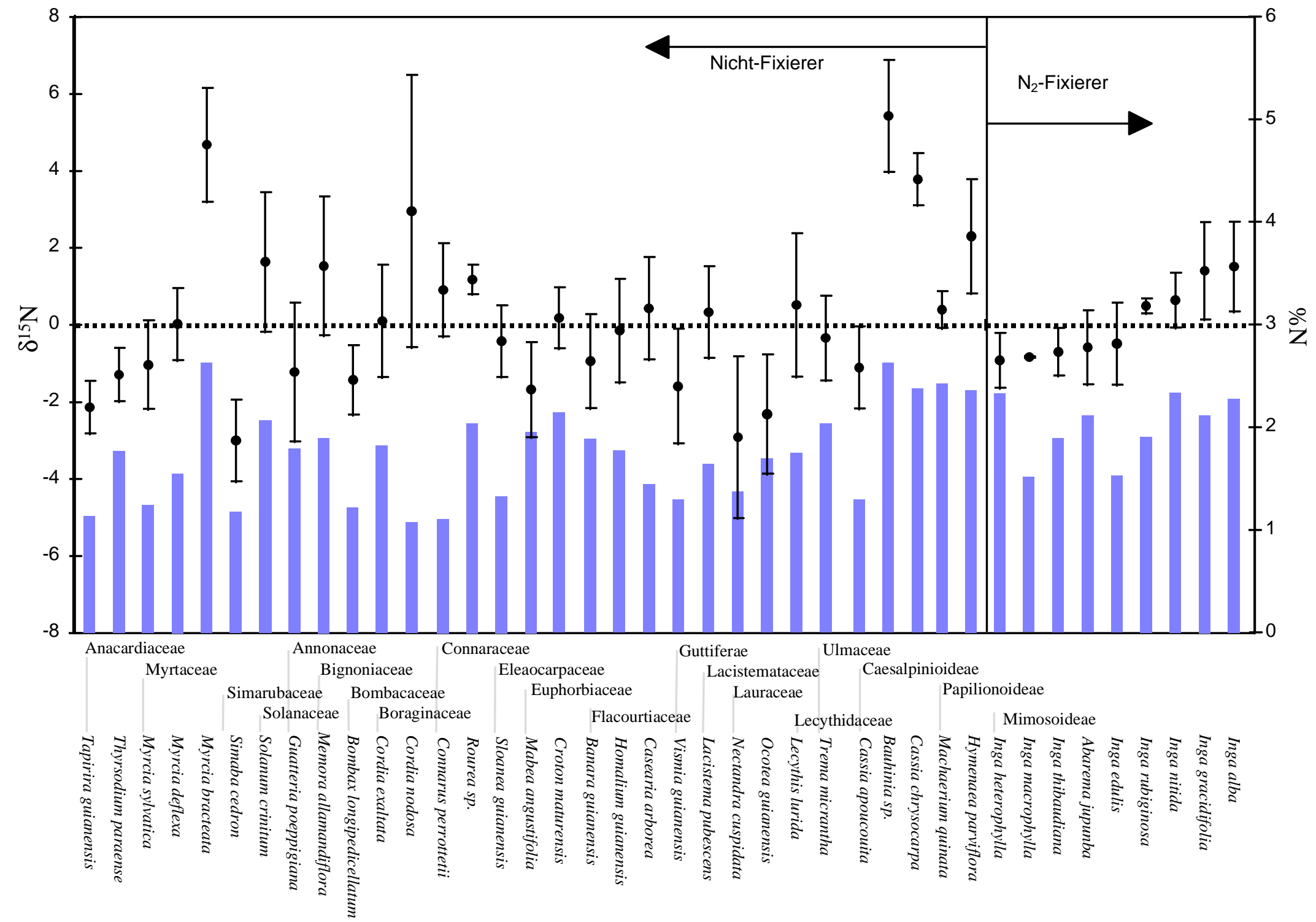

Abb. 16: Überblick über die $\delta^{15} \mathrm{~N}$-Werte (Punktmarker mit $s$ ) und N-Gehalte (Balken repräsentieren den Mittelwert für die jeweilige Art) in den Blättern der Sekundärvegetation in der Bragantina-Region. 


\section{$2 \quad \delta^{15} \mathrm{~N}$ im Mineralboden}

Zwischen den $\delta^{15} \mathrm{~N}$-Werten des Bodens und der Vegetation besteht ein Zusammenhang, denn die nicht fixierenden Pflanzen decken ihren N-Bedarf aus dem pflanzenverfügbaren $\mathrm{N}$ des Bodens. Zur Beurteilung der $\delta^{15} \mathrm{~N}-$ Werte in der Vegetation als eine Erklärung für die Variabilität des $\delta^{15} \mathrm{~N}$ und ihre Eignung für die ${ }^{15} \mathrm{NNAM}$ ist es daher von Bedeutung, folgende Parameter zu kennen:

- $\delta^{15} \mathrm{~N}$ des Boden-N generell und die Differenz zwischen dem $\delta^{15} \mathrm{~N}$ der Luft und der BodenN-Quelle

- Veränderung des $\delta^{15} \mathrm{~N}-$ Wertes mit der Bodentiefe

- Variabilität auf einzelnen Standorten

- Variabilität zwischen verschiedenen Standorten

- Zusammenhang von $\delta^{15} \mathrm{~N}$ mit den physikalischen und chemischen Bodencharakteristika

- Unterschiede in Regen- und Trockenzeit

\subsection{Räumliche Variabilität}

Eine dreifaktorielle Varianzanalyse der $\delta^{15} \mathrm{~N}$-Werte auf den Flächen M1 bis M5 zeigte bezüglich des Standortes und der Bodentiefe signifikante Unterschiede auf (Tab. 61 im Anhang). Kleinräumige Unterschiede hatten keine signifikante Bedeutung. Ob Boden unter einer Leguminose oder außerhalb ihrer Kronentraufe genommen wurde, spielte keine deutliche Rolle für den $\delta^{15} \mathrm{~N}$-Wert des $\mathrm{N}_{\text {tot. }}$. des Bodens. Diese Unterschiede waren jedoch auf einzelnen Flächen unterschiedlich stark ausgeprägt.

Die auf größere Entfernung liegenden 5 Flächen unterschieden sich in ihren $\delta^{15} \mathrm{~N}$-Werten jedoch signifikant voneinander. Dieser Unterschied blieb auch über die Bodentiefe erhalten.

Der $\delta^{15} \mathrm{~N}$-Wert veränderte sich auch mit der Bodentiefe signifikant, mit wenigen Ausnahmen auf allen 5 Flächen in gleicher Weise.

Die Signifikanzen in den Unterschieden der N-Gehalte der Böden wurden im Wesentlichen in gleicher Weise wie die der $\delta^{15} \mathrm{~N}$-Werte ausgewiesen. Die kleinräumigen Unterschiede im Oberboden veränderten sich naturgemäß auch hier mit der Bodentiefe. Es bestanden aber auch Wechselwirkungen zwischen allen getesteten Faktoren, was auf eine große Streuung der N-Gehalte schließen läßt. 


\subsubsection{Vertikale Variabilität von $\delta^{15} \mathrm{~N}$}

\section{Bis 1 m Bodentiefe}

In Abb. 17 ist die Entwicklung des $\delta^{15} \mathrm{~N}$-Wertes im oberen Boden anhand der Einzelwerte der Flächen dargestellt. Die Zunahme der $\delta^{15} \mathrm{~N}$-Werte folgte einem asymptotischen Verlauf, der für 0 bis $100 \mathrm{~cm}$ Bodentiefe mit folgender Formel beschrieben werden kann: $\mathrm{y}=\left(7.05^{*} \mathrm{x}\right) /(\mathrm{x}-$ 2.83); $\mathrm{s}_{\mathrm{y}, \mathrm{x}}=0.75 ; \mathrm{r}^{2}=0.74 ; \mathrm{df}=28 ; \mathrm{p} \leq 0.001 \%$. In den oberen $5 \mathrm{~cm}$ des Mineralbodens lagen die $\delta^{15} \mathrm{~N}$-Werte bei +2.5 bis +5 , in $30 \mathrm{~cm}$ Tiefe erreichten sie +7.5 . Von da an blieb der $\delta^{15} \mathrm{~N}$-Wert konstant. Die N-Gehalte sanken von durchschnittlich $0.15 \%$ in den oberen $5 \mathrm{~cm}$ auf $0.04 \%$ von $40 \mathrm{~cm}$ Bodentiefe und stehen damit im umgekehrt proportionalen Verhältnis zum $\delta^{15} \mathrm{~N}-$ Wert.

Ungefähr bei $40 \mathrm{~cm}$ Bodentiefe beginnt der B-Horizont der Untersuchungsflächen mit circa 20\% Tonanteil. Mit der Bodentextur ändert sich auch die Wasserhaltekapazität. In solchen Bereichen des Bodens, in denen Texturänderungen auftreten, können besondere Anreicherungen mit ${ }^{15} \mathrm{~N}$ auftreten (Delwiche und Steyn, 1970). In den Untersuchungsflächen waren in dieser Tiefe die höchsten $\delta^{15} \mathrm{~N}$-Werte anzutreffen.
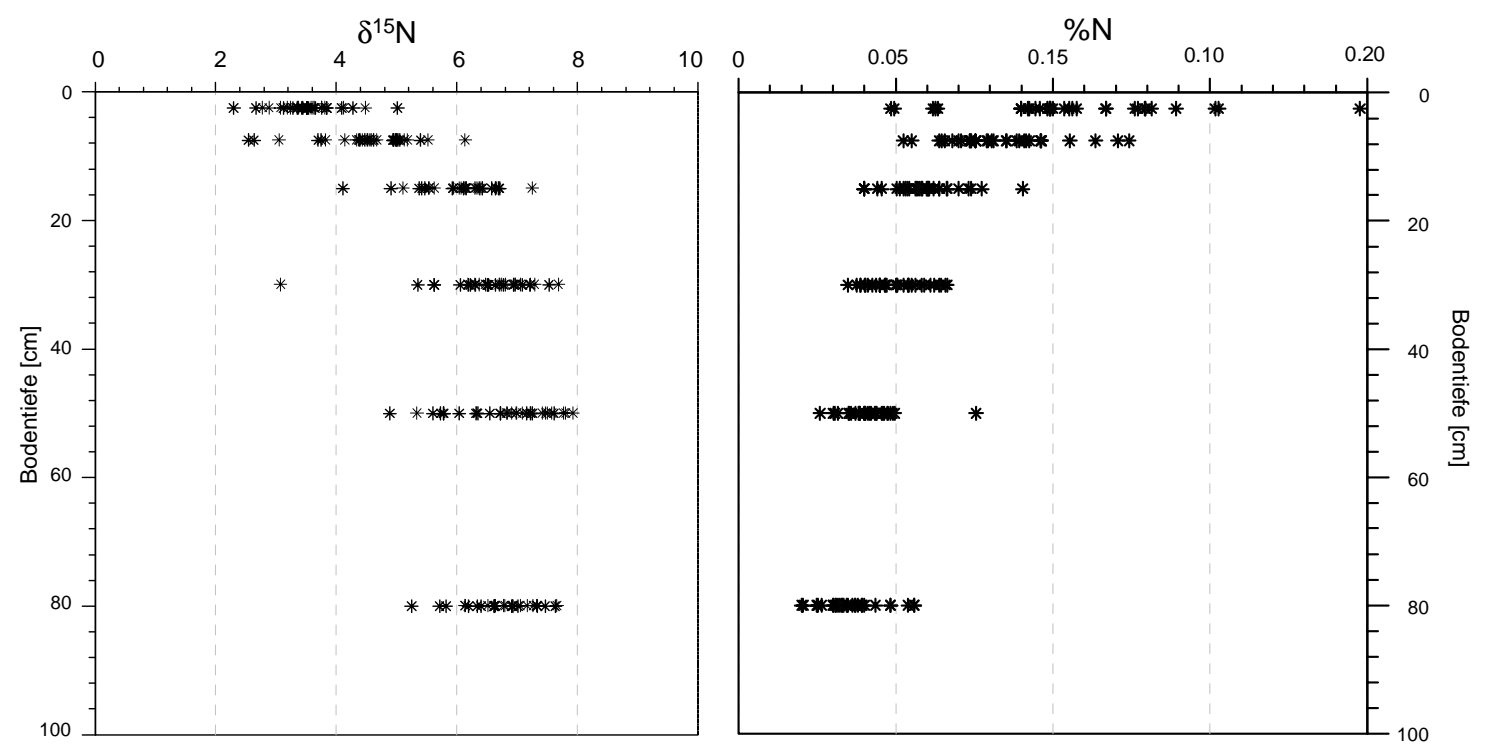

Abb. 17: $\delta^{15} \mathrm{~N}$ und N-Gehalte im A-Horizont unter junger Sekundärvegetation von Flächen M1 - M5

\section{Bis $6 m$ Bodentiefe}

In den Bodentiefen unter $1 \mathrm{~m}$ folgte der $\delta^{15} \mathrm{~N}-$ Verlauf einer Regression 1. Grades (Abb. 18).

Die Werte fielen von +7.5 in $1 \mathrm{~m}-1.50 \mathrm{~m}$ Tiefe bis +2.5 in $6 \mathrm{~m}$ Tiefe $a b$. Vereinzelt traten $\mathrm{ab}$ 
$5 \mathrm{~m}$ Bodentiefe negative $\delta^{15} \mathrm{~N}$-Werte auf. Dabei ist die hohe Streuung in tieferen Bodenschichten zu beachten, die durch meßtechnische Schwierigkeiten geringer N-Einwaagen bei der Analyse zustande kamen. Die Bodenproben der unteren Schichten hatten in der Regel weniger als $0.01 \% \mathrm{~N}_{\text {tot. }}$ aufzuweisen. Damit kam die massenspektrometrische Analyse an ihre Nachweisgrenzen. Der Trend kann aber als Indiz für die Verlagerung von löslichen $\delta^{15} \mathrm{~N}$ negativen Stickstoff-Verbindungen in tiefere Bodenschichten gelten.

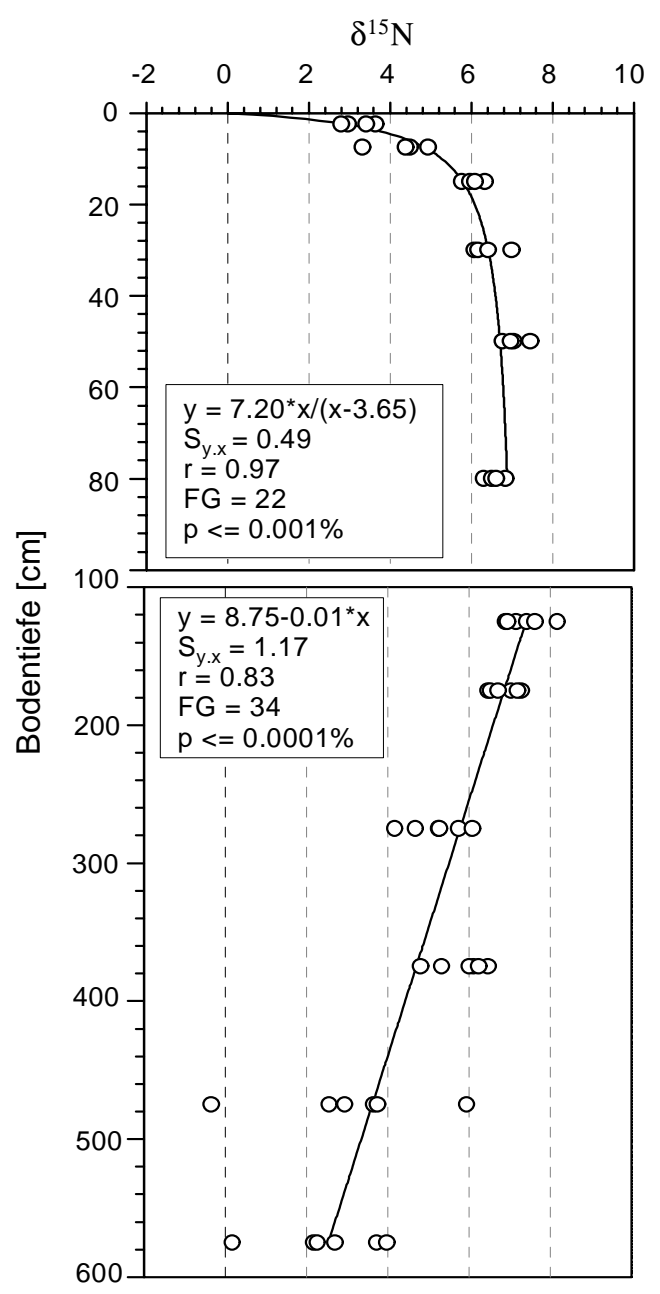

Abb. 18: $\delta^{15} \mathrm{~N}$ im $\mathrm{N}_{\text {tot. }}$ des Bodenprofils bis $6 \mathrm{~m}$ der Fläche M2S2, die Punkte geben die Einzelwerte der Proben an.

Die Ultisole der Untersuchungsregion besitzen eine geringe Wasserhaltekapazität und eine hohe Infiltrationsrate, die zu starken Auswaschungsprozessen führen kann. Während der Brandrodung und anschließenden Bewirtschaftung gibt es mehrfach Phasen, in denen $\mathrm{NO}_{3}{ }^{-}$ im Sickerwasser vorgefunden wurde (Hölscher, 1995). Erste ${ }^{15} \mathrm{~N}$-Analysen des pflanzenverfügbaren mineralischen $\mathrm{N}$ des Bodens von Fläche M1S1 bestätigen einen mit der Bodentiefe 
abnehmenden $\delta^{15} \mathrm{~N}$-Wert von anfänglich +4 bis +7 bis auf Werte von -4 in $60-100 \mathrm{~cm}$ Tiefe für $\mathrm{NH}_{4}{ }^{+}$. Für $\mathrm{NO}_{3}{ }^{-}$wurden $\delta^{15} \mathrm{~N}$-Werte von -27 in 10-20cm Bodentiefe gemessen. Diese Werte sind jedoch nur mit Vorsicht anzunehmen, da die N-Konzentration in den Proben für die ${ }^{15} \mathrm{~N}$-Analyse nahe an der Nachweisgrenze lagen (Paparcikova, 1996). Mit gleichen Analysen eines weiteren Bragantina-Bodens konnten die Resultate nicht reproduziert werden.

Bei dem Durchlauf durch eine Ionenaustauschersäule wird ${ }^{15} \mathrm{NO}_{3}{ }^{-}$mit einem $\beta$-Faktor von 1.0021 eher an die Austauscheroberflächen gebunden als ${ }^{14} \mathrm{NO}_{3}^{-}$(Delwiche und Steyn, 1970). Dieser chromatografische Effekt kann ebenfalls zu einer leichten ${ }^{15} \mathrm{~N}$-Verdünnung des $\mathrm{N}_{\text {tot. }}$ in 6m Bodentiefe beigetragen haben. Delwiche und Steyn (1970) schlossen auch den Einfluß ${ }^{15} \mathrm{~N}$-verdünnten alluvialen Stickstoffs in tieferen extrem N-verarmten Bodenzonen als mögliche Ursache niedriger $\delta^{15} \mathrm{~N}-$ Werte nicht aus.

Wie in Kap. VI.2.1.2 erläutert, lassen sich positive $\delta^{15} \mathrm{~N}$-Werte z.T. auf die ${ }^{15} \mathrm{~N}$-angereicherte organische Substanz zurückführen. Da ab einer Bodentiefe von 3.50m nur noch ein Kohlenstoffgehalt von höchstens 0.1\% nachzuweisen war (vergl. Tab. 10 und Sommer, 1996), kann eine Auswirkung des $\delta^{15} \mathrm{~N}$-Wertes der organischen Substanz auf den $\delta^{15} \mathrm{~N}$-Wert des $\mathrm{N}_{\text {tot. }}$ in Bodentiefen unterhalb von $3.50 \mathrm{~m}$ ausgeschlossen werden. Somit gelangt man in tieferen Bodenschichten an $\mathrm{N}$, dessen Isotopenverhältnisse durch weniger intensive Umsetzungsprozesse und seine Anreicherungsmechanismen nicht so stark verändert wurden.

\subsubsection{Zusammenhang von $\delta^{15} \mathrm{~N}$ mit bodenchemischen und -physikalischen Eigenschaften}

Eine multiple Regression mit den Bodenparametern Mn, Mg, K, Al, Basensättigung, C- und $\mathrm{N}$-Gehalt sowie Tonanteil ergab ein maximales Bestimmtheitsmaß von $r=0.69\left(\mathrm{r}^{2}=0.83\right.$, $\mathrm{F}(9.67)=16.72, \mathrm{p}<0.000, \sigma=0.75)$. Der Anteil der Tonfraktion an der Korngrößenverteilung korrelierte dabei positiv mit dem $\delta^{15} \mathrm{~N}$-Wert, Mg sowie Basensättigung negativ (Tab. 28).

Bei dieser stufenweisen Regression betrug, mit \% Ton beginnend, das Bestimmtheitsmaß von $\delta^{15} \mathrm{~N}$ zu Ton $\mathrm{r}^{2}=0.40,+\% \mathrm{~N}=0.61,+\mathrm{BS}=0.64,+\mathrm{Mg}=0.67$, während $\mathrm{Al}, \mathrm{K}, \mathrm{Mn}, \% \mathrm{C}$ und das $\mathrm{C} / \mathrm{N}-$ Verhältnis nur noch zu einer geringen Verbesserung (0.69) der Vorhersage führten. Über diese Faktoren können also 69\% der Varianz erklärt werden.

Ledgard et al. (1984) und auch Tiessen et al. (1984) erklärten hohe $\delta^{15} \mathrm{~N}$-Werte im Boden mit einem hohen Anteil an Fulvosäuren, Huminsäuren und Huminstoffen, deren Analysen 
$\delta^{15} \mathrm{~N}$-Werte von +4.7 bis +9.1 ergaben. Sie zeigten auch, daß das organische Bodenmaterial hauptsächlich mit der Tonfraktion verlagert wird, so daß die $\delta^{15} \mathrm{~N}$-Werte mit dem Tonanteil steigen, wie es auch mit dieser Untersuchung gezeigt werden konnte. Dieses Ergebnis wurde sowohl für einzelne Bodenschichten als auch für die gesamten Werte aller Bodentiefen gefunden.

Tab. 28: Zusammenfassung der multiplen Regression von $\delta^{15} \mathrm{~N}$ mit $\mathrm{Mn}, \mathrm{Mg}, \mathrm{K}, \mathrm{Al}$, Basensättigung (BS), C - und N - Gehalt und C/N-Verhältnis

\begin{tabular}{l|cl|cl|ll}
\hline Element & Korr.Koef. & $\sigma$ & Steigung & $\sigma$ & $\mathrm{t}(67)$ & $\mathrm{p}$ \\
\hline Intercept & & & 8.89 & 1.29 & 6.86 & $0.000^{* * *}$ \\
$\mathrm{Mn}$ & 0.04 & 0.17 & 0.04 & 0.15 & 0.27 & 0.78 \\
$\mathrm{Mg}$ & -0.25 & 0.11 & -1.31 & 0.57 & -2.30 & $0.02^{*}$ \\
$\mathrm{~K}$ & 0.02 & 0.18 & 1.29 & 11.50 & 0.11 & 0.91 \\
$\mathrm{Al}$ & -0.08 & 0.17 & -0.26 & 0.60 & -0.44 & 0.66 \\
$\mathrm{BS}$ & -0.37 & 0.17 & -0.03 & 0.01 & -2.18 & $0.03^{*}$ \\
$\% \mathrm{C}$ & 0.08 & 0.09 & 0.42 & 0.52 & 0.81 & 0.42 \\
$\%$ Ton & 0.33 & 0.11 & 0.05 & 0.02 & 2.78 & $0.007^{* *}$ \\
$\% \mathrm{~N}$ & -0.35 & 0.19 & -16.07 & 8.70 & -1.85 & 0.069 \\
$\mathrm{C} / \mathrm{N}$ & -0.13 & 0.08 & -0.08 & 0.05 & -1.55 & 0.13 \\
\hline
\end{tabular}

\subsubsection{Horizontale Variabilität}

\subsubsection{Kleinräumige Variabilität}

Eine Voraussetzung der NdfA-Bestimmung mit der ${ }^{15}$ NNAM ist der Zugriff sowohl der Leguminose als auch der Referenzpflanze auf den gleichen N-Pool. Daher wurden die $\delta^{15} \mathrm{~N}$-Werte im Profil des Bodens unter Leguminosen und einem 5m Radius, also außerhalb der Kronentraufe, der Leguminose verglichen ('kleine Distanz'), aus dem auch die monatlich beprobten Referenzpflanzen zur NdfA-Bestimmung stammten.

Unabhängig von den Standorten streuten die $\delta^{15} \mathrm{~N}$-Werte um \pm 1 Einheit. Signifikante Unterschiede konnten kleinräumig nur vereinzelt nachgewiesen werden (Tab. 29). Unterschiede in den $\delta^{15} \mathrm{~N}$-Werten wurden nur in den oberen $40 \mathrm{~cm}$ auf den Flächen M1 bis M3 gefunden, in den N-Gehalten war nur auf Fläche M2 in den obersten $5 \mathrm{~cm}$ ein Einfluß des Pflanzenbewuchses nachzuweisen. Da die Unterschiede auf kleinräumiger Distanz nur selten signifikant waren, bleiben sie ohne besondere Aussagekraft. Die Differenzen änderten sich mit der Bodentiefe auf den einzelnen Standorten nicht signifikant, während sich die \%N-Differenzen kleinräumig mehr und mehr anglichen. 
Tab. 29: Paarweiser $t$ - Test nach Student auf kleinräumige Unterschiede zwischen den Bodenproben der 5 M-Flächen unter Leguminosen und denen im $5 \mathrm{~m}$ - Radius (n.b.=nicht bestimmt, n.s.=nicht signifikant)

\begin{tabular}{|c|c|c|c|c|c|c|c|c|c|c|}
\hline Bodentiefe & M1 & M2 & M3 & M4 & M5 & M1 & M2 & M3 & M4 & M5 \\
\hline & \multicolumn{5}{|c|}{$\delta^{15} \mathrm{~N}$} & \multicolumn{5}{|c|}{$\% \mathrm{~N}$} \\
\hline $\mathrm{n}$ & 3 & 2 & 6 & 2 & 3 & 3 & 2 & 6 & 2 & 3 \\
\hline $0-5 \mathrm{~cm}$ & n.s. & $*$ & n.s. & n.s. & n.s. & n.s. & $*$ & n.s. & n.s. & n.s. \\
\hline $5-10 \mathrm{~cm}$ & n.s. & $*$ & n.s. & n.s. & n.s. & n.s. & n.s. & n.s. & n.s. & n.s. \\
\hline $10-20 \mathrm{~cm}$ & n.s. & n.s. & $*$ & n.b. & n.s. & n.s. & n.s. & n.s. & n.b. & n.s. \\
\hline $20-40 \mathrm{~cm}$ & $*$ & n.s. & n.s. & n.s. & n.s. & n.s. & n.s. & n.s. & n.s. & n.s. \\
\hline $40-60 \mathrm{~cm}$ & n.s. & n.s. & n.s. & n.s. & n.s. & n.s. & n.s. & n.s. & n.s. & n.s. \\
\hline $60-100 \mathrm{~cm}$ & n.s. & n.s. & n.s. & n.s. & n.s. & n.s. & n.s. & n.s. & n.s. & n.s. \\
\hline
\end{tabular}

Stellvertretend für alle Flächen sind in Abb. 19 die $\delta^{15} \mathrm{~N}$-Werte im Bodenprofil der Flächen M3 und M2 dargestellt (Tab. $61 \mathrm{im}$ Anhang gibt die Werte im einzelnen wieder). Obwohl nur selten statistisch signifikant war ein Trend des $\delta^{15} \mathrm{~N}$ von höheren Werten unter den nicht $\mathrm{N}_{2}$-fixierenden Referenzpflanzen zu 1 Einheit niedrigeren Werten unter der Leguminose zu erkennen. Die Unterschiede nehmen mit der Bodentiefe ab. Unterhalb von $20 \mathrm{~cm}$ waren sie überhaupt nicht mehr zu erkennen.

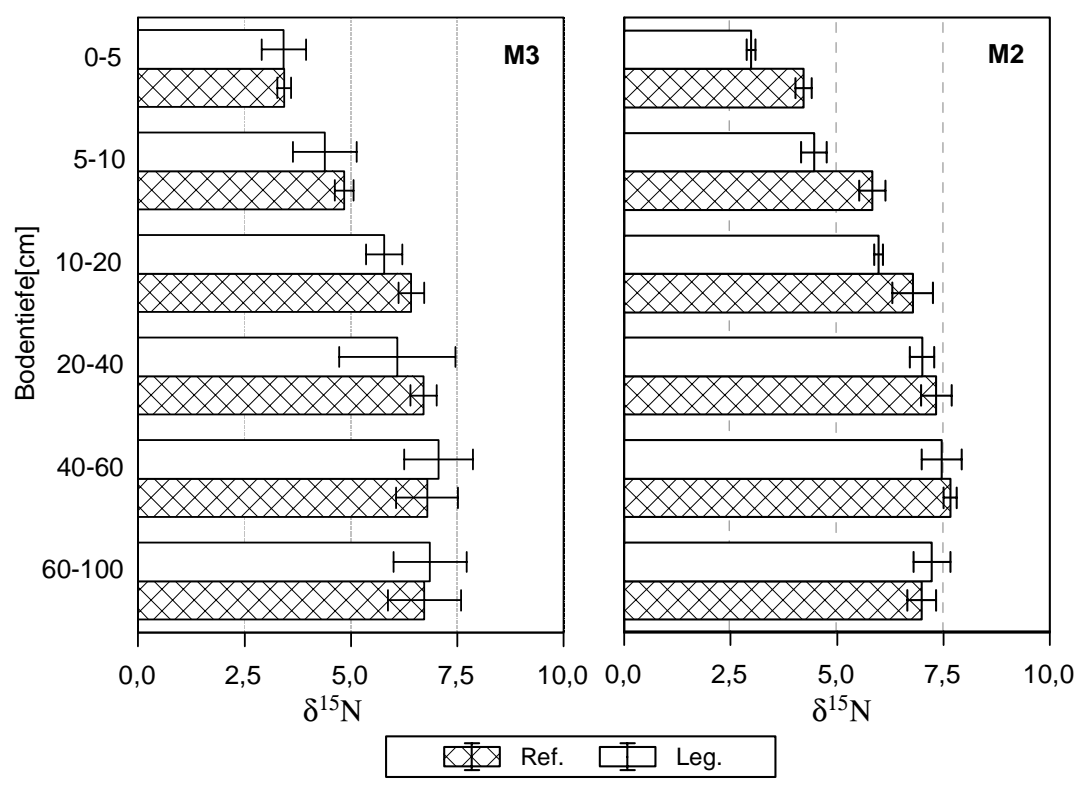

Abb. 19: Kleinräumige Unterschiede des $\delta^{15} \mathrm{~N}$-Wertes zwischen Boden unter Leguminosen (Leg.) und im 5m-Radius (Ref.) um sie herum in Abhängigkeit von der Bodentiefe auf Fläche M3 (n=6) und Fläche M2 (n=2) (Balken geben $s$ an) 


\subsubsection{Großräumige Variabilität}

\section{Igarapé Açu}

Die Unterschiede zwischen den Standorten M1 bis M5 in der Nähe von Igarapé Açu waren nicht sehr stark ausgeprägt, obwohl die Varianzanalyse (vergl. Tab. 60 im Anhang) Signifikanzen andeutete. Zwischen Fläche M1, M3 und M4 ergaben sich über die Bodentiefen keine signifikanten Unterschiede (Tab. 30). Lediglich Fläche M5 war in 5-10cm und 40-60cm Bodentiefe von den anderen signifikant verschieden. Diese Fläche wies auch die geringsten N-Gehalte auf. Mit zunehmender Bodentiefe verringern sich die bestehenden Unterschiede, blieben aber doch konstant. Auffällig ist die starke Zunahme des $\delta^{15} \mathrm{~N}$-Wertes auf Fläche M2 von durchschnittlich +3.6 in den ersten $5 \mathrm{~cm}$ bis auf +5.14 in $10 \mathrm{~cm}$ Bodentiefe. Im Mineralboden blieb diese Fläche aber diejenige mit den höchsten ${ }^{15} \mathrm{~N}$-Anreicherungen, während Fläche M5 die niedrigsten $\delta^{15} \mathrm{~N}-$ Werte hatte.

Tab. 30: $\delta^{15} \mathrm{~N}$ und $\mathrm{N}_{\text {tot. }}$ des Bodens von 5 Flächen in der Nähe von Igarapé Açu (Buchstaben hinter den Daten geben die Ränge innerhalb einer Bodentiefe nach Scheffé an, n=6 bei M1 und M5, n=12 bei M3, n=4 bei M2 und M4, in Klammern $s$ )

\begin{tabular}{|c|c|c|c|c|c|}
\hline Fläche & M1 & M2 & M3 & M4 & M5 \\
\hline \multicolumn{6}{|c|}{$\delta^{15} \mathrm{~N}$} \\
\hline 0 bis $5 \mathrm{~cm}$ & $3.63( \pm 0.13)^{a}$ & $3.60( \pm 0.14)^{\mathrm{a}}$ & $3.42( \pm 0.34)^{\mathrm{a}}$ & $4.06( \pm 0.37)^{\mathrm{a}}$ & $3.34( \pm 0.45)^{a}$ \\
\hline 5 bis $10 \mathrm{~cm}$ & $4.49( \pm 0.24)^{\mathrm{ab}}$ & $5.14( \pm 0.83)^{\mathrm{a}}$ & $4.62( \pm 0.48)^{\mathrm{ab}}$ & $4.98( \pm 0.22)^{\mathrm{a}}$ & $3.67( \pm 0.78)^{b}$ \\
\hline 10 bis $20 \mathrm{~cm}$ & $6.24( \pm 0.13)^{a}$ & $6.38( \pm 0.41)^{a}$ & $6.10( \pm 0.36)^{a}$ & $4.61( \pm 1.76)^{a}$ & $5.27( \pm 0.62)^{a}$ \\
\hline 20 bis $40 \mathrm{~cm}$ & $6.73( \pm 0.19)^{\mathrm{a}}$ & $7.17( \pm 0.32)^{a}$ & $6.40( \pm 0.84)^{\mathrm{a}}$ & $6.32( \pm 0.25)^{a}$ & $6.25( \pm 0.41)^{a}$ \\
\hline 40 bis $60 \mathrm{~cm}$ & $6.90( \pm 0.30)^{\mathrm{ab}}$ & $7.56( \pm 0.31)^{a}$ & $6.93( \pm 0.77)^{\mathrm{a}}$ & $6.60( \pm 0.37)^{\mathrm{ab}}$ & $5.89( \pm 0.49)^{\mathrm{b}}$ \\
\hline 60 bis $100 \mathrm{~cm}$ & $6.63( \pm 0.24)^{a}$ & $7.11( \pm 0.39)^{\mathrm{a}}$ & $6.80( \pm 0.86)^{\mathrm{a}}$ & $6.70( \pm 0.08)^{a}$ & $6.40( \pm 0.58)^{a}$ \\
\hline \multicolumn{6}{|c|}{$\% \mathrm{~N}$} \\
\hline 0 bis $5 \mathrm{~cm}$ & $0.11(0.02)^{\mathrm{a}}$ & $0.09(0.01)^{\mathrm{a}}$ & $0.16(0.10)^{\mathrm{a}}$ & $0.10(0.001)^{\mathrm{a}}$ & $0.06(0.01)^{a}$ \\
\hline 5 bis $10 \mathrm{~cm}$ & $0.09(0.02)^{a}$ & $0.06(0.001)^{\mathrm{a}}$ & $0.09(0.02)^{a}$ & $0.08(0.001)^{a}$ & $0.07(0.01)^{a}$ \\
\hline 10 bis $20 \mathrm{~cm}$ & $0.06(0.001)^{\mathrm{a}}$ & $0.07(0.001)^{a}$ & $0.06(0.01)^{\mathrm{a}}$ & $0.55(0.001)^{b}$ & $0.05(0.001)^{a}$ \\
\hline 20 bis $40 \mathrm{~cm}$ & $0.05(0.001)^{\mathrm{ab}}$ & $0.06(0.01)^{b}$ & $0.05(0.01)^{\mathrm{ab}}$ & $0.05(0.001)^{\mathrm{ab}}$ & $0.04(0.001)^{a}$ \\
\hline 40 bis $60 \mathrm{~cm}$ & $0.05(0.01)^{a}$ & $0.04(0.001)^{\mathrm{ab}}$ & $0.04(0.001)^{a b}$ & $0.04(0.001)^{a b}$ & $0.03(0.001)^{b}$ \\
\hline 60 bis $100 \mathrm{~cm}$ & $0.04(0.001)^{a b}$ & $0.04(0.001)^{\mathrm{ab}}$ & $0.04(0.001)^{b}$ & $0.05(0.01)^{b}$ & $0.02(0.001)^{\mathrm{a}}$ \\
\hline
\end{tabular}

\section{Peixe Boi}

Auf der Fazenda Monte Verde in der Nähe von Peixe Boi, 40km östlich von Igarapé Açu, waren Restbestände von Primärvegetation und eine 15-25jährige Sekundärvegetation anzutreffen. Hier können bisher noch wenig ackerbaulich genutzte Flächen vorausgesetzt werden, 
deren Böden daher in ihrer Nährstoffsituation weniger gestörter sind. Eine einmalige Beprobung mit 6 Einstichen für 1 Mischprobe (n=3 bzw. 4) wurde an diesen Stellen durchgeführt.

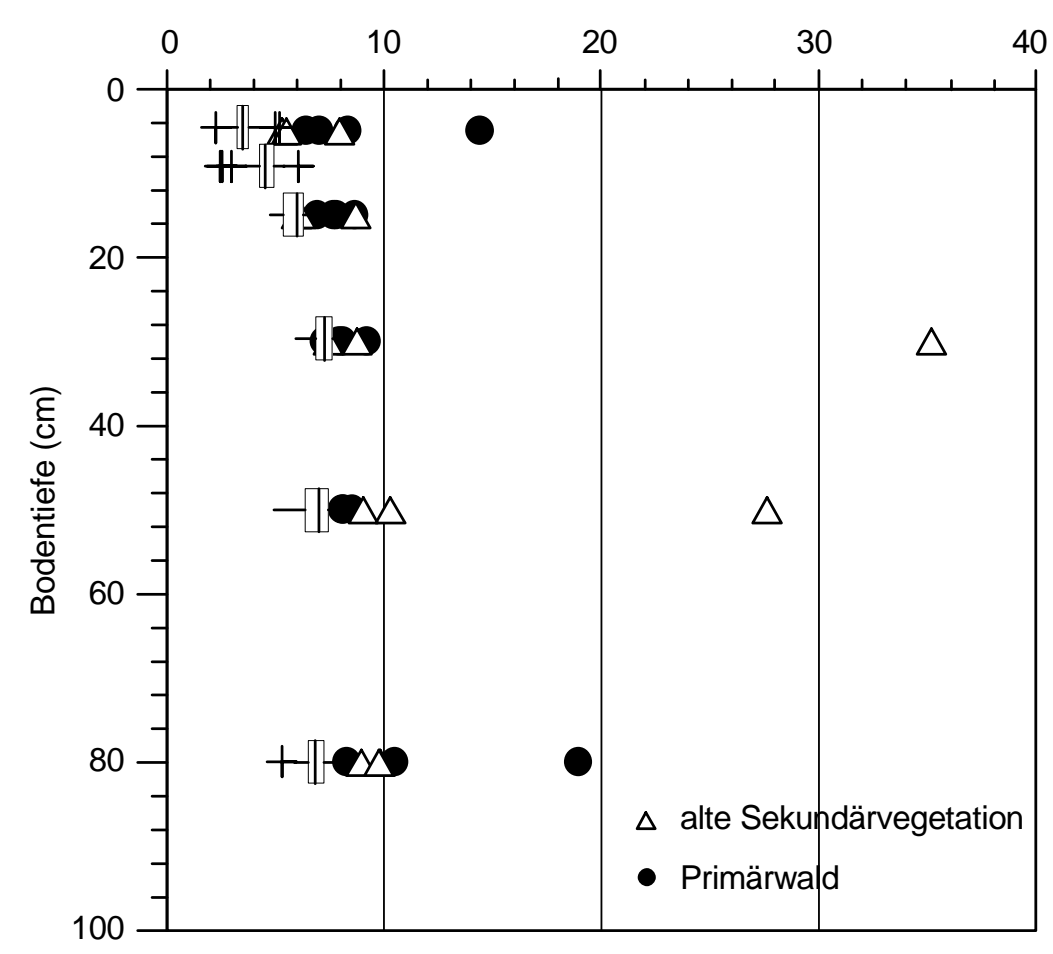

Abb. 20: $\delta^{15} \mathrm{~N}$ im Boden von Peixe Boi im Vergleich zu Igarapé Açu. Die Box-Whisker-Plots geben Mittelwert, Spannweite und Extremwerte der Igarape'Açu-Flächen ( $n=15)$ wieder. Marker stellen Einzelwerte der Flächen in Peixe Boi dar.

Der Verlauf von $\delta^{15} \mathrm{~N}$ im Boden entspricht dem der jungen Vegetationen in Igarapé Acu, insgesamt waren die Werte sowohl der alten Sekundär- als auch der Primärvegetation 2 Einheiten höher (Abb. 20). Vereinzelt wurden Werte bis zu $\delta^{15} \mathrm{~N}=+35$ gemessen, die wahrscheinlich auf z.B. den Einfluß von Blattschneiderameisen (Atta sp.) zurückzuführen sind. An diesen Stellen vereinzelt extrem hohe N-Gehalte weisen auf derartige Zusammenhänge hin (Tab. 31).

Ein signifikanter Unterschied im durchschnittlichen Boden- $\delta^{15} \mathrm{~N}$ zwischen der alten Sekundärvegetation und dem Primärwald konnte nach dem $H$-Test von Kruskal und Wallis aufgrund der geringen Wiederholungszahl und der hohen Streuung nicht verzeichnet werden (Tab. 31). 
Tab. 31: Mittelwerte (von drei Mischproben aus jeweils 6 Einstichen ) des Bodens in Peixe Boi (in Klammern stehen die Mittelwerte unter Einbezug der Extremwert)

\begin{tabular}{|l|cc|cc|cc|}
\hline Bodentiefe (cm) & $\begin{array}{c}\text { junge Sekundärvegetation } \\
\text { M1-M5, gemittelt }\end{array}$ & \multicolumn{2}{|c|}{$\begin{array}{c}\text { alte Sekundärvegetation } \\
\mathrm{PB}_{\mathrm{SW}}\end{array}$} & \multicolumn{2}{c|}{$\begin{array}{c}\text { Primärvegetation } \\
\mathrm{PB}_{\mathrm{PW}}\end{array}$} \\
\hline & $\delta^{15} \mathrm{~N}$ & $\% \mathrm{~N}$ & $\delta^{15} \mathrm{~N}$ & $\% \mathrm{~N}$ & $\delta^{15} \mathrm{~N}$ & $\% \mathrm{~N}$ \\
\hline $0-10 \mathrm{~cm}$ & $4.26(0.83)$ & $0.11(0.06)$ & 6.25 & 0.11 & $7.27(9.06)$ & 0.14 \\
$10-20 \mathrm{~cm}$ & $5.72(1.12)$ & $0.16(0.31)$ & 6.94 & 0.08 & 7.78 & 0.11 \\
$20-40 \mathrm{~cm}$ & $6.57(0.53)$ & $0.05(0.01)$ & $8.10(17.13)$ & 0.06 & 8.14 & 0.08 \\
$40-60 \mathrm{~cm}$ & $6.78(0.61)$ & $0.04(0.01)$ & $9.66(15.65)$ & 0.17 & 8.26 & 0.06 \\
$60-100 \mathrm{~cm}$ & $6.73(0.27)$ & $0.04(0.01)$ & 9.51 & 0.04 & $9.40(12.58)$ & 0.04 \\
\hline
\end{tabular}

Die $\delta^{15} \mathrm{~N}$-Werte des Bodens unter junger, alter und Primärvegetation wurden ebenfalls mit dem Kruskal-Wallis- $H$-Test verglichen, führten aber ebenfalls zu keinen signifikanten Unterschieden.

\section{$2.2 \quad \delta^{15} \mathrm{~N}$ in der Asche}

Eine mögliche Ursache für die negativen $\delta^{15} \mathrm{~N}-$ Werte der Vegetation kann im pflanzenverfügbaren $\mathrm{N}$, das durch jahrzehntelang praktizierte Brandrodungen via ${ }^{15} \mathrm{~N}$-Fraktionierung der Volatilisation verändert wurde, im $\mathrm{N}_{\text {tot }}$. des Bodens jedoch noch nicht klar zu erkennen ist, liegen. Analysen von Asche und der gerodeten Biomasse vor dem Brand zeigten aber keine signifikanten Änderungen des $\delta^{15} \mathrm{~N}$-Wertes des Pflanzenmaterials durch den Brand (Tab. 32). Der $\delta^{15} \mathrm{~N}$-Wert des Holzes betrug $+1.67( \pm 2.40)$ und des Blattmaterials $+0.19( \pm 0.73)$. Der $\delta^{15} \mathrm{~N}-$ Wert in der Asche verschob sich leicht Richtung Nullpunkt (+0.02 \pm 0.96$)$.

Tab. 32: $\delta^{15} \mathrm{~N}$ in Pflanzenmaterial und ihrer Asche (Werte in Klammern geben $s$ für $\mathrm{n}=10$ an; Buchstaben geben signifikante Unterschiede für p $<0.05$ nach Scheffé an)

\begin{tabular}{llll}
\hline Variabel & Asche & Holz & Blatt \\
\hline$\delta^{15} \mathbf{N}$ & $0.02( \pm 0.96)^{\mathrm{a}}$ & $1.67( \pm 2.40)^{\mathrm{a}}$ & $0.19( \pm 0.73)^{\mathrm{a}}$ \\
$\mathbf{\%} \mathbf{N}$ & $1.02( \pm 0.28)^{\mathrm{a}}$ & $0.46( \pm 0.10)^{\mathrm{b}}$ & $1.63( \pm 0.11)^{\mathrm{c}}$ \\
\hline
\end{tabular}

Vorgänge der Volatilisation und der Stofftransfer durch Brand werden als Mechanismen der ${ }^{15} \mathrm{~N}$-Anreicherungen beschrieben (Schulze et al., 1991; Handley und Raven, 1992; Turner et al., 1983). Bei einem Brand gehen 96 \% des Stickstoffs durch den Stofftransfer in die Atmo- 
sphäre über (Hölscher, 1995; Mackensen et al., 1996). In den verbliebenen 4\% in der Asche wurde hier keine Anreicherung festgestellt, so daß der ${ }^{15} \mathrm{~N}$ Fraktionierung via Volatilisation kein Einfluß auf die $\delta^{15} \mathrm{~N}$-Werte des Bragantina-Bodens zugeschrieben werden kann.

\subsection{Temporäre Variabilität}

Die Bodenbeprobung wurde in einem regenreichen Monat (Februar 1993) auf den Flächen M1 bis M5 unternommen und in einem regenarmen Monat (Juli 1993) auf dem Standort M2 wiederholt. Weder im Stickstoffgehalt noch in den $\delta^{15} \mathrm{~N}$-Werten konnten Unterschiede gefunden werden, die dem $t$-Test nach Student standhielten (Tab. 33). Die größere Streuung der feuchteren Proben der Regenzeit könnten durch den Trocknungsvorgang hervorgerufen worden sein.

Tab. 33: $\delta^{15} \mathrm{~N}$ und $\% \mathrm{~N}$ im Boden der Fläche $\mathrm{M} 2$ zu unterschiedlichen Jahreszeiten (in Klammern $s$ )

\begin{tabular}{l|c|cc|cc}
\hline $\begin{array}{l}\text { Bodentiefe } \\
\text { (cm) }\end{array}$ & n & \multicolumn{2}{|c|}{ Regenzeit } & \multicolumn{2}{c}{ Trockenzeit } \\
\hline & & $\delta^{15} \mathrm{~N}$ & $\% \mathrm{~N}$ & $\delta^{15} \mathrm{~N}$ & $\% \mathrm{~N}$ \\
0 bis $5 \mathrm{~cm}$ & 5 & $3.70( \pm 0.32)$ & $0.09( \pm 0.02)$ & $3.58( \pm 0.15)$ & $0.09( \pm 0.02)$ \\
5 bis $10 \mathrm{~cm}$ & 5 & $4.72( \pm 1.32)$ & $0.08( \pm 0.008)$ & $4.76( \pm 0.52)$ & $0.08( \pm 0.009)$ \\
10 bis $20 \mathrm{~cm}$ & 5 & $6.15( \pm 0.85)$ & $0.06( \pm 0.01)$ & $5.96( \pm 0.45)$ & $0.06( \pm 0.01)$ \\
20 bis $40 \mathrm{~cm}$ & 5 & $6.69( \pm 0.85)$ & $0.05( \pm 0.01)$ & $7.4( \pm 0.5)$ & $0.05( \pm 0.005)$ \\
40 bis $60 \mathrm{~cm}$ & 5 & $6.97( \pm 0.87)$ & $0.04( \pm 0.007)$ & $7.95( \pm 0.2)$ & $0.04( \pm 0.005)$ \\
60 bis $100 \mathrm{~cm}$ & 5 & $6.81( \pm 0.65)$ & $0.03( \pm 0.008)$ & $7.75( \pm 0.54)$ & $0.03( \pm 0.004)$ \\
\hline
\end{tabular}

\section{$3 \quad \delta^{15} \mathrm{~N}$ in der organischen Auflage der Sekundär - und Primärvegetation}

Der $\delta^{15} \mathrm{~N}$-Wert der organischen Auflage repräsentiert das ${ }^{15} \mathrm{~N} /{ }^{14} \mathrm{~N}-$ Verhältnis des teilweise abgebauten Stickstoffs. Trotz Umlagerungsprozesse während der Seneszenz spiegelt sich das vorhandene ${ }^{15} \mathrm{~N} /{ }^{14} \mathrm{~N}$-Verhältnis der Vegetation in ihr wider. Umsetzungsprozesse zu mineralischem Stickstoff bzw. organischen Substanzen haben begonnen und somit finden sich in der älteren organischen Auflage $\delta^{15} \mathrm{~N}$-Werte, die durch diese Vorgänge verändert wurden (Andreux et al, 1988). In diesem Kapitel soll nun beschrieben werden, welche $\delta^{15} \mathrm{~N}$-Werte in der Streu der Vegetation der Bragantina, junge, ältere und Primärvegetation, aufzufinden waren und ob ein Einfluß auf den $\delta^{15} \mathrm{~N}$-Wert des Bodens vorhanden war. 


\subsection{Räumliche Variabilität}

Auf größere Distanzen hin (ANOVA siehe Tab. 62 im Anhang) konnten signifikante Unterschiede im $\delta^{15} \mathrm{~N}$-Wert der organischen Auflage festgestellt werden. Die Differenz des $\delta^{15} \mathrm{~N}$-Wertes der organischen Auflage innerhalb zu außerhalb der Kronentraufe von Leguminosen („,kleine Distanz“) war auf den einzelnen Flächen unterschiedlich stark ausgeprägt, jedoch nie signifikant. Ebenso waren die Unterschiede zwischen frisch gefallenem und älterem, dem Zersetzungsprozeß unterworfenem Blattmaterial nicht signifikant. Die N-Gehalte veränderten sich jedoch in Abhängigkeit von dem Bewuchs mit Leguminosen oder Referenzpflanzen, sowie in Abhängigkeit von dem Grad der Zersetzung. Auch von Fläche zu Fläche wurden signifikante Unterschiede gefunden.

\section{Igarapé Açu}

Mit Ausnahme von Fläche M1 wurde die frische Streu unter Leguminosen durchgehend mit niedrigeren $\delta^{15} \mathrm{~N}$-Werten als unter Nichtfixierern gemessen (Tab. 34). Die Stickstoffkonzentrationen hingegen waren außer auf der Fläche M2 signifikant höher in der Leguminosenstreu. In der älteren Streu zeichnete sich auf allen Flächen unter Leguminosen sowie Nichtfixierern ein Trend - wiederum mit Ausnahme der Fläche M2 - zu höheren $\delta^{15} \mathrm{~N}-$ Werten ab.

Tab. 34: $\delta^{15} \mathrm{~N}$ und N-Gehalte im $\mathrm{N}_{\text {tot. }}$ der organischen Auflage in junger Sekundärvegetation (in Klammern $s$ * geben signifikante Unterschiede zwischen der Streu unter Leguminosen Leg.- und dem 5m-Radius -Ref.- nach Student an)

\begin{tabular}{|c|c|c|c|c|c|c|c|c|c|c|}
\hline & \multicolumn{2}{|c|}{ Fläche M1 } & \multicolumn{2}{|c|}{ Fläche M2 } & \multicolumn{2}{|c|}{ Fläche M3 } & \multicolumn{2}{|c|}{ Fläche M4 } & \multicolumn{2}{|c|}{ Fläche M5 } \\
\hline$\delta^{15} \mathbf{N}$ & Leg. & Ref. & Leg. & Ref. & Leg. & Ref. & Leg. & Ref. & Leg. & Ref. \\
\hline frische Streu & $\begin{array}{l}-0.67 * \\
(0.27)\end{array}$ & \begin{tabular}{|l|}
2.36 \\
$(0.94)$ \\
\end{tabular} & $\begin{array}{l}-0.84 \\
(0.49)\end{array}$ & $\begin{array}{l}-0.04 \\
(0.67)\end{array}$ & $\begin{array}{l}-0.69 \\
(0.31) \\
\end{array}$ & \begin{tabular}{|l|}
-0.47 \\
$(0.64)$ \\
\end{tabular} & $\begin{array}{l}-1.47 \\
(0.35) \\
\end{array}$ & -0.47 & $\begin{array}{l}-1.02 * \\
(0.21)\end{array}$ & \begin{tabular}{|l|}
1.08 \\
$(1.63)$ \\
\end{tabular} \\
\hline alte Streu & $\begin{array}{l}-0.10 \\
(0.22)\end{array}$ & \begin{tabular}{|l|}
-0.37 \\
$(0.56)$ \\
\end{tabular} & $\begin{array}{l}-1.64 \\
(0.58)\end{array}$ & $\begin{array}{l}-1.02 \\
(0.95)\end{array}$ & \begin{tabular}{|l}
-0.38 \\
$(0.56)$
\end{tabular} & \begin{tabular}{|l}
-0.38 \\
$(0.27)$ \\
\end{tabular} & \begin{tabular}{|l}
-0.58 \\
$(0.39)$
\end{tabular} & 0.20 & $\begin{array}{l}2.80 \\
(0.20)\end{array}$ & \begin{tabular}{|l}
0.54 \\
$(0.88)$ \\
\end{tabular} \\
\hline$\% \mathbf{N}$ & & & & & & & & & & \\
\hline frische Streu & $\begin{array}{l}1.34 * \\
(0.08)\end{array}$ & $\mid \begin{array}{l}0.80 \\
(0.17)\end{array}$ & $\begin{array}{l}1.46 \\
(0.23)\end{array}$ & $\begin{array}{l}0.90 \\
(0.18)\end{array}$ & $\begin{array}{l}1.56 * * \\
(0.18)\end{array}$ & $\begin{array}{l}1.10 \\
(0.15) \\
\end{array}$ & $\begin{array}{l}1.33^{*} \\
(0.10)\end{array}$ & 1.03 & $\begin{array}{l}1.76^{* *} \\
(0.15)\end{array}$ & $\begin{array}{l}1.15 \\
(0.21) \\
\end{array}$ \\
\hline alte Streu & $\begin{array}{l}0.87 \\
(0.06) \\
\end{array}$ & \begin{tabular}{|l|}
.18 \\
$(0.57)$ \\
\end{tabular} & $\begin{array}{l}0.98 * * \\
(0.01) \\
\end{array}$ & \begin{tabular}{|l}
1.24 \\
$(0.00)$ \\
\end{tabular} & \begin{tabular}{|l|}
1.25 \\
$(0.38)$ \\
\end{tabular} & \begin{tabular}{|l|}
.32 \\
$(0.19)$ \\
\end{tabular} & $\begin{array}{l}1.11^{*} \\
(0.09) \\
\end{array}$ & 0.82 & $\begin{array}{l}0.89 \\
(0.16) \\
\end{array}$ & \begin{tabular}{|l|}
1.00 \\
$(0.44)$ \\
\end{tabular} \\
\hline
\end{tabular}

Die Stickstoffkonzentrationen sind in der alten Streu niedriger und nur noch auf Fläche M3 und M5 war sie unter Leguminosen und Nichtfixierern signifikant verschieden. Auf Fläche M5 wurde die größte Differenz in den N-Konzentrationen zwischen frischer und älterer Streu gefunden, hier wurde auch die stärkste Anreicherung der älteren Streu mit ${ }^{15} \mathrm{~N}$ angetroffen. 
Diese kleinräumigen Unterschiede sind mit dem größeren Einfluß der Abbauprodukte der verrottenden, für den Mikrostandort spezifischen, organischen Substanz erklärbar, der sich mit zunehmender Bodentiefe verliert. Die hohe Variabilität der $\delta^{15} \mathrm{~N}$ in der organischen Auflage kann zum einen über die Entnahme unterschiedlich stark abgebauter organischer Substanz oder zum anderen über die unterschiedliche Artenzusammensetzung der jeweiligen Fläche erklärt werden. Die Biomasse der verschiedenen Arten wird je nach ihrer Größen- und Artenzusammensetzung in unterschiedlicher Geschwindigkeit und Art und Weise abgebaut (Downs et al., 1996). Jedoch spielen auch hier Fraktionierungsprozesse der Stickstoffisotopen eine Rolle. Durch den Abbau der organischen Substanz und die damit einhergehende ${ }^{15} \mathrm{~N}$-Diskriminierung wird das verbliebene organische Material sukzessive mit ${ }^{15} \mathrm{~N}$ angereichert, während das Produkt der Zersetzung und Mineralisation - $\mathrm{NH}_{4}{ }^{+}$und $\mathrm{NO}_{3}{ }^{-}-$weniger ${ }^{15} \mathrm{~N}$ mit sich führt (Andreux, 1988; Handley und Raven, 1992; Mordelet et al., 1996; Turner et al., 1983; Wada et al., 1975;).

\section{Peixe Boi}

Die organische Auflage der jungen Sekundärvegetation der Bragantina-Region wirde mit der einer 20-25jährigen Sekundärvegetation und eines Primärwaldes verglichen. Da keine signifikanten Unterschiede in den $\delta^{15} \mathrm{~N}$-Werten zwischen Leguminosen- und Nichtfixiererstreu sowie frischer und älterer Streu vorlagen, gingen diese Proben als Wiederholung für die jeweilige Fläche in den Hypothesen-Rang-Test der Flächen nach Scheffé mit ein (Tab. 35).

Tab. 35: $\delta^{15} \mathrm{~N}$ und N-Gehalte der Streuauflage auf den M-Flächen, einer alten Sekundärvegetation und eines Primärwaldes der Zona Bragantina ( $s$ in Klammern)

\begin{tabular}{cccccccc}
\hline Fläche & 1 & 2 & 3 & 4 & 5 & $\begin{array}{c}\text { Peixe Boi } \\
\text { alte Sekundärvegetation }\end{array}$ & $\begin{array}{c}\text { Peixe Boi } \\
\text { Primärwald }\end{array}$ \\
\hline $\mathrm{n}$ & 12 & 8 & 24 & 8 & 12 & 3 & 4 \\
\hline \multirow{2}{*}{$\delta^{15} \mathrm{~N}$} & $-0.89^{\mathrm{a}}$ & $-0.88^{\mathrm{a}}$ & $-0.48^{\mathrm{a}}$ & $-0.59^{\mathrm{a}}$ & $\begin{array}{c}\delta^{15} \mathrm{~N} \\
0.19^{\mathrm{b}}\end{array}$ & $2.55^{\mathrm{b}}(2.34)$ & $5.03^{\mathrm{c}}(0.49)$ \\
& $(1.08)$ & $(0.81)$ & $(0.46)$ & $(2.47)$ & $(1.16)$ & & \\
\hline$\% \mathrm{~N}$ & $1.02^{\mathrm{a}}$ & $1.14^{\mathrm{ab}}$ & $1.31^{\mathrm{ab}}$ & $1.07^{\mathrm{ab}}$ & $\begin{array}{c}\% \mathrm{~N} \\
1.20^{\mathrm{ab}}\end{array}$ & $1.54^{\mathrm{ab}}(0.08)$ & $1.58^{\mathrm{b}}(0.23)$ \\
& $(0.35)$ & $(0.26)$ & $(0.28)$ & $(0.20)$ & $(0.42)$ & & \\
\hline
\end{tabular}

Unter Primärwald zeigte die Streu ein wesentlich höheres ${ }^{15} \mathrm{~N} /{ }^{14} \mathrm{~N}$-Verhältnis als unter der Sekundärvegetation, wobei die alte Sekundärvegetation in Peixe Boi in ihrem $\delta^{15} \mathrm{~N}$ zwischen den jungen Vegetationen aus Igarapé Açu und dem Primärwald lagen. Die Sonderstellung 
Peixe Bois spiegelte sich auch in den höheren N-Gehalten wieder, die sich aber aufgrund der hohen Streuung nicht signifikant von den anderen Standorten abgrenzen ließ (Tab. 35).

In Abb. 21 sind die einzelnen $\delta^{15} \mathrm{~N}$-Werte der in Tab. 35 gegebenen Mittelwerte in Abhängigkeit vom Standort dargestellt. Hier wird ganz deutlich, daß die organische Auflage mit zunehmendem Alter der Vegetation immer höhere $\delta^{15} \mathrm{~N}$-Werte aufwies.

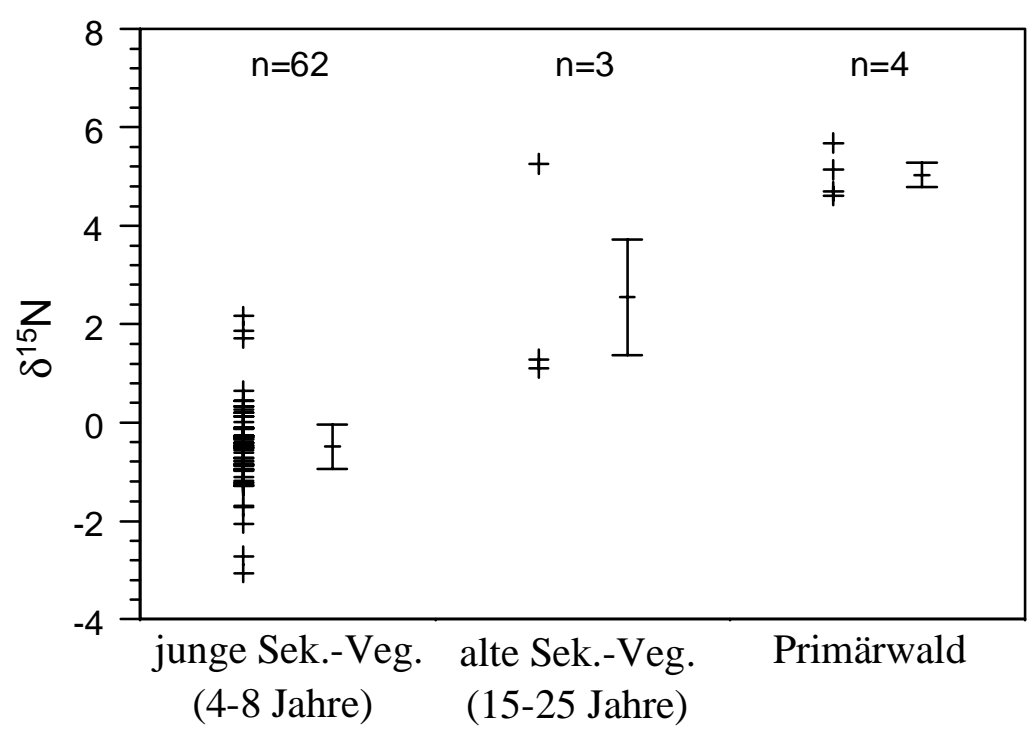

Abb. 21: $\delta^{15} \mathrm{~N}$ in der organischen Auflage verschiedener Vegetationssysteme (Balken geben $s$ an).

\subsection{Temporäre Variabilität im $\delta^{15} \mathrm{~N}$ der organischen Auflage}

Für Fläche M2 wurden die Proben der organischen Auflage sowohl in der Trocken - als auch Regenzeit analysiert. Bei einem $t$-Test wurden keine signifikanten Unterschiede in den einzelnen Bodenschichten festgestellt - die Streuung war zu hoch. Die Regenzeit läßt eine höhere Mineralisationsrate (Marrs et al., 1991) und damit einen höheren $\delta^{15} \mathrm{~N}-$ Wert in der verbliebenen organischen Substanz und eine höhere Abbaurate vermuten. Dies konnte hier jedoch nicht bestätigt werden.

Tab. 36: $\delta^{15} \mathrm{~N}$ und \% $\mathrm{N}$ der Streuauflage in Trocken - bzw. Regenzeit

\begin{tabular}{llll}
\hline & $\mathbf{n}$ & Regenzeit $(s)$ & Trockenzeit $(s)$ \\
\hline$\delta^{\mathbf{1 5}} \mathbf{N}$ & 5 & $-0.62(0.89)$ & $-0.16(0.93)$ \\
$\boldsymbol{\%} \mathbf{N}$ & 5 & $1.15(0.2)$ & $1.12(0.28)$ \\
\hline
\end{tabular}




\subsection{Beziehung von $\delta^{15} \mathrm{~N}$ der organischen Auflage zum $\delta^{15} \mathrm{~N}$ des Mineralbodens und der Vegetation}

Die Streu ist das Substrat, aus dem sich das mineralische N entwickelt. Bekannt ist seit langem, daß es zu Fraktionierungsprozessen während des Abbaus des organischen Materials kommt, die zu einer ${ }^{15} \mathrm{~N}$-Abreicherung des Produktes, also $\mathrm{NO}_{3}{ }^{-}$und in geringerem Maße $\mathrm{NH}_{4}{ }^{+}$, führt.

Die Differenz zwischen den $\delta^{15} \mathrm{~N}$-Werten, zu sehen an der Regressionskonstanten (Tab. 37), nahm mit der Bodentiefe von 3.7 auf maximal 6.7 zu. Während frische Streu in keinem $\mathrm{Zu}-$ sammenhang mit dem Mineralboden stand, konnte ein negativer Zusammenhang zwischen der alten organischen Substanz und dem Mineralboden gezeigt werden. Die Regressionsgleichungen der organischen Auflage mit dem Mineralboden sind für die beiden unterschiedlichen Zersetzungsgrade der Streu nahezu gleich, die Ausprägung des Zusammenhanges ist jedoch in der älteren Streuauflage deutlicher und daher häufiger signifikant.

Tab. 37: Regressionsanalysen des $\delta^{15} \mathrm{~N}$-Wertes der organischen Auflage und dem $\delta^{15} \mathrm{~N}$ des unterliegenden Bodens $(\mathrm{n}=10, \mathrm{~ns}=$ nicht signifikant $)$

\begin{tabular}{c|ccc|ccc}
\hline Bodentiefe & \multicolumn{2}{|c|}{ frische organische Auflage } & \multicolumn{3}{|c}{ ältere organische Auflage } \\
\hline & Modell & $\mathbf{r}(\mathbf{x}, \mathbf{y})$ & $\mathbf{r}^{2}$ & Modell & $\mathbf{r}(\mathbf{x}, \mathbf{y})$ & $\mathbf{r}^{2}$ \\
\hline $0-5 \mathrm{~cm}$ & $\delta^{15} \mathrm{~N}_{\text {tot. }}=3.7+0.1 \times \delta^{15} \mathrm{~N}_{\text {streu }}$ & 0.19 & $0.04^{\mathrm{ns}}$ & $\delta^{15} \mathrm{~N}_{\text {tot. }}=3.6+0.1 \times \delta^{15} \mathrm{~N}_{\text {streu }}$ & 0.08 & $0.01^{\mathrm{ns}}$ \\
$5-10 \mathrm{~cm}$ & $\delta^{15} \mathrm{~N}_{\text {tot. }}=4.9-0.03 \times \delta^{15} \mathrm{~N}_{\text {streu }}$ & -0.23 & $0.00^{\mathrm{ns}}$ & $\delta^{15} \mathrm{~N}_{\text {tot. }}=4.3-0.04 \times \delta^{15} \mathrm{~N}_{\text {streu }}$ & -0.87 & $0.76^{* * *}$ \\
$10-20 \mathrm{~cm}$ & $\delta^{15} \mathrm{~N}_{\text {tot. }}=5.6-0.2 \times \delta^{15} \mathrm{~N}_{\text {streu }}$ & -0.18 & $0.03^{\mathrm{ns}}$ & $\delta^{15} \mathrm{~N}_{\text {tot. }}=5.4-0.9 \times \delta^{15} \mathrm{~N}_{\text {streu }}$ & -0.50 & $0.25^{\mathrm{ns}}$ \\
$20-40 \mathrm{~cm}$ & $\delta^{15} \mathrm{~N}_{\text {tot. }}=6.5-0.2 \times \delta^{15} \mathrm{~N}_{\text {streu }}$ & -0.29 & $0.08^{\mathrm{ns}}$ & $\delta^{15} \mathrm{~N}_{\text {tot. }}=6.4-0.6 \times \delta^{15} \mathrm{~N}_{\text {streu }}$ & -0.71 & $0.50^{*}$ \\
$40-60 \mathrm{~cm}$ & $\delta^{15} \mathrm{~N}_{\text {tot. }}=6.7-0.2 \times \delta^{15} \mathrm{~N}_{\text {streu }}$ & -0.25 & $0.06^{\mathrm{ns}}$ & $\delta^{15} \mathrm{~N}_{\text {tot. }}=6.5-0.8 \times \delta^{15} \mathrm{~N}_{\text {streu }}$ & -0.87 & $0.76^{* * *}$ \\
$60-100 \mathrm{~cm}$ & $\delta^{15} \mathrm{~N}_{\text {tot. }}=6.7-0.01 \times \delta^{15} \mathrm{~N}_{\text {streu }}$ & -0.06 & $0.00^{\mathrm{ns}}$ & $\delta^{15} \mathrm{~N}_{\text {tot. }}=6.6-0.35 \times \delta^{15} \mathrm{~N}_{\text {streu }}$ & -0.84 & $0.69^{* *}$ \\
\hline
\end{tabular}

Ob ein Zusammenhang zwischen den Boden- $\delta^{15} \mathrm{~N}-$ Werten und dem $\delta^{15} \mathrm{~N}$ der Vegetation bestand, zeigen die Pearson-Korrelationen der beiden Parameter (Tab. 38). Es wurde mit den Mittelwerten der Arten, soweit auf allen Flächen vorhanden, für jeden Standort (S1 bis S6 und $\mathrm{PB}_{\mathrm{sw}}$ sowie $\mathrm{PB}_{\mathrm{pw}}$ ) eine Pearson-Korrelation mit dem Boden- $\delta^{15} \mathrm{~N}$ der Flächen aus Igarape'Açu und Peixe Boi durchgeführt (Tab. 38).

Eine deutlich positive Korrelation zwischen den $\delta^{15} \mathrm{~N}-$ Werten der Pflanzen mit dem $\delta^{15} \mathrm{~N}$ des Bodens von 0 bis $20 \mathrm{~cm}$, der Streu und der Bodenschicht von 60 bis $100 \mathrm{~cm}$ Tiefe bestand für 
Inga thibaudiana, Tapirira guianensis und Vismia guianensis. Offensichtlich dominieren die jeweiligen Arten mit ihrem Laub das $\delta^{15} \mathrm{~N}$ der oberen Bodenschichten, deren mineralisches $\mathrm{N}$ dann wieder in die Pflanzen aufgenommen wird. Die Vegetation und der Mineralboden beeinflussen ihre $\delta^{15} \mathrm{~N}-$ Werte gegenseitig durch das im System zirkulierende $\mathrm{N}$.

Dieser Zusammenhang wird jedoch durch die negative Korrelation der Streuauflage mit dem Mineralboden durchbrochen. An dieser Stelle des Abbaus finden offensichtlich Prozesse statt, die zu einer Aufspaltung von ${ }^{15} \mathrm{~N}$ und ${ }^{14} \mathrm{~N}$ zwischen Substraten und Produkten führen und so eine gegenläufige $\delta^{15} \mathrm{~N}$-Wert-Entwicklung verursachen.

Tab. 38: Pearson-Korrelation zwischen den $\delta^{15} \mathrm{~N}$-Werten der neuen Blätter und dem Boden des jeweiligen Standortes aus Igarape'Açu und Peixe Boi (Flächen $\mathrm{S} 1$ bis $\mathrm{S} 6$ und $\mathrm{Pb}_{\mathrm{sw}}$ und $\mathrm{Pb}_{\mathrm{pw}}$ )

\begin{tabular}{llllll}
\hline Bodentiefe & $\begin{array}{c}\text { Inga thibau- } \\
\text { diana }\end{array}$ & $\begin{array}{c}\text { Banara } \\
\text { guianensis }\end{array}$ & $\begin{array}{c}\text { Lacistema } \\
\text { pubescens }\end{array}$ & $\begin{array}{c}\text { Tapirira } \\
\text { guianensis }\end{array}$ & $\begin{array}{c}\text { Vismia } \\
\text { guianensis }\end{array}$ \\
\hline Streu & $0.96^{* * *}$ & 0.37 & 0.56 & $0.83^{*}$ & 0.64 \\
$0-10 \mathrm{~cm}$ & $0.94^{* * *}$ & 0.44 & 0.66 & $0.91^{* *}$ & $0.81^{* *}$ \\
$10-20 \mathrm{~cm}$ & $0.78^{*}$ & 0.19 & 0.30 & $0.77^{*}$ & 0.66 \\
$20-40 \mathrm{~cm}$ & 0.41 & -0.09 & 0.09 & 0.18 & 0.33 \\
$40-60 \mathrm{~cm}$ & 0.36 & -0.11 & 0.09 & 0.10 & 0.29 \\
$60-100 \mathrm{~cm}$ & $0.93^{* * *}$ & 0.35 & 0.66 & $0.84^{* *}$ & 0.69 \\
\hline
\end{tabular}

\section{$4 \quad \delta^{15} \mathbf{N}$ in den Pflanzen}

\subsection{Blattposition}

Blätter liefern von den Pflanzenorganen das geeignetste Material zur Ermittlung des $\delta^{15} \mathrm{~N}$-Wertes des von den Pflanzen aufgenommenen Stickstoffs, da sie nur eine relativ kurze Lebensdauer haben und somit nicht über Jahre Stickstoff akkumulieren. Durch die Blattbeprobung werden Bäume zudem am wenigsten in ihrem Wachstum beeinträchtigt.

Es stellte sich nun die Frage, ob es Unterschiede zwischen den neuen und alten Blättern einer Pflanze gibt und welches Blattalter am ehesten den $\delta^{15} \mathrm{~N}-$ Wert der N-Quelle wiedergibt und daher für die Bestimmung des \%Ndfa am günstigsten ist. Für die Genauigkeit der $\%$ Ndfa-Bestimmung war es weiterhin von Bedeutung, das Referenzorgan zu finden, dessen $\delta^{15} \mathrm{~N}$-Werte eine möglichst geringe Eigenstreuung mit sich brachte. 
Als Kriterien für die Wahl des geeigneten Blattalters können deshalb gelten und mußten untersucht werden:

1. Bestehen signifikante Unterschiede in den $\delta^{15} \mathrm{~N}-$ Werten zwischen alten und neuen Blättern?

2. Wie hoch ist das Ausmaß der $\delta^{15} \mathrm{~N}-$ Streuung der verschiedenen Blattalter?

3. Welches Blattalter liefert die größere Differenz im $\delta^{15} \mathrm{~N}$-Wert zwischen einer Referenzpflanze und der Leguminose.

$\mathrm{Zu} 1$.

Ein $t$-Test nach Student für abhängige Proben verdeutlichte, daß alte und junge Blätter meistens signifikant unterschiedliche $\delta^{15} \mathrm{~N}$-Werte hatten. Die Blätter von Tapirira guianensis und Banara guianensis waren nicht signifikant verschieden (Tab. 39).

Tab. 39: Unterschiede in den $\delta^{15} \mathrm{~N}$-Werten von neuen und alten Blättern von Leguminosen und Nichtleguminosen ( $t$-Test für abhängige Stichproben, $* * *=p<0.001)$

\begin{tabular}{lll}
\hline \multicolumn{2}{c|}{ Leguminosen } & \multicolumn{2}{|c}{ Nichtleguminosen } \\
\hline Abarema jupunba $\quad * * *$ & Banara guianensis & n.s. \\
Inga thibaudiana $\quad * * *$ & Lacistema pubescens & $* * *$ \\
Inga heterophylla $\quad * * *$ & Tapirira guianensis $\quad$ n.s. \\
& & Vismia guianensis $\quad * * *$ \\
\hline
\end{tabular}

Den Zusammenhang zwischen den alten und neuen Blättern kann man mit folgenden Gleichungen einer linearen Regression beschreiben:

Tab. 40: Regressionen zwischen den $\delta^{15} \mathrm{~N}-$ Werten von alten und neuen Blättern von 7 Sekundärwaldarten

\begin{tabular}{llcc}
\hline Spezie & \multicolumn{1}{c}{ Modell } & $\mathbf{r}(\mathbf{x}, \mathbf{y})$ & $\mathbf{r}^{2}$ \\
\hline Abarema jupunba & $\delta^{15} \mathrm{~N}_{\text {alt }}=-0.63+0.83 \times \delta^{15} \mathrm{~N}_{\text {neu }}$ & 0.71 & $0.50^{* *}$ \\
Banara guianensis & $\delta^{15} \mathrm{~N}_{\text {alt }}=-1.56-0.15 \times \delta^{15} \mathrm{~N}_{\text {neu }}$ & -0.18 & $0.03^{\mathrm{ns}}$ \\
Inga heterophylla & $\delta^{15} \mathrm{~N}_{\mathrm{alt}}=-0.61+0.79 \times \delta^{15} \mathrm{~N}_{\mathrm{neu}}$ & 0.88 & $0.78^{* * *}$ \\
Inga thibaudiana & $\delta^{15} \mathrm{~N}_{\mathrm{alt}}=-0.53+0.55 \times \delta^{15} \mathrm{~N}_{\mathrm{neu}}$ & 0.63 & $0.40^{*}$ \\
Lacistema pubescens & $\delta^{15} \mathrm{~N}_{\mathrm{alt}}=-1.14+1.00 \times \delta^{15} \mathrm{~N}_{\mathrm{neu}}$ & 0.91 & $0.84^{* * *}$ \\
Tapirira guianensis & $\delta^{15} \mathrm{~N}_{\mathrm{alt}}=+0.28+1.01 \times \delta^{15} \mathrm{~N}_{\mathrm{neu}}$ & 0.95 & $0.89 * * *$ \\
Vismia guianensis & $\delta^{15} \mathrm{~N}_{\mathrm{alt}}=-0.62+0.98 \times \delta^{15} \mathrm{~N}_{\mathrm{neu}}$ & 0.97 & $0.94 * * *$ \\
\hline
\end{tabular}


Die $\delta^{15} \mathrm{~N}$-Werte der alten Blätter lagen mindestens eine $1 / 2 \delta^{15} \mathrm{~N}$-Einheit niedriger als die der neuen Blätter, wie aus den negativen Werten für den y-Achsenabschnitt hervorgeht (vergl. auch Abb. 42 im Anhang). Daneben bestand für fast alle Arten eine positive, signifikante Korrelation zwischen den beiden Blattaltern. Es ist also zu erwarten, daß in den neuen Blättern stets höhere $\delta^{15} \mathrm{~N}$-Werte gemessen werden. Eine Ausnahme bildete jedoch Banara guianensis, für die kein Zusammenhang in den Blatt $-\delta^{15} \mathrm{~N}-$ Werten gefunden wurde.

Das Gefälle im $\delta^{15} \mathrm{~N}$-Wert von den neuen zu den alten Blättern kann durch Fraktionierungsprozesse während der Blattalterung, bzw. dem Abtransport von N-Verbindungen aus den alten Blättern in die anderen Gewebe der Pflanzen erklärt werden. In den neuen Blättern finden hauptsächlich anabolische Reaktionen mit einem $\beta$-Faktor von 1.0091 bis 1.0184 statt, in den alten Blättern die katabolischen Reaktionen mit einem $\beta$-Faktor von 1.0017 bis 1.0170 (Yoneyama, 1991a und b). Gestresste Pflanzen verwenden aber häufig die durch o.g. Prozesse ${ }^{15} \mathrm{~N}$-verdünnten $\mathrm{N}$-Verbindungen der alten Blättern für den Aufbau der neuen mit (Li, 1992).

$\mathrm{Zu} 2$.

Das Ausmaß der Streuung der $\delta^{15} \mathrm{~N}$-Werte war für die alten und neuen Blätter aller untersuchten Arten sehr gering (Tab. 41). Sowohl in der Gruppe der Leguminosen als auch der Referenzpflanzen war die über die jeweiligen Arten gemittelte Varianz der alten Blätter um 0.1 höher als in den neuen Blättern. Ein Ausblick auf artenspezifische Unterschiede gibt der Vergleich der Varianzen bezüglich der Arten: Nichtleguminosen wiesen eine wesentlich höhere Varianz als Leguminosen auf.

Tab. 41: Vergleich der Varianzen neuer und alter Blätter in der jungen Sekundärvegetation

\begin{tabular}{|c|c|c|c|c|c|}
\hline \multirow[t]{2}{*}{ Art } & & \multicolumn{2}{|c|}{ alte Blätter } & \multicolumn{2}{|c|}{ neue Blätter } \\
\hline & & $\mathrm{Mw}$ & Varianz & $\mathrm{Mw}$ & Varianz \\
\hline Abarema jupunba & (Leg.) & -0.40 & 0.52 & 0.05 & 0.21 \\
\hline Inga heterophylla & (Leg.) & -0.57 & 0.65 & 0.03 & 0.91 \\
\hline Inga thibaudiana & (Leg.) & -0.47 & 0.60 & -0.18 & 0.37 \\
\hline Banara guianensis & (Nichtleg.) & -0.54 & 1.46 & -0.48 & 1.44 \\
\hline Lacistema pubescens & (Nichtleg.) & 0.49 & 1.28 & 1.66 & 1.10 \\
\hline Tapirira guianensis & (Nichtleg.) & 0.49 & 2.42 & -2.11 & 2.56 \\
\hline Vismia guianensis & (Nichtleg.) & -0.72 & 3.65 & -0.26 & 3.48 \\
\hline \multicolumn{2}{|c|}{ Über die Arten gemittelte Varianz } & \multicolumn{2}{|c|}{1.51} & Leg.: 0.50, Ref.: 2.14 & 1.44 \\
\hline
\end{tabular}


$\mathrm{Zu} 3$.

Als drittes Entscheidungskriterium sollte die Differenz der alten bzw. neuen Blätter zwischen Referenzpflanzen und Leguminosen dienen. Diese Differenzen waren je nach Referenzart und Blattalter unterschiedlich stark ausgeprägt (Tab. 42). Banara guianensis wies mit 0.9 $\delta^{15} \mathrm{~N}$-Einheiten in den neuen Blättern (bzw. 0.7 in den alten Blättern) hierbei die geringsten, Tapirira guianensis mit zwei $\delta^{15} \mathrm{~N}$-Einheiten (bzw. 1.1 in den alten Blättern) die größten Differenzen auf. Lacistema pubescens mit 1.7 (1.1) und Vismia guianensis mit 1.6 (1.6) waren als intermediär anzusehen. Für drei der vier untersuchten Referenzarten war die größte Differenz in den $\delta^{15} \mathrm{~N}$-Werten der neuen Blättern zu finden. Eine Ausnahme bildete Vismia guianensis, die in neuen und alten Blättern die gleiche Differenz aufwies.

Tab. 42: Vergleich der $\delta^{15} \mathrm{~N}$-Differenzen zwischen den Mittelwerten der Referenzarten und den Leguminosen in den alten und neuen Blättern.

\begin{tabular}{|c|c|c|c|c|c|}
\hline \multirow[t]{2}{*}{ Leguminosen } & \multicolumn{5}{|c|}{ Referenzpflanzen } \\
\hline & $\begin{array}{c}\text { Banara } \\
\text { guianensis }\end{array}$ & $\begin{array}{l}\text { Lacistema } \\
\text { pubescens }\end{array}$ & $\begin{array}{l}\text { Vismia } \\
\text { guianensis }\end{array}$ & $\begin{array}{l}\text { Tapirira } \\
\text { guianensis }\end{array}$ & Gesamtdifferenz \\
\hline \multicolumn{6}{|c|}{ neue Blätter } \\
\hline Abarema jupunba & 1.0 & 1.6 & 1.6 & 2.1 & \\
\hline Inga heterophylla & 0.7 & 1.6 & 1.3 & 2.1 & \\
\hline Inga thibaudiana & 0.9 & 1.8 & 1.8 & 1.9 & \\
\hline $\begin{array}{l}\text { Mittelwert, auf die Refe- } \\
\text { renzarten bezogen }\end{array}$ & 0.9 & 1.7 & 1.6 & 2.0 & 1.5 \\
\hline \multicolumn{6}{|c|}{ alte Blätter } \\
\hline Abarema jupunba & 0.7 & 1.0 & 1.6 & 1.0 & \\
\hline Inga heterophylla & 0.7 & 1.2 & 1.6 & 1.2 & \\
\hline Inga thibaudiana & 0.7 & 1.1 & 1.5 & 1.1 & \\
\hline $\begin{array}{l}\text { Mittelwert, auf die Refe- } \\
\text { renzarten bezogen }\end{array}$ & 0.7 & 1.1 & 1.6 & 1.1 & 1.1 \\
\hline
\end{tabular}

Die einzelnen Kriterien zur Auswahl der die Boden-N-Quelle repräsentierenden Blattposition haben also folgendes ergeben: Die alten und neuen Blätter unterschieden sich in allen Arten außer auf einigen Standorten in Tapirira guianensis und Banara guianensis signifikant voneinander und standen in einem positiven Zusammenhang. Die alten Blätter wiesen mindestens eine $1 / 2 \delta^{15} \mathrm{~N}$-Einheit niedrigere Werte auf. Die Varianz als Maß für die Streuung ist in den neuen Blättern geringfügig niedriger. Ebenso fanden sich in den neuen Blättern die größeren Differenzen zwischen Leguminosen und Referenzen. Somit kann man davon ausgehen, 
daß die neuen Blätter zuverlässigere bzw. sensiblere Ergebnisse für die Bestimmung des $\%$ Ndfa liefern als die alten Blätter.

\subsection{Räumliche Variabilität}

Die $\delta^{15} \mathrm{~N}$-Werte in der jungen Sekundärvegetation der Bragantina-Region umfaßten eine Wertespanne von +6 bis -5 , wobei die meisten zwischen -2 und +2 lagen (Abb. 16). Letzterer ist ein sehr geringer Bereich. Die Streuung der meisten Arten war aber sehr hoch, so daß nur geringe Unterschiede zwischen den Arten aufgrund der $\delta^{15} \mathrm{~N}$-Werte zu erkennen waren. Die $\delta^{15} \mathrm{~N}$-Werte der Leguminosen streuten zwar weniger, jedoch auch in dieser Pflanzenfamilie kamen Arten vor mit einem $\delta^{15} \mathrm{~N}$ von +2 oder -2 .

Es stellte sich daher die Frage, ob diese Leguminosen überhaupt $\mathrm{N}_{2}$ fixieren, da sie doch innerhalb der $\delta^{15} \mathrm{~N}$-Spanne aller anderen untersuchten Arten lagen.

Eine für die ${ }^{15}$ NNAM geeignete Referenzpflanze sollte eine möglichst große Wertedifferenz $\mathrm{zu}$ den $\delta^{15} \mathrm{~N}$-Werten der Leguminose zeigen und gleichzeitig aus der gleichen Boden-N-Quelle schöpfen (Kohl et al., 1980). Da diese Wertedifferenz in der Vegetation der Bragantina-Region offensichtlich nicht sehr groß ausfallen würde, mußten Referenzpflanzen gesucht werden, die eine möglichst kleine innerartliche Streuung zeigten.

Mit einer vergleichenden Beprobung einer Primärvegetation (Peixe Boi), die noch nicht gebrannt wurde, sollte überprüft werden, ob die vorgefundenen $\delta^{15} \mathrm{~N}$-Werte der Sekundärvegetation eventuell mit der Brandrodung in Zusammenhang standen.

\subsubsection{Igarapé Açu}

Zur näheren Untersuchung der Streuung der $\delta^{15} \mathrm{~N}$-Werte in der Vegetation wurden 3 Leguminosen (Abarema jupunba, Inga thibaudiana, Inga heterophylla) und 4 Nichtleguminosen (Banara guianensis, Lacistema pubescens, Tapirira guianensis, Vismia guianensis), die sehr häufig in der Brachevegetation vorkamen, ausgewählt. Es wurde die innerartliche Variabilität auf einzelnen Flächen und zwischen verschiedenen Flächen sowie die Unterschiede zwischen den einzelnen Arten betrachtet (für die $\delta^{15} \mathrm{~N}$-Werte und N-Gehalte der einzelnen Arten auf den unterschiedlichen Flächen siehe im Anhang Abb. 42, Tab. 63 und 64 sowie die ANOVAErgebnisse in Tab. 65). 


\section{Standortabhängigkeit}

Die $\delta^{15} \mathrm{~N}$-Werte der neuen Blätter aller untersuchten Arten lagen in einem Bereich von -4 bis +4 (Abb. 22). Damit lagen sie im Wesentlichen niedriger als der $\delta^{15} \mathrm{~N}$-Wert des $\mathrm{N}_{\text {tot. }}$ Bodens (Abb. 17). Die $\delta^{15} \mathrm{~N}$-Werte der Leguminosen Abarema jupunba und Inga thibaudiana zeigten keine hohe standortabhängige Streuung und variierten auch innerhalb der einzelnen Flächen kaum. Die $\delta^{15} \mathrm{~N}$-Werte der neuen Blätter lagen für Abarema jupunba insgesamt zwischen -0.67 und -0.16 , für Inga thibaudiana zwischen -0.40 und +0.11. Die dritte Leguminose Inga heterophylla hatte auf den Flächen S2 und S3 mehr als $1 \delta^{15} \mathrm{~N}$-Einheit höhere Werte als auf den anderen Flächen (circa -0.5 gegenüber +1.05 ) und wies auch insgesamt eine höhere Standardabweichung als die beiden anderen Leguminosen auf.

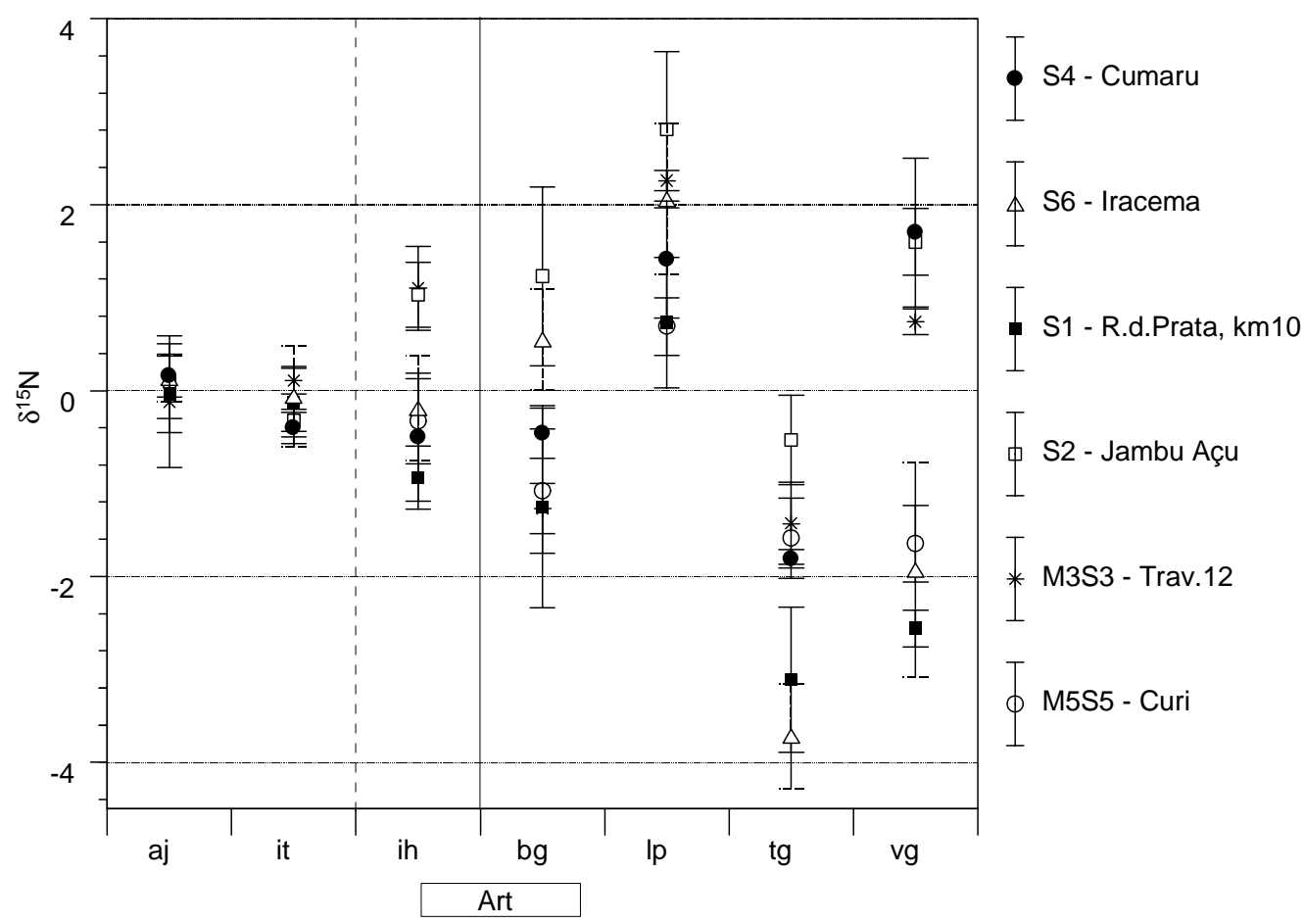

Abb. 22: $\delta^{15} \mathrm{~N}$ in den neuen Blättern von 7 Arten in Sekundärvegetationen gleichen Alters (aj = Abarema jupunba, it = Inga thibaudiana, ih = Inga heterophylla, $\mathrm{bg}=$ Banara guianensis, $\mathrm{lp}=$ Lacistema pubescens, $\operatorname{tg}=$ Tapirira guianensis, $\mathrm{vg}=$ Vismia guianensis, Balken geben $s$ an.

Die $\delta^{15} \mathrm{~N}$-Werte aller 4 Nichtleguminosen streuten sowohl innerhalb als auch zwischen den Flächen stärker als die Leguminosen. Auf Fläche S2 wurden bei allen Arten die höchsten, auf Fläche S1 die niedrigsten $\delta^{15} \mathrm{~N}$-Werte mit Ausnahme von Tapirira guianensis gefunden. Die standortabhängigen Unterschiede waren in Vismia guianensis am deutlichsten und in Lacistema pubescens am wenigsten ausgeprägt. Die $\delta^{15} \mathrm{~N}$-Werte der Fläche S2 unterschieden sich 
in Vismia guianensis sogar um fast $4 \delta^{15} \mathrm{~N}$-Einheiten (circa -2.0 gegenüber +1.6). Aus dieser Beprobung geht Tapirira guianensis als eine Art mit durchgehend negativen $\delta^{15} \mathrm{~N}$-Werten (Gesamtmittel=-2.11 \pm 1.12 ), Lacistema pubescens mit weitgehend positiven Werten $(+1.70$ $\pm 0.97)$ hervor. Vismia guianensis $(-0.26 \pm 1.88)$ hatte auf den Flächen S6, S1 und S5 ebenfalls stark negative Werte, auf der Fläche S4 jedoch positive, wodurch sie die größte Wertespanne umfaßte. Die Werte von Banara guianensis streuten zum Teil innerhalb einzelner Flächen am stärksten. Ihre $\delta^{15} \mathrm{~N}-$ Werte lagen zwischen +2 und -2 mit einem Durchschnittswert von $-0.8( \pm 1.19)$.

\section{Gruppierung der Arten aufgrund ihrer räumlichen $\delta^{15} N$-Schwankungen}

Um die Arten nach ihrem $\delta^{15} \mathrm{~N}$-Muster einzuordnen und voneinander abzugrenzen wurde eine Clusteranalyse nach dem Ward'schen Algorithmus mit der Euklid'schen Distanz als Distanzmaß durchgeführt (Abb. 23).

Die Clusteranalyse trennte als erstes Tapirira guianensis und Vismia guianensis von den anderen Arten ab. In einem zweiten und dritten Schritt wurden Lacistema pubescens und Banara guianensis von der Gruppe der Leguminosen abgesondert. Innerhalb der Leguminosen wurde noch einmal zwischen Inga heterophylla auf der einen Seite und Inga thibaudiana und Abarema jupunba auf der anderen Seite unterschieden.

Die beiden Leguminosen Abarema jupunba und Inga thibaudiana wurden von der Clusteranalyse deutlich von den Nichtleguminosen abgegrenzt. Auffällig an diesen beiden Arten war die sehr geringe Streuung unabhängig vom Standort und auch innerhalb einer Fläche. Die BNF könnte die Ursache dafür sein, denn dadurch werden Pflanzen unabhängig vom Stickstoff der Bodenquelle und bleiben in ihren $\delta^{15} \mathrm{~N}-$ Werten konstant.

Inga heterophylla nahm sowohl in der standortabhängigen Streuung als auch in der Clusteranalyse eine Mittelposition ein. Möglicherweise ist auch diese Art $\mathrm{N}_{2}$-fixierend, jedoch nicht in dem Ausmaß wie die beiden anderen Leguminosen. 


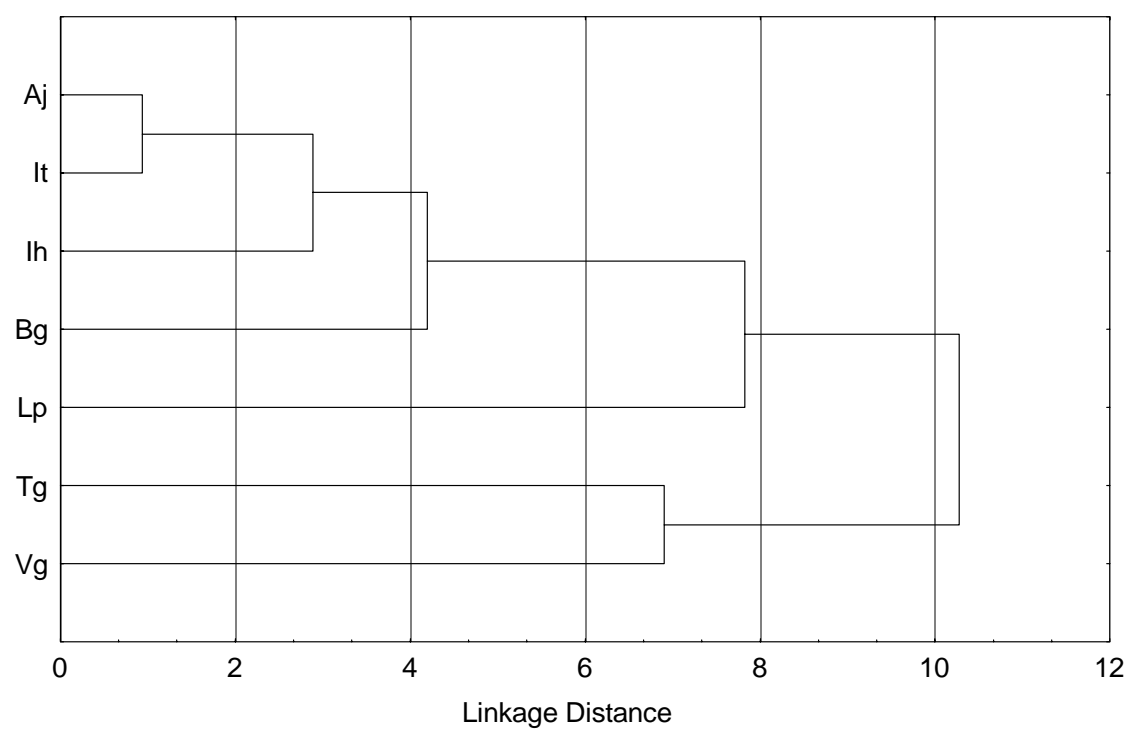

Abb. 23: Dendrogramm der Clusteranalyse nach Ward (Euclid'sche Distanz ) anhand der $\delta^{15} \mathrm{~N}$-Werte junger und alter Blätter von 7 Arten auf 6 verschiedenen Flächen.

Vismia guianensis und Tapirira guianensis kommen als einzige als Referenzpflanzen in Frage. Sie wurden von der Clusteranalyse als erstes und damit am deutlichsten von den Leguminosen getrennt. Tapirira guianensis hatte konstant negative $\delta^{15} \mathrm{~N}$-Werte und damit die größte Differenz zu den Leguminosen, Vismia guianensis auf drei der Flächen ebenso.

Folgende Gruppenaufteilung erschien daher sinnvoll:

\begin{tabular}{|c|c|c|}
\hline A & $\begin{array}{l}\text { Abarema jupunba } \\
\text { Inga thibaudiana }\end{array}$ & Stärker fixierende Leguminosen \\
\hline B & $\begin{array}{l}\text { Vismia guianensis } \\
\text { Tapirira guianensis }\end{array}$ & $\begin{array}{l}\text { Nicht-fixierende Arten mit deutlich von den Leguminosen ab- } \\
\text { gegrenzten } \delta^{15} \mathrm{~N} \text {-Werten, potentielle Referenzpflanzen }\end{array}$ \\
\hline $\mathrm{C}$ & $\begin{array}{l}\text { Inga heterophylla } \\
\text { Lacistema pubescens } \\
\text { Banara guianensis }\end{array}$ & $\begin{array}{l}\text { Arten mit } \delta^{15} \mathrm{~N}-\text { Werten im Bereich der Leguminosen oder kon- } \\
\text { stant positivem } \delta^{15} \mathrm{~N}\end{array}$ \\
\hline
\end{tabular}

\subsubsection{Peixe Boi}

Um einen Eindruck vom Einfluß der Brandrodung auf das ${ }^{15}$ N-Vorkommen der Bragantina-Region zu gewinnen, wurden ein noch nie anthropogen gebrannter Primärwald und zum Vergleich dazu in direkter Nachbarschaft eine alte Brachevegetation (15-25 Jahre), in üblicher Weise durch Brandrodung bewirtschaftet, untersucht ( Abb. 24 ). 


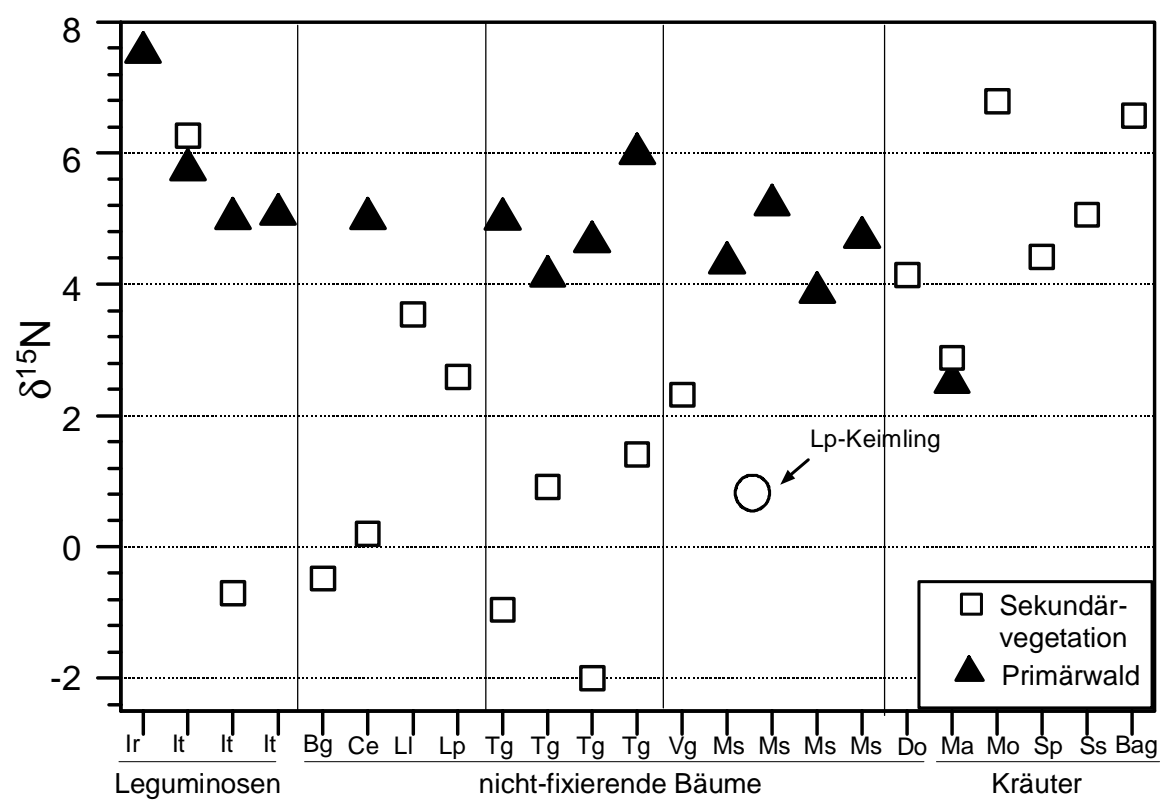

Abb. 24: $\delta^{15} \mathrm{~N}$ in den neuen Blättern von Primärwald und altem Sekundärwald der Fazenda Monte Verde, Peixe Boi (Abkürzungen sieheTab. 66 im Anhang)

Die Artenzusammensetzung in der Vegetation der Flächen in Peixe Boi war von der in Igarapé Açu sehr verschieden, so daß nur wenige übereinstimmende Spezies zur Verfügung standen. Deutlich wurde dennoch, daß Arten aus verschiedensten Familien inklusive der Leguminosen im Primärwald deutlich höhere Blatt- $\delta^{15} \mathrm{~N}$-Werte aufwiesen als in der benachbarten Sekundärvegetation oder der jungen Sekundärvegetation in Igarapé Açu (Abb. 24, Tab. 66 im Anhang, vergl. Abb. 22). Sämtliche Blatt- $\delta^{15} \mathrm{~N}-$ Wert von Primärwald-Arten bewegten sich im Bereich über $\delta^{15} \mathrm{~N}=+4$, während die Sekundärwald-Arten über eine Spanne von -2 bis $+7 \mathrm{zu}$ finden waren. In der alten Sekundärvegetation hatte Tapirira guianensis Werte zwischen +2 und -2 , im Primärwald jedoch zwischen +4 und +6 . Das gleiche wurde für Cordia exaltata und Inga thibaudiana beobachtet. Während die Nichtleguminose Tapirira guianensis in der alten Sekundärvegetation gleiche $\delta^{15} \mathrm{~N}$-Werte wie in der jungen Sekundärvegetation aus Igarapé Açu hatte, lag die Leguminose Inga thibaudiana im Primärwald mit durchschnittlich +5 extrem hoch. Möglicherweise fixiert diese Leguminose im Primärwald keinen Stickstoff. In der alten Sekundärvegetation waren für diese Leguminose sowohl positive als auch negative $\delta^{15} \mathrm{~N}$-Werte zu verzeichnen (+6 bzw. -1). Für Lacistema pubescens wurde in der alten Sekundärvegetation ein $\delta^{15} \mathrm{~N}-$ Wert von +2.5 gefunden, der den in der jungen Sekundärvegetation gefundenen Werten entsprach. Gleiches gilt für Banara guianensis. Interessant ist der $\delta^{15} \mathrm{~N}$-Wert der in der Grafik nicht eingetragenen Lacistema pubescens- Keimlinge von +0.91 (Tab. 66). Der Keimling spiegelt den $\delta^{15} \mathrm{~N}$-Wert des real verfügbaren $\mathrm{N}_{\text {min. }}$ der 
obersten Bodenschicht und Streuauflage entsprechend seiner Durchwurzelungstiefe wider, denn diese Pflänzchen wurden inklusive Wurzeln homogenisiert. Somit verlieren durch Diskriminierung entstandene Verschiebungen der ${ }^{15} \mathrm{~N} /{ }^{14} \mathrm{~N}$-Zusammensetzung innerhalb der Pflanze ihre Bedeutung. Die im Vergleich zu Bäumen geringere Durchwurzelungstiefe der Kräuter Bagassa guianensis, Memora allamandiflora, Selaginella stellaria, Sterculia pruriens und der Melastomataceae scheint sich auch in deren $\delta^{15} \mathrm{~N}$-Werten widerzuspiegeln, denn für diese Pflanzengruppe waren die $\delta^{15} \mathrm{~N}-$ Werte wie für die Keimlinge durchgehend positiv.

Die N-Gehalte der Blätter waren im Durchschnitt in der Vegetation von Peixe Boi höher als in der Sekundärvegetation der Umgebung von Igarapé Açu.

\subsection{Temporäre Variabilität}

Im untersuchten Vegetationstyp der Bragantina-Region waren saisonale Schwankungen der $\delta^{15} \mathrm{~N}$-Werte mit der regenreichen bzw. trockeneren Jahreszeit zu erwarten (Luizão et al., 1992; Marrs et al., 1991). Vergleiche der saisonalen $\delta^{15} \mathrm{~N}$-Muster der einzelnen Arten können daher eine weitere Entscheidungshilfe für die Eignung der Arten als Referenzen an die Hand geben, da sie auf den Zugriff der gleichen N-Quelle hinweisen.

Über ein Jahr wurden daher sowohl Leguminosen als auch im 5m-Umfeld von ihnen Nichtleguminosen beprobt, wobei neue und alte Blätter (auch als Blattposition bezeichnet) immer wieder von denselben Individuen geerntet wurden.

Mit dieser Beprobung sollten Informationen über den Zeitpunkt des höchsten N-Gehaltes in den Blättern, $\delta^{15} \mathrm{~N}$-Unterschiede und parallele ${ }^{15} \mathrm{~N}$-Muster mit der Jahreszeit von Leguminosen und Nichtleguminosen als ein Kriterium zur Auswahl weiterer Referenzpflanzen, und dem Zeitpunkt der größten $\delta^{15} \mathrm{~N}$-Differenz zwischen Leguminosen und Referenzen gewonnen werden.

\subsubsection{Generelle Tendenzen}

Um den besten Beprobungsmonat auszuwählen, wurde nach einer generellen Tendenz der $\delta^{15} \mathrm{~N}$-Werte und N-Gehalte im Jahresverlauf gesucht. In Abb. 25 sind die $\delta^{15} \mathrm{~N}$-Werte, gemittelt über alle Arten, zu den 7 ausgewerteten Beprobungszeitpunkten für die neuen und alten Blätter dargestellt. 
Generell unterschieden sich die Blattpositionen nur in ihren N-Gehalten deutlich, nicht aber in ihren $\delta^{15} \mathrm{~N}$-Werten (ANOVA siehe Tab. $67 \mathrm{im}$ Anhang). Der LSD je Probezeitpunkt ist so groß, daß bestehende Unterschiede zwischen neuen und alten Blättern nicht als signifikant nachgewiesen werden konnten. Die neuen Blätter lagen in ihren Werten wiederum konstant über denen der alten Blätter (vgl. Kap. VI.4.1) .

Sowohl die $\delta^{15} \mathrm{~N}$-Werte als auch die N-Gehalte nahmen von Oktober bis Dezember zu. Zu Beginn der Regenfälle im Januar '93 kam es zu einer deutlichen Erhöhung der N-Gehalte, die dann zum Juli hin wieder etwas abnahm. Die ANOVA, in der die einzelnen Probezeitpunkte als wiederholte Messungen eingingen, bestätigt einen signifikanten Effekt des Probezeitpunktes. In den jungen Blättern war diese Tendenz deutlicher zu erkennen als in den alten Blättern. Der $\delta^{15} \mathrm{~N}$-Wert zeigte in dem ersten Regenmonat eine durch die Absenkung von $\delta^{15} \mathrm{~N}$ in den neuen Blättern auffallend geringe Differenz zwischen neuen und alten Blättern. Im regenreichsten Monat - April'93 - waren die $\delta^{15} \mathrm{~N}$-Werte am höchsten. Sie fielen zum Juli hin wieder leicht $a b$.

Es kann also festgehalten werden, daß mit Einsetzen der Regenzeit generell eine höhere $\mathrm{N}$-Konzentration in den Blättern der Vegetation vorlag, die zu der leichten, nicht signifikanten Absenkung der $\delta^{15} \mathrm{~N}-$ Werte geführt haben könnte.

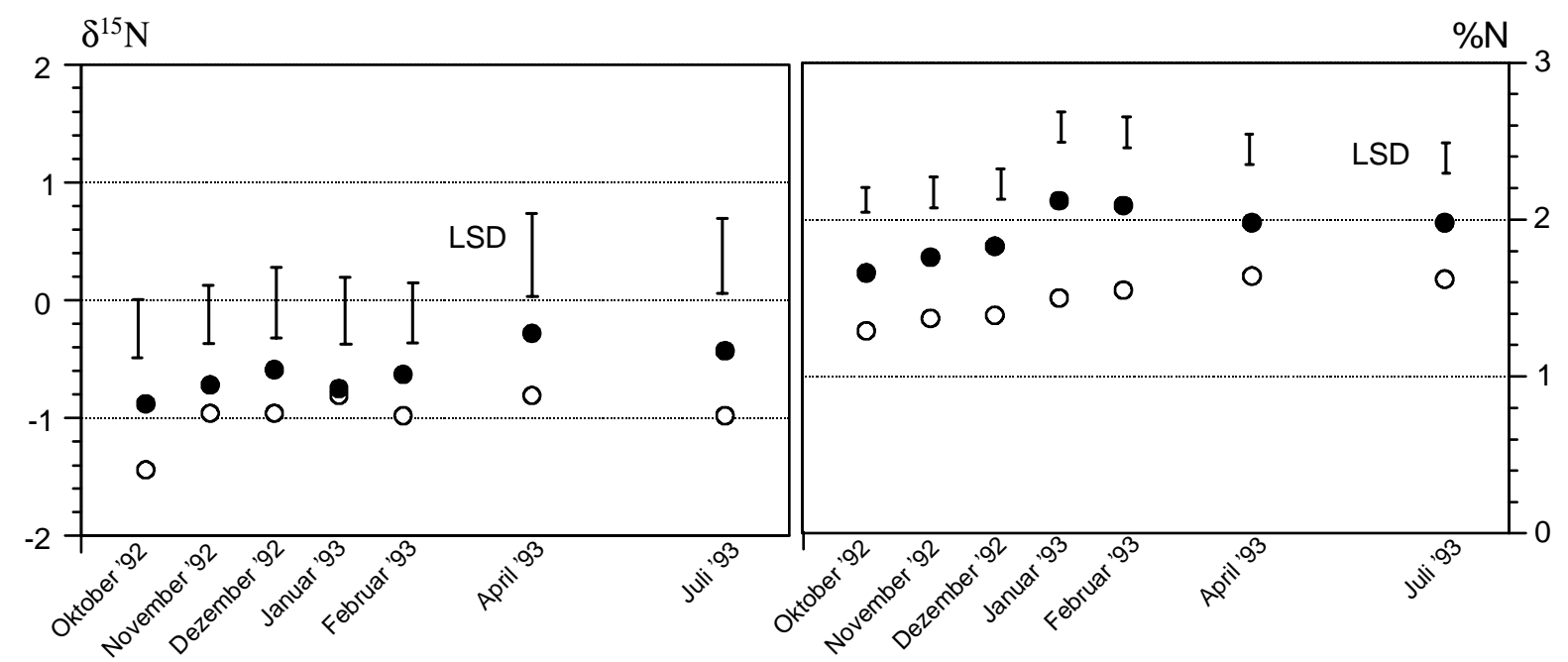

Abb. 25: $\delta^{15} \mathrm{~N}$-Werte und N-Gehalte der Blattpositionen zu den unterschiedlichen Probezeitpunkten neue Blätter, $\bigcirc$ alte Blätter) 


\subsubsection{Unterschiede im zeitlichen $\delta^{15} \mathrm{~N}-$ Muster der Arten}

Der Vergleich der $\delta^{15} \mathrm{~N}$-Werte der einzelnen Arten im Jahresgang ergab aufgrund der geringen $\delta^{15} \mathrm{~N}$-Distanzen zueinander und der sehr hohen Streuung nur einen schwachen Anhaltspunkt (Abb. 26). Es lassen sich daher allenfalls Tendenzen erkennen.

Die zeitliche Amplitude der Schwankungen war für die Leguminosen geringer als für die Nicht- $\mathrm{N}_{2}$-Fixierer.

Da für die \%Ndfa-Berechnung die neuen Blätter herangezogen werden, wurde das Hauptaugenmerk auf die Entwicklung der Isotopenverhältnisse dieser Blätter gelegt. Der Januar'93 hob sich bei allen Leguminosen und einigen Referenzen durch eine markante Erhöhung des $\delta^{15} \mathrm{~N}$-Wertes in den neuen Blättern hervor. In den Monaten April und Juli fanden sich in 6 von 10 Arten die höchsten $\delta^{15} \mathrm{~N}$-Werte, die niedrigsten $\delta^{15} \mathrm{~N}$-Werte in Januar und Februar. Bedeutende Schwankungen in allen Arten traten in den Monaten Dezember bis April ein, also mit Einsetzen der regenreichen Zeit und ihrem Fortschreiten.

Mit Einsetzen der Regenzeit wurde in Abarema jupunba, Inga heterophylla, Inga thibaudiana, Tapirira guianensis, Myrcia sylvatica, Vismia guianensis und Casearia arborea eine Zunahme des $\delta^{15} \mathrm{~N}$-Wertes in den jungen Blättern gefunden. Banara guianensis ließ eine eher gegenläufige Tendenz erkennen. Luizão et al. (1992) untersuchten die Entwicklung der Mineralisation im Boden einer einjährigen Brandrodungsfläche in Zentralamazonien und stellten eine Anhäufung von mineralisiertem N während der Trockenzeit fest, die mit einsetzender Regenzeit schnell immobilisiert wurde. Marrs et al.(1991) fanden die höchste Mineralisationsrate von terra firme-Böden im Norden Brasiliens im Übergang von der trockenen zur regenreichen Periode. Organische Substanz sammelte sich während der trockenen Zeit an und wurde bei beginnender Regenzeit mineralisiert. Das auf diese Weise mit dem Regen verfügbar gemachte $\mathrm{N}_{\text {min. }}$ könnte auch in der Bragantina-Region die in den Blättern vorgefundenen Tendenzen erklären, wenn man von negativen $\delta^{15} \mathrm{~N}$-Werten des mineralischen Stickstoffs ausgeht.

Ledgard et al. (1985) wiesen einen Fehler in der \%Ndfa-Berechnung nach, der aufgrund zeitlich versetzter Assimilationsoptima von Trifolium subterraneum und seinen Referenzgräsern entstand. Sie gingen davon aus, daß eine ähnliche Entwicklung des $\delta^{15} \mathrm{~N}$ im Jahresgang gleiche Assimilationsraten und die gleiche N-Quelle signalisieren. Banara guianensis ist eine Art, 


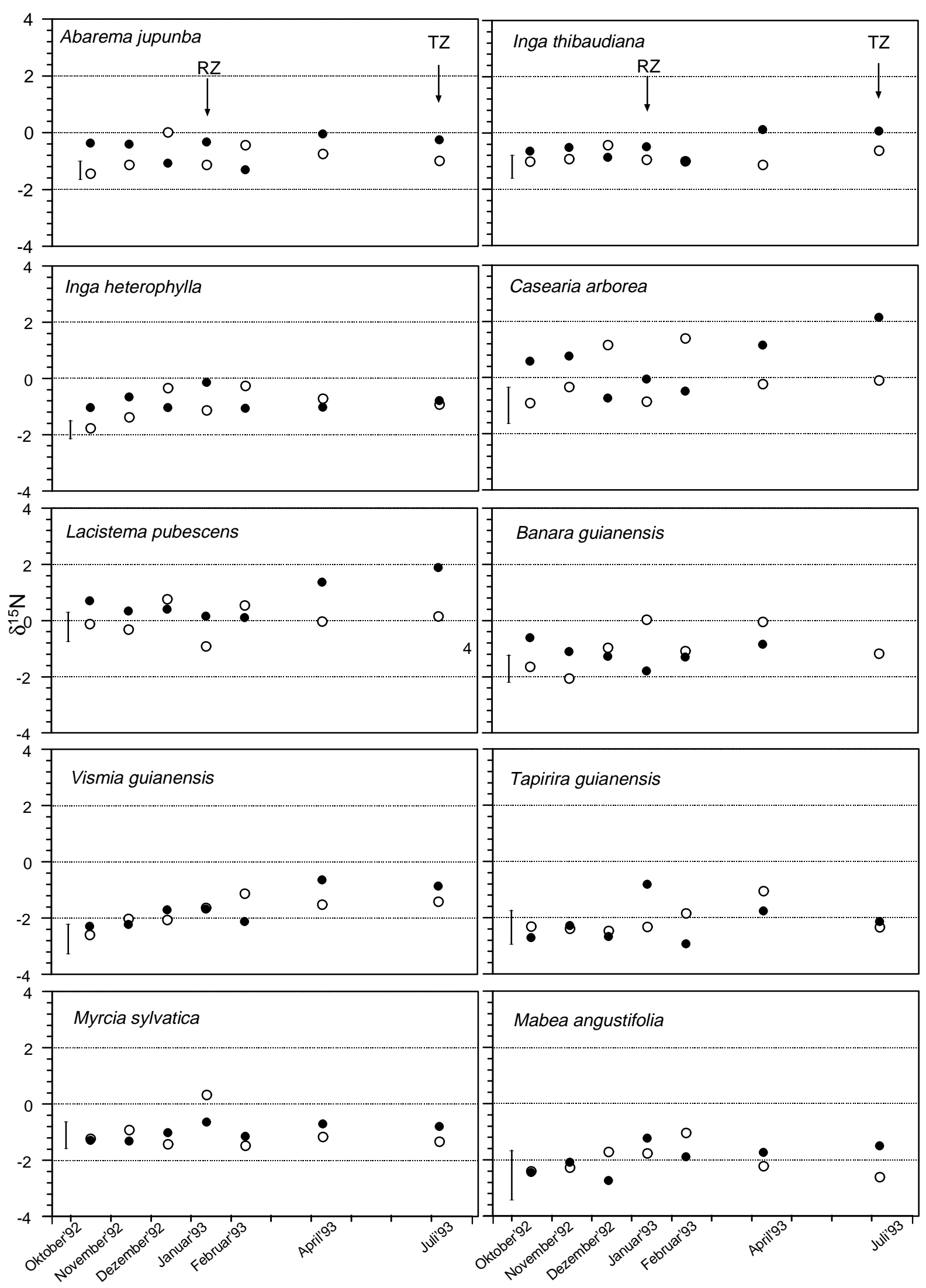

Abb. 26: $\delta^{15} \mathrm{~N}$ in den Blättern von Leguminosen (Abarema jupunba, Inga thibaudiana, Inga heterophylla) und Nichtleguminosen im Jahresverlauf. Die eingezeichneten Balken geben den LSD für $\mathrm{p} \leq 0.05$ an, RZ = Beginn der Regenzeit, TZ $=$ Beginn der Trockenzeit $(\bullet=$ neue Blätter, $\mathrm{o}=$ alte Blätter) 
die während der trockenen Zeit ihre Blätter fast vollständig abwirft. Der mit der beginnenden Regenzeit neue Aufbau der Blattmasse wirkt sich so über die Mobilisierung von Speicherstoffen auf die Entwicklung ihres $\delta^{15} \mathrm{~N}-$ Wertes in den Monaten Januar und Februar aus. Daher wurde Banara guianensis als Referenz für die \%Ndfa-Bestimmung ausgeschlossen.

Nichtleguminosen, die die gleiche, aber extremere Reaktion auf das Einsetzen der Regenzeit (Januar) zeigten wie die $\mathrm{N}_{2}$-Fixierer, lassen die Ausschöpfung der gleichen Boden-N-Quelle wie die Leguminosen vermuten. Daher wurde für Tapirira guianensis, Mabea angustifolia, und Myrcia sylvatica die gleiche N-Quelle wie für $\mathrm{N}_{2}$-Fixierer angenommen. Aufgrund des sehr inhomogenen Datensatzes können an dieser Stelle aber nur wenig fundierte Aussagen getroffen werden. Zum einen fehlen die Datenanalysen der Monate März, Mai, Juni, August und September, die Tendenzen wie die Erhöhung der $\delta^{15} \mathrm{~N}$-Werte in der regenreichen Zeit bestätigen könnten, zum anderen müßte der sprunghafte Anstieg der Werte zu Beginn der Regenzeit durch weitere Untersuchungen belegt werden.

In Abb. 27 sind als „Negativbeispiel“ zwei Arten dargestellt, die als Referenzpflanzen aufgrund ihrer enormen Streuung ungeeignet wären. Die Berechnung des \%Ndfa mit diesen $\delta^{15} \mathrm{~N}$-Werten ist problematisch, denn ihre $\delta^{15} \mathrm{~N}$-Werte würden mal oberhalb und mal unterhalb des $\delta^{15} \mathrm{~N}$ der Leguminosen liegen (vgl. Kap. VI.6). Da die Referenzpflanze jedoch den $\delta^{15} \mathrm{~N}$-Wert einer Leguminose repräsentieren soll, wenn diese nicht fixiert, wären $\%$ Ndfa-Zahlen über $100 \%$ bzw. unter $0 \%$ zu erwarten.

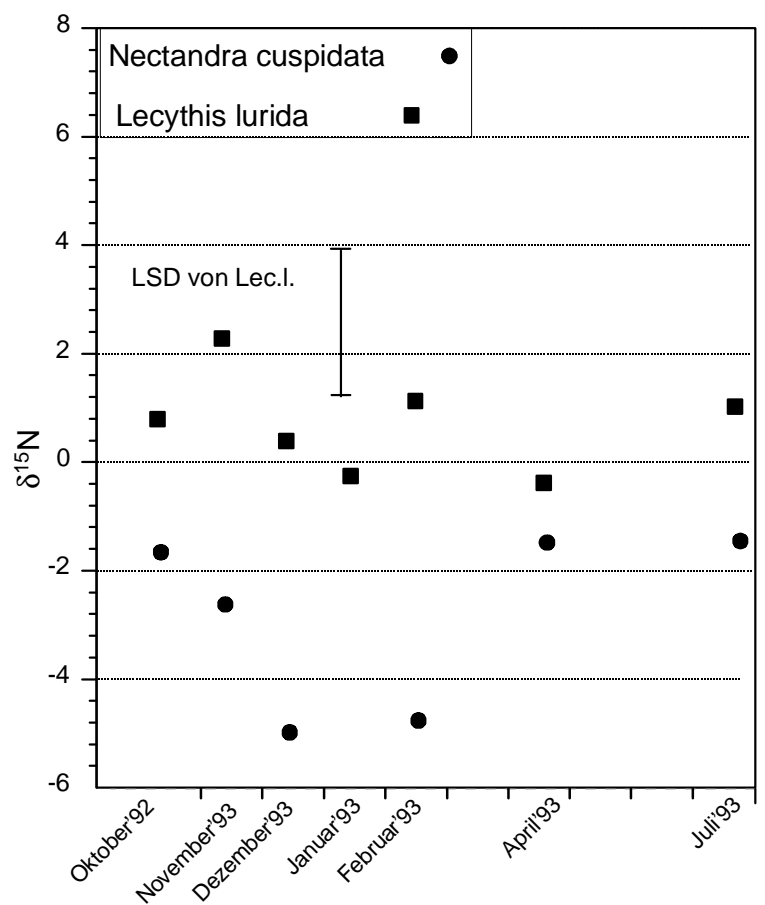

Abb. 27: $\delta^{15} \mathrm{~N}$ im Jahresverlauf der neuen Blätter von Nectandra cuspidata und Lecythis lurida 


\subsubsection{Pflanzengruppierung anhand zeitlicher Strukturen}

\subsubsection{Clusteranalyse}

Eine Clusteranalyse der $\delta^{15} \mathrm{~N}$-Werte und $\mathrm{N}$-Gehalte der sieben Zeitpunkte nach dem Ward'schen Algorithmus mit der Euclid'schen Distanz gruppierte die Arten nach ihren Gemeinsamkeiten bezüglich der zeitlichen Schwankungen, Wechselwirkungen mit der Blattposition und der Streuung im N-Gehalt und $\delta^{15} \mathrm{~N}$-Wert insgesamt (Abb. 28): Alle Arten mit negativen $\delta^{15} \mathrm{~N}$-Werten (Vismia guianensis, Tapirira guianensis, Mabea angustifolia, Tapirira guianensis, Myrcia sylvatica, Nectandra cuspidata) wurden als erstes in einer Gruppe zusammengefaßt, alle Arten mit Werten um $\delta^{15} \mathrm{~N}=0$ in eine zweite. Innerhalb dieser Gruppen spaltete sich jeweils eine Art aufgrund der hohen zeitlichen Amplituden - Nectandra cuspidata und Lecythis lurida- als Ausreißer ab (Abb. 27). Die Leguminosen wurden innerhalb der 2. Gruppe deutlich abgegrenzt von den Arten Lacistema pubescens, Casearia arborea und Banara guianensis. Deutlich wird, daß die Gruppierung auf die $\delta^{15} \mathrm{~N}$-Werte und auch $\mathrm{N}$-Gehalte zurückzuführen ist (Abb. 28b). Die Artengruppen, die aufgrund der zeitlichen $\delta^{15} \mathrm{~N}$-Muster gebildet wurden, bestätigen die Eingruppierung der Arten anhand der räumlichen Strukturen (siehe Abb. 23).
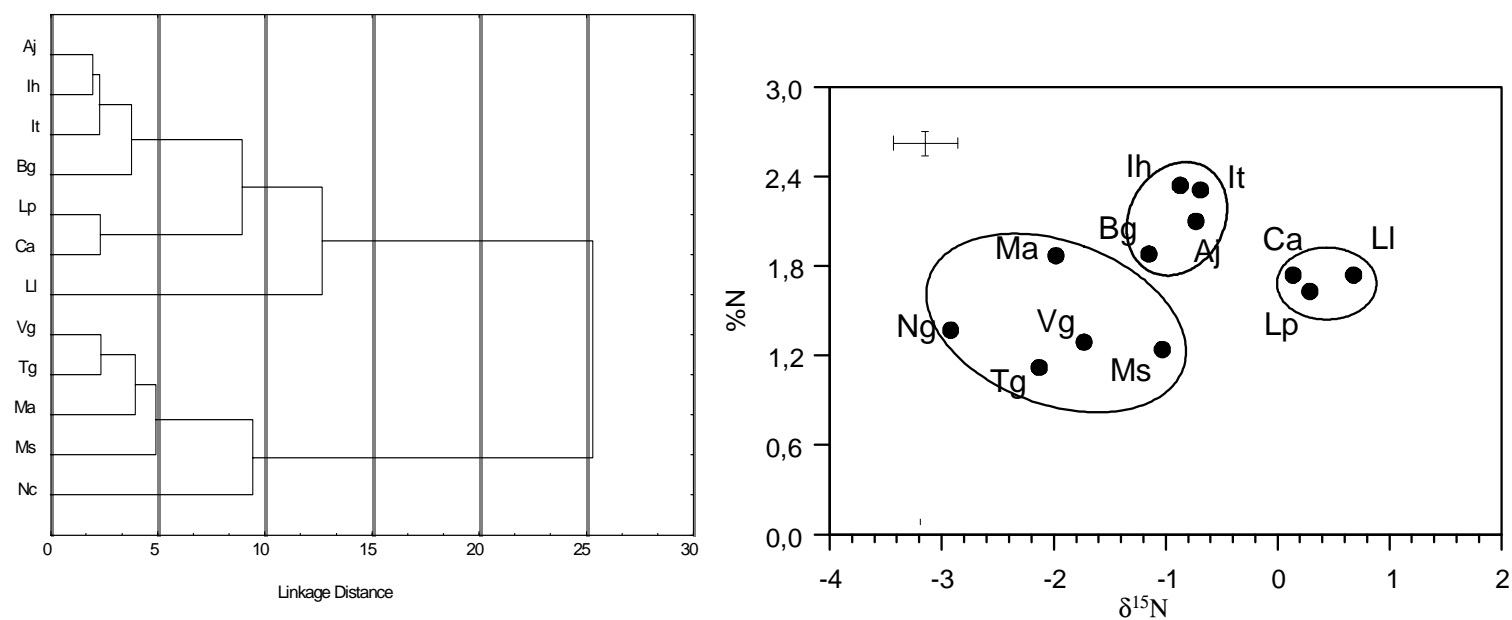

Abb. 28: a) Cluster Analyse der monatlichen $\delta^{15} \mathrm{~N}-$ Werte nach Ward mit dem Minimal-VarianzAlgorithmus und der Euclid'schen Distanz als Ähnlichkeitsmaß und b) $\delta^{15} \mathrm{~N}$ in Abhängigkeit vom NGehalt der in der Clusteranalyse ausgewiesenen Gruppen (Balken gibt die LSD für $\mathrm{p} \leq 0.05$ an) $(\mathrm{Aj}=$ Abarema jupunba, $\mathrm{Ih}=$ Inga heterophylla, $\mathrm{It}=$ Inga thibaudiana, $\mathrm{Bg}=$ Banara guianensis, $\mathrm{Lp}=$ Lacistema pubescens, $\mathrm{Ca}=$ Casearia arborea, $\mathrm{Ll}=$ Lecythis lurida, $\mathrm{Vg}=$ Vismia guianensis, $\mathrm{Tg}=$ Tapirira guianensis, $\mathrm{Ma}=$ Mabea angustifolia, $\mathrm{Ms}=$ Myrcia sylvatica, $\mathrm{Nc}=$ Nectandra cuspidata). 


\subsubsection{Differenzen zwischen Referenzpflanzen und Leguminosen}

Die Auswahl einer Referenzpflanze erfolgte auch anhand ihrer $\delta^{15} \mathrm{~N}$-Differenz zu der untersuchten Leguminose. Je größer die Streuung der einzelnen Arten ist, desto größer müssen die $\delta^{15} \mathrm{~N}$-Differenzen zwischen den Leguminosen und Referenzpflanzen sein. Bei einer $s$ von \pm 0.4 muß eine Differenz von $\delta^{15} \mathrm{~N}=4$ vorliegen, um nur einen $10 \%$ igen Fehler zu erreichen. Da in der Bragantina-Region mit einer durchschnittlichen Wertedifferenz von 1.5 und einer maximalen Differenz von $2.1 \delta^{15} \mathrm{~N}$-Einheiten gerechnet werden muß (vergl. Tab. 42 und Abb. 22), sollte also die Streuung und die größtmögliche Differenz zwischen Leguminosen und potentiellen Referenzpflanzen die entscheidenden Auswahlkriterien für die Bestimmung des Probenahmetermins sein. In Abb. 29 sind die saisonalen Veränderungen des $\delta^{15} \mathrm{~N}$-Wertes und ihr Standardfehler $(\sigma)$ dargestellt (siehe auch im Anhang Abb. 43, in der zusätzlich als Maß der Streuung die Varianz der $\delta^{15} \mathrm{~N}$-Werte und N-Gehalte für die einzelnen Arten in Abhängigkeit von der Jahreszeit dargestellt sind).

Allgemein traten die höchsten Schwankungen der $\delta^{15} \mathrm{~N}$-Werte der trockenen Jahreszeit im November und Juli, die geringsten im Oktober und Dezember auf. Die Monate der Regenzeit unterschieden sich nicht eindeutig. Im Januar war aufgrund der einsetzenden Regenzeit eine etwas höhere Variabilität zu verzeichnen als in den Monaten Februar und April. Monate, in denen die Streuung der N-Gehalte ebenfalls geringer ausfiel, deuten auf eine gleichmäßigere N-Verfügbarkeit auf den verschiedenen Flächen hin (Abb. 42 im Anhang). In der Regenzeit wäre dies der Monat April, in der Trockenzeit der Monat Oktober.

Die größten $\delta^{15} \mathrm{~N}$-Differenzen zwischen Leguminosen und Nichtleguminosen für die trockenere Zeit waren im Oktober zu lokalisieren. Für die Regenzeit war die Differenz zu den drei untersuchten Leguminosen unterschiedlich. Anhand von Durchschnittswerten für die Leguminosen konnte hier keine Unterscheidung gemacht werden. Der April war jedoch der regenreichste Monat, so daß davon ausgegangen werden kann, hier auch den Zeitpunkt der höchsten Biomasseproduktion zu finden - dem Argument von Pate et al. (1994) für die Auswahl des Beprobungszeitpunktes.

Anhand der Analyse der Meßzeitpunkte und der Clusteranalyse aufgrund zeitlicher Strukturen stellten sich die Arten Tapirira guianensis, Vismia guianensis sowie Mabea angustifolia und mit Einschränkungen Myrcia sylvatica als geeignete Referenzpflanzen dar. Sie wiesen eine ausreichende Differenz in ihren $\delta^{15} \mathrm{~N}$-Werten zu den Leguminosen auf und schienen ih- 
ren Stickstoff - aufgrund paralleler Schwankungen mit der Zeit - aus der gleichen Boden-N-Quelle zu beziehen. Als bester Zeitpunkt, um die $\mathrm{N}_{2}$-Fixierungskapazität der Leguminosen mit der ${ }^{15}$ NNAM zu bestimmen, stellte sich aufgrund der geringeren Streuung und größten Differenz zwischen Leguminosen und Referenzpflanzen der Monat Oktober (trockene Jahreszeit) heraus. In der regenreichen Zeit waren insgesamt größere Streuungen und geringere Wertedifferenzen zu erkennen und daher die Entscheidung für einen Probezeitpunkt problematisch. Aufgrund der etwas geringeren Schwankungen in den N-Gehalten der Blätter und der Annahme, daß der Zeitpunkt der höchsten Biomasseproduktion mit dem Zeitpunkt der höchsten Niederschlagsmengen zusammenfällt, könnte man daher den Monat April empfehlen.

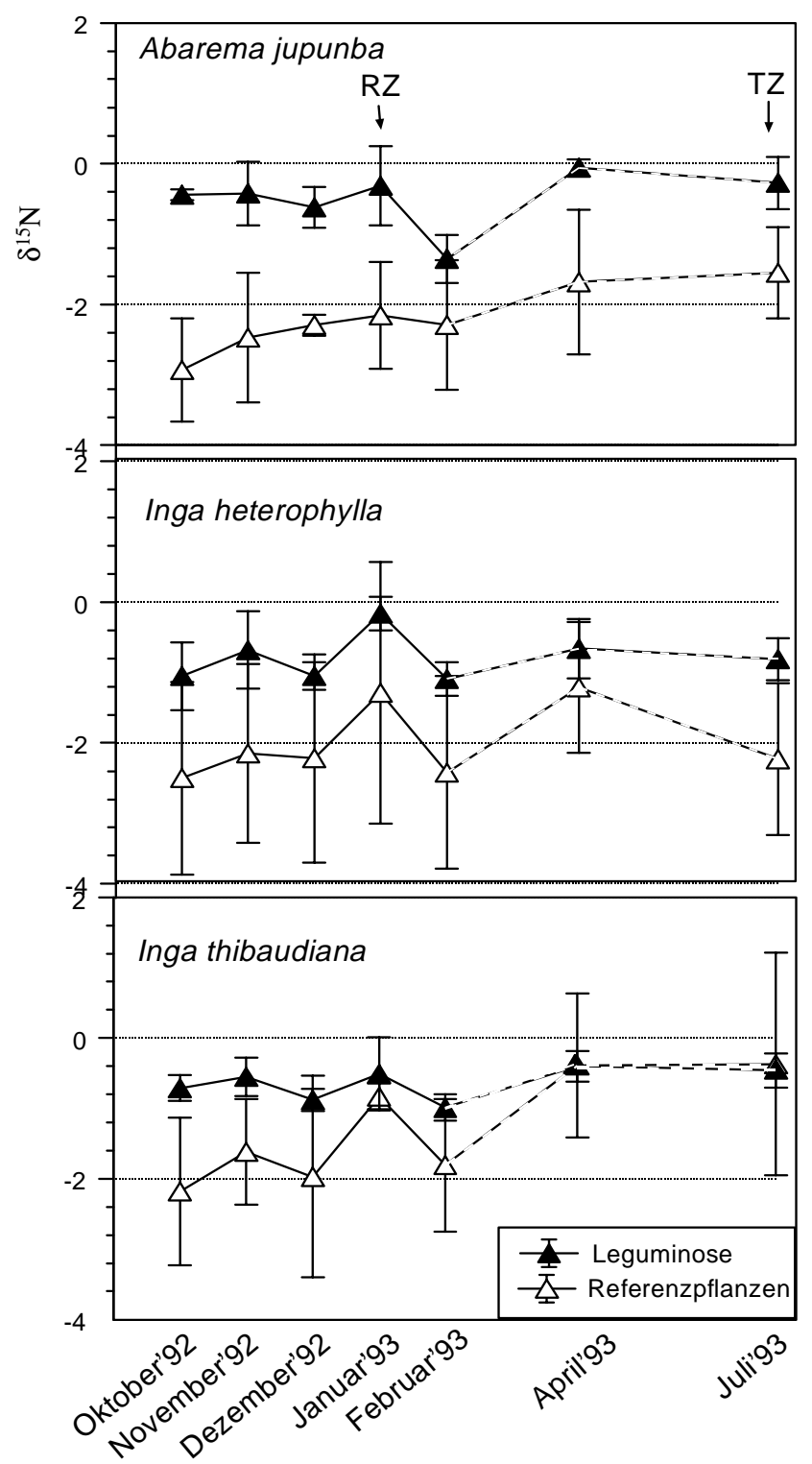

Abb. 29: Saisonale Veränderungen des $\delta^{15} \mathrm{~N}$-Wertes in den Blättern der Leguminosen im Vergleich zum $\delta^{15} \mathrm{~N}$-Mittelwert der $\delta^{15} \mathrm{~N}$-negativen Referenzpflanzen (Balken geben $\sigma$ wieder) 


\subsection{4 $\% \mathrm{~N}$ - und $\delta^{15} \mathrm{~N}$ - Unterschiede zwischen den Arten}

Da die in dieser Beprobung berücksichtigten Arten zwar Unterschiede in ihren $\delta^{15} \mathrm{~N}$-Werten aufwiesen, die jedoch nicht wesentlich vom Probezeitpunkt beeinflußt wurden, können sie hier noch einmal zusammengefaßt dargestellt werden. Die Arten mit den höchsten $\mathrm{N}-$ Gehalten waren die Leguminosen. Ein Zusammenhang der N-Gehalte mit dem $\delta^{15} \mathrm{~N}$-Wert konnte jedoch nicht hergestellt werden (Abb. 30). Die Arten unterschieden sich deutlich aufgrund ihres $\delta^{15} \mathrm{~N}$-Wertes. Lecythis lurida, Lacistema pubescens und Casearia arborea bildeten eine Gruppe mit positiven $\delta^{15} \mathrm{~N}$-Werten, die 3 Leguminosen (Abarema jupunba, Inga thibaudiana, Inga heterophylla) mit Myrcia sylvatica eine 2. Gruppe, Banara guianensis, Vismia guianensis und Tapirira guianensis bildeten wiederum eine Gruppe mit negativen $\delta^{15} \mathrm{~N}$-Werten. Nectandra cuspidata unterschied sich mit den niedrigsten $\delta^{15} \mathrm{~N}$-Werten $(-2.9)$ signifikant von allen anderen Arten.

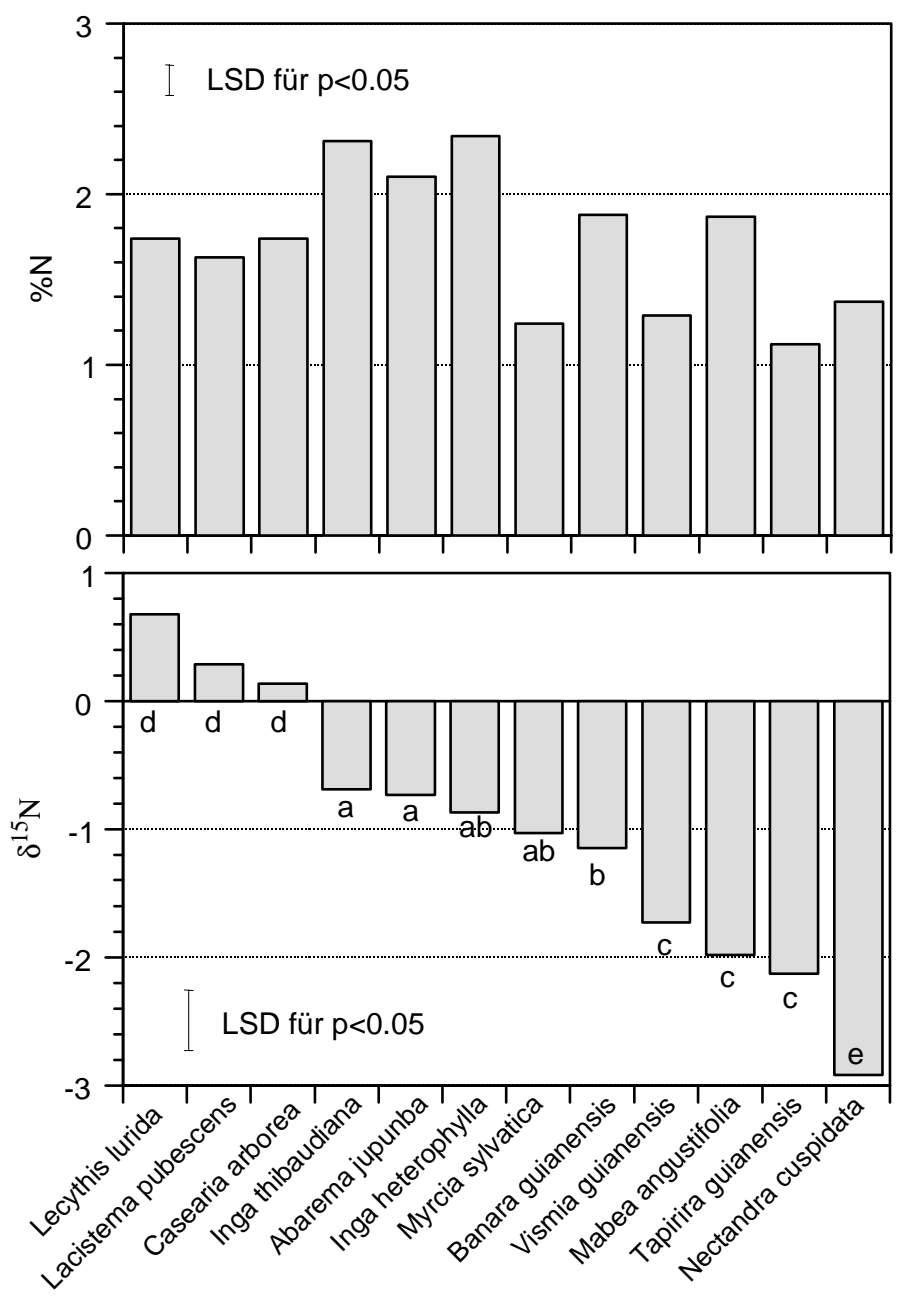

Abb. 30: $\delta^{15} \mathrm{~N}$ und N-Gehalte der untersuchten Arten $(30<\mathrm{n}<220)$, Buchstaben geben gleiche Ränge nach Scheffé an. 


\section{$5 \quad$ Verteilung von ${ }^{15} \mathrm{~N}$ in den Pflanzen}

Durch Fraktionierungsprozesse während der Aufnahme, $\mathrm{N}_{2}$-Fixierung und Assimilation von Stickstoff tritt ${ }^{15} \mathrm{~N}$ in unterschiedlichen Konzentrationen in den einzelnen Pflanzenorganen auf. Diese Isotopeneffekte müssen daher bei der Berechnung der $\mathrm{N}_{2}$-Fixierungsleistung mit einbezogen werden (Shearer et al. 1983). Für die Berechnung des \%Ndfa einer Leguminose muß man auch den $\delta^{15} \mathrm{~N}$-Wert derselben Art kennen, wenn sie ihren N-Bedarf ausschließlich aus der Luft decken muß. Man geht davon aus, daß sich das Fraktionierungsverhalten der Leguminosen im Feld und in hydroponischer Kultur gleicht und somit der Fehler, der bei der Berechnung des \%Ndfa entsteht durch die Berücksichtigung dieser sog. A-Pflanze verkleinert wird.

Die nicht fixierenden Referenzpflanzen sollen den $\delta^{15} \mathrm{~N}$-Wert der N-Quelle des Bodens widerspiegeln. Kennt man den $\beta$-Faktor, der die Veränderung der ${ }^{15} \mathrm{~N} /{ }^{14} \mathrm{~N}$-Zusammensetzung während des Weges des Stickstoffes aus dem Substrat in die Pflanzenorgane mit Hilfe eines definierten Gewächshausversuches beschreibt, so kann mit dieser Art über ihr Blatt- $\delta^{15} \mathrm{~N}$ im Feld auf das pflanzenverfügbare $\mathrm{N}$ ihrer N-Quelle geschlossen werden.

Mit Hilfe eines solchen Gewächshausversuches kann auch überprüft werden, ob die neuen Blätter tatsächlich den $\delta^{15} \mathrm{~N}$-Wert des Stickstoffs der Gesamtpflanze reflektieren. Im Feld ist die Bestimmung des $\delta^{15} \mathrm{~N}$-Wertes eines Gesamtbaumes nicht möglich, da die Wurzeln nicht mit in die Analysen einbezogen werden können. Sanginga et al. (1995) schätzten 50\% oder mehr des fixierten Stickstoffes von Baumleguminosen in der unterirdischen Biomasse. Dies könnte sich auch auf den $\delta^{15} \mathrm{~N}$-Wert der Blätter auswirken.

Zur Bestimmung des $\delta^{15} \mathrm{~N}$-Wertes von A-Pflanzen, dem Vergleich der $\delta^{15} \mathrm{~N}-$ Muster in einer Referenzpflanze und einer Leguminose, und dem $\beta$-Faktor des Weges des Stickstoffes von der Aufnahme bis zum Blatt einer Referenzpflanze wurde daher folgender Versuch angelegt:

Im Gewächshaus wurden Pflanzen von Abarema cochleatum und Abarema jupunba auf Nfreiem Medium (hier: Sand) über einen Zeitraum von 15 bzw. 20 Monate kultiviert. Parallel dazu wurden Tapirira guianensis und Abarema jupunba in einer Nährlösung mit $\mathrm{NO}_{3}{ }^{-}$des $\delta^{15} \mathrm{~N}$-Wertes -1.8 und auf Feldboden (die obersten $20 \mathrm{~cm}$ einer Brachefläche) kultiviert. Alle 2 bis 3 Monate wurden 3 Pflanzen geerntet, in die Kompartimente neue Blätter, alte Blätter, Holz, Rinde, Wurzeln und Knöllchen zerlegt und die Biomasse, den N-Gehalt und $\delta^{15} \mathrm{~N}$ bestimmt. 
Da es sich um ein offenes Gewächshaus handelte, konnten die äußeren Bedingungen nicht konstant gehalten und kein Schutz vor Befall durch Insekten und andere Schädlinge gewährleistet werden. Die Auswertung aller Entwicklungsstadien war so nicht möglich, da einige Individuen krank wurden. Auf statistische Absicherung wurde in diesem Versuch daher gänzlich verzichtet und für die Darstellung der Ergebnisse ein Entwicklungsstadium ausgewählt, das die beste Vergleichbarkeit mit den anderen im Gewächshaus angezogenen Arten und den Feldpflanzen darstellte. Als Kriterium für die Wahl des besten Entwicklungsstadiums wurde die N-Akkumulation/Monat während der Versuchsdauer der Gesamtpflanzen der verschiedenen Behandlungstypen herangezogen. Es wurde davon ausgegangen, daß Pflanzen, deren $\mathrm{N}$-Akkumulation noch steigend oder gleichbleibend hoch war, gesund waren bzw. sich im Wachstum befanden. Abnehmende Nährstoffakkumulation und Abbau der Biomasse wurde als ein Zeichen für einen Krankheitsbefall oder für eine saisonal bedingte Abnahme der Assimilationsrate interpretiert. Daher wurde für die A-Pflanze Abarema cochleatum der 10. Versuchsmonat und für Abarema jupunba der 12. Versuchsmonat ausgewählt. Für die anderen 4 Behandlungen wurde das Entwicklungsstadium nach 10 Monaten für die weiteren Auswertungen herangezogen. ( $\delta^{15} \mathrm{~N}$-Werte und akkumulierte N-Mengen siehe Tab. 68 im Anhang).

\subsection{A-Pflanzen}

Nach einer Wachstumsperiode von 12 bzw. 10 Monaten hatte sich eine klare Aufteilung von ${ }^{15} \mathrm{~N}$ zwischen den Pflanzenorganen der A-Pflanzen Abarema jupunba und Abarema cochleatum entwickelt (Abb. 31). Die beiden Arten zeigten in allen Organen ähnliche $\delta^{15} \mathrm{~N}$-Werte. Die niedrigsten $\delta^{15} \mathrm{~N}$-Werte wurden im Holz (-1.94 für Abarema jupunba) und in der Rinde (1.25 für Abarema jupunba), die höchsten in den Knöllchen gefunden (+5.09 für Abarema jupunba).

Unter Berücksichtigung der tatsächlich akkumulierten Stickstoffmenge der jeweiligen Kompartimente wurde der $\delta^{15} \mathrm{~N}$-Wert der Gesamtpflanze ermittelt. Er betrug für Abarema jupunba -0.2, für Abarema cochleatum -0.04, entsprechend dem $\delta^{15} \mathrm{~N}$-Wert des Luftstickstoffs. Dem $\delta^{15} \mathrm{~N}$-Wert der Gesamtpflanze kamen die Werte der Wurzeln sowie der alten und neuen Blätter am nächsten. Die Knöllchen unterschieden sich um circa $5 \delta^{15} \mathrm{~N}$-Einheiten vom Gesamtwert der Pflanzen. 


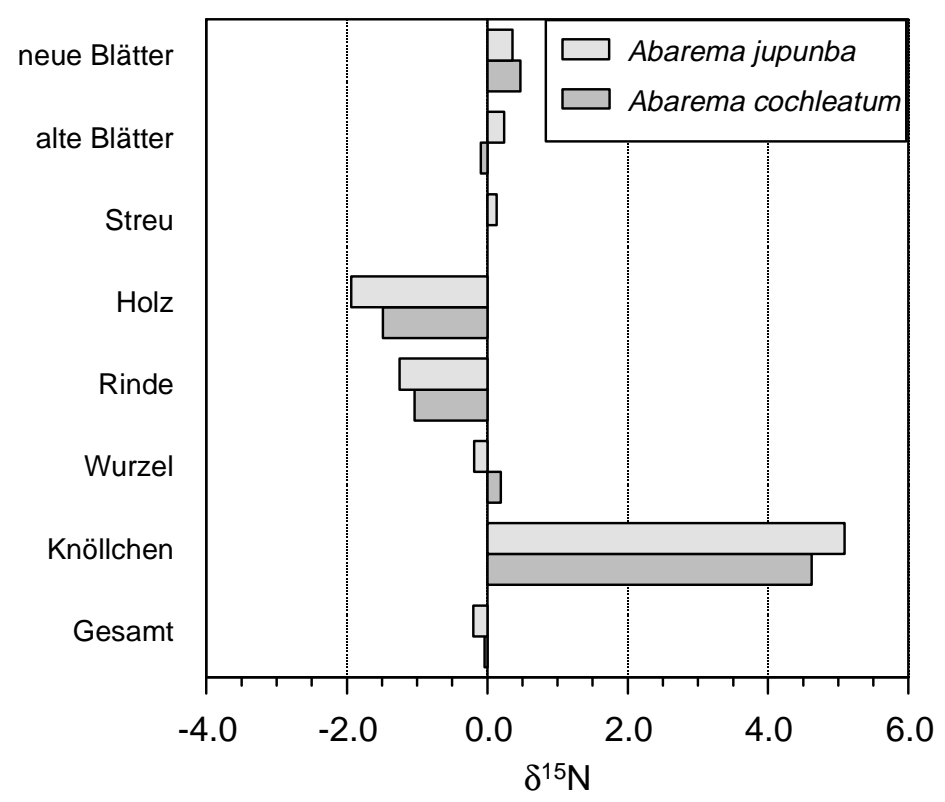

Abb. 31: $\delta^{15} \mathrm{~N}$ in den einzelnen Pflanzenorganen und der Gesamtpflanze von Abarema jupunba und Abarema cochleatum nach 10- bzw. 12-monatigem Wachstum in einer Sandkultur ohne N-Düngung $(\mathrm{n}<=3)$

Für die Berechnung des \%Ndfa von Feldleguminosen wurde der Mittelwert des $\delta^{15} \mathrm{~N}-\mathrm{Wertes}$ der neuen Blättern von Abarema jupunba und Abarema cochleatum herangezogen: $\underline{\mathbf{+ 0 . 4 2}}$

\section{$5.2 \quad{ }^{15} \mathrm{~N}$-Muster in den Pflanzenorganen}

Mit den $\delta^{15} \mathrm{~N}$-Werten der neuen Blätter und dem $\delta^{15} \mathrm{~N}$-Wert für die A-Pflanzen +0.42 wurde die Fixierungsrate für Abarema jupunba bestimmt. Sie betrug in der Sandkultur 45\% (mit $\mathrm{NO}_{3}{ }^{-}, \delta^{15} \mathrm{~N}=-1.8$ ), im Feldboden nur $19 \%$ (oberste $20 \mathrm{~cm}$ eines Bodens aus dem Projektgebiet, $\delta^{15} \mathrm{~N}=+3.8 \pm 1.3$ ). Die Hälfte des Stickstoffes nahm Abarema jupunba also aus der Nährlösung, was sich in den negativen $\delta^{15} \mathrm{~N}$-Werten von Wurzeln und Blättern manifestierte (Abb. 32). Allerdings ergab die Berechnung des $\delta^{15} \mathrm{~N}$-Wertes der Gesamtpflanze keinen Unterschied zum $\delta^{15} \mathrm{~N}$-Wert der A-Pflanze, womit die Unsicherheiten dieser Ergebnisse dokumentiert werden.

In den auf dem Feldboden gewachsenen Pflanzen wurden nur positive $\delta^{15} \mathrm{~N}$-Werte gefunden. In beiden Substraten wies Tapirira guianensis in den einzelnen Organen die gleiche Tendenz auf wie Abarema jupunba, in der Nährlösung um 0.5 bis $1.5 \delta^{15} \mathrm{~N}$-Einheiten zum negativen, auf dem Feldboden jedoch um 0.5 bis $2 \delta^{15} \mathrm{~N}$-Einheiten zum positiven hin verschoben. Durch die $\mathrm{N}_{2}$-Fixierung der Leguminose paßten sich also in beiden Fällen die $\delta^{15} \mathrm{~N}$-Werte in Rich- 
tung $\delta^{15} \mathrm{~N}=0$ an. Wie von Shearer et al. (1983) für Prosopis glandulosa wurde auch für diese beiden Arten eine geringere ${ }^{15} \mathrm{~N}$-Anreicherung in Holz und Rinde als in den Blättern gefunden. Ursache dafür sind die pflanzeninternen Stoffwechselprozesse, die den Stickstoff nach der Aufnahme und dem Transport in die jungen Blätter nur z.T. in stabile Strukturen in Holz und Rinde einbauen. Bei solchen Prozessen wird gegen ${ }^{15} \mathrm{~N}$ diskriminiert und auf diese Weise weniger ${ }^{15} \mathrm{~N}$-beladene Moleküle in Holz und Rinde eingearbeitet.

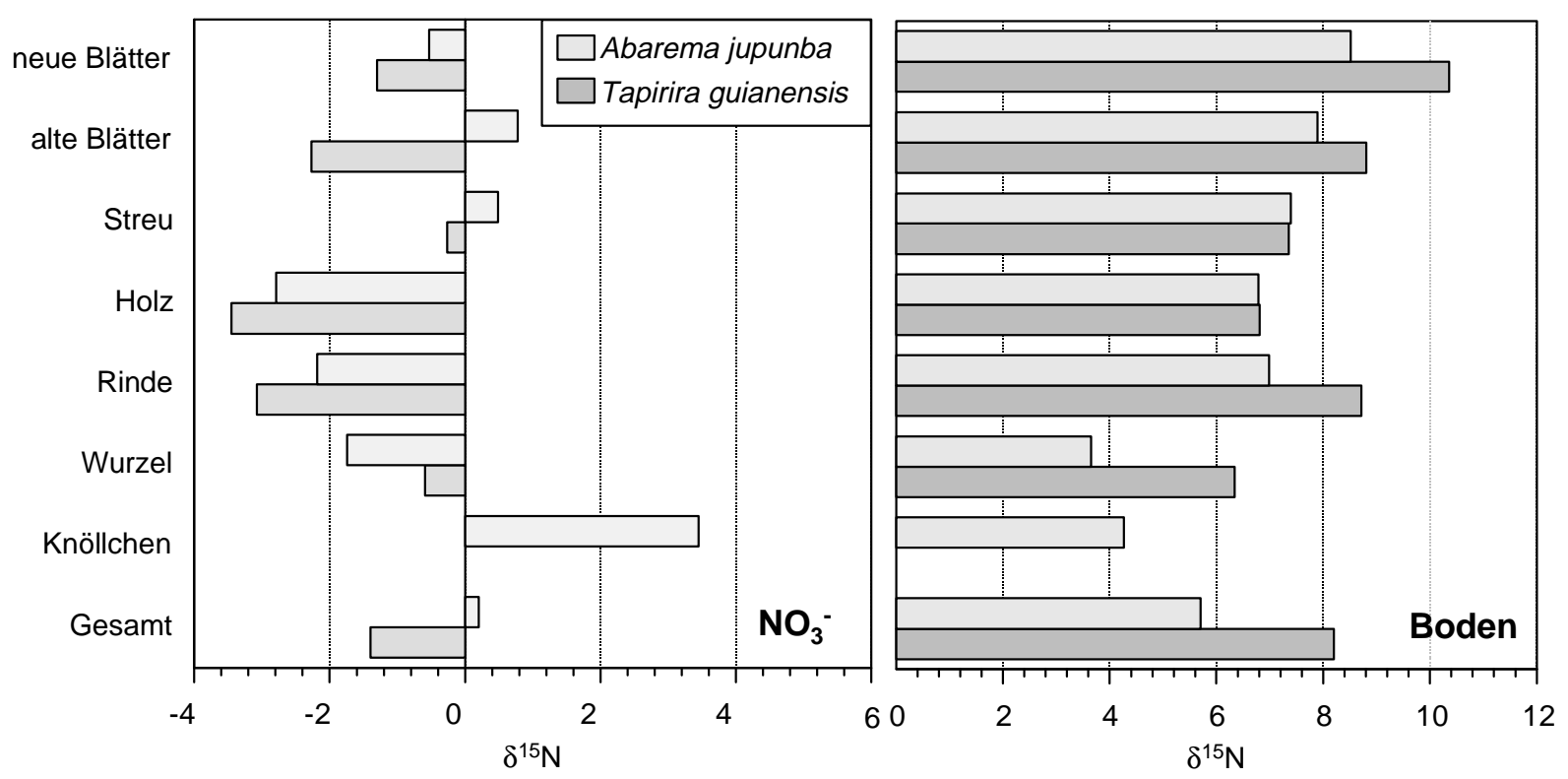

Abb. 32: $\delta^{15} \mathrm{~N}$ in den einzelnen Pflanzenorganen und der Gesamtpflanze von Abarema jupunba und Tapirira guianensis nach 10monatigem Wachstum auf Sand mit $\mathrm{NO}_{3}{ }^{-}$- Düngung $\left(\delta^{15} \mathrm{~N}=-1.8\right)$ bzw. Feldboden $\left(\delta^{15} \mathrm{~N}=+4\right)(\mathrm{n}<=3)$

Die in beiden Fällen gleichen Tendenzen in der Referenzpflanze und der Leguminose können als Indiz für gleiche Aufnahmemechanismen, Transport- und Stoffwechselwege von Tapirira guianensis und Abarema jupunba angesehen werden.

\section{$5.3 \quad \delta^{15} \mathrm{~N}$ des pflanzenverfügbaren Stickstoffs}

Zur Beschreibung des Grades der Fraktionierung einer Reaktion bedient man sich des sog. $\beta$-Faktors, der vereinfacht als Quotient aus Substrat und Produkt beschrieben werden kann. Er wird nach einer Formel berechnet, die in Kap. V.4.1 angegeben ist. 
Mit Hilfe der Kultivierung von Tapirira guianensis in einer Nährlösung mit einem definierten $\delta^{15} \mathrm{~N}$-Wert von -1.82 und der Analyse der $\delta^{15} \mathrm{~N}$-Werte der Gesamtpflanze war sowohl der $\delta^{15} \mathrm{~N}$-Wert der Gesamtpflanze $\left(\delta^{15} \mathrm{~N}_{\text {Produkt }}\right)$ als auch der Nährlösung $\left(\delta^{15} \mathrm{~N}_{\text {Substrat }}\right)$ mit den entsprechenden Atom $\%{ }^{15} \mathrm{~N}$ bekannt.

Mit der bis zum 10. Monat gedüngten Menge an Stickstoff und dem Gesamt-N von Tapirira guianensis wurde so ein $\beta$-Faktor von $\underline{1.0026}$ errechnet. Dieser Betrag ist bekannt für den Prozeß der Desaminierung bzw. Transaminierung und in etwas höheren Faktoren $(\beta=1.003)$ für die $\mathrm{NO}_{3}{ }^{-}$- Assimilation (Handley und Raven, 1992). Diese Prozesse schlugen sich in einer Differenz von $0.52 \delta^{15} \mathrm{~N}$-Einheiten $\left(\delta^{15} \mathrm{~N}_{\text {Blatt }}-\delta^{15} \mathrm{~N}_{\text {Nährlösung }}\right)$ in den Blättern von Tapirira guianensis nieder. Daher kann man davon ausgehen, daß das pflanzenverfügbare $\mathrm{N}$ in dem Feldboden des Gewächshausversuches einen $\delta^{15} \mathrm{~N}$-Wert von höchstens +9.84 hatte (10.360.52) (die einzelnen $\delta^{15} \mathrm{~N}$-Werte sind Tab. $68 \mathrm{zu}$ entnehmen). Paparcikova (1995) kam auf einen $\delta^{15} \mathrm{~N}$-Wert von +4.1 bis +7.4 für $\mathrm{N}_{\text {min. }}$ der oberen $20 \mathrm{~cm}$ eines Bodens aus der gleichen Region, wodurch der $\delta^{15} \mathrm{~N}-$ Wert von Tapirira guianensis im Gewächshausboden zum Teil erklärt wird. Im Feld selbst wies Tapirira guianensis jedoch negative $\delta^{15} \mathrm{~N}$-Werte auf (siehe Abb. 22 oder Tab. 63). Berücksichtigt man diese Werte und den $\beta$-Faktor, so müssen für die N-Quelle von Tapirira guianensis auf den Flächen S1 - S6 folgende $\delta^{15} \mathrm{~N}$-Werte angenommen werden (Tab. 43):

Tab. 43: $\delta^{15} \mathrm{~N}$ der pflanzenverfügbaren N-Quelle von $T a$ pirira guianensis auf Sekundärwaldflächen in der Bragantina-Region

\begin{tabular}{ccc}
\hline Fläche & $\delta^{15} \mathrm{~N}_{\text {Blatt }}$ & $\delta^{15} \mathrm{~N}_{\text {min. }}$ \\
\hline S1 & -3.11 & -3.63 \\
S2 & -0.53 & -1.05 \\
S3 & -1.43 & -1.95 \\
S4 & -1.81 & -2.33 \\
S5 & -1.59 & -2.11 \\
S6 & -3.72 & -4.24 \\
\hline Mittelwert & -2.03 & -2.55 \\
\hline
\end{tabular}

\section{$6 \%$ Ndfa der Leguminosen und die Präzision der Berechnung}

Der Anteil fixierten Stickstoffs am Gesamt-N einer $\mathrm{N}_{2}$-fixierenden Pflanze wird mit Hilfe des Vergleiches des $\delta^{15} \mathrm{~N}$-Wertes der Blätter von der zu untersuchenden Leguminose und einer 
oder mehrerer nicht fixierender Referenzpflanzen bestimmt (Formel siehe Kap. V.4.1). In diesem Kapitel soll nun mit den bisher gewonnenen Erkenntnissen und Referenzpflanzen ausgerechnet werden, wieviel $\% \mathrm{~N}$ von den Leguminosen fixiert wurde (\%Ndfa). Die Zuverlässigkeit dieser Berechnungen soll exemplarisch mit Hilfe der Daten des Monats Oktober überprüft werden (Tab. 44).

Tab. 44: $\delta^{15} \mathrm{~N}$-Werte der neuen Blätter der Leguminosen und ihrer jeweiligen Referenzpflanzen im Oktober 1992, und dem Mittelwert aller für eine Leguminose zuständigen Referenzpflanzen (Gesamtreferenz) (Leg = Leguminose, $\mathrm{Tg}=$ Tapirira guianensis, $\mathrm{Vg}=$ Vismia guianensis, $\mathrm{Ms}=$ Myrcia sylvatica, $\mathrm{Ma}=$ Mabea angustifolia, $\mathrm{Aj}=$ Abarema jupunba, $\mathrm{Ih}=$ Inga heterophylla, $\mathrm{It}=$ Inga thibaudiana $)$

\begin{tabular}{l|ccccccc}
\hline \multicolumn{7}{c}{$\delta^{15} \mathrm{~N}-$ Wert } \\
\hline & Leg & Tg & Vg & Ms & Ma & $\begin{array}{c}\text { Gesamt- } \\
\text { Referenz }\end{array}$ \\
\hline Aj1 & & & & & & -3.15 & -3.78 \\
Aj2 & -0.51 & - & -4.40 & - & -15 & -2.53 \\
Aj3 & -0.44 & - & -3.71 & -2.15 & -1.74 & -2.48 \\
Ih 1 & -1.62 & - & -2.49 & -1.20 & - & -1.85 \\
Ih 2 & -1.10 & - & - & - & -2.54 & -2.54 \\
Ih 3 & -0.64 & - & -2.64 & -0.21 & - & -1.43 \\
Ih 4 & -1.41 & - & -4.84 & - & - & -4.84 \\
Ih 5 & -0.49 & - & - & -1.84 & - & -1.84 \\
It 3 & -0.95 & - & -0.91 & - & - & -0.91 \\
It 4 & -0.53 & - & -2.49 & -1.04 & - & -1.77 \\
It 5 & -0.74 & -3.24 & - & - & - & -3.24 \\
It 6 & -0.62 & -2.72 & -2.87 & - & - & -2.80 \\
\hline
\end{tabular}

Deutlich wird zunächst das Problem der Abundanz der Referenzpflanzen. Es war nicht möglich, auf allen Standorten die gleichen Arten zu finden. So mußte auf die Arten zurückgegriffen werden, die sich vor Ort befanden.

Die mit den einzelnen Arten berechneten \%Ndfa-Werte wichen zum Teil stark von dem $\%$ Ndfa ab, der mit dem Mittelwert der vorgefundenen Referenzarten bestimmt wurde (Tab. 45) (die Umrandungen einzelner Felder in den Tab. 44 und Tab. 45 markieren die problematischen Daten). Dies traf hauptsächlich dann zu, wenn der $\delta^{15} \mathrm{~N}$-Wert der Referenzpflanze über dem der Leguminose lag oder die Differenz zwischen Leguminose und Referenzpflanze nicht groß genug war (siehe z.B. Ih 1 oder 3 und It 3). 
Tab. 45: \%Ndfa der einzelnen Leguminosen in Abhängigkeit von der in die Berechnung mit eingegangenen Referenzpflanzen und die Abweichungen dieser Berechnungen vom Gesamtmittel (+0.42 wurde als Wert für die A-Pflanze eingesetzt)

\begin{tabular}{|c|c|c|c|c|c|c|c|c|c|c|}
\hline \multicolumn{7}{|c|}{$\% \mathrm{Ndfa}$} & \multicolumn{4}{|c|}{$\begin{array}{l}\text { Abweichungen vom \%Ndfa, } \\
\text { berechnet mit Gesamt-Ref }\end{array}$} \\
\hline & Leg & $\mathrm{Tg}$ & $\mathrm{Vg}$ & Ms & $\mathrm{Ma}$ & $\begin{array}{l}\text { Gesamt- } \\
\text { Referenz }\end{array}$ & $\mathrm{Tg}$ & $\mathrm{Vg}$ & Ms & $\mathrm{Ma}$ \\
\hline$\overline{\mathrm{Aj} 1}$ & & & 81 & & 74 & 78 & & +3 & & -4 \\
\hline $\mathrm{Aj} 2$ & & & 80 & 67 & 61 & 71 & & +9 & -4 & -10 \\
\hline $\mathrm{Aj} 3$ & & 71 & 76 & & & 74 & -3 & +2 & & \\
\hline Ih 1 & & & 30 & -26 & & 10 & & +20 & -36 & \\
\hline Ih 2 & & & & & 49 & 49 & & & & \\
\hline Ih 3 & & & 66 & -70 & & 43 & & +23 & -113 & \\
\hline Ih 4 & & & 65 & & & 65 & & & & \\
\hline Ih 5 & & & & 60 & & 60 & & & & \\
\hline It 3 & & & -3 & & & -3 & & & & \\
\hline It 4 & & & 68 & 35 & & 57 & & +11 & -22 & \\
\hline It 5 & & 69 & & & & 69 & 0 & & & \\
\hline It 6 & & 67 & 69 & & & 68 & -1 & +1 & & \\
\hline
\end{tabular}

In einigen Fällen, wie z.B. Inga heterophylla 1 und Inga heterophylla 3, kam es zu Abweichungen vom \%Ndfa (mit den gesamten Referenzpflanzen berechnet) bis zu 113\%Ndfa. Die Ursache dafür lag hier in der Art Myrcia sylvatica, deren $\delta^{15} \mathrm{~N}-$ Werte an dieser Stelle zum Teil über denen der Leguminose waren und so zu groben Unterschätzungen führten. Wurde der $\%$ Ndfa mit Vismia guianensis als Referenzpflanze berechnet, lag der ermittelte Prozentsatz circa $10 \%$ über dem \%Ndfa, der unter Zuhilfenahme mehrerer Referenzpflanzen ermittelt wurde. \%Ndfa, unter Einbezug von Mabea angustifolia oder Myrcia sylvatica hingegen lagen stets unter dem Durchschnittswert, mit Tapirira guianensis errechnete \%Ndfa entsprachen weitgehend dem \%Ndfa der Gesamtreferenzen.

Eine Referenzpflanze soll den $\delta^{15} \mathrm{~N}$-Wert angeben, den die Leguminose hätte, wenn sie nicht fixieren würde (Abb. 33). Je mehr sie fixiert, desto mehr nähert diese Leguminose sich dem $\delta^{15} \mathrm{~N}$-Wert der A-Pflanze, um schließlich bei einer100\%igen Fixierungsleistung den $\delta^{15} \mathrm{~N}$ der A-Pflanze anzunehmen. Je größer die Differenz zwischen Leguminose und Referenzpflanze ist, desto genauer ist die Berechnung. Ein Vergleich der berechneten Fixierungsleistungen mit Hilfe von Tapirira guianensis oder Myrcia sylvatica verdeutlicht die Problematik: Schwankungen z.B. um $0.2 \delta^{15} \mathrm{~N}$-Einheiten führen im Falle von Tapirira guianensis zu einer Änderung von $7 \%$ Ndfa, im Falle von Myrcia sylvatica jedoch zu $16 \%$ Ndfa. Je höher die $\mathrm{N}_{2}$-Fixierungsleistung ist, desto ungenauer wird die Berechnung, denn die Differenz zwischen 
Leguminose und Referenzpflanze wird geringer. Bei einer für diese Region ungefähren Standardabweichung von $\delta^{15} \mathrm{~N}= \pm 0.7$ für Nicht-Fixierer und \pm 0.4 für $\mathrm{N}_{2}$-Fixierer (siehe Tab. 63) und einer maximalen Wertedifferenz von $2.5 \delta^{15} \mathrm{~N}$-Einheiten kann man jedoch nicht weniger als $20 \%$ Fehlerquote erwarten.

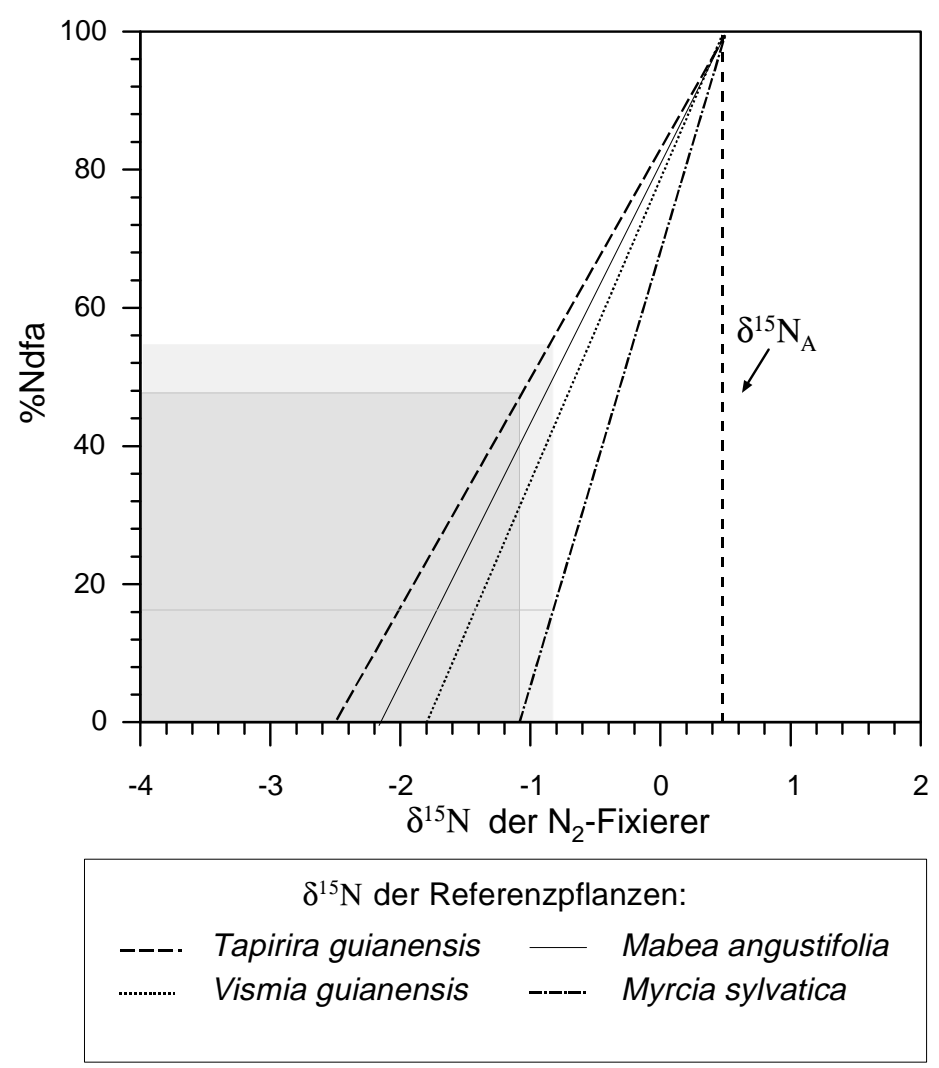

Abb. 33: Zusammenhang der \%Ndfa-Berechnung mit den $\delta^{15} \mathrm{~N}-$ Werten der in der Sekundärvegetation vorhandenen Referenzpflanzen (nach Unkovich et al., 1994; $\delta^{15} \mathrm{~N}_{\mathrm{A}}=\delta^{15} \mathrm{~N}$ der A-Pflanze), Schraffuren stellen Hilfslinien zum Ablesen der Werte dar, Erläuterungen siehe Text.

Mit Hilfe der $\delta^{15} \mathrm{~N}$-Werte von Vismia guianensis, Tapirira guianensis, Myrcia sylvatica und Mabea angustifolia wurden die monatlichen Fixierungsleistungen der Leguminosen Abarema jupunba, Inga thibaudiana und Inga heterophylla berechnet. Der Fehler der \%NdfaBerechnung wurde mit Hilfe der von Shearer und Kohl (1988a) zitierten Formel für die Ermittlung von Fehlerfortsetzungen in Summen und Quotienten von Mittelwerten für die 7 Meßzeitpunkte und 3 Leguminosen jeweils mit und ohne Einbeziehung von Myrcia sylvatica ermittelt (Tab. 46). 
Der bei Verwendung von Myrcia sylvatica erhöhte Fehler der \%Ndfa-Berechnung schlägt sich in der Fehlerberechnung der Fixierungsleistung von Inga heterophylla und Inga thibaudiana deutlich nieder. Daher werden im Folgenden die Berechnungen ohne Myrcia sylvatica zitiert.

Tab. 46: $\mathrm{N}_{2}$-Fixierungsleistung von 3 Leguminosen im Jahresverlauf. Die \%Ndfa-Berechnung und ihr Fehler wurden nach Shearer und Kohl (1988) berechnet. Für die A-Pflanze wurde $\delta^{15} \mathrm{~N}=+0.42$ eingesetzt. $\mathrm{n}$ in Klammern gibt die Wiederholungszahl für die Referenzen ohne Myrcia sylvatica an.

\begin{tabular}{|c|c|c|c|c|c|c|c|c|}
\hline \multirow[b]{2}{*}{ Monat } & \multirow[b]{2}{*}{$\mathrm{n}^{*}$} & \multirow[b]{2}{*}{$\delta^{15} \mathrm{~N}_{\text {Leg }}$} & \multicolumn{3}{|c|}{ mit Myrcia sylvatica } & \multicolumn{3}{|c|}{ ohne Myrcia sylvatica } \\
\hline & & & $\delta^{15} \mathrm{~N}_{\mathrm{Ref}}$ & $\%$ Ndfa & $\sigma$ & $\delta^{15} \mathrm{~N}_{\mathrm{Ref}}$ & $\%$ Ndfa & $\sigma$ \\
\hline \multicolumn{9}{|c|}{ Abarema jupunba } \\
\hline Oktober'92 & 3 & -0.44 & -2.93 & 74 & \pm 12 & -2.99 & 75 & \pm 11 \\
\hline November'92 & 3 & -0.42 & -2.47 & 73 & \pm 17 & -2.56 & 74 & \pm 16 \\
\hline Dezember'92 & 3 & -0.62 & -2.29 & 62 & \pm 14 & -2.41 & 64 & \pm 13 \\
\hline Januar'93 & 3 & -0.31 & -2.15 & 71 & \pm 22 & -2.12 & 71 & \pm 22 \\
\hline Februar'93 & 3 & -1.35 & -2.29 & 31 & \pm 18 & -2.36 & 32 & \pm 17 \\
\hline April'93 & 3 & -0.06 & -1.68 & 74 & \pm 20 & -1.66 & 74 & \pm 20 \\
\hline Juli'93 & 3 & -0.27 & -1.55 & 67 & \pm 21 & -1.55 & 67 & \pm 21 \\
\hline \multicolumn{9}{|c|}{ Inga thibaudiana } \\
\hline Oktober '92 & 4 & -0.71 & -2.18 & 48 & \pm 20 & -2.36 & 50 & \pm 18 \\
\hline November'92 & $4(3)$ & -0.55 & -1.62 & 49 & \pm 22 & -1.73 & 54 & \pm 22 \\
\hline Dezember'92 & $4(3)$ & -0.88 & -1.97 & 26 & \pm 32 & -2.40 & 42 & \pm 23 \\
\hline Januar'93 & $4(3)$ & -0.51 & -0.83 & 27 & \pm 40 & -0.88 & 41 & \pm 39 \\
\hline Februar'93 & $4(3)$ & -0.99 & -1.81 & 26 & \pm 26 & -2.08 & 39 & \pm 22 \\
\hline April'93 & $2(1)$ & -0.40 & -0.75 & 25 & \pm 31 & -1.28 & 68 & - \\
\hline Juli'93 & $2(1)$ & -0.46 & -0.22 & 93 & \pm 268 & -2.17 & 66 & - \\
\hline \multicolumn{9}{|c|}{ Inga heterophylla } \\
\hline Oktober '92 & $5(4)$ & -1.05 & -2.50 & 46 & \pm 29 & -3.13 & 53 & \pm 20 \\
\hline November'92 & $4(3)$ & -0.68 & -2.15 & 42 & \pm 31 & -2.61 & 48 & \pm 25 \\
\hline Dezember'92 & $4(3)$ & -1.05 & -2.22 & 20 & \pm 33 & -2.14 & 28 & \pm 26 \\
\hline Januar'93 & $4(3)$ & -0.16 & -1.29 & 103 & \pm 43 & -2.04 & 61 & \pm 23 \\
\hline Februar'93 & $5(4)$ & -1.09 & -2.42 & 37 & \pm 27 & -2.52 & 48 & \pm 15 \\
\hline April'93 & $4(4)$ & -0.66 & -1.21 & 31 & \pm 45 & -1.36 & 15 & \pm 38 \\
\hline Juli'93 & $5(4)$ & -0.81 & -2.23 & 47 & \pm 23 & -2.00 & 44 & \pm 25 \\
\hline
\end{tabular}

Alle Leguminosen fixierten einen erheblichen Teil des von ihnen aufgenommenen Stickstoffs. Die höchsten Raten waren für Abarema jupunba mit 32 bis $74 \%$ zu verzeichnen, Inga heterophylla bezogen zwischen $15 \%$ und 61\%, Inga thibaudiana $39 \%$ bis $68 \%$ ihres Gesamt-N aus der Luft. Der Standardfehler für Abarema jupunba liegt bei $\pm 11 \%$ bis $\pm 22 \%$, für Inga thibaudiana zwischen $\pm 18 \%$ und $39 \%$ und für Inga heterophylla zwischen $\pm 15 \%$ und $\pm 38 \%$.

Eine Abhängigkeit in der Fixierungsrate von der Jahreszeit war nicht zu erkennen. 
Die höchsten Standardfehler traten immer dann auf, wenn die Differenz zwischen der Leguminose und den Referenzpflanzen nicht groß genug war. Für Inga thibaudiana waren daher in den Monaten Januar, April und Juli und für Inga heterophylla im April die größten Standardfehler zu verzeichnen (vergl. Abb. 29). Der Standardfehler der Berechnungen für Abarema jupunba waren generell niedriger als die Berechnungen für die Inga-Arten. Bei dieser Art hoben sich die Monate Oktober und Dezember durch geringe Standardfehler hervor. In diesen Monaten war entweder die $\delta^{15} \mathrm{~N}$-Differenz Leguminose - Referenzpflanzen sehr groß oder die Streuung der $\delta^{15} \mathrm{~N}-$ Werte sehr gering.

Als Empfehlung für weitere Probenahmen sollte daher immer gelten, daß mehrere Proben von verschiedenen Arten gezogen und analysiert werden müssen, um eventuelle $\delta^{15} \mathrm{~N}$-Werte höher als die der Leguminose ausschließen zu können und eine genügend große Wertedifferenz zu garantieren. Eine Beprobung während der Trockenzeit scheint zuverlässigere Ergebnisse zu liefern, denn zu der Zeit sind die Differenzen Leguminosen zu Referenzpflanzen am größten. Für die Sekundärvegetation der Bragantina-Region kann mit der ${ }^{15}$ NNAM keine genauere Bestimmung der $\mathrm{N}_{2}$-Fixierungsleistung erreicht werden. Die Abweichungen von $\pm 20 \%$ müssen aufgrund der hohen Variabilität der $\delta^{15} \mathrm{~N}$-Werte innerhalb einer Art als Unsicherheit in Kauf genommen werden.

\section{Rolle der Biologischen $\mathbf{N}_{2}$-Fixierung in der Bragantina-Region}

\subsection{Infektionspotential eines Capoeirabodens}

Bisher liegen keine Informationen über die Knöllchenbildung von Leguminosen in der Sekundärvegetation der Bragantina-Region vor. Für den Südosten Brasiliens sind jedoch umfangreiche Untersuchungen von Faria et al. (1987 und 1989) durchgeführt worden. Sie fanden in den auch in der Bragantina-Region häufigen Gattungen wie Inga spp. und Pithecellobium spp. viele nodulierte Arten, jedoch immer an kleinen Pflanzen oder im Infektionsversuch im Gewächshaus, weniger an großen Bäumen. An Bäumen in tropischen Waldbeständen wurde bisher nur eine sehr spärliche Nodulation gefunden (Norris, 1969; Faria et al., 1984). Es wird angenommen, daß viele Arten potentiell fähig wären, Knöllchen auszubilden, jedoch durch die Nährstoffsituation (z.B. genügend N oder zu wenig Phosphor im Boden) oder allelopathische Substanzen an der Entwicklung einer Symbiose gehindert werden (Magalhães et al., 1982; Moreira et al, 1992). 
Die Brachevegetation befindet sich mit einem Alter bis circa 10 Jahren im Übergang von einer Buschvegetation zu einem Wald, so daß sich die Frage stellte, ob es in den ersten Jahren zur Ausbildung der Symbiose und damit zur Knöllchenbildung kommt. Aufgrund der bisher erarbeiteten $\delta^{15} \mathrm{~N}$-Ergebnisse kann man davon ausgehen, daß Baumleguminosen dieser Vegetationen Luftstickstoff binden. Die Untersuchung der Nodulation der Leguminosen könnte daher als eine Bestätigung dieser Ergebnisse dienen.

In 4-8jährigen Sekundärvegetationen wurde die Anzahl Knöllchen an den Wurzeln von Baumleguminosen ermittelt, indem um den Stamm herum die Erde ausgehoben und gesiebt wurde. Im Umkreis von $150 \mathrm{~cm}$ um den Hauptstamm wurde die Erde bis auf teilweise $1 \mathrm{~m}$ Tiefe auf Knöllchen abgesucht (Abb. 34).

Dabei wurde für die einzelnen Leguminosen unterschiedliche Knöllchenmengen gefunden. Allen gemeinsam war aber, daß in der organischen Auflage und den ersten $5 \mathrm{~cm}$ des Bodens die meisten Knöllchen zu finden waren. In 20-40cm Bodentiefe entwickelten sich kaum noch Knöllchen. In dieser Vegetation mit der höchsten Wurzeldichte in den obersten $20 \mathrm{~cm}$ war naturgemäß auch das höchste Rhizobium-Aufkommen im Oberboden zu finden.
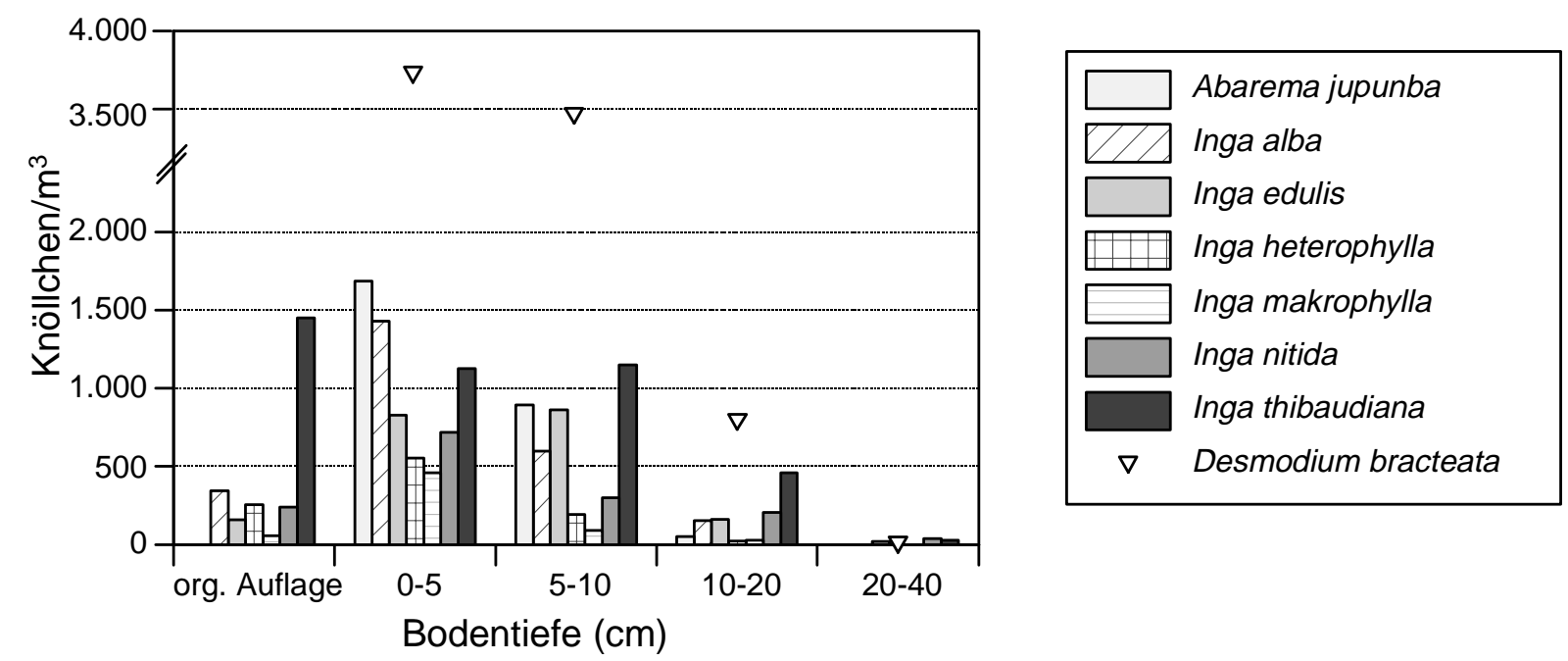

Abb. 34: Feldnodulation einiger nativer Baum-Leguminosen auf den Flächen M1,M2 und M5 in Abhängigkeit von der Bodentiefe.

Die Arten mit der stärksten Nodulation waren Inga thibaudiana, Inga alba und Abarema jupunba. Inga heterophylla und Inga macrophylla nodulierten hingegen nur schwach. In Abb. 34 wurde mit einem Dreiecksymbol auch die Knöllchenanzahl einer krautigen annuellen Leguminose, Desmodium bracteata, dargestellt. Desmodium bracteata zeichnete sich durch eine 
wesentlich höhere Knöllchenzahl gegenüber den Baumleguminosen aus (eine organische Auflage war im Falle dieser Leguminose nicht vorhanden, da sie in einer sechsmonatigen Sekundärvegetation mit niedrigem Deckungsgrad wuchs).

Viele Arten, wie z.B. Abarema jupunba, bildeten Wurzelausläufer dicht unter der Oberfläche, aus denen sie nach der Kulturphase als Wurzelsprosse wieder auswuchsen (Abb. 35). In anderen Beständen wurden große Baumstümpfe angetroffen, aus denen Abarema jupunba mit Hilfe von Adventivsprossen austrieb (Abb. 36). Inga thibaudiana hingegen ist für die gleichzeitige Ausbildung einer Pfahlwurzel und eines feineren horizontalen Wurzelsystems bekannt (pers. Mitt. Wiesenmüller). Dies könnte ein Grund für die ermittelte höhere Anzahl Knöllchen dieser Art sein.

Die Knöllchen verteilten sich häufig nesterweise an den Wurzelsträngen entlang der neu gebildeten Feinwurzeln. Obwohl darauf geachtet wurde, daß keine krautige Leguminose wie Desmodium bracteata in der näheren Umgebung wuchs, konnte in den meisten Fällen jedoch nicht mit absoluter Sicherheit bestimmt werden, ob die gefundenen Knöllchen von den Wurzeln der untersuchten Leguminose stammten. Aufgrund des Wurzelsystems von Abarema jupunba z.B. war es auch möglich, daß das Haupt-Feinwurzelsystem der jeweiligen Pflanze nicht direkt unterhalb und in der Nähe des Stammes gebildet wurde, sondern in einer größeren Entfernung an anderen Pflanzen der gleichen Art, die über die Wurzeln miteinander verbunden waren.

Die Knöllchenausbildung im Boden bis $1 \mathrm{~m}$ Tiefe an den untersuchten Sekundärwaldarten war insgesamt gesehen nicht sehr intensiv. Mit Hilfe eines Gewächshausversuches sollte daher die Information gewonnen werden, ob überhaupt ein hohes Vorkommen an Rhizobium in den Böden zu erwarten war und bis zu welcher Tiefe sie vorhanden waren. Dazu wurde ein Feldboden verschiedener Bodentiefen mit Vigna unguiculata bepflanzt. Die Anzahl Knöllchen an dieser „Fangpflanze“ pro 100g Boden wurde bestimmt und in dieser Arbeit als 'Infektionspotential' bezeichnet (Abb. 37). 

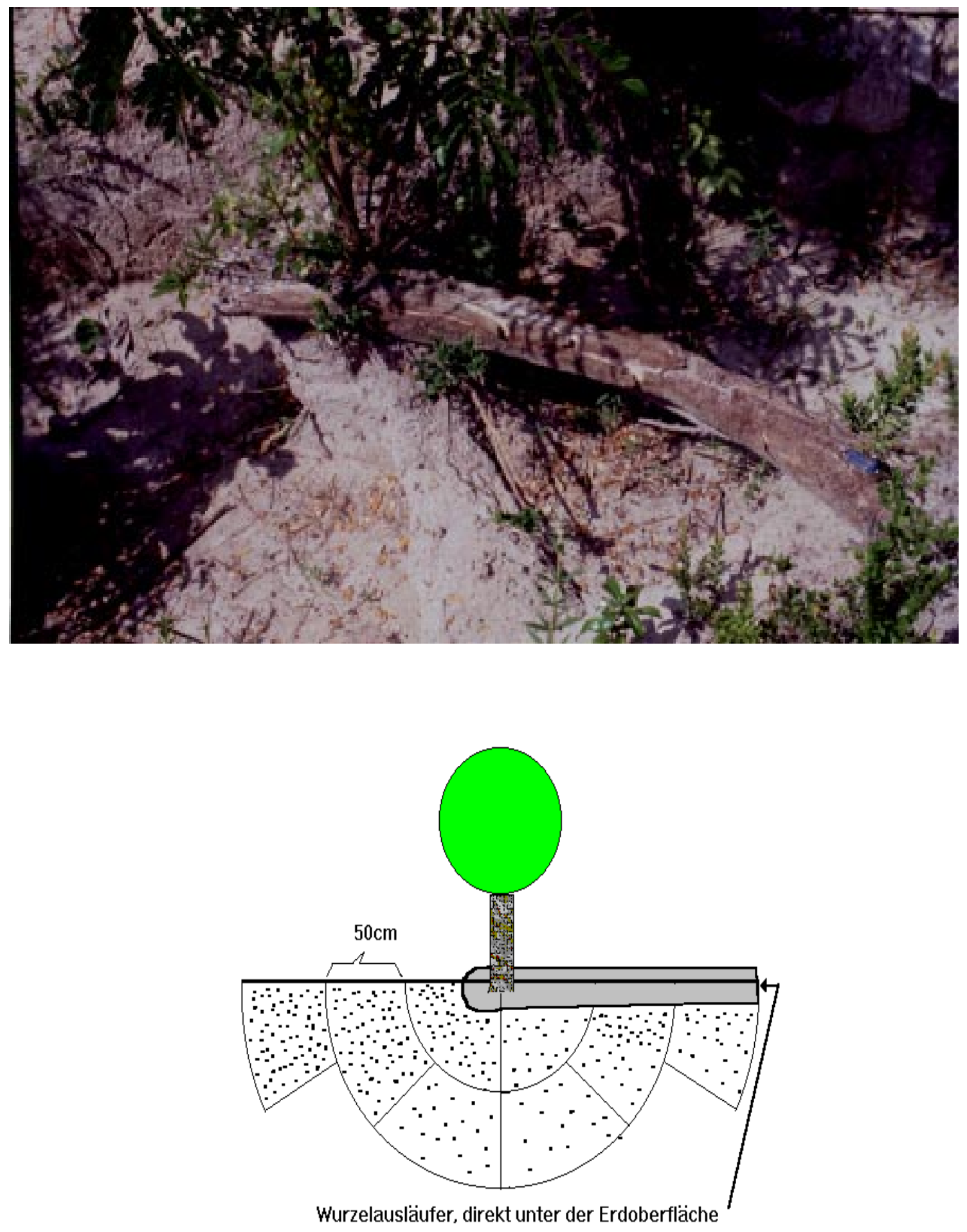

Abb. 35: Wurzelsystem und Knöllchenaufkommen (als Punkte eingezeichnet) von Abarema jupunba in $1 / 2$ jähriger Sekundärvegetation bis $40 \mathrm{~cm}$ Bodentiefe (Aufsicht auf den Boden im Halbkreis um den Stamm der Leguminose herum, Kantenlänge der Flächenuntereinheiten $=50 \mathrm{~cm}$ ) 

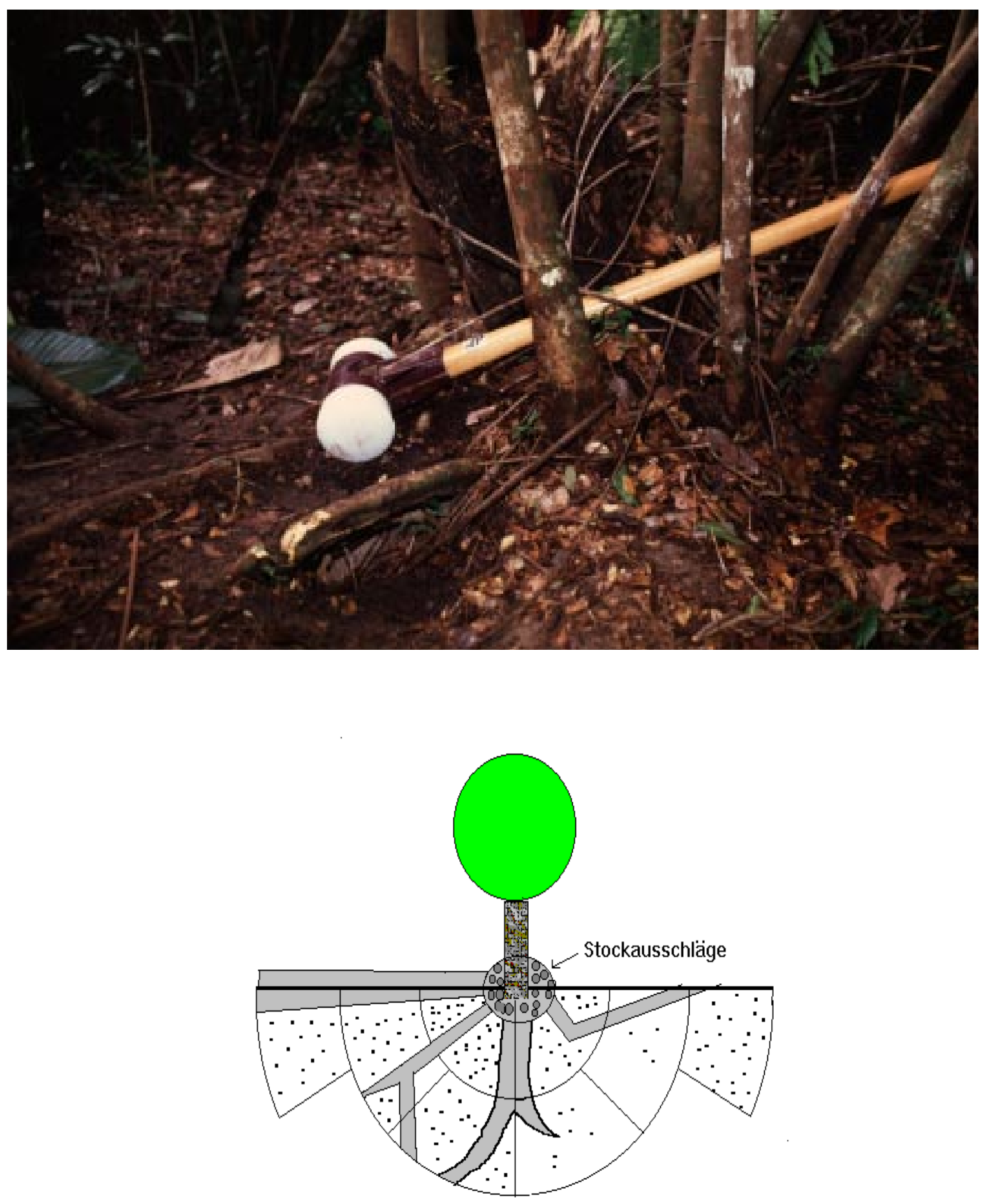

Abb. 36: Wurzelsystem und Knöllchenvorkommen von Abarema jupunba (als Punkte eingezeichnet) in 6jähriger Sekundärvegetation bis $40 \mathrm{~cm}$ Bodentiefe (Aufsicht auf den Boden im Halbkreis um den Stamm der Leguminose herum, Kantenlänge der Flächenuntereinheiten $=50 \mathrm{~cm}$ ) 
Das Infektionspotential war in den ersten $50 \mathrm{~cm}$ des Bodens mit 8.2 Knöllchen pro $100 \mathrm{~g}$ Boden am stärksten (Abb. 37, Ergebnisse des Wilcoxon-Rang-Testes im Anhang in Tab. 69). Schon in Boden aus 1m Tiefe war das Rhizobium-Aufkommen signifikant halbiert. In Boden aus 3m Tiefe war es auf unter 1 Knöllchen/100g Boden reduziert. Ein sehr kleines Infektionspotential blieb jedoch auch in $6 \mathrm{~m}$ Bodentiefe mit 0,9 Knöllchen/100g Boden bestehen. Trotz sorgfältiger Beprobung kann eine Kontamination während der Bodenbeprobung jedoch nicht ausgeschlossen werden, so daß zunächst nur von einem bemerkbaren Rhizobi$u m$-Vorkommen bis maximal 3m Bodentiefe ausgegangen werden kann.

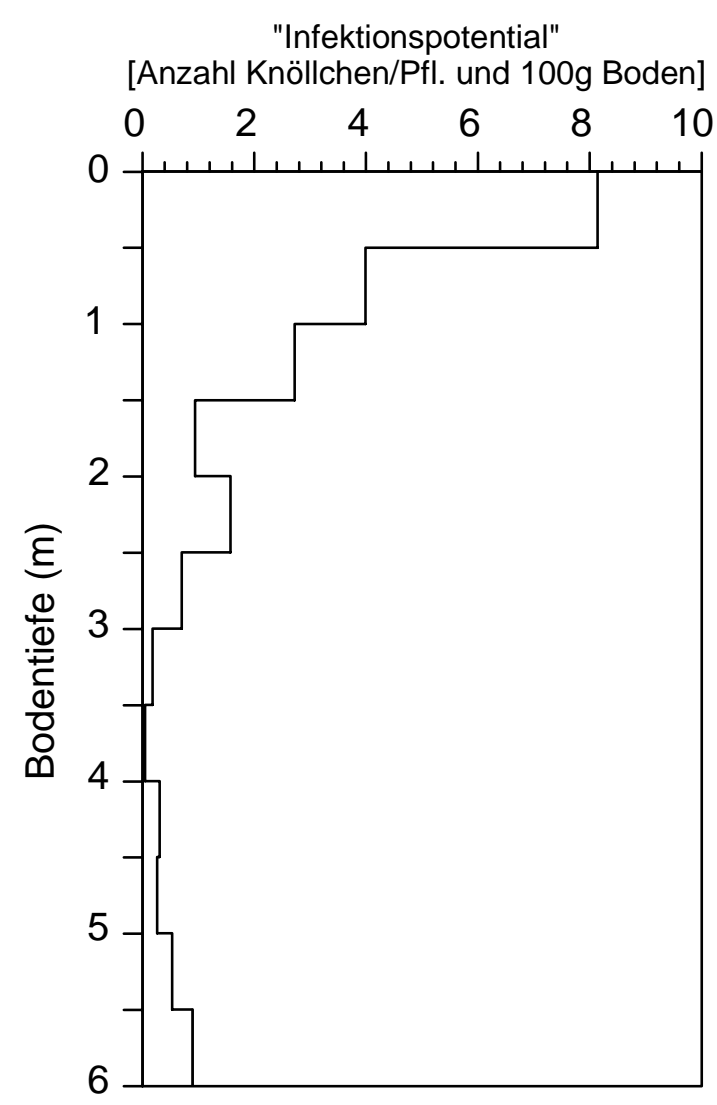

Abb. 37: Infektionspotential (Knöllchen an Vigna unguiculata in 100g Boden) im Bodenprofil von Fläche M1S2

In diesem Feldboden waren also Rhizobien bis in circa $3 \mathrm{~m}$ Bodentiefe vorhanden, die fähig waren, mit einer Fangpflanze (Vigna unguiculata) eine Symbiose zu bilden. Sowohl die Anzahl Knöllchen an den Bäumen im Feld als auch an Vigna unguiculata war jedoch nicht sehr hoch. Ob diese Symbiosen effektiv bzw. aktiv waren, kann an dieser Stelle nicht nachgewiesen werden. Wenn man die $\delta^{15} \mathrm{~N}$-Werte der Leguminosen, die häufig nahe $\delta^{15} \mathrm{~N}=0$ waren, als 
Maß für eine aktive BNF ansieht, so können das 'Infektionspotential' und die Anzahl Knöllchen an den Baumwurzeln als Bestätigung dieses Prozesses gelten.

\subsection{BNF in den verschiedenen Sukzessionsstadien der Entwicklung eines Tropenwaldes}

\subsubsection{Beziehung von Baumgröße und Fixierungsleistung}

Um eine Abschätzung der BNF einiger $\mathrm{N}_{2}$-fixierender Baumleguminosen durchzuführen, wurde die Idee entwickelt, mit Hilfe von Regressionsanalysen der Biomasse, der akkumulierten N-Menge in den oberirdischen Pflanzenteilen, des \%Ndfa und einem Maß für die Baumgröße dieser Art (hier $\pi^{*} r^{2}$, die Basalfläche) eine Berechnung des N-Inputs der Leguminosen pro Flächeneinheit zu versuchen. Dafür wurden verschieden große Individuen einer Art auf ihre Biomasse, akkumulierter N-Menge, Größenverhältnisse und Fixierungsraten (\%Ndfa) untersucht. Für die Berechnung des \%Ndfa wurde der mit dem Gesamt-N des jeweiligen Kompartiments gewichtete Mittelwert des $\delta^{15} \mathrm{~N}$ der jeweiligen Gesamtpflanze (oberirdische Pflanze) herangezogen. Mit diesen Daten wurden Funktionen erstellt, über die dann von der Basalfläche auf die Menge akkumulierten Stickstoffs in den oberirdischen Pflanzenteilen und die Fixierungsrate der Pflanze geschlossen werden konnte. Mit den Angaben zur Basalfläche und der Anzahl an Leguminosen eines Bestandes sollte dann auf die Menge fixierten Stickstoffs pro Flächeneinheit hochgerechnet werden.

Abarema jupunba ist eine häufig auftretende Art in der Sekundärvegetation, die für Untersuchungen der räumlichen und zeitlichen $\delta^{15} \mathrm{~N}$-Strukturen, sowie Fraktionierungen innerhalb einer Pflanze und als A-Pflanze untersucht wurde. Daher wurden auch diese Daten für Abarema jupunba erhoben und stellvertretend für fixierende Baumleguminosen der Sekundärvegetation zu den folgenden Berechnungen herangezogen (Abb. 38).

Begrenzender Faktor dieser Methode lag in der 3. Funktion (Abb. 38, 3A, 3B). Für diese Funktion war der Datensatz nicht groß genug. Es fehlten vor allem Werte für die Bäume mit einer Basalfläche von $10-14 \mathrm{~cm}^{2}$ und $>50 \mathrm{~cm}^{2}$ (siehe auch Tab. 70 im Anhang). Hier bleibt offen, ob die BNF mit zunehmendem Alter der Bäume konstant bleibt (sigmoide Kurve, 3A) oder ab einer noch fraglichen Größe wieder abnehmende Tendenzen annimmt (polynomische Funktion, Abb. 38 3B).

Daten von Nunez (1995) und Jorge (1994) wurden für die Berechnung der N-Akkumulation via BNF der Baumleguminosen in junge Sekundärwald-Bestände mit Hilfe der 3 Regressio- 
nen herangezogen. Ihre Biomasseerhebungen geben die genaue Größenverteilung der Leguminosen in verschieden alten Brachen an (siehe Tab. 71). Je nach Anwendung der sigmoiden oder polynomischen Funktion für die Berechnung des \%Ndfa steigerte sich der N-Input von 0 auf 21.2 bzw. $40.5 \mathrm{~kg} \mathrm{~N} \mathrm{ha}^{-1}$ in den ersten 10 Jahren der Brache (Tab. 47). Bis zu einer Basalfläche von $10 \mathrm{~cm}^{2}$ werden über die Funktion $3 b$ wesentlich höhere N-Mengen als über Funktion 3 a berechnet.
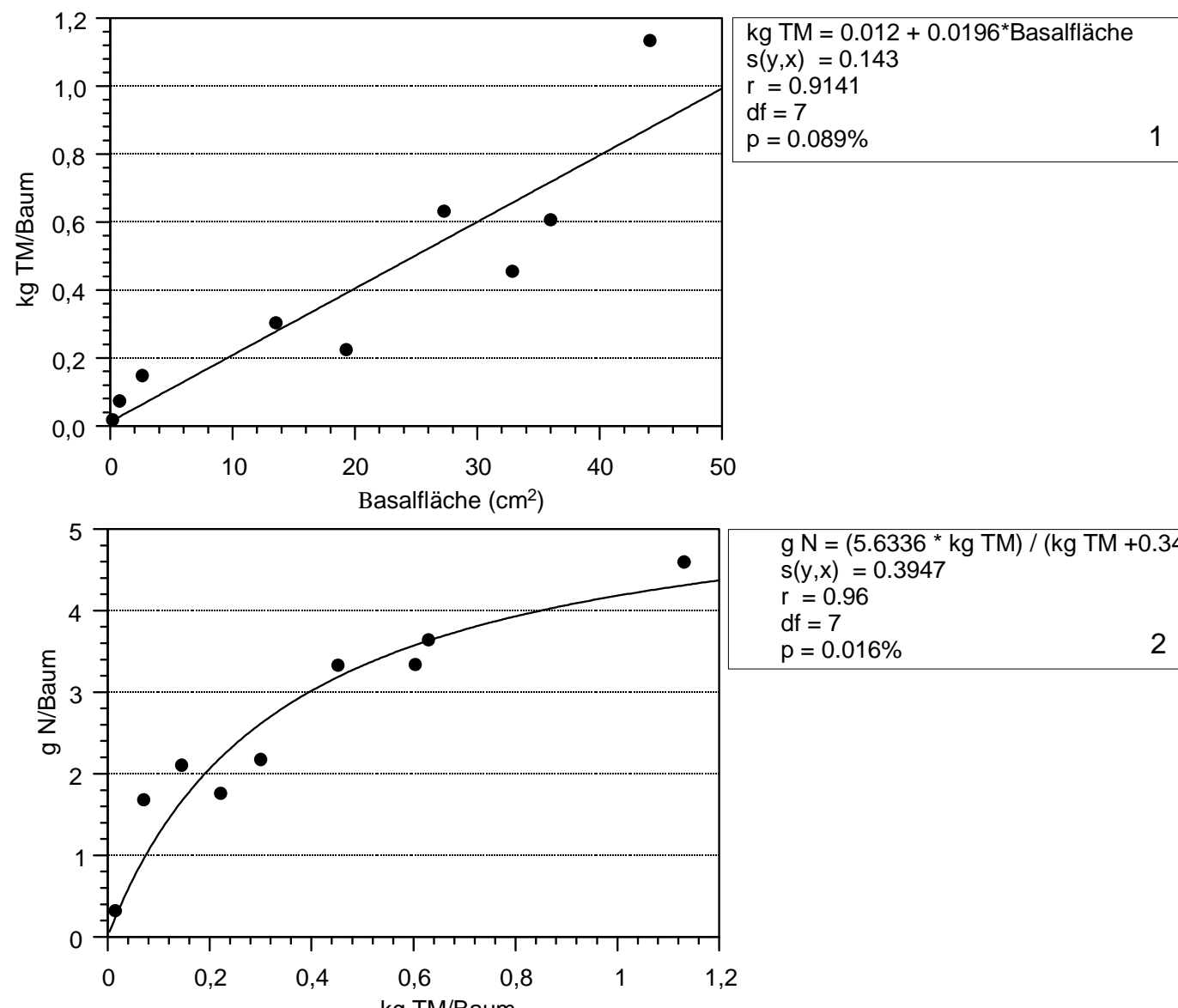

$\mathrm{g} \mathrm{N}=\left(5.6336{ }^{*} \mathrm{~kg} \mathrm{TM}\right) /(\mathrm{kg} \mathrm{TM}+0.347)$ $\mathrm{s}(\mathrm{y}, \mathrm{x})=0.3947$

$r=0.96$

$\mathrm{df}=7$

$\mathrm{p}=0.016 \%$

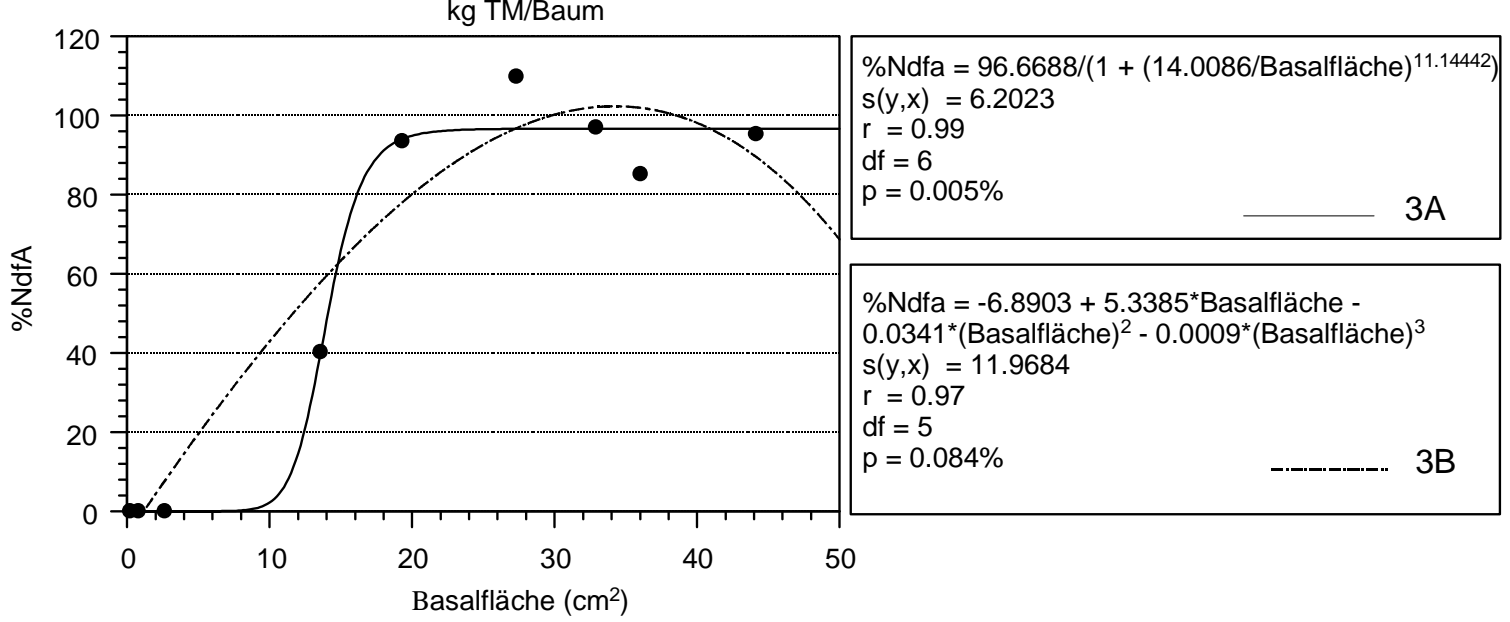

Abb. 38: Kurvenanpassung an die Biomassedaten von 9 verschieden großen Individuen der Abarema jupunba und der jeweiligen Fixierungsleistung in Abhängigkeit von der Basalfläche 
Für diese durch die kleinen Basalflächen repräsentierte anfängliche Wachstumsphase der Pflanzen ist es fraglich, ob die BNF erst ab einer bestimmten Pflanzengröße zugelassen wird oder aber sukzessive mit dem Wachstum zunimmt. Die hier beprobten Pflanzen wuchsen auf Böden, die durch P- und N-Mangel gekennzeichnet sind, die sich mit zunehmendem Alter des Bewuchses noch verstärken. In einem Düngeversuch einer neu wachsenden Sekundärvegetation zeigte Gehring (1997), daß das Wachstum von Inga macrophylla und Abarema jupunba lediglich durch Phosphor limitiert war. So erscheint eine BNF der hier untersuchten Jungpflanzen eher durch den permant vorhandenen P-Mangel als durch pflanzeninterne Regulationsmechanismen beeinflußt zu sein, die einen abrupten Anstieg der BNF verursachen könnten. Berechnungen der $\mathrm{N}_{2}$-Fixierungsleistung mit Hilfe der polynomischen Funktion $3 \mathrm{~b}$ sind daher für kleine Basalflächen wahrscheinlicher.

Die ermittelten Stickstoffmengen beziehen sich auf den bis zum Zeitpunkt der Messung akkumulierten Stickstoff. In Jahresraten ausgedrückt bedeutet der maximale Wert von $40.5 \mathrm{~kg} \mathrm{~N} \mathrm{ha}^{-1}$ jedoch nur einen N-Input von $4.05 \mathrm{~kg} \mathrm{~N} \mathrm{ha}^{-1} \mathrm{Jahr}^{-1}$, bei $24.1 \mathrm{~kg} \mathrm{~N} \mathrm{ha}^{-1} \mathrm{Jahr}^{-1}$ sogar nur $2.4 \mathrm{~kg} \mathrm{~N} \mathrm{ha}^{-1} \mathrm{Jahr}^{-1}$ (arithmet. Mittel).

Tab. 47: N-Akkumulation ( $\mathrm{kg} \mathrm{N} \mathrm{ha}^{-1}$ ) der Baumleguminosen (Subfamilie Mimosoideae und Papilionoideae) über ihre BNF in verschieden alten Sekundärvegetationen der Bragantina-Region. Zugrunde gelegt wurden Biomasseerhebungen und $\delta^{15} \mathrm{~N}$-Analysen von Abarema jupunba, stellvertretend für die Baumleguminosen, und Biomassedaten von Jorge (1993) und Nunez (1995) für drei verschieden alte Flächen.

\begin{tabular}{l|c|c|c}
\hline Formel & $1+2+3 \mathrm{a}$ & $1+2+3 \mathrm{~b}$ & Streuauflage \\
\hline Alter der Sekundärvegetation (Jahre) & \multicolumn{3}{|c}{$\mathrm{kg} \mathrm{N} \mathrm{ha}^{-1}$} \\
\hline 1 (Jorge) & 0.0 & 0.09 & 0.01 \\
7 (Nunez) & 8.5 & 23.2 & 1.0 \\
10 (Nunez) & 24.1 & 40.5 & 6.6 \\
\hline
\end{tabular}

$\mathrm{Zu}$ dieser N-Akkumulation muß der fixierte Stickstoff in der Streuauflage der Vegetationen addiert werden. Mit den Stickstoffreserven in der Streuauflage, dem prozentualen Anteil der Leguminosen an der oberirdischen Biomasse und der Voraussetzung der gleichen Größenverteilung in den von Nunez (1995) untersuchten Vegetationen beträgt der Anteil fixierten Stickstoff in der Streu $0.01 \mathrm{~kg} \mathrm{~N}^{-1}$ der 1 jährigen, $1 \mathrm{~kg} \mathrm{~N} \mathrm{ha}^{-1}$ der 7 jährigen und $6.6 \mathrm{~kg} \mathrm{~N} \mathrm{ha}^{-1}$ der 10jährigen Sekundärvegetation.

Über Funktion $3 b$ wurde für Pflanzen mit einer Basalfläche $>59 \mathrm{~cm}^{2}$ (sie kommt ab einem Vegetationsalter von 3-4 Jahren vor) keine $\mathrm{N}_{2}$-Fixierung mehr berechnet. Wäre diese Funktion die maßgebende, so würden Vegetationen mit Bäumen hauptsächlich größeren Durchmessers 
- den Unterwuchs nicht mitrechnend - keinen oder nur einen sehr geringen N-Input über BNF erbringen, denn die Anzahl fixierender kleinerer Bäume nähme mit der Anzahl größerer nicht fixierender Bäume ab. Dies zöge als logische Konsequenz nach sich, daß die Sekundärvegetation mit zunehmendem Alter aufgrund der Zusammensetzung mit den verschiedenen Baumgrößen, also dem Alter der Bäume, nicht mehr $\mathrm{N}_{2}$ fixierten.

Geht man jedoch davon aus, daß dies nicht der Fall ist, und die $\mathrm{N}_{2}$-Fixierung nicht über den Alterungsprozeß der Bäume sondern über systeminterne Regulationsmechanismen wie Konkurrenzfaktoren, Beschattung, Allelopathie etc. gesteuert wird, muß mit der sigmoiden Funktion zur Ermittlung des Stickstoff-Inputs weitergearbeitet werden.

\subsubsection{N-Akkumulation über BNF in verschieden alten Sekundärvegetationen und Pri- märwäldern}

Um einen Eindruck von der Bedeutung der $\mathrm{N}_{2}$-Fixierung in älteren Vegetationen in Bezug zu den früheren Sukzessionsstadien der Brachevegetation zu bekommen, wurden zusätzlich Daten von Saldarriaga et al. (1988) und Vieira (1996) als Grundlage für eine Hochrechnung der $\mathrm{N}$-Akkumulation der biologischen $\mathrm{N}_{2}$-Fixierung von verschiedenen, älteren Stadien der Vegetation bis hin zum Primärwald genommen. Die dazu verwendeten Daten und ihre Umrechnung für die Eingabe in die Funktionen (Abb. 38) befinden sich im Anhang in Tab. 70. Diese Kalkulationen können jedoch nur als Schätzung angesehen werden, da die genauen Angaben zur Verteilung der Durchmesserklassen unterhalb von $10 \mathrm{~cm}$ fehlten. Vieira (1996) nannte für Peixe Boi die Anzahl der Individuen mit kleineren Durchmessern gar nicht, während für die Rio Negro-Region nur die Aufschlüsselung der Verteilung für Durchmesser unter $10 \mathrm{~cm}$ fehlte. Für letztere wurde die gleiche Verteilung der Basalflächen wie bei Nunez (1995) angenommen. Die Aufteilung der Basalflächen in Klassen > 10cm wurde in Anlehnung an die von Vieira (1996) gefundenen vorgenommen. Die Daten von Vieira (1996) selbst wurden nicht weiter ergänzt.

Die Daten der Vegetationen in der Umgebung von Igarapé Açu und Peixe Boi und der Rio Negro Region zeigten eine N-Akkumulation über die BNF, die mit den verschiedenen Sukzessionsstadien variierte (Abb. 39).

In der Bragantina-Region akkumulierte der biologisch fixierte Stickstoff bis zu einem Vegetationsalter von 10 Jahren auf 24 bis $40 \mathrm{~kg} \mathrm{~N} \mathrm{ha}^{-1}$, während eine gleich alte Vegetation in der

Rio Negro-Region nur knapp $8 \mathrm{~kg} \mathrm{~N} \mathrm{ha}^{-1}$ fixierte. In Vegetationen mit einem Alter über 20 
Jahren nahm die fixierte Stickstoffmenge sukzessive mit dem Alter ab. Mit Erreichen des Vegetationsalter von 40 Jahren wurden nur noch $5 \mathrm{~kg} \mathrm{~N} \mathrm{ha}^{-1}$ aus der BNF berechnet.

$\mathrm{Zu}$ berücksichtigen ist jedoch, daß für Peixe Boi keine Daten über die Individuenzahl in den Basalflächenklassen $<5 \mathrm{~cm}$ vorlagen und somit nicht einbezogen werden konnten. Mit diesen für die BNF sehr entscheidenden Bäumen läge die fixierte N-Menge eher auf dem gleichen Niveau wie in Igarapé Açu. Die niedrige Menge in der 10jährigen Rio Negro-Vegetation kann auch auf die Vegetationsdichte zurückgeführt werden. Während Nunez (1995) fast 11330 Leguminosen auf einem ha vorfand, waren es bei Saldarriaga et al. (1988) nur knapp 5770 Leguminosen.

Eine weitere Einschränkung der hier vorgenommenen Abschätzungen durch eine fehlerhafte Annahme der Größenzusammensetzung innerhalb der Leguminosae muß in Betracht gezogen werden. Da keine direkte Größenzusammensetzung speziell für diese Familie in den Veröffentlichungen von Saldarriaga et al. (1988) und Vieira (1996) angegeben waren, wurde die Größenzusammensetzung der gesamten Vegetation zu Grunde gelegt. Es ist aber durchaus möglich, daß die Größenzusammensetzung Pflanzenfamilien-spezifisch ist und sich mit dem Alter der Vegetationen verändert hat. Daher kann es unabhängig von der angewendeten Formel zu einer Über- oder Unterschätzung der akkumulierten Menge fixierten Stickstoffs gekommen sein. Informationen bezüglich dieses Faktors lagen jedoch auch für andere Vegetations- und Biomasseaufnahmen nicht vor.

Die bisher vorgenommene Abschätzung der fixierten N-Menge bezog sich nur auf die vitale oberirdische Biomasse. Von Sanginga et al. (1990) wurde der Gesamt-Stickstoffanteil an der unterirdischen Biomasse von Leucaena-Pflanzen auf über $60 \%$ bestimmt; kalkuliert mit Daten von Nunez (1996) kann von 25\% der Gesamtbiomasse einer Sekundärvegetation ausgegangen werden. Sanchez (1982) geht von einem jährlichen Gesamt-N-Eintrag von $106 \mathrm{~kg}$ über den Streufall in eine Primärvegetation aus. Legt man dieselbe Größenverteilung wie für die Berechnung der über BNF akkumulierten N-Menge in der oberirdischen Biomasse der Vegetationen in Peixe Boi und am Rio Negro zu Grunde, müssen zusätzliche jährliche Raten von 1.5 bis $2.5 \mathrm{~kg}$ fixierten $\mathrm{N} \mathrm{ha}^{-1}$ in den Vegetationen bis 20 Jahre und 10 bis $14 \mathrm{~kg}$ fixiertem $\mathrm{N} \mathrm{ha}{ }^{-1}$ in Vegetationen über 40 Jahre einbezogen werden. Damit entstammte etwa 10\% des von Sanchez et al. (1982) kalkulierten N-Eintrags über den Streufall aus biologisch fixiertem Stickstoff. In einer Primärvegetation (entsprechendes Alter circa 200 Jahre) wären so mit den Jahren 2000 bis $2500 \mathrm{~kg}$ biologisch fixierter $\mathrm{N} \mathrm{ha}^{-1}$ in das System über den Streufall eingetragen worden. 


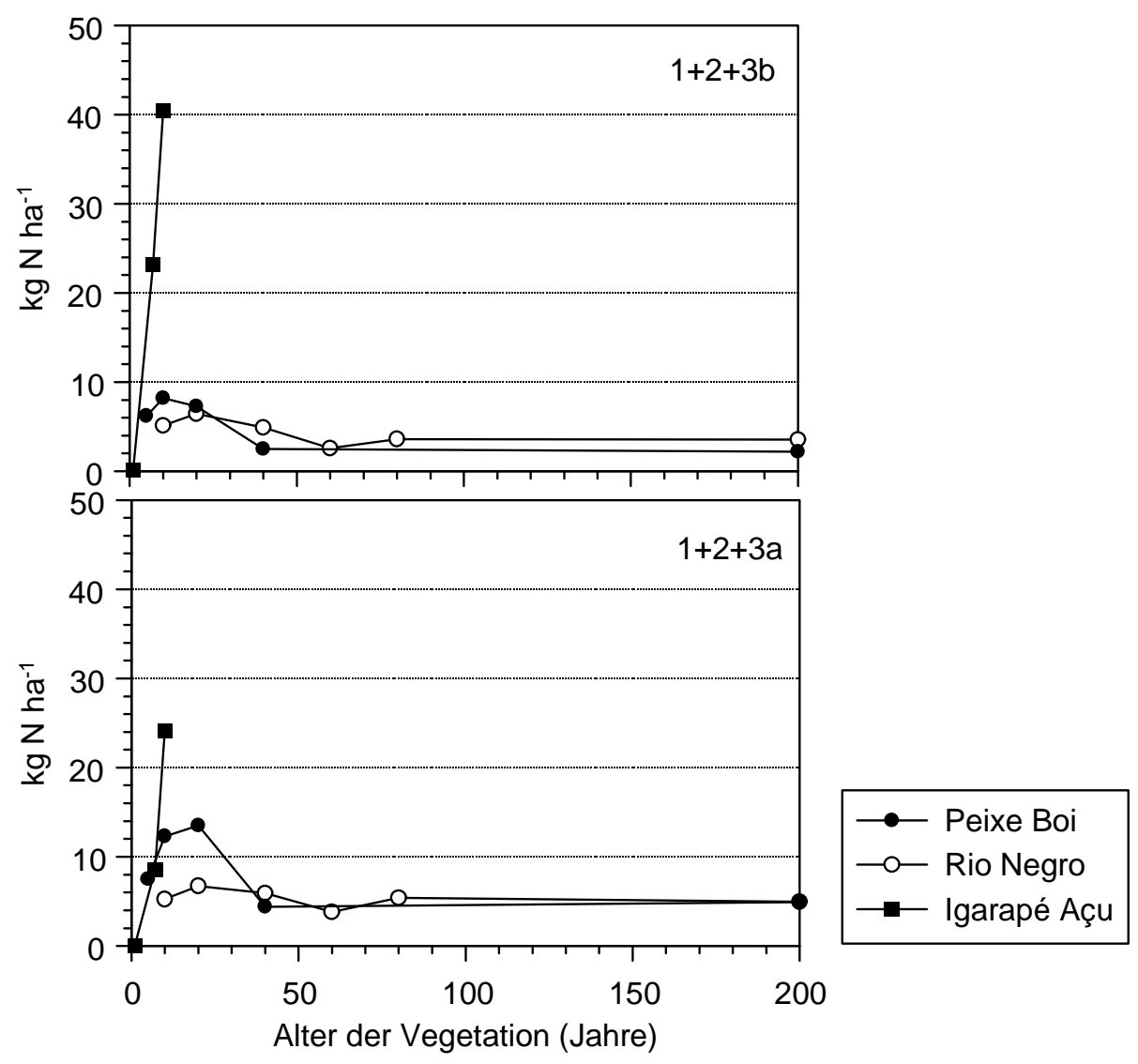

Abb. 39: Fixierter Stickstoff $\left(\mathrm{kg} \mathrm{N} \mathrm{ha}^{-1}\right)$ in den Baumleguminosen eines Bestandes in verschiedenen Sukzessionsstadien der Sekundärvegetation. Berechnungen für die Rio Negro-Region mit Daten von Saldarriaga et al. (1988), die für Peixe Boi mit Daten von Vieira (1996), die Daten aus Igarapé Açu entstammen dieser Arbeit bzw. aus Daten von Nunez (1995) und Jorge (1993)

\subsubsection{Vergleich der $\delta^{15} \mathrm{~N}$-Werte von Primärwald und Sekundärwald}

Ein Luft-N-Eintrag von mehr als $2000 \mathrm{~kg} \mathrm{~N} \mathrm{ha}^{-1}$, wie er für die Streu einer Primärvegetation geschätzt wurde, muß aber Konsequenzen für die Verteilung von ${ }^{15} \mathrm{~N}$ im System nach sich ziehen. Muß man hingegen davon ausgehen, daß systeminterne Regulationsmechanismen die BNF der Bäume steuern und nach circa 20 Jahren aufgrund genügenden N-Angebotes, P-Mangel oder Lichtmangel etc. zum Erliegen kommt, würde der N-Input via BNF über den Streufall lediglich 30 bis $50 \mathrm{~kg} \mathrm{~N} \mathrm{ha}^{-1}$ ausgemacht und keinen Einfluß auf den $\delta^{15} \mathrm{~N}$-Wert in Vegetation und Boden haben.

Eine vergleichende Betrachtung der $\delta^{15} \mathrm{~N}-$ Werte der untersuchten Vegetationen zeigt den $\mathrm{Zu}$ sammenhang der ${ }^{15} \mathrm{~N}$-Verteilung mit ihrer Vorgeschichte durch anthropogener Eingriffe und ihre Quantität (Abb. 40). Sowohl in den Böden, der organischen Auflage, den Blättern der Leguminosen und Nichtleguminosen zeigte sich eine abnehmende Tendenz in den 
$\delta^{15} \mathrm{~N}$-Werten von der Primärvegetation zur jungen Sekundärvegetation, wobei die ältere Sekundärvegetation eine Zwischenposition einnahm.

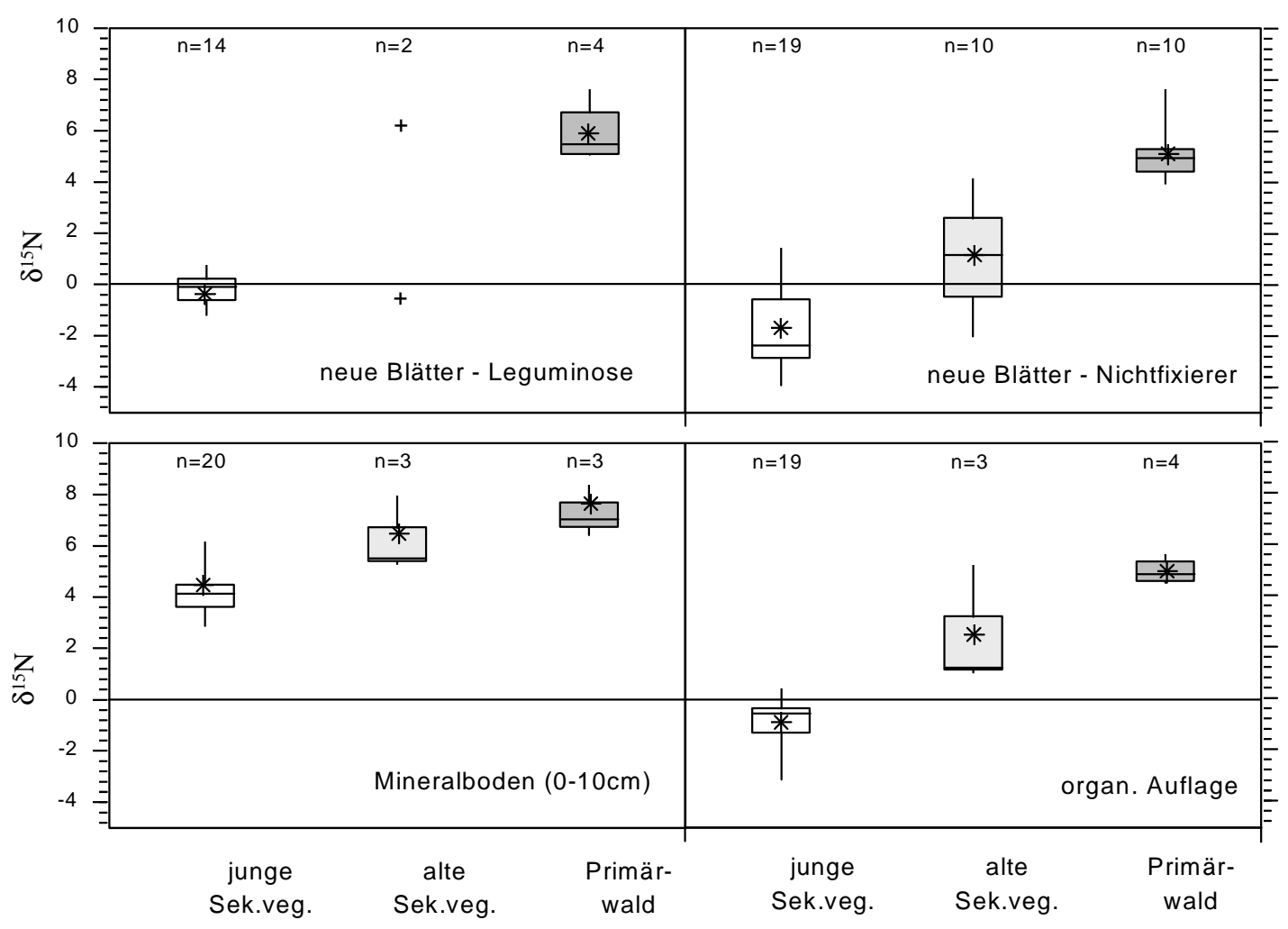

Abb. 40: $\delta^{15} \mathrm{~N}$ des Mineralbodens, der organischen Auflage, neue Blätter von Leguminosen und nicht fixierenden Bäumen in junger und alter Sekundärvegetation und Primärwald (die Box-Whisker-Plots geben Minimum, Maximum, oberes und unteres Quartil, Median und Mittelwert=* an)

Die $\delta^{15} \mathrm{~N}$-Werte zeigten in den Blättern eine klare Abnahme von +4 bis +8 im Primärwald auf -4 bis +2 in der jungen Sekundärvegetation. Im Mineralboden bildete sich dieser Trend am wenigsten deutlich aus $\left(\delta^{15} \mathrm{~N}=+7.5\right.$ auf durchschnittlich +5$)$. Die $\delta^{15} \mathrm{~N}$-Werte der organischen Auflage lagen zwischen denen der Vegetation und des Bodens.

Schlüsselposition in der Weitergabe der $\delta^{15} \mathrm{~N}$-Werte nimmt die organische Auflage ein. Ihre Isotopenzusammensetzung verändert sich mit dem Grad ihres Abbaus immer mehr von der ursprünglichen im Blatt zu der in der stabilen organischen Substanz vorhandenen. Diese Tendenz konnte in 4 der 5 untersuchten jungen Sekundärvegetationen am deutlichsten verfolgt werden (vergl. Tab. 34). Der dabei freigesetzte Stickstoff hat $\delta^{15} \mathrm{~N}$-Werte unter dem Niveau der Blattwerte, die in der jungen Sekundärvegetation negativ und in der Primärvegetation positiv waren. Die verbliebene organische Substanz kann so den $\delta^{15} \mathrm{~N}-\mathrm{Wert}$ des $\mathrm{N}_{\text {tot. }}$ des Bodens über ihre ${ }^{15} \mathrm{~N}$-Anreicherung dominieren. So scheinen die Isotopenverhältnisse, die über die 
BNF sowie die Fraktionierungsprozesse bei der Nitrifikation und Immobilisierung geschaffen wurden, in den Stickstoffpool weitergegeben zu werden und so über lange Jahre auch den $\delta^{15} \mathrm{~N}$-Wert des $\mathrm{N}_{\text {tot }}$ des Oberbodens zu beeinflussen.

Die Leguminosen zeigten im Primärwald ebenso hohe $\delta^{15} \mathrm{~N}$-Werte wie die Nichtfixierer, aber schon in der benachbarten alten Sekundärvegetation waren die gleichen Arten auch mit $\delta^{15} \mathrm{~N}$ um 0 nachzuweisen. Die $\delta^{15} \mathrm{~N}$-Werte implizieren, daß die Baumleguminosen in dem untersuchten terra firme-Primärwald nicht fixierten, aber in der Sekundärvegetation wohl. Der hohe $\delta^{15} \mathrm{~N}$-Wert legt die Vermutung nahe, daß die BNF der Baumleguminosen und die Akkumulation fixierten Stickstoffs über den Streufall in älteren Vegetationen vernachlässigbar ist, denn die oben vorgeschlagene N-Menge von mehr als $2000 \mathrm{~kg} \mathrm{~N}$ pro ha war nicht in entsprechend niedrigeren $\delta^{15} \mathrm{~N}$-Werten wiederzufinden. 


\section{Diskussion}

\section{Die biologische $\mathrm{N}_{2}$-Fixierung in der Bragantina-Region}

Berechnungen der biologisch fixierten N-Menge, die über die Baumleguminosen in junge Sekundärvegetationen der Bragantina-Region eingebracht wurden, ergaben eine in 10 Jahren maximale $\mathrm{N}$-Akkumulation von circa $40 \mathrm{~kg} \mathrm{~N} \mathrm{ha}^{-1}$ in der oberirdischen Biomasse zuzüglich circa $7 \mathrm{~kg} \mathrm{~N} \mathrm{ha}^{-1}$ in der Streuauflage. Dies entspräche einer jährlichen Rate von etwa $5 \mathrm{~kg} \mathrm{~N} \mathrm{ha}^{-1}$. In etwa vergleichbare Mengen der N-Akkumulation über die BNF wurden mit Hilfe der Daten von Vieira (1996) für weitere Flächen der Bragantina-Region ermittelt. Vegetationen der Rio Negro-Region (Daten von Saldarriaga et al., 1988) erbrachten hingegen einen maximalen $\mathrm{N}$-Akkumulation von $14 \mathrm{~kg} \mathrm{~N} \mathrm{ha}^{-1}$ in einer 20jährigen Sekundärvegetation. Bei diesen Bestandesaufnahmen können die N-Mengen, die durch Auswaschung der Blätter und der Streuauflage entzogen werden, nicht mit einbezogen werden. Allen Berechnungen gemeinsam ist, daß der über die BNF der Bäume akkumulierte $\mathrm{N}$ bis zu einem Vegetationsalter von circa 20 Jahren zunimmt, um dann mit dem Alter der Sukzession auf ein residuales Niveau von maximal $5 \mathrm{~kg} \mathrm{~N} \mathrm{ha}^{-1} \mathrm{zu}$ fallen. Im Vergleich $\mathrm{zu}$ den $\delta^{15} \mathrm{~N}$-Werten in den Vegetationen muß davon ausgegangen werden, daß die BNF der Bäume ab einem Vegetationsalter von etwa 20 Jahren generell eingestellt wurde.

Die geringe Menge an fixiertem Stickstoff in der alten Sekundärvegetation und dem Primärwald können einerseits auf die Größenzusammensetzung der Bäume zurückgeführt werden (vgl. Kap. VI.7.2.1). Mit dem Alter der Vegetation nimmt auch die Anzahl Bäume auf einem Hektar ab. Von einer gewissen Baumgröße an, verändert sich die individuelle jährlich akkumulierte N-Menge jedoch nicht mehr. Auf diese Weise wird eine bestimmte Menge fixierten Stickstoffs nicht überschritten. $\mathrm{N}_{2}$-fixierende Bäume benötigen eine hohe Lichtintensität (Izaguirre-Mayoral, 1995). Die in Peixe Boi nicht berücksichtigten Basalflächenklassen würden dem Unterwuchs angehören, der durch die größeren Bäume beschattet wäre. Daher ist es fraglich, ob und wieviel Stickstoff überhaupt über diese kleineren Bäume fixiert wurde und wie hoch daher der Fehler in der Berechnung einzuschätzen ist.

Andererseits kann die Nährstoffversorgung des Bodens älterer Wälder Einfluß auf die $\mathrm{N}_{2}$-Fixierungsaktivitäten der Bäume nehmen. In Plantagen geht die $\mathrm{N}_{2}$-Fixierungsleistung der Leguminosen häufig mit zunehmendem Alter zurück. So stellten Kessel et al. (1994) in ihren Leucaena-Pflanzungen schon im Laufe von 6 Jahren eine Abnahme der Fixierungsleistung 
fest, Parrotta et al. (1996) fanden diesen Trend sogar nach 3 Jahren. Kessel et al. (1994) und Parrotta et al. (1996) sind sich jedoch nicht einig darüber, ob dies auf die zunehmende Anreicherung von $\mathrm{N}$ im System durch die BNF oder durch absterbendes pflanzliches Material zurückzuführen ist, das mit dem Alter der Plantage zunehmend anfällt. Für die Baumleguminosen Inga jinicuil und Leucaena leucocephala fand Kessel (1983) einen Zusammenhang mit dem N-Angebot. Hansen und Pate (1987) zeigten an Acacia-Arten, daß die Fixierungsleistung dieser Bäume mit zunehmendem N-Angebot abnimmt. Mit dem Alter der Vegetation war in den Untersuchungen von Kessel et al. (1994) jedoch auch eine Abnahme des $\delta^{15} \mathrm{~N}$ Wertes zu verzeichnen, also eine Veränderung des $\delta^{15} \mathrm{~N}$-Wertes durch den Streueintrag der Leguminosen, der ${ }^{15} \mathrm{~N}$-verarmt war. Der Vergleich der $\delta^{15} \mathrm{~N}$-Werte der Sekundärvegetation und Primärvegetation in Peixe Boi machte deutlich, daß keine BNF im Primärwald, wohl aber im Sekundärwald ablief, denn die $\delta^{15} \mathrm{~N}$-Werte des pflanzenverfügbaren $\mathrm{N}$ (von den hohen $\delta^{15} \mathrm{~N}-$ Werten der nicht-fixierenden Pflanzen geschlossen) waren auf einem hohen Niveau. Bei dem Vergleich eines Regenwaldes mit einer subhumiden Feuchtsavannenvegetation, der die Nährstoffe ebenfalls durch Brand entzogen wurden, ermittelten Högberg und Alexander (1995) ähnliche Daten wie in der vorliegenden Arbeit. Im Primärwald konnten sie ausschließlich hohe $\delta^{15} \mathrm{~N}$-Werte sowohl für Fixierer als auch Nichtfixierer feststellen, welche in der Feuchsavannenvegetation jedoch insgesamt niedriger und für Leguminosen nahe 0 waren. Sie machten unter anderem den im dortigen Primärwald üblichen P-Mangel für das Ausbleiben der BNF verantwortlich.

Salati et al. (1982) verglichen verschiedene Primärvegetationen im Amazonasgebiet miteinander. Sie fanden auf mit Nährstoffen besser versorgten Böden gleichbleibend positive $\delta^{15} \mathrm{~N}$ Werte in der gesamten Vegetation, während die Leguminosen auf sandigen, nährstoffarmen Böden Werte nahe 0 annahmen. Dies impliziert eine BNF auch in alten Vegetationen unabhängig von der Größe der Bäume und weist darauf hin, daß systeminterne Regulationsmechanismen die BNF der Baumleguminosen steuern könnten.

Wälder akkumulieren Nährstoffe mit ihrem Wachstum, was sich auf Dauer in der Vegetation widerspiegelt (Sanchez und Palm, 1996). Sowohl im Boden als auch in der Vegetation der Primärwaldfläche, die noch nie gebrannt worden ist, sind höhere N-Gehalte angetroffen worden als auf den Sekundärwaldflächen. So erscheint der Primärwald mit genügend N versorgt und die BNF der Bäume wird unterdrückt. 
Faktoren, wie die Beschattung durch das Kronendach, ungünstige Bodenverhältnisse wie PMangel, fehlende Mikronährstoffe, hohe Al-Konzentrationen oder niedriger pH-Wert und allelopathische Effekte können sich ungünstig auf die Nodulation und Fixierungsaktivitäten einer Leguminose auswirken. Diese Faktoren sind im Rahmen des Projektes nicht eingehend untersucht worden. Sie sollten aber bei der weiteren Verfolgung der $\delta^{15} \mathrm{~N}$-Werte als Indikator für BNF in Primärvegetationen auf einen Zusammenhang mit den Isotopenverhältnissen und der $\mathrm{N}_{2}$-Fixierung hin untersucht werden. Sie könnten auch z.T. Ursache für die doch insgesamt recht geringen Fixierungsleistungen der Leguminosen in der Sekundärvegetation sein.

Für die Primärvegetation des Amazonasgebietes liegen einige Abschätzungen der BNF vor, die in den meisten Fällen jedoch nicht die Baumleguminosen betreffen (vgl. Tab. 1). Herrera et al. (1981) ermittelten eine BNF von 35-200 kg N ha ${ }^{-1} \mathrm{Jahr}^{-1}$ über freilebende Bakterien und Flechten auf verrottendem Holz und Rinde, in der Phyllosphäre und dem Boden eines sehr nährstoffarmen Caatinga-Waldes. Jordan et al. (1982) schätzten diesen Beitrag von Bakterien und Flechten in einem relativ gut versorgten terra firme-Wald hingegen auf nur $16 \mathrm{~kg} \mathrm{~N}$ $\mathrm{ha}^{-1} \mathrm{Jahr}^{-1}$. Sylvester-Bradley et al. (1980) kamen auf Fixierungsraten von bis zu $240 \mathrm{~kg} \mathrm{ha}^{-1}$ $\mathrm{Jahr}^{-1}$ in Primärvegetationen des Amazonasgebietes, die sie hauptsächlich den im Boden freilebenden und Rhizosphären-Bakterien zuschrieben. Schätzungen der BNF in der Phyllosphäre liegen zwischen $0.1 \mathrm{~kg} \mathrm{~N} \mathrm{ha}^{-1} \mathrm{Jahr}^{-1}$ (Carpenter, 1992) und $600 \mathrm{~kg} \mathrm{~N} \mathrm{ha}^{-1} \mathrm{Jahr}^{-1}$ (Paul, 1978, Ruinen, 1974). Diese Abschätzungen basieren auf Hochrechnungen von Inkubationsversuchen, Felderhebungen und indirekten Messungen der BNF der Bakterien (z.B. mit der Acethylen-Reduktions-Methode). Daher müssen diese Daten sehr kritisch betrachtet werden. Stewart et al. (1995) fanden bei der Untersuchung von Epiphyten in den Baumkronen verschiedener Primärwälder allerdings einen niedrigen $\delta^{15} \mathrm{~N}$-Wert, der einen indirekten Hinweis auf BNF in der Baumkrone über freilebende Bakterien und Flechten lieferte.

Welche Rolle die freilebenden $\mathrm{N}_{2}$-Fixierer in der Sekundärvegetation der Bragantina-Region einnehmen, kann an dieser Stelle nicht beantwortet werden. Wie schon zuvor erläutert, wird ein großes Potential an $\mathrm{N}_{2}$-fixierenden Bakterien der Phyllosphäre zerstört, andererseits wird die BNF der bodenbürtigen Bakterien hoch eingeschätzt. In 5 von 8 untersuchten Waldböden der Amazonasregion konnten Piccolo et al. (1996) deutlich höhere $\delta^{15} \mathrm{~N}$-Werte finden als in benachbarten Weideböden. Ihre Erklärung dafür suchten sie in der BNF freilebender Bakterien, die mit Weidegräsern assoziiert sind (Miranda und Boddey, 1987; Boddey und Döbereiner, 1988) und durch die Anhebung des pH-Wertes und bessere Phosphor-Verfügbarkeit nach 
dem die Weide vorbereitenden Brand gefördert wurden. Die $\mathrm{N}_{2}$-Fixierung durch bodenbürtige freilebende Bakterien und Flechten fanden Jordan et al. (1982) in einem durch slash and burn bearbeiteten Standort im Amazonasgebiet jedoch nicht wieder. Jordan (1989) vermutet, daß die $\mathrm{N}_{2}$-fixierenden Bodenmikrofauna, Bakterien und Flechten durch den Brand zerstört wurde. Vasquez et al. (1993) zeigten eine starke Reduktion autotropher $\mathrm{N}_{2}$-fixierender Bakterien in einem Boden nach Vegetationsbränden in Spanien. Andererseits konnten Piccolo et al. (1996) in 5 von 8 untersuchten Waldböden der Amazonasregion deutlich höhere $\delta^{15} \mathrm{~N}$-Werte finden als in benachbarten Weideböden. Ihre Erklärung dafür suchten sie in der BNF freilebender Bakterien, die mit Weidegräsern assoziiert sind (Miranda und Boddey, 1987; Boddey und Döbereiner, 1988) und durch die Anhebung des pH-Wertes und bessere PhosphorVerfügbarkeit nach dem die Weide vorbereitenden Brand gefördert wurden.

Die BNF auf Rinden, Blättern und im Boden der Primärvegetation kann also eine große Bedeutung haben, die durch den Vegetationsbrand stark herabgesetzt wird. Die neu wachsende Sekundärvegetation wird aber erst nach vielen Jahren wieder genügend Oberfläche (Phyllosphäre, Borken, abgestorbenes Holz etc.) bieten, auf der die nicht bodenbürtigen freilebenden $\mathrm{N}_{2}$-Fixierer bemerkbare Mengen Luftstickstoff binden können.

Hier kann die symbiontische $\mathrm{N}_{2}$-Fixierung der Baumleguminosen einen Beitrag zum NKreislauf leisten, denn die Symbiose in den Wurzeln der Pflanzen wird durch den Brand kaum beeinträchtigt. Das Wurzelsystem der Leguminosen ermöglicht einen schnellen Wiederaustrieb der Bäume und sorgt damit für die Nachlieferung von Nährstoffen für die Symbiose, die sich so schnell regenerieren kann. Pflanzen, die $\mathrm{N}_{2}$ fixieren, benötigen viel Energie, also Phosphor und eine hohe Lichtintensität (Izaguirre-Mayoral et al., 1995). In der Primärvegetation könnte die BNF durch diese Faktoren behindert werden. Der Brand liefert ihnen jedoch genau das, was sie brauchen, um aktiv zu werden - eine hohe Lichtintensität und Start-Nährstoffe. So könnte die symbiontische $\mathrm{N}_{2}$-Fixierung eine mögliche BNF der Phyllosphäre eines Primärwaldes in den frühen Jahren der Sukzession ersetzen, bis im System wieder Stickstoff akkumuliert ist und/oder die Nachfrage nach Stickstoff abnimmt.

Mit der ${ }^{15} \mathrm{~N}$ natural abundance Methode $\left({ }^{15} \mathrm{NNAM}\right)$ wurde mit den zuvor erarbeiteten Kriterien und Referenzpflanzen der \%Ndfa für Abarema jupunba, Inga thibaudiana und Inga heterophylla in 4-6jährigen Sekundärvegetationen in der Umgebung von Igarapé Açu berechnet. Der Gesamtstickstoff von Abarema jupunba wurde zu $75 \%( \pm 11 \%)$ aus der Luft genommen, von Inga thibaudiana zu 50\% ( $\pm 18 \%)$ und von Inga heterophylla zu $53 \%( \pm 20 \%)$. 
Unkovich (1994) bestimmte mit der ${ }^{15}$ NNAM etwa 70\%Ndfa für verschiedene Futter- und Weideleguminosen. Sprent et al. (1996) ermittelten 40-70\%Ndfa für Baumleguminosen im brasilianischen Cerrado. In Plantagenpflanzungen wurde für Leucaena leucocephala ebenfalls zwischen 50 und $70 \%$ Ndfa bestimmt ( $\delta^{15} \mathrm{~N}$ 'dilution method') (Sanginga et al., 1996). Der \%Ndfa für die drei Leguminosen liegt also auf dem gleichen Niveau, das häufig für Baumleguminosen gefunden wird.

Der Stickstoffgewinn über die BNF der Baumleguminosen von etwa $40 \mathrm{~kg} \mathrm{~N}^{-1}$ in $10 \mathrm{Jahren}^{-1}$ entspricht nur etwa 6\% des oberirdischen Gesamt-N einer Sekundärvegetation. Dies läßt sich auf die Artenzusammensetzung der Vegetation zurückführen. Denich (1989) ermittelte für Leguminosen zwar eine hohe Individualphytomasse, aber niedrige Abundanz, d.h. sie kamen auf den untersuchten Sekundärwald-Flächen in nur geringer Individuenzahl vor. Durch gezielte Managementpraktiken, die eine höhere Individuenanzahl fixierender Pflanzen zur Folge hätte, könnte diese Stickstoffbilanz beträchtlich verbessert werden. Das von Sanginga et al. (1996) und anderen durchgeführte regelmäßige Schneiteln der Baumkronen regt die BNF an und führt über diese Art der mehrfachen Gründüngung ebenfalls zu einer Vervielfachung des Stickstoffeintrages. Durchschnittlich erreichten sie so einen zusätzlichen Gewinn von $100 \mathrm{~kg}$ $\mathrm{N}^{-1} \mathrm{Jahr}^{-1}$. Denich (1989) schätzte die innerhalb eines Jahres durch Wiederaustrieb akkumulierte N-Menge von Abarema cochleatum, einer mit Abarema jupunba eng verwandten und physiologisch sehr ähnlichen Art, auf $5.3 \mathrm{~kg} \mathrm{ha}^{-1}$ bei circa 2100 Individuen $^{-1}$. Das entspricht $2.5 \mathrm{~g} \mathrm{~N}$ pro Pflanze, bzw. $1.9 \mathrm{~g}$ fixierter Stickstoff (bei $75 \% \mathrm{Ndfa}$ ). Dieser Input kann mit dem von Faidherbia albida oder Sesbania-Arten verglichen werden. Sanginga et al. (1996) erreichten 4-7g über BNF akkumulierten Stickstoff pro Jahr in einer Leucaena leucocephala-Pflanze. Im Vergleich zu den fixierten N-Mengen, die für gedüngte Gliricidia sepium bekannt sind (26g N - 64g N pro Baum, Liyanage et al., 1994), ist dies jedoch nur sehr wenig. Ob dieser $\mathrm{N}$-Input über die $\mathrm{N}_{2}$-Fixierung durch z.B. Abarema jupunba mit Hilfe von Praktiken wie wiederholtem Schneiden oder durch Düngung von Phosphor oder eventuell fehlenden Mikronährstoffen gesteigert werden könnte, bleibt weitergehenden Forschungen vorbehalten.

Eine zusätzliche Information über die BNF der Baumleguminosen liefert die Nodulation und die Rhizobienpopulation im Boden. Sowohl an den Wurzeln von Leguminosen wie Inga thibaudiana, Abarema jupunba und anderen wurde eine Nodulation bis in $40 \mathrm{~cm}$ Tiefe nachgewiesen. Bis in 3.5m Bodentiefe wurden auch infektiöse Rhizobien im Boden gefunden. Im Vergleich zu dem Nodulationspotential eines Bodens unter Prosopis glandulosa jedoch ist 
dieses Infektionspotential als gering einzustufen (Virginia et al., 1986). Virginia et al. (1986) fanden ein sehr hohes Nodulationspotential in 5m Bodentiefe direkt über dem Grundwasserspiegel und begründeten dies mit den Überlebensstrategien der Bakterien bei Wasserstreß und Nährstoffarmut. Zum einen lag der Grundwasserspiegel auf der hier untersuchten Fläche bei $8 \mathrm{~m}$, es wurde aber nur Boden bis $6 \mathrm{~m}$ Tiefe untersucht. Ob und wieviel Rhizobium in den Bodentiefen nahe des Grundwasserpegels leben, konnte daher nicht gezeigt werden. Zum anderen kann das Grundwasser in der Regenzeit bis auf die Bodentiefe von $4 \mathrm{~m}$ ansteigen, so daß die hier gefundene Grenze für das Vorkommen von Rhizobien bei $3.5 \mathrm{~m}$ als realistisch angesehen werden kann.

Über die Nodulation von Bäumen in Primärvegetationen liegen nur wenige Untersuchungen vor. Allen gemeinsam ist, daß Knöllchen an den Wurzeln großer Bäume kaum zu finden waren. Die seltenen Knöllchenfunde wurden in den Böden der Waldlichtungen gefunden (Vitousek und Denslow, 1986), am Waldrand oder an Keimlingen sowie kleinen Pflanzen. Sehr häufig war eine nachträgliche Nodulation von Pflänzchen zu beobachten, wenn sie der Primärwald-Umgebung entnommen wurden (Magalhães et al., 1982; Moreira et al., 1992; Norris, 1969; Souza et al., 1994). Sylvester-Bradley et al. (1980) fanden in Primärvegetationen in der Nähe von Manaus seltener nodulierte Wurzeln an Bäumen, die auf nährstoffreicheren Böden wuchsen als auf sandigeren, nährstoffärmeren Böden. Auch Magalhães et al. (1982) fanden unter Primärwaldkonditionen nur eine geringe Nodulation an Baumleguminosen, die sich in ihrer Quantität jedoch auf sandigeren Böden erhöhte. Magalhães et al. (1982) wiesen an Baumarten auch nach, daß die Effektivität der nativen Rhizobien-Stämme nur sehr niedrig war. Alle Autoren stellten letztlich einen umgekehrt proportionalen Zusammenhang zwischen der für die BNF relevanten Nährstoffversorgung des jeweiligen PrimärwaldSystems und der vorgefundenen Nodulation von Baumleguminosen her.

Bei den hier berechneten Fixierungsleistungen dürfen die methodischen Probleme der ${ }^{15}$ NNAM nicht außer Acht gelassen werden. Zum ersten Mal sollte mit dieser Arbeit in der Sekundärvegetation der Bragantina-Region die $\mathrm{N}_{2}$-Fixierungsleistung von Baumleguminosen überprüft werden. Dabei wurden Isotopenverhältnisse angetroffen, mit denen die Anwendung der Methode sehr erschwert war. Das auffälligste Ergebnis dieser Untersuchung war, daß im Boden durchgehend positive $\delta^{15} \mathrm{~N}$-Werte gefunden wurden, während die pflanzlichen $\delta^{15} \mathrm{~N}$ Werte zwischen -4 und (seltener) +4 lagen. Potentiell $\mathrm{N}_{2}$ fixierende Arten der Familie Leguminosae zeigten aber eine engere Spannweite $\left( \pm 2\right.$ um den $\left.\delta^{15} \mathrm{~N}_{\text {Luft }}=0\right)$. Die Leguminosen 
unterschieden sich nur um maximal $2.5 \delta^{15} \mathrm{~N}$-Einheiten von Nichtfixierern. Generell wies die Vegetation um 2 bis $6 \delta^{15} \mathrm{~N}$-Einheiten niedrigere Werte als der Boden auf. Einzige Ausnahme dabei machte die schon beschriebene Primärvegetation, deren Pflanzen ${ }^{15} \mathrm{~N}$-angereichert waren und den $\delta^{15} \mathrm{~N}-$ Werten des Bodens ähnelten.

\section{$2 \quad \delta^{15} \mathrm{~N}$ in den Böden der Bragantina-Region}

Die Böden der Bragantina-Region lassen sich mit Werten zwischen $\delta^{15} \mathrm{~N}=+2.9$ und +9.4 sowohl in der ${ }^{15} \mathrm{~N}$-Anreicherung als auch in der Spannbreite ihrer Werte im Oberboden in die Reihe weltweiter Untersuchungen eingliedern (vgl. Abb. 4 und Tab. 49 im Anhang). Für die Amazonasregion sind sie jedoch als besonders niedrig einzustufen. Piccolo et al. (1994 und 1996) führten Untersuchungen an brasilianischen Wald- und Weideböden des Amazonasgebietes durch und stießen dabei auf durchschnittlich um $5 \delta^{15} \mathrm{~N}$-Einheiten höhere Werte als die hier vorliegenden Ergebnisse der jungen Sekundärvegetation. Während auf den jungen Bragantina-Flächen $\delta^{15} \mathrm{~N}$-Werte von $+7.6 \pm 0.3$ nicht überschritten wurden, erreichten sowohl Weide- als auch Waldböden im benachbarten Paragominas und anderen Standorten des Amazonasbeckens $\delta^{15} \mathrm{~N}$-Werte von +13 in vergleichbarer Bodentiefe. In Sedimentanalysen der Várzea im mittleren Amazonaslauf fanden Martinelli et al. (1992) $\delta^{15} \mathrm{~N}$-Werte zwischen +3.8 und +4.4 - dieselben Sedimente, aus denen sich auch der Boden der Bragantina-Region entwickelte. In den tieferen Bodenschichten der untersuchten Bragantina-Böden sank der $\delta^{15} \mathrm{~N}$-Wert auf circa \pm 2 ab. Bis in Tiefen von $6 \mathrm{~m}$ reicht der Einfluß der stark angereicherten Huminstoffe und feinpartikulären organischen Substanzen nicht. So nehmen die $\delta^{15} \mathrm{~N}$-Werte des tieferen Bodens eine dem ursprünglichen Sedimentboden ähnlichere Form an.

Den positiven $\delta^{15} \mathrm{~N}$-Werten der oberen Bodenschichten gegenüber stehen erste Anzeichen für ein pflanzenverfügbares $\mathrm{N}$, dessen $\delta^{15} \mathrm{~N}$-Wert mit zunehmender Bodentiefe sukzessive negativer wird (Paparcikova, 1996). Das würde eine Erklärung für die $\delta^{15} \mathrm{~N}$-Werte der Vegetation liefern. Der Boden- $\delta^{15} \mathrm{~N}-$ Wert ist mit seinen positiven Werten von der stabilen organischen Substanz und ihrer ${ }^{15} \mathrm{~N}$-Konzentration dominiert. Das pflanzenverfügbare N, welches den kleinsten Teil an den N-Reserven des Bodens ausmacht, muß durch neuzeitliche Prozesse mit Luft-N oder aber durch Fraktionierungsprozesse bei Nitrifikation, Denitrifikation und Volatilisation auf ihren niedrigen $\delta^{15} \mathrm{~N}$-Wert verdünnt worden sein. 
Die Menge an verfügbarem N im Boden nach Eingriffen wie der Brandrodung, während der Kulturphase und der anschließenden Sekundärvegetation ist schwer nachvollziehbar. Hölscher (1995) wies für die junge Sekundärvegetation nach Unkrauthackungen, Brand und Ernten eine Erhöhung der $\mathrm{NO}_{3}{ }^{-}$-Konzentration in der Bodenlösung bis in $105 \mathrm{~cm}$ Tiefe nach (bei circa $30 \mathrm{mg} \mathrm{NO}_{3}^{-}-\mathrm{N}$ in 11 Bodenlösung sind dies etwa $9 \mathrm{~kg} \mathrm{~N}^{-1}$ ). Anfänglich auftretendes $\mathrm{NH}_{4}{ }^{+}$im Sickerwasser des untersuchten Bragantina-Bodens war schon 4 Monate nach dem Brand vollständig nitirifiziert bzw. von Pflanzen und Bakterien aufgenommen. Bei jedem Übergang von der Regen- zur Trockenzeit werden mindestens 10 bis $40 \mathrm{kgN} \mathrm{ha}{ }^{-1}$ durch die absterbende Feinwurzelmasse freigesetzt (pers. Mitt. Wiesenmüller). Innerhalb eines Jahres nach einer Brandrodung wird 75\% der unterirdischen Biomasse mit circa $170 \mathrm{~kg} \mathrm{~N}^{-1}$ (Daten von Nunez, 1995, für 10jährige Sekundärvegetation) abgebaut (Abbaurate aus Lessa et al., 1996). Wenn also zu solchen Zeitpunkten kurzfristig höhere Mengen an Stickstoff vorhanden sind, können Fraktionierungsprozesse der Nitrifikation den $\delta^{15} \mathrm{~N}$-Wert des pflanzenverfügbaren N zum negativen hin verschieben. Andererseits wies Gehring (1997) zwar sowohl N (sekundär limitierend) als auch P (primär limitierend) als wachstumslimitierende Faktoren für Kulturpflanzen nach, in Pflanzen der Sekundärvegetation jedoch traten diese Faktoren erst nach 2 Jahren Brachephase in Form einer Wachstumsförderung bei Applikation in Erscheinung. Wie groß der N-Mangel überhaupt ist, konnte er aufgrund des P-Defizits nicht bestimmen. Offenbar aber haben die Pflanzen der Sekundärvegetation ein sehr effektives, an die Nährstoffknappheit angepaßtes Wurzelsystem entwickelt, wodurch freier Stickstoff optimal absorbiert werden kann. Wieviel aktuell verfügbarer Stickstoff also während einer Bracheperiode im Boden vorhanden ist, läßt sich daher nicht deutlich sagen.

Die Rolle der Nitrifikation im Primärwald ist ebenfalls nicht eindeutig geklärt und wahrscheinlich je nach Waldsystem und Bodenverhältnissen verschieden. Während Jordan et al. (1979) aufgrund hoher Tanningehalte und dem niedrigen $\mathrm{pH}-$ Wert keine Nitrifikation in Primärvegetationen vermuten, fanden Vitousek und Matson (1988 und 1990) und Matson und Vitousek (1987) in zur Projektregion vergleichbaren terra firme-Wäldern nahe Manaus und in La Selva - Costa Rica - eine hohe $\mathrm{NO}_{3}{ }^{-}$-Produktion.

Insgesamt besteht noch ein sehr geringes Wissen über die Vorgänge der Nitrifikation in Sekundär- und Primärvegetationen, so daß der Mechanismus der ${ }^{15} \mathrm{~N}$-Verarmung über Nitrifikation als Ursache für die negativen $\delta^{15} \mathrm{~N}$-Werte in der Sekundärvegetation weder ausgeschlossen noch angenommen werden kann. 
Denitrifikation kann nur da eine Rolle spielen, wo genügend $\mathrm{NO}_{3}{ }^{-}$vorhanden ist. In Phasen der Bodenbearbeitung (s.o.) und direkt nach der Brandrodung oder in der Primärvegetation ist dieser Prozeß daher möglich. Die Denitrifikanten müssen anaerobe Verhältnisse vorfinden, um $\mathrm{NO}_{3}{ }^{-}$als terminalen Elektronenakzeptor nehmen zu können. Diese Verhältnisse finden sich in Bodenaggregaten, Teilen der organischen Substanz und da, wo über einen hohen $\mathrm{O}_{2}$-Bedarf kurzfristig anaerobe Verhältnisse geschaffen wurden (Robertson, 1989). Robertson und Tiedje (1988, in Groffman, 1995) ermittelten eine jährliche Denitrifikationsrate von 13.2 $\mathrm{N} \mathrm{kg} \mathrm{ha}^{-1}$ in Primärvegetationen, in einer jungen Biomasse aufbauenden Sekundärvegetation hingegen nur $3.6 \mathrm{~kg} \mathrm{~N}^{-1} \mathrm{Jahr}^{-1}$. Solche Bedingungen finden sich also offenbar eher in Primärvegetationen als in gerade gebrannten, vegetationsfreien und an organischem Material verarmten Böden oder Böden mit schnell wachsender Pflanzendecke. Treffen diese Zahlen auch auf die hiesigen Verhältnisse zu, so hätte die Denitrifkation in Peixe Boi einen größeren Einfluß auf die ${ }^{15} \mathrm{~N}$-Anreicherung des pflanzenverfügbaren $\mathrm{N}$ im Boden als in Igarapé Açu und trüge zu den vergleichsweise höheren $\delta^{15} \mathrm{~N}$-Werten in Peixe Boi bei.

Die neutralen $\delta^{15} \mathrm{~N}$-Werte der Asche, die sich nicht signifikant von der Vegetation unterschieden, sprechen für den Ausschluß der ${ }^{15} \mathrm{~N}$-Anreicherung durch den Stofftransfer in die Luft. Die N-Mengen der Asche betragen nur noch 3\% der ursprünglich in der oberirdischen Biomasse vorhandenen und sind vergleichsweise.

Neben schon in Kap. VI.2.1 erörterten Mechanismen spielen die Prozesse der Umwandlung der Streu zu mineralisierten $\mathrm{N}$ eine Schlüsselrolle in der ${ }^{15} \mathrm{~N}$-Fraktionierung. Die Korrelationsanalyse der $\delta^{15} \mathrm{~N}$-Werte der Blätter von Tapirira guianensis, Inga thibaudiana und Vismia guianensis mit der organischen Auflage und dem Boden der Bragantina-Region wiesen auf signifikante Wechselbeziehungen zwischen diesen Arten, dem Streufall und dem Boden hin. Für andere Arten konnte aber keine Beziehung festgestellt werden. Die Streu unter Leguminosen zeigte konstant niedrigere, jedoch statistisch nicht absicherbare, $\delta^{15} \mathrm{~N}$-Werte als unter nicht fixierenden Referenzpflanzen. Nadelhoffer und Fry (1988) erreichten über die Erhöhung der Streumenge eines Eichenwaldes eine Absenkung des $\delta^{15} \mathrm{~N}$-Wertes in den ersten $10 \mathrm{~cm}$ des Bodens und wiesen damit den Einfluß der Vegetation und deren Blattfall nach. Nadelhoffer und Fry (1988) schlossen Mechanismen wie unterschiedliche Fraktionierungen während des Streuabbaus sowie Verlagerungen und Änderung der N-Quelle als Ursachen für die sich vertikal ändernden ${ }^{15} \mathrm{~N}$-Verhältnisse aus. 
Für die Anwendung der ${ }^{15} \mathrm{NNAM}$ ist es also wichtig, die starke kleinräumige Inhomogenität der $\delta^{15} \mathrm{~N}$-Werte der organischen Auflage und den nachgewiesenen Einfluß einzelner Arten auf den $\delta^{15} \mathrm{~N}_{\text {Streu }}$ zu berücksichtigen. Indem vergleichende Beprobungen von Pflanzen für die Berechnung des \%Ndfa nur in unmittelbarer Umgebung zueinander vollzogen werden, könnte der Fehler aufgrund der Streuung der Boden $-\delta^{15} \mathrm{~N}-$ Werte vermindert werden. Wenn man von negativen $\delta^{15} \mathrm{~N}$-Werten im pflanzenverfügbaren $\mathrm{N}$ des Bodens ausgeht, müssen Referenzpflanzen mit ebenfalls negativen Blattwerten gesucht werden.

\section{$3 \quad \delta^{15} \mathbf{N}$ in der Vegetation der Bragantina-Region}

Es konnte eine deutliche Einteilung der Pflanzenarten nach ihren $\delta^{15} \mathrm{~N}$-Werten vorgenommen werden. Zum einen waren in den Blättern der Leguminosen sowohl räumlich als auch zeitlich geringe Streuungen, in den nicht-fixierenden Referenzpflanzen jedoch hohe Streuungen zu verzeichnen. Zum anderen gab es Arten mit durchgehend positiven $\delta^{15} \mathrm{~N}$-Werten wie Lacistema pubescens oder negativen Werten wie Tapirira guianensis, während Banara guianensis und Vismia guianensis in Abhängigkeit vom Standort Werte über oder unter $\delta^{15} \mathrm{~N}=0$ aufwiesen. Die untersuchten nicht-fixierenden Referenzpflanzen waren mit Ausnahme von Banara guianensis und Myrcia sylvatica signifikant von den Leguminosen verschieden.

Für die Untersuchungsregion bestätigte sich die von Amarger et al. (1979), Virginia und Delwiche (1982) oder auch Shearer et al. (1978) beschriebene größere Streuung der Referenzpflanzen in Abhängigkeit vom Standort gegenüber den Leguminosen. Sie führen dies auf die BNF der Leguminosen zurïck, die durch ihre $\mathrm{N}_{2}$-Fixierung unabhängiger vom Bodenstickstoff sind.

\section{Wurzelsystem der Pflanzen}

Von Clausing (1994) wurden sowohl Banara guianensis als auch Vismia guianensis als Flachwurzler, Lacistema pubescens als Flach-Tiefwurzler und Tapirira guianensis als Tiefwurzler ausgewiesen. Virginia et al. (1989) machten sich den mit der Bodentiefe verändernde $\delta^{15} \mathrm{~N}$-Wert zu nutze, um die Bodentiefe zu ermitteln, aus der Prosopis glandulosa ihren N bezieht. Indem sie die Wurzeln nahe der Oberfläche kappten, fanden sie - den Untersuchungen dieser Arbeit entsprechend - einen niedrigeren $\delta^{15} \mathrm{~N}$-Wert, der nur aus den tieferen Bo- 
denschichten stammen konnte. Virginia et al. (1988a) beziehen die niedrigen $\delta^{15} \mathrm{~N}$-Werte der Chaparral-Vegetation Kaliforniens auf die an den Brand angepasste tiefer wurzelnde Vegetation, die so an mineralisches $\mathrm{N}$ mit einem niedrigeren $\delta^{15} \mathrm{~N}$ gelangt. Dieser Mechanismus kann für die junge Sekundärvegetation der Bragantina-Vegetation in Erwägung gezogen werden, denn sowohl von Nepstad et al. (1993) als auch Carvalheiro und Nepstad (1996), Sommer (1996) sowie in eigenen Untersuchungen wurden Wurzeln bis zu 8m Bodentiefe gefunden.

Die Leguminose Abarema jupunba kann tiefe Wurzeln ausbilden (Jacobi, 1997). Oftmals entstanden diese Bäume jedoch aus dicht unter und parallel zu der Bodenoberfläche wachsenden Wurzelausläufern. In solchen Fällen konnten keine tiefergehenden Wurzeln beobachtet werden. Über diese Wurzelausläufer sind die Bäumchen mit einer großen 'Mutter'-Pflanze in unterschiedlicher Entfernung verbunden. Inga heterophylla bildet z.T. über weite Strecken Wurzelausläufer, die alle 2-3m Wurzeln in den Boden schicken. Wieviel Stickstoff über diese Wurzeln zu den einzelnen Bäumchen gelangt, ist nicht abschätzbar. Dieses Wurzelsystem wurde für viele Arten der Sekundärvegetation beobachtet und kann als typisch angesehen werden. Viele Leguminosen bildeten solche Wurzelsysteme aus, obwohl sie weltweit als Tiefwurzler bekannt sind (McKey, 1994).

Carvalheiro und Nepstad (1996) beschrieben verschiedene Typen von Bodenhohlräumen bis in 9m Tiefe, die durch Blattschneiderameisen - bzw. Wurzelgänge oder Ameisenbauten entstanden sind und in junger Sekundärvegetation am häufigsten in den oberen Bodenschichten bis $3 \mathrm{~m}$ auftraten. In diesen Bodenfenstern trafen sie eine höhere Mykorrhizierung und einen höheren Nährstoffgehalt als im umgebenden Boden an. Ein Teil der vorgefundenen Variabilität kann eventuell auch auf diese Hohlräume zurückgeführt werden, da an solchen Stellen andere ${ }^{15} \mathrm{~N}$-Verhältnisse vorherrschen können.

Garten (1993) fand für verschiedene Pflanzenarten standortabhängige ${ }^{15} \mathrm{~N}-$ Schwankungen und führt dies unter anderem auf eine unterschiedliche Ausprägung des Wurzelsystems zurück.

Auch Gebauer et al. (1994) erklärten die unterschiedlichen $\delta^{15} \mathrm{~N}$-Werte in einer Tundra-Vegetation Alaskas mit der Entnahme der Nährstoffe aus unterschiedlichen Bodentiefen, aber auch die Nutzung unterschiedlicher N-Quellen. Nadelhoffer et al. (1996) hielten solche, auch in der Bragantina-Region vorkommende Wurzelsysteme für eine Anpassung an die nährstoffarmen Böden der Tundra: Die Arten vermeiden so die Konkurrenz um den wenigen 
Stickstoff, indem sie die verschiedensten N-Quellen ausschöpfen. Eine solche optimale Ausschöpfung der N-Quellen ist im Boden der Bragantina-Region ebenso wahrscheinlich. Jedoch sind Informationen zu einzelnen Arten sehr spärlich vorhanden. Eine Vorstellung von der möglichen ${ }^{15} \mathrm{~N}$-Verteilung in den Pflanzen und dem Boden, den $\delta^{15} \mathrm{~N}$-Werten des $\mathrm{N}_{\text {min. }}$ wird in Abb. 41 gegeben.

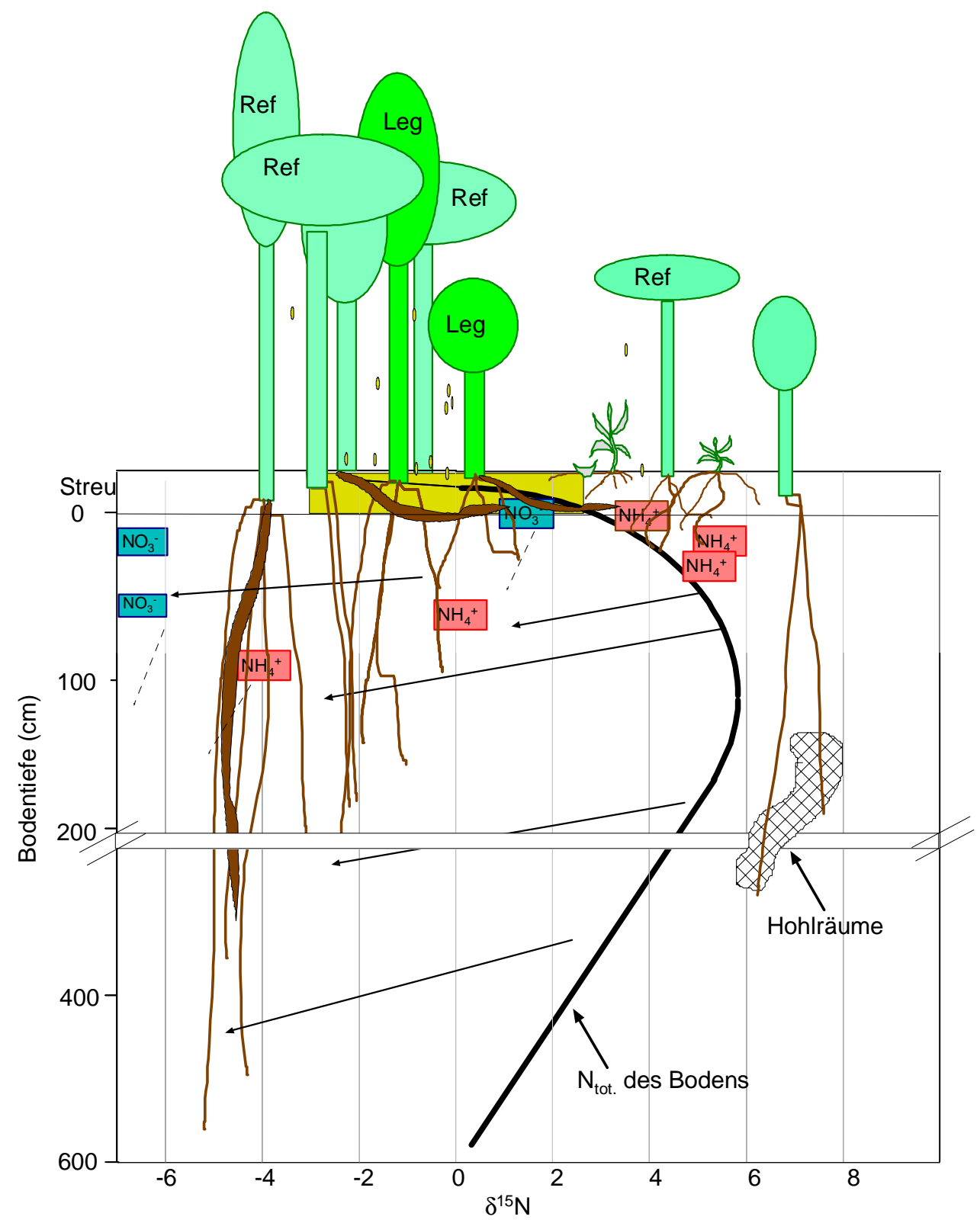

Abb. 41: Vertikale ${ }^{15} \mathrm{~N}$-Verteilung in der Sekundärvegetation der Bragantina-Region. (Der gelbe Balken gibt die Spannbreite der $\delta^{15} \mathrm{~N}$-Werte der organischen Auflage wieder, $\mathrm{NH}_{4}{ }^{+}$und $\mathrm{NO}_{3}{ }^{-}$-Werte sind der Arbeit von Paparcikova, 1996, entnommen) Mit „Hohlräume“ sind die Löcher und Gänge gemeint, die durch Blattschneiderameisen und Wurzeln geschaffen wurden. Pfeile links von der $\mathrm{N}_{\text {tot.-Kurve }}$ geben die möglichen ${ }^{15} \mathrm{~N}$-Verschiebungen im $\mathrm{N}_{\text {min. }}$. wieder, abwärts gerichtete Pfeile symbolisieren die Verlagerung von $\mathrm{N}_{\text {min. }}$ in tiefere Bodenschichten. 
Lacistema pubescens hatte durchgehend positive $\delta^{15} \mathrm{~N}$-Werte. Dies könnte ein Hinweis darauf sein, daß sie ihren Stickstoff aus den obersten $20 \mathrm{~cm}$ des Bodens bezieht, in denen mit hoher Wahrscheinlichkeit noch $\delta^{15} \mathrm{~N}$-positives verfügbarer Stickstoff vorliegt. Eine weitere Möglichkeit besteht in der direkten Nutzung von organischer Substanz, die positivere $\delta^{15} \mathrm{~N}$-Werte als das mineralisierte $\mathrm{N}$ annimmt. Tapirira guianensis zeigte durchgehend negative $\delta^{15} \mathrm{~N}$-Werte. Der $\delta^{15} \mathrm{~N}$-Wert der N-Quelle von Tapirira guianensis wurde mit durchschnittlich -2.5 bestimmt (siehe Kap. VI.5.3). Daher liegt die Annahme nahe, daß diese Art Stickstoff aus tieferem Boden beziehen kann, in dem der verfügbare Stickstoff ${ }^{15} \mathrm{~N}$-verarmt ist. Banara guianensis und Vismia guianensis zeigten in Abhängigkeit vom Standort positive oder negative $\delta^{15} \mathrm{~N}$-Werte. Beide Arten scheinen ihren N-Bedarf je nach Stickstoffangebot des Standortes aus verschiedenen Quellen oder Bodentiefen nehmen zu können.

Ein wichtiger Faktor, der sowohl den $\delta^{15} \mathrm{~N}$-Wert einer Pflanze als auch ihre N-Quelle beeinflußt, ist die Mykorrhiza (Högberg, 1990; Högberg und Alexander, 1995; Pate et al., 1993). Neuere Arbeiten von Michelsen et al. (1996) wiesen ${ }^{15} \mathrm{~N}$-Anreicherungen ektomykorrhizierter Pflanzen gegenüber erikoid-mykorrhizierten nach, was sie auch als Hinweis auf den Zugriff auf unterschiedliche N-Quellen werteten. Die meisten Baumarten der Tropen formen eine (vesikulär-) arbuskuläre Mykorrhiza ((V)AM) (Janos, 1983). Viele Arten der Familie Myrtaceae sind bekannt für eine Ektomykorrhizierung. Erkenntnisse zur Mykorrhizierung speziell der jungen Vegetation der Bragantina-Region sind jedoch nicht vorhanden.

\section{$\delta^{15} N$ in den Pflanzen als Indikator für das pflanzenverfügbare $N$}

Über die Betrachtung des Verhältnisses der $\delta^{15} \mathrm{~N}$-Werte der Vegetation zu denen des Bodens können weitere Rückschlüsse auf die $\delta^{15} \mathrm{~N}$-Werte des für Pflanzen verfügbaren $\mathrm{N}$ gezogen werden. Shearer et al. entwickelten 1974 ein steady state Modell der $\delta^{15}$ N-Flüsse im System, welches von Garten (1993) weiterentwickelt wurde. Letzterer stellte das Gleichgewicht zwischen Immobilisierung und Nitrifikation als den wichtigsten auf den $\delta^{15} \mathrm{~N}$-Wert des Bodens Einfluß nehmenden Faktor dar. Je nach Aktivität der mikrobiellen Biomasse wird bei der Immobilisierung mehrfach das gleiche Substrat/Produkt verarbeitet. Nitrifikation kann nur dann stattfinden, wenn $\mathrm{NH}_{4}{ }^{+}$vorhanden ist. An N-armen Standorten überwiegt die Immobilisierung die Nitrifikation. Die $\delta^{15} \mathrm{~N}$-Werte des pflanzenverfügbaren $\mathrm{N}$ sind negativer als an Orten mit besserer Stickstoffversorgung, denn die mehrfach wiederverwendeten Stick- 
stoff-Verbindungen führen zu einer größeren Aufsplittung des ${ }^{15} \mathrm{~N}$ zwischen immobilisierten und pflanzenverfügbarem N. Garten und Miegrot (1994) entwickelten daraus einen sogenannten Anreicherungsfaktor $\left(\delta^{15} \mathrm{~N}_{\text {Blättern }}-\delta^{15} \mathrm{~N}_{\text {Boden }}\right)$, der mit der Netto-Nitrifikation der von ihnen untersuchten Böden positiv korrelierte (je nach Art, von der die Blätter stammten, lag $r^{2}$ zwischen 0.83 und 0.96 ).

Für die Bragantina-Böden wurde dieser Anreicherungsfaktor folgendermaßen berechnet:

Tab. 48: Nach Garten und Miegrot (1994) berechnete Anreicherungsfaktoren der Untersuchungsflächen in der Bragantina- Region (basierend auf Mittelwert-Daten der Vegetationen der MFlächen im Monat Juli und des Bodens, vergl. Tab. 30 und 31)

\begin{tabular}{ll}
\hline Fläche & Anreicherungsfaktor \\
M1 & -5.45 \\
M2 & -4.77 \\
M5 & -4.75 \\
M3 & -4.29 \\
PB $_{\mathrm{SW}}$ & -3.97 \\
PB $_{\mathrm{PW}}$ & -2.41 \\
\hline
\end{tabular}

Je negativer dieser Anreicherungsfaktor ist, desto größer ist die Aufspaltung des ${ }^{15} \mathrm{~N}$ und ${ }^{14} \mathrm{~N}$ zwischen der Vegetation und dem Boden-Stickstoff-Pool. Das wiederum weist auf eine niedrigere Nitrifikationsrate und eine größere Bedeutung der Immobilisierung hin. Nach Garten und Miegrot (1994) sollten die jungen Sekundärwaldflächen aus Igarape'Açu in ihrer Nitrifikationsrate also niedriger einzustufen sein als die Flächen aus Peixe Boi, insbesondere der Primärwaldfläche. Nach der Brandrodung waren zwar zunächst geringe Mengen $\mathrm{NO}_{3}{ }^{-}-\mathrm{N}$ im Sickerwasser einer Sekundärwaldfläche nachzuweisen, 6 Monate nach der letzten Ernte jedoch nicht mehr (Hölscher, 1995). Das $\mathrm{NO}_{3}{ }^{-}$war von der schnell aufwachsenden Vegetation und der mikrobiellen Biomasse aufgenommen und Stickstoff geriet so ins Defizit. So überwog nun in der Brache die Immobilisierung die Nitrifikation. In Peixe Boi war dagegen ein gleichmäßigeres N-Angebot vorauszusetzen, daher wird die Immobilisierung dort eine geringere Bedeutung als die Nitrifikation haben.

Der Fraktionierungsfaktor $\beta$ des ${ }^{15} \mathrm{~N}$ zwischen der Nährlösung als Substrat und den $\delta^{15} \mathrm{~N}$-Werten des Blattes von Tapirira guianensis betrug 1.0026. Dieser $\beta$-Faktor entspricht dem bisher beschriebenen Fraktionierungsfaktor bei den pflanzeninternen Vorgängen der Stickstoffassimilation bis hin zum Einbau in organische Substanzen (Handley und Raven, 1992; siehe auch Tab. 3). Die Fraktionierung schlug sich in einer Differenz von 0.52 
$\delta^{15} \mathrm{~N}$-Einheiten zwischen dem zugegebenen $\mathrm{NO}_{3}{ }^{-}$und den Blättern nieder. Damit kommt ihr innerhalb der Pflanzen der Sekundärvegetation eine Bedeutung zu, da die Wertedifferenzen zwischen Leguminosen und Referenzen ohnehin nur maximal $2.5 \delta^{15} \mathrm{~N}$-Einheiten betrugen. Pate et al. (1994) testeten mehrere potentielle Referenzpflanzen für Weideleguminosen Australiens auf diese Fraktionierung, fanden jedoch nur in einer Grasart (Lolium rigidum) einen Unterschied von $1 \delta^{15} \mathrm{~N}$-Einheit zwischen der Nährlösung und dem Gras. Mariotti et al. (1980) konnten keine große Differenz zwischen dem $\delta^{15} \mathrm{~N}$-Wert des aufgenommenen Stickstoffs und den Blatt- $\delta^{15} \mathrm{~N}-$ Werten finden. Daher maßen Pate et al. (1994) der Fraktionierung durch die Aufnahme und den Transport von Stickstoff keine signifikante Bedeutung bei. Die Problematik der Referenzpflanzen sahen sie darin, daß sich auf dem Feld ein N-Transfer zwischen bestimmten Referenzpflanzen (hier Gräser) und den Leguminosen abspielen kann, der zu einer ungenauen \%Ndfa-Berechnung führt.

Für die \%Ndfa-Berechnungen wurde der $\delta^{15} \mathrm{~N}-$ Wert der Blätter der A-Pflanzen von Abarema jupunba und Abarema cochleatum ermittelt. Mit +0.42 lag er weit über den bisher in der Literatur beschriebenen Werte für Baumleguminosen (vgl. Tab. 4). Nur sehr vereinzelt wurden positive $\delta^{15} \mathrm{~N}$-Werte in den Blättern von krautigen Leguminosen gefunden (Turner und Bergersen, 1983). Die $\delta^{15} \mathrm{~N}$-Werte der Gesamtpflanzen der beiden getesteten Leguminosen hingegen betrugen -0.16 bzw. -0.04 , welche dem $\delta^{15} \mathrm{~N}$-Wert der Luft entsprechen und damit bestätigen, daß $100 \%$ des Stickstoffs dieser Pflanzen aus der $\mathrm{N}_{2}$-Fixierung stammten. Die Pflanzen waren zu Versuchsende 10-12 Monate alt. Eventuell hätte eine längere Kultivierung eine noch stärkere Aufteilung des ${ }^{15} \mathrm{~N}$ in den Pflanzenorganen und Knöllchen bewirkt und die Blatt- $\delta^{15} \mathrm{~N}$-Werte auch dieser Pflanzen wären negativ geworden. Die in der Literatur beschriebenen Pflanzen waren jedoch selten älter als die in dieser Untersuchung.

Aufgrund der Vielfältigkeit des Wurzelsystems einerseits und der Ungewißheit über die $\delta^{15} \mathrm{~N}$-Werte des pflanzenverfügbaren Stickstoffs andererseits, wurden in der vorliegenden Arbeit als Kriterium für die Auswahl der Referenzpflanzen nicht die Ähnlichkeit mit dem $\delta^{15} \mathrm{~N}$-Wert des $\mathrm{N}_{\text {tot. }}$ des Bodens herangezogen, sondern äußerlich sichtbare und überprüfbare Faktoren wie räumliche und zeitliche Muster des $\delta^{15} \mathrm{~N}$, ihre Variabilität und $\delta^{15} \mathrm{~N}$-Differenz zwischen Leguminosen und potentiellen Referenzpflanzen, welche im folgenden diskutiert werden. 


\section{Räumliche Variabilität von $\delta^{15} \mathrm{~N}$}

Die Gründe für die hohe innerartliche Variabilität der $\delta^{15} \mathrm{~N}$-Werte einiger Arten der jungen Sekundärvegetation der Bragantina-Region können in den kleinräumigen Unterschieden in der Beschaffenheit des Bodens gesucht werden. Die Bodentextur beeinflußt den Wasserhaushalt, das Durchwurzelungsvermögen und auch die mikrobielle Aktivität des Bodens. Kleinräumige Veränderungen im Nährstoffangebot, z.B. die schon zuvor erwähnten durch Blattschneiderameisen entstandenen Hohlräume, können Verschiebungen im $\delta^{15} \mathrm{~N}$-Wert der Pflanzen verursachen. Ist genug Stickstoff im Boden vorhanden, wird bei der Aufnahme durch die Pflanzen stärker gegen ${ }^{15} \mathrm{~N}$ diskriminiert als bei N-Mangel (Mariotti et al., 1982). In solchen Bodenbereichen verändert sich auch das schon oben beschriebene Verhältnis Nitrifikation zu Immobilisation, das von Garten und Miegrot (1994) für Änderungen in der Isotopenzusammensetzung des pflanzenverfügbaren Stickstoffs verantwortlich gemacht wird. Androsoff et al. (1995) fanden kleinräumig unterschiedliche Konzentrationen von $\mathrm{N}_{\min }$ im Boden und machten sie für die Schwankungen in der $\mathrm{N}_{2}$-Fixierung von Pisum sativum verantwortlich, da höhere Gehalte an $\mathrm{N}_{\min }$. hemmend auf die BNF wirken können.

Bremer und Kessel (1990) empfehlen eine Wertedifferenz von $5 \delta^{15} \mathrm{~N}$-Einheiten zwischen dem atmosphärischen Stickstoff und dem pflanzenverfügbaren mineralischen Stickstoffs des Bodens. Sie fanden innerhalb von $60 \mathrm{~m}$ Schwankungen im $\delta^{15} \mathrm{~N}-$ Wert der Vegetation von 6 Einheiten. Sutherland et al. (1991) maßen in Abhängigkeit vom Standort einmal eine Variation der $\delta^{15} \mathrm{~N}$-Werte, die innerhalb $\left(\delta^{15} \mathrm{~N}=+0.3\right.$ bis -0.3$)$ und einmal die außerhalb des $95 \%$ igen Vertrauensbereiches (+0.4 bis -0.6) lagen. Sutherland et al. (1991) ermittelten mit den gleichen Daten eine Wiederholungszahl von 11-25 für den homogenen und 170 für den heterogenen Standort, um auf einen nur 5\%igen Fehler zu kommen. Domenach und Corman (1984) ermittelten für Sojabohnen auf dem Feld eine durchschnittliche Wiederholungszahl von 10 bis 30 Einzelproben oder 3 Mischproben aus je 5 Pflanzen, um unter einen Fehler von 1 $\delta^{15} \mathrm{~N}$-Einheit zu kommen.

Auf den Flächen S1 bis S6 wurden in Abhängigkeit von den Referenzarten Standardabweichungen von \pm 0.1 bis \pm 1.15 berechnet, die einem durchschnittlichen Vertrauensbereich von \pm 0.5 , wie bei Sutherland et al. (1991), bedingten. Mit bis zu 5 Wiederholungen und einer maximalen Wertedifferenz von $\delta^{15} \mathrm{~N}=2.1$ lagen die in diesen Untersuchungen durchgeführten Wiederholungen daher weit unter den Ansprüchen von Sutherland et al. (1991). Unkovich et 
al. (1994) veranschaulichten die Problematik der Wertedifferenz und dem damit verbundenen Fehler in der Berechnung des \%Ndfa durch eine Grafik, die in Abb. 33 für die hier untersuchten Arten nachgestellt wurde. Die Fehlerberechnung nach Shearer und Kohl (1988) für die durchgeführten Kalkulationen lagen - der hohen Streuung und geringen Wertedifferenz Rechnung tragend - auch bei durchschnittlich 20\% (vgl. Tab. 46).

Eine Verringerung des hohen Fehlerwertes könnte mit einer gezielteren Auswahl der Probebäume erzielt werden. Der \%Ndfa für Abarema jupunba war bis zu einer Basalfläche von $20 \mathrm{~cm}^{2}$ baumgrößenabhängig. Bei den Beprobungen zur \%Ndfa-Berechnung wurden Bäume von Abarema jupunba mit Basalflächen von $12 \mathrm{~cm}^{2}$ bis etwa $20 \mathrm{~cm}^{2}$ erfaßt. Das entspricht dem Größenbereich, in dem sich der Anteil Stickstoff aus der BNF am Gesamt-N der Pflanze von $40 \%$ auf fast $100 \%$ Ndfa steigerte (vgl. Abb. 38). Mit der Auswahl von Bäumen mit gleich großen Basalflächen könnten die Kalkulationen verbessert werden.

Auf australischen Weiden gelang es erstmals, unter natürlichen Bedingungen \%Ndfa über die ${ }^{15}$ NNAM zu bestimmen (Sanford et al., 1993 und 1995). In einem langjährigen screening wurden Referenzpflanzen auf zeitliche und räumliche Variabilität, auf Fraktionierungsverhalten während der Aufnahme, N-Quellen und genetische Variabilität untersucht. Aufgrund einer für Weideleguminosen (Trifolium subterraneum, Medicago spp., Lotus spp.) gut geeigneten Referenzpflanze (Arctotheca calendula) und einer sehr hohen Wiederholungszahl (über 100 Proben) konnte in diesem Fall der Fehler der Berechnung auf etwa 7\% reduziert werden. Hier muß in Betracht gezogen werden, daß es sich um annuelle Pflanzen handelt, die einerseits leicht $\mathrm{zu}$ beproben sind und andererseits aus einer sehr gleichförmigen Vegetation stammen, die nicht den zwischenartlichen und räumlichen Schwankungen einer Sekundärvegetation der Bragantina-Region unterworfen ist.

Sanginga et al. (1996) stellten je nach Wahl der Referenzpflanze einen Unterschied von bis zu $10 \%$ Ndfa fest und erzielten bestenfalls eine LSD von 8 bis $10 \%$ Ndfa. Sprent et al. (1996) zeigten deutlich, daß die Berechnungen des \%Ndfa mit einer nicht fixierenden Leguminose als Referenz z.T. höhere und wesentlich gleichmäßigere Ergebnisse lieferte. Im Falle der Bragantina-Vegetation mußte jedoch je nach der - höchst unregelmäßigen - Frequenz der Arten immer auf mehrere mögliche Referenzarten verschiedener Familien zurückgegriffen werden. So wurde hierüber zusätzlich ein Fehler in die Berechnungen eingebracht, der bei weiterführenden Forschungen noch verbessert werden sollte. 


\section{Schlußfolgerungen}

Die Baumleguminosen der Bragantina-Region und ihre Streu können in Sekundärvegetationen bis zu $47 \mathrm{~kg} \mathrm{~N} \mathrm{ha}^{-1}$ über die biologische $\mathrm{N}_{2}$-Fixierung im System akkumulieren. Bei diesen Berechnungen konnte eine genaue Abschätzung des Eintrages von fixiertem Stickstoff einerseits über die Auswaschung und den Blattfall und der damit verbundenen periodischen Erneuerung der Blätter und andererseits der Umsetzung der Feinwurzelmasse nicht geleistet werden. Die Fixierungsrate einzelner Baumleguminosen schwankte zwischen etwa 30 und $75 \%$ Ndfa und erreichte somit eine hohe Effektivität. Es sollte daher überprüft werden, ob die insgesamt gering erscheinende BNF der Baumleguminosen nicht über Management-Praktiken verbessert werden und so einen Beitrag zum N-Haushalt der Sekundärvegetation leisten könnte. Eingriffe wie P-Düngung oder auch Mikronährstoff-Gaben, Erhöhung der Individuenanzahl fixierender Bäume, Baumkronenschnitt, Schaffung optimaler Lichtverhältnisse oder die Einführung einer allelopathisch verträglichen Artenzusammensetzung könnten dabei zu einer Steigerung der Biomasseproduktion und der BNF angewendet werden.

Für eine Primärvegetation der gleichen Region wurde keine bzw. nur eine sehr geringe Menge an fixiertem Stickstoff errechnet. Die These, daß in einer Primärvegetation eine ausgeglichene Nährstoffbilanz vorherrscht, die zu einem Ausbleiben der BNF der Bäume geführt hat, kann somit bestätigt werden. Allerdings bildete auch hier der mehrfach zirkulierende Stickstoff über den Blattfall und die unterirdische Biomasse nicht abschätzbare Faktoren, die aber einen großen Anteil an den N-Reserven dieses System einnehmen.

Inwieweit biologisch fixierter Stickstoff über freilebende Bakterien, Flechten und Algen im Boden und auf Pflanzen bedeutende Mengen zum Stickstoffhaushalt der Sekundärvegetation beitragen, kann über die ${ }^{15} \mathrm{NNAM}$ nicht erfaßt werden. Versuche mit ${ }^{15} \mathrm{~N}$-Anreicherungen könnten hier detaillierte Informationen liefern.

Die in der vorliegenden Arbeit vorgenommenen Berechnungen basierten auf Ergebnissen der ${ }^{15}$ NNAM. Diese Methode wurde für die natürliche Vegetation eines immergrünen Regenwaldes zum ersten Mal angewendet. Dabei wurden Probleme wie die hohe Variabilität der $\delta^{15} \mathrm{~N}$-Werte in der Vegetation, der Heterogenität des Bodens und des $\delta^{15} \mathrm{~N}$-Wertes des pflanzenverfügbaren $\mathrm{N}$ aufgeworfen.

In Vorarbeiten der vorliegenden Arbeit wurden Indizien gesammelt, die für mit der Bodentiefe zunehmend negative $\delta^{15} \mathrm{~N}$-Werte im pflanzenverfügbaren $\mathrm{N}$ des Bodens sprechen. Die Re- 
ferenzpflanzen, die für diese Methode von entscheidender Bedeutung sind, repräsentieren den $\delta^{15} \mathrm{~N}$-Wert der N-Quelle des Bodens und wurden folglich unter den $\delta^{15} \mathrm{~N}$-negativen Pflanzenarten ausgewählt. Innerhalb dieser Arbeit wurden aufgrund der insgesamt geringen Wertedifferenzen zwischen Leguminosen und Nichtleguminosen daher nur drei geeignete Referenzpflanzen (Tapirira guianensis, Vismia guianensis, Mabea angustifolia) benannt, die hinsichtlich ihrer räumlichen und zeitlichen Dynamik der Blatt- $\delta^{15} \mathrm{~N}-$ Werte und ihres $\mathrm{N}$-Aufnahmemodus geeignet erschienen. Es fehlten weitergehende Informationen über die Mykorrhizierung und das Wurzelsystem vieler Arten, um den realen $\delta^{15} \mathrm{~N}-$ Wert ihre Boden-N-Quelle zuordnen zu können. Das Problem der Referenzpflanzen wurde durch die z.T. geringe Präsenz der Arten verstärkt, die eine Beprobung der gleichen Arten an verschiedenen Standorten nicht zuließ. Die Erweiterung des Repertoires an geeigneten Referenzarten ist also zu empfehlen. Ein Versuch, nicht $\mathrm{N}_{2}$-fixierende Leguminosen als Referenzen einzusetzen, könnte zur Problemlösung beitragen. Die Frage nach dem $\delta^{15} \mathrm{~N}$-Wert der N-Quelle im Boden konnte in dieser Arbeit nur angerissen werden und muß für die Anwendung der ${ }^{15}$ NNAM untersucht werden.

Als weitere Referenz diente die sogenannte A-Pflanze, eine auf N-freiem Medium angezogene Leguminose, deren $\delta^{15} \mathrm{~N}$-Wert mit in die Berechnungen des $\% \mathrm{Ndfa}$ eingeht. In den hier beschriebenen Experimenten wurden positive Blattwerte ermittelt, ein für bisherige Forschungen ungewöhnlicher Sachverhalt. Diese Pflanzen waren maximal 12 Monate alt. Eine Weiterführung derartiger Experimente bis zu einem älteren Entwicklungsstadium von vielleicht 2 Jahren könnte die Unsicherheit über diesen Wert ausräumen.

Mit der vorliegenden Arbeit konnten semiquantitative Abschätzungen der BNF der Sekundärvegetation durchgeführt werden. Ungeklärt bleibt der Boden-interne N-Kreislauf und der Mechanismus der ${ }^{15} \mathrm{~N}$-Fraktionierung während der Umsetzung der Streu zu organischer Substanz, für den lediglich Hinweise erarbeitet werden konnten. Beide Prozesse könnten wichtige Indizien für die Umsetzung des Stickstoffs in der Sekundärvegetation der Bragantina-Region liefern. 


\section{Zusammenfassung}

Kernfrage der Arbeit war, ob die ${ }^{15} \mathrm{~N}$ natural abundance Methode $\left({ }^{15} \mathrm{NNAM}\right)$ für die $\mathrm{Ab}$ schätzung der $\mathrm{N}_{2}$-Fixierungsleistung von Baumleguminosen und ihrer N-Akkumulation in jungen Sekundärvegetationen über die biologische $\mathrm{N}_{2}$-Fixierung in tropischen Brachesystemen mit Brandrodung angewendet werden kann.

Dazu wurde das ${ }^{15} \mathrm{~N}$-Vorkommen in Brachevegetationen der Bragantina-Region, östliches Amazonasgebiet, hinsichtlich der Variabilität der $\delta^{15} \mathrm{~N}$-Werte in Boden und Pflanze, $\delta^{15} \mathrm{~N}$ der pflanzenverfügbaren N-Quelle und Fraktionierungsprozesse in der Pflanze untersucht.

$\delta^{15} N$ der Böden: Der Boden umfaßte $\delta^{15} \mathrm{~N}$-Werte von +2.9 in den obersten $5 \mathrm{~cm}$ bis zu +7.7 in $1 \mathrm{~m}$ Bodentiefe. Von $1 \mathrm{~m}$ bis $6 \mathrm{~m}$ Tiefe nimmt der $\delta^{15} \mathrm{~N}$-Wert wieder bis auf $+2.5 \mathrm{ab}$. Die $\delta^{15} \mathrm{~N}$-Werte korrelierten mit dem Tongehalt, der Basensättigung und den Magnesiumkonzentrationen des Bodens. Ein Hinweis auf die Entstehung negativer $\delta^{15} \mathrm{~N}$-Werte des mineralisierten $\mathrm{N}$ im Boden lieferte der mit Hilfe des $\delta^{15} \mathrm{~N}_{\text {Blatt }}$ von Tapirira guianensis über den $\beta$ Faktor errechnete $\delta^{15} \mathrm{~N}$-Werte für das pflanzenverfügbare $\mathrm{N}$ von durchschnittlich -2.55. Bis in $40 \mathrm{~cm}$ Bodentiefe konnte ein Einfluß der Leguminosen auf die $\delta^{15} \mathrm{~N}$-Werte des Bodens festgestellt werden, der jedoch nur in $10 \%$ der Fälle signifikant war.

Generell war ein „Infektionspotential“ von Rhizobium in den Böden der jungen Sekundärvegetation bis in tiefere Bodenschichten $(3 \mathrm{~m})$ vorhanden. Knöllchen an Wurzeln von Baumleguminosen wurden bis in $40 \mathrm{~cm}$ Tiefe gefunden.

$\delta^{15} \mathrm{~N}$-Unterschiede zwischen verschiedenen Standorten der Untersuchungsregion konnten nur vereinzelt signifikant nachgewiesen werden. Im Boden eines Primärwaldes lagen die $\delta^{15} \mathrm{~N}-$ Werte durchschnittlich 2 Einheiten höher als im Boden der Sekundärvegetation.

In der organischen Auflage des Bodens der Sekundärvegetation fanden sich die gleichen Tendenzen in ausgeprägterem Maße wie im Mineralboden wieder, also negative $\delta^{15} \mathrm{~N}$-Werte, die unter $\mathrm{N}_{2}$-fixierenden Leguminosen $1 / 2$ bis 1 Einheit niedriger waren als unter der nicht $\mathrm{N}_{2}$-fixierenden Vegetation. Die N-Gehalte der Auflage sind jedoch unter den Leguminosen höher.

$\delta^{15} N$ in der Vegetation: In der Sekundärvegetation waren in den Blättern $\delta^{15} \mathrm{~N}$-Werte zwischen -4 und +6 zu finden, wobei der durchschnittliche $\delta^{15} \mathrm{~N}-$ Wert bei -0.83 lag. 
Neue Blätter zeigten ${ }^{1 / 2}$ Einheit negativere $\delta^{15} \mathrm{~N}$-Werte als die alten Blätter. Die $\delta^{15} \mathrm{~N}$-Werte der alten Blätter streuten im Durchschnitt stärker als die der neuen. Die Differenz zwischen Blättern der Leguminosen und nicht $\mathrm{N}_{2}$-fixierenden Referenzpflanzen war in den neuen Blättern größer. Bei allen weiteren Fragestellungen wurden daher nur noch die neuen Blätter berücksichtigt.

Die Arten Abarema jupunba, Inga heterophylla, Inga thibaudiana, Banara guianensis, Lacistema pubescens, Tapirira guianensis und Vismia guianensis wurden auf die räumliche Variabilität ihrer $\delta^{15} \mathrm{~N}$-Werte untersucht. Die Streuung der Blattwerte innerhalb eines Standortes war artenabhängig. Die Leguminosen zeigten grundsätzlich eine geringere Streuung als die nicht $\mathrm{N}_{2}$-fixierenden Arten. Ihre $\delta^{15} \mathrm{~N}$-Werte reichten von +1.10 bis -0.94 . Die Nicht-Leguminosen zeigten Werte von -4 bis +3.5 .

Die $\% \mathrm{~N}$ - und ${ }^{15} \mathrm{~N}-$ Muster der einzelnen Arten über die verschiedenen Standorte und Jahreszeiten ermöglichte die Unterteilung der Arten in drei Gruppen: Die Leguminosen, die eine geringe inner- und zwischenstandörtliche sowie zeitliche Streuung und hohe N-Gehalte aufwiesen; die nicht $\mathrm{N}_{2}$-fixierenden Arten wie Tapirira guianensis, Mabea angustifolia, Vismia guianensis und Myrcia sylvatica, die stärker streuten, aber doch konstant negative $\delta^{15} \mathrm{~N}$-Werte auf allen Standorten und den meisten Probezeitpunkten zeigten; die nicht $\mathrm{N}_{2}$-fixierenden Arten, deren $\delta^{15} \mathrm{~N}$-Werte nahe 0 oder positiv waren und stark streuten.

Der Zeitpunkt der größten Differenz und geringsten innerartlichen Streuung der $\delta^{15} \mathrm{~N}$-Werte zwischen nicht-fixierenden und fixierenden Arten war der Monat Oktober. Dieser Monat ist daher als Probezeitpunkt für die Anwendung der ${ }^{15}$ NNAM zu wählen.

Neben den Ähnlichkeiten in räumlichen und zeitlichen $\% \mathrm{~N} /{ }^{15} \mathrm{~N}-$ Muster der Leguminosen und Referenzpflanzen wurden Ähnlichkeiten der pflanzeninternen $\delta^{15} \mathrm{~N}$-Muster für die Auswahl geeigneter Referenzpflanzen herangezogen. Das $\delta^{15} \mathrm{~N}$-Muster beispielsweise der Nichtleguminose Tapirira guianensis entsprach im Wesentlichen dem von Abarema jupunba.

Als nicht $\mathrm{N}_{2}$-fixierende Referenzpflanzen für die Berechnung der $\mathrm{N}_{2}$-Fixierungsleistung wurden daher insbesondere Tapirira guianensis, , Mabea angustifolia, Vismia guianensis und eingeschränkt Myrcia sylvatica herangezogen. Sie wiesen eine durchschnittliche negative Differenz von $2 \delta^{15} \mathrm{~N}$-Einheiten zu den Leguminosen, gleiche zeitliche Veränderungen der Blatt- ${ }^{15} \mathrm{~N}-$ Muster, und eine gleiche Fraktionierung von ${ }^{15} \mathrm{~N}$ innerhalb einer Pflanze (nur für Tapirira guianensis ermittelt) auf. 
Im Primärwald waren die durchschnittlichen $\delta^{15} \mathrm{~N}$-Werte bis zu 8 Einheiten höher als in der Sekundärvegetation der Bragantina-Region. Im Gegensatz zu der jungen Sekundärvegetation konnten im Primärwald keine Unterschiede zwischen Leguminosen und Referenzpflanzen gefunden werden, was auf das Fehlen der BNF der Bäume dieser Vegetation schließen läßt. Insgesamt erscheinen die $\delta^{15} \mathrm{~N}$-Werte in der hier untersuchten Vegetation dergestalt zu sein, daß Berechnungen der $\mathrm{N}_{2}$-Fixierungaseffektivität sinnvoll durchzuführen sind.

Quantifizierung der BNF durch Baumleguminosen des östlichen Amazonasgebietes

Zur Bestimmung des \% Ndfa (\% Nitrogen derived from atmosphere) mußte der Blatt- $\delta{ }^{15} \mathrm{~N}-$ Wert einer Leguminose bekannt sein, die ihren gesamten Stickstoff ausschließlich aus der Luft bezogen hatten. Es wurden $\delta^{15} \mathrm{~N}$-Werte von +0.42 für die 10monatige, in N-freier Nährlösung angezogene A-Pflanze Abarema jupunba und +0.71 für die 12 monatige Abarema cochleatum bestimmt.

Für die drei untersuchten Leguminosen wurden Fixierungsraten von 15 bis $75 \%$ bestimmt. Es zeichnete sich keine Saisonalität ab. Abarema jupunba bezog in allen Monaten mit Ausnahme des Februars (32\%) zwischen 64 und 75\% (durchschnittlich 65\%) ihres Stickstoffs aus der Luft, Inga thibaudiana zwischen 39 und 66\% (durchschnittlich 51\%), Inga heterophylla zwischen 15 und 61\% (durchschnittlich 40\%). Bei diesen Berechnungen lag der Standardfehler immer über $\pm 11 \%$ Ndfa, der durchschnittliche Standardfehler betrug $\pm 22 \%$ Ndfa. Aufgrund der geringen Wertedifferenzen und der hohen Streuung der $\delta^{15} \mathrm{~N}$-Werte ist mit den bisher genutzten Referenzpflanzen also nur eine semi-quantitative Bestimmung der $\mathrm{N}_{2}$-Fixierungsleistung gelungen.

Über den Zusammenhang zwischen der Basalfläche $\left(\mathrm{cm}^{2}\right)$ als Merkmal für die Größe eines Baumes, dem Gesamt-N (ohne Wurzeln) einer Baumleguminose und ihren \%Ndfa wurde die über BNF fixierte N-Menge des Baumes geschätzt. Mit Daten aus Biomasseerhebungen von Sekundärvegetationen der Bragantina-Region wurde die N-Akkumulation auf alle Baumleguminosen eines Bestandes hochgerechnet, wobei der Streufall nur sehr ungenau und die Auswaschung der Nährstoffe aus den Blättern nicht mit in die Kalkulationen einbezogen wurden. Die N-Akkumulation über BNF betrug $0.1 \mathrm{~kg} \mathrm{~N} \mathrm{ha}^{-1}$ für eine 1 jährige Sekundärvegetation, für eine 7jährige Brache wurden $24 \mathrm{~kg} \mathrm{~N} \mathrm{ha}^{-1}$, für eine 10jährige Brache $47 \mathrm{~kg} \mathrm{~N}$ ha $^{-}$ ${ }^{1}$ errechnet. 


\section{Summary}

Principal objectiv of this thesis was the question, whether the ${ }^{15} \mathrm{~N}$ natural abundance method $\left({ }^{15} \mathrm{~N}-\mathrm{NAM}\right)$ can be applied to estimate the $\mathrm{N}_{2}$ fixing efficiency and associated $\mathrm{N}$ accumulation via BNF of leguminous trees in the young natural secondary vegetation of a tropical slash and burn system. To this end the variability of the natural abundance of ${ }^{15} \mathrm{~N}$ in soil and plant material of the fallow vegetation of the Bragantina region, North-East-Pará, as well as the $\delta^{15} \mathrm{~N}$ of the plant available $\mathrm{N}$ source and the fractionation process in the plants were investigated.

$\delta^{15} N$ in the soil: The $\delta^{15} \mathrm{~N}$ of the $\mathrm{N}_{\text {tot }}(\%$ total $\mathrm{N}$ in the soil) ranged from +2.9 in the surface soil up to +7.7 at $1 \mathrm{~m}$ depth. Below this point down to $6 \mathrm{~m}$ depth the $\delta^{15} \mathrm{~N}$ value decreased continously to +2.5 . The $\delta^{15} \mathrm{~N}$ values were positively correlated with the $\%$ clay, base saturation and $\% \mathrm{Mg}^{2+}$. An estimate of the plant available ${ }^{15} \mathrm{~N}$ of the soil was gained by calculating the $\beta$-factor for the uptake of Nitrogen by Tapirira guianensis, indicating that the $\delta^{15} \mathrm{~N}$ values of plant available $\mathrm{N}$ could be -2.6 . Down to $40 \mathrm{~cm}$ soil depth an influence of leguminous litter could be seen, which was significant only in $10 \%$ of the cases.

A certain potential of infection by Rhizobium in the soils of the young secondary vegetation until a depth of $3 \mathrm{~m}$ was detected. Nodulation on the roots of tree legumes was found up to $40 \mathrm{~cm}$ depth.

$\delta^{15} \mathrm{~N}$ differences between soils of different stands existed, but were rarely significant. In the soil of a primary vegetation the $\delta^{15} \mathrm{~N}$ values were 2 units lower than in the soils of the young secondary vegetation. The litter of the secondary vegetation showed the same tendency as the mineral soil - under legumes lower $\delta^{15} \mathrm{~N}$ values than under non- $\mathrm{N}_{2}$-fixing species $(1 / 2$ to 1 unit). The nitrogen content in the litter was higher under legumes than under non-legumes.

$\delta^{15} N$ in the vegetation: the leaves of 40 species showed $\delta^{15} \mathrm{~N}$ values between -4 and +6 with an average of -0.83 . Young leaves were slightly more negativ ( $1 / 2$ unit) than old leaves. The variability in ${ }^{15} \mathrm{~N}$ was greater in the old than in the young leaves, whereas the difference between legumes and reference plants was greater in young than old leaves. As a result, only the $\delta^{15} \mathrm{~N}$ values of young leaves were considered in the avaluation and calculations of the experimental results. 
The spatial variability of the species Abarema jupunba, Inga heterophylla, Inga thibaudiana, Banara guianensis, Lacistema pubescens, Tapirira guianensis and Vismia guianensis was investigated. The variability of $\delta^{15} \mathrm{~N}$ in the leaves within a stand depended on the species under investigation. In generel, legumes showed less variation than non legumes. The former had $\delta^{15} \mathrm{~N}$ values between +1.1 and -0.94 , the non-legumes between -4 to +3.5 .

The $\% \mathrm{~N}$ and $\delta^{15} \mathrm{~N}$ pattern of the species in different stands and seasons led to a cluster model with three subdivisions: the legumes, whithout relevant seasonal or spatial variations and a high N content; the nonfixing species like Tapirira guianensis, Mabea angustifolia, Vismia guianensis and Myrcia sylvatica, that showed considerable variation and constantly negative $\delta^{15} \mathrm{~N}$ values in all stands and in most sampling seasons; the non fixing species, that had $\delta^{15} \mathrm{~N}$ values around 0 or positive and a very high variation.

The sampling period with the highest difference between fixing legumes and non-fixing reference plants and the smallest species-internal variation of $\delta^{15} \mathrm{~N}$ was October. Therefore this month is to be considered the best sampling period for the application of the ${ }^{15} \mathrm{~N}$ natural abundance method.

To evaluate the best reference plants we also examined the plant internal ${ }^{15} \mathrm{~N}$ pattern. The ${ }^{15} \mathrm{~N}$ pattern of Abarema jupunba and Tapirira guianensis were very similar. So we conclude a possibly similar uptake pattern and transport dynamic of these species and used it as argument to use Tapirira guianensis as reference plant.

For the calculation of the $\%$ Ndfa the non $\mathrm{N}_{2}$ fixing species Tapirira guianensis, Myrcia sylvatica, Mabea angustifolia and Vismia guianensis were used. They showed an overall difference of 2 units to the legumes, the same seasonal ${ }^{15} \mathrm{~N}$ pattern, and - in the case of Tapirira guianensis - the same ${ }^{15} \mathrm{~N}$ fractionation within the plant.

In the primary vegetation the average $\delta^{15} \mathrm{~N}$ of leguminous trees was 8 units higher than in the secondary vegetation. Contrary to the young secondary vegetation there were no $\delta^{15} \mathrm{~N}$ differences between legumes and non legumes in the primary vegetation, indicating the failure of BNF in trees. 
Quantification of BNF of tree legumes in the eastern Amazon region

The $\mathrm{N}$ fixing species under investigation cultivated on $\mathrm{N}$-free medium, the so called A-plant, is very important for calculating the $\% \mathrm{Ndfa}$ of the legume in the field. The $\delta^{15} \mathrm{~N}$ values of this plants serves as a reference for a $100 \% \mathrm{~N}_{2}$ fixing plant. In this case the $\delta^{15} \mathrm{~N}$ values of 10 month old Abarema jupunba was +0.43 and 12 month old Abarema cochleatum +0.71 .

The $\% \mathrm{Ndfa}$ of the 3 legumes investigated in the secondary vegetation was between $15 \%$ and 75\%. Seasonal patterns did not exist. Abarema jupunba derived 64\% to 75\% (average 65\%) of its nitrogen (except february with only 32\%), Inga thibaudiana 39\% to 66\% (average $51 \%$ ) and Inga heterophylla $15 \%$ to $61 \%$ (average 40\%) from the air.

The standard error never fell below $11 \% \mathrm{Ndfa}$, the average standard error was $\pm 22 \% \mathrm{Ndfa}$. The small differences of $\delta^{15} \mathrm{~N}$ values and the high variation of the reference plants investigated up to now, allowed only a semi quantitative estimation of \%Ndfa possible.

With the relationship of the basal area $\left(\mathrm{cm}^{2}\right)$ as an indicator of tree size, the total $\mathrm{N}$ of the tree legume (without roots) and its \%Ndfa the accumulated nitrogen via BNF of these trees was calculated to be around $2 \mathrm{~g}$. Based on biomass data of various secondary vegetation the $\mathrm{N}$ accumulation of all tree legumes in different stands were estimated. The inexact evaluation of the leaf litter and the leaching of nutrients from the canopy we ignored. The $\mathrm{N}$ accumulation via $\mathrm{BNF}$ of a 1 year old vegetation was $0.1 \mathrm{~kg} \mathrm{~N} \mathrm{ha}^{-1}$, of a 7 year old vegetation it was $24 \mathrm{~kg}$ $\mathrm{N} \mathrm{ha}^{-1}$ and of a 10 year old stand $47 \mathrm{~kg} \mathrm{~N} \mathrm{ha}^{-1}$. 


\section{Resumo}

O objetivo principal desta tese foi avaliar a aplicabilidade do método de abundância natural de ${ }^{15} \mathrm{~N}$ para estimar a capacidade da fixação de nitrogênio das leguminosas arbóreas nas vegetações secundárias novas e a acumulação de Nitrogênio via FBN (Fixação biológica de Nitrogênio) no sistema tropical de pousio com derruba e queima.

Para isso foi investigado a abundância natural de ${ }^{15} \mathrm{~N}$ no pousio da Zona Bragantina, região este da Amazônia, a variabilidade de $\delta^{15} \mathrm{~N}$ no solo e nas plantas, o $\delta^{15} \mathrm{~N}$ na fonte de Nitrogênio no solo acessível para a planta e os processos de fracionamento de ${ }^{15} \mathrm{~N}$ na planta.

$\delta^{15} N$ no solo: $\mathrm{O} \delta^{15} \mathrm{~N}$ nos solos apresentou valores de +2.9 na superfície à +7.7 em $1 \mathrm{~m}$ de profundidade. De 1 a $6 \mathrm{~m}$ o valor reduziu à +2.5 . O valor de $\delta^{15} \mathrm{~N}$ estava correlacionado ao contéudo de argila, a saturação de bases e a concentração de Magnésio. $\mathrm{O} \delta^{15} \mathrm{~N}$ do solo acessível as plantas foi conseguido pela determinação do $\beta$-factor da absorção, transporte e metabolismo de Nitrogênio atraves do $\delta^{15} \mathrm{~N}_{\text {folha }}$ da espécie Tapirira guianensis, com que o $\delta^{15} \mathrm{~N}$ do Nitrogênio acessível se calculou a -2.55 . Até uma profundidade de $40 \mathrm{~cm}$ o $\delta^{15} \mathrm{~N}$ do solo pareceu influenciada pela liteira das leguminosas, no entanto só $10 \%$ dos casos foram significante.

Um certo potencial de infecção do Rhizobium nos solos da vegetação secundária nova foi encontrado até uma profundidade de 3m. Nodulação nas raízes das leguminosas arbóreas foi encontrado até $40 \mathrm{~cm}$.

Diferenças entre diversas localidades da região raramente eram significantes. No solo da floresta primária os valores de $\delta^{15} \mathrm{~N}$ foram 2 unidades mais alto que na vegetação secundária.

Na matéria orgânica da vegetação secundária foram observadas as mesmas tendências em uma forma mais clara, valores de $\delta^{15} \mathrm{~N}$ negativos em geral, em baixo das leguminosas $1 / 2$ á 1 unidade mais negativo que em baixo da vegetação não fixadora. O contéudo de Nitrogênio na matéria orgânica porém era mais alto em baixo das leguminosas.

$\delta^{15} N$ na vegetação: Nas folhas da vegetação secundária se encontraram valores de $\delta^{15} \mathrm{~N}$ entre -4 a +6 e a media aritmética de -0.83 . Folhas novas eram uma meia unidade mais negativo que velhas. Os valores de $\delta^{15} \mathrm{~N}$ variaram mais nas folhas velhas que nas novas. A diferença 
entre plantas não fixadoras (referências) e as leguminosas era maior nas folhas novas que em velhas e assim decidiu-se usar só os dados ganhas com as folhas novas.

A variabilidade espacial de $\delta^{15} \mathrm{~N}$ nas espécies Abarema jupunba, Inga heterophylla, Inga thibaudiana, Banara guianensis, Lacistema pubescens, Tapirira guianensis e Vismia guianensis foi investigada. A variação nas folhas dentro de um local dependeu da espécie. As leguminosas com valores de $\delta^{15} \mathrm{~N}$ entre -0.94 e +1.10 geralmente variaram menos que espécies não fixadoras. As não fixadoras mostraram valores de -4 até +3.5.

$\mathrm{O}$ padrão de $\% \mathrm{~N} \mathrm{e}^{15} \mathrm{~N}$ de cada uma das espécies sobre os diversos locais e períodos do ano conduziu á uma divisão em tres grupos: As leguminosas com uma variação dentro e entre os locais e períodos muito baixo e um contéudo alto de N; As espécies não fixadoras como Tapirira guianensis, Mabea angustifolia, Vismia guianensis e Myrcia sylvatica, que mostraram valores de $\delta^{15} \mathrm{~N}$ constantamente negativos em relação aos locais e períodos e com grande variação; As espécies não fixadoras com valores em torno de 0 ou positivos e uma alta variação.

O mês, onde houve a maior diferença entre plantas referências e leguminosas e a menor variação dentro das espécies foi outubro. Este mês então foi considerado para a aplicação de ${ }^{15}$ NNAM.

Para a eleição de uma referência adequada, os padrões dentro de uma planta ao lado do semelhantes padrões de $\% \mathrm{~N} /{ }^{15} \mathrm{~N}$ nas leguminosas e não fixadoras foram incluido. Tapirira guianensis por exemplo correspondeu ao da Abarema jupunba.

Como plantas referências para os calculos da capacidade da fixação de nitrogênio então foram escolhidas especialmente Tapirira guianensis, também Mabea angustifolia e Vismia guianensis, em limite Myrcia sylvatica. Elas mostraram uma diferença negativa de pelo menos 2 unidades de $\delta^{15} \mathrm{~N}$, alterações dos padrões de ${ }^{15} \mathrm{~N}$ nas folhas similares e um fracionamento parecido de ${ }^{15} \mathrm{~N}$ dentro da planta (estimado apenas para Tapirira guianensis).

Em geral na mata primária os valores de $\delta^{15} \mathrm{~N}$ foram 8 unidades mais alto do que na vegetação secundària da Zona Bragantina. Ao contrario para a vegetação nova não se encontraram nemhuma diferença entre leguminosas e não fixadoras, indicando a ausência da fixação biológica de Nitrogênio. 


\section{Quantificação de FBN pelos leguminosas arbóreas na Amazônia oriental}

Para a avaliação de \%Ndfa (\%Nitrogênio derivado da atmosfera) há necessidade de se conhecer o valor de $\delta^{15} \mathrm{~N}$ nas folhas da leguminosa, quando $100 \%$ do Nitrogênio dela foi tirado da atmosfera. Foram estimado valores de $\delta^{15} \mathrm{~N}$ de +0.43 para Abarema jupunba, que foi cultivado 10 meses en uma solução de nutrientes sem Nitrogênio e +0.71 para Abarema cochleatum, cultivado por 12 meses da mesma maneira.

Para as 3 leguminosas examinadas foram avaliado taxas da fixação de Nitrogênio de $15 \%$ a 75\%. Nao houve saizonalidade. Abarema jupunba tirou em todos os meses, com uma exceção em fevereiro (32\%) entre 64\% e 75\% (a média foi 65\%) Nitrogênio do ar, Inga thibaudiana entre 39\% e 66\% (média=51\%), Inga heterophylla entre $15 \%$ e $61 \%$ (média $=40 \%$ ). Nessas calculações o padrão de error (standard error) ficou sempre em cima de $11 \% \mathrm{Ndfa}$, a mèdia foi $22 \% \mathrm{Ndfa}$. Isto significa, que com as referências usadas aqui apenas uma estimativa semi quantitativa em consequência da pequena diferênça dos valores de $\delta^{15} \mathrm{~N}$ e a alta variação foi conseguido.

A quantitade do Nitrogênio fixado por uma leguminosa arbórea foi estimado pela relação entre a área basal $\left(\mathrm{cm}^{2}\right)$ como uma característica do tamanho da arvore, o conteúdo total (sem raízes) de Nitrogênio e o \%Ndfa deste arvore. Com dados do levantamento da biomassa da vegetação secundária da Zona Bragantina a accumulação de Nitrogênio por todas as leguminosas de um local foi calculado, no qual a caída da liteira foi investigado apenas superficial e a lixiviação de nutrientes das folhas não foi incluido. A acumulação de Nitrogênio pela FBN foi $0.1 \mathrm{~kg} \mathrm{~N} \mathrm{ha}^{-1} \mathrm{em}$ uma vegetação de um ano, $24 \mathrm{~kg}$ em um pousio de 7 anos e $47 \mathrm{~kg} \mathrm{~N} \mathrm{ha}^{-1} \mathrm{em}$ um pousio de 10 anos. 


\section{Literatur}

Alexander, I.J. (1989a). Systematics and ecology of ectomycorrhizal Legumes. In: Stirton, C.H. und Zarucchi, J.L. (Hrsg.), Advances in legume biology 29, Monogr. Syst. Bot. Missouri Bot. Gard., pp. 607-624.

Alexander, I.J. (1989b). Mycorrhizas in tropical forests. In: Proctor, J. (Hrsg.), Mineral nutrients in tropical forest and savanna ecosystems. Blackwell Scientific Publications, Oxford, pp. 169-188.

Alexandratos, N., (1995). World Agriculture: towards 2010, an FAO study. Chichester, UK: Wiley and Sons. FAO, Rome.

Altabet, M.A., Francois, R., Murray, D.W. und Prell, W.L. (1995). Climate-related variations in denitrification in the Arabian sea from sediment ${ }^{15} \mathrm{~N} /{ }^{14} \mathrm{~N}$ ratios. Nature 373 (6514), pp. 506-509.

Amarger, N., Mariotti, A., Mariotti, F., Durr, J.C., Bourgignon, C. und Lagacherie, B. (1979). Estimate of symbiotically fixed Nitrogen in field grown soybeans using variations in ${ }^{15} \mathrm{~N}$ natural abundance. Plant and Soil 52, pp. 269-280.

Andreux, F., Cerri, C., Vose, P.B. und Vitorello, V.A. (1988). Potential of stable isotope, ${ }^{15} \mathrm{~N}$ and ${ }^{13} \mathrm{C}$ methods for determining input and turnover in soils. In: Harrison, A.F., Ineson, P., Med, O.W. (Hrsg.), Nutrient cycling in terrestrial ecosystem. Elsevier Applied Science, pp. 259-274.

Androsoff, G.L., Kessel, C.van und Pennock, D.J. (1995). Landscape-scale estimates of Dinitrogen fixation by Pisum sativum by ${ }^{15} \mathrm{~N}$ natural abundance and enrichment isotope dilution. Biology and Fertility of Soils 20 (1), pp. 33-40.

Awoneike, K.O., Danso, S.K.A. und Zapata, F. (1993). The use of a double $\left({ }^{15} \mathrm{~N}\right.$ and $\left.{ }^{34} \mathrm{~S}\right)$ labelling technique to assess the suitability of various reference crops for estimating Nitrogen fixation in Gliricidia sepium and Leucaena leucocephala. Plant and Soil 155 (156), pp. 325-328.

Baar, R. (1997). Vegetationskundliche und -ökologische Untersuchungen der Buschbrache in der Feldumlagewirtschaft im östlichen Amazonasgebiet. Ph. D. Thesis, Universität Göttingen.

Bidigare, R.R., Kennicutt, M.C., Keeney-Kennicutt, W.L. und Macko, S.A. (1991). Isolation and purification of chlorophyll $\mathrm{a}$ and $\mathrm{b}$ for the determination of stable Carbon and Nitrogen isotope compositions. Analytical Chemistry 63, pp. 130-133.

Bigarella, J.J. und Ferreira, A.M.M. (1985). Amazon geology and the pleistocene and the cenozoic environments and paleoclimates. Key Environments: Amazonia (Hrsg.: Prance, G.T., Lovejoy, T.E.), Pergamon Press, Oxford.

Binkley, D., Sollins, P. und McGill, W.B. (1985). Natural abundance of Nitrogen-15 as a tool for tracing alder-fixed Nitrogen. Soil Science Society of America Journal 49, pp. 444-447.

Blair, G., Catchpoole, D. und Horne, P. (1990). Forage tree legumes: their management and contribution to the Nitrogen economy of wet and humid tropical environments. Advances in Agronomy 44, pp. 27-54.

Boddey, R.M. und Döbereiner, J. (1988). Nitrogen fixation associated with grasses and cereals: recent results and perspectives for future research. Plant and Soil 108, pp. 53-65. 
Boletim Agrometeorológico 1977-1987. EMBRAPA/CPATU, Belém.

Brandes, J.A., Mcclain, M.E. und Pimentel, T.P. (1996). ${ }^{15} \mathrm{~N}$ evidence for the origin and cycling of inorganic Nitrogen in a small Amazonian catchment. Biogeochemistry 34 (1), pp. 45-56.

Bremer, E., Gehlen, H., Swerhone, G.D.W. und Kessel, C.van (1993). Assessment of reference crops for the quantification of $\mathrm{N}_{2}$ fixation using natural and enriched levels of ${ }^{15} \mathrm{~N}$ abundance. Soil Biology and Biochemistry 25 (9), pp. 1197-1202.

Bremer, E. und Kessel, C. van (1990). Appraisal of the ${ }^{15} \mathrm{~N}$ natural abundance method for quantifying Dinitrogen fixation. Soil Science Society of America Journal 54, pp. 404-411.

Bremner, J.M. und Tabatabai, M.A. (1973). Nitrogen-15 enrichment of soils and soil-derived nitrate. Journal of Environmental Quality 2 (3), pp. 363-365.

Brewbaker, J.L., Beldt, R. van den und MacDicken, K. (1981). Nitrogen fixing tree resources: Potentials and limitations. In: Graham, P.H. und Harris, S.C. (Hrsg.), BNF technology for tropical agriculture papers presented at a workshop held at CIAT 9.-13.März 1981,Cali, Columbia, CIAT, pp. 413-425.

Broadbent, F.E., Rauschkolb, R.S., Lewis, K.A. und Chang, G.H. (1980). Spatial variability of

${ }^{15} \mathrm{~N}$ and total Nitrogen in some virgin and cultivated soils. Soil Science Society of America Journal 44, pp. 524-427.

Buchmann, N., Schulze, E.-D. und Gebauer, G. (1995). ${ }^{15} \mathrm{~N}$-Ammonium and ${ }^{15} \mathrm{~N}-\mathrm{Nitrate}$ uptake of a 15-year-old Picea abies plantation. Oecologia 102, pp. 361-370.

Burris, R.H. und Miller, C.E. (1941). Application of ${ }^{15} \mathrm{~N}$ to the study of biological Nitrogen fixation. Science 93, pp. 2405-2406.

Carpenter, E.J. (1992). Nitrogen fixation in the epiphyllae and root nodules of trees in the lowland tropical rainforest of Costa Rica. Acta Ecologica 13, (2), pp. 153-160.

Carvalheiro, K. do und Nepstad, D.C. (1996). Deep soil heterogeneity and fine root distribution in forests and pastures of eastern Amazon. Plant and Soil 182, pp. 279-285.

Chalk, P.M. (1985). Review: Estimation of $\mathrm{N}_{2}$ fixation by isotope dilution: An appraisal of techniques involving ${ }^{15} \mathrm{~N}$ enrichment and their application. Soil Biology and Biochemistry 17 (4), pp. 389-410.

Chalk, P.M., Smith, C.J., Hopmans, P. und Hamilton, S.D. (1996). A yield-independent ${ }^{15} \mathrm{~N}$ isotope dilution method to estimate legume symbiotic dependence without a non- $\mathrm{N}_{2}$-fixing reference plant. Biology and Fertility of Soils 23 (2), pp. 196-199.

Cheng, H.H., Bremner, J.M. und Edwards, A.P. (1964). Variations of Nitrogen-15 abundance in soils. Science 146, pp. 1574-1575.

Clark, F.E. (1981). The Nitrogen cycle, viewed with poetic licence. In: Clark, F.E. und Rosswall, T. (Hrsg.), Ecological Bulletin - Terrestrial Nitrogen Cycles 33, pp. 13-24.

Clausing, G. (1994). Frühe Regeneration und Wiederbesiedlung auf Kulturflächen der WaldFeld-Wechselwirtschaft im östlichen Amazonasgebiet. Göttinger Beiträge zur Land-und Forstwirtschaft in den Tropen und Subtropen 97, (M.Sc. Thesis).

Clinton, P.W., Newman, R.H. und Allen, R.B. (1995). Immobilization of ${ }^{15} \mathrm{~N}$ in forest litter studied by ${ }^{15} \mathrm{~N}$ CPMAS NMR spectroscopy. European Journal of Soil Science 46 (4), pp. 551-556. 
Coomes, D.A. und Grubb, P.J. (1996). Amazonian caatinga and related communities at La Esmeralda, Venezuela: Forest structure, physiognomy and floristics and control by soil factors. Vegetatio 122 (2), pp. 167-191.

Danso, S.K.A. (1995). Assessment of biological Nitrogen fixation. Fertilizer Research 42 (13), pp. 33-41.

Danso, S.K.A., Zapata, F., Bowen, G.D., and Sanginga, N. (1991). Applications of ${ }^{15} \mathrm{~N}$ methods for measuring Nitrogen fixation in trees. In: Stable Isotopes in Plant Nutrition, Soil Fertility and Environmental Studies, Proceedings of a Symposium, IAEA, Vienna, pp. 499.

Dauchez, S., Legendre, L. und Fortier, L. (1995). Assessment of simultaneous uptake of nitrogenous nutrients $\left({ }^{15} \mathrm{~N}\right)$ and inorganic carbon $\left({ }^{13} \mathrm{C}\right)$ by natural phytoplankton populations. Marine Biology 123 (4), pp. 651-666.

Delwiche, C.C. und Steyn, P.L. (1970). Nitrogen isotope fractionation in soils and microbial reactions. Environmental Science and Technology 4 (11), pp. 929-935.

Delwiche, C.C., Zinke, P.J., Johnson, C.M. und Virginia, R.A. (1979). Nitrogen isotope distribution as a presumptive indicator of $\mathrm{N}_{2}$-fixation. Botanical Gazette 140, pp. 565-569.

Denich, M. (1989). Untersuchungen zur Bedeutung junger Sekundärvegetation für die Nutzungssystemproduktivität im östlichen Amazonasgebiet, Brasilien. Göttinger Beiträge zur Land-und Forstwirtschaft in den Tropen und Subtropen 46.

Denich, M. und Kanashiro M. (1995). Secondary vegetation in the agricultural landscape of Northeast Pará, Brazil. In: Parrotta, J.A. and Kanashiro, M.. Proceedings of an International Symposium/Workshop "Management and Rehabilitation of Degraded Lands and Secondary Forests in Amazonia" 18.-22.April 1993, Santarém, Para [EMBRAPA/CPATU]. International Institute of Tropical Forestry, USDA Forest Service: Rio Piedras, PR. 1995, pp. $12-20$

Depinto, G.L., Martinez, M., Degotera, O.G., Vera, A., Rivas, C. und Ocando, E. (1995). Comparison of two Pithecellobium gum exudates. Biochemical Systematics and Ecology 23, (7-8), pp. 849-853.

Diniz, T.D. de S. (1986). Caracterisação climática da Amazônia oriental. EMBRAPA/CPATU-GTZ, pp. 3-13.

Döbereiner, J., Burris, R.H. und Hollander, A. (1979). Limitations and potentials for biological Nitrogen fixation in the tropics. Plenum Press.

Döbereiner, J., Urquiaga, S. und Boddey, R.M. (1995). Alternatives for Nitrogen nutrition of crops in tropical agriculture. Fertilizer Research 42 (1-3), pp. 339-346.

Domenach, A.-M., Beaupied, H. und Moiroud, A. (1992). Variability of longevity and isotopic Nitrogen value of alder (Alnus glutinosa) leaves through the growth period. Acta Oecologica 13, pp. 387-393.

Domenach, A.-M. und Corman, A. (1984). Dinitrogen fixation by field grown soybeans: Statistical analysis of variations in $\delta^{15} \mathrm{~N}$ and proposed sampling procedure. Plant and Soil 78 , pp. 301-313.

Domenach, A.-M. und Kurdali, F. (1989). Influence des réserves azotées sur la formation des feuilles de Alnus glutinosa et ses conséquences dans l'estimation de la fixation d'azote. Canadian Journal of Botany 67, pp. 865-871. 
Domenach, A.-M., Kurdali, F. und Bardin, R. (1989). Estimation of symbiotic $\mathrm{N}_{2}$-fixation in alder forest by the method based on natural ${ }^{15} \mathrm{~N}$ abundance. Plant and Soil 118, pp. 51-59.

Domenach, A.-M., Kurdali, F., Danière, C. und Bardin, R. (1988). Détermination de l'identité isotopique de l'azote fixé par le Franckia associé au genre Alnus. Canadian Journal of Botany 66, pp. 1241-1247.

Doughton, J.A., Saffigna, P.G., Vallis, I. und Mayer, R.J. (1995). Nitrogen fixation in chickpea. 2. Comparison of ${ }^{15} \mathrm{~N}$ enrichment and ${ }^{15} \mathrm{~N}$ natural abundance methods for estimating Nitrogen fixation. Australian Journal of Agricultural Research 46 (1), pp. 225236.

Doughton, J.A., Vallis, I. und Saffigna, P.G. (1992). An indirect method for estimating ${ }^{15} \mathrm{~N}$ isotope fractionation during Nitrogen fixation by a legume under field conditions. Plant and Soil 144, pp. 23-29.

Downs, M.R., Nadelhoffer, K.J., Melillo, J.M. und Aber, J.D. (1996). Immobilization of a ${ }^{15} \mathrm{~N}$-labelled nitrate addition by decomposing forest litter. Oecologia 105 (2), pp. 141-150.

Duivenvoorden, J.F. (1995). Tree species composition and rain forest environment relationships in the middle Caqueta area, Colombia, NW Amazonia. Vegetatio 120 (2), pp. 91-113.

Enquete-Kommission "Schutz der Erdatmosphäre" des 12. Deutschen Bundestages (Hrsg.) (1994). Schutz der grünen Erde: Klimaschutz durch umweltgerechte Landwirtschaft und Erhalt der Wälder. Economica-Verlag, Bonn.

Evans, R.D. und Ehleringer, J.R. (1993). A break in the Nitrogen cycle in aridlands? Evidence from $\delta^{15} \mathrm{~N}$ of soils. Oecologia 94, pp. 314-317.

Evans, R.D. und Ehleringer, J.R. (1994). Water and Nitrogen dynamics in an arid woodland. Oecologia 99, pp. 233-242.

Evans, R.D. und Ehleringer, J.R. (1994). Plant $\delta^{15} \mathrm{~N}$ values along a fog gradient in the Atacama Desert, Chile. Journal of Arid Environments 28, pp. 189-193.

Faria, S.M.,de, Franco, A.A., Jesus, R.M.,de, S.Merandro, M.,de, Baitello, J.B., Mucci, E.S.F., Döbereiner, J., and Sprent, J.I., (1984). New nodulating legume trees from south east Brazil. New Phytologist 98, pp. 317-328.

Faria, S.M. de, Lewis, G.P., Sprent, J.I. und Sutherland, J.M. (1989). Occurence of nodulation in the Leguminosae. New Phytologist 111, pp. 607-619.

Faria, S.M., de, Lima, A.C., de, Franco, A.A., Nucct, F.N.F. und Sprent, J.I. (1987). Nodulation of legume trees from south east Brazil. Plant and Soil 99, pp. 347-356.

Focht, D.D. (1973). Isotope fractionation of ${ }^{15} \mathrm{~N}$ and ${ }^{14} \mathrm{~N}$ in microbial Nitrogen transformations: A theoretical model. Journal of Environmental Quality 2 (2), pp. 247-252.

France, R. (1995). Stable Nitrogen isotopes in fish: Literature synthesis on the influence of ecotonal coupling. Estuarine Coastal and Shelf Science 41 (6), pp. 737-742.

Francisco, B.H.R., Loewenstein, P., Silva, O.F. da und Silva, G.G. da (1971). Contribuição à geologia da folha São Luis (SA 23) no estado do Pará. Boletim do Muséu Paraense Emilio Goeldi, Ciências da Terra 17, pp. 1-40.

Freney, J.R., Simpson, J.R. (Hrsg.) 1983. Gaseous loss of Nitrogen from plant-soil systems. Martinus Nijhoff, Dr.W.Junk., New York. 
Frey, B. und Schüepp, H. (1992). Transfer of symbiotically fixed Nitrogen from berseem (Trifolium alexandrinum L.) to maize via VAM hyphae. New Phytologist 122, pp. 447-454.

Fried, M., Danso, S.K.A. und Zapata, F. (1983). The methodology of measurement of $\mathrm{N}_{2}$ fixation by nonlegumes as inferred from field experiments with legumes. Canadian Journal of Microbiology 29, pp. 1053-1062.

Gamble, T.N., Betlach, M.R. und Tiedje, J.M. (1977). Numerically dominant denitrifying bacteria from world soils. Applied and Environmental Microbiology 33 (4), pp. 926-939.

Garrity, D.P. und Mercado, A.R. (1994). Nitrogen fixation capacity in the component species of contour hedgerows: How important? Agroforestry Systems 27 (3), pp. 241-258.

Garten, C.T. Jr. (1992). Nitrogen isotope composition of Ammonium and Nitrate in bulk precipitation and forest throughfall. International Journal of Environmental Analytical Chemistry 47, pp. 33-45.

Garten, C.T. Jr. (1993). Variation in foliar ${ }^{15} \mathrm{~N}$ abundance and the availability of soil Nitrogen on Walker Branch Watershed. Ecology 74 (7), pp. 2098-2113.

Garten, C.T. Jr. und Miegroet, H. van (1994). Relationships between soil Nitrogen dynamics and natural ${ }^{15} \mathrm{~N}$ abundance in plant foliage from Great Smoky Mountains National Park. Canadian Journal of Forestry Research 24 (8), pp. 1636-1645.

Gebauer, G., Giesemann, A., Schulze, E.-D. und Jager, H.J. (1994). Isotope ratios and concentrations of sulfur and Nitrogen in needles and soils of Picea abies stands as influenced by atmospheric deposition of Sulfur and Nitrogen compounds. Plant and Soil 164 (2), pp. 267-281.

Gebauer, G. und Schulze, E.-D. (1991). Carbon and Nitrogen isotope ratios in different compartments of a healthy and a declining Picea abies forest in the Fichtelgebirge. Oecologia 87, pp. 198-207.

Gehring, C. (1997). Die Bedeutung der Nährstoffversorgung für die Regeneration einer Sekundärvegetation im östlichen Amazonasgebiet. M. Sc. Thesis am Institut für Pflanzenbau und Tierhygiene in den Tropen und Subtropen, Georg August Universität Göttingen.

Gibson, T.A. und Waring, S.A. (1994). The soil fertility effects of leguminious ley pastures in north-east Thailand - I: Effects on the growth of roselle (Hibiscus sabdarrifa cv. altissima) and cassava (Manihot esculenta). Field Crops Research 39, pp. 119-127.

Giller, K.E. und Wilson, K.J. (1991). Nitrogen fixation in tropical cropping systems. CAB International, Wallingford.

Gorham, E., Vitousek, P.M., and Reiners, W.A (1979). The regulation of chemical budgets over the course of terrestrial ecosystem succession. Annual Review of Ecologicy and Systematics 10, pp. 53-84.

Groffman, P.M. (1995). A conceptual assessment of the importance of denitrification as a source of soil Nitrogen loss in tropical agro-ecosystems. Fertilizer Research 42 (1-3), pp. 139-148.

Guimarães, G.de, Bastos, J.B. und Lopes, E. de (1970). Métodos de análises física, química e instrumental de solos. Série: Química de solos 1(1), (IPEAN), pp. 1-112.

Gutteridge, R.C. und Shelton, H.M. (1993). The scope and potential of tree legumes in agroforestry. Agroforestry Systems 23 (2-3), pp. 177-194. 
Halsall, D.M., Leigh, J.H., Gollasch, S.E. und Holgate, M. (1995). The role of allelopathy in legume decline in pasture, crop and weed residues on germination, nodulation and root growth. Australian Journal of Agricultural Research 46, pp. 189-207.

Hamel, C., Nesser, C., Cartin, U.B. und Smith, D.L. (1991). Endomycorrhizal fungal species mediate ${ }^{15} \mathrm{~N}$ transfer from soybean to maize in non-fumigated soil. Plant and Soil 138, pp. 41-47.

Hamilton, S.D., Chalk, P.M., Smith, C.J. und Hopmans, P. (1992). Evaluation of reference plants by labelling with ${ }^{35} \mathrm{~S}$ in the estimation of $\mathrm{N}_{2}$ fixation by ${ }^{15} \mathrm{~N}$ isotope dilution. Plant and Soil 145, pp. 177-185.

Handley, L.L., Daft, M.J., Wilson, J., Scrimgeour, C.M., und Ingleby, K.,S. (1993). Effects of the Ecto-and VA-Mycorrhizal Fungi Hydnagium Carneum and Glomus Clarum on the $\delta^{15} \mathrm{~N}$ and $\delta^{13} \mathrm{C}$ values of Eucalyptus globulus and Ricinus Communis. Plant, Cell and Environment 16(589), pp. 375-382.

Handley, L.L., Odee, D. und Scrimgeour, C.M. (1994). $\delta^{15} \mathrm{~N}$ and $\delta^{13} \mathrm{C}$ patterns in savanna vegetation: Dependence on water availability and disturbance. Functional Ecology 8, pp. 306-314.

Handley, L.L. und Raven, J.A. (1992). The use of natural abundance of Nitrogen isotopes in plant physiology and ecology. Plant, Cell and Environment 15, pp. 965-985.

Hansen, A.P. und Pate, J.S. (1987). Evaluation of the ${ }^{15} \mathrm{~N}$ natural abundance method and xylem sap analysis for assessing $\mathrm{N}_{2}$-fixation of understory legumes in Jarrah (Eucalyptus marginata Donn ex Sm.) forest in SW Australia. Journal of Experimental Botany 38 (194), pp. 1446-1458.

Hauck, R.D. (1973). Nitrogen tracers in Nitrogen cycle studies - past use and future needs. Journal of Environmental Quality 2 (3), pp. 317-327.

Hauck, R.D., Melsted, S.W. und Yankwich, P.E. (1958). Use of N-Isotope distribution in Nitrogen gas in the study of denitrification. Soil Science 86, pp. 287-291.

Haystead, A., Malajczuk, N. und Grove, T.S. (1988). Underground transfer of Nitrogen between pasture plants infected with vesicular-arbuscular mycorrhizal fungi. New Phytologist 118, pp. 417-423.

Heaton, T.H.E. (1987). The ${ }^{15} \mathrm{~N} /{ }^{14} \mathrm{~N}$ ratios of plants in South Africa and Namibia: Relationship to climate and coastal/saline environments. Oecologia 74, pp. 236-246.

Herman, D.J. und Rundel, P.W. (1989). Nitrogen Isotope fractionation in burned and unburned chaparral soils. Soil Science Society of America Journal 53, pp. 1229-1236.

Herrera, R. und Jordan, C.F. (1981). Nitrogen cycle in a tropical Amazonian rain forest: The caatinga of low mineral nutrient status. In: Clark, F.E., Rosswall, T. (Hrsg.), Terrestrial Nitrogen Cycles - Ecological Bulletin 33, pp. 493-505.

Herridge, D.F., Bergersen, F.J. und Peoples, M.B. (1990). Measurement of Nitrogen fixation by soybean in the field using the ureide and natural ${ }^{15} \mathrm{~N}$ abundance methods. Plant Physiology 93, pp. 708-716.

Herridge, D.F. und Danso, S.K.A. (1995). Enhancing crop legume $\mathrm{N}_{2}$ fixation through selection and breeding. Plant and Soil 174, pp. 51-82.

Högberg, P. (1986). Nitrogen fixation and nutrient relations in savanna woodland trees (Tanzania). Journal of Applied Ecology 23, pp. 675-688. 
Högberg, P. (1990). ${ }^{15} \mathrm{~N}$ natural abundance as a possible marker of the ectomycorrhizal habit of trees in mixed african woodlands. New Phytologist 115, pp. 483-486.

Högberg, P. und Alexander, I.J. (1995). Roles of root symbiosis in african woodland and forest: Evidence from ${ }^{15} \mathrm{~N}$ abundance and foliar analysis. Journal of Ecology 83, pp. 217 224.

Högberg, P. und Johannisson, C. (1993). ${ }^{15} \mathrm{~N}$ abundance of forests is correlated with losses of Nitrogen. Plant and Soil 157 (1), pp. 147-150.

Högberg, P., Johannisson, C., Högberg, M. und Högbom, L. (1995). Measurements of abundances of ${ }^{15} \mathrm{~N}$ and ${ }^{13} \mathrm{C}$ as tools in retrospective studies of $\mathrm{N}$ balances and water stress in forests: A discussion of preliminary results. Plant and Soil 168-169, pp. 125-133.

Högberg, P. und Kvarnström, M. (1982). Nitrogen fixation by the woody legume Leucaena leucocephala in Tanzania. Plant and Soil 66, pp. 21-28.

Högberg, P., Näsholm, T., Högbom, L. und Stahl, L. (1994). Use of ${ }^{15} \mathrm{~N}$ labelling and ${ }^{15} \mathrm{~N}$ natural abundance to quantify the role of mycorrhizas in $\mathrm{N}$ uptake by plants: Importance of seed $\mathrm{N}$ and of changes in the ${ }^{15} \mathrm{~N}$ labelling of available N. New Phytologist 127, pp. 515519.

Hölscher, D. (1995). Wasser- und Stoffhaushalt eines Agrarökosystems mit Waldbrache im östlichen Amazonasgebietes. Göttinger Beiträge zur Land-und Forstwirtschaft in den Tropen und Subtropen 106.

Hoering, T.C. (1955). Variations of Nitrogen- ${ }^{15}$ abundance in naturally occurring substances. Science 122, pp. 1233-1234.

Hoering, T.C. und Ford, H.T. (1960). The isotope effect in the fixation of Nitrogen by Azotobacter. Journal of the American Chemical Society 82, pp. 376-378.

Hoghjensen, H. und Kristensen, E.S. (1995). Estimation of biological $\mathrm{N}_{2}$ fixation in a clovergrass system by the ${ }^{15} \mathrm{~N}$ dilution method and the total-N difference method. Biological Agriculture and Horticulture 11 (1-4), pp. 203-219.

Hoghjensen, H. und Schjoerring, J.K. (1994). Measurement of biological Dinitrogen fixation in grassland: Comparison of the enriched ${ }^{15} \mathrm{~N}$ dilution and the natural ${ }^{15} \mathrm{~N}$ abundance methods at different Nitrogen application rates and defoliation frequencies. Plant and Soil 166 (2), pp. 153-163.

Hossain, S.A., Waring, S.A., Strong, W.M., Dalal, R.C. und Weston, E.J. (1995). Estimates of Nitrogen fixations by legumes in alternate cropping systems at Warra, Queensland, using enriched ${ }^{15} \mathrm{~N}$ dilution and natural ${ }^{15} \mathrm{~N}$ abundance technique. Australian Journal of Agricultural Research 46, pp. 493-505.

Humphreys, L.R. (1995). Diversity and productivity of tropical legumes. Tropical legumes in animal nutrition. CAB International, Wallingford, pp. 1-21.

IDESP (1990). Anuário estatístico do Estado do Pará 1990. Instituto do Desenvolvimento Economico e Social do Pará, 11(1).

Izaguirre-Mayoral, M.L., Vivas, A.I. und Oropeza, T. (1995). New insights into the symbiotic performance of native tropical legumes: I. Analysis of the response of thirty-seven native legume species to artificial shade in a neotropical savanna. Symbiosis 19, pp. 111-129. 
Jacobi, I. (1997). Der Beitrag von Keimlingen zur Regeneration der holzigen Brachevegetation in der östlichen Amazonasregion. Dissertation an der Universität Hamburg, Fachbereich Angewandte Botanik.

Janos, D.P. (1983). Tropical mycorrhizas, nutrient cycles and plant growth. In: Sutton, S.H., Whitmore, T.C. und Chadwick, A.C. (Hrsg.), Tropical rain forest: Ecology and management. Blackwell, Oxford, pp. 327-345

Jonsson, K., Stahl, L. und Högberg, P. (1996). Tree fallows: A comparison between five tropical tree species. Biology and Fertility of Soils 23 (1), pp. 50-56.

Jordan, C.F. (Hrsg.) (1989). An Amazonian rainforest - The structure and function of a nutrient stressed ecosystem and the impact of slash-and-burn agriculture. Man and the Biosphere Series 2, UNESCO and The Parthenon Publishing Group.

Jordan, C.F., Caskey, W., Escalante, G., Herrera, R., Montagnini, F., Todd, R., and Uhl, C. (1982). The Nitrogen cycle in a 'terra firme' rainforest on oxisol in the Amazon territory of Venezuela. Plant and Soil 67, pp. 325-332.

Jordan, C.F., Todd, R.L. und Escalante, G. (1979). Nitrogen conservation in a tropical rain forest. Oecologia 39, pp. 123-128.

Jorge, A.L.F.A. (1993). Phytomass development in the secondary vegetation of abandoned fields in the eastern Amazon. M. Sc. Thesis (Technische Universität, Karlsruhe).

Junk, G. und Svec, H.J. (1958). The absolute abundance of the $\mathrm{N}$ isotope in the atmosphere and compressed gas from various sources. Geochimica et Cosmochimica Acta 14, pp. 234243.

Kadiata, B.D. und Mulongoy, K. (1995). Early Nitrogen fixation and utilization in Albizia lebbeck, Leucaena leucocephala, and Gliricidia sepium using Nitrogen $\left({ }^{15} \mathrm{~N}\right)$ labelling. Communication of Soil Science and Plant Analysis 26, (9-10), pp. 1397-1409.

Karamanos, R.E. und Rennie, D.A. (1978). Nitrogen isotope fractionation during Ammonium exchange reactions with soil clay. Canadian Journal of Soil Science 58, pp. 53.

Karamanos, R.E. und Rennie, D.A. (1980). Variations in natural ${ }^{15} \mathrm{~N}$ abundance as an aid in tracing fertilizer Nitrogen transformations. Soil Science Society of America Journal 44, pp. $57-62$.

Karamanos, R.E., Voroney, R.P. und Rennie, D.A. (1981). Variations in natural ${ }^{15} \mathrm{~N}$ abundance of central Saskatchewan soils. Soil Science Society of America Journal 45, pp. 826-828.

Kauffman, J.B., Cummings, D.L. und Ward, D.E. (1994). Relationship of fire, biomass and nutrient dynamics along a vegetation gradient in the brazilian cerrado. Journal of Ecology 82, pp. 519-531.

Kauffman, J.B., Cummings, D.L., Ward, D.E. und Babbitt, R. (1995). Fire in the brazilian Amazon: Biomass, nutrient pools, and losses in slashed primary forests. Oecologia 104 (4), pp. 397-408.

Kessel, C.van, Farrell, R.E. und Pennock, D.J. (1994). Carbon-13 and Nitrogen-15 natural abundance in crop residues and soil organic matter. Soil Science Society of America Journal 58, pp. 382-389. 
Kessel, C.van, Farrell, R.E., Roskoski, J.P. und Keane, K.M. (1994). Recycling of the naturally-occurring ${ }^{15} \mathrm{~N}$ in an established stand of Leucaena leucocephala. Soil Biology and Biochemistry 26 (6), pp. 757-762.

Kessel, C.van und Roskoski, J.P. (1983). Nodulation and $\mathrm{N}_{2}$ fixation by Inga jinicuil, a woody legume in coffee plantations. III. Effect of fertilizers and soil shading on nodulation and Nitrogen fixation (acetylene reduction) of Inga jinicuil seedlings. Plant and Soil 72, pp. 95105.

Kim, K.R. and Craig, H. (1993). Nitrogen-15 and Oxygen-18 characteristics of Nitrous Oxide - A global perspective. Science 262(5141), pp. 1855-1857.

Knowles, R. (1980). 9. Denitrification in soils. In: Advances in agricultural microbiology, pp. 243-266.

Kohl, D.H., Shearer, G. und Harper, J.E. (1980). Estimates of $\mathrm{N}_{2}$ fixation based on differences in the natural abundance of ${ }^{15} \mathrm{~N}$ in nodulating and nonnodulating isolines of soybeans. Plant Physiology 66, pp. 61-65.

Kurdali, F., Domenach, A.-M., Bouvarel, L. und Moiroud, A. (1993). Field comparison of $\delta^{15} \mathrm{~N}$ values and growth of Alder provenances and species. Soil Science and Plant Nutrition 39 (4), pp. 635-643.

Ladha, J.K., Peoples, M.B., Garrity, D.P. und Dart, P.J. (1993). Estimating Dinitrogen fixation of hedgerow vegetation using the Nitrogen-15 natural abundance method. Soil Science Society of America Journal 57, pp. 732-737.

Lajtha, K. und Marshall, J.D. (1994). Sources of variation in the stable isotopic composition of plants. In: Lajtha,K. und Michener, R.H.. Stable isotopes in ecology and environmental science, Blackwell Scientific Publications, Oxford, pp. 1-21.

Ledgard, S.F. (1989). Nutrition, moisture and rhizobial strain influence isotopic fractionation during $\mathrm{N}_{2}$ fixation in pasture legumes. Soil Biology and Biochemistry 21 (1), pp. 65-68.

Ledgard, S.F., Freney, J.R. und Simpson, J.R. (1984). Variations in natural enrichment of ${ }^{15} \mathrm{~N}$ in the profiles of some australian pasture soils. Australian Journal of Soil Research 22, pp. 155-164.

Ledgard, S.F. and Giller, K.E. (1995). Atmospheric $\mathrm{N}_{2}$ fixation as an alternative $\mathrm{N}$ source. In: Bacon, P.E. (Hrsg.), Nitrogen Fertilization in the Environment. Marcel Dekker Inc., NY, pp. 443-486.

Ledgard, S.F. and Peoples, M.B. 1988. Measurement of Nitrogen fixation in the field. In: Wilsons, J.R. (Hrsg.), Advances in N cycling in agricultural ecosystems, Proceedings of the symposium held in Brisbane, Australia, 11.-15. Mai 1987, CAB International, p. 351-367.

Ledgard, S.F., Simpson, J.R., Freney, J.R. und Bergersen, F.J. (1985). Effect of reference plant on estimation of Nitrogen fixation by subterranean clover using ${ }^{15} \mathrm{~N}$ methods. Australian Journal of Agricultural Research 36, pp. 663-676.

Lehmann, J., Schroth, G. und Zech, W. (1995). Decomposition and nutrient release from leaves, twigs and roots of three alley-cropping tree legumes in central Togo. Agroforestry Systems 29, pp. 21-36.

Leigh, J.H., Halsall, D.M. und Holgate, M.D. (1995). The role of allelopathy in legume decline in pasture. I. Effects of pasture and crop residues on germination and survival of subterranean clover in the field and nursery. Australian Journal of Agricultural Research 46, pp. 179-188. 
Lenthe, H.R. und Cordeiro, J.C. (1985). Explicações sobre o sistema brasileiro de classificação de solos. Ministério do Interior, Dep. Nacional de Obras contra Secas, Ministério da Cooperação Económica, GTZ, Teresina-Pauí.

Lessa, A.S.N., Anderson, D.W. und Moir, J.O. (1996). Fine root mineralization, soil organic matter and exchangeable cation dynamics in slash and burn agriculture in the semi-arid northeast of Brazil. Agriculture, Ecosystems and Environment 59, pp. 191-202.

Li, Y.S., Redmann, R.E. und Kessel, C.van (1992). Nitrogen budget and ${ }^{15} \mathrm{~N}$ translocation in a perennial wheatgrass. Functional Ecology 6, pp. 221-225.

Liu, K.K., Su, M.J., Hsueh, C.R. und Gong, G.C. (1996). The Nitrogen isotopic composition of Nitrate in the Kuroshio water northeast of Taiwan: Evidence for Nitrogen fixation as a source of isotopically light nitrate. Marine Chemistry 54 (3-4), pp. 273-292.

Liyanage, M.d., Danso, S.K.A. und Jayasundara, H.P.S. (1994). Biological Nitrogen fixation in four Gliricidia sepium genotypes. Plant and Soil 161, pp. 267-274.

Luizão, R., Bonde, T.A. und Rosswall T. (1992). Seasonal variation of soil microbial biomass - the effects of clear felling a tropical rainforest and establishment of pasture in the Central Amazon. Soil Biology and Biochemistry 6, pp. 1-22.

Lunaorea, P. und Wagger, M.G. (1996). Management of tropical legume cover crops in the bolivian Amazon to sustain crop yields and soil productivity. Agronomy Journal 88 (5), pp. 765-776.

Mackensen, J., Hölscher, D., Klinge, R. und Fölster, H. (1996). Nutrient transfer to the atmosphere by burning of debris in eastern Amazonia. Forest Ecology and Management 86, pp. 121-128.

Magalhães, F.M.M., Magalhães, L.M.S., Oliveira, L.A. de und Döbereiner, J. (1982). Occorência de nodulação em leguminosas florestais de terra firme nativas da região de Manaus-AM. Acta Amazônica 12 (3), pp. 509-514.

Mallarino, A.P., Wedin, W.F., Goyenola, R.S., Perdomo, C.H. und West, C.P. (1990). Legume species and proportion effects on symbiontic Dinitrogen fixation in legume-grass mixtures. Agronomy Journal 82, pp. 785-789.

Mallarino, A.P., Wedin, W.F., Perdomo, C.H., Goyenola, R.S. und West, C.P. (1990). Nitrogen transfer from white Clover, red Clover, and Birdfoot Trefoil to associated grass. Agronomy Journal 82, pp. 790-795.

Mariotti, A. (1983). Atmospheric Nitrogen is a reliable standard for natural ${ }^{15} \mathrm{~N}$ abundance measurements. Nature 303, pp. 685-687.

Mariotti, A., Mariotti, F., Amarger, N., Pizelle, G., Ngambi, J.M., Champigny, M.L., und Moyse, A. (1980a). Fractionements isotopique de l'azote lors de processus d'absorption des nitrates et de fixation de l'azote atmosphérique par les plantes. Physiologie vegetale 18 (1), pp. 163-181.

Mariotti, A., Mariotti, F., Champigny, M.L., Amarger, N. und Moyse, A. (1982). Nitrogen isotope fractionation associated with Nitrate reductase activity and uptake of $\mathrm{NO}_{3}{ }^{-}$by Pearl Millet. Plant Physiology 69, pp. 880-884.

Mariotti, A., Pierre, D., Vedy, J.C., Bruckert, J. und Guillemot, J. (1980b). The abundance of natural $\mathrm{N}^{15}$ in the organic matter of soils along an altitudinal gradient. Catena 7, pp. 293-300. 
Marrs, R.H., Thompson, J., Scott, D. und Proctor, J. (1991). Nitrogen mineralization and nitrification in terra-firme-forest and savanna soils on Ilha de Maracá, Roraima, Brazil. Journal of Tropical Ecology 7, pp. 123-137.

Martinelli, L.A., Victoria, R.L., Trivelin, P.C.O., Davol, A.H. und Richey, J.E. (1992). ${ }^{15} \mathrm{~N}$ natural abundance of plants of the Amazon river floodplain and potential atmospheric $\mathrm{N}_{2}$ fixation. Oecologia 90 (4), pp. 591-596.

Matson, P.A. und Vitousek, P.M. (1987). Cross-system comparisons of soil Nitrogen transformations and Nitrous oxide flux in tropical forest ecosystems. Global Biochemical Cycles 1 (2), pp. 163-170.

Matson, P.A., Vitousek, P.M., Livingstone, G.P. und Swanberg, N.A. (1990). Sources of variation in nitrous oxide flux from Amazonian ecosystems. Journal of Geophysical Research 95 (d10), pp. 789-816.

McAuliffe, C., Chamblee, D.S., Uribe-Arango, H., und Woodhouse, W.W.J. (1958). Influence of inorganic Nitrogen on Nitrogen fixation by Legumes as revealed by ${ }^{15} \mathrm{~N}$. Agronomy Journal 50, pp. 334-337.

McKey, D. (1994). Legumes and Nitrogen: The evolutionary ecology of a Nitrogendemanding lifestyle. In: Sprent, J.I., McKey, D. (Hrsg.), Advances in Legume Systematics 5: The Nitrogen Factor. Royal Botanical Garden, pp. 211-228.

Michelsen, A., Schmidt, I.K., Jonasson, S., Quarmby, C. und Sleep, D. (1996). Leaf ${ }^{15} \mathrm{~N}$ abundance of subarctic plants provides field evidence that ericoid, ectomycorrhizal and nonand arbuscular mycorrhizal species access different sources of soil Nitrogen. Oecologia 105, pp. 53-63.

Michelsen, A. und Sprent, J.I. (1994). The influence of vesicular-arbuscular mycorrhizal fungi on the Nitrogen fixation of nursery-grown Ethiopian Acacias estimated by the $15 \mathrm{~N}$ natural abundance method. Plant and Soil 160, pp. 249-257.

Minagawa, M. und Wada, E. (1984). Stepwise enrichment of ${ }^{15} \mathrm{~N}$ along food chains: Further evidence and the relation between $\delta^{15} \mathrm{~N}$ and animal age. Geochímica et Cosmochímica Acta 48, pp. 1135.

Minami, H., Minagawa, M. und Ogi, H. (1995). Changes in stable Carbon and Nitrogen isotope ratios in sooty and short-tailed shearwaters during their northward migration. The Condor 97, pp. 565-574.

Miranda, C.H.B. und Boddey, R.M. (1987). Estimation of BNF associated with 11 ecotypes of Panicum maximum grown in Nitrogen ${ }^{15}$ labelled soil. Agronomy Journal 79 (3), pp. 558563.

Mordelet, P., Cook, G., Abbadie, L., Grably, M. und Mariotti, A. (1996). Natural ${ }^{15} \mathrm{~N}$ abundance of vegetation and soil in the Kapalga savanna, Australia. Australian Journal of Ecology 21 (3), pp. 336-340.

Moreira, F.M. de S., Silva, M.F. da und Faria, S.M. de, (1992). Occurence of nodulation in legume species in the Amazon region of Brazil. New Phytologist 121, pp. 563-570.

Mosier, A.R. und Schimel, D.S. (1993). 7. Nitrification and denitrification. In: Knowles, R. und Blackburn, T.H. (Hrsg.), Nitrogen Isotope Techniques. Academic Press, San Diego, USA, pp. 181-208. 
Muhr, L., Leihner, D.E., Hilger, T.H. und Müller-Sämann, K.M. (1995). Intercropping of Cassava with herbaceous legumes. II. Yields as affected by below-ground competition. Angewandte Botanik 69, pp. 22-26.

Muhr, L., Leihner, D.E., Hilger, T.H. und Müller-Sämann, K.M. (1995). Intercropping of Cassava with herbaceous legumes. I. Rooting patterns and their potential importance for below-ground competition. Angewandte Botanik 69, pp. 17-21.

Myers, N. (1991). Tropical forests: present status and future outlook. In: Myers, N. (Hrsg.), Tropical forests and climate. Kluwer Academic Publishers, Dordrecht, pp. 3-32.

Nadelhoffer, K.J. und Fry, B. (1988). Controls on natural Nitrogen-15 and Carbon-13 abundances in forest soil organic matter. Soil Science Society of America Journal 52, pp. 1633-1640.

Nadelhoffer, K.J. und Fry, B. (1994). Nitrogen isotope studies in forest ecosystems. in: Lajtha, K. und Michener, R.H., Stable isotopes in ecology and environmental science. Blackwell Scientific Publications, Oxford, pp. 22-45.

Nadelhoffer, K.J., Shaver, G., Fry, B., Giblin, A., Johnson, L. und Mckane, R. (1996). ${ }^{15} \mathrm{~N}$ natural abundances and $\mathrm{N}$ use by tundra plants. Oecologia 107 (3), pp. 386-394.

Nair, P.K.R. (1986). Fruit trees in tropical agroforestry systems. Working paper 32, (ICRAF), Nairobi.

National Academy of Science, (1979). Tropical legumes: resources for the future. Washington DC.

Neill, C., Piccolo, M.C., Steudler, P.A., Melillo, J.M., Feigl, B.J. und Cerri, C.C. (1995). Nitrogen dynamics in soils of forests and active pastures in the western brazilian Amazon basin. Soil Biology and Biochemistry 27 (9), pp. 1167-1175.

Nepstad, D.C., Decarvalho, C.R., Davidson, E.A., Jipp, P.H. und Lefe, (1994). The role of deep roots in the hydrological and carbon cycles of Amazonian forests and pastures. Nature 372 (6507), pp. 666-669.

Nommik, H., Pluth, D.J., Larrson, K. und Mahendrappa, M.K. (1994). Isotopic fractionation accompanying fertilizer Nitrogen transformations in soil and trees of a Scots Pine ecosystem. Plant and Soil 158, pp. 169-182.

Norris, D.O. (1969). Observations on the nodulation status of rainforest leguminous species in Amazonia and Guyana. Tropical Agriculture 46, pp. 145-151.

Nosko, P., Bliss, L.C. und Cook, F.D. (1994). The association of free-living Nitrogen fixing bacteria with the roots of high arctic graminoids. Arctic and Alpine Research 26, pp. 180186.

Nunez, J.B.H. (1995). Fitomassa e estoque de bioelementos das diversas fases da vegetação secundária, provenientes de diferentes sistémas de uso da terra no nordeste Paraense, Brasil. M.Sc. Thesis (UFPa, Belém, Brasilien).

Ovalle, C., Longeri, L., Aronson, J., Herrera, A. und Avendano, J. (1996). $\mathrm{N}_{2}$-fixation, nodule efficiency and biomass accumulation after two years in three chilean legume trees and Tagasaste (Chamaecytisus proliferus subsp palmensis). Plant and Soil 179 (1), pp. 131-140.

Palm, C.A. und Sanchez, P.A. (1990). Decompostion and nutrient release patterns of the leaves of three tropical legumes. Biotropica 22 (4), pp. 330-338. 
Paparcikova, L. (1996). Bestimmung von $\delta^{15} \mathrm{~N}$ in der anorganischen N-Fraktion von Böden aus dem östlichen Amazonasgebiet Brasiliens. M. Sc. Thesis (Institut für Pflanzenbau und Tierproduktion in den Tropen und Subtropen, Göttingen).

Parrotta, J.A., Baker, D.D. und Fried, M. (1994). Application of ${ }^{15} \mathrm{~N}$-enrichment methodologies to estimate Nitrogen fixation in Casuarina equisetifolia. Canadian Journal of Forestry Research 24, pp. 201-207.

Parrotta, J.A., Baker, D.D., and Fried, M. (1996). Changes in Dinitrogen fixation in maturing stands of Casuarina equisetifolia and Leucaena leucocephala. Canadian Journal of Forestry Research 26(9), pp. 1684-1691.

Pate, J.S., Stewart, G.R. und Unkovich, M.J. (1993). ${ }^{15} \mathrm{~N}$ natural abundance of plant and soil components of a Banksia woodland ecosystem in relation to nitrate utilization, life form, mycorrhizal status and $\mathrm{N}_{2}$-fixing abilities of component species. Plant, Cell and Environment 16, pp. 365-373.

Pate, J.S., Unkovich, M.J., Armstrong, E.L. und Sanford, P. (1994). Selection of reference plants for $\delta^{15} \mathrm{~N}$ natural abundance assessment of $\mathrm{N}_{2}$ fixation by crop and pasture legumes in South-west Australia. Australian Journal of Agricultural Research 45, pp. 133-147.

Paul, E.A. (1978). Contribution of Nitrogen fixation to ecosystem functioning and Nitrogen fluxes on a global basis. Ecological Bulletin 26, pp. 282-293.

Pearson, J. und Stewart, G.R. (1993). The deposition of atmospheric Ammonia and its effects on plants. New Phytologist 125 (2), pp. 283-305.

Peoples, M.B., Bergersen, F.J., Brockwell, J., Fillery, I.R.P. und Herridge, D.F. (1995b). Management of Nitrogen for sustainable agricultural systems. Proceedings of an FAO/IAEA regional seminar for Asia and the Pacific "Nuclear methods in soil-plant aspects of sustainable agriculture" 5.-9.4.1993, (IAEA), Colombo, Sri Lanka, pp. 17-35.

Peoples, M.B., Bergersen, F.J., Turner, G.L., Sampet, C., Rerkasem, B., Bhromsiri, A., Nurhayati, D.P., Faizah, A.W., Sudin, M.N., Norhayati, M., and Herridge, D.F. (1991). Use of the natural enrichment of ${ }^{15} \mathrm{~N}$ in plant available soil $\mathrm{N}$ for the measurement of symbiotic $\mathrm{N}_{2}$ fixation. in: Stable isotopes in plant nutrition, soil fertility and environmental studies (IAEA, Wien), pp. 117-128.

Peoples, M.B., Faizah, A.W., Rerkasem, B. und Herridge, D.F. (1989). Methods for evaluating Nitrogen fixation by nodulated legumes in the field. ACIAR Monograph Series 11VII, (ACIAR), Canberra, pp. 1-76.

Peoples, M.B., Herridge, D.F. und Ladha, J.K. (1995a). Biological Nitrogen fixation: An efficient source of Nitrogen for sustainable agricultural production. Plant and Soil 174, pp. $3-28$.

Peoples, M.B., Palmer, B., Lilley, D.M., Duc, L.M. und Herridge, D.F. (1996). Application of ${ }^{15} \mathrm{~N}$ and xylem ureide methods for assessing $\mathrm{N}_{2}$ fixation of three shrub legumes periodically pruned for forage. Plant and Soil 182 (1), pp. 125-137.

Peoples, M.B., Sudin, M.N. und Herridge, D.F. (1987). Translocation of Nitrogenous compounds in symbiotic and Nitrate-fed Amide-exporting legumes. Journal of Experimental Botany 38 (189), pp. 567-579.

Peterson, B.J. and Fry, B. (1987). Stable isotopes in ecosystem studies. Annual Review of Ecologicy and Systematics 18, pp. 293-320. 
Piccolo, M.C., Neill, C. und Cerri, C.C. (1994a). Natural abundance of ${ }^{15} \mathrm{~N}$ in soils along forest-to-pasture chronosequences in the western brazilian Amazon Basin. Oecologia 99, pp. 112-117.

Piccolo, M.C., Neill, C. und Cerri, C.C. (1994b). Net Nitrogen mineralization and Net nitrification along a tropical forest-to-pasture chronosequence. Plant and Soil 162, pp. 61-70.

Piccolo, M.C., Neill, C., Melillo, J.M., Cerri, C.C. und Steudler, P.A. (1996). ${ }^{15} \mathrm{~N}$ natural abundance in forest and pasture soils of the brazilian Amazon basin. Plant and Soil 182, pp. 249-258.

Prieto Fernandez, A., Villar, M.C., Carballas, M. und Carballas, T. (1993). Short-term effects of a wildfire on the Nitrogen status and its mineralization kinetics in an atlantic forest soil. Soil Biology and Biochemistry 25 (12), pp. 1657-1664.

Rao, A.V. und Giller, K.E. (1993). Nitrogen fixation and its transfer from Leucaena to grass using ${ }^{15}$ N. Forest Ecology and Management 61 (3-4), pp. 221-227.

Raven, J.A. und Sprent, J.I. (1989). Phototrophy, diazotrophy and paleoatmospheres: Biological catalysis and the H, C, N and O cycles. Journal of the Geological Society 146, pp. 161-170.

Redmon, L.A., Rouquette, F.M., Smith, G.R. und Stuth, J.W. (1994). Nitrogen transfer from warm-season annual legumes to pearl millet. Journal of Plant Nutrition 18 (4), pp. 803-813.

Rego, R.S., Silva, B.R.N.d. und Junior, R.S.O. (1993). Detailed soil survey in an area in the municipality of Igarapé Açu. Summeries and lectures presented at the 1st SHIFT- workshop in Belém, February 1992.

Reineking, A., Langel, R. und Schikowski, J. (1993). ${ }^{15} \mathrm{~N},{ }^{13} \mathrm{C}$-on-line measurement with an elemental analyser (Carlo Erba, NA 1500), a modified trapping box and a gas isotope mass spectrometer (Finnigan MAT 251). Isotopenpraxis Environmental Health Studies 29, pp. 169-174.

Rennie, D.A., Paul, E.A. und Johns, L.E. (1976). Natural Nitrogen-15 abundance of soil and plant samples. Canadian Journal of Soil Science 56, pp. 43-50.

Rennie, R.J. und Rennie, D.A. (1983). Techniques for quantifying $\mathrm{N}_{2}$ fixation in association with nonlegumes under field and greenhouse conditions. Canadian Journal of Microbiology 29, pp. 1022-1035.

Rice, E.L. (1984). Allelopathy. Academic Press, Inc., Orlando, Florida.

Riga, H., Praag, H.J. van und Brigode, N. (1971). Rapport isotopique naturel de l'azote dans quelques sols forestieres et agricoles de belgique soumis à divers traitements culturaux. Geoderma 6, pp. 213-222.

Robertson, G.P. (1989). Nitrification and denitrification in humid tropical ecosystems: potential controls on Nitrogen retention. Mineral nutrients 178, pp.55-69.

Rondon, M.A. und Thomas, R.J. (1994). A piston-action ball mill for the rapid preparation of plant and soil samples for the automated analysis of Nitrogen $\left({ }^{15} \mathrm{~N}\right)$ and Carbon $\left({ }^{13} \mathrm{C}\right)$. Communication of Soil Science and Plant Analysis 25 (3+4), pp. 435-445.

Rosecrance, R.C., Brewbaker, J.L. und Fownes, J.H. (1992). Alley cropping of maize with nine leguminous trees. Agroforestry Systems 17, pp. 159-168.

Rosetti, D.F., Goes, A.M. und Truckenbrodt, W. (1990). A influência marinha nos sedimentos barreiras. Boletim do Museu Paraense Emílio Goeldi, Ciências da Terra 2, (17), pp. 29. 
Ruinen, J. (1974). Nitrogen fixation in the phyllosphere. In: Quispel, A. (Hrsg.), NorthHolland Research Monographs 'Frontier of Biology' The Biology of Nitrogen Fixation 33. Amsterdam, pp. 121-167.

Salati, E., Sylvester-Bradley, R. und Victoria, R.L. (1982). Regional gains and losses of Nitrogen in the Amazon basin. Plant and Soil 67, pp. 367-376.

Saldarriaga, J.G., West, C.D., Tharp, M.L. und Uhl, C. (1988). Long-term chronosequence of forest succession in the upper Rio Negro of Colombia and Venezuela. Journal of Ecology 76, pp. 938-958.

Sanchez, P.A., Bandy, D.E., Villachica, J.H. und Nicholaides, J.J. (1982). Amazon basin soils: management for continous crop production. Science 216, pp. 821-827.

Sanchez, P.A. und Palm, C.A. (1996). Nutrient cycling and agroforestry in Africa. Unasylva 47(2), (185), pp. 24-28.

Sanford, P., Pate, J.S. und Unkovich, M.J. (1993). A Survey of porportional dependence of Subterranean Clover and other pasture legumes on $\mathrm{N}_{2}$ fixation in South-West Australia utilizing ${ }^{15} \mathrm{~N}$ natural abundance. Australian Journal of Agricultural Research 45, pp. 165181.

Sanford, P., Pate, J.S., Unkovich, M.J. und Thompson, A.N. (1995). Nitrogen fixation in grazed and ungrazed subterranean clover pasture in south-west Australia assessed by the ${ }^{15} \mathrm{~N}$ natural abundance technique. Australian Journal of Agricultural Research 46, (7), pp. 1427 1443.

Sanginga, N. (1995). Management of biological processes in alley farming: need for more research. In: Proceedings of an FAO/IAEA regional seminar for Asia and the Pacific "nuclear methods in soil-plant aspects of sustainable agriculture" 5.-9.4.1993, (IAEA), Colombo, Sri Lanka, pp. 113-119.

Sanginga, N., Danso, S.K.A. und Bowen, G.D. (1989). Nodulation and growth response of Allocasuarina and Casuarina species to Phosphorus fertilization. Plant and Soil 118, pp. 125-132.

Sanginga, N., Danso, S.K.A. und Zapata, F. (1996). Field measurements of Nitrogen fixation in leguminous trees used in agroforestry systems: influence of ${ }^{15} \mathrm{~N}$-labeling approaches and reference trees. Biology and Fertility of Soils 23, pp. 26-32.

Sanginga, N., Danso, S.K.A., Zapata, F. und Bowen, G.D. (1990). Influence of reference trees on $\mathrm{N}_{2}$-fixation estimates in Leucaena leucocephala and Acacia albida using ${ }^{15} \mathrm{~N}$-labelling techniques. Biology and Fertility of Soils 9, pp. 341-346.

Sanginga, N., Vanlauwe, B. und Danso, S.K.A. (1995). Management of biological $\mathrm{N}_{2}$ fixation in alley cropping systems: estimation and contribution to $\mathrm{N}$ balance. Plant and Soil 174, pp. 119-141.

Sanginga, N., Zapata, F., Danso, S.K.A. und Bowen, G.D. (1990). Effect of successive cutting on nodulation and Nitrogen fixation of Leucaena leucocephala using ${ }^{15} \mathrm{~N}$ dilution and the difference methods. Plant Nutrition - Physiology and Application, pp. 667-674.

Sanginga, N., Zapata, F., Danso, S.K.A. und Bowne, G.D. (1990). Effect of successive cuttings on uptake and partitioning of ${ }^{15} \mathrm{~N}$ among plant parts of Leucaena leucocephala. Biology and Fertility of Soils 9, pp. 37-42. 
Sanginga, N., Zapata, N. und Danso, S.K.A. (1992). Nitrogen fixation in tropical trees: estimations based on ${ }^{15} \mathrm{~N}$ techniques. Maximisez la FBA pour la Production et Forestiére en Afrique.

Sanhueza, E. (1982). The role of the atmosphere in Nitrogen cycling. Plant and Soil 67, pp. 61-71.

Schachtschabel, P., Blume, H.-P., Brümmer, G., Hartge, K.-H., and Schwertmann, U. (1989). Lehrbuch der Bodenkunde. Ferdinand Enke Verlag Stuttgart.

Schlegel, H.G. (1992). Allgemeine Mikrobiologie. Georg Thieme Verlag, Stuttgart.

Schmidt-Lorenz, R. (1986). Die Böden der Tropen und Subtropen. Handbuch der Landwirtschaft und Ernährung in den Entwicklungsländern 3, (Hrsg.: Rehm, S.), Ulmer, Stuttgart.

Schöneberger, M.M., Volk, R.J. und Davey, C.B. (1989). Selection of reference plants and methods for estimating $\mathrm{N}_{2}$ fixation in mycorrhizal leguminous plants. Soil Science Society of America Journal 53, pp. 1425-1429.

Schulze, E.-D., Chapin III, F.S. und Gebauer, G. (1994). Nitrogen nutrition and isotope differences among life forms at the northern treeline of Alaska. Oecologia 100, pp. 406-412.

Schulze, E.-D., Gebauer, G., Ziegler, H. und Lange, O.L. (1991). Estimates of Nitrogen fixation by trees in an aridity gradient in Namibia. Oecologia 88, pp. 451-455.

Selles, F., Karamanos, R.E. und Kachanoski, R.G. (1986). The spatial variability of Nitrogen15 and its relation to the variability of other soil properties. Soil Science Society of America Journal 50, pp. 105-110.

Shearer, G. und Kohl, D.H. (1986). $\mathrm{N}_{2}$-fixation in field settings: Estimations based on natural

${ }^{15} \mathrm{~N}$ abundance. Australian Journal of Plant Physiology 13, pp. 699-756.

Shearer, G. und Kohl, D.H. (1988a). 20: Estimates of $\mathrm{N}_{2}$ fixation in ecosystems: the need for and basis of the ${ }^{15} \mathrm{~N}$ natural abundance method. Ecological Studies 68, pp. 342-374.

Shearer, G. und Kohl, D.H. (1988b). Natural ${ }^{15} \mathrm{~N}$ abundance as a method of estimating the contribution of biologically fixed Nitrogen to $\mathrm{N}_{2}$-fixing systems: Potential for non-legumes. Plant and Soil 110, pp. 317-327.

Shearer, G. und Kohl, D.H. (1993). 4. Natural abundance of ${ }^{15} \mathrm{~N}$ : Fractional contribution of two sources to a common sink and use of isotope discrimination. In: Knowles, R., Blackburn, T.H. (Hrsg.), Nitrogen isotope techniques, Academic Press, pp. 89-125.

Shearer, G., Kohl, D.H. und Chien, S.-H. (1978). The Nitrogen- ${ }^{15} \mathrm{~N}$ abundance in a wide variety of soils. Soil Science Society of America Journal 42, pp. 899-902.

Shearer, G., Kohl, D.H. und Commoner, B. (1974a). A steady state model of isotopic fractionation accompanying Nitrogen transformations in soil. Soil Science Society of America Proceedings 38, pp. 315-322.

Shearer, G., Kohl, D.H. und Commoner, B. (1974b). The precision of determinations of the natural abundance of Nitrogen-15 in soils, fertilizer and shelf chemicals. Soil Science 118, pp. 308-316.

Shearer, G., Kohl, D.H. und Harper, J.E. (1980). Distribution of ${ }^{15} \mathrm{~N}$ among plant parts of nodulating and nonnodulating isolines of soybeans. Plant Physiology 66, pp. 57-60. 
Shearer, G., Kohl, D.H., Virginia, R.A., Bryan, B.A., Skeeters, J.L., Nilsen, E.T., Sharifi, M.R., und Rundel, P.W., (1983). Estimates of $\mathrm{N}_{2}$-fixation from variation in the natural abundance of ${ }^{15} \mathrm{~N}$ in Sonoran desert ecosystems. Oecologia 56, pp. 365-373.

Sioli, H. (1968). Zur Ökologie des Amazonasgebietes. Serie: Biogeography and Ecology in South America (Hrsg.: Fittkau, E.J., Illies, J., Klinge, H., Schwabe, G.H., Sioli, H.), Dr.W.Junk, Den Haag.

Singh, R. und Möller, M.R.F. (1984). Disponibildade de micronutrientes em classes de solos dominantes do trópico úmido brasileiro. Zinco. EMBRAPA-CPATU, Belém. Boletim de Pesquisa 55, pp. 1-43.

Somasegaran, P. und Hoben, H.J. (1985). Methods in legume-Rhizobium technology. University of Hawaii NifTAL Project and MIRCEN.

Sommer, R. (1997). Kohlenstoffvorräte in Böden unter intensiv genutzten Sekundärwaldflächen im östlichen Amazonasgebiet. Diplomarbeit, Universität Göttingen.

Souza, L.A.G. de, Silva, M.F. da und Moreira, F.W. (1994). Capacidade de nodulação de cem Leguminosas da Amazônia. Acta Amazônica 24, (1/2), pp. 9-19.

Sprent, J.I. (1995). Legume trees and shrubs in the Tropics: $\mathrm{N}_{2}$ fixation in perspective. Soil Biology and Biochemistry 27 (4-5), pp. 401-407.

Sprent, J.I., Geoghegan, I.E., Whitty, P.W. und James, E.K. (1996). Natural abundance of ${ }^{15} \mathrm{~N}$ and ${ }^{13} \mathrm{C}$ in nodulated legumes and other plants in the cerrado and neighbouring regions of Brazil. Oecologia 105, pp. 440-446.

Sprent, J.I. (1994). Evolution and diversity in the legume-Rhizobium symbiosis: Chaos theory? Plant and Soil 161, pp. 1-10.

Stacey, G., Burris, R.H. und Evans, H.J. (Hrsg.) (1992). Biological Nitrogen fixation. Chapman und Hall, New York, London.

Steele, K.W., Bonish, P.M. und O'Hara, G.W. (1983). Effect of rhizobial strain and host plant on Nitrogen isotopic fractionation in legumes. Plant Physiology 72, pp. 1001-1004.

Steele, K.W., Wilson, A.T. und Saunders, W.M.H. (1981). Nitrogen isotope ratios in surface and sub-surface horizons of New Zealand improved grassland soils. New Zealand Journal of Agricultural Research 24, pp. 167-170.

Stephenson, F.J. (1986). Cycles of soil - Carbon, Nitrogen, Phosphorus, Sulfur, Micronutrients. J.Wiley and Son, New York.

Stephenson, R.A. and Raisson, R.J. (1988). Nitrogen cycling in tropical evergreen tree crop ecosystems. In: Wilsons, J.R. (Hrsg.), Advances in $\mathrm{N}$ cycling in agricultural ecosystems. Brisbane, Australia, CAB international, pp. 315-332.

Stevens, P.R. und Walker, T.W. (1970). The chronosequence concept and soil formation. The Quarterly Review of Biology 45, pp. 333-350.

Stewart, G.R., Schmidt, S., Handley, L.L., Turnbull, M.H. und Erskin, (1995). ${ }^{15} \mathrm{~N}$ natural abundance of vascular rainforest epiphytes: Implications for Nitrogen source and acquisition. Plant and Cell and Environment 18 (1), pp. 85-90.

Stock, W.D., Wienand, K.T. und Baker, A.C. (1995). Impacts of invading $\mathrm{N}_{2}$-fixing Acacia species on patterns of nutrient cycling in two cape ecosystems: Evidence from soil incubation studies and ${ }^{15} \mathrm{~N}$ natural abundance. Oecologia 101, pp. 375-382. 
Sutherland, R.A., Kessel, C. van, Farrell, R.E. und Pennock, D.J. (1993). Landscape-scale variations in plant and soil Nitrogen-15 natural abundance. Soil Science Society of America Journal 57, pp. 169-178.

Sutherland, R.A., Kessel, C. van und Pennock, D.J. (1991). Spatial variability of Nitrogen-15 natural abundance. Soil Science Society of America Journal 55, pp. 1339-1247.

Sylvester-Bradley, R., Oliveira, L.A.d., Podestá Filho, J.A. und St.John, T.V. (1980). Nodulation of legumes, Nitrogenase activity of roots and occurrence of Nitrogen-fixing Azospirillum spp. in representative soils of Central Amazonia. Agro-Ecosystems 6, pp. 249266.

Tamm, C.O. (1990). Nitrogen in terrestrial ecosystems. Springer Verlag Berlin.

Thompson, J., Proctor, J., Viana, V., Milliken, W., Ratter, J.A. und Scott, D.A. (1992). Ecological studies on a lowland evergreen rain forest on Maracá Island, Roraima, Brazil. I. Physical environment, forest structure and leaf chemistry. Journal of Ecology 80, pp. 689703.

Tiessen, H., Karamanos, R.E., Stewart, J.W.B. und Selles, F. (1984). Natural Nitrogen-15 abundance as as indicator of soil organic matter transformations in native and cultivated soils. Soil Science Society of America Journal 48, pp. 312-315.

Todd, R.L., Meyer, R.D. und Waide, J.B. (1978). Nitrogen fixation in a decidous forest in the south-eastern United States. Ecological Bulletin 26, pp. 172-177.

Tortoso, A.C. und Hutchinson, G.L. (1990). Contribution of autotrophic and heterotrophic nitrifiers to soil $\mathrm{NO}$ and $\mathrm{N}_{2} \mathrm{O}$ emissions. Applied Environmental Microbiology 56, pp. 17991805 .

Turner, G.L. und Bergersen, F.J. (1983). Natural abundance of ${ }^{15} \mathrm{~N}$ in root nodules of soybean, lupin, subterranean clover and lucerne. Soil Biology and Biochemistry 15 (5), pp. 525-530.

Turner, G.L., Bergersen, F.J. und Tantala, H. (1983). Natural enrichment of ${ }^{15} \mathrm{~N}$ during decomposition of plant material in soil. Soil Biology and Biochemistry 14 (4), p.495

Turner, G.L., Gault, R.R., Morthope, L., Chase, D.L. und Bergersen, F.J. (1987). Difference in the natural abundance of ${ }^{15} \mathrm{~N}$ in the extractable mineral Nitrogen of cropped and fallowed surface soils. Australian Journal of Agricultural Research 38, pp. 15-25.

Unkovich, M.J., Pate, J.S., Sanford, P. und Armstrong, E.L. (1994). Potential precision of the $\delta^{15} \mathrm{~N}$ natural abundance method in field estimates of Nitrogen fixation by crop and pasture legumes in South-West Australia. Australian Journal of Agricultural Research 45, pp. 119132.

Vasquez, F.J., Acea, M.J. und Carballas, T. (1993). Soil microbial populations after wildfire. FEMS Microbiology Ecology 13, pp. 93-104.

Vesterager, J.M., Osterby, S., Jensen, E.S. und Schjoerring, J.K. (1995). Symbiotic $\mathrm{N}_{2}$ fixation by the cover crop Pueraria phaseoloides as influenced by litter mineralization. Plant and Soil 177 (1), pp. 1-10.

Vieira, I.C.G. (1996). Forest succession after shifting cultivation in Eastern Amazonia. Ph. D. Thesis (University of Stirling), Scotland.

Vincent, J.M. (1970). A manual for the practical study of root-nodule bacteria. IBP Handbook No.15, Blackwell Scientific Publication, Oxford. 
Virginia, R.A. (1972). Natural abundance of Nitrogen-15 in selected ecosystems. Ph. D. Thesis, University of California, Davis.

Virginia, R.A. und Delwiche, C.C. (1982). Natural ${ }^{15} \mathrm{~N}$ abundance of presumed $\mathrm{N}_{2}$-fixing and non $\mathrm{N}_{2}$-fixing plants from selected ecosystems. Oecologia 54, pp. 317-325.

Virginia, R.A., Jarrell, W.M., Rundel, P.W., Shearer, G. und Kohl, D.H. (1989). The use of variations in the natural abundance of ${ }^{15} \mathrm{~N}$ to assess symbiotic Nitrogen fixation by woody plants. In: Rundel, R.W., Ehleringer, J.R., Nagy, K.A (Hrsg.), Stable isotopes in ecological research - Ecological Studies 68, Springer Verlag, New York, pp. 375-394.

Virginia, R.A., Jenkins, M.B. und Jarrell, W.M. (1986). Depth of root symbiont occurrence in soil. Biology and Fertility of Soils 2, pp. 127-130.

Vitousek, P.M. und Denslow, J.S. (1986). Nitrogen and Phosphorus availability in treefall gaps of a lowland tropical rainforest. Journal of Ecology 74, pp. 1167-1178.

Vitousek, P.M. und Matson, P.A. (1988). Nitrogen transformations in a range of tropical forest soils. Soil Biology and Biochemistry 20 (3), pp. 361-367.

Vitousek, P.M. und Matson, P.A. (1991). Gradient analysis of ecosystems. In: Cole, J., Covett, G. und Findley, S. (Hrsg.), Comparative Analysis of Ecosystems, Patterns, Mechanisms, and Theories. Springer Verlag, New York, pp. 287-298.

Vitousek, P.M., Shearer, G. und Kohl, D.H. (1989). Foliar ${ }^{15} \mathrm{~N}$ Natural Abundance in Hawaiian Rainforest: Patterns and possible Mechanisms. Oecologia 78, pp. 383-388.

Wada, E., Kadonaga, T. und Matsuo, S. (1975). ${ }^{15} \mathrm{~N}$ abundance in Nitrogen of naturally occuring substances and global assessment of denitrification from isotopic viewpoint. Geochemical Journal 9, pp. 139-148.

Wahlund, T.M. und Madigan, M.T. (1993). Nitrogen fixation by the thermophilic green sulphur bacterium Chlorobium tepidum. Journal of Bacteriology 175, pp. 474-478.

Walter, H. (1977). Vegetationszonen und Klima. Eugen Ulmer Verlag, Stuttgart.

Watrin, O. dos S. (1994). Estudo da dinâmica na paisagem da Amazônia oriental através de técnicas de geoprocessamento. M. Sc. (INPE-5631-TDI/555, INPE Sao José dos Campos), Brasilien.

Wellmann, R.P., Cook, F.D. und Krouse, H.R. (1968). Nitrogen-15 microbial alteration of abundance. Science 161, pp. 269-270.

Yoneyama, T. (1987). $\mathrm{N}_{2}$ fixation and natural ${ }^{15} \mathrm{~N}$ abundance of leguminous plants and Azolla. Bull. Nat. Inst. Agrobiol. Resourc. 3, pp. 59-87.

Yoneyama, T. (1996). Characterization of natural ${ }^{15} \mathrm{~N}$ abundance of soils. In: Boutton, T.W. und Yamasaki, S. (Hrsg.), Mass spectrometry of soils. Marcel Dekker, NY, pp. 205-223.

Yoneyama, T., Fujita, K., Yoshida, T., Matsumaoto, T., Kambayashi, I., and Yazaki, J. (1986). Variation in natural abundance of ${ }^{15} \mathrm{~N}$ among plant parts and in ${ }^{15} \mathrm{~N} /{ }^{14} \mathrm{~N}$-fractionation during $\mathrm{N}_{2}$ fixation in the legume-Rhizobia symbiotic system. Plant and Cell Physiology 27, (5), pp. 791-799.

Yoneyama, T., Kamachi, K., Yamaya, T. und Mae, T. (1993a). Fractionation of Nitrogen isotopes by Glutamine Synthetase isolated from Spinach leaves. Plant and Cell Physiology 34 (3), pp. 489-491. 
Yoneyama, T. und Kaneko, A. (1989). Variations in the natural abundance of the ${ }^{15} \mathrm{~N}$ in nitrogenous fractions of Komatsuna plants supplied with nitrate. Plant and Cell Physiology 30 (7), pp. 957-962.

Yoneyama, T., Murakami, T., Boonkerd, N., Wadisirésuk, P., Siripin, S. und Kouno, K. (1990). Natural ${ }^{15} \mathrm{~N}$ abundance in shrubs and tree legumes, Casuarina and non $\mathrm{N}_{2}$-fixing plants in Thailand. Plant and Soil 128, pp. 287-292.

Yoneyama, T., Muraoka, T., Murakami, T. und Boonkerd, N. (1993b). Natural abundance of

${ }^{15} \mathrm{~N}$ in tropical plants with emphasis on tree legumes. Plant and Soil 153, pp. 295-304.

Yoneyama, T., Nambiar, P.T.C., Lee, K.K., Rao, B.S. und Williams, J., H. (1990). Nitrogen accumulation in three legumes and two cereals with emphasis on estimation of $\mathrm{N}_{2}$ fixation in the legumes by the natural ${ }^{15} \mathrm{~N}$ abundance technique. Biology and Fertility of Soils 9, pp. 2530 .

Yoneyama, T., Omata, T., Nakata, S. und Yazaki, J. (1991a). Fractionation of Nitrogen isotopes during the uptake and assimilation of ammonia by plants. Plant and Cell Physiology 32 (8), pp. 1211-1217.

Yoneyama, T., Uchiyama, T., Sasakawa, H., Gamo, T., Ladha, J. und Watanabe, I. (1991b). $\mathrm{N}_{2}$-accumulation and changes in natural ${ }^{15} \mathrm{~N}$ abundance in the tissues of legumes with emphasis on $\mathrm{N}_{2}$ fixation by stem-nodulating plants in upland and paddy Fields. Soil Science and Plant Nutrition. 37, pp. 75-82. 
XII Anhang 
Anhang Tab. 49: $\delta^{15} \mathrm{~N}$ in Vegetation, Boden, organischer Substanz (SOM) und $\mathrm{N}_{\text {min. }}$ in den verschiedenen Regionen der Welt

\begin{tabular}{|c|c|c|c|c|c|c|c|c|}
\hline Ort, Klimazone & & Vegetation & \multicolumn{5}{|c|}{ Boden } & \multirow[t]{2}{*}{ Referenz } \\
\hline & Beschreibung & & Tiefe (cm) & $\mathbf{N}_{\text {tot. }}$ & $\mathrm{NO}_{3}^{-}$ & $\mathrm{NH}_{4}^{+}$ & SOM & \\
\hline Nordküste Alaskas & Tundra-Ökosystem, Tussok & $\begin{array}{c}\mathrm{R}:-7 \Leftrightarrow+3 \\
\mathrm{~L}:-1.5 \Leftrightarrow-0.1\end{array}$ & $0-30$ & $-3 \Leftrightarrow+3$ & & & $-3 \Leftrightarrow 0$ & $\begin{array}{l}\text { Nadelhoffer et } \\
\text { al.,1996 }\end{array}$ \\
\hline Nordalaska & Nadelwald & $-10 \Leftrightarrow+1.7$ & & & & & & $\begin{array}{l}\text { Schulze et al., } \\
1994\end{array}$ \\
\hline Lappland, Schweden & Tundra, montan, subarktisch & $\begin{array}{c}\mathrm{R}:-9 \Leftrightarrow+2 \\
\mathrm{~L}:-2\end{array}$ & $0-10$ & $-1 \Leftrightarrow+1$ & & & & $\begin{array}{l}\text { Michelsen et al., } \\
1996\end{array}$ \\
\hline $\begin{array}{l}\text { Süd Saskatchewan, } \\
\text { Canada }\end{array}$ & glazial-lakustrine Sedimente, Eichenwald & $+4 \Leftrightarrow+6$ & $0-10$ & +8.2 & & & & $\begin{array}{l}\text { Sutherland et al., } \\
1993\end{array}$ \\
\hline $\begin{array}{l}\text { Süd Saskatchewan, } \\
\text { Canada }\end{array}$ & Chernozem, Getreide & $+3.2 \Leftrightarrow+7.4$ & $0-10$ & $+7.7 \Leftrightarrow+8.3$ & $+1.9 \Leftrightarrow+7.4$ & & & $\begin{array}{l}\text { Sutherland et al., } \\
1991\end{array}$ \\
\hline Saskatchewan, Canada & Haploboroll & $+3.2 \Leftrightarrow+7.8$ & $0-20$ & & & & $+8.4 \Leftrightarrow+8.7$ & Kessel, 1994 \\
\hline \multirow[t]{2}{*}{ Saskatchewan, Canada } & Luvisol, Chernozem, Gleysol, Solonetz, Alfisol & & $0-20$ & $+8.4 \Leftrightarrow+9.4$ & & & & \multirow{2}{*}{$\begin{array}{l}\text { Karamanos et } \\
\text { al., } \\
1981\end{array}$} \\
\hline & Schwarzer Chernozem & & $0-20$ & $+3.5 \Leftrightarrow+25.2$ & & & & \\
\hline Saskatchewan, Canada & Agriborol, lehmiger Boden & & $0-20$ & $+7.7 \Leftrightarrow+8.8$ & & & & $\begin{array}{l}\text { Selles et al., } \\
1986\end{array}$ \\
\hline \multirow[t]{2}{*}{ Canada } & Grasland-Boden (schwarzer Chernozem) & $\begin{array}{l}\mathrm{R}:+1.3 \Leftrightarrow+4.2 \\
\mathrm{~L}:+0.7 \Leftrightarrow+1.6\end{array}$ & $0-20$ & $+6.1 \Leftrightarrow+8.7$ & $+2.7 \Leftrightarrow+12.8$ & & & \multirow[t]{2}{*}{$\begin{array}{l}\text { Rennie et al., } \\
1976\end{array}$} \\
\hline & Luvisolic Waldboden & & $0-20$ & $+4.6 \Leftrightarrow+6.2$ & +7 & & & \\
\hline Vancouver Island & Mischwald,unfruchtbarer Lehmton & $\begin{array}{l}\mathrm{R}:-5 \Leftrightarrow+9 \\
\mathrm{~L}:-4 \Leftrightarrow+9\end{array}$ & $0-60$ & $+1 \Leftrightarrow+6$ & $-3 \Leftrightarrow+1$ & $-5 \Leftrightarrow+2$ & & \multirow[t]{2}{*}{$\begin{array}{l}\text { Binkley et al., } \\
1985\end{array}$} \\
\hline Vancouver Island & Mischwald, fruchtbarer Lehmton & $\begin{array}{l}\mathrm{R}:-3 \Leftrightarrow+16 \\
\mathrm{~L}:-1 \Leftrightarrow+0.5\end{array}$ & $0-60$ & $+1 \Leftrightarrow+3$ & $-8 \Leftrightarrow-7$ & $-4 \Leftrightarrow-2$ & & \\
\hline USA, Maine & Caribou, Waldboden & & $0-15$ & $+3 \Leftrightarrow+8.5$ & & & & $\begin{array}{l}\text { Shearer et al., } \\
1978\end{array}$ \\
\hline \multirow[t]{2}{*}{ Wisconsin, Madison } & \multirow[t]{2}{*}{ Lehmton } & \multirow[t]{2}{*}{-1.8 (Wurzel) } & $0-10$ & $+2.6( \pm 0.33)$ & & & $-3.8( \pm 0.22)$ & \multirow{2}{*}{$\begin{array}{l}\text { Nadelhoffer } \\
\text { und Fry, } 1988\end{array}$} \\
\hline & & & $10-20$ & $+5.3( \pm 0.18)$ & & & & \\
\hline \multirow[t]{4}{*}{ USA, Iowa } & Edina schluffiger Lehm & & $0-15$ & $+1 \Leftrightarrow+2$ & & & & \multirow{4}{*}{$\begin{array}{l}\text { Cheng et al., } \\
1964\end{array}$} \\
\hline & tiefgründiger schluffig-toniger Lehm & & $0-15$ & $+16 \Leftrightarrow+20$ & $+1 \Leftrightarrow+6$ & $+3 \Leftrightarrow+7$ & & \\
\hline & schluffig-toniger Lehm, N-gedüngter Boden & & $0-45$ & $+11 \Leftrightarrow+12$ & & & & \\
\hline & Fargo schluffiger Lehm & & $0-15$ & -2 & & & & \\
\hline
\end{tabular}


Fortsetzung Anhang Tab. 49: $\delta^{15} \mathrm{~N}$ in Vegetation, Boden, organischer Substanz (SOM) und $\mathrm{N}_{\min .}$ in den verschiedenen Regionen der Welt

\begin{tabular}{|c|c|c|c|c|c|c|c|c|}
\hline Ort, Klimazone & & Vegetation & \multicolumn{5}{|c|}{ Boden } & Referenz \\
\hline & Beschreibung & & Tiefe $(\mathbf{c m})$ & $\mathbf{N}_{\text {tote }}$ & $\mathrm{NO}_{3}^{-}$ & $\mathbf{N H}_{4}{ }^{+}$ & SOM & \\
\hline \multirow[t]{4}{*}{ USA Iowa } & unbearbeitet & & $0-15$ & $-4.4 \Leftrightarrow-1.1$ & & & & \multirow{4}{*}{\begin{tabular}{|l} 
Bremner und \\
Tabatai, 1973
\end{tabular}} \\
\hline & kultiviert & & $0-15$ & $0 \Leftrightarrow+3$ & & & & \\
\hline & & & $0-15$ & $-4.1 \Leftrightarrow+1.6$ & & $-7.9 \Leftrightarrow-0.5$ & & \\
\hline & & & $15-30$ & $-0.8 \Leftrightarrow+1.9$ & & $-9.6 \Leftrightarrow+6.3$ & & \\
\hline Salt Lake City, USA & Wachholder-Wald & & $0-120$ & $+4.0 \Leftrightarrow+9.0$ & & & & \begin{tabular}{|l} 
Evans und \\
Ehleringer, 1993
\end{tabular} \\
\hline USA, Utah & Coral Pink Sand Dunes state Park & $\mathrm{R}:+0.2 \Leftrightarrow+2.9$ & & & & & & \begin{tabular}{|l} 
Evans und \\
Ehleringer, 1994 \\
\end{tabular} \\
\hline USA, Utah & schluffig-toniger Lehm, Boden unter Kräutern & & $0-15$ & $+7.8 \Leftrightarrow+12.7$ & & & & $\begin{array}{l}\text { Shearer et al., } \\
1978\end{array}$ \\
\hline Deutschland & Fichtelgebirge, Nadelwald & $-1 \Leftrightarrow-5$ & $0-15$ & $-3 \Leftrightarrow+4$ & & & $-4 \Leftrightarrow 0$ & \begin{tabular}{|l} 
Gebauer et al., \\
1994 und \\
Gebauer und \\
Schulze, 1991
\end{tabular} \\
\hline \multirow[t]{5}{*}{ Belgien } & Ardennes, Braunerde & & $0-22$ & $-6.6 \Leftrightarrow+0.7$ & & & & \multirow{5}{*}{\begin{tabular}{|l} 
Riga et al., \\
1971
\end{tabular}} \\
\hline & Waldboden & & $22-100$ & $+0.9 \Leftrightarrow+2.6$ & & & & \\
\hline & Braunerde, Mikrogley & & $0-25$ & $-7.0 \Leftrightarrow-0.1$ & & & & \\
\hline & Waldboden & & $25-110$ & $+1.0 \Leftrightarrow+3.5$ & & & & \\
\hline & Ackerboden & & $0-60$ & $+1.2 \Leftrightarrow+4.4$ & & & & \\
\hline \multirow[t]{4}{*}{ Französische Alpen } & eutrophe Braunerde - hohe Humifikation & -1.9 & $0-50$ & $+1.4 \Leftrightarrow+5.9$ & & & +1.4 & \multirow{4}{*}{$\begin{array}{l}\text { Mariotti et al., } \\
1980\end{array}$} \\
\hline & mesotrophe Braunerde - hohe Humifikation & -1.8 & $0-100$ & $+1.1 \Leftrightarrow+5.8$ & & & +1.1 & \\
\hline & gelbbraune Braunerde - niedrige Humifikation & -5.0 & $0-90$ & $-2.3 \Leftrightarrow+5.1$ & & & -2.3 & \\
\hline & gelbbraune Podsol - niedrige Humifikation & $-3.5 \Leftrightarrow-1.8$ & $0-80$ & $-4.0 \Leftrightarrow+5.1$ & & & $-4.0 \Leftrightarrow-2.8$ & \\
\hline Französische Alpen & Mischwald mitAcacia und Alnus etc. & $\begin{array}{c}\mathrm{R}:-7 \Leftrightarrow-2.5 \\
\mathrm{~L}:-2.7 \Leftrightarrow-1.5\end{array}$ & & & & & +10.5 & $\begin{array}{l}\text { Domenach et al., } \\
\text { 1989; Kurdali et } \\
\text { al., 1993 }\end{array}$ \\
\hline \multirow[t]{2}{*}{ Süd-Appalachien, USA } & \multirow[t]{2}{*}{ Ultisol, saurer Waldboden, } & \multirow[t]{2}{*}{$-4 \Leftrightarrow+1$} & $0--20$ & $+3.8 \Leftrightarrow+4.1$ & $-4 \Leftrightarrow-4.4$ & $+3 \Leftrightarrow+7$ & & \multirow{2}{*}{ Garten, 1993} \\
\hline & & & Oi-Horizont & $-4 \Leftrightarrow 0$ & & & & \\
\hline Süd-Appalachien, USA & Regen & & & & $-3 \Leftrightarrow+7$ & $-9 \Leftrightarrow+1$ & & Garten, 1992 \\
\hline
\end{tabular}


Fortsetzung Anhang Tab. 49: $\delta^{15} \mathrm{~N}$ in Vegetation, Boden, organischer Substanz (SOM) und $\mathrm{N}_{\text {min. }}$ in den verschiedenen Regionen der Welt

\begin{tabular}{|c|c|c|c|c|c|c|c|c|}
\hline Ort, Klimazone & & Vegetation & \multicolumn{5}{|c|}{ Boden } & Referenz \\
\hline & Beschreibung & & Tiefe $(\mathbf{c m})$ & $\mathbf{N}_{\text {tot. }}$ & $\mathrm{NO}_{3}^{-}$ & $\mathrm{NH}_{4}{ }^{+}$ & SOM & \\
\hline \multirow[t]{2}{*}{ San Joaquim Valley } & Entisol & & $0-30$ & $+0.5( \pm 2.3)$ & & & & \multirow{6}{*}{$\begin{array}{l}\text { Broadbent, } \\
1980\end{array}$} \\
\hline & & & $270-300$ & $-8.4( \pm 9.4)$ & & & & \\
\hline \multirow{2}{*}{$\begin{array}{l}\text { Georgetown, Sierra } \\
\text { foothill, } 730 \mathrm{~m},\end{array}$} & Mariposa Ultisol, steinig & & $0-15$ & $+3.8( \pm 4.5)$ & & & & \\
\hline & & & $105-120$ & -5.4 & & & & \\
\hline \multirow[t]{2}{*}{ San Joaquim Valley } & Inceptisol & & $0-15$ & $-0.4 \Leftrightarrow+2.5$ & & & & \\
\hline & & & $105-120$ & $+1.8 \Leftrightarrow+2.8$ & & & & \\
\hline Kalifornien & Sequoia National Park & $\begin{array}{l}\text { R: }-6 \Leftrightarrow-1 \\
\text { L: }-2.5 \Leftrightarrow 0\end{array}$ & & & & & & \begin{tabular}{|l} 
Virginia et al., \\
1989
\end{tabular} \\
\hline Kalifornien, Mexico & Wüsten-Ökosystem & $\begin{array}{c}\text { R: }+4 \Leftrightarrow+13 \\
\mathrm{~L}:+2 \Leftrightarrow+8\end{array}$ & & & & \multirow[b]{2}{*}{$-10 \Leftrightarrow-1$} & & $\begin{array}{l}\text { Virginia et al., } \\
1989 \\
\end{array}$ \\
\hline \multirow[t]{2}{*}{$\begin{array}{l}\text { Kalifornien, Sequoia } \\
\text { National Park }\end{array}$} & Boden nach Brand & & $0-20$ & -0.94 & $-8 \Leftrightarrow+18$ & & & \multirow[t]{2}{*}{$\begin{array}{l}\text { Herman und } \\
\text { Rundel, } 1989\end{array}$} \\
\hline & Boden vor Brand & & $0-20$ & -1.28 & $-2 \Leftrightarrow-5$ & $-15 \Leftrightarrow-5$ & & \\
\hline \multirow[t]{2}{*}{ Kalifornien, Davis } & Entisol & & $0-15$ & $-6.0( \pm 3.3)$ & & & & \multirow{4}{*}{$\begin{array}{l}\text { Broadbent, } \\
1980 \\
\text { Broadbent, } \\
1980 \\
\end{array}$} \\
\hline & & & $270-300$ & $+1.7( \pm 1.8)$ & & & & \\
\hline \multirow[t]{2}{*}{ Kalifornien Sierra, } & Koniferen-Erle-Weide-Wald, Entisol, 2080m Höhe & & 0.15 & $+2.8( \pm 4.6)$ & & & & \\
\hline & & & $105-120$ & $+9.2( \pm 12.2)$ & & & & \\
\hline $\begin{array}{l}\text { Great Smoky Mountains } \\
\text { National Park, USA }\end{array}$ & $\begin{array}{l}\text { Boden unter Fichte, Buche, Hartholz, Pappel, Pinie, } \\
\text { Eiche }\end{array}$ & $-4.5 \Leftrightarrow-0.6$ & $0-10$ & $+3.4 \Leftrightarrow+5.7$ & & & & $\begin{array}{l}\text { Garten und } \\
\text { Miegrot, } 1994 \\
\end{array}$ \\
\hline \multirow[t]{2}{*}{ Hawaii } & Boden vulkanischen Ursprungs, 28 Jahre alt & $-4.6 \Leftrightarrow+4.8$ & $0-20$ & $-3.4 \Leftrightarrow-1.1$ & & & & \multirow{2}{*}{\begin{tabular}{|l} 
Vitousek et al., \\
1989
\end{tabular}} \\
\hline & Boden vulkanischen Ursprungs, 460000 Jahre alt & $-0.9 \Leftrightarrow-0.6$ & $0-20$ & $+3.1 \Leftrightarrow+4.2$ & & & & \\
\hline Panama & Barro Colorado, Primärwald & $\begin{array}{c}\mathrm{R}:+6 \Leftrightarrow+9 \\
\mathrm{~L}:+4.5 \Leftrightarrow+7\end{array}$ & & & & & & \begin{tabular}{|l} 
Virginia et al., \\
1989 \\
\end{tabular} \\
\hline Thailand & & $\begin{array}{l}\text { R: }+1.7 \Leftrightarrow+8.0 \\
\mathrm{~L}:-0.5 \Leftrightarrow+3.0\end{array}$ & $0-20$ & $+3.4 \Leftrightarrow+8.4$ & & & & $\begin{array}{l}\text { Peoples et al., } \\
1991\end{array}$ \\
\hline Thailand & & $\begin{array}{l}\mathrm{R}:+1 \Leftrightarrow+10 \\
\mathrm{~L}:-2 \Leftrightarrow+10\end{array}$ & $0-20$ & $+3.6 \Leftrightarrow+8.4$ & & & & \begin{tabular}{|l} 
Yoneyama et al., \\
1990
\end{tabular} \\
\hline
\end{tabular}


Fortsetzung Anhang Tab. 49: $\delta^{15} \mathrm{~N}$ in Vegetation, Boden, organischer Substanz (SOM) und $\mathrm{N}_{\text {min. }}$ in den verschiedenen Regionen der Welt

\begin{tabular}{|c|c|c|c|c|c|c|c|c|}
\hline Ort, Klimazone & & Vegetation & & & Boden & & & Referenz \\
\hline & Beschreibung & & Tiefe $(\mathrm{cm})$ & $\mathbf{N}_{\text {tot}}$ & $\mathrm{NO}_{3}^{-}$ & $\mathrm{NH}_{4}^{+}$ & SOM & \\
\hline \multirow[t]{3}{*}{ Phillippinen } & & & $0-20$ & $+6.6 \Leftrightarrow+14.9$ & & & & \multirow{2}{*}{$\begin{array}{l}\text { Peoples et al., } \\
1991\end{array}$} \\
\hline & unter Leguminosen & & $0-20$ & +2.7 & & & & \\
\hline & Ultic Haplorthox & & $0-100$ & $+5.5 \Leftrightarrow+7.3$ & & & & $\begin{array}{l}\text { Ladha et al., } \\
1993\end{array}$ \\
\hline \multirow[t]{2}{*}{ Malaysia } & natürlicher Waldboden & & $0-20$ & +16.6 & & & & \multirow{3}{*}{$\begin{array}{l}\text { Peoples et al., } \\
1991\end{array}$} \\
\hline & unter Plantagen & & $0-20$ & +3.5 & & & & \\
\hline Indonesien & & & $0-20$ & $+4.5 \Leftrightarrow+7.1$ & & & & \\
\hline \multirow[t]{2}{*}{ Amazonas-Varzea, } & Andensedimente, Überschwemmungsebene & $\begin{array}{c}\mathrm{R}:+1.3 \Leftrightarrow+10.4 \\
\mathrm{~L}:+0.1 \Leftrightarrow+4.4\end{array}$ & & $+3.8 \Leftrightarrow+4.4$ & & & & \multirow{2}{*}{$\begin{array}{l}\text { Martinelli et } \\
\text { al., } 1992\end{array}$} \\
\hline & Flußschwebstoffe & & & +5.3 & & & & \\
\hline \multirow{2}{*}{$\begin{array}{l}\text { West-Rondonia, } \\
\text { Brazilien, } \\
\text { Amazonasgebiet }\end{array}$} & Tropudult, Weide & & $0-20$ & $+9.8 \Leftrightarrow+13.6$ & & & +9.2 & \multirow[t]{2}{*}{ Piccolo, 1994} \\
\hline & Wald & +5.1 & $0-20$ & $+7.8 \Leftrightarrow+15$ & & & +3.3 & \\
\hline Brasilien, Cerrado & Waldvegetation, natürliche & $\begin{array}{l}\text { R: }-0.61 \Leftrightarrow+1.82 \\
\text { L: }-1.85 \Leftrightarrow+0.86\end{array}$ & & & & & & \multirow{3}{*}{\begin{tabular}{|l} 
Sprent et al., \\
1996
\end{tabular}} \\
\hline Amazonia, Manaus & Waldboden & & $0-160$ & $+7 \Leftrightarrow+22$ & & & & \\
\hline Pará, Rondonia & Waldboden & & $0-160$ & $+8 \Leftrightarrow+13$ & & & & \\
\hline Manaus, Amazonia, & Campina-Vegetation, sandy soil, Ultisol & $\begin{array}{c}\text { R:-7.0 } \\
\text { L:- } 1.6 \Leftrightarrow-2.2\end{array}$ & & & & & & $\begin{array}{l}\text { Salati et al., } \\
1982\end{array}$ \\
\hline Brasilien & Reserva Ducke, clayey soil, Oxisol & $\begin{array}{c}\mathrm{R}:+5.8 \Leftrightarrow+7 \\
\mathrm{~L}:+3.9 \Leftrightarrow+10\end{array}$ & & & & & & \multirow{3}{*}{$\begin{array}{l}\text { Brandes et al., } \\
1996\end{array}$} \\
\hline \multirow[t]{2}{*}{$\begin{array}{l}\text { Barro branco, } \\
\text { Amazonas, Brasilien }\end{array}$} & Grundwasser im Hochland & & & & $+6.25 \pm 0.9$ & & $+3.5 \pm 1.2$ & \\
\hline & $\begin{array}{l}\text { Grundwasser unter dem Fluß } \\
\text { Flußwasser }\end{array}$ & & & & & $\begin{array}{r}+9.17 \pm 1 \\
+4.52 \pm 0.8 \\
\end{array}$ & & \\
\hline Brasilien, Pará & Sekundärvegetationen & $\begin{array}{c}\mathrm{R}:+1.7 \Leftrightarrow+9 \\
\mathrm{~L}:-2 \Leftrightarrow+7\end{array}$ & $0-20$ & $+5.1 \Leftrightarrow+10.9$ & & & & $\begin{array}{l}\text { Yoneyama et al. } \\
1993\end{array}$ \\
\hline \multirow[t]{4}{*}{ Brasilien, Pará, } & \multirow[t]{4}{*}{ Ultisol, mit Brandvorgeschichte, Sekundärwald } & $\mathrm{R}:-4.5 \Leftrightarrow+3.5$ & $0-10$ & $+3.4 \Leftrightarrow+5.0$ & +1.5 & +4.1 & $-2.4 \Leftrightarrow+2.8$ & \multirow{4}{*}{$\begin{array}{l}\text { vorliegende } \\
\text { Arbeit } \\
\text { und } \\
\text { Paparcikova, } \\
1995 \\
\end{array}$} \\
\hline & & $\mathrm{L}:-2 \Leftrightarrow+1$ & & & & & & \\
\hline & & & $60-100$ & $+6.4 \Leftrightarrow+7.1$ & -7.8 & -4.3 & & \\
\hline & & & $550-600$ & $+0.5 \Leftrightarrow+4.0$ & & & & \\
\hline
\end{tabular}


Fortsetzung Anhang Tab. 49: $\delta^{15} \mathrm{~N}$ in Vegetation, Boden, organischer Substanz (SOM) und $\mathrm{N}_{\text {min. }}$ in den verschiedenen Regionen der Welt

\begin{tabular}{|c|c|c|c|c|c|c|c|c|}
\hline Ort, Klimazone & & Vegetation & & & Boden & & & Referenz \\
\hline & $\begin{array}{r}\text { Beschreibung } \\
\end{array}$ & & Tiefe $(\mathrm{cm})$ & $\mathbf{N}_{\text {tot. }}$ & $\mathrm{NO}_{3}^{-}$ & $\mathrm{NH}_{4}{ }^{+}$ & SOM & \\
\hline \multirow[t]{2}{*}{ Pará, Brasilien } & \multirow[t]{2}{*}{ Ultisol, ohne Brandvorgeschichte, Primärwald } & $\mathrm{R}:+3 \Leftrightarrow+5$ & $0-10$ & $+7.3 \Leftrightarrow+9.1$ & & & $+5.0( \pm 0.5)$ & \\
\hline & & $\mathrm{L}:+5 \Leftrightarrow+8$ & $60-100$ & $+9.4 \Leftrightarrow+12.6$ & & & +2.5 & \\
\hline Afrika, Kamerun & Tiefland Regenwald, Korup & $\begin{array}{c}\mathrm{R}:+4.4 \Leftrightarrow+5 \\
\mathrm{~L}:+3.3\end{array}$ & & & & & & $\begin{array}{l}\text { Högberg und } \\
\text { Alexander,1995 }\end{array}$ \\
\hline Afrika Tanzania & Miombo Wald & $\begin{array}{l}\text { R: }-0.60 \Leftrightarrow+1.73 \\
\text { L: }-0.81 \Leftrightarrow+0.62\end{array}$ & & & & & & $\begin{array}{l}\text { Yoneyama et al., } \\
1990\end{array}$ \\
\hline Afrika Tanzania & Savanna Wald & $\begin{array}{l}\text { R: }-1.64 \Leftrightarrow+3.5 \\
\text { L: }+1.4 \Leftrightarrow-1.09\end{array}$ & & & & & & Högberg, 1986 \\
\hline Afrika, Zambia & Miombo Wald & $\begin{array}{c}\mathrm{R}:+2 \Leftrightarrow+3 \\
\mathrm{~L}:+0.2\end{array}$ & & & & & & $\begin{array}{l}\text { Högberg und } \\
\text { Alexander,1995 }\end{array}$ \\
\hline \multirow[t]{2}{*}{ Südafrika, Namibia } & saliner Standort & $\begin{array}{c}\mathrm{R}:+4.8 \Leftrightarrow+14.6 \\
\mathrm{~L}:+5.1\end{array}$ & & & & & & \multirow[t]{2}{*}{ Heaton, 1987} \\
\hline & nicht saliner Standort & $\begin{array}{c}\mathrm{R}:+3.3 \Leftrightarrow+6.3 \\
\mathrm{~L}:+4.9\end{array}$ & & & & & & \\
\hline \multirow[t]{4}{*}{ Afrika, Cape } & nährstoffarmer saurere Sandboden & $\mathrm{R}:-2 \Leftrightarrow+5$ & $0-10$ & $+3 \Leftrightarrow+6$ & & & & \multirow{4}{*}{$\begin{array}{l}\text { Stock et al., } \\
\mid 1995\end{array}$} \\
\hline & Acacia-dominiert & $\mathrm{L}:-1.5 \Leftrightarrow 0$ & $0-10$ & $+2 \Leftrightarrow+4.5$ & & & & \\
\hline & nährstoffreicher Boden & $\mathrm{R}:-0.5 \Leftrightarrow+0.5$ & $0-10$ & $+5 \Leftrightarrow+6.5$ & & & & \\
\hline & Acacia-dominiert & $\mathrm{L}:-2 \Leftrightarrow+0.5$ & $0-10$ & $+2 \Leftrightarrow+5$ & & & & \\
\hline Chile & Atacama Wüste & $\begin{array}{c}\mathrm{R}:+3.8 \Leftrightarrow+11.9 \\
\mathrm{~L}:+12\end{array}$ & & & & & & \begin{tabular}{|l|} 
Evans und \\
Ehleringer,1994
\end{tabular} \\
\hline Australien & Weide & $\begin{array}{c}\mathrm{L}:-0.36 \Leftrightarrow+0.45 \\
\mathrm{R}:+2.8 \Leftrightarrow+4.0\end{array}$ & & & & & & $\begin{array}{l}\text { Unkovich et al., } \\
1994\end{array}$ \\
\hline Australien & Weide & $\begin{array}{l}\mathrm{L}:-1 \Leftrightarrow-+2 \\
\mathrm{R}:+1 \Leftrightarrow+8\end{array}$ & & & $+4 \Leftrightarrow+8$ & $+6.5 \Leftrightarrow+10.5$ & & Pate et al., 1994 \\
\hline \multirow[t]{2}{*}{ Australien } & unter Weide & $\mathrm{R}:+5.3 \Leftrightarrow+11.9$ & $0-20$ & $+4.6 \Leftrightarrow+7$ & & & & \multirow{2}{*}{$\begin{array}{l}\text { Peoples et al., } \\
1991\end{array}$} \\
\hline & unter Leguminosen & $\mathrm{L}:-3.1 \Leftrightarrow-0.2$ & $0-20$ & $+3 \Leftrightarrow+6.4$ & & & & \\
\hline Südwest Australien, & Banksia Waldland & $\begin{array}{c}\mathrm{R}:-1 \Leftrightarrow+5 \\
\mathrm{~L}:-1 \Leftrightarrow+0.4\end{array}$ & $0-20$ & $+0.7 \Leftrightarrow+1.7$ & & $+3.1 \Leftrightarrow+11$ & & Pate et al., 1993 \\
\hline Australien, Kapalga & Savanna Waldland, Eucalyptus-dominiert & $\begin{array}{c}\mathrm{R}:-1 \Leftrightarrow 0 \\
\mathrm{~L}:-2 \Leftrightarrow-0.5\end{array}$ & $0-40$ & $+2.4 \Leftrightarrow+6$ & & & $+2.5 \Leftrightarrow+6.5$ & $\begin{array}{l}\text { Mordelet et al., } \\
1996\end{array}$ \\
\hline Neuseeland & toniger Lehm, Grasland & & $0-7.5$ & $-1 \Leftrightarrow+7$ & & & & $\begin{array}{l}\text { Steele et al., } \\
1981\end{array}$ \\
\hline
\end{tabular}


Fortsetzung Anhang Tab. 49: $\delta^{15} \mathrm{~N}$ in Vegetation, Boden, organischer Substanz (SOM) und $\mathrm{N}_{\text {min. }}$ in den verschiedenen Regionen der Welt

\begin{tabular}{|c|c|c|c|c|c|c|c|c|}
\hline Ort, Klimazone & & Vegetation & \multicolumn{5}{|c|}{ Boden } & Referenz \\
\hline & Beschreibung & & Tiefe $(\mathrm{cm})$ & $\mathbf{N}_{\text {tot }}$ & $\mathrm{NO}_{3}{ }^{-}$ & $\mathrm{NH}_{4}{ }^{+}$ & SOM & \\
\hline \multirow[t]{2}{*}{$\begin{array}{l}\text { Australien, Canberra, } \\
\text { Pejar Dam }\end{array}$} & $\begin{array}{l}\text { gelber Duplex - Granit, - Sediment, toter Duplex - } \\
\text { Basalt, gelber Podsol }\end{array}$ & & $\begin{array}{c}\text { Granit, } \\
\text { Basalt, } \\
\text { Sediment }\end{array}$ & $\begin{array}{l}+5.3 \\
+1.8 \\
+3.9\end{array}$ & & & & $\begin{array}{l}\text { Ledgard et al., } \\
1984\end{array}$ \\
\hline & Granit, & $+2.5 \Leftrightarrow+3.5$ & $0-15$ & $+5.6 \Leftrightarrow+9$ & $+0.5 \Leftrightarrow+4$ & & $+4.1 \Leftrightarrow+9.1$ & \\
\hline \multirow[t]{2}{*}{ Australien, Canberra } & kultuvierter Boden & \multirow[t]{2}{*}{$+5.3 \pm 0.2$} & \multirow[t]{2}{*}{$0-10$} & \multirow[t]{2}{*}{$+5.3 \Leftrightarrow+8.4$} & \multicolumn{2}{|c|}{$+10.6 \Leftrightarrow+11.3$} & & Turner et al \\
\hline & kontinuierliche Brache, Yanco & & & & \multicolumn{2}{|c|}{$+7.9 \Leftrightarrow+11.9$} & & 1987 \\
\hline
\end{tabular}


Tab. 50: Physikalische und chemische Eckdaten des Bodens der Untersuchungsfläche M1S1 für Bodentiefen von 0 bis $100 \mathrm{~cm}$ (es sind die Mittelwerte aus 6 Mischproben je Bodentiefe mit dem Standardfehler angegeben)

\begin{tabular}{|c|c|c|c|c|c|c|}
\hline Bodentiefe $[\mathrm{cm}]$ & $0-5$ & $5-10$ & $10-20$ & $20-40$ & $40-60$ & $60-100$ \\
\hline Grobsand [\%] & $58.5(0.5)$ & $52.5(1.5)$ & $47.5(2.5)$ & $40.5(1.5)$ & $37.0(0.0)$ & $34.5(1.5)$ \\
\hline Feinsand [\%] & $29.5(1.5)$ & $33.5(0.5)$ & $32.5(1.5)$ & $31.0(2.0)$ & $29.0(0.0)$ & $26.5(0.5)$ \\
\hline Schluff [\%] & $7.0(3.0)$ & $3.5(2.5)$ & $7.0(1.0)$ & $9.0(2.0)$ & $7.0(0.0)$ & $9.0(2.0)$ \\
\hline Ton [\%] & $5.0(1.0)$ & $10.5(3.5)$ & $13.0(0.0)$ & $19.5(1.5)$ & $27.0(0.0)$ & $30(1.0)$ \\
\hline $\mathrm{pH}\left(\mathrm{H}_{2} \mathrm{O}\right)$ & 5.6 & 5.5 & $5.2(0.05)$ & $5.05(0.05)$ & $4.9(0.2)$ & $4.8(0.1)$ \\
\hline $\mathrm{pH}(\mathrm{KCl})$ & 4.6 & 4.5 & $4.2(0.0)$ & $4.1(0.0)$ & $4.05(0.05)$ & $4.05(0.05)$ \\
\hline $\mathrm{C}[\%]$ & $0.65(0.40)$ & $0.86(0.04)$ & $0.78(0.10)$ & $0.48(0.04)$ & $0.36(0.04)$ & $0.26(0.005)$ \\
\hline $\mathrm{N}[\%]$ & $0.07(0.00)$ & $0.06(0.005)$ & $0.05(0.01)$ & $0.04(0.01)$ & $0.04(0.005)$ & $0.03(0.01)$ \\
\hline $\mathrm{C} / \mathrm{N}$ & $16.5(1.5)$ & $16.0(2.0)$ & $16.5(5.5)$ & $13.0(4.0)$ & $10.5(2.5)$ & $9.0(3.0)$ \\
\hline $\mathrm{P}[\mathrm{ppm}]$ & $2.50(0.50)$ & $2.50(0.50)$ & $2.00(0.00)$ & $2.00(1.00)$ & $1.50(0.50)$ & $2.00(1.00)$ \\
\hline $\mathrm{Ca}^{++}[\mathrm{mval} / 100 \mathrm{~g}]^{*}$ & $1.50(0.30)$ & $1.05(0.15)$ & $0.65(0.05)$ & $0.65(0.05)$ & $0.40(0.00)$ & $0.45(0.25)$ \\
\hline $\mathrm{Mg}^{++}[\mathrm{mval} / 100 \mathrm{~g}]$ & 1.10 & $0.75(0.05)$ & $0.55(0.05)$ & & & \\
\hline $\mathrm{K}^{+}[\mathrm{mval} / 100 \mathrm{~g}]$ & 0.05 & 0.04 & $0.03(0.00)$ & $0.02(0.00)$ & $0.02(0.005)$ & $0.02(0.005)$ \\
\hline $\mathrm{Na}^{+}[\mathrm{mval} / 100 \mathrm{~g}]$ & $0.04(0.005)$ & 0.04 & $0.02(0.005)$ & $0.03(0.00)$ & $0.02(0.00)$ & $0.02(0.005)$ \\
\hline $\mathrm{Al}^{+++}[\mathrm{mval} / 100 \mathrm{~g}]$ & $0.10(0.10)$ & 0.00 & $0.03(0.10)$ & $0.75(0.05)$ & $0.85(0.15)$ & $1.00(0.00)$ \\
\hline $\mathrm{H}^{+}[\mathrm{mval} / 100 \mathrm{~g}]$ & $2.60(0.40)$ & 2.80 & $2.60(0.20)$ & $2.15(0.25)$ & $2.20(0.00)$ & $1.95(0.55)$ \\
\hline $\mathrm{Ak}[\mathrm{mval} / 100 \mathrm{~g}]$ & $5.30(0.70)$ & $4.70(0.10)$ & $3.65(0.35)$ & $3.55(0.25)$ & $3.60(0.00)$ & $6.55(2.85)$ \\
\hline Ake $[\mathrm{mval} / \mathrm{g}]$ & $2.70(0.30)$ & $1.90(0.10)$ & $1.25(0.05)$ & $0.65(0.05)$ & $0.40(0.00)$ & $0.45(0.25)$ \\
\hline Basensättigung [\%] & $51.0(1.0)$ & $40.5(1.5)$ & $34.5(4.5)$ & $18.5(2.5)$ & $11.0(0.0)$ & $6.0(1.0)$ \\
\hline $\mathrm{Zn}$ [ppm] & $0.17(0.03)$ & $0.20(0.08)$ & $0.07(0.01)$ & $0.74(0.11)$ & $0.21(0.05)$ & $0.17(0.08)$ \\
\hline $\mathrm{Cu}[\mathrm{ppm}]$ & 0.01 & 0.01 & $1.00(1.00)$ & $0.04(0.03)$ & $0.02(0.005)$ & $0.02(0.00)$ \\
\hline Mn [ppm] & $3.32(0.44)$ & $1.61(0.25)$ & $0.64(0.10)$ & $0.18(0.02)$ & $0.20(0.06)$ & $0.20(0.005)$ \\
\hline
\end{tabular}

\footnotetext{
* leere Zellen $=\mathrm{Ca}^{++}$und $\mathrm{Mg}^{++}$wurden zusammen bestimmt
} 
Tab. 51: Physikalische und chemische Eckdaten des Bodens der Untersuchungsfläche M2S1 für Bodentiefen von 0 bis $100 \mathrm{~cm}$ (es sind die Mittelwerte aus 6 Mischproben je Bodentiefe mit dem Standardfehler angegeben)

\begin{tabular}{|c|c|c|c|c|c|c|}
\hline Bodentiefe [\%] & $0-5$ & $5-10$ & $10-20$ & $20-40$ & $40-60$ & $60-100$ \\
\hline Grobsand [\%] & $49.5(1.5)$ & $50.5(0.5)$ & $47.5(0.5)$ & $44.0(5.0)$ & $36.0(1.0)$ & $36.0(1.1)$ \\
\hline Feinsand [\%] & $35.5(0.5)$ & $35.5(0.5)$ & $32.0(0.0)$ & $30.5(1.5)$ & $30.5(0.5)$ & $26.5(1.0)$ \\
\hline Schluff [\%] & $8.5(2.5)$ & $5.0(0.0)$ & $10.0(1.0)$ & $6.0(3.0)$ & $7.5(0.5)$ & $6.5(0.6)$ \\
\hline Ton $[\%]$ & $6.5(0.5)$ & $9.0(0.0)$ & $10.5(0.5)$ & $19.5(0.5)$ & $26.0(1.0)$ & $31.0(1.29)$ \\
\hline $\mathrm{pH}(\mathrm{H} 20)$ & & $5.6(0.0)$ & $5.4(0.1)$ & $5.2(0.1)$ & $4.8(0.5)$ & $4.9(0.1)$ \\
\hline $\mathrm{pH}(\mathrm{KCl})$ & & $4.5(0)$ & $4.2(0.1)$ & $4.1(0.0)$ & $4.0(0.1)$ & $4.1(0.0)$ \\
\hline $\mathrm{C}[\%]$ & $1.60(0.20)$ & $0.90(0.03)$ & $0.70(0.05)$ & $0.52(0.00)$ & $0.41(0.04)$ & $0.56(0.09)$ \\
\hline $\mathrm{N}[\%]$ & $0.12(0.00)$ & $0.10(0.01)$ & $0.09(0.00)$ & $0.07(0.00)$ & $0.06(0.00)$ & $0.07(0.01)$ \\
\hline $\mathrm{C} / \mathrm{N}$ & $12.5(1.5)$ & $10.0(0.0)$ & $7.5(0.5)$ & $7.0(0.0)$ & $6.5(0.5)$ & $7.4(0.8)$ \\
\hline $\mathrm{P}[\mathrm{ppm}]$ & $4(0)$ & $3(0)$ & $2(0)$ & $2(0)$ & $1(0)$ & $1(0)$ \\
\hline $\mathrm{Ca}^{++}[\mathrm{mval} / 100 \mathrm{~g}]^{*}$ & $1.65(0.05)$ & $0.90(0.10)$ & $0.50(0.10)$ & $0.60(0.10)$ & $0.70(0.00)$ & $0.23(0.07)$ \\
\hline $\mathrm{Mg}^{++}[\mathrm{mval} / 100 \mathrm{~g}]$ & $1.6(0.1)$ & $0.7(0.0)$ & $0.6(0.1)$ & & & \\
\hline $\mathrm{K}^{+}[\mathrm{mval} / 100 \mathrm{~g}]$ & $0.07(0.00)$ & $0.04(0.01)$ & $0.03(0.00)$ & $0.02(0.00)$ & $0.01(0.00)$ & $0.01(0.00)$ \\
\hline $\mathrm{Na}^{+}[\mathrm{mval} / 100 \mathrm{~g}]$ & $0.01(0.00)$ & $0.04(0.00)$ & $0.04(0.005)$ & $0.03(0.00)$ & $0.02(0.005)$ & $0.03(0.003)$ \\
\hline $\mathrm{Al}^{+++}[\mathrm{mval} / 100 \mathrm{~g}]$ & $0.00(0.00)$ & $0.30(0.10)$ & $0.40(0.00)$ & $0.50(0.10)$ & $0.70(0.10)$ & $0.97(0.08)$ \\
\hline $\mathrm{H}^{+}[\mathrm{mval} / 100 \mathrm{~g}]$ & $3.50(0.10)$ & $2.60(0.20)$ & $2.20(0.00)$ & $2.20(0.00)$ & $2.00(0.00)$ & $1.77(0.18)$ \\
\hline Ak [mval/100g] & $6.85(0.05)$ & $4.60(0.20)$ & $3.80(0.20)$ & $3.30(0.20)$ & $3.40(0.10)$ & $2.97(0.20)$ \\
\hline Ake $[\mathrm{mval} / \mathrm{g}]$ & $3.35(0.05)$ & $1.70(0.10)$ & $1.20(0.20)$ & $0.60(0.10)$ & $0.70(0.00)$ & $0.23(0.07)$ \\
\hline Basensättigung [\%] & $49.0(1.0)$ & $36.5(0.5)$ & $31.5(3.5)$ & $18.0(2.0)$ & $2.5(0.5)$ & $7.7(2.5)$ \\
\hline $\mathrm{Zn}$ [ppm] & $0.36(0.06)$ & $0.20(0.01)$ & $0.12(0.01)$ & $0.15(0.04)$ & $0.28(0.14)$ & $0.52(0.18)$ \\
\hline $\mathrm{Cu}[\mathrm{ppm}]$ & $0.01(0.00)$ & & & & $0.04(0.00)$ & $0.04(0.01)$ \\
\hline Mn [ppm] & $5.74(1.32)$ & $2.39(0.28)$ & 0.880 .23 & 0.400 .06 & $0.33(0.04)$ & $0.30(0.03)$ \\
\hline
\end{tabular}

\footnotetext{
* leere Zellen $=\mathrm{Ca}++$ und $\mathrm{Mg}++$ wurden zusammen bestimmt
} 
Tab. 52: Physikalische und chemische Eckdaten des Bodens der Untersuchungsfläche M3S3 für Bodentiefen von 0 bis $100 \mathrm{~cm}$ (es sind die Mittelwerte aus 6 Mischproben je Bodentiefe mit dem Standardfehler angegeben)

\begin{tabular}{|c|c|c|c|c|c|c|}
\hline Bodentiefe [\%] & $0-5$ & $5-10$ & $10-20$ & $20-40$ & $40-60$ & $60-100$ \\
\hline Grobsand [\%] & $48.3(1.8)$ & $44.8(1.0)$ & $42.0(1.7)$ & $33.3(1.2)$ & $32.7(0.3)$ & $33.0(0.3)$ \\
\hline Feinsand [\%] & $38.0(1.2)$ & $40.8(1.1)$ & $38.0(0.6)$ & $36.0(0.6)$ & $35.0(0.0)$ & $33.6(0.4)$ \\
\hline Schluff [\%] & $5.7(0.7)$ & $5.5(0.5)$ & $5.0(1.0)$ & $7.3(1.2)$ & $5.0(0.6)$ & $4.6(0.5)$ \\
\hline Ton [\%] & $8.0(1.2)$ & $9.0(0.6)$ & $15.0(0.6)$ & $23.3(0.7)$ & $27.3(0.7)$ & $28.8(0.5)$ \\
\hline $\mathrm{pH}\left(\mathrm{H}_{2} \mathrm{O}\right)$ & $5.8(0.4)$ & $5.5(0.15)$ & $5.3(0.07)$ & $5.1(0.08)$ & $5.02(0.08)$ & $5.06(0.04)$ \\
\hline $\mathrm{pH}(\mathrm{KCl})$ & $4.7(0.3)$ & $4.43(0.12)$ & $4.22(0.47)$ & $4.2(0.05)$ & $4.12(0.02)$ & $4.2(0.02)$ \\
\hline $\mathrm{C}[\%]$ & $1.6(0.16)$ & $1.0(0.15)$ & $0.71(0.09)$ & $0.47(0.03)$ & $0.37(0.06)$ & $0.22(0.01)$ \\
\hline $\mathrm{N}[\%]$ & $0.12(0.03)$ & $0.10(0.03)$ & $0.09(0.03)$ & $0.08(0.03)$ & $0.08(0.03)$ & $0.06(0.02)$ \\
\hline $\mathrm{C} / \mathrm{N}$ & $14.8(3.8)$ & $13(4.8)$ & $10.8(3.4)$ & $8.5(3.1)$ & $7.25(2.56)$ & $5.4(1.32)$ \\
\hline $\mathrm{P}[\mathrm{ppm}]$ & $5(0.4)$ & $2.5(0.3)$ & $1.5(0.29)$ & $1.25(0.25)$ & $1.25(0.25)$ & $1.2(0.2)$ \\
\hline $\mathrm{Ca}^{++}[\mathrm{mval} / 100 \mathrm{~g}]^{*}$ & $2.78(0.51)$ & $1.22(0.41)$ & $0.52(0.09)$ & $0.45(0.1)$ & $0.38(0.08)$ & $0.34(0.08)$ \\
\hline $\mathrm{Mg}^{++}[\mathrm{mval} / 100 \mathrm{~g}]$ & $1.225(0.232)$ & $0.82(0.02)$ & $0.63(0.03)$ & $0.8(0)$ & & \\
\hline $\mathrm{K}^{+}[\mathrm{mval} / 100 \mathrm{~g}]$ & $0.078(0.011)$ & $0.04(0.01)$ & $0.02(0.003)$ & $0.04(0.03)$ & $0.02(0.01)$ & $0.01(0.00)$ \\
\hline $\mathrm{Na}^{+}[\mathrm{mval} / 100 \mathrm{~g}]$ & $0.04(0.01)$ & $0.03(0.00)$ & $0.01(0.00)$ & $0.02(0.00)$ & $0.02(0.00)$ & $0.02(0.00)$ \\
\hline $\mathrm{Al}^{+++}[\mathrm{mval} / 100 \mathrm{~g}]$ & $0.05(0.05)$ & $0.10(0.10)$ & $0.48(0.12)$ & $0.82(0.06)$ & $1.00(0.10)$ & $0.84(0.07)$ \\
\hline $\mathrm{H}^{+}[\mathrm{mval} / 100 \mathrm{~g}]$ & $3.22(0.25)$ & $3.00(0.40)$ & $2.70(0.37)$ & $2.48(0.29)$ & $2.05(0.19)$ & $1.46(0.11)$ \\
\hline $\mathrm{Ak}[\mathrm{mval} / 100 \mathrm{~g}]$ & $7.40(0.80)$ & $5.20(0.80)$ & $4.20(0.50)$ & $4.00(0.40)$ & $3.45(0.22)$ & $2.64(0.09)$ \\
\hline Ake [mval/g] & $4.10(0.64)$ & $2.12(0.44)$ & $1.00(0.19)$ & $0.70(0.20)$ & $0.40(0.06)$ & $0.34(0.07)$ \\
\hline Basensättigung [\%] & $54.8(3.6)$ & $40.0(4.1)$ & $24.0(4.1)$ & $16.2(3.3)$ & $11.8(2.2)$ & $13.2(3.0)$ \\
\hline $\mathrm{Zn}$ [ppm] & $0.28(0.06)$ & $0.21(0.07)$ & $0.15(0.01)$ & $0.30(0.10)$ & $0.34(0.17)$ & $0.28(0.14)$ \\
\hline $\mathrm{Cu}[\mathrm{ppm}]$ & & $0.02(0.00)$ & $0.01(0.00)$ & $0.04(0.02)$ & $0.02(0.00)$ & $0.04(0.03)$ \\
\hline Mn [ppm] & $4.79(0.78)$ & $1.86(0.49)$ & $0.44(0.08)$ & $0.21(0.06)$ & $0.31(0.03)$ & $0.43(0.06)$ \\
\hline
\end{tabular}


Tab. 53: Physikalische und chemische Eckdaten des Bodens der Untersuchungsfläche M4 für Bodentiefen von 0 bis $100 \mathrm{~cm}$ (es sind die Mittelwerte aus 6 Mischproben je Bodentiefe mit dem Standardfehler angegeben)

\begin{tabular}{|c|c|c|c|c|c|c|}
\hline Bodentiefe [\%] & $0-5$ & $5-10$ & $10-20$ & $20-40$ & $40-60$ & $60-100$ \\
\hline Grobsand [\%] & & $48.0(0.0)$ & $44.0(1.0)$ & $37.3(1.8)$ & $38.0(0.0)$ & $36.0(0.0)$ \\
\hline Feinsand [\%] & & $36.0(0.0)$ & $39.0(0.0)$ & $37.3(0.3)$ & $36.0(0.0)$ & $35.5(0.5)$ \\
\hline Schluff $[\%]$ & & $6.0(0.0)$ & $7.0(0.0)$ & $6.3(1.4)$ & $7.0(0.0)$ & $5.0(0.0)$ \\
\hline Ton [\%] & & $10.0(0.0)$ & $10.0(1.0)$ & $19.0(2.5)$ & $19.0(0.0)$ & $23.5(0.5)$ \\
\hline $\mathrm{pH}\left(\mathrm{H}_{2} \mathrm{O}\right)$ & & $5.5(0.0)$ & $5.5(0.2)$ & $4.97(0.12)$ & $4.95(0.15)$ & $4.95(0.15)$ \\
\hline $\mathrm{pH}(\mathrm{KCl})$ & & $4.5(0.0)$ & $4.4(0.1)$ & $4.13(0.03)$ & $4.15(0.05)$ & $4.2(0.1)$ \\
\hline $\mathrm{C}[\%]$ & $1.30(0.08)$ & $0.84(0.08)$ & $0.60(0.02)$ & $0.42(0.06)$ & $0.34(0.02)$ & $0.29(0.01)$ \\
\hline $\mathrm{N}[\%]$ & $0.08(0.01)$ & $0.06(0.01)$ & $0.05(0.00)$ & $0.04(0.00)$ & $0.04(0.01)$ & $0.04(0.01)$ \\
\hline $\mathrm{C} / \mathrm{N}$ & $15.0(0.0)$ & $15.0(4.0)$ & $12.0(0.0)$ & $11.3(0.9)$ & $9.5(0.5)$ & $8.0(1.0)$ \\
\hline$P[\mathrm{mg} / 100 \mathrm{~g}]$ & $4.0(0.0)$ & $2.0(0.0)$ & $2.0(0.0)$ & $2.3(0.3)$ & $2.0(0.0)$ & $2.0(0.0)$ \\
\hline $\mathrm{Ca}^{++}[\mathrm{mval} / 100 \mathrm{~g}]^{*}$ & $2.00(0.40)$ & $1.00(0.20)$ & $0.45(0.15)$ & $0.43(0.18)$ & $0.35(0.15)$ & $0.35(0.15)$ \\
\hline $\mathrm{Mg}^{++}[\mathrm{mval} / 100 \mathrm{~g}]$ & $1.10(0.00)$ & $0.60(0.00)$ & $0.85(0.05)$ & & & \\
\hline $\mathrm{K}^{+}[\mathrm{mval} / 100 \mathrm{~g}]$ & $0.06(0.01)$ & $0.04(0.01)$ & $0.02(0.01)$ & $0.01(0.00)$ & $0.01(0.00)$ & $0.01(0.00)$ \\
\hline $\mathrm{Na}^{+}[\mathrm{mval} / 100 \mathrm{~g}]$ & $0.05(0.00)$ & $0.03(0.00)$ & $0.04(0.01)$ & $0.03(0.00)$ & $0.02(0.01)$ & $0.02(0.01)$ \\
\hline $\mathrm{Al}^{+++}[\mathrm{mval} / 100 \mathrm{~g}]$ & $0.10(0.10)$ & $0.10(0.10)$ & $0.10(0.10)$ & $0.60(0.11)$ & $0.60(0.20)$ & $0.60(0.20)$ \\
\hline $\mathrm{H}^{+}[\mathrm{mval} / 100 \mathrm{~g}]$ & $3.40(0.20)$ & $2.45(0.05)$ & $2.10(0.10)$ & $2.00(0.11)$ & $2.20(0.00)$ & $1.80(0.00)$ \\
\hline $\mathrm{Ak}[\mathrm{mval} / 100 \mathrm{~g}]$ & $6.70(0.50)$ & $4.25(0.15)$ & $3.55(0.05)$ & $3.03(0.17)$ & $3.15(0.05)$ & $2.75(0.05)$ \\
\hline Ake $[\mathrm{mval} / 100 \mathrm{~g}]$ & $3.20(0.40)$ & $1.70(0.20)$ & $1.35(0.15)$ & $0.43(0.18)$ & $0.35(0.15)$ & $0.35(0.15)$ \\
\hline Basensättigung [\%] & $47.5(2.5)$ & $39.5(3.5)$ & $37.5(4.5)$ & $13.7(5.2)$ & $11.0(5.0)$ & $12.5(5.5)$ \\
\hline Zn [ppm] & $0.21(0.03)$ & $0.10(0.01)$ & $0.18(0.09)$ & $0.15(0.07)$ & $0.14(0.06)$ & $0.11(0.01)$ \\
\hline $\mathrm{Cu}[\mathrm{ppm}]$ & & $0.01(0.00)$ & $0.02(0.01)$ & $0.01(0.00)$ & & \\
\hline $\mathrm{Mn}[\mathrm{ppm}]$ & $2.45(0.21)$ & $1.14(0.22)$ & $0.39(0.24)$ & $0.18(0.06)$ & $0.12(0.02)$ & $0.17(0.00)$ \\
\hline
\end{tabular}

\footnotetext{
* leere Zellen $=\mathrm{Ca}^{++}$und $\mathrm{Mg}^{++}$wurden zusammen bestimmt
} 
Tab. 54: Physikalische und chemische Eckdaten des Bodens der Untersuchungsfläche M5 für die Bodentiefen von 0 bis $100 \mathrm{~cm}$ (es sind die Mittelwerte aus 6 Mischproben je Bodentiefe mit $\sigma$ angegeben)

\begin{tabular}{|c|c|c|c|c|c|c|}
\hline Bodentiefe [\%] & $0-5$ & $5-10$ & $10-20$ & $20-40$ & $40-60$ & $60-100$ \\
\hline Grobsand [\%] & $84.0(0)$ & $79.0(0$ & $81.50 .5)$ & $74.5(0.5)$ & $69.5(1.5)$ & $67.0(0)$ \\
\hline Feinsand [\%] & $12.0(0)$ & $16.0(0)$ & $12.5(1.5)$ & $17.5(0.5)$ & $19.5(1.5)$ & $19.0(1.0)$ \\
\hline Schluff [\%] & $1.0(0)$ & $2.0(0)$ & $1.0(0.0)$ & $2.5(0.5)$ & $4.0(0.0)$ & $3.0(1.0)$ \\
\hline Ton [\%] & $0.0(0)$ & $3.0(0)$ & $5.0(1.0)$ & $5.5(0.5)$ & $7.0(0.0)$ & $11.0(0)$ \\
\hline $\mathrm{pH}\left(\mathrm{H}_{2} 0\right)$ & $5.4(0)$ & $5.6(0)$ & $5.6(0.3)$ & $5.4(0.2)$ & $5.2(0.1)$ & $5.0(0.1)$ \\
\hline $\mathrm{pH}(\mathrm{KCl})$ & $4.6(0)$ & $4.5(0)$ & $4.5(0.3)$ & $4.4(0.2)$ & $4.2(0.1)$ & $4.3(0.0)$ \\
\hline $\mathrm{C}[\%]$ & $0.60(0.02)$ & $0.56(0.02)$ & $0.57(0.07)$ & $0.44(0.04)$ & $0.28(0.01)$ & $0.22(0.00)$ \\
\hline $\mathrm{N}[\%]$ & $0.10(0.01)$ & $0.09(0.00)$ & $0.10(0.0) 1$ & $0.08(0.01)$ & $0.08(0.01)$ & $0.08(0.02)$ \\
\hline $\mathrm{C} / \mathrm{N}$ & $6.0(0.0)$ & $6.0(0.0)$ & $5.5(0.5)$ & $5.0(0.0)$ & $3.5(0.5)$ & $3.5(0.5)$ \\
\hline $\mathrm{P}[\mathrm{ppm}]$ & $2.5(0.5)$ & $2.5(0.5)$ & $3.0(0.0)$ & $4.0(0.0)$ & $2.5(0.5)$ & $2.0(0.0)$ \\
\hline $\mathrm{Ca}^{++}[\mathrm{mval} / 100 \mathrm{~g}]^{*}$ & $0.75(0.15)$ & $0.4(0)$ & $0.3(0.1)$ & $0.45(0.05)$ & $0.3(0.1)$ & $0.2(0.00)$ \\
\hline $\mathrm{Mg}^{++}[\mathrm{mval} / 100 \mathrm{~g}]$ & $0.85(0.25)$ & $1.00(0.30)$ & $0.70(0.00)$ & $0.70(0.00)$ & & \\
\hline $\mathrm{K}^{+}[\mathrm{mval} / 100 \mathrm{~g}]$ & $0.03(0.00)$ & $0.02(0.00)$ & $0.02(0.00)$ & $0.02(0.00)$ & $0.01(0.00)$ & $0.01(0.00)$ \\
\hline $\mathrm{Na}^{+}[\mathrm{mval} / 100 \mathrm{~g}]$ & $0.04(0.02)$ & $0.02(0.01)$ & $0.01(0.00)$ & $0.02(0.00)$ & $0.02(0.01)$ & $0.02(0.01)$ \\
\hline $\mathrm{Al}^{+++}[\mathrm{mval} / 100 \mathrm{~g}]$ & $0.00(0.00)$ & $0.00(0.00)$ & $0.10(0.10)$ & $0.10(0.10)$ & $0.45(0.05)$ & $0.45(0.05)$ \\
\hline $\mathrm{H}^{+}[\mathrm{mval} / 100 \mathrm{~g}]$ & $1.60(0.20)$ & $1.20(0.20)$ & $1.10(0.10)$ & $1.30(0.10)$ & $1.25(0.25)$ & $1.40(0.10)$ \\
\hline $\mathrm{Ak}[\mathrm{mval} / 100 \mathrm{~g}]$ & $3.25(0.35)$ & $2.60(0.10)$ & $2.20(0.10)$ & $2.20(0.10)$ & $2.00(0.20)$ & $2.05(0.15)$ \\
\hline Ake $[\mathrm{mval} / \mathrm{g}]$ & $1.65(0.15)$ & $1.40(0.30)$ & $1.00(0.10)$ & $0.80(0.30)$ & $0.30(0.10)$ & $0.2(0.00)$ \\
\hline Basensättigung [\%] & $51.0(1.0)$ & $53.5(9.5)$ & $45.5(6.5)$ & $36.0(12.0)$ & $15.5(6.5)$ & $9.5(0.5)$ \\
\hline $\mathrm{Zn}[\mathrm{ppm}]$ & $0.10(0.01)$ & $0.06(0.02)$ & $0.04(0.02)$ & $0.15(0.07)$ & $0.08(0.03)$ & $0.08(0.02)$ \\
\hline $\mathrm{Cu}[\mathrm{ppm}]$ & $0.01(0.00)$ & $0.01(0.00)$ & $0.01(0.00)$ & $0.01(0.00)$ & $0.01(0.00)$ & $0.01(0.00)$ \\
\hline Mn [ppm] & $1.55(0.06)$ & $1.25(0.41)$ & $0.80(0.12)$ & $0.78(0.10)$ & $0.64(0.19)$ & $0.48(0.08)$ \\
\hline
\end{tabular}

* leere Zellen $=\mathrm{Ca}^{++}$und $\mathrm{Mg}^{++}$wurden zusammen bestimmt 
Tab. 55: Physikalische und chemische Eckdaten des Bodens der Untersuchungsfläche $\mathbf{S 2}$ für die Bodentiefen von 0 bis $100 \mathrm{~cm}$ (es sind die Mittelwerte aus 6 Mischproben je Bodentiefe mit dem Standardfehler angegeben)

\begin{tabular}{|c|c|c|c|c|c|}
\hline Bodentiefe [\%] & $0-10$ & $10-20$ & $20-40$ & $40-60$ & $60-100$ \\
\hline \multicolumn{6}{|l|}{ Grobsand [\%] } \\
\hline \multicolumn{6}{|l|}{ Feinsand [\%] } \\
\hline \multicolumn{6}{|l|}{ Schluff [\%] } \\
\hline \multicolumn{6}{|l|}{ Ton $[\%]$} \\
\hline $\mathrm{pH}\left(\mathrm{H}_{2} \mathrm{O}\right)$ & 5.1 & 5.2 & 5.2 & 5.2 & 5.3 \\
\hline $\mathrm{pH}(\mathrm{KCl})$ & 4.0 & 4.1 & 4.2 & 4.2 & 4.2 \\
\hline $\mathrm{C}[\%]$ & 0.87 & 0.58 & 0.37 & 0.25 & 0.20 \\
\hline $\mathrm{N}[\%]$ & 0.12 & 0.08 & 0.06 & 0.05 & 0.04 \\
\hline $\mathrm{C} / \mathrm{N}$ & 7 & 7 & 6 & 5 & 5 \\
\hline $\mathrm{P}[\mathrm{ppm}]$ & 3 & 3 & 1 & 1 & 1 \\
\hline $\mathrm{Ca}^{++}[\mathrm{mval} / 100 \mathrm{~g}]^{*}$ & 0.60 & 0.70 & 0.70 & 0.70 & 0.50 \\
\hline $\mathrm{Mg}^{++}[\mathrm{mval} / 100 \mathrm{~g}]$ & 0.40 & & & & \\
\hline $\mathrm{K}^{+}[\mathrm{mval} / 100 \mathrm{~g}]$ & 0.04 & 0.03 & 0.02 & 0.01 & 0.02 \\
\hline $\mathrm{Na}^{+}[\mathrm{mval} / 100 \mathrm{~g}]$ & 0.04 & 0.04 & 0.02 & 0.01 & 0.01 \\
\hline $\mathrm{Al}^{+++}[\mathrm{mval} / 100 \mathrm{~g}]$ & 0.60 & 0.70 & 0.40 & 0.40 & 0.40 \\
\hline $\mathrm{H}^{+}[\mathrm{mval} / 100 \mathrm{~g}]$ & 3.20 & 3.00 & 1.70 & 1.50 & 1.20 \\
\hline $\mathrm{Ak}[\mathrm{mval} / 100 \mathrm{~g}]$ & 4.90 & 4.40 & 2.80 & 2.60 & 2.10 \\
\hline Ake [mval/g] & 1.10 & 0.70 & 0.70 & 0.70 & 0.50 \\
\hline Basensättigung [\%] & 22 & 16 & 25 & 27 & 24 \\
\hline $\mathrm{Zn}[\mathrm{ppm}]$ & 0.45 & 0.40 & 0.40 & 0.45 & 0.26 \\
\hline $\mathrm{Cu}[\mathrm{ppm}]$ & 0.001 & 0.001 & 0.02 & 0.03 & 0.04 \\
\hline Mn [ppm] & 2.09 & 1.24 & 1.07 & 0.62 & 0.20 \\
\hline
\end{tabular}

* leere Zellen $=\mathrm{Ca}^{++}$und $\mathrm{Mg}^{++}$wurden zusammen bestimmt 
Tab. 56: Physikalische und chemische Eckdaten des Bodens der Untersuchungsfläche S4 für die Bodentiefen von 0 bis $100 \mathrm{~cm}$ (es sind die Mittelwerte aus 6 Mischproben je Bodentiefe mit dem Standardfehler angegeben, durch Werte von U. Diekmann ergänzt)

\begin{tabular}{|c|c|c|c|c|c|}
\hline Bodentiefe [\%] & $0-10$ & $10-20$ & $20-40$ & $40-60$ & $60-100$ \\
\hline Grobsand [\%] & 66.1 & 56.9 & & & \\
\hline Feinsand [\%] & 20.1 & 23.7 & & & \\
\hline Schluff [\%] & 3.6 & 4.9 & & & \\
\hline Ton [\%] & 10.1 & 14.6 & & & \\
\hline $\mathrm{pH}\left(\mathrm{H}_{2} \mathrm{O}\right)$ & 5.8 & 5.2 & 4.9 & 4.9 & 4.9 \\
\hline $\mathrm{pH}(\mathrm{KCl})$ & 4.6 & 4.02 & 4.0 & 4.1 & 4.1 \\
\hline $\mathrm{C}[\%]$ & 1.02 & 0.64 & 0.43 & 0.34 & 0.29 \\
\hline $\mathrm{N}[\%]$ & 0.09 & 0.08 & 0.06 & 0.05 & 0.04 \\
\hline $\mathrm{C} / \mathrm{N}$ & 11 & 8.03 & 7 & 7 & 7 \\
\hline $\mathrm{P}[\mathrm{ppm}]$ & 5 & 2 & 2 & 1 & 1 \\
\hline $\mathrm{Ca}^{++}[\mathrm{mval} / 100 \mathrm{~g}]^{*}$ & 1.5 & 0.53 & 0.4 & 0.4 & 0.4 \\
\hline $\mathrm{Mg}^{++}[\mathrm{mval} / 100 \mathrm{~g}]$ & 0.3 & & & & \\
\hline $\mathrm{K}^{+}[\mathrm{mval} / 100 \mathrm{~g}]$ & 0.05 & 0.03 & 0.02 & 0.01 & 0.01 \\
\hline $\mathrm{Na}^{+}[\mathrm{mval} / 100 \mathrm{~g}]$ & 0.03 & 0.03 & 0.03 & 0.03 & 0.02 \\
\hline $\mathrm{Al}^{+++}[\mathrm{mval} / 100 \mathrm{~g}]$ & 0.00 & 0.23 & 1.00 & 1.20 & 1.00 \\
\hline $\mathrm{H}^{+}[\mathrm{mval} / 100 \mathrm{~g}]$ & 2.7 & 2.87 & 2.20 & 2.00 & 1.70 \\
\hline Ak [mval/100g] & 4.60 & 3.70 & 3.60 & 4.10 & 3.10 \\
\hline Ake $[\mathrm{mval} / 100 \mathrm{~g}]$ & 1.91 & 0.69 & 0.40 & 0.90 & 0.40 \\
\hline Basensättigung [\%] & 41.7 & 16.2 & 11 & 22 & 13 \\
\hline $\mathrm{Zn}[\mathrm{ppm}]$ & 0.37 & 0.41 & 0.37 & 0.38 & 0.40 \\
\hline $\mathrm{Cu}[\mathrm{ppm}]$ & 0.001 & 0.001 & 0.01 & 0.02 & 0.02 \\
\hline Mn [ppm] & 2.25 & 0.50 & 0.28 & 0.25 & 0.33 \\
\hline
\end{tabular}

\footnotetext{
* leere Zellen $=\mathrm{Ca}^{++}$und $\mathrm{Mg}^{++}$wurden zusammen bestimmt
} 
Tab. 57: Physikalische und chemische Eckdaten des Bodens der Untersuchungsfläche $\mathbf{S 6}$ für die Bodentiefen von 0 bis $100 \mathrm{~cm}$ (es sind die Mittelwerte aus 6 Mischproben je Bodentiefe mit dem Standardfehler angegeben. durch Werte von U. Diekmann ergänzt)

\begin{tabular}{|c|c|c|c|c|c|c|}
\hline Bodentiefe [\%] & $0-5$ & $0-10$ & $10-20$ & $20-40$ & $40-60$ & $60-100$ \\
\hline Grobsand [\%] & & 45.1 & 28.3 & & & \\
\hline Feinsand [\%] & & 39 & 41.6 & & & \\
\hline Schluff [\%] & & 5.6 & 7.6 & & & \\
\hline Ton [\%] & & 10.3 & 12.6 & & & \\
\hline $\mathrm{pH}\left(\mathrm{H}_{2} 0\right)$ & 5.2 & 5.5 & 5.2 & 5.0 & 5.1 & 5.0 \\
\hline $\mathrm{pH}(\mathrm{KCl})$ & 4.1 & 4.3 & 4.1 & 4.2 & 4.3 & 4.2 \\
\hline $\mathrm{C}[\%]$ & 1.11 & 1.12 & 0.72 & 0.46 & 0.33 & 0.22 \\
\hline $\mathrm{N}[\%]$ & 0.08 & 0.11 & 0.07 & 0.06 & 0.05 & 0.05 \\
\hline $\mathrm{C} / \mathrm{N}$ & 14 & 11 & 10 & 8 & 7 & 4 \\
\hline $\mathrm{P}[\mathrm{ppm}]$ & 3 & 3 & 2 & 1 & 1 & 1 \\
\hline $\mathrm{Ca}^{++}[\mathrm{mval} / 100 \mathrm{~g}]^{*}$ & 0.80 & 1.21 & 0.40 & 0.20 & 0.20 & 0.20 \\
\hline $\mathrm{Mg}^{++}[\mathrm{mval} / 100 \mathrm{~g}]$ & 0.40 & 0.37 & & & & \\
\hline $\mathrm{K}^{+}[\mathrm{mval} / 100 \mathrm{~g}]$ & 0.04 & 0.05 & 0.03 & 0.02 & 0.01 & 0.01 \\
\hline $\mathrm{Na}^{+}[\mathrm{mval} / 100 \mathrm{~g}]$ & 0.04 & 0.03 & 0.02 & 0.03 & 0.02 & 0.02 \\
\hline $\mathrm{Al}^{+++}[\mathrm{mval} / 100 \mathrm{~g}]$ & 0.40 & 0.70 & 0.40 & 0.80 & 0.80 & 0.80 \\
\hline $\mathrm{H}^{+}[\mathrm{mval} / 100 \mathrm{~g}]$ & 3.30 & 3.2 & 2.90 & 2.20 & 1.50 & 1.20 \\
\hline $\mathrm{Ak}[\mathrm{mval} / 100 \mathrm{~g}]$ & 5.00 & 4.90 & 3.75 & 3.20 & 2.50 & 2.20 \\
\hline Ake [mval/g] & 1.30 & 1.70 & 0.43 & 0.20 & 0.20 & 0.20 \\
\hline Basensättigung [\%] & 26 & 34 & 11 & 6 & 8 & 9 \\
\hline Zn [ppm] & 0.43 & 0.59 & 0.27 & 0.54 & 0.55 & 0.22 \\
\hline $\mathrm{Cu}[\mathrm{ppm}]$ & 0.001 & 0.02 & 0.02 & 0.01 & 0.03 & 0.05 \\
\hline $\mathrm{Mn}$ [ppm] & 1.65 & 0.55 & 0.19 & 0.19 & 0.20 & 0.28 \\
\hline
\end{tabular}

* leere Zellen $=\mathrm{Ca}^{++}$und $\mathrm{Mg}^{++}$wurden zusammen bestimmt 
Tab. 58: Liste aller beprobten Arten, ihre Familienzugehörigkeit, Wuchsform und Zuordnung zu den Abkürzungen

\begin{tabular}{|c|c|c|c|c|}
\hline wissenschaftlicher Name & Familie & Abk. & Trivialname & Wuchsform \\
\hline Abarema jupunba (WILLD.)BRITTON und KILLIP & Mimosaceae & $\mathrm{Aj}$ & Saboueira & Baum, bis $35 \mathrm{~m}$ \\
\hline Aegiphila racemosa $(V E L L)$ & Verbenaceae & & & Bäumchen, bis $4 \mathrm{~m}$ \\
\hline Bagassa guianensis & Moraceae & & & \\
\hline Banara guianensis AUBL. & Flacourtiaceae & Ban & Quebra faca & Baum, bis $12 \mathrm{~m}$ \\
\hline Bauhinia spp. & Caesalpiniaceae & $\mathrm{Bau}$ & Unha da vaca & Kletterpflanze \\
\hline Bombax longipedicellatum (ROBYNS) DUCKE & Bombacaceae & $\mathrm{B} 1$ & & Baum, bis $35 \mathrm{~m}$ \\
\hline Casearia arborea & Flacourtiaceae & $\mathrm{Ca}$ & & Baum, bis $10 \mathrm{~m}$ \\
\hline Cassia apoucouita AUBL. & Caesalpiniaceae & Cap & Coracão de negro & Baum, bis $30 \mathrm{~m}$ \\
\hline Cassia chrysocarpa DESV. & Caesalpiniaceae & $\mathrm{Cc}$ & & holzige Kletterpflanze \\
\hline Cordia exaltata (KTZE) LAM. & Boraginaceae & $\mathrm{Ce}$ & Chapeau de sol & Baum \\
\hline Croton matourensis (AUBL.) & Euphorbiaceae & $\mathrm{Cm}$ & Maravuvuia & Baum, bis $25 \mathrm{~m}$ \\
\hline Cordia nodosa (KTZE) LAM. & Boraginaceae & $\mathrm{Cn}$ & Pau de formiga & Baum, bis $10 \mathrm{~m}$ \\
\hline Connarus perrottetii (D.C.) PLANCH & Connaraceae & $\mathrm{Cp}$ & Marximbe & Baum, bis $8 \mathrm{~m}$ \\
\hline Desmodium bracteata & Fabaceae & & & Kraut \\
\hline Dipteryx odorata & Fabaceae & & & Baum, \\
\hline Guatteria poeppigiana MART. & Annonaceae & Gp & & Baum \\
\hline Homalium guianensis AUBL.OKEN & Flacourtiaceae & $\mathrm{Hg}$ & & \\
\hline Hymenaea parviflora $H A B E R$ & Fabaceae & Hym & Jutaí-mirim & Baum, \\
\hline Inga gracidifolia & Mimosaceae & Ig & & Baum, \\
\hline Inga alba (SW) WILLD. & Mimosaceae & Ina & Ingá vermelha & Baum, bis $15 \mathrm{~m}$ \\
\hline Inga edulis MART. & Mimosaceae & Ine & Ingá cipó & Baum, bis $30 \mathrm{~m}$ \\
\hline Inga heterophylla WILLD. & Mimosaceae & Inh & Inga xixíca & Baum, bis $12 \mathrm{~m}$ \\
\hline Inga macrophylla H.B.K. & Mimosaceae & Inm & & Baum, bis $15 \mathrm{~m}$ \\
\hline Inga nitida WILLD. & Mimosaceae & Inn & & Baum, \\
\hline Inga rubiginosa & Mimosaceae & Inr & Ingá peludá & Baum, bis $25 \mathrm{~m}$ \\
\hline Inga thibaudiana $D C$ & Mimosaceae & Int & Ingá xixí & Baum,bis $25 \mathrm{~m}$ \\
\hline Lacistema pubescens & Lacistemataceae & Lac & Caferana & Baum, bis $10 \mathrm{~m}$ \\
\hline Lecythis lurida (MIERS.) MORI & Lecythidaceae & $\mathrm{Ll}$ & Jarana & Baum, bis $10 \mathrm{~m}$ \\
\hline Mabea angustifolia BENTH. & Euphorbiaceae & Ma & & Bäumchen \\
\hline $\begin{array}{l}\text { Memora allamandiflora (BENTH.) } \\
\text { BUR. }+ \text { K.SCHUM. }\end{array}$ & Bignoniaceae & Mal & & Kraut \\
\hline Myrcia bracteata (L.C.RICH) DC. & Myrtaceae & $\mathrm{Mb}$ & Murta-cabeluda & Baum \\
\hline Myrcia cuprea (BERG) KIAERS & Myrtaceae & & & Baum \\
\hline Myrcia sylvatica $(M E Y) D C$ & Myrtaceae & Ms & Murta & Baum, bis $6 \mathrm{~m}$ \\
\hline Myrcia deflexa (POIR) DC. & Myrtaceae & Myd & Murta & Baum, bis $20 \mathrm{~m}$ \\
\hline Machaerium quinata (AUBL.) SANDW. & Fabaceae & $\mathrm{Mq}$ & & Strauch \\
\hline Nectandra cuspidata (MART.ex NEES) MEZ & Lauraceae & $\mathrm{Nc}$ & Louro preto & Baum, bis $30 \mathrm{~m}$ \\
\hline Ocotea guianensis AUBL. & Lauraceae & Oc & Louro prata & Baum, bis $25 \mathrm{~m}$ \\
\hline Rollinia exsucca & Annonaceae & & Envira preta & Baum, bis $15 \mathrm{~m}$ \\
\hline Rourea sp. & Connaraceae & Rs & & holzige Kletterpflanze \\
\hline Selaginella stellaria & Selaginellaceae & & & Kraut \\
\hline Solanum caavurana VELL & Solanaceae & & & Strauch \\
\hline Solanum crinitum LAM. & Solanaceae & Sc & & Baumstrauch, bis $5 \mathrm{~m}$ \\
\hline Sloanea guianensis (AUBL.) BENTH. & Elaeocarpaceae & $\mathrm{Sg}$ & & Baum \\
\hline Simaba cedron PLANCK & Simarubaceae & Sic & Jurubebão & Bäumchen \\
\hline Sterculia pruriens & Sterculiaceae & & & Kraut \\
\hline Tapirira guianensis AUBL. & Anacariaceae & Tap & Tapiririca & Baum, bis $45 \mathrm{~m}$ \\
\hline Trema micrantha (L.) BLUME & Ulmaceae & $\mathrm{Tm}$ & Corindiba & Baum, bis $8 \mathrm{~m}$ \\
\hline Thyrsodium paraense HUBER & Anacardiaceae & $\mathrm{Tp}$ & Amaparana & Baum, bis $10 \mathrm{~m}$ \\
\hline Vismia guianensis (AUBL.) CHOISY & Guttiferae & Vis & Lacre & Baum, bis 9m \\
\hline
\end{tabular}


Tab. 59: $\delta^{15} \mathrm{~N}$-Werte und $\mathrm{N}$-Gehalte in den Blättern der Sekundärvegetation der Bragantina-Region

\begin{tabular}{|c|c|c|c|c|}
\hline Art & Familie & Trivialname & $\% \mathrm{~N}$ & $\delta^{15} \mathrm{~N}$ \\
\hline Abarema jupunba (WILLD.)BRITTON und KILLIP & Mimosaceae & Saboueira & $2.11 \pm 0.50$ & $-0.60 \pm 0.96$ \\
\hline Banara guianensis AUBL. & Flacourtiaceae & Quebra faca & $1.88 \pm 0.59$ & $-0.96 \pm 1.22$ \\
\hline Bauhinia spp. & Caesalpiniaceae & Unha da vaca & $2.62 \pm 0.08$ & $5.42 \pm 1.45$ \\
\hline Bombax longipedicellatum (ROBYNS) DUCKE & Bombacaceae & & $1.22 \pm 0.32$ & $-1.43 \pm 0.91$ \\
\hline Casearia arborea & Flacourtiaceae & & $1.45 \pm 0.93$ & $0.42 \pm 1.33$ \\
\hline Cassia apoucouita AUBL. & Caesalpiniaceae & Coracão de negro & $1.29 \pm 0.35$ & $-1.13 \pm 1.07$ \\
\hline Cassia chrysocarpa DESV. & Caesalpiniaceae & & $2.37 \pm 0.02$ & $3.77 \pm 0.67$ \\
\hline Cordia exaltata (KTZE) LAM. & Boraginaceae & Chapeau de sol & $1.82 \pm 0.69$ & $0.11 \pm 1.46$ \\
\hline Croton matourensis (AUBL.) & Euphorbiaceae & Maravuvuia & $2.14 \pm 0.60$ & $0.17 \pm 0.80$ \\
\hline Cordia nodosa (KTZE) LAM. & Boraginaceae & Pau de formiga & $1.08 \pm 0.24$ & $2.96 \pm 3.54$ \\
\hline Connarus perrottetii (D.C.) PLANCH & Connaraceae & Marximbe & $1.11 \pm 0.36$ & $0.92 \pm 1.21$ \\
\hline Guatteria poeppigiana MART. & Annonaceae & & $1.79 \pm 0.70$ & $-1.22 \pm 1.80$ \\
\hline Homalium guianensis AUBL.OKEN & Flacourtiaceae & & $1.77 \pm 0.86$ & $-0.17 \pm 1.34$ \\
\hline Hymenaea parviflora HABER & Fabaceae & Jutaí-mirim & $2.36 \pm 0.06$ & $2.30 \pm 1.48$ \\
\hline Inga gracidifolia & Mimosaceae & & $2.12 \pm 0.08$ & $1.42 \pm 1.26$ \\
\hline Inga alba $(S W)$ WILLD. & Mimosaceae & Ingá vermelha & $2.28 \pm 0.11$ & $1.54 \pm 1.16$ \\
\hline Inga edulis MART. & Mimosaceae & Ingá cipó & $1.52 \pm 0.37$ & $-0.51 \pm 1.07$ \\
\hline Inga heterophylla WILLD. & Mimosaceae & Inga xixíca & $2.33 \pm 0.61$ & $-0.94 \pm 0.71$ \\
\hline Inga macrophylla H.B.K. & Mimosaceae & & $1.51 \pm 0.10$ & $-0.86 \pm 0.02$ \\
\hline Inga nitida WILLD. & Mimosaceae & & $2.34 \pm 0.35$ & $0.66 \pm 0.71$ \\
\hline Inga rubiginosa & Mimosaceae & Ingá peludá & $1.91 \pm 0.15$ & $0.52 \pm 0.19$ \\
\hline Inga thibaudiana $D C$ & Mimosaceae & Ingá xixí & $1.89 \pm 0.56$ & $-0.72 \pm 0.62$ \\
\hline Lacistema pubescens & Lacistemataceae & Caferana & $1.64 \pm 0.50$ & $0.32+1.19$ \\
\hline Lecythis lurida (MIERS.) MORI & Lecythidaceae & Jarana & $1.75 \pm 0.56$ & $0.51 \pm 1.86$ \\
\hline Mabea angustifolia BENTH. & Euphorbiaceae & & $1.96 \pm 0.54$ & $-1.66 \pm 1.24$ \\
\hline $\begin{array}{l}\text { Memora allamandiflora (BENTH.) } \\
\text { BUR. }+ \text { K.SCHUM. }\end{array}$ & Bignoniaceae & & $1.90 \pm 0.43$ & $1.53 \pm 1.80$ \\
\hline Myrcia bracteata (L.C.RICH) DC. & Myrtaceae & Murta-cabeluda & $2.63 \pm 0.40$ & $4.69 \pm 1.48$ \\
\hline Machaerium quinata (AUBL.) SANDW. & Fabaceae & & $2.43 \pm 0.48$ & $0.39 \pm 0.48$ \\
\hline Myrcia sylvatica $(M E Y) D C$ & Myrtaceae & Murta & $1.25 \pm 0.32$ & $-1.03 \pm 1.15$ \\
\hline Myrcia deflexa (POIR) DC. & Myrtaceae & Murta & $1.55 \pm 0.13$ & $0.03 \pm 0.94$ \\
\hline Nectandra cuspidata (MART.ex NEES) MEZ & Lauraceae & Louro preto & $1.37 \pm 0.41$ & $-2.93 \pm 2.10$ \\
\hline Ocotea guianensis AUBL. & Lauraceae & Louro prata & $1.70 \pm 0.49$ & $-2.33 \pm 1.55$ \\
\hline Rourea sp. & Connaraceae & & $2.05 \pm 0.15$ & $1.20 \pm 0.38$ \\
\hline Solanum crinitum LAM. & Solanaceae & & $2.07 \pm 0.47$ & $1.65 \pm 1.81$ \\
\hline Sloanea guianensis (AUBL.) BENTH. & Elaeocarpaceae & & $1.34 \pm 0.21$ & $-0.40 \pm 0.93$ \\
\hline Simaba cedron PLANCK & Simarubaceae & Jurubebão & $1.18 \pm 0.26$ & $-3.00 \pm 1.06$ \\
\hline Tapirira guianensis AUBL. & Anacariaceae & Tapiririca & $1.14 \pm 0.39$ & $-2.13 \pm 0.69$ \\
\hline Trema micrantha (L.) BLUME & Ulmaceae & Corindiba & $2.04 \pm 0.65$ & $-0.35 \pm 1.10$ \\
\hline Thyrsodium paraense HUBER & Anacardiaceae & Amaparana & $1.77 \pm 0.39$ & $-1.29 \pm 0.69$ \\
\hline Vismia guianensis (AUBL.) CHOISY & Guttiferae & Lacre & $1.29 \pm 0.38$ & $-1.61 \pm 1.49$ \\
\hline
\end{tabular}


Tab. 60: Einfluß von kleinräumigem und großräumigem Standortunterschieden und der Bodentiefe auf den Prozentsatz $\mathrm{N}$ und $\delta^{15} \mathrm{~N}$ des $\mathrm{N}_{\text {tot. }}$ des Bodens

\begin{tabular}{l|c|cc|cc|cc}
\hline & FG & \multicolumn{2}{|c|}{ QS } & \multicolumn{2}{c|}{ MQS } & \multicolumn{2}{c}{ F-Wert } \\
\hline Hauptwirkungen: & & $\delta^{15} \mathrm{~N}$ & $\% \mathrm{~N}$ & $\delta^{15} \mathrm{~N}$ & $\% \mathrm{~N}$ & $\delta^{15} \mathrm{~N}$ & $\% \mathrm{~N}$ \\
kleine Distanzen & 1 & 1.12 & 0.000 & 1.12 & 0.000 & $1.98^{\text {n.s. }}$ & $0.01^{\text {n.s. }}$ \\
Fläche & 4 & 15.42 & 0.98 & 3.85 & 0.24 & $6.79 * * *$ & $27.29 * * *$ \\
Bodentiefe & 5 & 279.74 & 5.41 & 55.95 & 1.08 & $98.48^{* * *}$ & $121.19^{* * *}$ \\
Wechselwirkungen: & & & & & & & \\
kleine Distanz x Fläche & 4 & 10.04 & 0.22 & 2.51 & 0.06 & $4.42^{* *}$ & $6.30^{* * *}$ \\
kleine Distanz x Bodentiefe & 5 & 1.77 & 0.23 & 0.35 & 0.05 & $0.62^{\text {n.s. }}$ & $5.27 * * *$ \\
Fläche x Bodentiefe & 20 & 15.59 & 1.30 & 0.78 & 0.06 & $1.37^{\text {n.s. }}$ & $7.27 * * *$ \\
kl.Distanz x Fläche x Bodentiefe & 20 & 12.69 & 1.24 & 0.63 & 0.06 & $1.12^{\text {n.s. }}$ & $6.93 * * *$ \\
\hline Residuen & 126 & 71.58 & 1.12 & 0.56 & 0.009 & & \\
Total & 185 & 407.96 & 10.51 & & & & \\
\hline
\end{tabular}


Tab. 61: Kleinräumige Unterschiede in den $\delta^{15} \mathrm{~N}$-Werten und N-Gehalten der Böden von Fläche M1 bis M5 (Leg. = Boden unter einer Leguminose, Ref. $=$ Boden unter Referenzpflanzen im 5m Radius um die Leguminose herum, es ist das arithmetische Mittel und in Klammern die Standardabweichung angegeben)

\begin{tabular}{|c|c|c|c|c|c|c|c|c|c|c|}
\hline \multirow[t]{2}{*}{ Bodentiefe $[\mathrm{cm}]$} & \multicolumn{2}{|c|}{ M1 } & \multicolumn{2}{|c|}{ M2 } & \multicolumn{2}{|c|}{ M3 } & \multicolumn{2}{|c|}{ M4 } & \multicolumn{2}{|c|}{ M5 } \\
\hline & Leg. & Ref. & Leg. & Ref. & Leg. & Ref. & Leg. & Ref. & Leg. & Ref. \\
\hline \multicolumn{11}{|c|}{$\delta^{15} \mathrm{~N}-\mathrm{Wert}$} \\
\hline $0-5$ & $3.66(0.08)$ & $3.61(0.18)$ & $2.98(0.10)$ & $4.22(0.19)$ & $3.42(0.52)$ & $3.43(0.16)$ & $4.27(0.74)$ & $3.84(0.00)$ & $2.80(0.48)$ & $3.88(0.43)$ \\
\hline $5-10$ & $4.50(0.34)$ & $4.48(0.14)$ & $7.66(0.09)$ & $5.83(0.31)$ & $4.39(0.75)$ & $4.84(0.22)$ & $4.95(0.44)$ & $5.00(0.00)$ & $3.33(0.56)$ & $4.00(1.00)$ \\
\hline $10-20$ & $6.10(0.06)$ & $6.39(0.21)$ & $5.98(0.35)$ & $6.78(0.48)$ & $5.78(0.43)$ & $6.42(0.30)$ & $6.34(0.23)$ & $2.87(3.28)$ & $5.00(0.63)$ & $5.55(0.62)$ \\
\hline $20-40$ & $6.42(0.21)$ & $7.03(0.17)$ & $7.00(0.29)$ & $7.33(0.36)$ & 6.09 (1.37) & $6.71(0.31)$ & $7.03(0.50)$ & $5.62(0.00)$ & $6.18(0.10)$ & $6.32(0.72)$ \\
\hline $40-60$ & $6.78(0.32)$ & $7.03(0.28)$ & $7.46(0.47)$ & $7.66(0.15)$ & $7.06(0.81)$ & $6.79(0.73)$ & $6.97(0.25)$ & $6.23(0.49)$ & $5.75(0.62)$ & $6.03(0.36)$ \\
\hline $60-100$ & $6.52(0.00)$ & $6.75(0.49)$ & $7.23(0.43)$ & $6.99(0.34)$ & $6.86(0.86)$ & $6.73(0.86)$ & $6.62(0.00)$ & $6.77(0.16)$ & $6.31(0.54)$ & $6.50(0.63)$ \\
\hline \multicolumn{11}{|c|}{ N-Gehalte } \\
\hline $0-5$ & $0.12(0.02)$ & $0.10(0.01)$ & $0.13(0.00)$ & $0.35(0.00)$ & $0.21(0.17)$ & $0.12(0.02)$ & $0.11(0.01)$ & $0.09(0.00)$ & $0.06(0.02)$ & $0.06(0.00)$ \\
\hline $5-10$ & $0.10(0.02)$ & $0.08(0.01)$ & $0.04(0.00)$ & $0.07(0.00)$ & $0.09(0.02)$ & $0.08(0.01)$ & $0.09(0.01)$ & $0.08(0.00)$ & $0.07(0.02)$ & $0.06(0.01)$ \\
\hline $10-20$ & $0.06(0.00)$ & $0.06(0.01)$ & $0.07(0.00)$ & $0.06(0.00)$ & $0.06(0.01)$ & $0.06(0.01)$ & $0.06(0.00)$ & $1.03(0.00)$ & $0.05(0.00)$ & $0.05(0.00)$ \\
\hline $20-40$ & $0.06(0.00)$ & $0.05(0.00)$ & $0.06(0.00)$ & $0.05(0.01)$ & $0.05(0.01)$ & $0.05(0.01)$ & $0.05(0.00)$ & $0.04(0.00)$ & $0.04(0.01)$ & $0.04(0.00)$ \\
\hline $40-60$ & $0.04(0.00)$ & $0.05(0.01)$ & $0.04(0.00)$ & $0.04(0.00)$ & $0.04(0.00)$ & $0.04(0.01)$ & $0.04(0.00)$ & $0.04(0.00)$ & $0.04(0.01)$ & $0.03(0.00)$ \\
\hline $60-100$ & $0.04(0.00)$ & $0.04(0.01)$ & $0.04(0.00)$ & $0.04(0.00)$ & $0.04(0.00)$ & $0.04(0.00)$ & $0.05(0.01)$ & $0.05(0.01)$ & $0.02(0.00)$ & $0.03(0.00)$ \\
\hline
\end{tabular}


Tab. 62: Einfluß von kleinräumigen und großräumigen Standortunterschieden und des Alters der organischen Auflage auf den N-Gehalt und $\delta^{15} \mathrm{~N}$ des $\mathrm{N}_{\text {tot }}$. der Streuauflage (Flächen M1 - M5)

\begin{tabular}{l|c|cc|cc|cc}
\hline & FG & \multicolumn{2}{|c|}{ QS } & \multicolumn{2}{|c}{ MQS } & \multicolumn{2}{c}{ F-Wert } \\
\hline Hauptwirkungen: & & $\delta^{15} \mathrm{~N}$ & $\% \mathrm{~N}$ & $\delta^{15} \mathrm{~N}$ & $\% \mathrm{~N}$ & $\delta^{15} \mathrm{~N}$ & $\% \mathrm{~N}$ \\
kleine Distanzen & 1 & 4.19 & 0.59 & 4.19 & 0.59 & $3.59 \mathrm{~ns}$ & $9.85^{*}$ \\
Fläche & 4 & 12.27 & 0.77 & 3.07 & 0.19 & $3.42^{*}$ & $3.23^{*}$ \\
Alter organ. Auflage & 1 & 0.45 & 0.37 & 0.45 & 0.37 & $0.50 \mathrm{~ns}$ & $6.05^{*}$ \\
Wechselwirkungen: & & & & & & & \\
kleine Distanz x Fläche & 4 & 17.82 & 0.04 & 4.46 & 0.01 & $4.07 * *$ & $0.11 \mathrm{~ns}$ \\
kleine Distanz x Alter organ. Auflage & 1 & 1.41 & 1.40 & 1.41 & 1.40 & $1.57 \mathrm{~ns}$ & $23.54^{* * *}$ \\
Fläche x Alter organ. Auflage & 4 & 8.55 & 0.52 & 2.14 & 0.13 & $2.38 \mathrm{~ns}$ & $2.18 \mathrm{~ns}$ \\
kl.Distanz x Fläche x Alter organ. & 4 & 8.32 & 0.26 & 2.08 & 0.06 & $2.32 \mathrm{~ns}$ & $1.09 \mathrm{~ns}$ \\
Auflage & & & & & & & \\
\hline Residuen & 43 & 38.62 & 2.57 & 0.90 & 0.06 & & \\
Total & 62 & 91.24 & 6.53 & & & & \\
\hline
\end{tabular}


Tab. 63: $\delta^{15} \mathrm{~N}$-Werte der Blätter von 7 Arten auf verschiedenen Flächen mit junger Sekundärvegetation (Mittelwerte mits in Klammern, $\mathrm{n}=5$ )

\begin{tabular}{|c|c|c|c|c|c|c|c|c|}
\hline Fläche & $\begin{array}{l}\text { Abarema } \\
\text { jupunba }\end{array}$ & $\begin{array}{c}\text { Inga } \\
\text { thibaudiana }\end{array}$ & $\begin{array}{c}\text { Inga } \\
\text { heterophylla }\end{array}$ & $\begin{array}{c}\text { Banara } \\
\text { guianensis }\end{array}$ & $\begin{array}{l}\text { Lacistema } \\
\text { pubescens }\end{array}$ & $\begin{array}{c}\text { Tapirira } \\
\text { guianensis }\end{array}$ & $\begin{array}{c}\text { Vismia } \\
\text { guianensis }\end{array}$ & $\begin{array}{c}\text { LSD } \\
(\mathrm{p}<0.05)\end{array}$ \\
\hline \multicolumn{9}{|c|}{ neue Blätter } \\
\hline S1 & $-0.04( \pm 0.41)$ & $-0.13( \pm 0.37)$ & $-0.94( \pm 0.34)$ & $-1.25( \pm 1.09)$ & $0.72( \pm 0.70)$ & $-3.11( \pm 0.78)$ & $-2.56( \pm 0.20)$ & 0.48 \\
\hline $\mathrm{S} 2$ & - & - & $1.03( \pm 0.34)$ & $1.23( \pm 0.97)$ & $2.81( \pm 0.84)$ & $-0.53( \pm 0.48)$ & $1.60( \pm 0.36)$ & 0.67 \\
\hline $\mathrm{S} 3$ & $0.10( \pm 0.40)$ & $-0.32( \pm 0.12)$ & $1.10( \pm 0.45)$ & $-1.27( \pm 0.27)$ & $2.26( \pm 0.11)$ & $-1.43( \pm 0.44)$ & $0.74( \pm 0.14)$ & 0.57 \\
\hline S4 & $0.16( \pm 0.23)$ & $-0.40( \pm 0.17)$ & $-0.50( \pm 0.69)$ & $-0.46( \pm 0.27)$ & $1.41( \pm 0.63)$ & $-1.81( \pm 0.10)$ & $1.69( \pm 0.79)$ & 0.61 \\
\hline S5 & $-0.12( \pm 0.71)$ & $0.11( \pm 0.15)$ & $-0.33( \pm 0.46)$ & $-1.08( \pm 0.66)$ & $-0.69( \pm 0.31)$ & $-1.59( \pm 0.43)$ & $-1.51( \pm 0.41)$ & 0.29 \\
\hline S6 & $0.13( \pm 0.26)$ & $-0.06( \pm 0.54)$ & $-0.19( \pm 0.56)$ & $-0.54( \pm 0.54)$ & $2.06( \pm 0.81)$ & $-3.72( \pm 0.56)$ & $-1.93( \pm 1.15)$ & 0.42 \\
\hline $\begin{array}{c}\text { LSD } \\
(\mathrm{p}<0.05)\end{array}$ & 0.44 & 0.35 & 0.48 & 0.73 & 0.60 & 0.54 & 0.59 & \\
\hline \multicolumn{9}{|c|}{ alte Blätter } \\
\hline S1 & $-0.67( \pm 0.55)$ & $-0.76( \pm 0.11)$ & $-1.10( \pm 0.41)$ & $-0.93( \pm 0.43)$ & $-0.47( \pm 0.84)$ & $-3.08( \pm 0.66)$ & $-3.08( \pm 0.71)$ & 0.61 \\
\hline $\mathrm{S} 2$ & - & - & $-0.17( \pm 0.30)$ & $1.28( \pm 1.08)$ & $1.61( \pm 0.99)$ & $-1.79( \pm 0.66)$ & $1.83( \pm 0.50)$ & 1.07 \\
\hline $\mathrm{S} 3$ & $-0.56( \pm 0.58)$ & $-0.63( \pm 0.13)$ & $0.48( \pm 0.77)$ & $-1.22( \pm 0.59)$ & $1.18( \pm 0.40)$ & $-1.10( \pm 0.90)$ & $-0.16( \pm 0.26)$ & 0.53 \\
\hline S4 & $-0.16( \pm 0.21)$ & $-0.35( \pm 0.19)$ & $-0.77( \pm 0.67)$ & $-0.52( \pm 0.18)$ & $1.04( \pm 1.42)$ & $-1.19( \pm 0.50)$ & $1.16( \pm 0.50)$ & 0.94 \\
\hline S5 & $-0.30( \pm 0.92)$ & $-0.27( \pm 0.25)$ & $-1.00( \pm 0.29)$ & $-1.39( \pm 0.46)$ & $-0.17( \pm 0.38)$ & $-0.88( \pm 0.66)$ & $-2.04( \pm 0.57)$ & 0.62 \\
\hline S6 & $-0.73( \pm 0.02)$ & $-0.95( \pm 0.23)$ & $-1.45( \pm 0.49)$ & $-1.02( \pm 1.74)$ & $0.24( \pm 1.05)$ & $-2.65( \pm 1.21)$ & $-2.74( \pm 0.59)$ & 0.94 \\
\hline $\begin{array}{c}\text { LSD } \\
(\mathrm{p}<0.05)\end{array}$ & 0.43 & 0.36 & 0.51 & 0.96 & 0.87 & 0.61 & 0.53 & \\
\hline
\end{tabular}


Tab. 64: N-Gehalte der Blätter von 7 Arten auf verschiedenen Flächen mit junger Sekundärvegetation (Mittelwerte mit Standardabweichungen in Klammern, $\mathrm{n}=5$ )

\begin{tabular}{|c|c|c|c|c|c|c|c|c|}
\hline Fläche & $\begin{array}{r}\text { Abarema } \\
\text { jupunba }\end{array}$ & $\begin{array}{c}\text { Inga } \\
\text { thibaudiana }\end{array}$ & $\begin{array}{c}\text { Inga } \\
\text { heterophylla }\end{array}$ & $\begin{array}{c}\text { Banara } \\
\text { guianensis }\end{array}$ & $\begin{array}{l}\text { Lacistema } \\
\text { pubescens }\end{array}$ & $\begin{array}{c}\text { Tapirira } \\
\text { guianensis }\end{array}$ & $\begin{array}{c}\text { Vismia } \\
\text { guianensis }\end{array}$ & $\begin{array}{c}\text { LSD } \\
(p<0.05)\end{array}$ \\
\hline \multicolumn{9}{|c|}{ neue Blätter } \\
\hline S1 & $2.15( \pm 0.36)$ & $2.45( \pm 0.31)$ & $2.23( \pm 0.17)$ & $1.89( \pm 0.15)$ & $1.93( \pm 0.20)$ & $0.96( \pm 0.07)$ & $1.47( \pm 0.14)$ & 0.20 \\
\hline S2 & - & - & $3.89( \pm 1.07)$ & $2.06( \pm 0.29)$ & $2.34( \pm 0.19)$ & $1.97( \pm 0.41)$ & $1.46( \pm 0.15)$ & 0.31 \\
\hline S3 & $2.41( \pm 0.08)$ & $2.58( \pm 0.20)$ & $2.62( \pm 0.49)$ & $1.89( \pm 0.31)$ & $2.31( \pm 0.16)$ & $1.70( \pm 0.46)$ & $1.79( \pm 0.17)$ & 0.20 \\
\hline $\mathrm{S} 4$ & $1.96( \pm 0.23)$ & $2.07( \pm 0.09)$ & $1.87( \pm 0.13)$ & $2.21( \pm 0.21)$ & $2.05( \pm 0.41)$ & $1.35( \pm 0.11)$ & $1.55( \pm 0.09)$ & 0.51 \\
\hline S5 & $2.12( \pm 0.22)$ & $2.51( \pm 0.13)$ & $3.26( \pm 0.98)$ & $1.98( \pm 0.40)$ & $2.18( \pm 0.25)$ & $1.43( \pm 0.29)$ & $1.42( \pm 0.19)$ & 0.28 \\
\hline S6 & $2.60( \pm 0.67)$ & $2.33( \pm 0.23)$ & $2.73( \pm 0.45)$ & $2.57( \pm 0.18)$ & $2.56( \pm 0.43)$ & $1.31( \pm 0.25)$ & $1.41( \pm 0.25)$ & 0.42 \\
\hline $\begin{array}{c}\text { LSD } \\
(p<0.05)\end{array}$ & 0.36 & 0.22 & 0.28 & 0.91 & 0.24 & 0.30 & 0.16 & \\
\hline \multicolumn{9}{|c|}{ alte Blätter } \\
\hline S1 & $1.96( \pm 0.29)$ & $1.85( \pm 0.14)$ & $2.09( \pm 0.18)$ & $1.65( \pm 0.22)$ & $1.37( \pm 0.15)$ & $0.86( \pm 0.07)$ & $1.10( \pm 0.16)$ & 0.19 \\
\hline $\mathrm{S} 2$ & - & - & $2.12( \pm 0.04)$ & $1.81( \pm 0.16)$ & $1.57( \pm 0.14)$ & $1.11( \pm 0.11)$ & $1.12( \pm 0.11)$ & 0.25 \\
\hline $\mathrm{S} 3$ & $1.78( \pm 0.26)$ & $2.29( \pm 0.23)$ & $2.04( \pm 0.28)$ & $1.51( \pm 0.27)$ & $1.75( \pm 0.21)$ & $1.13( \pm 0.22)$ & $1.55( \pm 0.10)$ & 0.18 \\
\hline $\mathrm{S} 4$ & $1.97( \pm 0.17)$ & $1.83( \pm 0.16)$ & $2.01( \pm 0.21)$ & $1.82( \pm 0.12)$ & $1.67( \pm 0.19)$ & $1.24( \pm 0.12)$ & $1.27( \pm 0.16)$ & 0.38 \\
\hline S5 & $2.11( \pm 0.18)$ & $2.16( \pm 0.11)$ & $2.06( \pm 0.13)$ & $1.65( \pm 0.35)$ & $1.34( \pm 0.10)$ & $0.90( \pm 0.24)$ & $1.04( \pm 0.13)$ & 0.24 \\
\hline S6 & $1.90( \pm 0.06)$ & $1.98( \pm 0.08)$ & $2.16( \pm 0.19)$ & $1.66( \pm 0.29)$ & $1.60( \pm 0.12)$ & $1.11( \pm 0.06)$ & $1.14( \pm 0.19)$ & 0.39 \\
\hline $\begin{array}{c}\text { LSD } \\
(p<0.05)\end{array}$ & 0.33 & 0.17 & 0.21 & 0.93 & 0.21 & 0.20 & 0.16 & \\
\hline
\end{tabular}




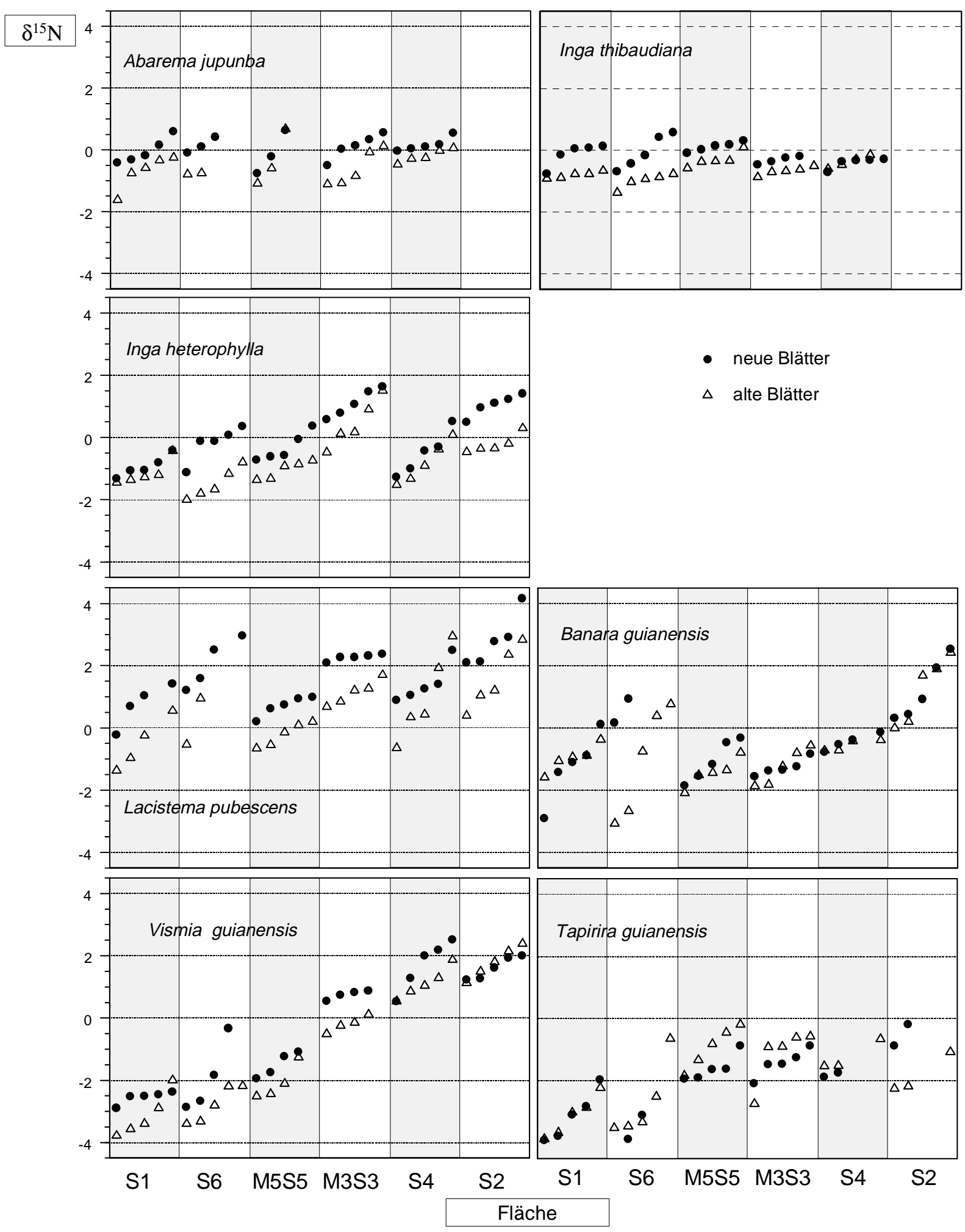

Abb. $42: \delta^{15} \mathrm{~N}$ - Einzelwerte der alten und neuen Blätter von 7 Pflanzenarten auf den Flächen S1 bis S6. (S1= R.d.Prata, S2= Jambu Açu, S3= Trav.12, S4= Cumarú, S5= Curí, S6= Iracema) 
Tab. 65: Zweifaktorielle Varianzanalyse der $\delta^{15} \mathrm{~N}$-Werte und $\mathrm{N}-$ Gehalte in neuen Blättern von 7 Sekundärwaldarten auf 6 Flächen

\begin{tabular}{l|cc|cc|cc|cc}
\hline & \multicolumn{2}{|c|}{ FG } & \multicolumn{2}{c|}{ QS } & \multicolumn{2}{c|}{ MQS } & \multicolumn{2}{c}{ F-Wert } \\
& $\% \mathrm{~N}$ & $\delta^{15} \mathrm{~N}$ & $\% \mathrm{~N}$ & $\delta^{15} \mathrm{~N}$ & $\% \mathrm{~N}$ & $\delta^{15} \mathrm{~N}$ & $\% \mathrm{~N}$ & $\delta^{15} \mathrm{~N}$ \\
Hauptwirkungen: & & & & & & & & \\
Fläche & 5 & 5 & 6.06 & 101.5 & 1.21 & 30.5 & $99.37 * * *$ & $9.3^{* * *}$ \\
Art & 6 & 6 & 36.3 & 183.3 & 6.05 & 20.3 & $66.07 * * *$ & $46.4^{* * *}$ \\
Wechselwirkungen: & & & & & & & & \\
Fläche x Art & 28 & 28 & 14.2 & 65.2 & 0.51 & 2.3 & $7.57^{* * *}$ & $3.9^{* * *}$ \\
\hline Fehler & 188 & 42.4 & 17.9 & 138 & 0.13 & 0.31 & & \\
Korr.Total & 177 & 392.4 & 74.5 & 177 & & & & \\
\hline
\end{tabular}

Tab. 66: $\delta^{15} \mathrm{~N}$-Werte und N-Gehalte der Pflanzen in der näheren Umgebung von Peixe Boi $\left({ }^{*}\right.$ wo Wiederholungen vorhanden, wurde der Mittelwert eingesetzt)

\begin{tabular}{|c|c|c|c|c|c|c|}
\hline \multirow[t]{2}{*}{ Art } & \multirow[t]{2}{*}{ Familie } & \multirow[t]{2}{*}{ Abk. } & \multicolumn{2}{|c|}{ Primärwald } & \multicolumn{2}{|c|}{ Sekundärwald } \\
\hline & & & $\% \mathrm{~N}$ & $\delta^{15} \mathrm{~N}$ & $\% \mathrm{~N}$ & $\delta^{15} \mathrm{~N}$ \\
\hline$?$ & Melastomataceae & Mo & & & 2.40 & 5.39 \\
\hline Bagassa guianensis & Moraceae & Bag & & & 3.64 & 6.53 \\
\hline Banara guianensis & Flacourtiaceae & $\mathrm{Bg}$ & & & 3.46 & -0.48 \\
\hline Cordia exaltata & Boraginaceae & $\mathrm{Ce}$ & 3.51 & 5.08 & 2.22 & 0.20 \\
\hline Dipteryx odorata & Papilionoideae & Do & & & 1.91 & 4.15 \\
\hline Inga rubiginosa & Mimosoideae & Ir & 2.73 & 7.61 & & \\
\hline Inga thibaudiana & Mimosoideae & It & 3.21 & $5.35^{*}$ & 2.92 & $2.79 *$ \\
\hline Lacistema pubescens & Lacistemataceae & $\mathrm{Lp}$ & & & 2.59 & 2.60 \\
\hline Lac. pubescens - Keimling & Lacistemataceae & & & & 2.18 & 0.93 \\
\hline Lecythis lurida & Lecythidaceae & $\mathrm{Ll}$ & & & 1.81 & 3.54 \\
\hline Memora allamandiflora & Bignoniaceae & $\mathrm{Ma}$ & 4.35 & 2.58 & 5.78 & 2.88 \\
\hline Myrcia sylvatica & Myrtaceae & Ms & 1.49 & $4.61 *$ & & \\
\hline Neea oppositifolia & Nyctaginaceae & No & 1.70 & 5.01 & 5.69 & 6.79 \\
\hline Selaginella stellaria & Selaginellaceae & Ss & & & 2.82 & 5.06 \\
\hline Sterculia pruriens & Sterculiaceae & $\mathrm{Sp}$ & & & 1.62 & 4.42 \\
\hline Tapirira guianensis & Anacardiaceae & $\mathrm{Tg}$ & 1.69 & $5.02 *$ & 1.59 & $-0.64 *$ \\
\hline Vismia guianensis & Guttiferae & $\mathrm{Vg}$ & & & 3.06 & 2.32 \\
\hline
\end{tabular}


Tab. 67: Zweifaktorielle ANOVA der $\delta^{15} \mathrm{~N}$-Werte und N-Gehalte in den Blättern von zwei Positionen am Baum (die ersten 3 Blätter vom Stamm aus und von der Astspitze aus gesehen) von 8 Arten, bei der die wiederholten Beprobungen an denselben Individuen von 7 Monaten (Oktober 1992 bis Februar 1993, April 1993 und Juli 1993) als Meßwiederholungen eingeht.

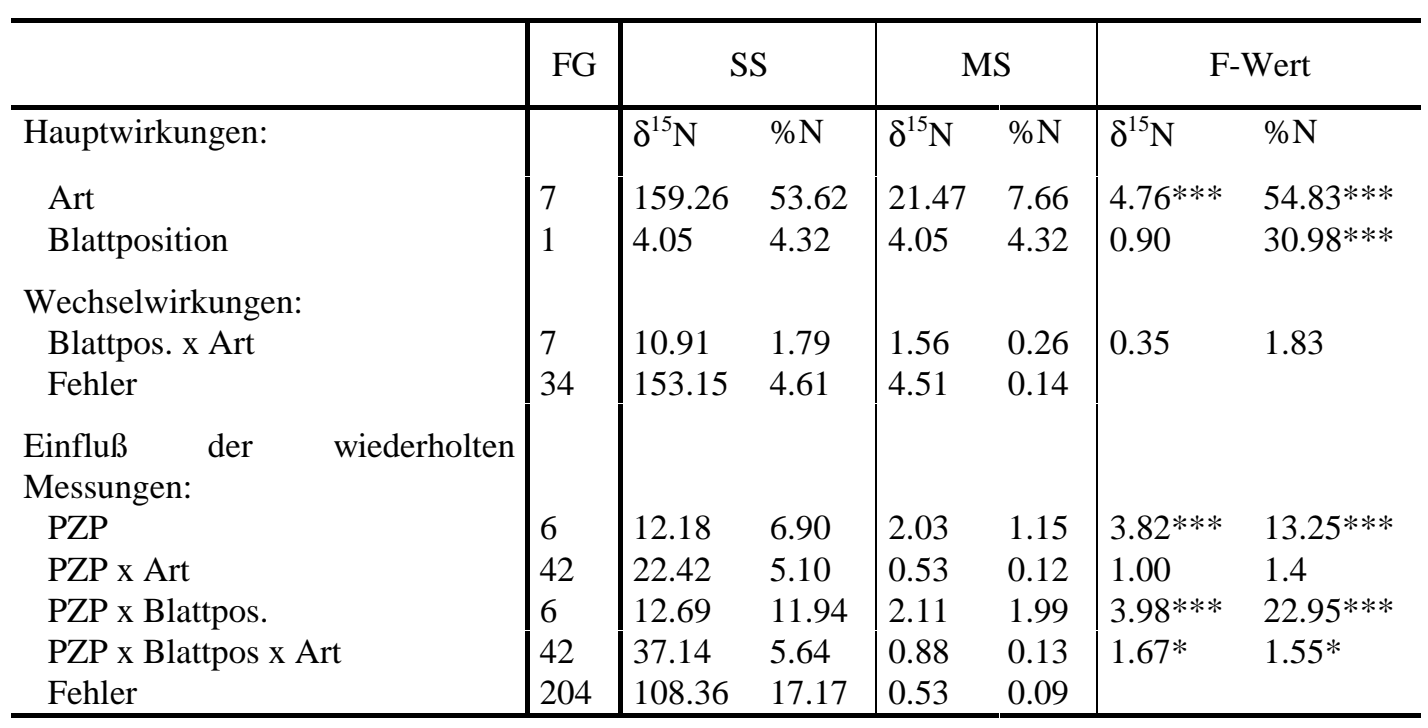

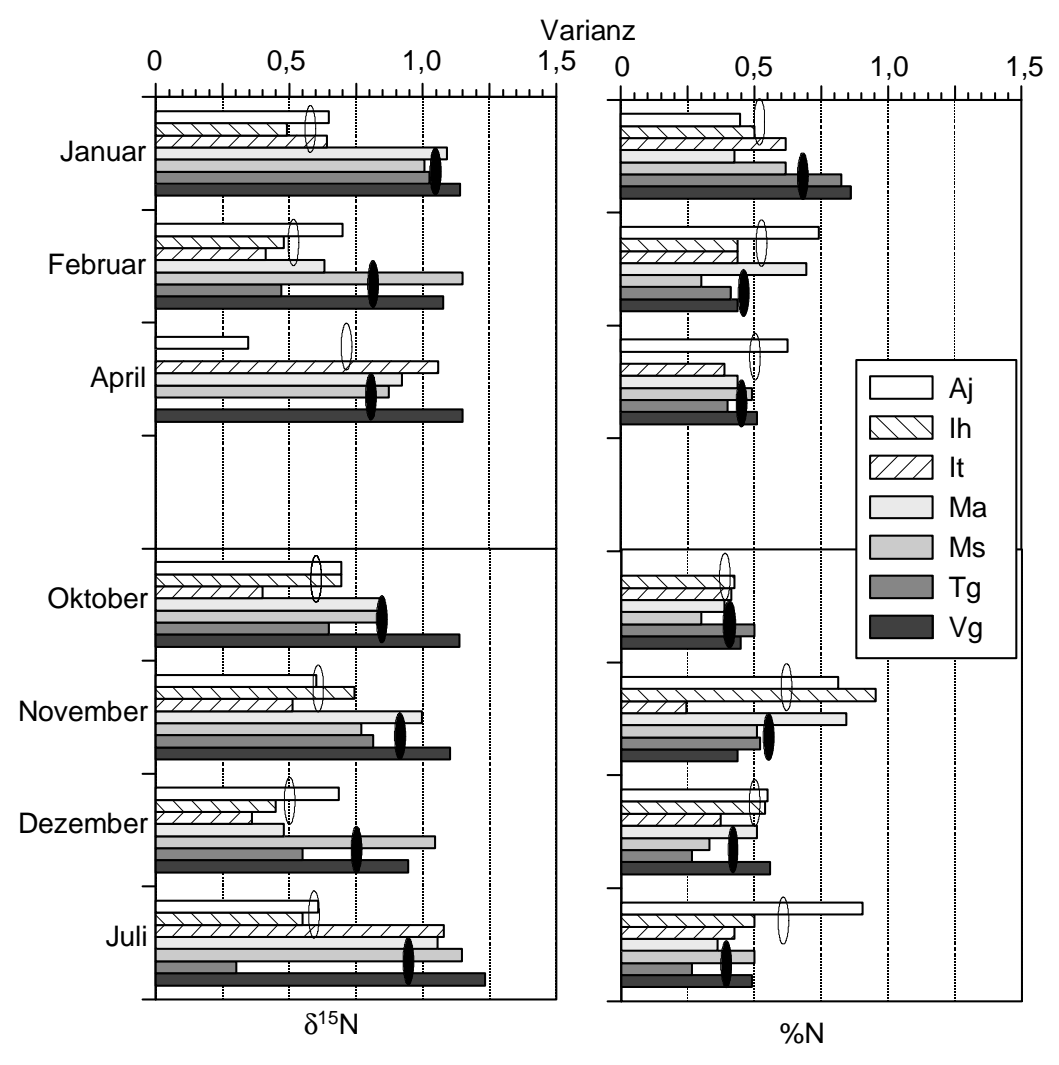

Abb. 43: Varianz der $\delta^{15} \mathrm{~N}-$ Werte und $\mathrm{N}$-Gehalte in Abhängigkeit von den einzelnen Arten und Probezeitpunkten $(\mathrm{Aj}=$ Abarema jupunba, $\mathrm{Ih}=$ Inga heterophylla, $\mathrm{It}=$ Inga thibaudiana, $\mathrm{Ma}=$ Mabea angustifolia, $\mathrm{Ms}=$ Myrcia sylvatica, $\mathrm{Tg}=$ Tapirira guianensis, $\mathrm{Vg}=$ Vismia guianensis) 
Tab. 68: $\delta^{15} \mathrm{~N}$-Werte und mg akkumulierter Stickstoff von Abarema cochleatum, Abarema jupunba und Tapirira guianensis nach 10- bzw. 12-monatigem Wachstum auf Sand mit und ohne $\mathrm{NO}_{3}{ }^{-}$sowie auf Feldboden ( $\mathrm{n}<=3$; für den $\delta^{15} \mathrm{~N}$-Wert der Gesamtpflanzen ist die Standardabweichung angegeben)

\begin{tabular}{|c|c|c|c|c|c|c|}
\hline \multirow[b]{2}{*}{ Art } & \multicolumn{2}{|c|}{ Sandkultur ohne $\mathrm{NO}_{3}^{-}$} & \multicolumn{2}{|c|}{ Sandkultur mit $\mathrm{NO}_{3}^{-}$} & \multicolumn{2}{|c|}{ Feldboden } \\
\hline & $\begin{array}{l}\text { Abarema } \\
\text { jupunba }\end{array}$ & $\begin{array}{c}\text { Abarema } \\
\text { cochleatum }\end{array}$ & $\begin{array}{l}\text { Abarema } \\
\text { jupunba }\end{array}$ & $\begin{array}{c}\text { Tapirira } \\
\text { guianensis }\end{array}$ & $\begin{array}{l}\text { Abarema } \\
\text { jupunba }\end{array}$ & $\begin{array}{l}\text { Tapirira } \\
\text { guianensis }\end{array}$ \\
\hline \multicolumn{7}{|c|}{$\delta^{15} \mathrm{~N}$} \\
\hline neue Blätter & +0.36 & +0.47 & -0.53 & -1.30 & +8.51 & +10.36 \\
\hline alte Blätter & +0.24 & -0.09 & +0.77 & -2.27 & +7.89 & +8.81 \\
\hline Streu & +0.13 & - & +0.49 & -0.27 & +7.39 & +7.35 \\
\hline Holz & -1.94 & -1.49 & -2.79 & -3.45 & +6.79 & +6.81 \\
\hline Rinde & -1.25 & -1.04 & -2.18 & -3.07 & +6.99 & +8.71 \\
\hline Wurzel & -0.19 & +0.19 & -1.74 & -0.59 & +3.66 & +6.34 \\
\hline Knöllchen & +5.09 & +4.62 & +3.45 & - & +4.27 & - \\
\hline Gesamt- $\delta^{15} N$ & $-0.16 \pm 0.18$ & $-0.04 \pm 0.07$ & $+0.18 \pm 0.75$ & $-1.37 \pm 0.58$ & $+5.71 \pm 1.17$ & $+8.24 \pm 0.47$ \\
\hline \multicolumn{7}{|c|}{ mg akkumulierter Stickstoff } \\
\hline neue Blätter & 16.8 & 33.9 & 73.5 & 59.7 & 50.7 & 78.8 \\
\hline alte Blätter & 168.1 & 184.8 & 714.7 & 55.3 & 120.9 & 103.1 \\
\hline Streu & 22.6 & - & 3.8 & 7.4 & 12.4 & 21.0 \\
\hline Holz & 27.3 & 29 & 21.8 & 12.9 & 24.4 & 12.9 \\
\hline Rinde & 62.3 & 66.3 & 35.0 & 53.2 & 56.6 & 28.4 \\
\hline Wurzel & 207.1 & 205.7 & 145.4 & 141.6 & 258.6 & 70.6 \\
\hline Knöllchen & 45.3 & 67 & 12.9 & - & 0.4 & - \\
\hline Gesamt-N & $549.7 \pm 113$ & $586.7 \pm 50$ & $1007.1 \pm 556$ & $330.1 \pm 19$ & $524.0 \pm 44$ & $314.8 \pm 1$ \\
\hline
\end{tabular}

Tab. 69: Infektionspotential der nativen Rhizobiumpopulation eines Capoeirabodens - Ergebnisse des paarweisen Wilcoxon-Rang-Testes $(\mathrm{n}=10)$ :

\begin{tabular}{|c|c|c|c|c|c|c|c|c|c|c|c|c|}
\hline & $\begin{array}{l}\text { bis } \\
50 \mathrm{~cm}\end{array}$ & $\begin{array}{l}\text { bis } \\
100 \mathrm{~m}\end{array}$ & $\begin{array}{l}\text { bis } \\
150 \mathrm{~cm}\end{array}$ & $\begin{array}{l}\text { bis } \\
200 \mathrm{~cm}\end{array}$ & $\begin{array}{l}\text { bis } \\
250 \mathrm{~cm}\end{array}$ & $\begin{array}{l}\text { bis } \\
300 \mathrm{~cm}\end{array}$ & $\begin{array}{l}\text { bis } \\
350 \mathrm{~cm}\end{array}$ & $\begin{array}{l}\text { bis } \\
400 \mathrm{~cm}\end{array}$ & $\begin{array}{l}\text { bis } \\
450 \mathrm{~cm}\end{array}$ & $\begin{array}{l}\text { bis } \\
500 \mathrm{~cm}\end{array}$ & $\begin{array}{l}\text { bis } \\
550 \mathrm{~cm}\end{array}$ & $\begin{array}{l}\text { bis } \\
600 \mathrm{~cm}\end{array}$ \\
\hline bis $50 \mathrm{~cm}$ & & $*$ & ns & $* *$ & $* *$ & $* *$ & $* *$ & $* * *$ & $* *$ & $* *$ & $* * *$ & $* *$ \\
\hline bis $100 \mathrm{~m}$ & & & ns & $*$ & $*$ & $*$ & $*$ & $*$ & * & $*$ & $* *$ & $* *$ \\
\hline bis $150 \mathrm{~cm}$ & & & & ns & ns & ns & $* *$ & $* * *$ & $*$ & $*$ & $* *$ & ns \\
\hline bis $200 \mathrm{~cm}$ & & & & & ns & ns & $*$ & $* * *$ & ns & ns & $*$ & ns \\
\hline bis $250 \mathrm{~cm}$ & & & & & & ns & $*$ & ns & $* *$ & ns & ns & ns \\
\hline bis $300 \mathrm{~cm}$ & & & & & & & ns & $* *$ & ns & ns & ns & ns \\
\hline bis $350 \mathrm{~cm}$ & & & & & & & & ns & ns & ns & ns & ns \\
\hline bis $400 \mathrm{~cm}$ & & & & & & & & & $*$ & ns & ns & ns \\
\hline bis $450 \mathrm{~cm}$ & & & & & & & & & & ns & ns & ns \\
\hline bis $500 \mathrm{~cm}$ & & & & & & & & & & & ns & ns \\
\hline $\begin{array}{l}\text { bis } 550 \mathrm{~cm} \\
\text { bis } 600 \mathrm{~cm}\end{array}$ & & & & & & & & & & & & ns \\
\hline
\end{tabular}


Tab. 70: Basalflächen $\left(\pi^{*} \mathrm{r}^{2}\right)$, Gesamt-Biomasse, -N-Reserven, $-\delta^{15} \mathrm{~N}$-Werte und $-\mathrm{N}_{2}$-Fixierungsleistung der 9 Pflanzen von Abarema jupunba, die für die Regressionsanalysen verwendet wurden

\begin{tabular}{c|cccccc}
\hline Wdh. & $\mathrm{g} \mathrm{N}$ & $\mathrm{kg}_{\text {Biom }}$ & $\mathrm{pi}^{*} \mathrm{r}^{2}\left(\mathrm{~cm}^{2}\right)$ & $\delta^{15} \mathrm{~N}_{\text {Leg }}$ & $\delta^{15} \mathrm{~N}_{\text {Ref }}$ & $\%$ NdfA \\
& & & & & & \\
\hline 1 & 0.31 & 0.016 & 0.24 & 2.30 & 1.24 & 0 \\
2 & 1.67 & 0.072 & 0.80 & 2.97 & 1.69 & 0 \\
3 & 2.09 & 0.146 & 2.66 & 4.50 & 2.31 & 0 \\
4 & 2.16 & 0.301 & 13.58 & -0.58 & -0.93 & 40 \\
5 & 1.75 & 0.223 & 19.31 & -0.15 & -1.36 & 93 \\
6 & 3.63 & 0.630 & 27.33 & 0.05 & -1.33 & 110 \\
7 & 3.32 & 0.453 & 32.91 & -0.11 & -1.39 & 97 \\
8 & 3.33 & 0.605 & 36.03 & -0.28 & -1.47 & 85 \\
9 & 4.59 & 1.132 & 44.16 & -0.13 & -1.32 & 95 \\
\hline
\end{tabular}

Tab. 71: Zugrunde gelegte Daten von Saldarriaga et al. (1988) und ihre Umrechnung für den N-Input einer Sukzession von 10jähriger Sekundärvegetation bis zum Primärwald der Rio Negro Region in Columbien und Venezuela. unter der Vorraussetzung, daß 27\% der Biomasse der Vegetation aus Leguminosen bestehen (nach Denich, 1989 und Vieira, 1996)

\begin{tabular}{|c|c|c|c|c|c|c|c|c|c|c|c|}
\hline \multirow[b]{3}{*}{$\begin{array}{c}\text { Alter } \\
\text { (Jahre) }\end{array}$} & \multirow{2}{*}{\multicolumn{2}{|c|}{$\begin{array}{c}\text { Originaldaten } \\
\text { Stämme/0.03ha in } \\
\text { den Größenklassen: }\end{array}$}} & \multicolumn{9}{|c|}{ artifizielle Aufteilung in Anzahl Leguminosen pro 1 ha } \\
\hline & & & \multicolumn{3}{|c|}{$\mathrm{r}<5 \mathrm{~cm}$ nach Nunez (1995) } & \multicolumn{6}{|c|}{$\mathrm{r}>5 \mathrm{~cm}$ nach Vieira (1996) } \\
\hline & $\mathrm{r}<5 \mathrm{~cm}$ & $\mathrm{r}>5 \mathrm{~cm}$ & $\mathrm{r}<1$ & $\mathrm{r} \approx 2$ & $\mathrm{r} \approx 4$ & $\mathrm{r} \approx 7.5$ & $r \approx 10$ & $\mathrm{r} \approx 12.5$ & $\mathrm{r} \approx 15$ & $\mathrm{r} \approx 17.5$ & $>20$ \\
\hline 9 & 621 & 21 & 1508 & 3350 & 726 & 142 & 42 & 6 & 0 & 0 & 0 \\
\hline 11 & 458 & 17 & 1112 & 2471 & 535 & 115 & 34 & 5 & 0 & 0 & 0 \\
\hline 12 & 485 & 58 & 1177 & 2616 & 567 & 391 & 115 & 16 & 0 & 0 & 0 \\
\hline 14 & 472 & 27 & 1146 & 2546 & 552 & 182 & 53 & 7 & 0 & 0 & 0 \\
\hline 20 & 649 & 54 & 1575 & 3501 & 759 & 272 & 184 & 29 & 0 & 0 & 0 \\
\hline 20 & 953 & 25 & 2313 & 5141 & 1114 & 126 & 85 & 13 & 0 & 0 & 0 \\
\hline 20 & 498 & 38 & 1209 & 2687 & 582 & 191 & 130 & 20 & 0 & 0 & 0 \\
\hline 20 & 458 & 49 & 1112 & 2471 & 535 & 247 & 167 & 26 & 0 & 0 & 0 \\
\hline 30 & 629 & 29 & 1527 & 3393 & 735 & 175 & 78 & 8 & 0 & 0 & 0 \\
\hline 35 & 389 & 49 & 944 & 2098 & 455 & 295 & 132 & 13 & 0 & 0 & 0 \\
\hline 35 & 450 & 53 & 1092 & 2428 & 526 & 319 & 143 & 14 & 0 & 0 & 0 \\
\hline 40 & 487 & 63 & 1182 & 2627 & 569 & 380 & 170 & 17 & 0 & 0 & 0 \\
\hline 60 & 221 & 32 & 536 & 1192 & 258 & 155 & 83 & 26 & 6 & 3 & 14 \\
\hline 60 & 285 & 43 & 692 & 1537 & 333 & 209 & 112 & 35 & 8 & 4 & 19 \\
\hline 60 & 267 & 44 & 648 & 1440 & 312 & 214 & 115 & 36 & 8 & 4 & 20 \\
\hline 80 & 323 & 54 & 784 & 1742 & 378 & 194 & 136 & 78 & 19 & 15 & 44 \\
\hline 80 & 471 & 74 & 1143 & 2541 & 551 & 266 & 186 & 106 & 27 & 20 & 60 \\
\hline 80 & 287 & 58 & 697 & 1548 & 335 & 209 & 146 & 83 & 21 & 16 & 47 \\
\hline 80 & 354 & 50 & 859 & 1910 & 414 & 180 & 126 & 72 & 18 & 13 & 40 \\
\hline Prim.w. & 272 & 51 & 660 & 1467 & 318 & 183 & 128 & 73 & 18 & 14 & 41 \\
\hline Prim.w. & 394 & 55 & 956 & 2125 & 461 & 198 & 138 & 79 & 20 & 15 & 45 \\
\hline Prim.w. & 478 & 51 & 1160 & 2579 & 559 & 183 & 128 & 73 & 18 & 14 & 41 \\
\hline Prim.w. & 264 & 48 & 641 & 1424 & 309 & 173 & 121 & 69 & 17 & 13 & 39 \\
\hline
\end{tabular}




\section{Danksagung}

Diese Arbeit wurde durch das Bundesministerium für Bildung, Wissenschaft, Forschung und Technologie im Rahmen des Projektes „Sekundärwald und Brachevegetation in der Kulturlandschaft des östlichen Amazonasgebietes - Funktion und Manipulierbarkeit" (Fördernr. 39389A) gefördert.

Herrn Prof. Dr. P.L.G. Vlek danke ich für die freundliche Überlassung des Themas, seine Hilfestellung und Beratung bei den verschiedensten Fragestellungen und sein Verständnis auch für die finanziellen und zeitlichen Engpässe einer Doktorandin.

Mein besonderer Dank gilt Dr. Manfred Denich für die geduldige Betreuung dieser Arbeit, die durch seine Diskussionsbereitschaft, sein fachliches know how und - zu guter letzt - dem leidigen Korrekturlesen viel gewonnen hat. Für wertvolle Hinweise bei der statistischen Auswertung bedanke ich mich bei Dr. Ronald Kühne.

Allen Mitarbeitern des Institutes für tropischen Pflanzenbau, die zum Gelingen dieser Arbeit beigetragen haben, möchte ich meinen Dank aussprechen. Derselbe Dank gilt meinen Helfern João, Messias, Adna, Vânia und Socorro in Igarapé Açu und Belém.

Die ${ }^{15} \mathrm{~N}$-Analysen wurden vom Isotopenlaboratorium für Biologische und Medizinische Forschung in Göttingen durchgeführt. Für die tolle Zusammenarbeit und Beratung in den Isotopenfragen möchte ich mich daher ganz herzlich bei Rainer Langel und Dr. August Reineking bedanken.

Professorin M.M. de Lourdes Silva Santos danke ich für die Überlassung ihres mikrobiologischen Labors und des Gewächshausplatzes für meine Studien.

Herzlichen Dank gilt meinen Freunden, die mir Rückhalt gaben und zu guter Letzt das Korrekturlesen übernahmen.

Mein besonderer Dank gilt meinem Mann, der mir immer vorbehaltlos zur Seite stand, Korrektur gelesen hat, mir mit Rat und Tat die Computer-technischen Details erleichtert und mich bei unserem Sohn vertreten hat.

Für seine liebevolle Anteilnahme und die Geduld, die er mit einer oft übelgelaunten Mutter haben mußte, möchte ich mich bei meinem Sohn bedanken. Für wen sonst als für unsere Kinder lohnt sich der Aufwand so einer Arbeit? 


\section{Lebenslauf}

\section{Persönliche Daten}

Name:

Geburtsdatum:

Geburtsort:

Familienstand:

Staatsangehörigkeit:

\section{Schulausbildung}

1968 - 1972

1972 - 1981

16.06.1981

\section{Studium}

$1981-1982$

$1982-1984$

17.10.1984

$1984-1988$

Juni 1986 - Dezember 1987

28.07.1987

18.12.1987

10.11.1988

WS $88 / 89$ - WS 89/90
Antje Thielen-Klinge

23.01.1962

Gütersloh

verheiratet, ein Kind

deutsch
Besuch der Grundschule Eichendorff in Gütersloh

Besuch des städtischen Gymnasiums in Gütersloh

Abitur

Studuim der Biologie und Textiles Gestalten für Lehramt Sek. I an der WWU Münster, abgebrochen

Gundstudium der Biologie an der WWU Münster

Diplom-Biologen-Vorprüfung

Diplomstudium der Biologie an der Universität Göttingen

Diplomarbeit $\mathrm{zu}$ dem Thema „Untersuchungen $\mathrm{zu}$ suppressiven Böden bei bodenbürtigen Erregern von Fußkrankheiten an Weizen und Gerste" am Institut für Pflanzenpathologie und Pflanzenschutz, Abt. Mykologie von Prof. Dr. H. Fehrmann, Göttingen

Geburt des Sohnes Jakob

Abgabe der Diplomarbeit

Diplomprüfung

Belegung von Seminaren des Aufbaustudiums „Ökologische Umweltsicherung“ in Witzenhausen (GH Kassel) 
Febr. 1989 - Sept. 1991

Januar 1992 - März 1994

April 1994 - Mai 1997
Wissenschaftliche Mitarbeiterin am Institut für Pflanzenpathologie und Pflanzenschutz, Abt. Physiologie von Professor Dr. G. Wolf, Göttingen.

* Entwicklung eines Screeningverfahrens zur Erfassung keimungsfördernder Substanzen (Suppline) von Bakterien an Zuckerrübe, Getreide und Phaseolus-Bohne, in Zusammenarbeit mit der Kleinwanzlebener Saatzucht $\mathrm{AG})$

* Biologische Kontrolle von Pflanzenkrankheiten: Untersuchung zu Pathotypen von Pseudocercosporella herpotrichoides

Aufenthalt in Belém, Pará, Brasilien, zur Datenerhebung für die Promotion zum Thema „Role of leguminous trees for the fallow vegetation of Amazônia, Brazil - application of the ${ }^{15} \mathrm{~N}$ natural abundance method"“

Auswertung und Fertigstellung der Promotion

Göttingen, den 25. März 1997 